\title{
Biodiversität und Waldvermehrung
}

\section{Ein Verfahren zur naturschutzfachlichen Evaluation von Erstaufforstungen}

\author{
Dissertation \\ zur Erlangung des Doktorgrades \\ der Fakultät für Forstwissenschaften und Waldökologie \\ der Georg-August-Universität Göttingen
}

vorgelegt von

Ralf Eisenbeiß

geboren in Kehl am Rhein

Göttingen 2002 


\section{7}

1. Berichterstatter:

2. Berichterstatter:
Prof. Dr. Renate Bürger-Arndt

Prof. Dr. Achim Dohrenbusch

Tag der mündlichen Prüfung: $\quad$ 07. Oktober 2002

Diese Arbeit wurde durch die Niedersächsische Staats- und Universitätsbibliothek, Göttingen unter folgender Internetadresse veröffentlicht:

http//webdoc.sub.gwdg.de/diss/eisenbeiss/index.html 

Für Silke und Bendiks

\section{Danksagung}

Ich möchte mich bei all denen Bedanken, die mich in der Schaffensphase zu dieser Arbeit tatkräftig unterstützt haben. Allen voran meine Frau Silke, deren liebevoller Rückhalt mir sehr viel bedeutet, und meiner Mutter für den aufmunternden Zuspruch aus der Ferne.

Außerdem bedanke ich mich bei Frau Prof. Bürger-Arndt für den organisatorischen Freiraum und die gute Kooperation während meiner Tätigkeit am Institut.

Die Erstellung der Arbeit wäre natürlich kaum möglich gewesen, ohne die Kooperationsbereitschaft diverser Behörden und Personen aus dem Bereich Forstwirtschaft und Naturschutz. Besonderer Dank gebührt den Herren Schwerhoff und Stiefel von der Thüringer Landesanstalt für Wald und Forstwirtschaft, Gotha für die Bereitstellung von Daten und Luftbildern sowie den Umweltämtern der Kreise Weimarer Land und Northeim für die Bereitstellung von Karten und Informationen. Erwartungsgemäß große Hilfe erfuhr ich auch von den Akteuren des Naturschutzes im Bereich Forst und Ornithologie, insbesondere den Forstämtern Bad Berka (Thüringen) und Dassel (Niedersachsen) sowie der Nabu Ortsgruppe Dassel-Einbeck. Ich hoffe, ihnen allen mit dieser Arbeit nicht nur meinen Dank ausdrücken, sondern auch ein paar interessante Impulse für die weitere Arbeit geben zu können. 


\section{Inhalt}

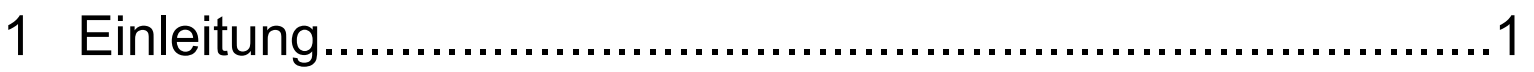

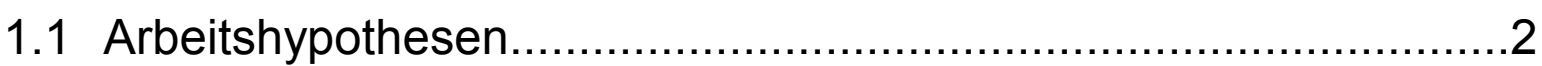

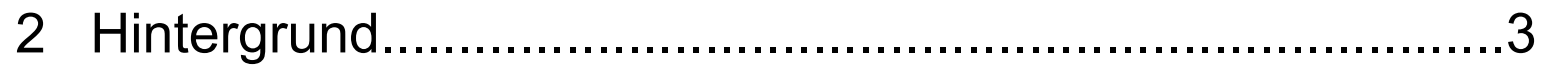

2.1 Erstaufforstung in der Diskussion..............................................

2.2 Naturschutzfachliche Bewertung zwischen Soll und Ist.................

2.3 Regionales Konzept als Grundlage für Einzelfallentscheidungen..12

3 Theoretischer Rahmen und Methodik...............................17

3.1 Planungsmethoden für den Naturschutz...................................17

3.2 Leitbilder des Arten- und Biotopschutzes.....................................21

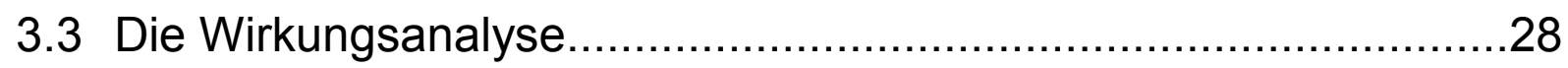

3.3.1 Waldbauliche Prämissen..................................................................39

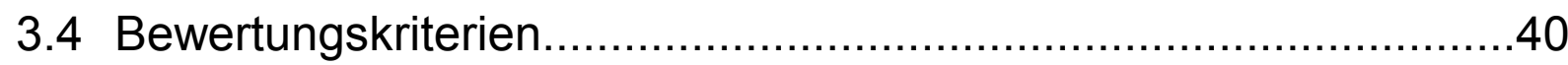

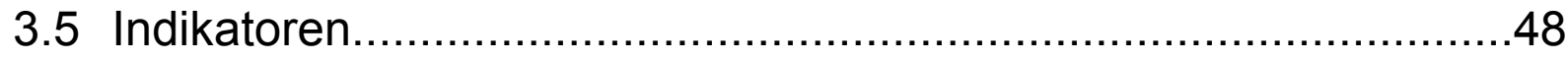

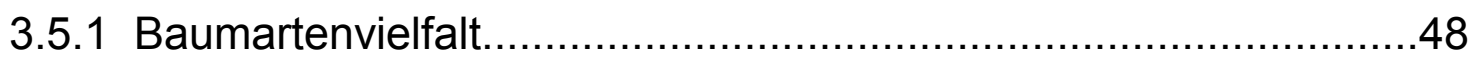

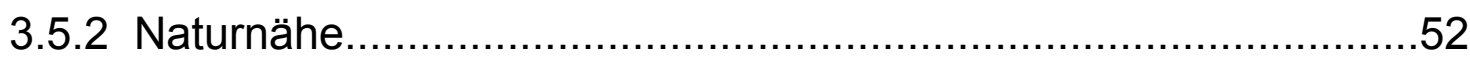

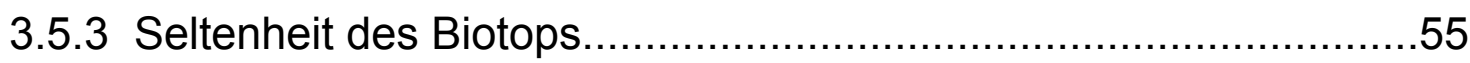

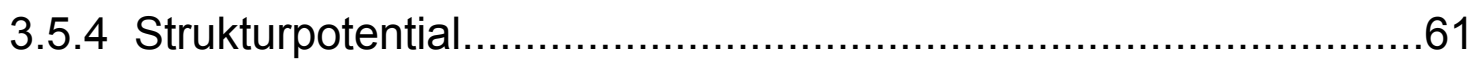

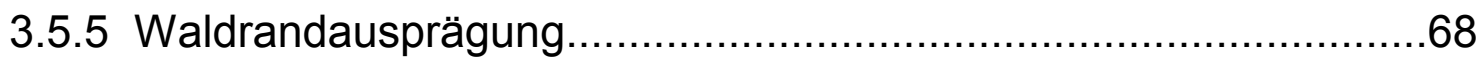

3.5.6 Biotopverbund.........................................................................

3.5.7 Landnutzungsverhältnis............................................................

3.5.8 Randlinien-Vorkommen............................................................77 
4 Ergebnisse im USG 1 - Region Apolda

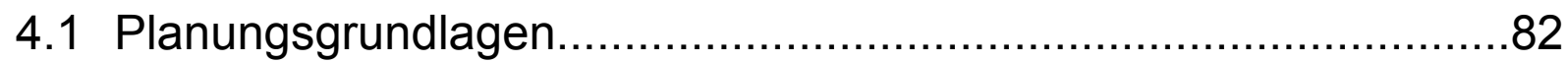

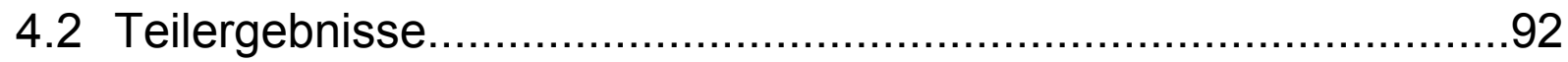

4.2.1 Flächenspezifische Ergebnisse - Aufforstungsvarianten und

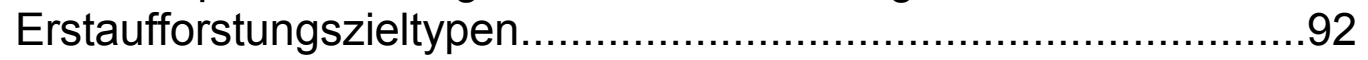

4.2.2 Flächenspezifische Ergebnisse - Raumbezug..........................96

4.3 Gesamtergebnisse für die Untersuchungsflächen in der Region

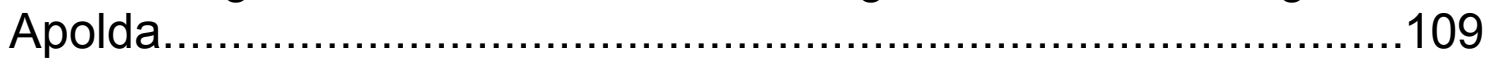

5 Ergebnisse im USG 2 - Region Dassel........................113

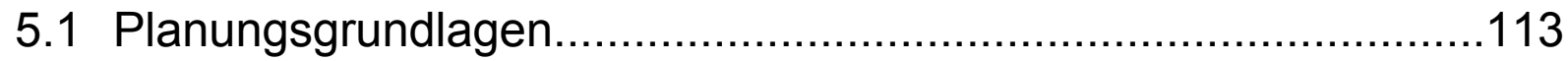

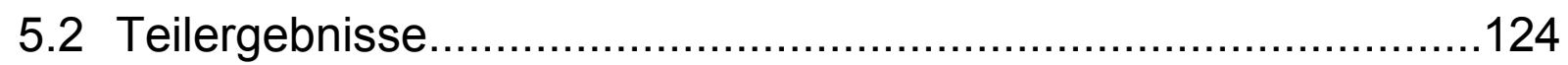

5.2.1 Flächenspezifische Ergebnisse - Aufforstungsvarianten und Waldentwicklungstypen........................................................ 124

5.2.2 Flächenspezifische Ergebnisse - Raumbezug.........................128

5.3 Gesamtergebnisse für die Untersuchungsflächen in der Region

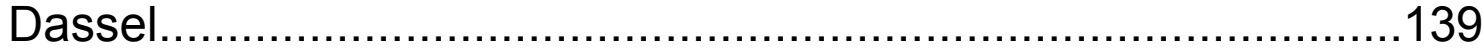

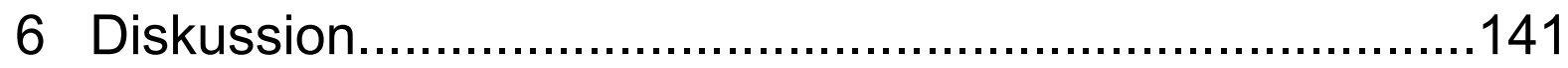

6.1 Instrumentalisierung der naturschutzfachlichen Bewertung.........141

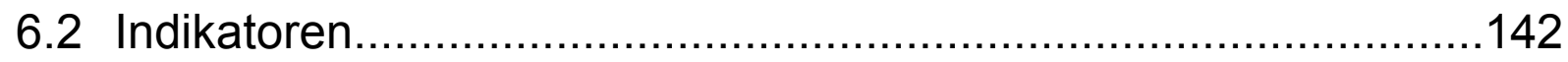

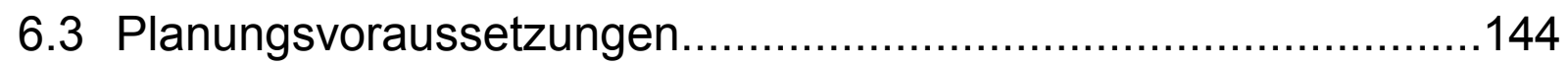

6.4 Transferierbarkeit des Verfahrens..........................................146

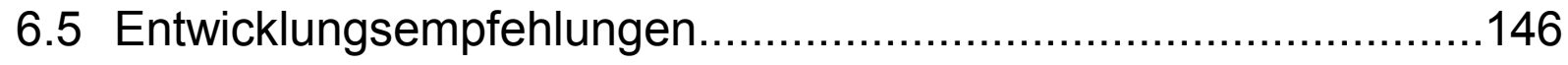

7 Zusammenfassung............................................. 148

8 Literaturverzeichnis............................................150

9 Anhang......................................................... 157 


\section{Tabellenverzeichnis}

2.1-1 Kostenzuschuss bei der Erstaufforstung, gestaffelt nach Baumarten, nach der Richtlinie des Sächsischen Staatsministeriums für Umwelt und Landwirtschaft zur Förderung der ökologischen Waldmehrung im Freistaat Sachsen (RL-Nr. 93/2000)

3.1-1 Die Beiträge des Naturschutzes zur Landschaftsplanung auf verschiedenen Ebenen 18

3.3-1 Verflechtungsmatrix - Elemente der 1. Systemebene (Aufforstungsfläche) 33

$\begin{array}{ll}\text { 3.3-2 } & \text { Verflechtungsmatrix - Elemente der 2. und 3. Systemebene } \\ \text { (Ökologisches Umfeld und erweiterter Planungsraum) } & 36\end{array}$

3.4-1 Synoptischer Überblick zu Bewertungskriterien im Naturschutz - nach
unterschiedlichen Autoren

3.5-1 Vergleich der geplanten Baumartenmischung mit der potentiell natürlichen des
angesprochenen Standortes; Auszug aus dem Erhebungsbogen

3.5-2 Synopse der festgestellten Biotoptypen in den Kartierungsverfahren Thüringen sowie der Biotoptypen der Roten Liste der gefährdeten Biotoptypen der Bundesrepublik Deutschland

3.5-3 Auswirkung der Mischung von Baumarten unterschiedlicher sukzessionaler Stellung (nach OTTO 1994)

3.5-4 Strukturpotential der Baumarten nach artspezifischen Charakteristika 66

3.5-5 Checkliste zum Indikator Waldrandausprägung; Bewertung nach der Summe der festgestellten Merkmale bei Waldrandbegründung

4.1-1 Übersicht zu den vorkommenden Bodentypen im Untersuchungsgebiet und den zugehörigen natürlichenWaldgesellschaften

4.1-2 Flächennutzungsanteile in der Region Apolda / Niedertreba 86

4.1-3 Regionales Leitbild für das Innerthüringer Ackerhügelland 89

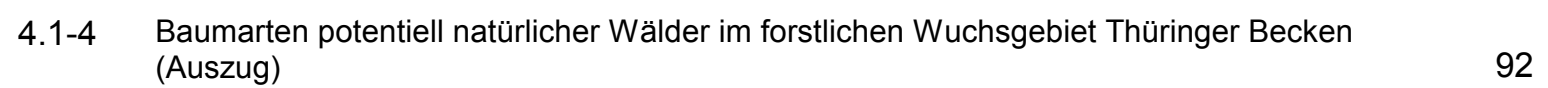

4.2-1 Aktuelle und angestrebte Flächennutzungsanteile in der Region Apolda / Niedertreba 97

4.2-2 Daten zum Bezugsraum Apolda-1 und zur Aufforstungsfläche AP-1 99

4.2-3 Daten zum Bezugsraum Apolda-2 und zur Aufforstungsfläche AP-2 101

4.2-4 Daten zum Beuzgsraum Apolda-3 und zur Aufforstungsfläche AP-3 103

4.2-5 Daten zum Bezugsraum Apolda-4 und zur Aufforstungsfläche AP-4 106

4.2-6 Daten zum Bezugsraum Apolda-5 und zur Aufforstungsfläche AP-5 108

5.1-1 Die potentiell natürliche Vegetation (PNV) im Untersuchungsraum Dassel und ihre
forstlichen Ersatzgesellschaften

$\begin{array}{ll}\text { 5.1-2 Die den Aufforstungsflächen zuzuordnenden natürlichen Waldgesellschaften mit Haupt- } & 116 \\ \text { und Nebenbaumarten }\end{array}$

$\begin{array}{ll}\text { 5.1-3 Landwirtschaftliche Nutzungsfläche in Relation zur Gesamtfläche der } & 117\end{array}$

5.2-1 Daten zum Bezugsraum Dassel-1 und zur Aufforstungsfläche DA-1 129

5.2-2 Daten zum Bezugsraum Dassel-2 und zur Aufforstungsfläche DA-2 131

5.2-3 Daten zum Bezugsraum Dassel-3 und zur Aufforstungsfläche DA-3 133

5.2-4 Daten zum Bezugsraum Dassel-4 und zur Aufforstungsfläche DA-4 135

5.2-5 Daten zum Bezugsraum Dassel-5 und zur Aufforstungsfläche DA-5 137 


\section{Abbildungsverzeichnis}

2.1-I Zusammensetzung der Erstaufforstungsförderung im Freistaat Thüringen (LAWUF 1999)

3.1-I Flächennutzungsevaluation als operationalisiertes Planungsinstrument auf der Grundlage einer ökologischen Wirkungsanalyse. verändert; nach BECHMANN (1977) verändert in BUCHWALD/ENGELHARDT (1980)

3.3-I Der Transformationsprozess in einem System 31

3.3-II Das Transformationsmodell der 1. Systemebene (Aufforstungsfläche) 35

3.3-III Das Transformationsmodell der 2. Systemebene (Ökologisches Umfeld) 36

$\begin{array}{lll}\text { 3.4-I Übersicht zu den Kriterien, Indikatoren, Leitbildern und Datenquellen des } & 46 \\ \text { Evaluationsverfahrens }\end{array}$

$\begin{array}{lll}\text { 3.5-I } & \text { Bewertungsschema des Indikators Baumartenvielfalt; Vergleich der realen Vielfalt mit } \\ \text { der potentiellen Vielfalt der natürlichen Waldgesellschaft } & 51\end{array}$

3.5-II Bewertungsstufen zum Indikator Baumartenvielfalt $\quad 52$

3.5-III Die Einordnung des potentiellen Waldbiotops in die Liste der gefährdeten und

3.5-IV Bewertungsstufen zu den Strukturpotential-Klassen im Indikator Struktur 67

3.5-V Feststellung der Biotopverbundwirkung und Habitatergänzung durch die Aufforstung; Auszug aus dem Erhebungsbogen

3.5-VI Die Nutzung des Waldrandes als Lebensraum durch Tierarten des Waldes, des Offenlandes und der Übergangsbereiche

3.5-VII Bewertung der Veränderung des Randlinienvorkommens unter Berücksichtigung der Randlinien-Dichte (Ifm/ha) und der effektiven Waldrandverlängerung; Auszug aus dem Erhebungsbogen

4.2-I Die Beziehung zwischen der Baumartenzahl und dem Indikatorwert Baumartenvielfalt 93

4.2-II Indikatorsummen in Abhängigkeit vom Pflanzplan (EAZT und AV für Apolda) 94

4.2-III Raumbezogene Indikatorenwerte für den Bezugsraum Apolda-1 100

4.2-IV Raumbezogene Indikatorenwerte für den Bezugsraum Apolda-2 102

4.2-V Raumbezogene Indikatorenwerte für den Bezugsraum Apolda-3 105

4.2-VI Raumbezogene Indikatorenwerte für den Bezugsraum Apolda-4 107

4.2-VII Raumbezogene Indikatorenwerte für den Bezugsraum Apolda-5 109

4.3-I Mindest- und Maximalpunktzahl auf verschiedenen Flächen (USG Apolda) 111

4.3-II Waldrandsummen mit und ohne Doppelbewertung der Süd- und West-Exposition 112

$\begin{array}{lll}\text { 5.2-I } & \text { Flächenbezogene Indikatorwerte für die PNV-optimierten Erstaufforstungsvarianten } & 125\end{array}$

5.2-II Flächenbezogene Indikatorwerte für die Erstaufforstung nach Waldentwicklungstypen (USG Dassel)

5.2-III Raumbezogene Indikatorenwerte für den Bezugsraum Dassel-1 130

5.2-IV Raumbezogene Indikatorenwerte für den Bezugsraum Dassel-2 132

5.2-V Raumbezogene Indikatorenwerte für den Bezugsraum Dassel-3

5.2-VI Raumbezogene Indikatorenwerte für den Bezugsraum Dassel-4 136

5.2-VII Raumbezogene Indikatorenwerte für den Bezugsraum Dassel-5 138

5.3-I Mindest- und Maximalpunktzahl auf verschiedenen Flächen (USG Dassel) 140 


\section{Einleitung}

Die Idee zu der vorliegenden Arbeit entstand im Rahmen eines internationalen Projektes, das vom Institut für Forstpolitik, Forstgeschichte und Naturschutz koordiniert wurde. Das Projekt befasste sich mit unterschiedlichen Fragestellungen zur Erstaufforstung in unterbewaldeten Regionen. In verschiedenen Landstrichen Europas ist die Aufforstung landwirtschaftlicher Flächen ein wichtiges Thema. Nicht zuletzt aufgrund der Bedeutung, die der Erstaufforstung als flankierende Maßnahme in der Gemeinsamen Agrarpolitik der E.U. zugedacht wird. Internationale Richtlinien und nationale Förderprogramme sollen die Neustrukturierung des landwirtschaftlich geprägten Raumes ordnen. Meist erfordert die Aufforstungsmaßnahme aber eine vorhergehende, interdisziplinär abgestimmte Genehmigung. Und obwohl sich die Fachdisziplinen generell einig sind, dass ein gewisser Prozentsatz der landwirtschaftlichen Nutzfläche in Wald umgewandelt werden soll - die Angaben reichen von etwa 1 bis 3 Prozent - herrschen am konkreten Einzelfall unterschiedliche Meinungen vor.

Verschiedene Gespräche mit den für die Aufforstungsgenehmigung zuständigen Behörden führten immer wieder zu den gleichen Schlussfolgerungen: die Forstwirtschaft propagiert Aufforstungen in den meisten Fällen. Nimmt die Landwirtschaft eine ablehnende Haltung ein, so erfolgt dies meist mit einem Hinweis auf die regionalen Strukturen und die ökonomischen Zwänge in der Bewirtschaftung. Spricht sich aber der Naturschutz gegen eine Aufforstung aus, so können unterschiedliche Gründe vorliegen. Häufig geht es um den Schutz einer besonderen, gefährdeten Art. Manchmal ist es das Landschaftsbild, manchmal auch ein angrenzendes Biotop, das vor negativen Auswirkungen der Aufforstung bewahrt werden soll. Insgesamt betrachtet, führt die wechselnde Argumentation dazu, dass die Entscheidung des Naturschutzes schwer kalkulierbar ist und so - vor allem auf Seiten der Grundbesitzer Misstrauen entsteht. Es wird beklagt, dass der Naturschutz kein klares, allgemein gültiges Konzept zur Beurteilung von Erstaufforstungen besitzt.

Tatsächlich ist es so, dass der Naturschutz verschiedene Leitbilder besitzt, die nicht immer miteinander harmonieren. Eine Prioritätensetzung ist kaum möglich. Die räumliche Konkretisierung durch die Definition regionaler Entwicklungsziele ist schwierig. Selbst ein Konsens im innerfachlichen Diskurs bringt noch keine Garantie für die Implementierung der Planungsergebnisse, da letztere nur über den Grundeigentümer erfolgen kann (Ausnahme: Schutzgebiete).

In diesem Zusammenhang soll mit der vorliegenden Arbeit ein Versuch unternommen werden, entscheidende Kriterien für die ökologische Bewertung einer Fläche zu definieren und in einem Verfahren zusammenzuführen. Dies dient der Darstellung naturschutzfachlicher Ansprüche an den Landnutzungswandel im Rahmen der Agrarstrukturreform. Darüber hinaus bietet es eine Chance, durch die Entwicklung eines transparenten Verfahrens Anstöße zu geben für eine Planung ökologisch wertvoller Aufforstungen. 


\subsection{Arbeitshypothesen}

- Der Transfer von Leitbildern der regionalen Planung in ein allgemeines methodisches Verfahren mit regionalem Maßstab ermöglicht die gleichzeitige Berücksichtigung überregionaler Interessen sowie regionaler Entwicklungsziele und Bedingungen.

Der Naturschutz hat längst eine nationale und internationale Dimension erreicht, die in Initiativen und Förderprogrammen zum Ausdruck kommen. Bei der Umsetzung der überregionalen Ziele müssen auch die regionalen Bedürfnisse und lokalen Bedingungen berücksichtigt werden. Ein Bewertungsverfahren muss daher den Ansprüchen der verschiedenen Verwaltungsebenen gerecht werden.

- Ökologische Auswirkungen von Erstaufforstungen können anhand einer ordinalen Skala bewertet werden.

Durch die Verwendung von ordinalen Skalen im Rahmen der Bewertung, kann das Verfahren Hinweise für eine Befürwortung oder Förderung beantragter Maßnahmen liefern, wodurch den Entscheidungsträgern im Genehmigungsverfahren ein neues Hilfsmittel entsteht.

- Unterschiedliche Ziele können in einem einheitlichen Verfahren berücksichtigt und bewertet werden.

Innerhalb des Naturschutzes bestehen eine Reihe unterschiedlicher, teilweise konkurrierender Zielsetzungen. Die Durchsetzung eines Zieles auf Kosten anderer, ebenfalls berechtigter Interessen ist zu vermeiden. Daher soll aufgezeigt werden, dass die Bewertung eines Nutzungswandels dessen Beitrag zur Erreichung verschiedener Ziele nebeneinander berücksichtigen kann.

- Generelle Ansprüche des Naturschutzes sind in unterschiedliche Landschaften transferierbar.

Es soll gezeigt werden, dass in unterschiedlich stark bewaldeten Landesteilen ein einheitliches Verfahren zur Anwendung kommen kann, welches auf die wichtigsten Kriterien und Indikatoren des wissenschaftlichen Diskurses Rücksicht nimmt.

- $\quad$ Ein transparentes Bewertungsverfahren fördert nicht nur die Nachvollziehbarkeit, sondern erhöht auch die Möglichkeit der Einflussnahme durch den Grundeigentümer.

Der Antragsteller kann die Chancen einer Genehmigung selbst abschätzen und durch gezielte Flächenwahl sowie einen an ökologischen Kriterien orientierten Aufforstungsplan beeinflussen.

- Ein Verfahren mit externen Bewertungsschlüsseln schafft eine hohe Flexibilität gegenüber variablen Anwendungsbedingungen.

Landschaftspläne und andere Entwicklungspläne formulieren die Entwicklungsziele anhand der Interessenlage zum Zeitpunkt der Planerstellung. Veränderungen der relevanten Bedingungen, z.B. Eigentümerinteressen durch Generationenwechsel, Landschaftsbild durch fortgeschrittene Einflussnahmen, machen eine Revision der Ziele und Überarbeitung des Planes notwendig. Ein hinsichtlich der Umgebungsbedingungen flexibles Verfahren kann dagegen unabhängig von detaillierten Plänen agieren, wenn die regionalen Leitbilder feststehen. 


\section{Hintergrund}

\subsection{Erstaufforstung in der Diskussion}

Biodiversität ist ein aktuelles und modernes Thema. Aktuell, weil viel davon gesprochen wird - modern, weil kaum eine Veranstaltung, eine Vereinbarung oder ein Abkommen zu Umweltund Naturschutz heute ohne dieses Thema auszukommen scheint. Spätestens seit der Biodiversitäts-Konvention (Rio de Janeiro, 1992) sind Schutz und Nutzen der Biodiversität in aller Munde. Dabei lässt die Vielschichtigkeit des Begriffes viel Raum für politische Interpretationen. Unbestritten ist, dass sich die Biodiversität auf verschiedenen ökologischen Ebenen, von den Genen bis zu Ökosystemen beschreiben lässt. Weitgehend unbestritten ist auch, dass der umfassende Charakter der Biodiversität eine Vielzahl von Ressourcen einschließt. Sie stellt eine Generalressource dar, deren nachhaltiges Management zu einer zentralen Aufgabe der globalen Politik und Kooperation wird (SPELLERBERG/SAWYER 1996). Somit müssen sich alle landverbrauchenden oder ökosystemverändernden Entwicklungen an deren Auswirkungen auf die Biodiversität messen lassen.

Die Erstaufforstung landwirtschaftlich genutzter Flächen kann ebenfalls als aktuelles Thema bezeichnet werden. Modern ist es deshalb nicht. Seit den großen Heideaufforstungen im späten 18. Jahrhundert spielte die Waldvermehrung immer wieder eine mehr oder weniger große, doch immer landschaftsprägende Rolle. Auch eine Begrenzung des Betrachtungszeitraumes auf das 20. Jahrhundert zeigt, dass es mehrere Aufforstungswellen gab, die zumindest regional bedeutende Ausmaße annahmen. In diesem Zusammenhang sei nur an die großflächigen Rekultivierungsprojekte der Bergbaufolgelandschaften oder an die Großflächenaufforstungen entwässerter, abgetorfter Moorgebiete in Norddeutschland erinnert. Das aktuellste Beispiel für eine flächig landschaftsprägende Aufforstungswelle ist wohl Irland, welches in Bezug auf das Verhältnis zwischen Aufforstung und existierender Waldfläche in Europa eine Spitzenreiter-Rolle übernimmt (IDF 2001). In den vergangenen Jahrzehnten spielten aber zunehmend auch gesetzliche Bestimmungen und öffentliche Förderprogramme eine wichtige Rolle als Motor für die Waldvermehrung. Damit wandelten sich auch die gesellschaftlichen Ansprüche an die Umwandlungsmaßnahmen. Neue Wälder müssen heute in Planung, Anlage und Entwicklungsmöglichkeiten den Zielen der Agenda 21 entsprechen und neben ökonomischen und ökologischen vor allem auch gesellschaftspolitischen Zielen verpflichtet sein (FRIEDRICHSDORF 1999).

Noch in den achtziger Jahren des vergangenen Jahrhunderts sah es die Europäische Gemeinschaft als eine ihrer vordringlichsten Aufgaben an, den Stand der Nahrungsmittelproduzenten als Garant für die Versorgung der Bürger vor den Unbilden der freien Marktwirtschaft zu schützen. Anfang der 1990er Jahre bestand der EG-Haushalt zu $75 \%$ aus Agrarausgaben. Die verfehlte Agrarpolitik der 1970er und 1980er Jahre führte zu einer gemeinschaftsweiten Überproduktion an landwirtschaftlichen Erzeugnissen. 1992 startete eine EG-Agrarreform (Gemeinsame Agrarpolitik - GAP) mit den Zielen, die staatlich gestützten Erzeugerpreise auf Weltmarktniveau abzusenken und die Überproduktion zu verringern. Durch eine Verringerung der Stützpreise und Interventionsmengen für Getreide, Ölsaaten und Hülsenfrüchte wurde zunächst Druck auf die Landwirte ausgeübt. Gleichzeitig wurden aber direkte Beihilfen als Ausgleich für Einkommensverluste bei bestimmten Fruchtarten (Getreide, Hülsenfrüchte, Ölsaaten) in Aussicht gestellt. Den Anspruch auf diese Beihilfen erwarb der Agrarbetrieb durch das Einrichten von Rotations- oder Dauerbrachflächen.

Zusätzliche Möglichkeiten zur Verringerung der Überproduktion erhoffte sich die Gemeinschaft von einer Förderung der Erstaufforstungen (VO EWG 2080/92, später VO EG 1257/99). Diese sollten den Landwirten langfristige Perspektiven bieten. Zur Verringerung des Einkommensverlustes in den ersten Jahren, wurden Aufforstungs- und Pflegeprämien eingeführt. In der Folge wurde ein Großteil der in Deutschland ausgeführten 
Erstaufforstungen über dieses Förderprogramm im Rahmen einer Kofinanzierung zwischen Bundesländern und EU gefördert.

Bei den aus der Produktion ausscheidenden Flächen handelte (und handelt es sich noch immer) in erster Linie um jene, die unter den Bedingungen des EU-Binnenmarktes keine hohe landwirtschaftlichen Erträge mehr versprechen. Diese bilden keineswegs einen repräsentativen Querschnitt durch die verschiedenen Standortbedingungen (FINCK/SCHRÖDER 1997), sondern können mit dem Begriff Grenzertragsböden umschrieben werden. Sie zeichnen sich durch besondere Nährstoffarmut, Feuchtigkeit oder Trockenheit, bzw. durch eine Kombination dieser Merkmale aus. Gerade auf diesen Flächen sind aber für den Artenschutz besonders wertvolle Biotope und Lebensgemeinschaften zu finden, weshalb eine Umwandlung in eine andere Nutzungsart aus Naturschutzgründen zu vermeiden wäre.

Die Verordnung EWG 2080/92 war so konzipiert, dass sie vor allem die klassische Aufforstung nach forstökonomischer Planung förderte. Neben einer Kostenbeihilfe für die Initialphase gab es weitere Finanzmittel für die zugehörigen Pflege- und Bewirtschaftungsmaßnahmen sowie eine Erstaufforstungsprämie als Ausgleich für Einkommensverluste aus bisher landwirtschaftlicher Nutzung (vgl. Abb. 2.1-I). Die Förderung erfolgte im Rahmen der Kofinanzierung aus Mitteln der EU, des Bundes und der Länder.

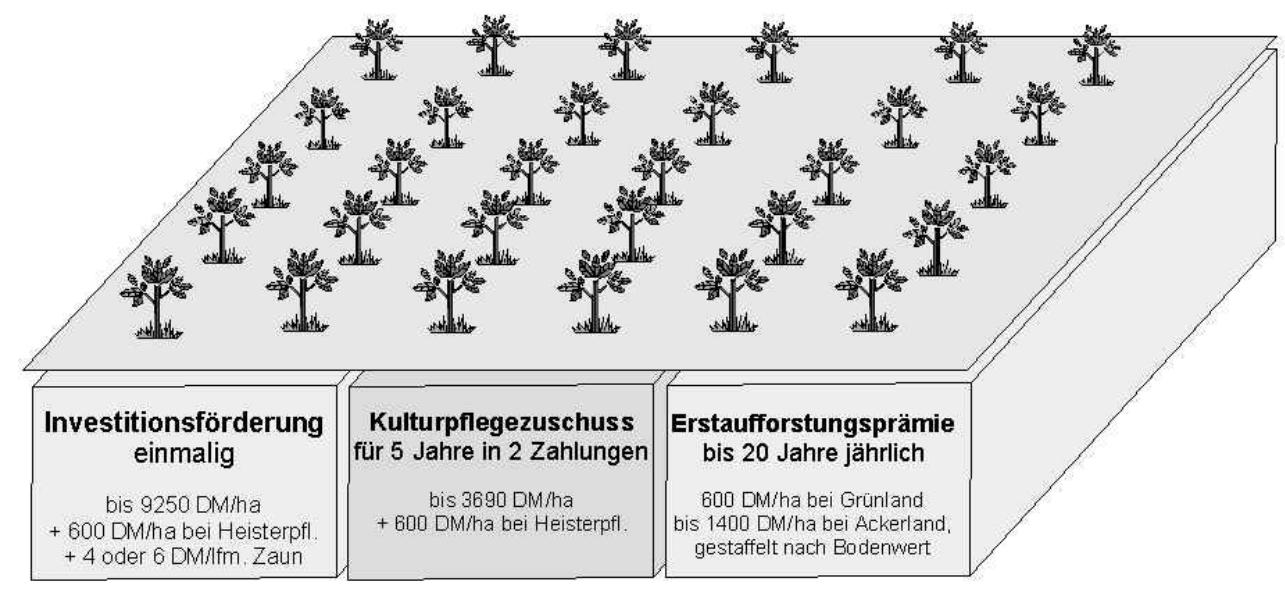

Abb.2.1-I: Zusammensetzung der Erstaufforstungsförderung im Freistaat Thüringen (LAWUF 1999)

Investitionsförderung und Pflegezuschuss wurden in Abhängigkeit vom Bestandestyp gezahlt. Die Finanzhilfe sollte die Pflegemaßnahmen der ersten fünf Jahre abdecken. Zusätzlich konnten Grundeigentümer eine Prämie erhalten, die den Einkommensverlust aus dem Übergang von der landwirtschaftlichen zur forstlichen Nutzung in den ersten Jahren ausgleichen sollte. Für diese Prämie erfolgte eine Bemessung des Zahlungszeitraumes am gewählten Bestandestyp. Die Höhe der Zahlungen wurde dagegen über die Qualität des Bodens errechnet.

Obwohl der gewählte Bestandestyp bei der Bemessung der Fördermittel berücksichtigt wurde, kann nicht ernsthaft von einer Berücksichtigung naturschutzfachlicher Aspekte gesprochen werden. Es wurden weder Anreize für besonders vielfältige Bestände geschaffen, noch schlug sich eine naturschutzorientierte Gestaltung, beispielweise durch einen strauchreichen, breiten Waldrand, in der Fördersumme nieder.

Auch die Orientierung der Aufforstungsprämie an den Bodenwerten führten zu einer ökologischen Fehlentwicklung. In Verbindung mit einer maximalen Prämie von DM 1400 pro ha führte diese Bemessungsweise nämlich dazu, dass die Grundeigentümer in aller Regel nur zur Aufforstung von Grenzertragsstandorten bereit waren. 
Unter Hinweis auf das Missverhältnis zwischen den Aufforstungsprämien und den potentiell möglichen landwirtschaftlichen Erträgen zieht KLEIN (1997) daher ein negatives Fazit aus der Entwicklung: Auf schlechten Standorten liegen die Aufforstungsförderungen über den Erträgen einer extensiven Bewirtschaftung der Fläche. Sie verdrängen somit eine ökologisch sinnvolle Nutzung. Auf den guten Standorten reichen die Fördergelder dagegen nicht aus, den Einkommensverlust gegenüber einer landwirtschaftlichen Nutzung auszugleichen, weswegen die produktiven Standorte kaum in Wald umgewandelt werden.

Diese Einschätzungen werden auch von MIRZA (2001) bestätigt, wonach eine Expertenbefragung an Forstbehörden ergab, dass die aufgeforsteten landwirtschaftlichen Flächen eine durchschnittliche Bodenpunktzahl von 33 Punkten besitzen (Skala von 1 bis 100 BP). Dabei wird erst einem Boden mit mindestens 35 Punkten eine Qualität zugesprochen, die einen durchschnittlichen landwirtschaftlichen Ertrag erwarten lässt (ARBEITSAUSSCHUSS RICHTZAHLEN 2000).

Fehlende Anreize für naturgemäße Baumartenzusammensetzungen, keine Berücksichtigung der Waldrandsituationen und passive Förderung der Aufforstung von Grenzertragsböden der Naturschutz stand bei der Formulierung der Verordnung EWG 2080/92 offensichtlich nicht Pate. Im Gegenteil beklagt zum Beispiel der DEUTSCHE RAT FÜR LANDSCHAFTSPFLEGE (DRL, 1997; S.19), dass "die Umsetzung dieser Verordnung vor Ort oft entgegengesetzt zu Naturschutzzielen erfolgt".

Verschiedene Auswege aus diesem Dilemma sind denkbar. Einer davon könnte in der Orientierung der Fördermittelhöhe an der ökologischen Qualität der Aufforstungsmaßnahme bestehen. Einen Versuch hierzu unternimmt beispielsweise der Freistaat Sachsen durch die Richtlinie zur „Förderung der ökologischen Waldmehrung“ (RL-Nr. 93/2000). Sie staffelt den finanziellen Kostenzuschuss zur Erstaufforstung nach den gepflanzten Baumarten. Die Förderrichtlinie kann aber nur in sehr begrenztem Umfang auf die ökologischen Anforderungen an eine Aufforstung eingehen. Schon der große Geltungsbereich, ein ganzes Bundesland, verhindert eine Klassifizierung nach regionalen Kriterien. Die Einteilung der Aufforstungsbestände in sechs Förderklassen (siehe Tab. 2.1-1) kann den naturschutzfachlichen Anspruch an die Bildung naturgemäßer Waldgesellschaften nicht wiedergeben. Weitere Aspekte des Arten- und Biotopschutzes, etwa der Biotopverbund oder die Waldrandgestaltung bleiben völlig außer Acht.

Der Deutsche Rat für Landespflege (DRL 1997) schlägt als verbessernde Passi im europäischen Verordnungstext die Förderung standortheimischer Waldbestände, die Untersagung der Aufforstung auf bereits in Sukzession befindlichen Flächen, die finanzielle Besserstellung von Sukzession gegenüber Aufforstungen auf "Intensivflächen“ und die Modifizierung der Bewaldungstechniken vor. Nähere Ausführungen zur Bestimmung der förderungswürdigen standortheimischen Waldbestände werden allerdings nicht gemacht. Die Abwägung einer Aufforstung gegenüber der Sukzession wird auch von anderen Autoren gelegentlich gefordert (ZUNDEL 2000, REIF 1997, STURM 1993). Dabei sind allerdings verschiedene Einschränkungen zu machen, wann eine Sukzession tatsächlich das bessere Ergebnis erwarten lässt. In isolierten Lagen dauert es sehr lange, bis sich über eine ungelenkte Sukzession tatsächlich eine Waldgesellschaft entwickelt, die als repräsentativ hinsichtlich ihrer Artenzusammensetzung gelten wird. Die ökologische Wirksamkeit von Wald kann indes durch eine Aufforstung wesentlich schneller erreicht werden (SCHÖLMERICH 1999), zumal durch Platz für begleitende Sukzession und durch eine an der natürlichen Waldgesellschaft orientierte Baumartenwahl ökologische Akzente bewusst gesetzt werden können.

Die Bestimmung des Beitrages einer Aufforstung zur Biodiversität erfordert zunächst die Definition des Terminus. Das Konzept der Biodiversität „bezieht sich auf die Gesamtheit aller Formen von Leben in einem System und bewegt sich damit in einer Bandbreite von organischen Molekülen bis hin zu Pflanzen- und Tierarten, Pflanzengesellschaften, Landschaften und Lebensräumen. Wird Biodiversität nicht nur als statisches sondern auch als dynamisches Konzept aufgefasst, muss ebenfalls die genetische Variabilität der Arten berücksichtigt werden." (KÖHL/ZINGG 1995, S. 77). Es geht also um ganz unterschiedliche 
Betrachtungsebenen und -objekte, die in ein gemeinsames Bewertungsverfahren zu integrieren sind. Die Biodiversität lässt sich nicht auf die Merkmale des Gen-Pools, des Arten- oder des Lebensraumspektrums reduzieren. Alle drei Kategorien werden durch einen Landnutzungswandel lokal, meist auch im weiteren räumlichen Zusammenhang beeinflusst. Daher muss eine adäquate Beurteilung von Nutzungsänderungen klar zwischen den funktionalen Betrachtungsebenen differenzieren. Eine Reduktion der betrachteten Zusammenhänge, beispielsweise auf die Artenzahl einer festgelegten Fläche, würde zwar die Erfassbarkeit deutlich verbessern, andererseits aber eine immense Beschränkung in der Aussagekraft der Evaluation bedeuten. Genetik, Artenvielfalt und lebensraumtypische Systemzusammenhänge ergeben somit die Grundbausteine einer umfassenden, prognostischen Bewertung von Erstaufforstungsmaßnahmen aus Sicht des Naturschutzes.

Tab. 2.1-1: Kostenzuschuss bei der Erstaufforstung, gestaffelt nach Baumarten; nach der Richtlinie des Sächsischen Staatsministeriums für Umwelt und Landwirtschaft zur Förderung der ökologischen Waldmehrung im Freistaat Sachsen (RL-Nr. 93/2000)

\begin{tabular}{|c|c|c|}
\hline Baumart & $\begin{array}{c}\text { Mindeststückzahl } \\
\text { Stück / ha }\end{array}$ & $\begin{array}{c}\text { Betrag } \\
\boldsymbol{\epsilon} / \text { ha }\end{array}$ \\
\hline $\begin{array}{c}\text { Fichte, Douglasie, sonstige } \\
\text { Nadelbäume }\end{array}$ & 1.500 & 2.045 \\
\hline Kiefer & 5.000 & 2.556 \\
\hline $\begin{array}{c}\text { Rotbuche, Traubeneiche, } \\
\text { Stieleiche }\end{array}$ & 5.000 & 5.420 \\
\hline $\begin{array}{c}\text { Linde, sonstige Hartlaubbäume } \\
\text { Pappel, Weide u. a. } \\
\text { schnellwachsende Arten }\end{array}$ & 2.000 & 4.090 \\
\hline Sonstige Laubbäume, Sträucher & 1.000 & 1.020 \\
\hline
\end{tabular}

Die Aufforstung landwirtschaftlicher Flächen wird häufig auch als Maßnahme zur Bindung von Kohlenstoff, und damit im Zusammenhang mit dem globalen Klimaschutz genannt. Hierzu ist zunächst festzustellen, dass der Nutzungswandel zu einer Verringerung des Stickstoffeintrages führt, da im Wald keine Stickstoff-Düngung erfolgt. Ein entscheidender Beitrag zur Kohlenstoffbindung durch die aufgeforstete Fläche muss - zumindest für die aktuelle Aufforstungsrate in Deutschland - wohl verneint werden (PRIES 1997, DOHRENBUSCH 1996). Die im Zeitraum 1992 bis 1999 über die VO 2080/92 geförderten Erstaufforstungen bewirkten gerade einmal eine Verringerung der Kohlendioxid-Belastung um 0,02 \% der im Kyoto-Protokoll für die Bundesrepublik festgesetzten Reduktionsquote (MIRZA 2001).

In Bezug auf den Strukturwandel können Erstaufforstungen auf landwirtschaftlichen Flächen dennoch als gesamtökologisch positiver Beitrag gesehen werden. Die Umwandlung bewirkt - in noch stärkerem Maße als die Extensivierung - einen Verzicht auf den Einsatz von Mineraldünger und vor allem von Pflanzenschutzmitteln bei der Produktion (AMMER/PRÖBSTL 1988). Auch KEDING (1999) bewertet Aufforstungen im Rahmen der Gemeinsamen Agrarpolitik generell als „für den Naturschutz überwiegend positiv“. Er stellt weiter fest, dass eine ökologische Waldmehrung im öffentlichen Interesse liegt, dies aber nicht ohne gleichsinnige Ziele des Grundeigentümers umsetzbar sei. Daher muss der Gesetzgeber durch die Anwendung politischer Instrumente einen entsprechenden Anreiz schaffen. Insbesondere die mancherorts langwierigen Genehmigungsverfahren beeinträchtigen allerdings die Aufforstungswilligkeit der Landwirte. Eine Verringerung des restriktiv wirkenden bürokratischen Aufwands könnte dem Wunsch der Landes- und Bundesregierungen zur Vermehrung des Waldanteils deutlich unterstützen. Folgerichtig weist auch das Nationale Forstprogramm Deutschland (BMELF 2000) auf eine notwendige Beschleunigung und Vereinfachung des Genehmigungsverfahrens hin. Es fordert darüber hinaus, dass die Förderung der Erstaufforstung nicht weniger attraktiv sein darf, als die Stillegung landwirtschaftlicher Flächen.

Die von den Landeswaldprogrammen angestrebten Aufforstungsraten könnten eine neue, landschaftsgestaltende Phase einläuten. In Niedersachsen beispielsweise, soll der 
Waldanteil langfristig (innerhalb von 50 Jahren) von $23 \%$ auf $25 \%$ gesteigert werden (KEDING 1994). Dies bedeutet für den angegebenen Zeitraum eine Erstaufforstungsfläche von ca. 2000 ha jährlich. In Thüringen, dem ebenfalls in dieser Arbeit behandelten Bundesland, bestehen seit 1992 Bestrebungen der Landesregierung, den Waldbestand jährlich um ca. 1000 ha Aufforstungen zu mehren. Das Ziel konnte bisher noch nicht erreicht werden. Auch wenn in den ersten Jahren bedeutende Waldflächenzunahmen zu verzeichnen waren, sank die Aufforstungsrate danach wieder kontinuierlich ab. Im Jahre 1999 war sogar ein realer Waldflächenverlust zu bilanzieren (TRIEBEL 2000). Das Land Sachsen strebt mit einer Erhöhung des Waldanteils von derzeit $27 \%$ auf künftig $30 \%$ ebenfalls einen bemerkenswerten Waldzuwachs an, der sich in einer Aufforstungsfläche von immerhin 50.000 ha ausdrückt (KÖPF 2002). Da die politischen Erfolge allerdings noch zu Wünschen übrig lassen, sind Durchhaltewille und neue Konzepte gefragt.

Der politische Wille sucht generell, also auch in den behandelten Bundesländern, vor allem die unterbewaldeten Landesteile mit neuen Waldflächen anzureichern. In den dicht bewaldeten Gebieten ist eine Waldzunahme dagegen zu vermeiden (LAWUF 1999, KEDING 1994). Folglich ist von landschaftsprägenden Einflüssen dieser Maßnahmen auszugehen.

Bei der Neuanlage von Wald sind neben seiner visuellen Eingliederung in die Landschaft auch seine zukünftigen Funktionen zu berücksichtigen. Das Management bestehender Wälder orientiert sich am Leitbild der ökosystemgerechten Waldnutzung. Dieses umfasst eine standortsgerechte, nachhaltige und umweltschonende Bewirtschaftung. Dabei sind die Regelungs-, Lebensraum-, Nutzungs- sowie Kultur- und Sozialfunktionen im Rahmen einer Mehrzweckforstwirtschaft gleichberechtigt zu erfüllen (BEESE 1996). Aufforstungen sind so anzulegen, dass der entstehende Wald die vielen Aufgaben im Sinne der ökosystemgerechten Waldnutzung erfüllen kann.

Ein Genehmigungsverfahren, wie es durch die Forst- und Waldgesetze vorgesehen ist, dient der Abwägung sozialer, ökologischer und sozioökonomischer Interessen. Es erfolgt nach landesspezifischen Verfahren, wobei in den meisten Bundesländern die Forstbehörden schriftführend sind. Gleichzeitig fungieren sie häufig als Antragsbehörde für Fördermittel.

Die Genehmigungsverfahren zeichnen sich einheitlich durch einen großen Ermessensspielraum aller beteiligten Behörden aus. Klare Vorgaben für den Vollzug fehlen. Die Ämter können die Genehmigung mit mehr oder minder tragfähigen Argumenten ablehnen oder befürworten (THOROE 1997). Eine Identifikation des politischen Willens hinter einer derartigen Amtsentscheidung ist schwierig. Die Entscheidungsfindung ist für den Bürger weder transparent noch prognostizierbar.

Der Staat versucht mit drei verschiedenen politischen Instrumenten die Aufforstungstätigkeiten zu lenken. Zunächst durch die Beratung des Grundeigentümers bei der Planung und Durchführung der Umwandlungsmaßnahme. Dieser Teil der Tätigkeit ist in seiner Qualität von der betreuenden Forstsachverständigen vor Ort abhängig und kann deshalb nicht generell beurteilt werden. Das zweite Instrument ist das beschriebene Genehmigungsverfahren, in dem Eigentümerinteressen und öffentliches Interesse gegeneinander abgewogen werden sollen. Das dritte Instrument ist schließlich die finanzielle Aufforstungsförderung. Die diesbezüglichen Programme verfolgen die Umsetzung öffentlicher Interessen durch die Bezuschussung bestimmter Maßnahmen. Sie können allerdings nur dann Wirkung zeigen, wenn die darin enthaltenen Anreize auch die persönlichen Interessen der Grundeigentümer treffen. Der aufforstungswillige Landwirt muss sprichwörtlich dort abgeholt werden, wo er steht.

Wie schwierig allerdings die Einschätzung der tatsächlichen Beweggründe für eine Nutzungsänderung ist, zeigt die Arbeit von MIRZA (2001). Sie vergleicht die Ergebnisse einer Umfrage unter Grundeigentümern, die eine Aufforstungsförderung nach VO 2080/92 beantragten, mit den Ergebnissen einer Expertenbefragung unter den für die Bewilligung zuständigen Forstbehörden. Letztere schätzten zu $56 \%$, dass finanzielle Gründe sehr wichtig für den Entscheidungsprozess des Grundeigentümers waren. Dagegen gaben lediglich $6 \%$ der Aufforstungswilligen an, dass finanzielle Gründe tatsächlich einen derart 
hohen Stellenwert besitzen. Auch hohe Fördersummen stellen keine Garantie für den Erfolg einer Richtlinie dar. Die Reduktion der Eigentümerinteressen auf monetäre Beweggründe ist eine unzulässige Simplifizierung, die nicht selten zu einer Fehleinschätzung der tatsächlichen Lage führt. Dies kann im Nachhinein auch für die Feststellung von ERLBECK (1993, S.232) gelten, der nach Inkrafttreten der Verordnung EWG 2080/92 mutmaßte: „Die Bereitschaft zur Aufforstung wird also aus rein betriebswirtschaftlichen Gründen steigen, weil jeder, der rechnen kann, sich für die Aufforstung entscheiden wird, auch wenn die Prämien für die Freihaltung gleich hoch wären."

Komplementär zur Überbewertung der finanziellen Beweggründe wurden die ökologischen Ambitionen der Eigentümer unterschätzt. Mehr als zwei Drittel der befragten Behörden (MIRZA 2001) gingen davon aus, dass sich Flächeneigentümer nicht von ökologischen Gründen (Boden-, Arten-, Wasser- und Klimaschutz) leiten ließen. Demgegenüber bezeichneten jeweils deutlich mehr als 50 \% der Grundeigentümer solche ökologischen Überlegungen als sehr wichtig bei der persönlichen Entscheidungsfindung.

Diese Beispiele zeigen, dass es für Behörden häufig schwierig ist, die Beweggründe der Grundeigentümer zu prognostizieren. Wenn solche Prognosen dann aber als Grundlage für die Erstellung räumlicher Entwicklungskonzepte dienen, verhindern die Fehleinschätzungen der sogenannten Experten eine optimale Effektivität der Maßnahmen.

Neben der Integration der Grundeigentümer bei der Planerstellung besteht die zweite Möglichkeit zur Lösung des Problems in der Entwicklung flexibler, planunabhängiger Instrumente für die Raumplanung. Diese könnten beispielsweise bei den Interessen der Eigentümer ansetzen und dann im Einzelfall die aktuelle und die neu zu schaffende Situation erörtern. Wenn das Verfahren dann die Leitbilder der regionalen Raumplanung und des Naturschutzes berücksichtigt, kann auf die Zonierung im Sinne von Aufforstungsgewannen verzichtet werden.

Zur Einschätzung der ökologischen Wertigkeit gegenüber anderen Landnutzungen fehlen bisher detaillierte Konzepte. Bundesweite Konzepte (Bundeswaldgesetz, Nationales Forstprogramm) bleiben zu diesem Themenbereich unkonkret und geben zu wenig Anhaltspunkte für die Umsetzung. Auch von Seiten des Verbandsnaturschutzes wird wenig Konkretes angeboten. Positions- und Hintergrundpapiere zur Aufforstungsthematik liegen nur im Einzelfall vor und sind kaum aussagekräftig bezüglich Flächenauswahl oder Bewaldungstechnik (vgl. z.B. NABU 1997).

Untersuchungen, wie etwa diejenige von KORNECK/SUKOPP (1988), welche von einer bedeutenden Beteiligung der Forstwirtschaft am Rückgang der Artenvielfalt sprechen, werden in ihren Ergebnissen von den forstwirtschaftlichen Praktiken zurückliegender Jahrzehnte stark beeinflusst. Nadelbaum-Monokulturen und Aufforstungen von Sonderstandorten oder entwässerten Flächen sind heute sehr selten geworden. Deshalb kann aus derartigen Beschuldigungen keine generell negative Einschätzung von Aufforstungen abgeleitet werden. Das in dieser Arbeit entwickelte Verfahren kann im Gegenteil Hinweise geben, unter welchen Voraussetzungen eine Aufforstung positive Auswirkungen auf die Artenvielfalt erwarten lässt.

Eine objektive Abwägung der ökologischen Folgen einer Aufforstung kann nicht allein aufgrund eines vorhandenen Raumplanes erfolgen. Es muss die Möglichkeit bestehen, einen Nutzungswandel anhand der aktuellen Situation zu erörtern. Zu diesem Zwecke bedarf es einer systematischen Evaluation der Erstaufforstung anhand einheitlicher Kriterien mit regionalisiertem Bewertungsmaßstab.

Unter den für die Genehmigung von Erstaufforstungen zuständigen Behörden (Forstbehörden, Landwirtschaftskammern) ist die Meinung weit verbreitet, dass Erstaufforstungen landwirtschaftlicher Flächen einen positiven Beitrag zur Biodiversität leisten (HERREN/BAUR 1993, MIRZA 2001). Diese Wirkungen sollten dann aber bereits in einem Genehmigungsverfahren systematisch betrachtet und prognostiziert werden. Dabei kann es nicht nur darum gehen, eine Für-oder-Wider-Entscheidung zu treffen, sondern darüber hinaus auch eine Abwägung zwischen unterschiedlichen Varianten zu ermöglichen. 
Ist das Verfahren schließlich noch transparent und plausibel, so dient es auch dem Grundeigentümer zur Einschätzung der Genehmigungsfähigkeit, bzw. zur Gestaltung der Aufforstungspläne nach ökologischen Kriterien.

\subsection{Naturschutzfachliche Bewertung zwischen Soll und Ist}

Planung hat immer den Sinn zielorientierten Handelns, d.h. ein gegebenes Ziel von einem aktuellen Standpunkt aus anzustreben. Die dafür notwendigen Pfeiler sind einerseits die Zielvorgabe, bzw. das Leitbild und andererseits die Erfassung der augenblicklichen Situation, die Beschreibung des Zustandes. Aus diesen beiden Strängen, Zielentwicklung und Situationsanalyse, ergeben sich die Pole „Soll“ und „Ist“. Ein Vergleich der beiden führt zu einer Bewertung der augenblicklichen Situation in Relation zum avisierten Zustand. Grundbedingung dabei ist, dass für Zielbeschreibung und Datenerfassung die gleiche Sprache verwendet wird. Dies drückt sich beispielsweise in gleichen Messgrößen und einheitlicher, räumlicher Bezugsskala aus. Liegen sektorale Ziele vor, so ergibt sich aus den Bewertungen unmittelbar der Handlungsbedarf als Differenz zwischen Soll und Ist (WIEGLEB 1998). Bei komplexen Zielen (unterschiedliche Leitbilder) ist dagegen zwingend ein formales Bewertungsverfahren zur Entscheidungsunterstützung zu erarbeiten.

Im Naturschutz existieren gleich mehrere Leitbilder mehr oder weniger gleichberechtigt nebeneinander (Kap. 3.2), weshalb eine Bewertung ohne Berücksichtigung dieser Zielvorgaben nicht möglich oder zumindest nicht sinnvoll ist. Zu diesem Schluss kommt auch WIEGLEB (1998), der weiter folgert, dass eine Hierarchie der Leitbilder seitens der Planungsträger vorgegeben sein muss, die dann in das Bewertungsverfahren zu übernehmen wäre. Ist die Hierarchie nicht vorgegeben, so kann es zu Widersprüchen zwischen verschiedenen Werten kommen. Die Leitbildentwicklung löst zwar nicht das Bewertungsproblem, strukturiert es aber wesentlich vor. Kommt sie dieser Aufgabe nicht nach, so sind die Kriterien als gleichberechtigt nebeneinander stehend zu betrachten. Einer weiteren Unterteilung in Merkmale oder Indikatoren muss dann eine ebenfalls gleichberechtigte Wertung folgen.

Die Frage, ob nun in der Naturschutzplanung zunächst das Ist festzustellen und dann das Soll zu formulieren ist, oder umgekehrt, kann nicht abschließend geklärt werden. Ziel und Situationsbeschreibung müssen jedenfalls die gleiche Sprache und Struktur verwenden. Es ist folglich ein langwieriger, sich ständig verändernder Prozess, in dem Situationen beschrieben, Ziele formuliert, die Wirklichkeit neu erfasst, Ziele revidiert werden, und so weiter. Die Leitbilder müssen auf den aktuellen Kenntnisstand zur Ist-Situation aufbauen. Kommen neue Zielvorstellungen hinzu, so müssen eventuell fehlende Daten nachträglich erhoben werden.

Bei der Erfassung und Bewertung von Wäldern im Sinne des Naturschutzes wird häufig auf pflanzensoziologische Einheiten zurückgegriffen. Sie spielen eine wichtige Rolle bei der Ansprache von Waldbiotopen. Auf der Grundlage erfasster Biotope erfolgt dann die Planung zur Erhaltung des Zustandes und eventuell einer bestimmten Entwicklung. Hier können weiterhin zwei Vorgehensweisen unterschieden werden. Erstens der Schutz einzelner Arten und Ökosysteme (Waldtypen). Ein Beispiel für diesen Typ sind die Biotopkartierungen, die mittlerweile in ganz Deutschland durchgeführt werden (DRACHENFELS 1994). Zweitens die Kartierung von Wirtschaftsweisen und Merkmalen forstlicher Produktionssysteme, die flächendeckend stattfindet, aber auch nur bestimmte Parameter von Ökosystemen abbildet (LAWUF 1996).

Ein methodisches Problem der Beschreibung von Wäldern, insbesondere hinsichtlich deren ökologischer Bedeutung, tritt in Mosaik-Landschaften auf. Inwieweit können Gehölzgruppen und kleine Wäldchen zu Einheiten zusammengefasst werden? Und welche Rolle spielen dabei unterschiedliche Baumartenzusammensetzungen, Wirtschaftsweisen, Altersstrukturen oder Vegetationsschichten? Es liegt auf der Hand, dass diese Fragen nicht generell, sondern 
nur in Abhängigkeit vom zentralen Interesse der jeweiligen Biotoperfassung oder -bewertung erfolgen kann. Aus diesen Sachverhalten ergibt sich für natürlich entstehende oder neu angelegte Wälder die Frage: Welche Rolle können neue Waldflächen spielen in der Erweiterung und Verknüpfung bestehender Wälder und welche Merkmale müssen sie hierzu aufweisen?

Offensichtlich nimmt ein Bestand im Jungwuchs- und Dickungsalter andere Funktionen wahr als ein reifer oder überalterter Bestand gleichartiger Baumartenzusammensetzung. Andererseits dauert die Jungwuchsphase nicht ausreichend lange, um eine gesonderte Bewertung im Rahmen einer langfristigen Planung zu rechtfertigen. Ein ökologisch orientierter Waldbau, wie er von den meisten Forstverwaltungen propagiert und auch von vielen Privatwaldbesitzern betrieben wird, setzt auf lange Umtriebszeiten unter Ausnutzung von Naturverjüngung. Ein Bestandesalter von mehr als 100 Jahren ist also zu erwarten. Damit nähme aber die Jungwuchsphase lediglich 10-20 \% der Entwicklungszeit ein. Eine Bewertung von Aufforstungen muss daher vor allem die längerfristige Entwicklung prognostizieren und in die Urteilsfindung einfließen lassen.

Wie bereits erwähnt, spielt die Art und Detailliertheit der Datenerfassung eine wichtige Rolle für die Möglichkeit, adäquate und umsetzungsfähige Zielvorstellungen zu formulieren. Bei der Bewertung in reich strukturierten Landschaften spielt die Verwendung von Lebensraumkartierungen, Fernerkundungsdaten und verschiedenen thematischen Karten eine wichtige Rolle (KIRBY 1994). Studien zur Veränderung der Situation durch Aufforstungen in strukturreichen Landschaftstypen gibt es bereits, allerdings beschäftigen sich diese nicht mit der Frage der Biodiversität im betrachteten Raum, sondern lediglich mit der Auswirkung der Maßnahme auf einzelne Tierarten des Offenlandes, deren Habitat durch eine neue Waldfläche beeinflusst oder zerschnitten werden könnte (z.B. GRUTTKE/WILLECKE 1993, ZENKER 1982).

Je komplizierter ein Bewertungsschema ist, desto schwieriger sind dessen Ergebnisse zu interpretieren. Klare Strukturen bei der Wertermittlung mit möglichst reduziertem Ermessensspielraum für den Gutachter vereinfachen den Bewertungsgang zu Gunsten von Transparenz und Nachvollziehbarkeit. Ein praxistaugliches Bewertungsverfahren sollte in seiner Komplexität der Planungsaufgabe angepasst sein. Eine logische Struktur und die Befreiung von erfahrungsbezogenen, gutachtlichen Einschätzungen ermöglicht die Durchführung der Bewertung auch durch Personen, die nur über Grundkenntnisse des Naturschutzes verfügen. Wird der Interpretations- und Ermessensspielraum des Gutachters beschnitten, so kommt dies der Reproduzierbarkeit der Ergebnisse unbedingt zu Gute. Liegt kein hierarchisches Leitbild-Konstrukt vor, so sind die verwendeten Bewertungskriterien einheitlich zu gewichten. Auch die Skalen für die erfassten Merkmale sollten einheitliche Größen aufweisen. Innerhalb einer Elementbewertung (z.B. Arteninventar) sollten alle Elemente (Arten) gleich bewertet werden. Im Falle einer Punktebewertung würde dies bedeuten, dass eine vorhandene Art mit einer fixen Punktzahl in die Berechnung einfließt und keine Unterscheidung in mehr oder weniger bedeutend getroffen wird (KIRBY 1994). Ein wesentlicher Beitrag zur objektiven Wertfindung ist auch die Ermittlung des Wertes anhand von Idealzuständen, also beispielsweise der Vergleich der Artenvielfalt mit der Vielfalt in der potentiell natürlichen Vegetation des Standortes, statt mit der Artenvielfalt eines anderen Bestandes.

Die Landschaftsplanung verlangt nach einfachen, gut handhabbaren und gleichzeitig hinreichend komplexen Informationen über den Planungsgegenstand. Daher rührt die Tendenz, mehrere Eigenschaften eines Ökosystems zu erfassen, zu beurteilen und zu einem Gesamtwert zu aggregieren. Die Verknüpfung unterschiedlicher Parameter folgt subjektiven Erwägungen und ist dabei naturwissenschaftlich kaum begründbar (BASTIAN 1999). Andererseits versetzt es den Planer in die Lage, einen wesentlichen Teil des Systems abzubilden, zu abstrahieren und für die weitere Planung zu analysieren. Abstriche in der wissenschaftlichen Aussagetiefe und -schärfe sind dabei meist unvermeidbar. Nur auf diese Weise ist es aber möglich, trotz der häufig schmalen Datenbasis für größere Gebiete eine ausreichend detaillierte Planungsgrundlage zu erhalten. Schließlich kann die 
Landschaftsplanung keinen jahrelangen Vorlauf der Grundlagenforschung abwarten. In der Literatur findet sich ein weites Spektrum von Biotopansprachen von der einfachen, verbalen Beschreibung der Biotoptypen bis zur komplizierten mathematischen Berechnung (vgl. BASTIAN 1999). Gegenwärtig sind in der landschaftsplanerischen Praxis einige additive Modelle in breiter Variation gebräuchlich. Die Einzelmerkmale werden über Verflechtungsmatrizen hergeleitet und miteinander verknüpft. Unter Umständen werden die Bewertungskriterien dann noch in höherrangigen Matrizen nutzwertanalytisch zum Gesamtwert aggregiert. Mit zunehmender Größe und höherem Rang der Matrize steigt auch der Grad der Komplexität. Gleichzeitig geht die Überschaubarkeit des Verfahrens verloren. Zusätzlich ist die Art der Verknüpfung von Bedeutung. Komplizierte Formeln sind zu vermeiden, die Grundrechenarten sollten als mathematische Hilfsmittel ausreichen.

Darüber hinaus weist BASTIAN (1999) darauf hin, dass die Problematik bei der Bestimmung komplexer Biotopwerte in der Schwierigkeit besteht, dass mehrere Sachverhalte (Einzelkriterien) nur verbal zu beschreiben sind, zudem teilweise miteinander korrelieren und sich demzufolge einer sinnvollen mathematischen Behandlung weitgehend entziehen. Als Alternative schlägt er eine Wertermittlung vor, die auf einem komplexen und vielarmigen Entscheidungsbaum beruht, bei dem der Bearbeiter aufgrund vieler Abwägungen, bzw. auf dem Hintergrund seiner fundierten, landschaftsökologischen Vorkenntnisse einen Biotopwert erarbeitet. Die Wertfindung erfolgt unter inhaltlichen Gesichtspunkten. Der entscheidende Nachteil liegt dann erneut in der fehlenden Transparenz und Nachvollziehbarkeit für Außenstehende (s.o.). Des weiteren ist zu beachten, dass eine Aggregation numerischer Bewertungen verschiedener Merkmale nur dann statthaft ist, wenn diese untereinander unabhängig sind (KIRBY 1994). Bei Flächengröße und Artenzahl wäre dies beispielsweise nicht der Fall.

Die Aggregation und Vereinfachung von Indikatoren ist generell vorsichtig zu behandeln. Nach KIRBY (1994) und PLACHTER (1990) kann die sogenannte Leistungsfähigkeit des Naturhaushalts nicht mit Hilfe eines oder weniger Indikatoren bestimmt werden. Auch die Verwendung aggregierter Parameter bringt für generelle Fragestellungen nur zweifelhafte Vorteile, da Primärdaten und Detailinformationen verloren gehen. Stattdessen ist ein möglichst breites Spektrum unterschiedlicher Parameter einzusetzen. Allerdings muss auch gesagt werden, dass eine Aggregation umso eher möglich erscheint, je spezieller die Fragestellung ist.

Da Landschaften einander ähneln können, sich aber praktisch nie gleichen, ist es von Bedeutung, dass bei der Bewertung von Flächen nach einem neuen Verfahren zunächst eine repräsentative Auswahl an Flächen gewählt wird, um das gesamte Bewertungsspektrum darzustellen. Es ist einerseits notwendig um die Funktionsfähigkeit des Verfahrens von beiden Bewertungsextremen her zu erläutern. Andererseits liefert es einen ersten Referenzrahmen zur Einschätzung einzelner Fallbeispiele. Je komplizierter das Bewertungsschema ist, desto mehr Fallbeispiele müssen zur Erläuterung untersucht werden.

Bei allen Bemühungen um Ausgewogenheit und Objektivität können „subjektive Entscheidungen und Urteile [...] nicht völlig ausgeschlossen werden, doch lässt sich die Bewertung von Wäldern dadurch bis zu einem gewissen Grade objektivieren, dass die Bewertung - soweit möglich - systematisch und vorzugsweise quantitativ durchgeführt wird" (KIRBY 1994, S. 185). Eine Kombination quantitativer und qualitativer Elemente ist also durchaus möglich. Wo qualitative Beschreibungen und gutachtliche Einschätzungen notwendig sind, können formale Vorgaben gemacht werden, die dem Außenstehenden den Entscheidungsgang erläutern und diesen somit nachvollziehbar machen.

Die genannten Eigenschaften einer naturschutzfachlichen Bewertung werden im Hinblick auf die Fragestellung, welchen Beitrag eine Erstaufforstung zur Biodiversität der Fläche an sich und der Umgebung leisten kann, in der hier vorliegenden Arbeit aufgegriffen. Da gutachtliche Feststellungen im Zusammenhang mit der Situationsanalyse nicht ausgeklammert werden können, ist ein subjektives Moment im Ergebnis nicht immer zu vermeiden. Andererseits ist durch die Übernahme von Wertermittlungsverfahren und Idealwerten aus anderen 
Planungsvorlagen (z.B. Standort- und Biotopkartierung) sowie gleichberechtigte Leitbilder ein Höchstmaß an Flexibilität und Objektivität zu erreichen (vgl. Kap. 3.4).

\subsection{Regionales Konzept als Grundlage für Einzelfallentscheidungen}

Diverse internationale Abkommen und Vereinbarungen befürworten eine generelle Steigerung des Waldanteils (vgl. Kap. 2.1). Auf europäischer Ebene wird diesem politischen Willen durch finanzielle Förderprogramme Ausdruck verliehen. Die Bundesregierung gibt die Aufgabe der Programmbildung an die Länder weiter. Da Aufforstungen einen eher lokalen bis regionalen Bezug besitzen, könnten nationale Programme auch nur generelle Aussagen machen. Folgerichtig regeln die Bundesländer durch eigene Programme und Richtlinien den Sachverhalt der Genehmigung und Förderung von Erstaufforstungen. Die Länder versuchen ihrerseits, die Bemühungen auf einzelne Regionen zu konzentrieren. Zum einen, da finanzielle Mittel zur Aufforstung nicht unbegrenzt zur Verfügung stehen. Zum anderen, da politische Erfolge schneller zu erreichen und eindrucksvoller zu demonstrieren sind, wenn der Aktionsschauplatz geographisch eingeschränkt wird.

In Deutschland besitzen Aufforstungen landwirtschaftlicher Flächen regionale Schwerpunkte. Eine Aufforstungswelle, die bundesweit zu einer signifikanten Änderung der Bewaldung führt, ist nicht gegeben. Daher besteht seitens des Naturschutzes und der Landschaftspflege auch keine Notwendigkeit zur Entwicklung eines nationalen Konzeptes oder eines Programms zur Steuerung von Erstaufforstungen. Anders wäre die Situation beispielsweise für Irland anzusprechen, wo die Erhöhung des Waldanteils um fast zwanzig Prozent in den 1990er Jahren eine nationale Steuerung durchaus sinnvoll erscheinen lässt. Die politische Situation sowie die geographische und naturräumliche Gliederung Deutschlands legen dagegen eine regionale Steuerung der Aufforstungsaktivitäten nahe. Die Landschaftsplanung kann hier als Fachplanung des Naturschutzes durch die Entwicklung von regionalen NaturschutzLeitbildern einen Beitrag zur Steuerung leisten (FINCK/SCHRÖDER 1997). Solche Leitbilder werden allerdings meist für das gesamte thematische Spektrum der Landschaftsplanung definiert. Die Waldvermehrung spielt häufig eine untergeordnete Rolle (vgl. Kap 3.2 und Tab. 4.1-3), weshalb die Entwicklungsziele nicht immer konkrete Hinweise auf einen möglichen Landnutzungswandel geben.

Eine weitere, ebenfalls häufig vorgeschlagene Methode der regionalen Konzeptionierung, ist die Ausweisung von Aufforstungsgewannen (AMMER 1997). Anträgen zur Aufforstung ist eine Genehmigung in diesen Landschaftsteilen sicher, weil landschaftliche Verträglichkeit bereits a priori festgestellt wurde. Das Gegenstück zum Aufforstungsgewann sind die Ausschlussflächen oder "Tabu-Bereiche“, in denen eine Neuanlage von Wald zu untersagen ist. Die Ausweisung derartiger Vorrang- oder Vermeidungsgebiete ist ein beliebtes Mittel zur Steuerung der Waldvermehrung in der Forstlichen Rahmenplanung. Dieses Planungsinstrument ist in Deutschland allerdings nicht flächendeckend vorhanden. Regionale Forstliche Rahmenpläne sind derzeit lediglich für 41,2 \% der Bundesfläche vorhanden oder in Bearbeitung. Gleichzeitig werden damit nur 34,1 \% der bislang unbewaldeten Flächen von diesem Planungsinstrument erfasst (BMELF 1999).

Außerhalb von Aufforstungsgewannen sind Erstaufforstungen prinzipiell genehmigungspflichtig ( $\$ 10$ BWaldG). Die zuständige Behörde hat öffentliches und privates Interesse gegeneinander abzuwägen. Der Blick sollte jedoch nicht nur auf die Fläche selbst gerichtet sein, sondern weit darüber hinaus greifen. Die Bewertung soll sich an der Förderung des Allgemeinguts Natur und Landschaft orientieren (KLEIN 1997a).

Die gesellschaftlichen Ansprüche, soweit sie den Raum betreffen, können durch Leitbilder dargestellt werden. Diese sind aus mehreren Gründen für einen begrenzten, regionalen 
Raum zu formulieren. Zunächst liegen in einer Region verhältnismäßig homogene Bedingungen vor. Die naturräumliche Einheit zeichnet sich durch ein bestimmtes Landschaftsbild, ein gemeinsames Klima, Geländerelief und Ähnliches aus. Die sozialen Strukturen der Landnutzer sind sich innerhalb dieses Raumes ähnlicher als im Vergleich zu anderen Räumen. Auch die Menschen, ihre Mentalität und ihr Heimatempfinden ähneln sich - zumindest soweit es sich um hier gebürtige Personen handelt. Neben der naturräumlichen Homogenität ist daher auch von einer regionalen Wertestruktur auszugehen. Deren langfristige Entwicklung spiegelt sich in der Landschaftsgeschichte, bzw. der historischen Entwicklung der Kulturlandschaft wieder. Natur, Kultur und Gesellschaft stehen in engen wechselseitigen Beziehungen und verändern sich miteinander.

Ein Nutzungswandel wirkt sich direkt auf das Landschaftsbild aus. Diese Auswirkung ist dort erfahrbar, wo die Landschaft „überschaubar“ ist - also im lokalen Bereich. Die Bewertung der Aufforstung durch den Menschen und die Gesellschaft hat indes einen über den lokalen Horizont hinausgehenden, meist regionalen Bezugsraum. Der ortsansässige Beobachter im Thüringer Becken wird die landschaftliche Veränderung durch eine Aufforstung nicht an jenem Landschaftsbild messen, welches er vielleicht bei einem Ausflug in den Hochharz kennen lernte. Ein Vergleich mit der Landschaft der umliegenden Gemarkungen wird dagegen automatisch stattfinden, da das persönliche Landschaftsbild durch diesen größeren Radius bestimmt wird.

Auch unter den Gesichtspunkten des Arten- und Biotopschutzes ist die Entwicklung regionaler Leitbilder naheliegend. Die Region ist der Bezugsraum, in welchem ein bestimmtes Set an Arten und Biotopen als Inventar feststellbar ist. Hier können Bemühungen um Biotopverbund und -pflege Erfolge erzielen, die den einzelnen Populationen zugute kommen. Der genetische Austausch zwischen diesen Populationen ist auf regionaler Ebene möglich und notwendig, um den langfristigen Erhalt der Arten zu sichern. Der regionale Maßstab ermöglicht darüber hinaus ein Monitoring der Arten und somit eine Erfolgskontrolle für den Naturschutz. Auf lokaler Ebene würde die Bezugsgröße zur Einordnung der Ergebnisse fehlen. Ein landesweiter Erfassungsraum würde dagegen die Bedeutung der Einzelvorkommen relativieren und die regionalen Besonderheiten (zumindest im unteren bis mittleren Bereich der Gefährdungskategorien) vernachlässigen. Außerdem gilt auch für den Naturschutz, dass eine regionale Konzentration und Abstimmung der Aktivitäten besonders schnell zu vorzeigbaren Ergebnissen führt.

Schließlich spricht auch die Planungspraxis für die Entwicklung von Leitbildern auf regionaler Ebene, da hier bereits flächengenaue Aussagen gemacht werden können und da für alle von der Landnutzung betroffenen Fachbereiche Planungskompetenzen oder Behörden angesiedelt sind. Somit können Zielkonflikte angesprochen und gelöst, komplementäre Entwicklungen gefördert und interdisziplinäre Zielvorstellungen entwickelt werden. Die Integration der Bevölkerung in die Leitbildentwicklung ist auf dieser Ebene bereits möglich, wodurch Akzeptanzproblemen schon früh begegnet werden kann.

Der Landschaftsplan dient unter anderem der Implementierung des regionalen naturschutzfachlichen Leitbildes und kann als solcher Vorschläge für aufzuforstende Flächen oder Aufforstungsgewanne machen. Dieser Fachplan der Naturschutz- oder Landespflegebehörde ist vergleichbar mit dem Forstlichen Rahmenplan. Landschaftspläne liegen für große Bereiche des Bundesgebietes, allerdings noch nicht flächendeckend vor (AMMER 1997). Leider sind sie in ihren Zielaussagen hinsichtlich der Aufforstungsbereiche nicht immer deckungsgleich mit den forstlichen Rahmenplänen.

Das entscheidende Manko von Fachplänen und regionalen Konzepten ist deren Statik, die eine schnelle Reaktion auf wechselnde Zielsetzungen und Eigentümerinteressen verhindert (BROGGI 1999). Selbst wenn es gelingt, die augenblicklichen Interessen der Bevölkerung über ein partizipatives Verfahren zu berücksichtigen, entsteht daraus mit der Festlegung von Gewannen oder Tabubereichen eine statische Vorgabe. Wechseln die Interessen der Eigentümer, etwa durch Restrukturierung des Betriebes oder durch Eigentumsübergang, so kann eine Aufforstung plötzlich dort gewollt sein, wo der Plan keine vorsieht - und umgekehrt. Eine Revision der Pläne in regelmäßigen Abständen, etwa alle zehn Jahre, wäre 
zwar theoretisch möglich, würde jedoch in der Praxis einen enormen Aufwand bedeuten, da die Diskussionen mit den Grundeigentümern und zwischen den beteiligten Fachdisziplinen stets neu zu führen wären. Dies entspricht dem Ansatz der „offenen Planung“, bei der eine Festlegung von Details vermieden wird.

$\mathrm{Zu}$ den Aufgaben der Raumplanung gehört es, die Interessen der Raumnutzer und die Auswirkungen der Flächennutzungen zu prüfen, Interessenkonflikte aufzuzeigen und Lösungen vorzuschlagen. Die Landschaftsplanung beschäftigt sich als Fachplanung des Naturschutzes in diesem Zusammenhang insbesondere mit den ökologischen Auswirkungen der unterschiedlichen Flächennutzungen. Sie entwickelt landschaftliche Leitbilder und schlägt dingliche Maßnahmen vor, beispielsweise die Neuanlage von Biotopen und Strukturelementen. Auch die Forstliche Rahmenplanung kann Vorschläge zur Landschaftsentwicklung machen, soweit diese in Zusammenhang mit der forstlichen Bewirtschaftung stehen. Statt regionaler Leitbilder verwendet die forstliche Fachplanung eher die Ausweisung besagter Aufforstungsgewanne und Ausschlussflächen als Instrument. Eine Übernahme der Bereiche in die Landschaftsplanung ist möglich. Noch sinnvoller ist allerdings eine gemeinsame Festlegung dieser Bereiche durch die betroffenen Fachdisziplinen.

In der Planungspraxis stoßen Aufforstungsgewanne und Ausschlussflächen häufig auf Akzeptanzprobleme seitens der Grundeigentümer, die aus mangelnder Partizipation in der Planungsphase resultieren. Versteht sich die Raumplanung jedoch als Prozess der interdisziplinären Lösungsfindung, so scheint es folgerichtig, dass auch für den speziellen Bereich der Erstaufforstungsplanung nach einer gemeinsamen Zielformulierung gesucht wird (PREEN 1996, AMmER 1997). Das Handicap besteht dabei in der vergleichbar geringen Bedeutung der Erstaufforstung gegenüber anderen Aufgabenstellungen in der Raumplanung (Siedlungsentwicklung, Infrastruktur, u.a.) bei gleichzeitig hohem Diskussionsbedarf und Planungsaufwand. Beteiligte Fachbereiche bringen bereits eigene Vorstellungen für die räumliche Entwicklung in Form von fachlichen Entwicklungsplänen mit. Die fachspezifischen Leitbilder setzen dabei ganz unterschiedliche Schwerpunkte. Gegenüber der bisher üblichen, monodisziplinären Formulierung eines Erstaufforstungsleitbildes durch den Forstbereich, steigt die Bedeutung des Leitbildes mit jeder zusätzlich beteiligten Fachdisziplin. Ein interdisziplinäres Erstaufforstungsleitbild würde allerdings nur einen Kompromiss aus den sektoralen Zielformulierungen von Land-, Forst-, Wasserwirtschaft, Naturschutz, Siedlungsplanung, Tourismus und anderen Raumnutzern darstellen. Gleichzeitig müssten Bedingungen formuliert werden, unter denen dieses Erstaufforstungsleitbild Vorrang gegenüber anderen Interessen erhält, wenn Zielkonflikte auftreten.

Es ist fraglich, wie viele Fachdisziplinen sich mit dem Gedanken eines interdisziplinär festgesetzten, allgemeingültigen Leitbildes anfreunden könnten. Allein im Naturschutz bestehen oft mehrere, teilweise konkurrierende Leitbilder nebeneinander (vgl. Kap. 3.2). Für andere Landnutzergruppen, wie z.B. die Forstwirtschaft und die Landwirtschaft, sieht es ähnlich aus. Besser wäre es daher, die unterschiedlichen Leitbilder und Zielvorstellungen der beteiligten Raumnutzer in einem Verfahren zu berücksichtigen, auf welches man sich im interdisziplinären Austausch verständigt. Dieses Verfahren sollte dann anhand einer Einzelfallentscheidung die Bedingungen und Entwicklungspotentiale abfragen und innerhalb eines vorgegebenen Bewertungsrahmens einordnen. Bei der Entwicklung eines derartigen Instrumentes müssten Grundsatzdiskussionen nur einmal geführt werden. Einzelfallentscheidungen würden transparenter, kalkulierbarer und könnten mit geringerem Aufwand durchgeführt werden.

Grundsätzlich können in Naturschutz und Landschaftspflege zwei verschiedene Planungsweisen unterschieden werden. Die deduktive Methode der Planentwicklung führt über verschiedene politische Ebenen, von der internationalen Gemeinschaft bis zur Gemeindeebene. Dagegen befasst sich die Einzelfallplanung mit einer speziellen Problematik lokalen Ausmaßes. Sie nimmt ebenfalls Rücksicht auf die gesetzlichen Reglementierungen und verbindlichen Festlegungen übergeordneter Ebenen, kann jedoch ein anderes Leitbild als diese verfolgen. 
Die top-down-Entwicklung von Plänen aus dem Bereich der Landschaftsplanung, verlangt auch eine deduktive Entwicklung der Leitbilder. Sie schließt eine stetige Verfeinerung und Präzisierung der Ziele ein. Sind die Leitbilder der oberen Planungsebenen wenig aussagekräftig oder fehlt diesen Plänen die Verbindlichkeit, so kommt es zu mangelhafter Stringenz der Zielaussagen. Andererseits dürfen die Vorgaben nicht zu eng gefasst sein, da die regionalen und lokalen Besonderheiten geschützt werden müssen. Nichts liegt dem Naturschutz ferner, als eine Nivellierung der Verhältnisse auf großer Fläche.

Eine konsistente Erstellung von Leitbildern, Konzepten und Programmen im Sinne einer deduktiven Hierarchie ermöglicht hingegen eine Steigerung der politischen Umsetzungsfähigkeit. Der landwirtschaftliche Strukturwandel in der EU führt - in welchem Umfang sei momentan dahingestellt - zur Umwandlung einer sehr großen Fläche. Der Naturschutz hat mit den Schwerpunktfeldern standörtlicher Ressourcenschutz, biotischer Ressourcenschutz und Landschaftsästhetik drei unterschiedliche Ansprüche zu vertreten. Gelingt es ihm, Leitbilder und Konzepte zu erstellen, die diese Ansprüche verdeutlichen, so kann er dadurch effektiv auf die Entwicklung jener Flächen Einfluss nehmen, die außerhalb von Schutzgebieten liegen und daher nicht per se dem Naturschutz gewidmet sind.

Bei der Umsetzung theoretischer Naturschutzideen in konkrete Vorhaben stellt sich häufig die Frage, ob der Arten- oder der Biotopschutz das grundsätzliche Vorgehen leiten soll. Gerade bei Einzelfallentscheidungen, wie sie auch im Rahmen von Genehmigungsverfahren vorkommen, greift die Argumentation gerne auf grundsätzliche Leitbilder zurück. Der Artenschutz, der sich im Einzelfall auf bestimmte, meist besonders gefährdete oder mit positiven Bildern besetzte Arten konzentriert, bringt trotz seiner Praxistauglichkeit eine gewisse Beschränkung mit sich. Er berücksichtigt eben nur einen kleinen Teil der Fauna und Flora. Von den Insekten werden beispielweise gerade einmal 4000 bis 5000 Arten in solchen Konzepten berücksichtigt, was maximal $10 \%$ der heimischen Insektenfauna repräsentiert (JEDICKE 1994). Eine Möglichkeit, den Kreis der profitierenden Arten zu vergrößern, besteht in der Identifikation und dem Schutz von Leitarten. Stehen diese für ein bestimmtes Set an Biotopmerkmalen und eine besondere Zustandsqualität, so können vergesellschaftete Arten von den Schutzbemühungen um diese Leitart profitieren. Dieses Vorgehen leitet über zum Biotopschutz, der bestimmte Biotope als typische und/oder gefährdete Elemente der Kulturlandschaft aus Gründen der Landschaftspflege, aber auch wegen ihrer Bedeutung für die darin vorkommenden Tier- und Pflanzenarten in Qualität und Umfang fördern möchte. Für die Landschaftsplanung ist der Biotopschutz außerdem umsetzungsfreundlicher. Zum einen sind die Biotope ganzjährig und mit geringerem Aufwand erfassbar als einzelne Arten, was sich im Monitoring und in der Zielkontrolle positiv auswirkt. Zum anderen ist deren Präsenz auch für den Laien augenscheinlich, wodurch der Naturschutz an Plastizität gewinnt.

Zur Erreichung prinzipieller Ziele der Landschaftsplanung ist eine Standardisierung der Verfahren und Entscheidungsfindungen unumgänglich. An diesen Prozess sind allerdings bestimmte Ansprüche zu richten (KNICKREHM et al. 2000), damit die Planung einen hohen Effizienzgrad in der Umsetzung erreichen kann. Dazu gehört eine möglichst pragmatische Ausrichtung der Standardisierung an den gegebenen Bedingungen. Vorhandene Datenquellen (z.B. Biotopkartierungen, Standortkartierungen, Arterfassungsprotokolle) sind zu erschließen und zu integrieren, damit zusätzliche Aufnahmen minimiert und Doppelerfassungen vermieden werden. Der Formalisierungsgrad des Vorgehens soll sich an der Komplexität der Fragestellung orientieren. Die Bewertung der Umwandlung geschützter Landschaftsbestandteile kann beispielsweise unterbleiben, da sie nicht genehmigungsfähig scheint und daher nicht praxisrelevant ist. Ein problemadäquates Vorgehen spiegelt sich in der Strukturierung des Vorgehens unter gleichzeitiger Belassung von Spielräumen zur Adaption an die regionalen Verhältnisse wieder. Schließlich ist an die Instrumente der Landschaftsplanung die Forderung nach einem hohen Maß an Flexibilität zu stellen (KNICKREHM et al. 2000), damit auf lokale Prozesse und Umsetzungsbedingungen eingegangen werden kann. Der Ruf nach Ermessensspielraum für den Planer wird laut. All diese Anforderungen sprechen für die Entwicklung von Verfahren und Vorgehensweisen, die grundsätzliche Ziele des Naturschutzes und der Landschaftspflege im Rahmen ihrer 
methodischen Struktur integrieren, gleichzeitig aber die Flexibilität zur Berücksichtigung regionaler Bedingungen und spezieller Interessen gewährleisten.

Gemäß ihrer bestimmenden Elemente - Umwelteinflüsse, Standortbedingungen, Ausstattung und Nutzung - muss die Landschaftsplanung alle Einflussgrößen berücksichtigen. Auf lokaler Ebene (Gemeinde oder Gemeindeverbund) kann eine Integration der ökologischen, ökonomischen und sozialen Ansprüche an die Nutzung der Ressourcen mit ausreichender Flächengenauigkeit angegangen werden, um auch Grundeigentümer ausreichend zu berücksichtigen. Letzteres erfordert aber die Partizipation des Eigentümers am Planungsprozess, damit seine Ziele berücksichtigt werden können.

Eine andere Möglichkeit, eine entsprechende Planungsverbindlichkeit bis auf die Ebene der Einzelparzellen zu erreichen, besteht in der Kombination von regionalen Plänen mit transparenten Entscheidungsmodellen für die Landnutzung. Dazu gehört beispielsweise die Definition von Entscheidungskriterien für Genehmigungsverfahren bei Landnutzungsumwandlungen. Dabei tritt die langfristige Festlegung auf regionale Ziele in den Hintergrund, zugunsten einer gesteigerten Operationalität. BROGGI (1999, S. 23) formuliert: „Im Sinne des Naturschutzes sind (darum) Überlegungen vorrangig, wie die Naturschutz-Anliegen effizient integriert werden könnten. Sie stützen sich auf eine generelle Vorsorgepolitik im Bereich der Nutzungen wie in der konkreten Integration des Naturschutzes in Prozesse der Planung."

Ein transparentes Entscheidungsmodell für die planerische Praxis soll mit der vorliegenden Arbeit zur naturschutzfachlichen Evaluation von Erstaufforstungen vorgeschlagen werden. Es bietet eine Möglichkeit, die Folgen eines beabsichtigen Nutzungswandels für den Artenund Biotopschutz sowie die Landschaftsveränderung zu prognostizieren und zu bewerten. Dabei werden einerseits politische Willenserklärungen der nationalen und internationalen Ebene berücksichtigt. Andererseits beinhaltet das Vorgehen eine regionalisierte Bewertung der Erstaufforstungsmaßnahme, wie sie von verschiedenen Autoren (z.B. REIF 1997, KLEIN 1997a, AMMER 1997) gefordert wird. Das Verfahren zeigt darüber hinaus, wie der Entscheidungsprozess innerhalb von Aufforstungs-Genehmigungsverfahren systematisiert und transparent gestaltet werden kann.

Schließlich ist das neue Verfahren der Aufforstungsevaluation richtungsweisend in der Flexibilität, mit der auf wechselnde Gesellschafts- aber vor allem auch Eigentümerinteressen eingegangen werden kann. Ersteres wird durch veränderbare Bewertungsmaßstäbe und -klassen erreicht, letzteres durch die Loslösung von starren Planvorgaben. Es zeigt dem Grundeigentümer darüber hinaus, welche Faktoren des Genehmigungsverfahrens er durch entsprechende Gestaltung und Zusammensetzung der Anpflanzung oder durch die Flächenwahl beeinflussen kann. 


\section{Theoretischer Rahmen und Methodik}

\subsection{Planungsmethoden für den Naturschutz}

Die gesetzlichen Bestimmungen des Bundes und der Länder statten den Naturschutz, genauer gesagt die Fachdisziplin Landespflege mit dem Instrument der Landschaftsplanung aus. Dieses Instrument kann unterschiedliche Formen annehmen und auf verschiedenen Ebenen wirken. Zentrales Anliegen ist es dabei, auf eine Sicherung und Entwicklung der Landschaft als Lebensraum der Gesellschaft derart hinzuwirken, dass die ökologischen Werte erhalten bleiben und der Naturhaushalt gleichzeitig optimal im Sinne der ökologischen Nachhaltigkeit genutzt werden kann. Darüber hinaus gilt ein besonderes Augenmerk der Landschaft als Erholungsraum für den Menschen. Die Landschaftsstruktur als visuell erfassbarer Teil der Umgebung ist daher ebenfalls Bestandteil der Landschaftsplanung. Der gesetzgeberische Auftrag zur Landschaftsplanung erging erstmals durch das Bundesnaturschutzgesetz (BNatSchG) von 1976 und wurde in den folgenden Jahren durch die Naturschutz- und Landespflegegesetze der Länder näher ausgeführt. Mit diesem Schritt löste sich die Gesellschaft vom bis dato praktizierten konservierenden Flächenschutz. Fortan wurde die Vorsorge zum zentralen Handlungsziel der Fachdisziplin.

Die Landschaftsplanung versteht die menschliche Umwelt als ökologisch-strukturelles Wirkungsgefüge und sucht dieses hinsichtlich der nachhaltigen und optimalen Nutzung durch die Gesellschaft zu erfassen, bewerten und beplanen. Dabei stellen Formen der Oberfläche, Nutzungstypen oder die Art der Ressourcen keine Abgrenzungskriterien dar. Die Landschaftsplanung muss im Gegenteil nicht nur die unterschiedliche Strukturen in sich vereinen, sondern auch die Zusammenhänge und Wechselwirkungen zwischen diesen Strukturen deuten und integrieren.

\section{Das Feld der Landschaftsplanung}

Die Planungsaktivitäten der Landespflege können anhand der Zielgruppen in querschnittsorientierte und sektorale Planungen unterschieden werden (Tab. 3.1-1)

Als querschnittsorientierte Planungen sind jene $z u$ verstehen, welche unter den verschiedenen Gesichtspunkten der Ökologie, Ökonomie und der Sozialwissenschaft die Verträglichkeit unterschiedlicher Nutzungsansprüche untersuchen und bewerten. Als Ziel kann eine unter ökologischen Aspekten optimale Nutzungskombination angesehen werden. Diese Resultate ergeben, in einem Planungsbericht zusammengefasst, schließlich den Fachbeitrag der Landespflege zur räumlichen Entwicklung auf der jeweiligen politischen Ebene (Gesamtplanung auf Bundes-, Landes-, regionaler oder kommunaler Ebene).

In den sektoralen Planungen zum Naturschutz und der Erholung soll die Landespflege nicht nur eine schnelle Umsetzung wissenschaftlicher Erkenntnisse des Fachbereiches verfolgen, sondern sie muss auch die eigenen fachlichen Zielsetzungen in die Pläne einarbeiten. Andere Fachverwaltungen und Fachplanungsbehörden sind anschließend gehalten, die auf diese Weise postulierten und im Rahmen der Planung konkretisierten Ziele von Naturschutz und Landschaftspflege $\mathrm{zu}$ berücksichtigen, $\mathrm{zu}$ unterstützen und gegebenenfalls umzusetzen, wobei allerdings keine Verpflichtung hierzu besteht.

Zur Fachplanung des Naturschutzes ist auch die Biotopverbundplanung zu rechnen. NITSCHE und NITSCHE (1994) beschreiben diese als wichtigstes Planungsinstrument des Naturschutzes neben der Landschaftsplanung. Bemerkenswert ist, dass die Biotopverbundplanung diese hohe Wertschätzung trotz ihres verhältnismäßig jungen Alters erlangen konnte. Erst seit den 1980er Jahren kennen wir derartige Planungen und in vielen Regionen besteht in dieser Hinsicht noch hoher Nachholbedarf. Die hohe Wertschätzung begründet sich weniger auf der großen Verbreitung dieser Planungsart als vielmehr auf der guten Übertragbarkeit der Planungsergebnisse in nachfolgende und fachfremde Planungen. 
Auch die Bedeutung, die dem Biotopschutz heute von der Gesellschaft beigemessen wird, trägt zur Anerkennung der Naturschutzplanung bei.

Tab. 3.1-1: Die Beiträge des Naturschutzes zur Landschaftsplanung auf verschiedenen Ebenen

\begin{tabular}{|c|c|}
\hline Querschnittsorientierte Planung & Sektorale Planung \\
\hline $\begin{array}{l}\text { Beitrag zur Gesamtplanung auf } \\
\text { - Bundesebene } \\
\text { - Landesebene } \\
\text { - Regionaler Ebene } \\
\text { - Kommunaler Ebene }\end{array}$ & $\begin{array}{l}\text { - Naturschutzplanung } \\
\text { (z.B. Arten- und Biotopschutzkonzepte, } \\
\text { Schutzgebietskonzepte) } \\
\text { - Erholungsplanung } \\
\text { - Beitrag zu anderen Fachplanungen } \\
\text { - Grünplanung } \\
\text { - Landschaftsplanerische Gutachten und } \\
\text { Stellungnahmen } \\
\text { - Fachliche Unterlagen zu außersektoralen } \\
\text { Planungen }\end{array}$ \\
\hline
\end{tabular}

Die Biotopverbundplanung ergänzt das Konzept der differenzierten Bodennutzung in vier Ansätzen (JEDICKE 1994, S. 85), die unterschiedliche Adressaten ansprechen:

1) großflächiges Schutzgebietssystem als Dauerhabitat stabiler Populationen

2) Netz mit Trittsteinbiotopen als Ausgangsbiotope und Zwischenstationen für den genetischen Austausch zwischen benachbarten Populationen

3) Verbund punktförmiger Lebensräume durch lineare Landschaftselemente (z.B. Hecken, Bäche) als Korridorbiotope für die Ausbreitung der Populationen

4) flächendeckende Extensivierung der Flächennutzung

Diese mehrfache Zielkonzeption spricht gleichzeitig verschiedene Ebenen der Landschaftsplanung an, vom Landschaftsprogramm auf Landesebene bis zur großmaßstäblichen Grünordnungs- und Bauleitplanung. Eine Konkretisierung auf wenige Leitarten und -biotope einer Region scheint aus strategischen Gründen angeraten. Sie erleichtert die Integration der Biotopverbundplanung in andere Pläne. Darüber hinaus bleibt das Konzept durch die Konzentration auf bestimmte Arten und Biotope übersichtlich, was wiederum die Akzeptanz durch Fachbehörden und Gesellschaft zu fördern vermag.

Eine Sonderform der sektoralen Planung stellt das landschaftsplanerischen Gutachten dar. Es ist zu erstellen, wenn sich bestimmte Vorhaben im Bereich von Nutzungsumwandlungen, Siedlungstätigkeiten oder Infrastrukturmaßnahmen ankündigen, die zum Zeitpunkt der Erstellung eines übergeordneten Landschaftsplanes noch nicht, oder nicht in vollem Umfang absehbar waren und folglich nicht mitbehandelt wurden. Derartige Gutachten oder Stellungnahmen behandeln in erster Linie gestalterische Elemente des Landschaftsbildes sowie Fragestellungen nach ökologischen Zusammenhängen und Auswirkungen auf andere Nutzungen, bzw. den Naturhaushalt. Aus dieser Form der landschaftsplanerischen Gutachten und Stellungnahmen entwickelte sich auch die Umweltverträglichkeitsprüfung, welche nach einem entsprechenden Gesetz (UVPG) für alle dort aufgeführten privaten und öffentlichen Maßnahmen durchgeführt werden muss.

Schließlich kann der sektoralen Planung auch die Erstellung von Unterlagen für außersektorale Planungen zugeordnet werden. Hier findet quasi im Rahmen einer Informationsbereitstellung eine Vermittlung eigener Daten und Einschätzungen statt, die dann von anderen Fachdisziplinen aufgegriffen werden sollen. Diese Form der Zielvermittlung ist sicherlich die unverbindlichste, kann sich aber dennoch als sehr fruchtbar erweisen, wenn die Kooperation mit anderen Fachbehörden gut entwickelt ist. 


\section{Einordnung des dargestellten Verfahrens}

Aus der Komplexität der landschaftsplanerischen Ansprüche ergibt sich die Notwendigkeit zur Betrachtung von Wechselwirkungen zwischen Medien und Schutzgütern, also zwischen Boden, Wasser, Luft, Klima, Pflanzen- und Tierwelt, aber auch der Betrachtung von Landschaftsbild und Erlebniswert einer Landschaft nach gesellschaftlicher Wertschätzung. Natur und Landschaft müssen als ein System betrachtet und behandelt werden, in welchem unterschiedliche Komponenten in Abhängigkeiten und Beziehungen zueinander stehen.

Es sollen die augenblicklichen, aber insbesondere auch alle geplanten Nutzungen und Beeinflussungen dieses Systems erfasst und hinsichtlich ihrer Rückwirkungen auf das System beurteilt werden. Die Landschaftsplanung findet dabei schutzgutübergreifend und querschnittsorientiert statt. Dadurch wird allerdings eine Abgrenzung von Untersuchungseinheiten wesentlich erschwert. Nur im Ausnahmefall oder mit Bezug auf bestimmte Untersuchungsfragen können Abgrenzungen aus Teildisziplinen übernommen werden.

Die Betrachtung von Natur und Landschaft als System findet sich in allen oben beschriebenen Planungsarten der Landespflege wieder. Dennoch kann nicht auf eine allgemeingültige Systemdefinition als Grundlage für sämtliche Planungen zurückgegriffen werden. Vielmehr ist das jeweils zu betrachtende System genau zu beschreiben und in dieser Beschreibung auf die Ziele der jeweiligen Planungsart abzustimmen.

Für alle querschnittsorientierten und für jene sektoralen Planungen, die in nachfolgenden Planungen anderer Fachdisziplinen berücksichtigt werden sollen, erfolgt die Vermittlung der landespflegerischen Ziele auch unter der Vorstellung von Leitbildern und Entwicklungskonzepten. Diese können eine deduktive Hierarchie bilden, in welcher bei einem Ebenenwechsel jeweils die Unterziele aus den Oberzielen abgeleitet werden (näheres in Kap. 3.2). Gleichzeitig findet eine Konkretisierung in Bezug auf den geographischen Raum und den fachlichen Inhalt statt.

Fachgutachten und Stellungnahmen - zu denen auch die Beurteilung eines Erstaufforstungsantrages durch die Naturschutzbehörde zu zählen ist - greifen Leitbilder der eigenen und gegebenenfalls auch anderer Fachplanungen auf, um dann anhand des Einzelfalles zu einer Empfehlung zu kommen. Im Idealfall repräsentiert die Landschaftsplanung sämtliche Belange von Naturschutz und Landschaftspflege im Sinne einer Zusammenfassung und mit einer situationsabgestimmten Prioritätensetzung. Dadurch wären die Ergebnisse unmittelbar in die anfallenden Planungsentscheidungen und Genehmigungen einzubringen. Beispielsweise könnte ein Forstbeamter, der im Rahmen seiner Tätigkeit einen Aufforstungsantrag bescheiden soll, die Ziele aus dem zugehörigen Landschaftsplan übernehmen und bei seiner Beurteilung ausreichend berücksichtigen. Hier zeigt die Planungspraxis allerdings, dass dies in der Regel nicht möglich ist. Immer wieder stoßen Anträge auf nicht vorhergesehene Einwände seitens des Naturschutzes. Diese Einwände stammen in den meisten Fällen aus dem Bereich des Biotop- und Artenschutzes, obwohl dem Naturschutz doch gerade in diesen Bereichen an allgemeinverständlichen, übergreifenden und regionalisierten Schutzkonzepten sehr gelegen sein müsste. Da diese Konzepte aber häufig fehlen, sind Probleme vorprogrammiert.

\section{Bewertungsmaßstäbe}

Fachgutachten und Stellungnahmen greifen die Umweltqualitätsziele aus den Landschaftsplänen und -programmen auf und entwickeln daraus Maßstäbe zur Beurteilung von Folgewirkungen zu bewertender Vorhaben. Selbstverständlich gilt auch in diesem Falle, dass die Maßstäbe den jeweiligen Bedingungen angepasst sein sollen. Andererseits kann verlangt werden, dass die Maßstäbe für ähnliche Sachverhalte innerhalb des Geltungsraumes desselben Landschaftsplanes übertragbar sind.

Das in dieser Arbeit vorgestellte Verfahren zur naturschutzfachlichen Bewertung von Erstaufforstungen stellt in diesem Zusammenhang ein Instrument dar, welches dem 
Naturschutz im engeren Sinne, aber auch dem entsprechend geschulten Bearbeiter einer verwandten Fachdisziplin die Bewertung einer Nutzungsumwandlung von landwirtschaftlich genutzter Fläche in Wald nach naturschutzrelevanten Gesichtspunkten ermöglicht. Zur Anwendbarkeit des Verfahrens wird in Kapitel 3.2 Stellung genommen. Das Verfahren selbst reiht sich in den oben beschriebenen Planungsarten des Naturschutzes ein zwischen dem landschaftsplanerischen Gutachten und der Erstellung von Unterlagen für außersektorale Planungen. Es gelten für dieses Verfahren weitgehend die gleichen Grundzüge, die BUCHWALD für die Wirkungs- und Risikoanalyse als Bestandteile der Landschaftsplanung beschreibt (BUCHWALD/ENGELHARDT 1980). Als Grundlage der Verfahrensanwendung erfolgt zunächst eine Zustandserfassung, in der die wesentlichen Merkmale des zu betrachtenden Systems erfasst werden. Als ökologisch-gestalterische Planung geht die Landschaftsplanung von den aktuellen Flächennutzungen aus und projiziert die geplanten Änderungen in das entstandene Bild hinein. Sie erfasst und analysiert sodann die ökologischen Auswirkungen auf den Standort sowie die umgebenden Nutzungsflächen/Ökosysteme. Schließlich gibt sie eine Einschätzung der Entwicklung wieder. Das hier behandelte Verfahren greift die zentralen Aspekte der Landschaftsplanung auf, wenn auch der Ressourcenschutz nur indirekt mitbehandelt wird. Ökonomische Fragestellungen bleiben ebenso außen vor wie beispielsweise Fragen der Betriebsstruktur oder der technischen Erschließung des Grundstückes. Im Zentrum der Aufmerksamkeit steht die Biodiversität der betrachteten, potentiellen Aufforstungsfläche und deren Beitrag zur Biodiversität der umgebenden Ökosysteme. Außerdem geht es in diesem Schritt nicht um die Bewertung der augenblicklichen Flächennutzung, sondern um die Bewertung einer geplanten, künstlichen Bewaldung.

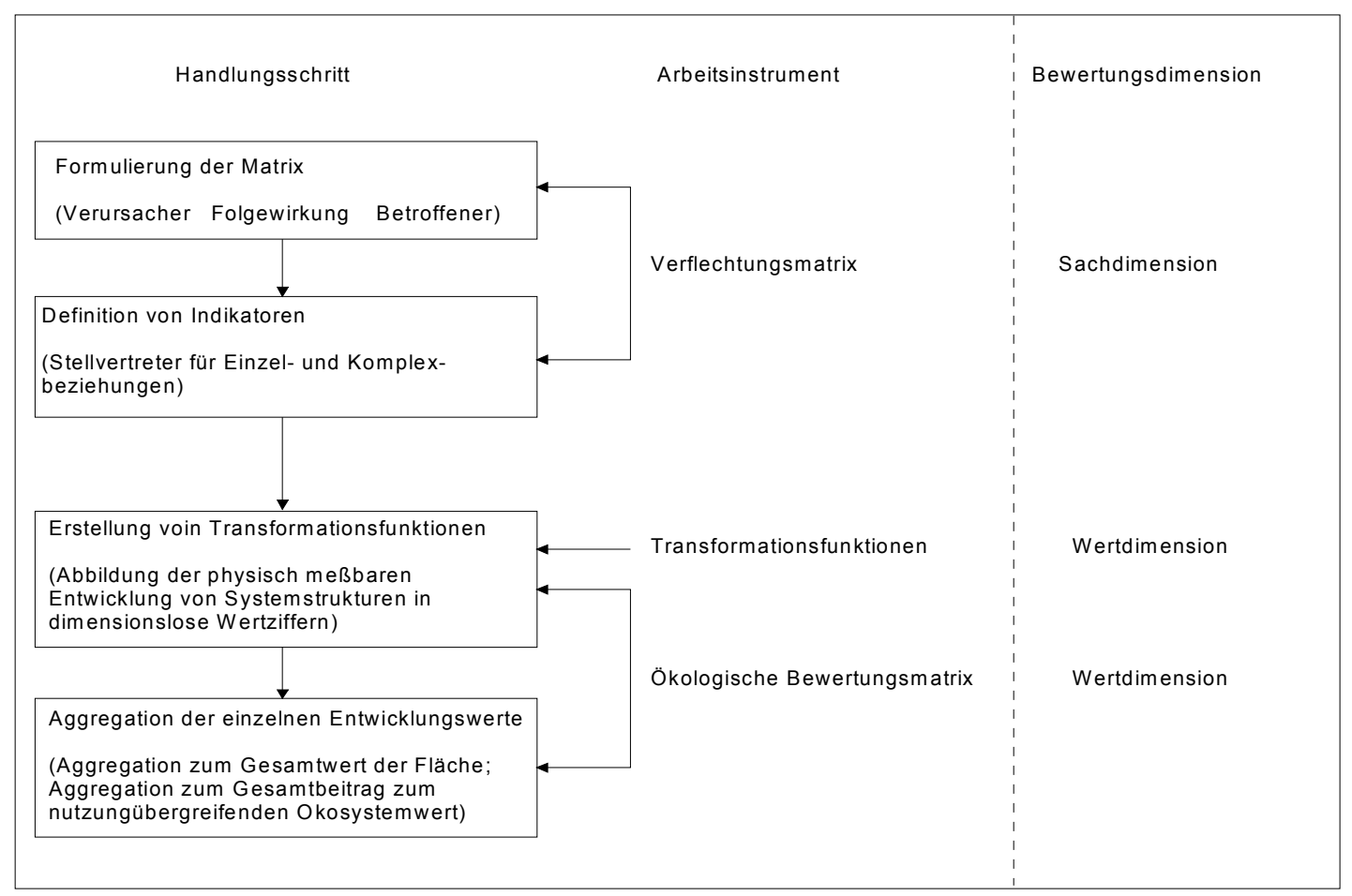

Abb. 3.1-I: Flächennutzungsevaluation als operationalisiertes Planungsinstrument auf der Grundlage einer ökologischen Wirkungsanalyse; verändert; nach BECHMANN (1977) verändert in BUCHWALD/ENGELHARDT (1980)

Daraus ergibt sich eine Modifizierung des bei BUCHWALD beschriebenen Verfahrens der ökologischen Wirkungsanalyse nach folgendem Muster: Das Verfahren beginnt mit der Erstellung einer Verflechtungsmatrix. Diese soll die Wirkungskette Verursacher $\rightarrow$ Folgewirkung $\rightarrow$ Betroffener wiedergeben und die wichtigsten Einzel- oder Komplexbeziehungen hervorheben (Abb. 3.1-1). Die Matrix gilt gleichzeitig als Hilfsmittel zur 
Definition von Indikatoren für die gefundenen Wechselwirkungen (Kap. 3.5). Auf diesen aufbauend werden sodann Transformationsfunktionen beschrieben zur Abbildung der physisch messbaren Parameter in dimensionslose Wertziffern. Mit Hilfe dieser Transformationsfunktionen können die Veränderungen im Ökosystem in das abstrakte Beziehungsgefüge des Modells übertragen und nachvollziehbar gemacht werden.

Die dimensionslosen Wertziffern können sodann für jede geplante Aufforstung und nach regionalen Kriterien nachvollziehbar ermittelt werden. Der regionale Bezug besteht in der Ableitung der Teilziele (=Kriterien) aus den jeweiligen Planungsgrundlagen, z.B. ein im Regionalplan angegebenes Flächenutzungsprozent als Entwicklungsziel, an dem die Veränderung durch den Nutzungswandel gemessen wird. Eine gutachtliche Stellungnahme könnte allein auf dieser Grundlage bereits Aussagen machen zur erwarteten Entwicklung der Biodiversität, bzw. den Auswirkungen auf den Arten- und Biotopschutz. Eine konkrete Befürwortung oder Ablehnung der geplanten Maßnahme bedingt aber die Einordnung in einen fundierten Bezugsrahmen. Dieser wird geschaffen durch die mehrfache Durchführung des Verfahrens anhand von geplanten und durchgeführten Erstaufforstungen in unterschiedlichsten Verhältnissen der Region. Hierbei kommt es insbesondere auf die unterschiedlichen Grade und Arten der Verflechtungen mit anderen Biotoptypen an. Die Anzahl der notwendigen Untersuchungen ist stark von der Heterogenität des Planungsgebietes abhängig. Da sich die zur Erstaufforstung anstehenden landwirtschaftlichen Flächen aber in der Regel sehr ähneln, dürften schon wenige Untersuchungen ein Ergebnis liefern, welches eine Befürwortung oder Ablehnung der Maßnahme auf dieser Datengrundlage ermöglicht.

\subsection{Leitbilder des Arten- und Biotopschutzes}

In der Raumplanung werden Leitbilder im Allgemeinen eingesetzt, um Entwicklungsziele zu illustrieren. Sie haben immer etwas Visionäres und Ideales in sich. Die Aussagen eines Leitbildes zielen auf die Zukunft und beziehen sich nicht unmittelbar auf die augenblickliche Situation. Leitbilder sollen langfristig geltende Vorstellungen über den erwünschten Zustand eines Raumes beschreiben. Die Landschaft kann sich diesen Vorstellungen unter bestimmten Voraussetzungen annähern. Von der Vision zur Realität ist es aber gemeinhin ein langer Weg. Dieser zieht sich von den überregionalen (international, national) Planungsebenen, über die regionalen bis zur lokalen Ebene. Dabei werden jeweils Unterziele aus Oberzielen abgeleitet und es entsteht eine deduktive Hierarchie. Es erfolgt in diesem Rahmen eine zunehmende Konkretisierung der Ideen und Ziele. In der Literatur wird der Begriff Leitbild mit einer enormen Bandbreite von der Vision bis zum raumkonkreten Pflegeziel verwendet (HAAREN 1999). Eine nähere Betrachtung der deduktiven Leitbildentwicklung scheint daher angebracht.

Auf überregionaler Ebene werden Ideen/Visionen sowie Leitprinzipien/Leitlinien ausformuliert. Sie beschreiben die grundlegenden Ziele des Naturschutzes und zeigen besonderen Handlungsbedarf auf (HAAREN 1999). Für die lokale Naturschutzpraxis sind die überregionalen Leitlinien schwer umsetzbar, da diese in der Regel keine raumkonkreten Angaben machen. Im Zuge der Leitlinienentwicklung werden außerdem Umweltqualitätsstandards und Umweltqualitätsziele vorgeschlagen, die sich dann schon deutlich von einer Vision abgrenzen und in überschaubaren Zeiträumen erreichbar sein müssen. Auf überregionaler Ebene sind die Ziele noch nicht mit den Belangen anderer Fachbereiche abgewogen.

Auf regionaler und kommunaler Ebene erfolgt zunächst die Datenerhebung (Status quo, Potentiale, Beeinträchtigungen) zur Situation des Naturschutzes. Auf dieser Basis und unter Berücksichtigung der übergeordneten Leitlinien, Umweltqualitätsstandards und -ziele werden anschließend Entwicklungsziele formuliert. Nun erfordert eine realitätsangepasste Naturschutzkonzeption die Integration bzw. Berücksichtigung der Ziele anderer Raumnutzer, beispielsweise im Rahmen eines partizipativen Prozesses. Rechtliche und administrative Bedingungen sind zusätzlich zu beachten. In diesem Sinne erfolgt eine Adaption der 
visionären, landschaftlichen Leitbilder an die reale Situation durch die Ableitung realisierbarer, naturschutzfachlicher Entwicklungsziele.

Eine vollständige Befreiung vom visionären Charakter ist jedoch auch bei deduktiver Herleitung von Entwicklungszielen aus Leitbildern selten möglich. Im Rahmen der Raumplanung wird dem Leitbild deshalb ein Konzept nachgeordnet. Ein solches Konzept verfeinert die Oberziele nochmals und definiert ein vernünftiges Ziel-Maßnahmen-Bündel. Es schlägt sozusagen die Brücke zwischen Ideal und Realität. Das Konzept wird schließlich umsetzbar durch die Erstellung eines Programms. Dieses wiederum ordnet die Teilziele nach Prioritäten und setzt eine Reihenfolge für die durchzuführenden Maßnahmen fest.

Aus planungsmethodischer Sicht ist es daher nicht notwendig, dass ein Leitbild auch tatsächlich erreichbar sein muss. Es gilt eher als Ideal, dem man sich weitmöglichst annähern sollte. Diese Annäherung findet jedoch unter diversen Sachzwängen statt und ist damit verschiedenen Rahmenbedingungen unterworfen, z.B. gegensätzlicher Interessen oder der Existenz mehrerer fachspezifischer Leitbilder für den gleichen Raum.

Neben der Präsentation der naturschutzfachlichen Ziele im Rahmen der interdisziplinären Raumplanung besitzen die Leitbilder auch eine wichtige Aufgabe für den ideologischen Ordnungsprozess des eigenen Fachbereiches: Als Hilfsmittel stehen die besagten Konzepte und Programme zur Verfügung (BROGGI 1999), die übergeordnete Ziele für die Naturschutzpraxis konkretisieren. Adressaten sind somit die Beschäftigten und Aktiven innerhalb des Fachbereiches. Gleichzeitig liefern die Konzepte für den Landeigentümer eine Hilfe zur ökologisch orientierten Entscheidungsfindung.

Die Bezugseinheit eines Leitbildes oder nachgeordneten Programms bietet bereits erste Anhaltspunkte für dessen Umsetzbarkeit. Zum Schutze der Vielfalt an Arten und Biotopen sowie der landschaftlichen Besonderheiten, bietet die Kulturlandschaft die wohl zweckmäßigste Einteilung der Erdoberfläche. Eine naturräumliche Einheit grenzt sich durch verschiedene Elemente und Faktoren (Makroklima, Relief, u.a.) gegenüber der Umwelt ab. Bei der Kulturlandschaft kommen Einflüsse der anthropogenen Nutzung hinzu, die das Erscheinungsbild des Naturraumes und dessen Standortbedingungen überprägen. Die Kulturlandschaft selbst ist mehr als ein bestimmtes Arrangement von Flächennutzungen, Strukturelementen und geologischen Formen. Sie ist eine wichtige Grundlage für den Biotopund Artenschutz, gleichzeitig aber auch eine „identifikationsfördernde und heimatstiftende Kraft“ (BURGGRAF/HEIN 1999) für die Bevölkerung. Egal wie schwierig eine detaillierte Beschreibung erscheinen mag, ihre Bedeutung als plastisches Leitbild in der Landschaftsplanung fordert die Benennung ihrer Elemente und Charaktermerkmale. Für den Arten- und Biotopschutz ist es dabei besonders wichtig, dass die aktuelle und die angestrebte Rolle der jeweiligen Flächennutzungen und Strukturelemente geklärt werden.

Leitbilder finden sich auf allen unterschiedlichen Planungsebenen. Für den Naturschutz gilt, dass er einerseits eigene Leitbilder entwirft und im Rahmen der Planungsaktivitäten stärker detailliert. Andererseits muss er sich als Fachdisziplin auch jener Leitbilder annehmen, die von übergeordneten Planungsträgern vorgegeben werden oder die im fachübergreifenden Diskurs festgelegt wurden. Abhängig von der Art der betrachteten naturschutzfachlichen Planung, können die peripheren Leitbilder eine mehr oder weniger wichtige Rolle spielen. Welche Leitbilder im Rahmen einer Aufforstungsplanung zu berücksichtigen sind, soll im Folgenden geklärt werden.

\section{Raumordnerische Leitbilder}

Die Bundesraumordnung wartet mit einem Set eigener Leitbilder auf, welche vom Naturschutz als nachgeordnete Disziplin angemessen berücksichtigt werden sollen. Die Aussagekraft dieser Leitbilder für den Arten- und Biotopschutz ist jedoch eher gering. BUCHWALD (1980) unterscheidet vier hauptsächliche Leitbilder der Bundesraumordnung. Diese geben Ordnungsvorstellungen für den nationalen Raum wieder, auf die sich Bund und Länder geeinigt haben. 
Ein erstes Leitbild wird durch das Bundesraumordnungsprogramm selbst definiert und zielt auf gleichwertige Lebensbedingungen aller Bundesbürger ab. Zu den Lebensbedingungen gehört eine Lebensqualität im Sinne von Erholungsmöglichkeiten, sauberer und nachhaltig gesicherter Umwelt und entsprechender Zugänglichkeit für alle. Diese Qualitätsmerkmale besitzen räumlich betrachtet eine graduelle Ausprägung, wobei die Raumordnung versucht, sie anhand von Achsen und Zentren zu gliedern. Nach der Situationsanalyse folgt die Planung, mit dem Ziel, allen Räumen einen Mindeststandard, besser noch eine ausgeglichene Wertigkeit zu verschaffen. Aus der fachspezifischen Perspektive des Naturschutzes betrachtet, würde das raumordnerische Prinzip - konsequent durchgehalten zu einer großflächigen strukturellen und ökologischen Nivellierung der Landschaftsräume, aber auch der Lebensqualität führen. Da es nicht möglich ist, den Grad an Lebensqualität beliebig zu steigern, beispielsweise die Artenvielfalt überall auf einen bestimmten Level anzuheben, würde aus diesem Prinzip letztlich auch eine Verringerung der Lebensqualität in den Gebieten, wo sie heute besonders hoch ist, auf ein geringeres, wenn auch einheitlich niedriges Niveau folgen (BUCHWALD 1980).

Das zweite Leitbild der Bundesraumordnung steht für einen Ausgleich zwischen den Funktionsräumen. Vereinfacht gesagt, basiert dieses Bild auf der dinglichen Trennung aber räumlichen Verknüpfung von Arbeits- und Erholungsräumen. Dadurch kommt es zu lokalen Konzentrationen unterschiedlichen Ausmaßes und zur Ausbildung von Vorranggebieten. Die Vielfalt der Arbeits- und Erholungsgebiete kann durchaus erhalten oder gar gestärkt werden. Darüber hinaus liefert dieses zweite Leitbild aber ebenfalls keine richtungsweisenden Vorgaben für den Arten- und Biotopschutz.

Das dritte Leitbild der Bundesraumordnung lässt sich als jenes der passiven Sanierung bezeichnen. Es beinhaltet die Möglichkeit, die Bevölkerung in wenigen Räumen zu konzentrieren und dafür andere Räume zu „entleeren“, damit sie sich selbst regenerieren können. Dieses Leitbild würde einen Naturschutz der Enthaltung, bzw. die Entwicklung der „Urwälder von morgen“ durch großflächigen Prozessschutz stützen. Allerdings scheint dieses Leitbild eher theoretisch und nicht umsetzbar. Allein aus politischen Gründen wäre eine derartige Umsiedlung großer Teile der Bevölkerung niemals durchführbar.

Das vierte Leitbild bringt schließlich den Gedanken einer großräumigen funktionalen Arbeitsteilung auf. Darunter ist ein Mosaik von großräumigen Vorranggebieten mit unterschiedlichen Nutzungskombinationen zu verstehen, die sich gegenseitig ergänzen. Dabei können großräumige Vorranggebiete, mit regionaler Bedeutung und entsprechender Ausdehnung, von kleinräumigen, auf Gemeindeebene ausgewiesenen Vorranggebieten unterschieden werden. In diesem Sinne wurden bislang vor allem Landschaftsräume mit Freiraumfunktionen als ökologische Vorranggebiete ausgewiesen. Ihnen gegenüber stehen die landwirtschaftlichen und die industriellen Vorranggebiete, die Großflugplätze und Gewerbeparks, die Wohngebiete und ähnliche bauliche Agglomerationen. Für den Biotopund Artenschutz spielen ökologische Vorranggebiete eine besondere Rolle. In innen sollte es generell einfacher sein, die Ziele des Naturschutzes durchzusetzen. Dennoch bedeutet dies natürlich nicht, dass seine Ziele in anderen Vorranggebieten nicht relevant wären. Sie werden dort nur nicht mit Vordringlichkeit verfolgt, sondern stehen in der zweiten Reihe.

Innerhalb der groß- und kleinräumigen ökologischen Vorranggebiete soll ein ökologisch günstiges Verteilungsmuster der Landschaftsformen Feld, Wald und Wiese angestrebt werden. Damit wird das Ziel einer optimalen Vielfalt der Landschaftsräume im Sinne der ökologisch-biologischen und der strukturell-visuellen Vielfalt verfolgt. BUCHWALD (a.g.O.) spricht von einer ökologisch-optimalen Feinstruktur bzw. „Korngröße“, die in diesen Vorranggebieten angestrebt wird. Damit gibt aber auch dieses Leitbild aus der Bundesraumordnung nur einen Strukturierungshinweis für die ökologische Flächenordnung im Raum. Konkrete Hinweise für die Flächenplanung sind daraus nicht zu entnehmen, noch nicht einmal abzuleiten.

Der Naturschutz bleibt demnach weitgehend frei in der Erstellung und Ausformulierung eigener Leitbilder. Dies gilt insbesondere für den Bereich des Arten- und Biotopschutzes. Die Leitbilder der Bundesraumordnung, insbesondere das der ausgeglichenen Funktionsräume 
und das der großräumigen funktionalen Arbeitsteilung, unterstützen den naturschützerischen Anspruch zur Gestaltung der Flächennutzungsstruktur in der Landschaft nur mit Blick auf eine Optimierung der ökologischen und strukturellen Vielfalt.

\section{Erstaufforstungsleitbilder}

Für das Ziel der Waldvermehrung können für größere Gebiet auch spezielle Erstaufforstungsleitbilder entworfen werden (PREEN 1996). Diese sollen den Aufforstungswilligen eine Hilfestellung bei der Auswahl geeigneter Flächen bieten. VON PREEN fordert dabei eine Erstellung der Leitbilder unter Berücksichtigung von natürlichen Voraussetzungen, landschaftlichen Gegebenheiten sowie allen betroffenen Interessengruppen. Die Grenze zwischen Leitbild und Entwicklungsziel wird damit aufgeweicht. Der Schutz der vorhandenen Ressourcen ist ebenso zu integrieren wie die zukunftsweisende Gestaltung der Landschaft entsprechend der wirtschaftlich-strukturellen Entwicklung in der Region. Da das Leitbild unmittelbar für die Praxis entworfen wird, also auch den Grundstückseigentümer anspricht, ist besonders auf eine realitätsnahe Ausrichtung zu achten. Dies bedeutet, dass neben den visionären Überlegungen der Fachdisziplin Naturschutz auch die verschiedenen Interessen anderer Nutzergruppen vorausschauend zu bedenken sind. Das Leitbild soll nicht auf historische Landschaftsbilder zurückgreifen, sondern muss vom aktuellen Landschaftsbild ausgehend eine Zielperspektive für einen mittelfristigen Planungszeitraum (fünf bis zehn Jahre) aufzeigen. VON PREEN (1996) fordert aus gutem Grunde eine Flexibilität des Leitbildes gegenüber politischen Vorgaben. Nur wenn das Leitbild unter geänderten politischen Voraussetzungen, einschließlich veränderter Förderungsbedingungen für Erstaufforstungen, anwendbar bleibt, lohnt sich die Erstellung. Darüber hinaus muss aber auch eine Flexibilität gegenüber den veränderten Eigentümer- und Nutzerinteressen gefordert werden. Der aktuell stattfindende Strukturwandel in der Landwirtschaft und der latente, aber stets präsente Wechsel von Eigentümerverhältnissen in der Landschaft (insbesondere durch Erbfolge) bringen häufig auch einen Wandel der Eigentümerinteressen mit sich. Deshalb darf das Erstaufforstungsleitbild keine zu starren Vorgaben machen, an welchen Orten aufgeforstet werden darf und an welchen nicht.

Gegen ein Erstaufforstungsleitbild spricht außerdem, dass dieses auch dann noch Bestand haben müsste, wenn sich gegen Ende des Planungszeitraumes die Landschaftsverhältnisse durch verschiedene Aufforstungen bereits verändert haben. Da die tatsächliche Entwicklung aber nicht absehbar ist, ist dieser Anspruch nicht realisierbar.

\section{Naturschutzfachliche Leitbilder}

Der Naturschutz besitzt eine Reihe von Leitbildern, die sehr unterschiedliche Bereiche abdecken (s.u.). Sie können kaum kompromisslos nebeneinander verfolgt werden. Tendenziell sieht die Mehrheit der Betroffenen wohl die Lösung in einem „sowohl-als-auch“ (WOLF 1992). Dies erleichtert es dem Außenstehenden allerdings nicht, herauszufinden, was "der Naturschutz" nun eigentlich will. Deshalb sei bereits an dieser Stelle darauf hingewiesen, dass es - auch mit Bezug auf das oben Ausgeführte - nicht bei der Definition von Leitbildern bleiben darf. Konzepte, Programme und Entwicklungsziele gehören zur Verdeutlichung der disziplinären Ideen und Ansprüche dazu.

Zur inhaltlich-strategischen Diskussion tragen verschiedene Grundmotive, auch „Protoleitbilder" bei. Diese sind in der öffentlichen Diskussion vorhanden und dienen als Ansatzpunkt für die weitere Präzisierung der Ziele, in Richtung spezifischer Leitbilder (WIEGLEB et al. 1998). Die wichtigsten Protoleitbilder - sie entsprechen den „sektoralen Leitbildern“ bei PLACHTER und REICH (1994) - werden hier im Anhalt an BROGGI (1999) erläutert: Sie begründen sich aus dem vielgestaltigen Naturschutzauftrag mit ganz unterschiedlichen, nicht konvergenten, teilweise sogar widersprüchlichen Teilzielen. 


\section{Das historische Leitbild}

Die Erhebung eines Landschaftsbildes, wie es vor einer längeren Zeit einmal existent war, zum Ziel der Naturschutzbemühungen entspricht einer rückwärtsgerichteten Betrachtung bei der Prioritätensetzung für die Zukunft. Dabei scheiden sich die Geister schon sehr schnell an der Frage, wo der Referenzzeitpunkt zu setzen ist. Die höchste Artenvielfalt war vermutlich irgendwann zwischen 1800 und 1900 erreicht (LFU 1992) und ist sowohl auf die Vielfalt der Landnutzungsarten und die weichen Übergänge zwischen diesen zurückzuführen, als auch auf die Kleinparzellierung der Nutzflächen und die schadstoffarmen Umweltbedingungen. Andere Autoren (BROGGI / SCHLEGEL 1989) schlagen hingegen die Situation um 1960 als Umweltqualitätsziel vor. Selbst dieses vermeintlich realistischere Ziel würde bereits bedeuten, dass der als naturnah zu bezeichnende Flächenanteil (von BROGGI und SCHLEGEL untersucht am Beispiel des Schweizer Mittellandes), gegenüber der derzeitigen Situation in den intensiv landwirtschaftlich genutzten Regionen, auf das Dreifache ansteigen müsste.

Unabhängig vom Referenzzeitpunkt des historischen Leitbildes ist festzustellen, dass dieses Ziel nach einer Verringerung der Landwirtschaftsintensität auf einen Bruchteil des heutigen Niveaus verlangt. Zusätzlich ist eine Verkleinerung der Nutzflächen, eine Steigerung der Anbauvielfalt und eine Schaffung sanfterer Übergänge zu fordern. Dadurch wirkt dieses Leitbild als atavistisch und kaum umsetzbar.

\section{Das ästhetische Leitbild}

Es zielt ab auf ein allgemeines Schönheits- und Geborgenheitsempfinden des Menschen beim Anblick bestimmter landschaftlicher Strukturen und Konstellationen. Inwieweit das subjektive Schönheitsideal verallgemeinerbar ist, ob seine Merkmale nur regional oder auch überregional einheitlich sind, bleibt eine offene Frage. Auch ist die Ästhetik einem gesellschaftlichen Wertewandel unterworfen, welcher die Landschaftselemente nicht ausnimmt. Von Tacitus über Fontane bis Berndorf zeigt die Literatur das sich verändernde Gefühl des Menschen gegenüber dem Wald. Einst Wildnis und gesetzloser Raum, in der Romantik dann verklärtes Sinnbild der Freiheit und Raum der Erhabenheit, heute ein Rückzugsraum für den Menschen und verkörperte Heimat. Die Werbung bringt das populärste Bild der Landschaft und damit das am ehesten verallgemeinerbare Landschaftsideal zum Vorschein. Es ist geprägt durch freie Räume, fein strukturiert, nicht bedrohlich, jedoch mit Entdeckungsräumen in Form von Gehölzen, Wäldern und Gewässern. Doch wenn der Mensch das Heimatgefühl in die Wagschale wirft, welches er im Laufe seiner Jugend und seines Lebens entwickelte, so wird das ästhetische Empfinden wohl regionale Schattierungen kaum verhehlen können.

\section{Das biotische Leitbild}

Das biotische Leitbild umfasst das Streben nach einer höchstmöglichen Vielfalt allen Lebens. Die Umsetzung des Zieles bedingt, dass der Mensch zunächst alles Leben erkennen und erfassen lernt, um es anschließend auch schützen zu können. Angesichts der großen Vielfalt an Pflanzen- und Tierarten, sie dürfte in Mitteleuropa bereits mehr als 20.000 Arten umfassen, wäre ein auf alle Arten abgestimmter Schutz nicht praktikabel. Stattdessen stützt sich der Naturschutz auf das integrierende Indikationspotential einzelner Organismengruppen. Zu vielfältig sind allerdings die Strukturen und Zusammenhänge in verschiedenen Ökosystemen um Gewissheit zu erlangen, dass tatsächlich alle dort potentiell vorkommenden Spezies von den Schutzbemühungen um die Indikatorarten profitieren.

Ein weiteres, grundlegendes Problem dieses Ansatzes ist die darin implizierte Statik des Arteninventars. Ein Fortschreibung der Evolution ist im Ansatz nicht inbegriffen. Arten, deren natürlich Verbreitung durch den Landschaftswandel gefährdet ist, sollen durch Pflegeeingriffe und Abwehrmaßnahmen gesichert werden. Die Roten Listen der gefährdeten Tier- und Pflanzenarten demonstrieren eindrucksvoll, dass dies auf eine Vielzahl der Arten zutrifft. Die Landschaft wird damit zu einem gesamtheitlichen Pflegefall.

\section{Das Naturleitbild}

Das Naturleitbild stellt den Gegenpol zum biotischen Leitbild dar. Es beinhaltet die Forderung nach Raum für ungestörte natürliche Prozesse und Dynamiken. Als eine Grundlage dieses Ansatzes kann die von REMMERT formulierte "Mosaik-Zyklus-Theorie“ gelten (BAYER. 
AKADEMIE F. NATURSCHUTZ U. LANDSChAFTSPFlege 1991). Es ist das Prinzip des Werdens und Vergehens im Rahmen eines großen, nie endenden Kreislaufes, in dem alles seine Zeit und seinen Platz besitzt. Großflächige Naturlandschaften gibt es in Mitteleuropa heute nicht mehr. Die Landschaft wurde durch die menschliche Nutzung überprägt. Im Sinne des Naturleitbildes würde es allerdings auch ausreichen, den menschlichen Einfluss heute auf möglichst großen Flächen zu eliminieren. Trotz Agrarreform wird dies aber nur in Einzelfällen, beispielsweise in bestimmten Nationalparks, möglich sein. So fordern die Verfechter dieses Leitbildes zumindest eine repräsentative Schaffung von Freiräumen für ungelenkte Entwicklungen in allen Landschaftsteilen. Als Referenzflächen sollen diese Räume schließlich Hinweise geben, die in einer integrierten und naturgemäßen Nutzung der übrigen Landesteile zu berücksichtigen sind.

\section{Das abiotische (standörtliche) Leitbild}

Es berücksichtigt vorrangig den Schutz primärer Lebensgüter im Bereich Boden-WasserLuft. Die standörtlichen Bedingungen bilden die Rahmenbedingungen für die Entwicklung bestimmter Ökosysteme und der damit zusammenhängenden Artvorkommen. Ihnen muss deshalb, gerade im Zuge der Extensivierung oder Restrukturierung in der Landwirtschaft, entsprechendes Gewicht beigemessen werden. Wieder geht es um einen repräsentativen Schutz. Alle vorkommenden Standortseinheiten, unabhängig vom System der Klassifizierung, sind zu erhalten. Eine Nivellierung ist ebenso zu vermeiden wie eine fallweise Melioration bestimmter Standorte durch Entwässerung oder Düngung, welche dem Leitbild entgegen liefe.

\section{Regionale Leitbilder}

Im Rahmen der Landschaftsplanung kommen heute sehr häufig regionale Leitbilder zur Anwendung. Sie stellen eine naturraumspezifische Konkretisierung dar. Hier fließen mehrere der sektoralen Leitbilder oder Protoleitbilder ein. Ihr Charakter kann jedoch durchweg als konservativ oder konservierend beschrieben werden (DRL 1997). Diese regionalen, Statusquo-orientierten Leitbilder versinnbildlichen im Regelfalle einen Schutz der augenblicklichen Biotopsituation nach Qualität und Quantität, insbesondere mit Bezug auf die Biotope langer Genese, eingebettet in Bemühungen um eine mit Strukturelementen angereicherte und mehr oder weniger extensiv genutzte Kulturlandschaft. Da die Praxis der Landschaftsplanung dabei zunehmend die aktuelle Situation bei der Erstellung der Leitbilder berücksichtigt und auch die Beteiligung von Grundeigentümern und Interessengruppen praktiziert wird, wird eine begriffliche und inhaltliche Trennung bei regionalen Leitbildern und Entwicklungszielen schwieriger.

\section{Praxisrelevanz von Leitbildern}

Leitbilder dienen wie beschrieben der Verdeutlichung der Anliegen und Ansprüche des Naturschutzes. Sie müssen, damit sie die betroffenen Landnutzer und die Öffentlichkeit ansprechen können, nicht nur verbal präzise formuliert sein, sondern sollten zur Unterstützung auch visuell illustriert werden. Die Akzeptanz der Leitbilder hängt allerdings von weiteren Faktoren ab. Eine Grundvoraussetzung ist dabei, dass sich die Naturschützer zunächst in den eigenen Reihen auf ein oder mehrere Leitbilder verständigen. Wenn „der Naturschutz", welcher von Außenstehenden mal in einem Verbandsmitglied, mal in einer Zeitschrift und mal in einem staatlichen Vertreter erkannt wird, nach außen hin eine einheitliche Stellung einnimmt, stärkt er dadurch nicht nur die eigene Position, sondern macht es den Verhandlungspartnern auch einfacher, ihm zu folgen und seine Ansprüche zu akzeptieren.

Wie bereits im Zusammenhang mit den Erstaufforstungsleitbildern erwähnt, muss ein Leitbild im Sinne der Praxistauglichkeit auch stets ein gewisses Maß an Flexibilität aufweisen (DRL 1997). Es sind immer verschiedene Szenarien der Entwicklung, ausgehend vom Status Quo 
zu berücksichtigen. Ein Leitbild ist dann besonders gut anwendbar, wenn zumindest die wahrscheinlichsten Entwicklungen der Landschaft bei seiner Erstellung bedacht und integriert wurden. Damit hängt seine Qualität auch eng mit jener der Situationsanalyse im betroffenen Gebiet zusammen. Eine sorgfältige Vorarbeit ist Voraussetzung für eine zielsichere Planung.

Zur Anwendungsfreundlichkeit der Leitbilder gehört außerdem, dass eine Koexistenz mehrerer sektoraler Leitbilder für den gleichen Planungsraum nur akzeptiert werden kann, wenn diese sich nicht widersprechen oder zumindest flächenmäßig nebeneinander realisiert werden können. Bei derartiger Sachlage bedarf es auch einer einzelfallweisen, wiederum internen Abwägung, welche Ziele an welcher Stelle verwirklicht werden sollen. Eine Zusammenfassung verschiedener, sektoraler Leitbilder zu einem einzelnen, regionalen (Landschafts-)Leitbild für den Naturschutz ist nur in einheitlich strukturierten Landschaften möglich. Wo mehrere Landschaftstypen (vgl. Kap. 3.5.6) nebeneinander vorkommen, ist die Reduktion auf ein einzelnen Landschaftsleitbild nicht sinnvoll. Dabei ist zu beachten, dass die notwendige naturschutzinterne Diskussion über die Konflikte, bzw. Widersprüche zwischen sektoralen Leitbildern vor der Publikation des regionalen Leitbildes erfolgen muss. Es darf in diesem Zusammenhang nicht nur um den kleinsten gemeinsamen Nenner gehen, weil sonst eine Reduktion der Aussagen auf eine mehr oder weniger vom Planungsraum losgelöste Grundausrichtung des Naturschutzes droht, wodurch der Sache wenig gedient wäre (MENGEL 1999).

Schließlich darf sich der Naturschutz auch nicht scheuen, konkret zu werden. Verschiedene Autoren beklagen gerade im Zusammenhang mit Leitbildern und Zielsetzungen die Konzeptionslosigkeit des Naturschutzes für große Räume (KRAHL 1999; BROGGI 1999). In diesem Sinne ist die oben beschriebene Informationshierarchie mit Leitbild, Konzept und Programm nur ein erster Schritt zur Manifestierung der eigenen Ansprüche. Umweltqualitätsziele und Umweltqualitätsstandards ergänzen diese Instrumente. Die Aussagen für einen Planungsraum bleiben dadurch aber dennoch allgemeingültig (Leitbilder, Standards, Qualitätsziele) oder beruhen auf einem bestimmten Szenario (Konzepte und Programme, teilweise auch Leitbilder). Sie geben keine oder nur unzureichende Hinweise, welche Leitbilder oder Zielvorgaben anzuwenden sind, wenn die Entwicklung dem Szenario nicht entspricht. Dem Außenstehenden ist kaum zuzumuten, die Leitbilder anhand der vorhandenen Pläne zu analysieren und theoretisch auf die neue Situation zu übertragen. Selbst wenn die Diskussion in einen Zirkel von Naturschützern zurückgegeben würde, müsste eine neue Feststellung der Zielhierarchie stattfinden. Um diesen zeit- und arbeitsaufwendigen Prozess zu vermeiden, sollten neue Instrumente geschaffen werden, die eine Übertragung der Kernideen festgestellter Leitbilder auf eine neue Situation ermöglichen. „Wenn der Naturschutz etwas für die „Natur" erreichen will, dann ist es nicht nur wichtig, dass er mit einer Zunge spricht, sondern auch, dass er seine Zielvorstellungen und Bewertungsmaßstäbe darlegt" (KRAHL, 1999; S. 30).

Aus regionalen/raumspezifischen Zielvorstellungen und Bewertungsmaßstäben ließe sich ein entsprechendes Instrument konstruieren, welches zum Beispiel bei geänderten LandnutzerInteressen angewandt werden könnte, um der neuen Situation und gleichzeitig den festgestellten Leitbildern gleichermaßen zu entsprechen. Selbstverständlich bedingt ein solches Instrument wiederum den innerfachlichen Konsens der Naturschützer, damit es auch für andere Landnutzer verlässlich wird. Ein solches Instrument könnte beispielsweise eine Bewertung von Landnutzungsumwandlungen nach regionalen Maßstäben (abgeleitet aus der Regionalplanung) ermöglichen. Bezüglich der Kooperation mit anderen Fachbereichen im Rahmen der Raumplanung ist darüber hinaus zu beachten, dass in der großräumigen Landschaftsplanung auch fachfremde Leitbilder $\mathrm{zu}$ berücksichtigen sind. Eine interdisziplinäre Leitbildentwicklung ist allerdings ein langwieriger, häufig auch sehr konfliktträchtiger Prozess. Er erfordert nicht selten die Aufgabe bestimmter Forderungen oder eine andere Prioritätensetzung. Beides wird von einer Fachbehörde aber vermieden. Einen Ausweg aus der Misere kann in bestimmten Fällen die Loslösung vom starren Großkonzept ideologischer Prägung sein. Stattdessen bietet sich die Entwicklung eines flexibel anwendbaren Verfahrens der Entscheidungsfindung auf der Grundlage eine 
multikriteriellen Leitbildes (WIEGLEB et al. 1999) an. Ein derartiges Vorgehen verfolgt die hier vorgeschlagene Methode der Evaluierung von Erstaufforstungen unter Berücksichtigung verschiedener Einzelparameter oder Indikatoren, die jeweils integriert und miteinander verrechnet werden.

\subsection{Die Wirkungsanalyse}

Wirkungsanalysen stellen ein verbreitetes und häufig erprobtes Instrumentarium der Landschaftsplanung dar. Ihre Aufgabe ist es, die zu erwartenden Folgen einer Planung aufzuzeigen, beziehungsweise zu beschreiben, welche Entwicklungen durch eine gezielte Planung beeinflusst oder verhindert werden können. Es kommt darauf an, zunächst die Ausgangssituation zu erfassen und die wissenschaftstheoretische Problemstellung zu definieren. Aufgrund dieser Problemstellung lässt sich ein System abgrenzen und beschreiben, in welchem die zu erwartenden Auswirkungen der geplanten Handlung stattfinden werden. Verschiedene Szenarien können anhand der Wirkungsanalyse durchgespielt und miteinander verglichen werden. Dazu bedarf es der Feststellung der Systemstruktur und der Beschreibung des Systemverhaltens. Anschließend wird dieses theoretische System durch die Daten der augenblicklichen Systemsituation (IST-Zustand) konkretisiert. Die definierten Systemstrukturen werden mit virtuellen Inputs konfrontiert und die Veränderung des Systems wird als Wirkungsprognose beschrieben. Schließlich erfolgt eine Bewertung der simulierten Entwicklung anhand der zuvor festgesetzten Planungsziele (BECHMANN 1981).

Für die Praxis der Aufforstungsplanung ist eine prognostische Wirkungsanalyse anzusetzen, da die Maßnahme ex-ante bewertet werden soll, also noch vor ihrer Durchführung. Es bedarf der Feststellung der im betrachteten System wirkenden Zusammenhänge und Gesetzmäßigkeiten, damit die Wertbildung im neu entstehenden Waldbiotop nachvollzogen werden kann. Daraus wird ein Szenario aufgebaut, das die geplante Aufforstung unter den gegebenen Umweltbedingungen betrachtet. Zur Entwicklung der Bestandesmerkmale ist ein längerer Zeitraum notwendig. Der Entwicklungsgang wird bis zu einem Bestandesalter von 50 Jahren prognostiziert. Dieser Zeitraum ist ausreichend zur Entwicklung eines Bestandesklimas, einer weitgehenden Differenzierung der Baumschicht sowie der Entwicklung der Randstrukturen. Auch eine Besiedelung mit Waldtieren und -pflanzen sollte erkennbar sein. Lediglich die typischen Elemente eines reifen und überreifen Bestandes, v.a. Totholz und starke Baumdimensionen, fehlen in diesem Stadium noch. Die im Bestandesalter von 50 Jahren zu erwartenden Biotopmerkmale werden also im hier behandelten Fall anhand der durch den Naturschutz vorgegebenen Idealvorstellungen bewertet. Wie die Biotopmerkmale und die Idealvorstellungen aussehen, wird in den folgenden Kapiteln erläutert.

Die Systembeschreibung konzentriert sich auf jene Merkmale und Zusammenhänge, die für die Fragestellung der Wirkungsanalyse entscheidend sind. Es handelt sich also um ein abstraktes, dabei modellartiges Bild der Wirklichkeit. Eine besonders gute Abbildung des realen Systems wird als Wirklichkeitstreue bezeichnet und stellt ein Qualitätsmerkmal der Wirkungsanalyse dar. Ihr steht die Systemrationalität gegenüber, also die korrekte Wiedergabe von Zusammenhängen und Beziehungen innerhalb des betrachteten Systems.

\footnotetext{
${ }^{1}$ Für die im vorliegenden Fall interessierende Frage der Wirkungen einer Erstaufforstung bedeutet dies, dass zunächst einmal festzustellen ist, welche Merkmale des entstehenden Bestandes und seiner unmittelbaren Umgebung im Sinne der Analyse von Bedeutung und wie sie untereinander korreliert sind. Anschließend werden die Daten, z.B. Standortstyp, vorhandene Nutzung, angrenzende Biotope, Artvorkommen, u.a. erfasst. Das Variantenstudium wird anhand unterschiedlicher Pflanzpläne durchgespielt. Diese können sich beispielsweise nach Baumartenmischung, Pflanzabstand und Waldrandgestaltung unterscheiden. Die Entwicklung der fiktiven Bestände wird auf einen bestimmten Zeitpunkt hin prognostiziert und schließlich anhand der eingangs festgestellten Merkmale untereinander und mit der Ausgangssituation verglichen.
} 
Eine ausgeprägte Systemrationalität gilt als Garant für Praxisnähe und ist somit Voraussetzung für die Umsetzung der Untersuchungsergebnisse. KRAUSE beschreibt drei Qualitätsmerkmale (in KRAUSE/HENKE 1980, S. 29/30), die stellvertretend für den TheoriePraxis-Bezug stehen, also den Wert einer systemtheoretischen Betrachtung für die Landschaftsplanung verdeutlichen:

1) Das systemtheoretische Verständnis des Untersuchungsobjektes ist eine wertvolle Hilfe zur geordneten Betrachtungsweise. Die Entwicklung von Gestaltungs- und Konstruktionsmodellen, die Abstraktion der Zusammenhänge und die Definition der entscheidenden Komponenten schaffen eine hohe Ordnungsqualität. Als Grundvoraussetzung für eine interdisziplinäre Koordination und Kooperation, in welche die Landschaftsplanung beispielsweise als Teil der Regionalplanung eingebunden ist, gilt dabei, dass der abstrakte Bezugsrahmen für alle Fachdisziplinen und für die gesamte Betrachtungsdauer unverändert bleibt.

2) Die systemtheoretische Vorstellung erleichtert die ganzheitliche Betrachtungsweise, da der Planer gezwungen wird, alle auftretenden Zusammenhänge plastisch darzustellen und Verknüpfungen zu analysieren. Gleichzeitig erfolgt eine Kontrolle der Vollständigkeit an Elementen. Die Nachzeichnung des Beziehungsgefüges ist ein wichtiger Schritt zur Entwicklung der Integrations- und Koordinierungsqualität.

3) Auf der Grundlage ganzheitsbezogener Systemvorstellungen können die darin eingebundenen Elemente und Funktionen bei Bedarf partiell untersucht werden, ohne dabei den Gesamtzusammenhang aus den Augen zu verlieren. Aus der systemtheoretischen Betrachtung ergibt sich damit eine Legitimation zur Konzentration auf bestimmte Schlüsselelemente des Systems bei der Umsetzung in die Praxis.

\section{Systemebenen}

Bei der Abgrenzung des Systems gegenüber der Umgebung können die Untersuchungseinheiten nicht losgelöst von dieser betrachtet werden, wenn Wechselbeziehungen vorhanden sind. Eine Wirkungsanalyse muss daher sowohl die internen als auch die nach außen führenden Wirkungsketten des Systemausschnittes erkennen und interpretieren. Die Identifikation der Zusammenhänge geht einher mit der Offenlegung von Prämissen und Prinzipien, welche für das betrachtete System eine allgemeine Gültigkeit besitzen (Kap. 3.3.1).

Der Systembegriff bleibt in der Landschaftsplanung nur grob umrissen, da die vielfältigen Beziehungen auf unterschiedlichen geographischen, physikalischen und biologischen Ebenen eine genaue und in sich konsistente Abgrenzung in der Praxis unmöglich machen. Dies wird besonders deutlich, wenn als Komponenten eines Systems auch Tierarten betrachtet werden, die nicht an dieses System gebunden sind, sondern immer oder auch nur gelegentlich unterschiedliche Systeme neben- oder nacheinander nutzen. Die Systemelemente können folglich Beziehungen nach außen besitzen und sind nicht zwingend auf die betrachtete Fläche fixiert. Ein komplexes Gebilde, bei dem einzelne Teile mit der Umgebung interagieren, wird in der Theorie als offenes System bezeichnet. Ein weiteres Merkmal sind die nach außen praktisch undeterminierbaren Grenzen. Das System selbst strebt einem Fließgleichgewicht そu, dessen Stabilität aber von der Umwelt abhängig ist (KRAUSE/HENKE 1980, S. 20-29).

\footnotetext{
2 Im Fallbeispiel der Erstaufforstung stellt die Entwicklung des Bestandes mit all seinen Charaktermerkmalen den in sich geschlossenen Systembereich dar. Er beinhaltet jene Pflanzen und Tierarten, deren Lebenszyklus vollständig innerhalb der Aufforstungsfläche abläuft. Zusätzlich bestehen aber Wechselwirkungen mit der Umgebung. Dazu gehören in erster Linie die Bedeutung als Teil- oder temporärer Lebensraum für Tiere. Der entstehende Wald wirkt aber auch auf die abiotischen Ressourcen (z.B. Wasserreinigung) und die Standortbedingungen der angrenzenden Biotope (Windlenkung, Beschattung). Damit stellt die Erstaufforstung ein offenes System dar.
} 
Die Landschaftsplanung tut sich schwer, ein offenes System als Grundlage für ihre Aktivitäten zu beschreiben. Die Beziehungsdichte zwischen den einzelnen Elementen einer Landschaft lässt sich häufig nur erahnen, nur selten aber qualitativ oder gar quantitativ beschreiben. Je größer der Landschaftsausschnitt gewählt wird, desto komplexer zeigt sich dessen Struktur. Auch die Vielfalt der Elemente wächst - wenn auch nicht unbedingt linear mit der Flächengröße. Je kleiner die Fläche aber wird, desto häufiger muss mit den oben genannten Beziehungen zwischen Elementen und Systemumwelt gerechnet werden.

Der Idealfall für alle Planungsvorhaben wäre demnach ein geschlossenes System, in welchem die Elemente nur untereinander gekoppelt sind. Hier bestehen keine Wechselwirkungen mit der Umgebung. Die Komplexität innerhalb des Systems muss deshalb nicht weniger groß sein. Allein die Tatsache, dass keine unvorhersehbaren Störfaktoren oder Veränderungen der Umwelt beachtet werden müssten, würde allerdings einen klaren Gewinn an Planungssicherheit bedeuten.

Der Landschaftsplaner sieht sich mit dem Problem konfrontiert, eine Planung für einen mehr oder weniger gut umrissenen Teilbereich eines stets offenen Systems zu erstellen. Er neigt dennoch dazu, den betrachteten Systembereich im Sinne eines halbgeschlossen Systems zu behandeln. Dies bedeutet, im Mittelpunkt steht ein quasi offenes System, dessen Umwelt nur nach ihrem Störpotential für die innere Sicherheit des Systems, sprich für die Erhaltung des Fließgleichgewichts beurteilt wird. Sämtliche Elemente außerhalb des beplanten Landschaftsausschnittes, welche zwar mit den Elementen innerhalb des Systems in Wechselbeziehung stehen, die Sicherheit desselben aber nicht gefährden, werden ignoriert oder zumindest als zufällig / systemunabhängig betrachtet. KRAUSE (in KRAUSE/HENKE 1980, S. 20-29) erklärt diese Reduktion des Betrachtungsobjektes mit der Angst vor unüberschaubaren Zusammenhängen und mangelnden Handlungsmöglichkeiten zur Einordnung des Systems in eine übergeordnete Systemkonstellation.

Für das vorliegende Verfahren zur naturschutzfachlichen Bewertung von Erstaufforstungen wird ein System als Betrachtungsobjekt auf verschiedenen Ebenen abgegrenzt. Dieses Vorgehen respektiert die Umwelteinflüsse auf das Systeminnere nach unterschiedlichen Intensitäten und Verbindlichkeiten. Auf einer ersten, „introvertierten“ Ebene werden die Beziehungen der einzelnen Elemente einer Aufforstung (Pflanzenarten, Strukturmerkmale, Naturnähe, etc.) untereinander als geschlossenes System betrachtet: die Aufforstungsfläche. Die Prämisse dieser Abgrenzung ist, dass eine Bewirtschaftung der Fläche derart erfolgt, dass Umwelteinwirkungen ausgeschlossen oder minimiert werden können. Die Aufforstung würde demnach durch geeignete Pflegemaßnahmen so modelliert, dass der Charakter der Fläche sich entsprechend der Zielsetzung zum Zeitpunkt der Pflanzung entwickelt. Diese Prämisse erscheint im Hinblick auf die aktuelle Förderungspraxis gerechtfertigt, wonach nicht nur die Pflanzung selbst, sondern auch die Pflegemaßnahmen der ersten Jahre bezuschusst werden. Eine zielgerichtete Pflege wird also vorausgesetzt. Die flächenbezogene Begrenzung dieser Systemebene ergibt sich aus der Größe der Aufforstungsfläche.

Auf einer zweiten, „extrovertierten“ Systemebene werden die Beziehungen des AufforstungsBiotops zu gleichartigen oder ähnlichen Biotopen im Sinne eines Biotopverbundes betrachtet: das Aufforstungsumfeld. Der Betrachtungsraum wird hier so weit vergrößert, dass die Aktionsräume der Tiere in den umliegenden Lebensräumen zumindest mit Teilflächen integriert werden (siehe Kap. 3.5.6). Von besonderem Interesse ist die Distanz zwischen gleichartigen Biotopen, die von diesen Tierarten im Rahmen der individuellen Aktivitäten und der Populationsausbreitung über biotopfremde Strukturen hinweg überquert werden kann. Die Landschafts- und Biotopverbundplanung geht von einer für die meisten Tierarten überbrückbaren Distanz von 200 - 300 m aus (TLL 1997, RIESS 1986, HEYDEMANN 1986, ZENKER 1982). Daher wird eine Untersuchungsfläche für die zweite Systemebene bestimmt, die eine quadratische Grundform mit zwei Kilometern Kantenlänge besitzt und deren Zentrum im mathematischen Zentrum der Aufforstung liegt. Somit ist die Berücksichtigung der erreichbaren Biotope sichergestellt.

Eine dritte, ebenfalls „extrovertierte“ Systemebene ergibt sich schließlich aus dem erweiterten Planungsraum. Dieser wird vorgegeben durch bereits vorhandene Planungen mit 
konkreten, nutzungstypen- und naturschutzbezogenen Aussagen zum Untersuchungsgebiet (Bsp.: Landschaftsplan, Regionalplan, Arten- und Biotopschutzprogramm). Häufig bedienen sich die Pläne einer Kombination aus Naturräumen und Verwaltungseinheiten. Diese Einteilung kann übernommen werden. Der Agrarraumnutzungsplan Niedertreba (TLL 1997) unterscheidet beispielsweise drei unterschiedliche Landschaftscharaktere und hält diese Unterscheidung auch im Rahmen des vorgeschlagenen Konzeptes kontinuierlich durch. Im konkreten Fall kann die betrachtete dritte Systemebene daher auf den betroffenen Landschaftscharaktertyp eingegrenzt werden.

Die dritte Systemebene kommt nicht als Untersuchungsgebiet zum Tragen, sondern lediglich als Quelle zur Ableitung der Planungsziele. Sie wird terminologisch mit der zweiten Ebene zum „Bezugsraum“ zusammengefasst. Hier werden Entwicklungsziele aus übergeordneten Planungen, z.B. einem Arten- und Biotopschutzprogramm oder einem Landschaftsplan übernommen und als Soll-Werte für die Daten des Aufforstungsumfeldes verwendet. Die Integration der verfahrensexternen Zielvorgaben ermöglicht eine dynamische Anpassung an Veränderungen in der gesellschaftlichen Wertschätzung der Landschaft.

Mit der Betrachtung des Untersuchungsobjektes auf drei Systemebenen (Aufforstungsfläche, Aufforstungsumfeld und erweiterter Planungsraum) steht das Verfahren im methodischen Einklang zur allgemeinen Praxis der Landschaftsplanung. Bei der Entwicklung von Leitbildern und Konzepten ist diese Dreigliederung in Objektebene, Landschaftsebene und regionale Ebene ein gängiges Vorgehen (WIEGLEB et al. 1999). Sie wird auch hier angewandt. Thematisch beschränkt sich die Betrachtung beim vorliegenden Evaluierungsverfahren auf den Nutzungswandel von Landwirtschaft zu Forstwirtschaft. In der allgemeinen Landschaftsplanung ist das Untersuchungsgebiet dagegen weiter gefasst und deckt häufig einen größeren geographischen Raum ab. Funktional entsprechen sich daher die Ebenen Aufforstungsumfeld und Landschaftsebene, bzw. erweiterter Planungsraum und regionale Ebene sehr gut.

\section{Wirkungszusammenhang}

Innerhalb eines Systems laufen mehrere Vorgänge ab, durch die die Elemente untereinander verknüpft oder voneinander abhängig sind. Ein Hilfsmittel der Systemanalyse ist die kybernetische Deutungsweise des Wirkungszusammenhangs. Sie unterteilt die Elemente des Systems in den Operator als aktiv handelnde Größe. Dieser beeinflußt (zumindest) ein Objekt, den sogenannten Operanden. Der Operand erfährt eine Verwandlung (Transition) in einen neuen Zustand, die sogenannte Transformierte. Aus dieser Veränderung (Abb. 3.3-I) ergibt sich der Grundbaustein des Wirkungszusammenhangs.

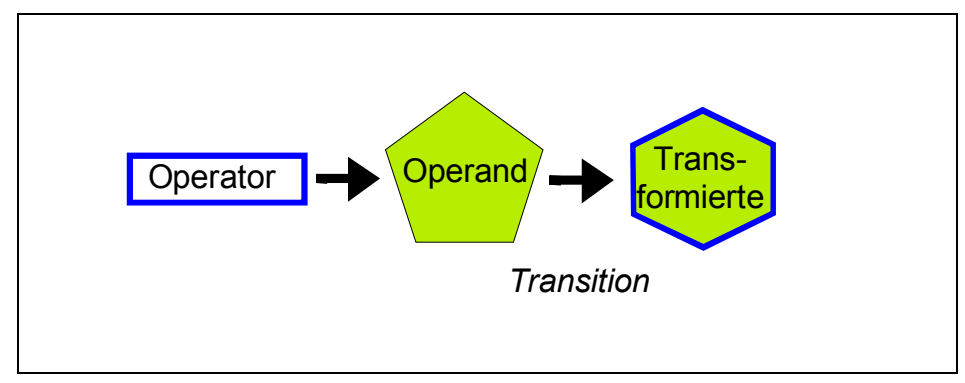

Abb.3.3-I: $\quad$ Der Transformationsprozess in einem System

Der Operator kann auf mehrere Operanden einwirken und Veränderungen hervorrufen. Er kann auch auf einen Operanden und dessen Transformierte mehrfach in gleichen oder unterschiedlichen Abständen und Intensitäten einwirken. Es können aber auch Transformierte auf andere Operanden oder Transformierte einwirken und neue Transitionen veranlassen. Die Summe aller Veränderungen / Transitionen innerhalb des Systems wird schließlich als Transformation desselben zusammengefasst (Transformationsbegriff nach ASHBY 1956 / 1974, zitiert in KRAUSE/HENKE 1980). 
Durch die Analyse des Systems kann der Planer die Schlüsselfaktoren definieren. Die Frage, die dabei im Mittelpunkt der Betrachtung steht, sucht einerseits nach den Kräften des dynamischen Ablaufes und andererseits nach den typischen Prozesseigenschaften. Das Wirkungsgeschehen wird charakterisiert und die prozesshafte Komponente herausgestellt. Die sorgfältige Freilegung des Wirkungsgeschehens ermöglicht dem Planer eine Reduktion von Ballastinformation. Er trifft eine Auswahl aktiver Elemente, die direkt oder indirekt miteinander reagieren.

Zur Umsetzung des systemtheoretischen Transformationsmodells in die Praxis der Landschaftsplanung ist es sinnvoll, den von KRAUSE (s.o.) vorgeschlagenen Weg der drei Schritte zu gehen:

a) Indentifizierung der aktiven Elemente als Operator und Operand

b) Charakterisierung des Wirkungsgeschehens im Sinne der Transformationsstruktur (nach AsHBY)

c) Korrelierung des Wirkungsgeschehens mit den Systemverhältnissen aktiver Elemente

Dieses Vorgehen wird im vorliegenden Fall anhand der Erstaufforstungs-Evaluation umgesetzt. Zur Charakterisierung des Wirkungsgeschehens werden die drei Systemebenen zunächst aufgelöst, damit eine objektive Betrachtung aller Vorgänge, Beziehungen und Verknüpfungen innerhalb des Systems erfolgen kann. Eine Analyse der Abhängigkeiten der Elemente untereinander bietet den Einstieg in das Beziehungsgefüge. Dies erfolgt mit Hilfe einer Matrix. Daraus lassen sich Knotenpunkte der Verknüpfungen herauslesen, welche sich in der Matrix als Punktewolken abzeichnen und sozusagen die Gelenke des Systems darstellen. In einem erweiterten Ansatz werden dann die Elemente im Sinne von Input, Zwischenzuständen und Outputgrößen dargestellt. Die einzelnen, zu erwartenden Transitionen werden definiert und zusammen mit den Elementen als Transformationsmodell dargestellt (siehe Abb. 3.3-II).

Im betrachteten Modell eines Nutzungswandels durch eine Erstaufforstung stellt diese selbst bereits den Operator dar. Durch die Erstaufforstung erfolgt ein Impuls, welcher Systemelemente beeinflusst. Es werden neue Bedingungen geschaffen.

\section{Systemebene}

Die Aufforstung wird als Inputgröße genau charakterisiert durch den zugehörigen Pflanzplan mit Arten, Pflanzverband, Pflanzengröße und -zahl, die als Operatoren auftreten.

Die Operanden sind auf den genannten drei Ebenen, Aufforstungsfläche, Aufforstungsumfeld und erweiterter Planungsraum, zu unterscheiden. Die vielfältigsten Veränderungen finden sicherlich direkt auf der Fläche statt. Hier wird eine Vielzahl an Elementen direkt durch die Aufforstung beeinflusst, wie z.B. die Artenanzahl, die horizontale und vertikale Struktur der Vegetation, die Bodenverhältnisse, das Mikroklima. Diese Elemente werden als Operanden innerhalb des Systems interpretiert, die im Rahmen der weiteren Bestandesentwicklung auch selbst die Rolle von Operatoren übernehmen können.

Auf der Ebene des Aufforstungsumfeldes, also im Sinne des direkten Biotopgefüges, werden insbesondere die Vielfalt der Biotoptypen, der Verbund und die funktionale Vernetzung gleichartiger Biotope beeinflusst. Für den erweiterten Planungsraum ergeben sich Veränderungen in der Biotoptypenvielfalt sowie im Landschaftsbild.

Eine ausführlichere Auflistung von Operanden findet sich in Tab. 3.3-1. Es handelt sich bei dieser Tabelle um eine Matrix, in welcher die Elemente aufgelistet sind, die das entstehende Biotop genauer beschreiben. Sie sind somit alle auf der ersten Systemebene zu suchen. Unter den Elementen fallen zunächst jene auf, die durch die Aufforstungsmaßnahme direkt gesteuert werden, z.B. die verwendeten Gehölzarten, die Pflanzdichte und die Form der Mischung. Des weiteren sind die Rahmenbedingungen als Elemente aufgeführt, also alle Faktoren, die den Standort selbst bestimmen. Letztere können natürlichen oder anthropogenen Ursprungs sein. 
Tab. 3.3-1: Verflechtungsmatrix - Elemente der 1. Systemebene (Aufforstungsfläche); die gefüllten Zellen zeigen jeweils die Abhängigkeit des Zeilen-Elementes vom SpaltenElement an; die Punktewolken werden nach Indikatoren zusammengefasst (V1,V2 Vielfalt ; NN1, NN2 - Naturnähe; SR1,SR2 - Strukturpotential; WR1,WR2,WR3 Waldrandausprägung)

\begin{tabular}{|c|c|c|c|c|c|c|c|c|c|c|c|c|c|c|c|c|c|c|c|c|c|}
\hline $\begin{array}{l}\text { 1. System- } \\
\text { ebene }\end{array}$ & & 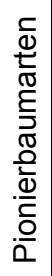 & 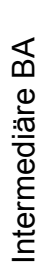 & 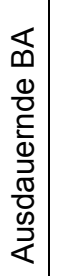 & 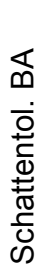 & 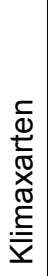 & 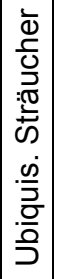 & 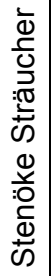 & 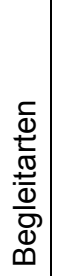 & 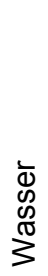 & $\begin{array}{l}\frac{1}{0} \\
\frac{0}{0} \\
\frac{\infty}{0}\end{array}$ & 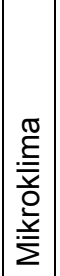 & 를 & $\mid \begin{array}{l}0 \\
0 \\
\frac{1}{0} \\
.0 \\
\frac{0}{0} \\
\frac{\pi}{0} \\
\frac{0}{0} \\
\frac{0}{2} \\
\Sigma\end{array}$ & 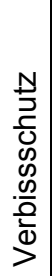 & 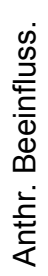 & 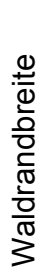 & 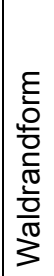 & 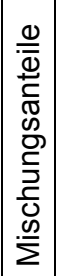 & 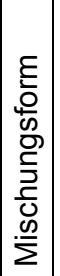 & 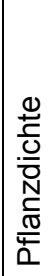 \\
\hline & & $\bar{w}$ & జ̃ & $\stackrel{m}{\tilde{w}}$ & 岌 & & $\stackrel{\leftrightarrow}{\ddot{H}}$ & $\hat{w}$ & $\stackrel{\infty}{w}$ & 요 & $\stackrel{\circ}{\dot{w}}$ & $\check{\leftarrow}$ & $\stackrel{N}{\leftarrow}$ & $\stackrel{m}{\varpi}$ & $\stackrel{⿱}{\amalg}$ & $\frac{\omega}{\tilde{w}}$ & $\frac{\bullet}{\dot{w}}$ & $\frac{N}{\dot{w}}$ & $\frac{\infty}{\dot{w}}$ & $\frac{\Omega}{\dot{\varpi}}$ & พั \\
\hline Pionierbaumarten & E1 & & $\Delta$ & $\diamond$ & $\diamond$ & $\diamond$ & & & & 1 & $\diamond$ & $\diamond$ & $\diamond$ & & & $\diamond$ & $\diamond$ & & $\checkmark$ & $\diamond$ & $\diamond$ \\
\hline $\begin{array}{c}\text { Intermediäre } \\
\text { Baumarten }\end{array}$ & E2 & & & $\diamond$ & $\diamond$ & $\diamond$ & & & & $\diamond$ & $\diamond$ & $\diamond$ & $\diamond$ & $\diamond$ & $\diamond$ & $\diamond$ & $\diamond$ & & $\diamond$ & $\diamond$ & $\diamond$ \\
\hline $\begin{array}{l}\text { Ausdauernde } \\
\text { Baumarten }\end{array}$ & E3 & $\bullet$ & $\nabla$ & & $\Delta$ & $\bullet$ & & & & $\diamond$ & $\diamond$ & $\diamond$ & $\diamond$ & $\diamond$ & $\diamond$ & & & & $\diamond$ & $\diamond$ & $\diamond$ \\
\hline $\begin{array}{c}\text { Schattentolerante } \\
\text { Baumarten }\end{array}$ & E4 & $\bullet$ & $\diamond$ & $\bullet$ & & $\diamond$ & & & & $\diamond$ & $\diamond$ & $\diamond$ & $\diamond$ & $\diamond$ & $\diamond$ & & & & 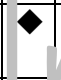 & 8 & $\diamond$ \\
\hline Klimaxarten & E5 & $\diamond$ & $\checkmark$ & $\diamond$ & $\diamond$ & 1 & & & & $\diamond$ & $\diamond$ & 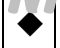 & $\diamond$ & $\diamond$ & $\diamond$ & 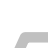 & & & $\diamond$ & $\diamond$ & $\diamond$ \\
\hline $\begin{array}{l}\text { Ubiquisitäre } \\
\text { Sträucher }\end{array}$ & E6 & $\diamond$ & $\diamond$ & $\diamond$ & $\diamond$ & $\diamond$ & & & & $\diamond$ & $\diamond$ & $\diamond$ & $\diamond$ & $\diamond$ & & $\bullet$ & $\diamond$ & $\diamond$ & $\diamond$ & $\diamond$ & $\diamond$ \\
\hline $\begin{array}{c}\text { Stenöke } \\
\text { Sträucher }\end{array}$ & E7 & $\diamond$ & $\diamond$ & $\diamond$ & $\diamond$ & $\diamond$ & $\diamond$ & & & $\diamond$ & $\diamond$ & $\diamond$ & $\diamond$ & $\diamond$ & & 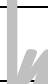 & - & 4 & $\diamond$ & $\diamond$ & $\diamond$ \\
\hline Begleitarten & E8 & $<$ & $\diamond$ & $\diamond$ & $\diamond$ & $>$ & $\diamond$ & $\diamond$ & & $\diamond$ & $\diamond$ & $\diamond$ & $\bullet$ & & & $\bullet$ & & $\diamond$ & $\bullet$ & $\diamond$ & 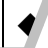 \\
\hline Wasser & E9 & 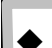 & $\diamond$ & $>$ & $\diamond$ & $\diamond$ & & & & & $\diamond$ & $\diamond$ & & $\diamond$ & & & & & & & \\
\hline Boden & E10 & 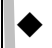 & $\diamond$ & 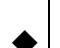 & $\diamond$ & $\diamond$ & 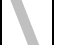 & & $\diamond$ & $\diamond$ & & $\diamond$ & & $\diamond$ & & & & & & & $\diamond$ \\
\hline Mikroklima & E11 & $\diamond$ & $\diamond$ & $\diamond$ & $\downarrow$ & 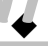 & $\diamond$ & & & & & & & & & & $\diamond$ & $\diamond$ & & & $\diamond$ \\
\hline hpnV & E12 & & & & & & & & & $\bullet$ & $\bullet$ & $\diamond$ & & $\bullet$ & $\bullet$ & $\diamond$ & & & & & \\
\hline $\begin{array}{l}\text { Melioration / } \\
\text { Düngung } \\
\end{array}$ & E13 & & & & & & & & & & $\Delta$ & $\Delta$ & & & & 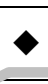 & & & & & \\
\hline Verbissschutz & E14 & & & & & & & & & & & & & & & $\diamond$ & & & & & \\
\hline $\begin{array}{l}\text { Anthropogene } \\
\text { Beeinflusssung }\end{array}$ & E15 & & & & & & 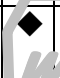 & $\Delta$ & & 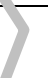 & & & & & $\diamond$ & & 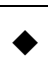 & $<$ & & $\diamond$ & \\
\hline Waldrandbreite & E16 & & & & & & $\Delta$ & $\bullet$ & & & & & & & & $\diamond$ & & & & & \\
\hline Waldrandform & E17 & & & & & & & & & & & & & & & 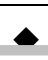 & & & & & \\
\hline Mischungsanteile & E1\& & $\diamond$ & $\diamond$ & $\diamond$ & $\diamond$ & $\diamond$ & $\diamond$ & $>$ & & & & & & & & & & & & & \\
\hline Mischungsform & E1c & $\bullet$ & $\diamond$ & $\diamond$ & 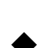 & $\nabla$ & $\diamond$ & $\diamond$ & & & & & & & & & & & & & \\
\hline Pflanzdichte & $\mathrm{E} 2 \mathrm{C}$ & $\diamond$ & $\diamond$ & $\diamond$ & $\diamond$ & $\diamond$ & $\diamond$ & $\diamond$ & & $\bullet$ & & & & & & & & & & & \\
\hline
\end{tabular}


Die Matrix stellt ein Hilfsmittel dar, um die Abhängigkeiten der Elemente untereinander systematisch zu hinterfragen. Das Ergebnis dieser Untersuchungen zeigt Schwerpunktbildungen im Beziehungsgefüge der Elemente, welche durch Punktewolken verdeutlicht werden. 3 Aufgrund der Systemtheorie lässt sich folgern, dass die Zusammenhänge, die zwischen den einzelnen Elementgruppen bestehen, quasi als Gelenke eines Organismus veränderbare, in ihren Relationen voneinander abhängige Knotenpunkte darstellen. Wenn es also gelingt, die Punktewolken im Sinne von Indikatoren zu operationalisieren, so könnte über deren Ausprägung das System als Ganzes nachgezeichnet werden.

Die Matrix dient darüber hinaus als Grundlage für eine Einordnung der Elemente in das Transformationsmodell (Abb. 3.3-II). Die Grafik gibt das Beziehungsgefüge der ersten Systemebene wieder. Wir finden die durch die Aufforstung direkt gesteuerten Elemente hier als aktive Elemente, bzw. Operanden der erster Generation wieder (linke Seite des Schaubildes). Diese bestimmen zusammen die Ausprägung der ersten Output-Elemente, also der Operanden zweiter Generation. Die höchste Verknüpfung von Elementzusammenhängen finden wir in den Begriffen Diversität und Naturnähe, welche als Operanden vierter Generation quasi am Ende der Verknüpfungen stehen (rechte Seite des Schaubildes

Die Rahmenbedingungen des Systems werden mit der anthropogenen Beeinflussung und den Standortsbedingungen vorgegeben. Beide stellen ein Kontinuum dar, das zwar über die Zeit verändert werden kann, jedoch im Rahmen der Aufforstung zumindest mittelfristig stabil ist und somit eine gewisse Planungssicherheit ermöglicht. Als anthropogene Beeinflussung zählen in diesem Sinne sowohl Einflüsse auf die Umweltbedingungen (Melioration, Düngung, Grundwasserabsenkung, etc.), als auch Einflüsse auf die Vegetation, beispielsweise durch waldbauliche Maßnahmen. Eine entscheidende Prämisse für die Anerkennung der anthropogenen Einflüsse als mittelfristig gleichbleibenden Einfluss besteht darin, dass die Planung der Aufforstung (Gehölzarten, Pflanzplan) und die waldbauliche Behandlung über die ersten Jahrzehnte aufeinander sowie auf die Standortsgegebenheiten abgestimmt sind.

Die Begriffe Diversität und Naturnähe besitzen eine hohe Symbolkraft als naturschutzfachliche Wertkriterien, stehen sie doch für eine Reihe von Systemzusammenhängen. Dies weist bereits auf die Gefahr hin, welche bei der alleinigen Betrachtung dieser beiden Elemente bestehen würde. Sie würde lediglich eine oberflächliche Einschätzung des Systems ermöglichen. Eine Beschreibung der Vorgänge und Veränderungen ist auf diesem Wege nicht möglich. Letztere ist aber zwingend notwendig, wenn der Mensch planend und lenkend eingreifen möchte. Aus diesem Grunde wird versucht, die Systemebene anhand der wichtigsten Operanden abzubilden.

\footnotetext{
${ }^{3}$ Beispiel: Die Punktewolke V1 zeigt an, dass Vorkommen und Ausprägung von Baum-, Strauch- und Begleitarten jeweils von der Konkurrenz durch andere, konkurrenzkräftigere Arten abhängig ist. Dies kann zum Ausfall der konkurrenzschwächeren Arten führen, wodurch die Vielfalt des Bestandes (V) beeinflusst wird. Umgekehrt können schwächere Baumarten die konkurrenzstärkeren auch beeinflussen. Dies wird allerdings bei einer Aufforstung weniger zur Entmischung als vielmehr zu einer Beeinflussung der Struktur (SR1) führen.

Gegenbeispiel: Die Überlebenschancen von Baum-, Strauch- und Begleitarten sind, je nach deren Konkurrenzkraft, von den gewählten Mischungsanteilen, der Mischungsform und der Pflanzdichte abhängig (Punktewolke V2 -Vielfalt). Umgekehrt wird sich der Waldbesitzer bei der Bestimmung von Mischungsanteilen, -form und Pflanzdichte nach der Konkurrenzkraft der gewünschten Baumarten richten. Dies wirkt sich wiederum auf die Struktur des Bestandes aus (SR2)
} 


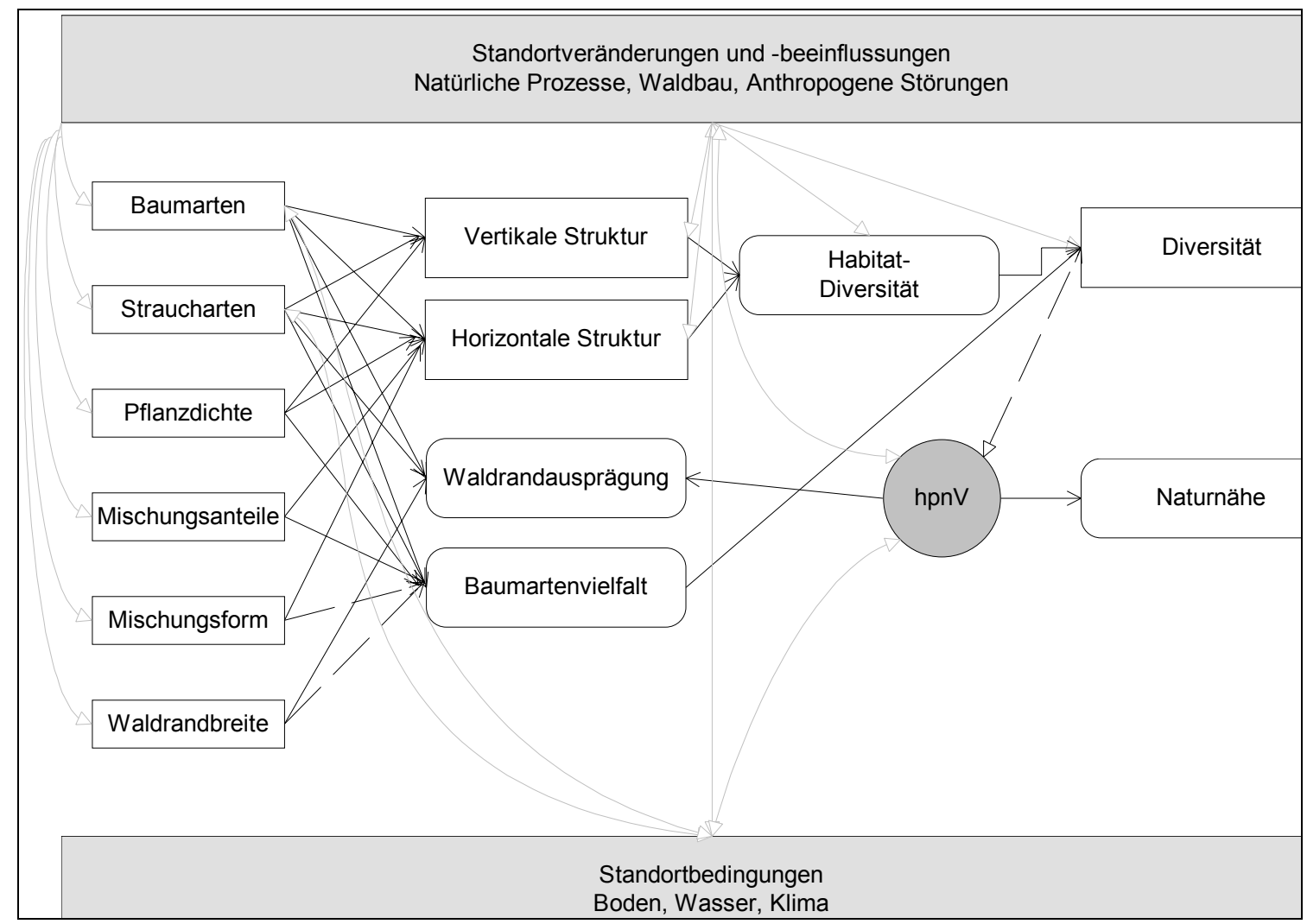

Abb.3.3-II: Das Transformationsmodell der 1. Systemebene (Aufforstungsfläche) zeigt die Verknüpfung zwischen den Operanden unterschiedlicher Generation an

Relativ eindeutig ist die Situation für die Operanden Waldrand(-form) und Naturnähe, da beide einfach zu erfassen sind. Die Diversität eines Bestandes beinhaltet allerdings neben den einfach erfassbaren Baumarten auch die faunistischen und floristischen Begleitarten. Da allerdings im Rahmen der Landschaftsplanung nicht alle Arten eines Bestandes erfassbar sind, muss nach operationalen, aussagekräftigen Indikatoren für diese Werte gesucht werden. Das Beziehungsgefüge im System der Aufforstung legt den Schluss nahe, dass die Diversität auch aus den leichter erfassbaren Elementen Baumartenvielfalt und Habitatdiversität zusammengesetzt werden kann. In diesem Zusammenhang bezeichnet der Begriff Habitat die Summe der Lebensraumbedingungen, die einer Art das Leben ermöglichen. Finden also besonders viele Arten günstige, ihrer ökologischen Nische entsprechende Lebensbedingungen vor, so ist dies als eine hohe Diversität an Habitaten zu bezeichnen. Damit kann der Bestand auch potentiell eine höhere Gesamtdiversität erreichen. Es bleibt allerdings zu prüfen, ob Baumartenvielfalt und Habitatdiversität einen hinreichend genauen Ansatz zur Bestimmung der Gesamtdiversität bieten.

\section{2. und 3. Systemebene}

Auf der übergeordneten zweiten und dritten Systemebene, also bei Betrachtung in biotopübergreifenden bis regionalen Maßstäben, wird ein neues Set an Elementen betrachtet (Tab. 3.3-2, Abb.3.3-III). Bedingt durch den neuen Maßstab müssen neue Operatoren und Operanden definiert werden. Sie sollen die Beziehungen zwischen den Biotopen beschreiben und messbar machen. Einerseits steht die quantitative Vielfalt im Zentrum des Interesses. Andererseits ist die Verknüpfung verschiedener Lebensräume entscheidend für die Qualität des Ökosystems und gibt wichtige Informationen für den Landschaftsplaner ab. Entsprechend der Leitbildwahl in Naturschutz und Landschaftspflege wären zwei Ansätze zur Wertfindung denkbar: zunächst der als objektivistisch geltende Ansatz der höchstmöglichen Vielfalt in der Landschaft zur Schaffung einer ebensolchen Artenvielfalt (WAGNER 1992), mit stabilen Populationen und großer genetischer Vielfalt innerhalb dieser Populationen. Dieser Ansatz kann als ökozentrisch bezeichnet werden, weil er jeder (noch) 
existierenden Art ein Überlebensrecht einräumt und eine Unterteilung der Arten in "Gut und Böse“, bzw. erhaltenswert und geduldet vermeidet.

Tab. 3.3-2 Verflechtungsmatrix - Elemente der 2. und 3. Systemebene (Aufforstungsumfeld und erweiterter Planungsraum); die gefüllten Zellen zeigen jeweils die Abhängigkeit des Zeilen-Elements vom Spalten-Element (SB - Seltenheit d. Biotops ; BV Biotopverbund; R1,R2 - Randlinien; LV - Landnutzungsverhältnis)

\begin{tabular}{|c|c|c|c|c|c|c|c|c|c|c|c|}
\hline Systemebene 2 und 3 & & 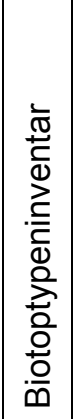 & 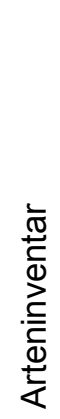 & $\begin{array}{l}\frac{0}{0} \\
\frac{0}{0} \\
\frac{0}{0} \\
0 \\
\frac{1}{0} \\
0 \\
\overline{0} \\
0 \\
0 \\
\frac{1}{0} \\
0\end{array}$ & 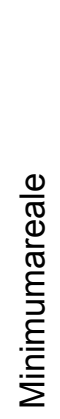 & 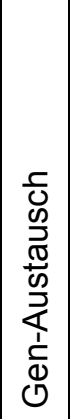 & 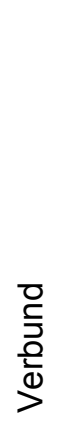 & 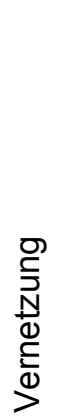 & 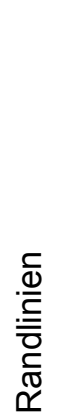 & 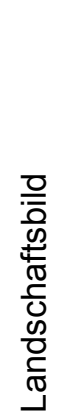 & 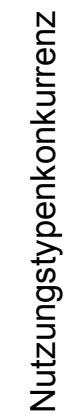 \\
\hline & & S1 & $\mathrm{S} 2$ & S3 & S4 & S5 & S6 & S7 & S8 & s9 & $\mathrm{S} 10$ \\
\hline Biotoptypeninventar & S1 & $\pi$ & & & & & & & 12 & & $\diamond$ \\
\hline Arteninventar & S2 & $\diamond$ & & & & 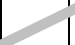 & $\diamond$ & $\bullet$ & $\bullet$ & & $\bullet$ \\
\hline Gen-Pool der Population & S3 & & & 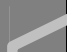 & & $\diamond$ & $\diamond$ & $\diamond$ & & & $\bullet$ \\
\hline Minimumareale & S4 & $\diamond$ & $\diamond$ & $\diamond$ & & 4 & $\bullet$ & $\diamond$ & & & $\diamond$ \\
\hline Gen-Austausch & S5 & $\diamond$ & & $\bullet$ & & 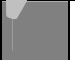 & $\diamond$ & $\bullet$ & & $\diamond$ & $\diamond$ \\
\hline Verbund & S6 & $S B$ & & - & $\bullet$ & $\bullet$ & & 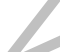 & & & $\diamond$ \\
\hline Vernetzung & S7 & $\bullet$ & & & - & $\bullet$ & 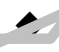 & & $\bullet$ & & 0 \\
\hline Randlinien & S8 & $\bullet$ & & & & & $\diamond$ & $\diamond$ & 1 & & $\diamond$ \\
\hline Landschaftsbild & s9 & $\diamond$ & & & & & $\bullet$ & $\diamond$ & $\diamond$ & & $\bullet$ \\
\hline Nutzungstypenkonkurrenz & S10 & & & & & & - & & $\bullet$ & & \\
\hline
\end{tabular}

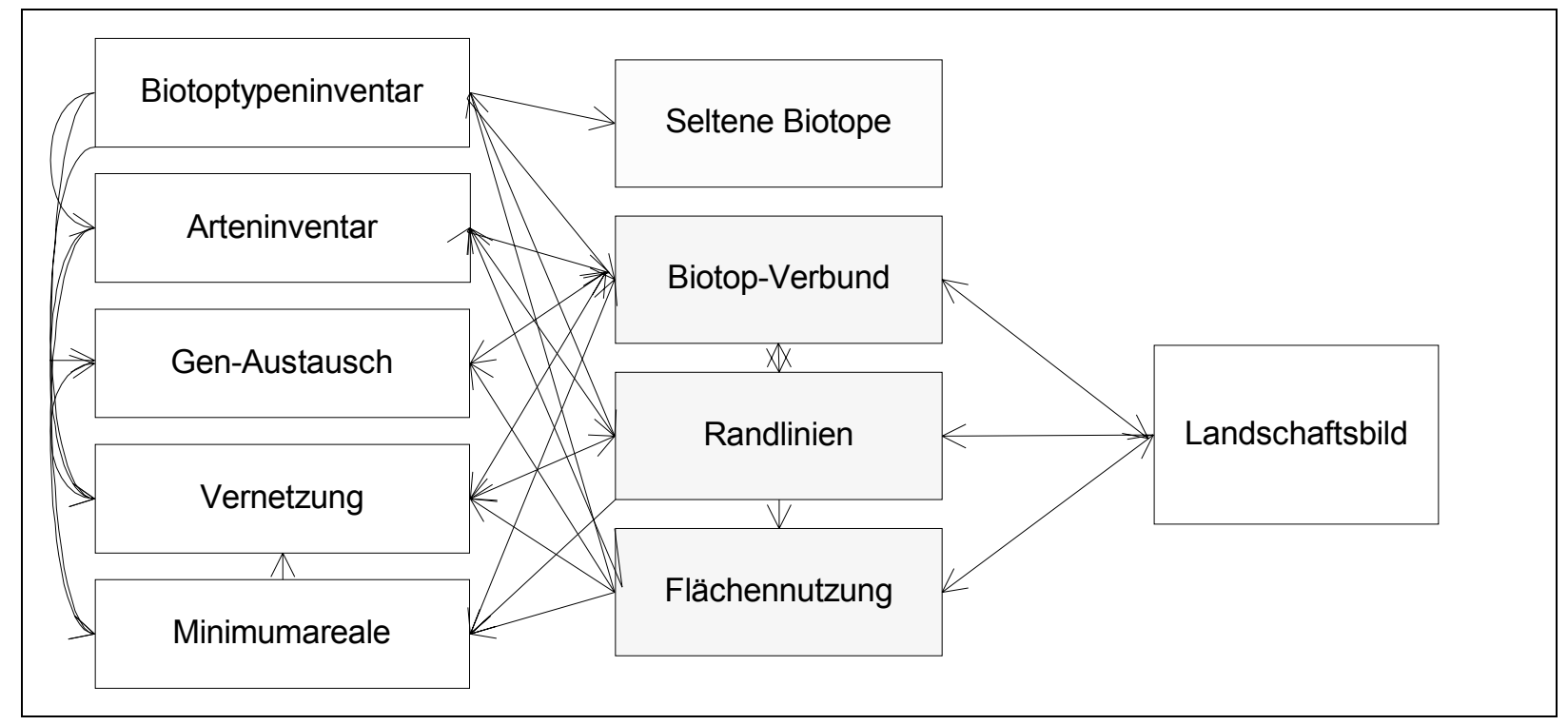

Abb. 3.3-III: Das Transformationsmodell der 2. und 3. Systemebene (Aufforstungsumfeld und erweiterter Planungsraum) 
Daneben gibt es einen zweiten, eher anthropozentrischen Ansatz. Er integriert das ästhetische Ziel eines besonderen Landschaftsbildes mit bestimmtem Wald-Feld-Anteil und einer häufig historisch begründeten Landschaftsgliederung. Des weiteren verfolgt er die Entwicklung regionaltypischer Charakteristika und Merkmale der Landschaft. Dies kann zum Beispiel eine besonders hohe Verzahnung bestimmter Biotope miteinander sein oder auch die Koppelung bestimmter Biotope aneinander. Dazu gehören aber auch Richtlinien des Biotopverbundes, welche aus einer Abstraktion des Naturraumes abgeleitet werden.

Die beiden Ansätze dürfen allerdings nicht getrennt voneinander angewandt werden. Die Ansprüche an den Lebensraum sind artspezifisch. Es müsste also zur Verwendung des anthropozentrischen Ansatzes zunächst einmal geklärt werden, welche Art nun das räumliche Leitbild für die Entwicklung der Landschaft bestimmen soll. Soll gleichzeitig der ökozentrische Ansatz berücksichtigt werden, so ist eine Benachteiligung anderer Arten unbedingt zu vermeiden. Der Königsweg ist daher in einer Verknüpfung der beiden Ansätze zu suchen: die Erhaltung einer für ein kulturgeschichtlich gewachsenes Landschaftsbild maximale Artenvielfalt. Die Praxis der Landschaftsplanung erfordert andererseits die Konzentration der Bemühungen auf wenige Interessenspunkte. Dies ermöglicht mittelfristige Erfolgskontrollen - ein wichtiges Kriterium für jegliches Wirtschaften. Außerdem können die Aktivitäten gezielter eingesetzt, Ressourcen schonender genutzt werden.

Die hier vorliegende Arbeit versucht deshalb, Leitarten für den jeweiligen Landschaftstyp zu benennen. Diese Leitarten zeichnen sich gemäß der Definition nach FLADE (1994) in ihrer Beziehung zum Landschaftstypus durch eine relativ hohe Stetigkeit aus. In der Regel erreicht die jeweilige Art in jenen Landschaftstypen, für die sie eine Leitartenrolle einnimmt, wesentlich höhere Siedlungsdichten als in allen anderen Landschaftstypen. Diese Beobachtung ist eng verknüpft mit der Feststellung, dass Leitarten in den von innen präferierten Landschaftstypen die von innen benötigten Habitatstrukturen und Requisiten wesentlich häufiger und regelmäßiger vorfinden als in allen anderen Landschaftstypen. Dadurch ist eine gewisse Indikatorfunktion impliziert, da von einem tatsächlichen oder zumindest wahrscheinlichen Vorkommen jener Arten ausgegangen werden kann, die ähnliche, aber keine höheren Ansprüche an den Lebensraum stellen, wie eben die betrachteten Leitarten.

\section{Von der Systemtheorie zur Praxis der Landschaftsplanung}

In der Systemtheorie wird häufig der Weg vom Ganzen ins Detail anschließend wieder zurückgegangen, indem versucht wird, dem Wirkungsgeschehen eine zusammenfassende Begriffkonstruktion zuzuordnen. Diese soll den formallogischen Hintergrund und die dingliche Vielfalt der Elemente in sich vereinen. Der Begriff, bzw. die Begriffskonstruktion soll eine assoziative Leistungskraft besitzen hinsichtlich der systemimmanenten Aktivität einerseits und der dynamischen Schärfeeinstellung zur Problemabbildung andererseits (KRAUSE/HENKE 1980). Im Rahmen des ausgewählten Beispieles aus der Landschaftsplanung, nämlich einer potentiellen Nutzungsänderung, ist dieser Begriff schnell gefunden: die Biodiversität. Im aktuellen Sprachgebrauch, national wie international, steht dieser Begriff für ganz unterschiedliche Merkmale und Zusammenhänge. Das Naheliegendste ist dabei die Artenvielfalt innerhalb eines Biotops, Ökosystems oder auch eines größeren Landschaftsraumes. Weiterhin wird unter diesem Begriff häufig auch die sogenannte Eveness, also die gleichmäßige Verteilung von Individuen auf die vorkommenden Arten des betrachteten Objektes, einbezogen. Auf die Unterschiede in der Erfassung, welche sich durch das Raster der Untersuchung ergeben (Erfassung nach Rassen, Arten, Familien, oder vielleicht nur nach taxonomischen Ordnungen), soll hier nicht näher eingegangen werden.

Der Begriff Biodiversität muss aber, gerade im Naturschutz, auch immer mit Blick auf die Dynamik der Systeme und der Landschaftsentwicklung betrachtet werden. Sei es nun bezüglich des mittel- und langfristigen Landschaftswandels, der sukzessionalen Abläufe innerhalb eines Biotops oder in Hinsicht auf die jahreszeitlich bedingten Veränderungen auf der gleichen Fläche. Die Biodiversität erfüllt den Anspruch, sowohl die Vielfalt der Elemente innerhalb des theoretischen Systems, als auch deren Verknüpfungen und die Dynamik des 
Geschehens gleichzeitig zu umfassen. Eine derartig weit gefasste Begrifflichkeit wird von Seiten der Planungsträger und insbesondere der Politik nur allzu gerne übernommen. Dies zeigt sich deutlich in der Vielzahl nationaler und internationaler Programme und Abkommen (Agenda 21, Biodiversitätskonvention, Ministerkonferenz zum Schutz der Wälder in Europa), welche die Förderung der Biodiversität als Haupt- oder Teilziel benennen, ohne in ihren Ausführungen so konkret zu werden, dass sich daraus ein Entscheidungskriterium für die Landnutzungsplanung vor Ort ergäbe. Gleichzeitig ist in diesem Zusammenhang aber auch zu erkennen, wie wichtig es ist, das System nach den Wirkungszusammenhängen zu durchleuchten und die Verknüpfungen aufzuzeigen. Letztere dienen schließlich als Ansatzpunkte für jedwede Maßnahme zur Veränderung des Systemoutputs.

Da das Wirkungsgefüge zu komplex und vielschichtig ist, als dass alle Einzelheiten erfasst werden könnten, macht die Umsetzung der Systemtheorie in die Praxis der Landschaftsplanung eine pragmatische Vereinfachung notwendig. Zur Erreichung eines simplifizierten Abbildes bieten sich drei unterschiedliche Operationsebenen an, auf welchen ein Untersuchungsprozess stattfinden kann.

Auf der kausalen Ebene soll eine Aufschlüsselung der Ursache-Wirkungs-Zusammenhänge stattfinden. Gleichzeitig ist eine Vielzahl von Variablen zu erfassen und zu verarbeiten. Beide Schritte werden im vorliegenden Fall durch die Erstellung der Verflechtungsmatrizen (Tab. 3.3-1 und 3.3-2) auf den verschiedenen Systemebenen zusammengefasst. Sämtliche Elemente, die im Rahmen einer Untersuchung erfasst werden könnten, werden dabei zunächst aufgelistet, unabhängig von ihrer Position im Rahmen des Wirkungsgefüges (Reihenfolge des Operanden). Anschließend wird in Form einer ja/nein-Entscheidung gutachtlich eingeschätzt, ob eine Abhängigkeit der jeweiligen Einzelelemente untereinander besteht.

Auf der zielorientierten Ebene soll eine zielsuchende Anpassung der Darstellung erfolgen. Sie dient der Feststellung imperativer Inputs. Durch eine entsprechende Anordnung der Elemente wird eine Abbildung von Abhängigkeitszentren gefördert und somit eine weitere Vereinfachung des Systems ermöglicht. Verschiedene Abhängigkeiten können nun nämlich zu Relationsindikatoren zusammengefasst werden, also zu Sachverhalten, die eine Reihe von Abhängigkeiten miteinander verbinden und somit das Wirkungsgefüge an bestimmten Punkten nachzeichnen. Dies erfolgt im vorliegenden Fall durch die Übertragung der Tabelle in das zugehörige Schaubild (Abb. 3.3-II, bzw. 3.3-III) mit Darstellung der entscheidenden Variablen.

Die Erfassung der Elemente und insbesondere die Feststellung der gegenseitigen Abhängigkeiten ist natürlich abhängig von den betrachteten Zielfunktionen eines Systems. Im vorliegenden Fall sollen für das System der Erstaufforstung vorrangig die Ziele der Förderung der Vielfalt und des räumlichen Biotopverbunds betrachtet werden, wobei letzterem eine Wirkung der funktionalen Vernetzung unterstellt wird. Darüber hinaus kommen noch andere Gründe für eine Aufforstung in Frage. Beispielswiese kann die Förderung bestimmter Waldfunktionen, wie die Erholungsfunktion, der Ressourcenschutz oder der Objektschutz gefördert werden. Diese Zielsetzungen würden sicherlich ein anderes Set an Elementen und Variablen bedingen, wenn sie optimal behandelt werden sollten. Auch ökonomisch orientierte Ziele sind denkbar, wie etwa Vermögensanlage, zusätzliches Arbeitseinkommen oder Arrondierung von Flächen (ZUNDEL 2000). Ob der Grundeigentümer die Aufforstung aus emotionalen Beweggründen anstrebt oder damit eine Veränderung der Betriebsstruktur beabsichtigt, ist für die Systemzusammenhänge nicht relevant. Für die vorliegende Arbeit stehen die Ziele der Biodiversität im Vordergrund der Betrachtung. Erholungs- und Schutzfunktionen können im Kielwasser der Aufforstung erreicht werden, werden hier aber nicht gesondert ermittelt.

Auf der normativen Ebene erfolgt schließlich eine Erfassung der Abweichungen von den vermuteten Zusammenhängen anhand eines vorgegeben Rahmens. Dadurch können Schwachstellen der Systemabbildung aufgedeckt werden. Dieser Schritt erfordert die Durchführung des Verfahrens an verschiedenen Orten und unter gleichartigen Bedingungen. Im Rahmen dieser Arbeit kann leider nur ein geringer Stichprobenumfang bearbeitet werden. 
Letzte Gewissheit darüber, inwieweit das Verfahren der Wirklichkeit gerecht wird und die systemaren Zusammenhänge hinreichend genau erfasst werden, kann nur in einer groß angelegten Stichprobe oder im Rahmen langjähriger Erfahrungen erreicht werden.

\subsubsection{Waldbauliche Prämissen}

Die Konzentration der Betrachtungen auf einen Teil der Wirklichkeit im Rahmen der Wirkungsanalyse erfordert die Definition der Rahmenbedingungen (Abb. 3.3-II). Deren Stabilität über den betrachteten Zeitraum ist die Grundbedingung für die Bewertung. Die Standortbedingungen Klima, Boden und Wasser sind im langfristigen Mittel des Betrachtungszeitraumes (50 Jahre) stabil und erfordern kein regulatives Eingreifen. Natürliche Prozesse und anthropogene Veränderungen der Standortbedingungen sollten dagegen so weit ausgeglichen werden, dass der Bestand sich zielgerecht entwickeln kann. Mit anderen Worten: waldbauliche Maßnahmen sollen die Bestandesentwicklung so beeinflussen, dass die zum Zeitpunkt der Bewertung festgestellten Merkmale über den gesamten Betrachtungszeitraum erhalten bleiben. Dies bezieht sich in erster Linie auf die Baumartenanteile und Mischungsformen, beinhaltet aber unter Umständen auch die Pflege des Waldrandes.

Der Waldbau tritt in dieser Betrachtung als Vermittler zwischen den unterschiedlichen Interessen auf, die an eine Aufforstung gestellt werden. Auf der einen Seite steht das Interesse an der ökologischen und visuellen Wirkung des entstehenden Waldbiotops. Auf der anderen Seite stehen wirtschaftliche Überlegungen. Die unterschiedlichen Zielsetzungen können und sollen über waldbauliche Eingriffe umgesetzt werden. Dies kommt bereits in der Aufforstungsplanung zum Ausdruck, wenn Baumartenwahl, Mischungsform und Pflanzverband unter Berücksichtigung eben dieser Ziele bestimmt werden.

Die naturschutzfachliche Bewertung einer Aufforstung konzentriert sich auf die ökologischen Aspekte der Maßnahme. Ökonomische Betrachtungen bleiben außen vor. Politische Vereinbarungen und Programme (vgl. Kap. 2.1) belegen ein öffentliches Interesse an der Waldvermehrung. Die diesbezügliche Argumentation unterstreicht immer wieder die erhofften ökologische Wirkungen. Folglich ist es auch Aufgabe der Regierungen, die ökonomischen Rahmenbedingungen so zu setzen, dass ökologische Aspekte bei der Aufforstungsplanung berücksichtigt werden können und nicht unter einem wirtschaftlichen Zwang zur Rentabilität leiden müssen. Damit ist gerechtfertig, dass ein naturschutzfachlich orientiertes Bewertungsverfahren sich nicht mit den ökonomischen Rahmenbedingungen beschäftigt.

Neben den wirtschaftlichen Bedingungen können theoretisch auch die standörtlichen Voraussetzungen die Möglichkeiten begrenzen. In der Praxis sieht es allerdings so aus, dass vor allem bislang landwirtschaftlich genutzte Flächen aufgeforstet werden. Diese zeichnen sich wiederum durch einen relativ guten Nährstoffvorrat (gegenüber den bereits bewaldeten Standorten) aus. Selbst für sogenannte „Grenzertragsböden" trifft diese Aussage meist zu. Zumindest für die ackerbaulich genutzten Flächen kann daher von sehr guten Bedingungen ausgegangen werden, die die Baumartenwahl kaum einschränken (DOHRENBUSCH 1996). $\mathrm{Da}$ sich die naturschutzfachlichen Ansprüche aber sowieso an den natürlichen Bedingungen orientieren, ist selbst auf den schwächeren Standorten eine Berücksichtigung ökologischer Wertvorstellungen möglich.

Die Aufgabe des Waldbaus kann folglich zusammengefasst werden als Umsetzung vorgegebener Ziele unter gegebenen, natürlichen Bedingungen. Dazu stehen ihm eine Reihe von Maßnahmen zur Verfügung, die von der Flächenvorbereitung über die Pflanzplanung und den Kulturschutz bis hin zu lenkenden Eingriffen in die Bestandesstruktur reichen.

Die Technik hat sich dabei in den vergangenen Jahrzehnten ebenso verändert wie die Zielsetzungen. Ausdruck dieser Veränderung sind sowohl die Beiträge zur wissenschaftlichpraktischen Diskussion als auch die ökologisch orientierten Wald(bau)programme und richtlinien der Bundesländer (z.B. LÖWE). 
Aus der Perspektive des Naturschutzes ist diesbezüglich vor allem die Verschiebung in den gepflanzten Baumarten von Nadelholz zu Laubholz und von Rein- zu Mischbeständen hervorzuheben.

Auch die Beteiligung von Pionier- und Vorwaldbaumarten bei der Pflanzung ist zu begrüßen. Diese Baumarten sorgen für eine frühe Strukturentwicklung auf der Fläche und dienen gleichzeitig als Füll- und Treibholz für Hauptwirtschaftsbaumarten. Ihre Beteiligung im Rahmen von Aufforstungen hat in den vergangenen Jahren deutlich zugenommen, wie die Berichte verschiedener Autoren belegen (SCHÖLMERICH 1999, FRIEDRICHSDORF 1999, WAGNER 1995).

Die Entwicklung zu weiteren Pflanzverbänden und geringeren Pflanzenzahlen kann unter ökologischen Gesichtspunkten begrüßt werden. Sie bietet zumindest in den ersten Jahren etwas mehr Raum für natürlich ankommende (Begleit-) Vegetation. Dieser Effekt lässt allerdings nach, sobald die Kultur den Dichtschluss erreicht hat.

Eine längerfristige, ebenfalls ökologisch positive Wirkung ist von der Gestaltung der Waldränder zu berichten. Viele Aufforstungen berücksichtigen breite, strauchreiche Randzonen im Übergangsbereich zu landwirtschaftlichen Flächen und schaffen somit wertvolle Ökotone.

Weitere Hinweise zur Weiterentwicklung der Technik für Erstaufforstungen landwirtschaftlich genutzter Flächen sind in der Literatur zu finden (z.B. LEDER 1996, PETERSEN/FISCHER 1999, VOGEL 1999, WAGNER 1995) . Deren Bezug zu ökologischen Auswirkungen ist allerdings zu gering, als dass an dieser Stelle näher darauf eingegangen werden soll. Nichtsdestotrotz zeigt sich aus der Fülle der Beiträge die zunehmende Bedeutung der Umwandlung von Ackerflächen in Wald, die nicht nur unter ökonomischen, sondern auch unter ökologischen Gesichtspunkten zu betrachten ist.

Das Bild der Aufforstungen hat sich - sowohl im übertragenen Sinn als auch in der tatsächlichen Erscheinung - in den vergangenen Jahrzehnten geändert. Eintönige, häufig auch standortsfremde Nadelholzreinbestände werden heute kaum noch angelegt. Stattdessen bestimmen vielerorts baumartenreiche Mischbestände mit teilweise deutlich ausgeprägten Waldrändern das Erscheinungsbild der Erstaufforstungen. Seitens des Naturschutzes ist diese Entwicklung unbedingt zu begrüßen und zu unterstützen. Wo dies möglich ist, sollte er mit konstruktiven Anregungen und fundierten Konzepten seinen Beitrag dazu leisten.

\subsection{Bewertungskriterien}

Die Systemanalyse führt nach der dargelegten Methode zur Definition der Schlüsselelemente, mit deren Hilfe die Wirkungszusammenhänge nachgezeichnet werden. Diese werden über die Verflechtungsmatrix eingegrenzt. Die Umwandlung der Operanden in echte Indikatoren ist nur vor dem Hintergrund der beabsichtigten Wertfeststellung möglich. Die anzustrebenden Werte werden durch rechtliche Vorgaben sowie durch die wissenschaftliche Diskussion (im Sinne eines state-of-the-art) vorgegeben. Jedes Charaktermerkmal eines Systems muss auf dessen Bezug zu diesen Werten überprüft werden. Daraus ergibt sich die Aussage und Bedeutung der Indikatoren.

Zur Entwicklung eines Evaluationsverfahrens werden zunächst die Wertvorgaben unterschiedlicher Quellen betrachtet. Sie geben die Kriterien für eine naturschutzfachliche Bewertung vor. Aus den Kriterien können anschließend die Indikatoren abgeleitet werden. Dabei tauchen Probleme auf, wenn die Kriterien nicht unabhängig voneinander sind. In diesem Falle ist es sinnvoller, die Indikatoren direkt aus der Systemanalyse zu entwickeln und den Bezug zu den Bewertungskriterien nachträglich festzustellen. Dadurch kann die Unabhängigkeit der Indikatoren - nach KIRBY (1994) ein sehr entscheidendes Merkmal einer Bewertung - gewahrt werden. Dieser Schritt der Ableitung von Indikatoren aus der Systemanalyse erfolgt auch in der vorliegenden Arbeit (Kap. 3.5). Die Definition der 
Indikatoren muss auf alle Fälle in Zusammenhang mit der von innen erwarteten Zielaussage erfolgen und empirisch oder logisch begründet sein.

Die Naturschutzgesetze des Bundes und der Länder geben in den einleitenden Paragraphen die Ziele und Grundsätze des Naturschutzes vor. Die diversen Interessenschwerpunkte kommen in unterschiedlichen Schutzgegenständen zum Ausdruck. Eine zentrale Rolle spielt der Artenschutz, sowohl als Individuenschutz (Handel, Haltung, Transport) als auch mit Bezug zu Populationen und Lebensraumbedingungen (Lebensraumelemente, Biozönosen). Eine zweite Hauptrolle kommt dem Gebietsschutz zu. Hierunter ist die Landschaftsausstattung mit mehr oder weniger gebietstypischen Elementen zu verstehen, die gleichzeitig auch die Grundlage für das Vorkommen unterschiedlicher Tier- und Pflanzenarten bilden (biogene Erscheinungsformen im Arten- und Biotopschutz). Ergänzt wird der Schutz der biogenen Elemente durch die Erhaltung der geogenen Erscheinungsformen, also mehr oder weniger statischen Formen, die einen eigenen Beitrag zur Stabilität und Vielfalt der Landschaft leisten. Schließlich gehört zum Gebietsschutz auch der Schutz komplexer Landschaftsformen, die sich aus den unterschiedlichen und miteinander verknüpften Biotopen ergeben. Diese sind in Mitteleuropa in ihrer Zusammensetzung und Erscheinungsform sehr stark beeinflusst durch viele Jahrhunderte relativ intensiver Bewirtschaftung. Die Kultivierung und Nutzung der Natur ist gleichzeitig Bedrohung und Chance für das Vorkommen und die Entwicklung einzelner Arten. Natur und Kultur (im Sinne von Landnutzung) können daher nicht losgelöst voneinander betrachtet werden.

Die Vielfalt der gesetzlich benannten Ziele des Naturschutzes weist bereits auf die unterschiedlichen Interessen hin, die bei Planung und Umsetzung zu wahren sind. Eine große Interessenvielfalt bewirkt wiederum regelmäßig das Auftreten von Zielkonflikten. Die Ableitung sogenannter Legalkriterien für die Bewertung aus den gesetzlichen Naturschutzzielen ist theoretisch möglich. In der Praxis scheitert dieses Vorgehen jedoch an der unterschiedlichen Gewichtung von Zielen durch die beteiligten Interessengruppen. Hinzu kommt, dass die Naturschutzpraxis stets projektorientiert arbeitet. Ob es nun um eine bestimmte Art, ein Biotop, ein Schutzgebiet oder eine ganze Region geht, es werden stets die relevanten Ziele des Naturschutzes sondiert und die Kriterien dementsprechend festgelegt. In der fachlichen Diskussion tauchen deshalb immer wieder neue Listen von Bewertungskriterien auf, die von unterschiedlicher Detailliertheit geprägt sind (z.B. BUCHWALD 1980; WAGNER 1992). Die Tabelle 3.4-1 gibt einen synoptischen Überblick zu einigen dieser Listen. Daraus wird ersichtlich, dass sich einige Kriterien in allen Quellen wiederfinden, dass aber auch subjektive Schwerpunkte der Autoren feststellbar sind. Allgemein kann festgehalten werden, dass die fachlichen Kriterien in großem Maße die unterschiedlichen Naturschutzziele berücksichtigen.

\section{Naturnähe}

Unter Naturnähe oder Natürlichkeit ist ein Kriterium zu verstehen, welches den Grad der Annäherung eines Naturelementes an den Naturzustand wiedergibt. Ein Urwald stellt hinsichtlich dieses Kriteriums einen idealen Vegetationstyp dar, also einen Wald, der noch nie durch anthropogene Nutzung in seiner Zusammensetzung oder Struktur beeinflusst wurde. Urwälder sind in Mitteleuropa allerdings nicht mehr vorhanden. Die gesamte Landschaft wurde durch die Kultivierung und Nutzung beeinflusst und mehr oder weniger stark überprägt. Auch ursprünglich waldfreie Gebiete, wie z.B. Hochmoore, wurden durch Meliorationsmaßnahmen oder Nutzungen bereits stark verändert und häufig in land/forstwirtschaftliche Kulturflächen umgewandelt.

Die Naturnähe hat jedoch für die Forstwirtschaft heute eine hohe Bedeutung. Sowohl in der waldbaulichen Pflege als auch in der Neuanlage von Waldflächen gelten naturnahe Artenzusammensetzung und einzelne Strukturparameter des Naturwaldes (Totholzreichtum) als Zielvorgaben. Die angestrebte Artenzusammensetzung drückt sich dabei in der potentiellen natürlichen Vegetation (PNV) aus. Damit werden die langfristigen anthropogenen Beeinflussungen der Ökosysteme und der Landschaft durch 
jahrhundertelange Nutzung der Natur als Ausgangssituation berücksichtigt. Das Idealziel PNV ist durch die aktuellen Standortbedingungen vorgegeben und wird durch die Waldbewirtschaftung nicht mehr beeinflusst. Damit gehört sie zu den Rahmenbedingungen des Systems (näheres siehe Kap. 3.5.2).

Tab. 3.4-1: $\quad$ Synoptischer Überblick zu Bewertungskriterien im Naturschutz - nach unterschiedlichen Autoren

\begin{tabular}{|c|c|c|}
\hline WAGNER, H. (1992) & BUCHWALD, K. (1980) & USHER, M. I ERZ, W. (1994) \\
\hline Bewertungskriterien & Zielkriterien & Bewertungskriterien \\
\hline $\begin{array}{ll}\text { - } & \text { Vielfalt } \\
\text { (von Biotopen, Arten, } \\
\text { Landschaftselementen) } \\
\text { - Schönheit } \\
\text { (des Landschaftsbildes) } \\
\text { - Eigenart } \\
\text { (der Landschaft) } \\
\text { - } & \text { Anturnähe } \\
\text { Anzahl und } \\
\text { Gefährdungsgrad von } \\
\text { Arten der Roten Liste } \\
\text { - Seltenheit und } \\
\text { - Gefährdung von Biotopen } \\
\text { Ausprägungsform von } \\
\text { Ökosystemtypen } \\
\text { Repräsentanz } \\
\text { (für den Naturraum) } \\
\text { Alter und } \\
\text { Regenerierbarkeit von } \\
\text { Ökosystemen }\end{array}$ & $\begin{array}{l}\text { - Natürlichkeit } \\
\text { - Gefährdung } \\
\text { - Unersetzbarkeit } \\
\text { - Sielfalt } \\
\text { - Voltenheit } \\
\text { - Repräsentanz }\end{array}$ & $\begin{array}{l}\text { - } \text { Diversität } \\
\text { - } \text { Fläche } \\
\text { - } \text { Seltenheit } \\
\text { - Reprrlichkeit } \\
\text { - Zusäsentanz } \\
\text { nach Verfahren und } \\
\text { Objekt der Bewertung, } \\
\text { z.B. } \\
\circ \quad \text { Empfindlichkeit } \\
\circ \text { Stabilität } \\
\circ \text { Veg.-Struktur } \\
\circ \text { Sukzession } \\
\circ \text { Dauer der Entwicklung } \\
\circ \text { Endemismus }\end{array}$ \\
\hline
\end{tabular}

Die Naturnähe wird im Allgemeinen durch Stufen von natürlich bis unnatürlich/ künstlich angegeben. Anzahl und Benennung der einzelnen Stufen sind autorenspezifisch. Die Bestimmung des Wertes kann dabei zwei verschiedenen Ansätzen folgen. Meist nähert sich der Betrachter dem Wert von Seiten des natürlichen (Ideal-)Zustandes an (ELLENBERG 1982, WESTHOFF in BUCHWALD/ENGELHARDT 1980, HORNSTEIN 1958). Der andere, nicht ganz so häufig vorzufindende Ansatz, wählt die Hemerobie (aktualistische Naturveränderung) als Maßstab und bestimmt den Wert indirekt über den Grad künstlicher Veränderung (KOCH et al. 1999, GRABHERR et al. 1995).

\section{Vielfalt}

Der Begriff der Vielfalt oder Mannigfaltigkeit beschreibt zunächst nur die Existenz unterschiedlicher Elemente eines betrachteten Systems. Seine Wertschätzung erfährt das Kriterium durch den Vergleich mit anderen Flächen gleicher Grundbedingungen. Die Betrachtung konzentriert sich dabei auf einen bestimmten Systemausschnitt. Während sich in der allgemeinen Diskussion die Vielfalt aus den Elementen Arten, Biotope und Landschaftselemente zusammensetzt (vgl. Tab. 3.4-1), ist die Möglichkeit, diese zu beeinflussen, unterschiedlich groß. Der Schutz, bzw. die Schaffung von Landschaftselementen (z.B. Solitärbäume, Hecken, Steinwälle) ist wesentlich einfacher zu realisieren als die Schaffung neuer Biotope oder gar der Schutz gesamter Populationen, da der selektive Artenschutz unterschiedlicher Maßnahmen und Regelungen mit verschiedenen Wirkungszeiträumen bedarf. Der Schutz einer bestimmten Population ist nur dann 
erfolgreich, wenn die von ihr benötigten Biotoptypen in ausreichender Zahl und Qualität vorhanden sind, gleichzeitig der genetische Austausch gewährleistet ist und Bestandesbedrohungen (wie z.B. durch eine Bejagung oder durch eingeschleppte Konkurrenten) auszuschließen sind.

Die Artenvielfalt eines Biotops drückt sich in verschiedenen Elementen aus. Dazu gehören unterschiedlichste Tier- und Pflanzengruppen, von den Mikroorganismen bis zu den Großsäugern, von den Moosen bis den Bäumen.

Die Biotopvielfalt eines Landschaftsteiles umfasst die dort vorhandenen Biotoptypen, aber auch die unter Naturschutzgesichtspunkten wichtigen Übergangszonen (Ökotone). Je nach Detailliertheit der zugrunde gelegten Klassifizierung, erfordert die Erfassung der Biotoptypen einen mehr oder weniger großen Aufwand. Eine Unterteilung der Wiesen in Typische Glatthafer-Wiese, Kohldistel-Glatthafer-Wiese, Salbei-Glatthafer-Wiese und GoldhaferBergwiese (NITSCHE / NITSCHE 1994) vervielfacht demnach die Aufnahmetätigkeiten und erfordert unter Umständen auch einen größeren Maßstab in der kartographischen Darstellung der Flächen.

Jede Vegetationsform besitzt einen eigenen Ausschnitt der Biodiversität. Dieser umfasst einiges mehr, als nur die Anzahl der vorkommenden Tier- und Pflanzenarten. Vielmehr ist unter dem Begriff der Biodiversität auch die Vielfalt adäquater Lebensbedingungen für unterschiedliche Arten und die Verteilung der Individuen auf die einzelnen Arten zu sehen. Eine allgemeine Definition der Biodiversität fällt schwer, die genaue Erfassung derselben ist wohl unmöglich. Selbst eine Beschränkung auf den faunistischen Teil des Ökosystems würde den Wissenschaftler noch vor eine unmögliche Aufgabe stellen. So gibt es derzeit beispielsweise keine vollständige Erfassung eines faunistischen Arteninventars für Wälder. Nach SCHÄFER (2001) ist die Anzahl der Arten aus den verschiedenen Taxa von den Protozoen bis zu den Säugetieren für einen Laubwald im temperierten Klimabereich zwischen 2000 und 5000 anzusiedeln. Daraus ist die Problematik der Erfassbarkeit leicht abzulesen. Die Annäherung an die Biodiversität muss folglich über Hilfsgrößen, bzw. Indikatoren erfolgen.

Mit besonderer Rücksicht auf die Vielfalt der Nischen für Kleintiere, werden Wälder oberirdisch gekennzeichnet durch die Straten Kraut- und Strauchschicht, durch die Stammregion und durch verschiedene Kronenschichten. Anzahl und Besiedelungsdichte verschiedener Tierarten hängen von der Größe des Waldes, der regionalen Faunenzusammensetzung, der Waldstruktur, dem jeweiligen Alter der Waldbestände und vom Waldinnenklima ab. Wie in anderen Ökosystemen nutzen die Tierarten im Wald nicht das gesamte Ökosystem, sondern vielmehr nur bestimmte Teile davon, beispielsweise bestimmte Baumarten, Baumschichten, Altersstadien, Mikrohabitate oder Mikroklimabereiche in artspezifischer Weise. Das Set der entsprechenden Lebensbedingungen wird als Authabitat bezeichnet. Ein Wald, welcher diese Merkmale in großer Varietät enthält, ist besonders nischenreich und damit potentiell für viele Tierarten ein geeignetes Habitat.

Für eine Reihe von Tieren gibt das Verhältnis von Waldfläche zu Flächenanteilen des Offenlandes den Ausschlag für die Besiedlung der Landschaft, wobei die Beschaffenheit der Übergangszone ein zusätzliches Kriterium ist. Daher ist auch die Verzahnung von Wald und Offenland bei der Landschaftsplanung zur Biodiversitätsförderung zu beachten (Kap. 3.5.8).

\section{Seltenheit und Gefährdung}

Obwohl sie in der Naturschutzpolitik häufig als qualitatives Kriterium behandelt wird, ist die Seltenheit zunächst ein quantitatives Kriterium. Sie macht eine Aussage über das tatsächliche oder vermutete Vorkommen einer Art oder eines Biotops. Dabei können die Gründe für das spärliche Vorkommen sehr unterschiedlich sein. Eine Art ist naturgemäß am Rande ihres Verbreitungsgebietes seltener als in dessen Zentrum. Die Seltenheit kann ihre Ursache auch in der anthropogenen Beeinflussung der Biotope besitzen. Beispielsweise 
führte die systematische „Melioration“ von Mooren durch Entwässerung für die Bewohner der Hochmoore zu einer extremen Bedrohung, da die Lebensbedingungen verändert wurden. Stenöke Moorarten wurden daraufhin gleichermaßen mit den Biotopen selten. Und schließlich können bestimmte Biotoptypen, z.B. aufgrund deren zeitlicher Rolle im Rahmen der Sukzession, von Natur aus seltener anzutreffen sein. So spielt die Pionierphase zeitlich eine deutlich geringere Rolle als die Alters- und Zerfallsphase eines Waldes.

Für die Seltenheit von Arten und Biozönosen können unter anderem Endemismus, Arealfaktoren, Stenökie und paläohistorische Entwicklung entscheidende Einflussgrößen darstellen. BUCHWALD (1980) zeigt anhand der Lachseeschwalbe auf, dass es nicht einfach festzustellen ist, ob die ermittelte Seltenheit einer Art natürlichen oder anthropogenen Ursachen folgt. Die Lachseeschwalbe tritt in Deutschland am Rande ihres Vorkommens auf, zeichnet sich durch stenöke Lebensraumansprüche aus und ihre Ausbreitung wurde vermutlich auch durch historische Gründe mitbeeinflusst.

Die Gefährdung wird bei einigen Autoren als getrenntes Kriterium betrachtet (vgl. Tab. 3.41), führt allerdings immer zu einer Beeinflussung der Seltenheit. Die Gefährdung drückt die Verringerung einer Art oder eines Biotops in dessen Bestand aus. Ihre Ursachen können natürlich (Klimawandel), indirekt anthropogen (z.B. Schadstoffeintrag) oder direkt anthropogen (z.B. Umwandlung von bestimmten Biotopen) sein. Einen Ausdruck für die Gefährdungssituation von Arten und Biotopen findet sich in der Klassifizierung der Roten Listen. Solche für Biotoptypen unterscheiden dabei noch zwischen der Gefährdung durch direkte Bedrohung (Umwandlung) und einer Gefährdung durch Degradation, also eine schleichende qualitative Verschlechterung der Biotope (RIECKEN et al. 1994).

Seltenheit und Gefährdung sind also direkt miteinander gekoppelt. Auch innerhalb der Teilkriterien bestehen in der Regel Abhängigkeiten zwischen den Biotopen und den darin vorkommenden Arten oder zwischen den sukzessional aufeinander folgenden Entwicklungsstadien einer Vegetationsgesellschaft. Eine Reduktion der Bewertung auf die Arten der Roten Liste, wie sie aus dem Kriterienkatalog von WAGNER (vgl. Tab. 3.4-1) hervorgeht, erscheint nicht sinnvoll, da deren Vollständigkeit fraglich ist und auch dort nicht vorkommende Arten regional durchaus förderungswürdig sein können.

\section{Repräsentanz}

Die Repräsentanz ist sowohl ein qualitatives als auch ein quantitatives Kriterium zur Beschreibung des Vorkommens einer Art, eines Biotops oder eines Lebensraumelementes in Bezug auf dessen „typische“ Ausprägung. Sehr häufig werden danach vor allem Elemente und Bewohner der Kulturlandschaft bewertet. Fraglich ist, welches Landschaftsbild und Elementinventar als typisch zu betrachten ist. Dies kann einerseits das derzeitige Erscheinungsbild der Landschaft sein, was gleichzeitig das am einfachsten zu bewahrende wäre. Typisch könnte aber auch ein Erscheinungsbild sein, welches durch Veränderungen in der jüngsten Vergangenheit bereits überprägt wurde und nun wieder hergestellt werden soll.

An die Feststellung des historischen Zeitpunktes, dessen Erscheinungsbild als Bewertungsmaßstab für die Repräsentanz gesetzt wird, schließt sich die Frage nach dem Elementinventar. Es kann davon ausgegangen werden, dass die Häufigkeit der Biotope und Elemente untereinander nicht ausgeglichen ist. Der optische Eindruck einer Landschaft verändert sich zum Beispiel sehr stark, wenn statt $15 \%$ der Fläche nur noch $5 \%$ der Fläche mit einem auffälligen Biotoptyp wie der Streuobstwiese bestanden sind.

In der naturschutzfachlichen Bewertung wird die Rote Liste häufig in Zusammenhang mit der Repräsentanz genannt. Dies erscheint nachvollziehbar vor dem Hintergrund der Erstellung Roter Listen als Instrument im Sinne von Expertengutachten über das Vorkommen von Arten und Biotopen. In den Listen kommen alle Elemente vor, die für einen Lebensraum typisch sind. Allerdings ist nicht sicher, ob die Gefährdungseinstufung auch lokal ihre Richtigkeit besitzt. Wiederum stellt sich das Problem der Unterscheidung zwischen natürlichen und anthropogenen Faktoren bei Seltenheit und Gefährdung. 
BUCHWALD (1980) benennt die Vollkommenheit als zusätzliches, eigenständiges Kriterium in Bezug auf die vollständige/optimale Ausprägung einer Landschaft oder deren Teile. Bezogen auf ein Biotop ist damit dessen Arteninventar angesprochen. Beschreibungen der typischen Ausprägungen von Pflanzengesellschaften finden sich in der einschlägigen Literatur (ELLENBERG 1982; HOFMEISTER 1990; u.a.).

Im landschaftsökologischen Sinn ist eine Landschaft dann vollkommen, wenn allen Elemente eine entscheidende Funktion im Ökosystem erfüllen. Eine Nisthilfe für Störche füllt den Landschaftsraum an und ergibt somit eine höheren Grad der Vollständigkeit an Landschaftselementen. Wenn sich dort aber keine Störche einfinden, weil andere Störfaktoren auftreten oder die Lebensbedingungen nicht entsprechend sind, so erscheint auch dieser höhere Grad an Vollkommenheit wertlos.

Das Kriterium der Vollkommenheit unterscheidet nicht zwischen wichtigen und weniger wichtigen Arten. Da alle Elemente vorhanden sein sollen, gibt es keine per se förderungswürdigen Arten. Genau diese Gleichrangigkeit erschwert allerdings die Umsetzung des Kriteriums in die Praxis, da diese stets Ziele sucht, auf die sich Maßnahmen konzentrieren lassen. Erfahrungsgemäß ist die Vervollständigung eines Arten- oder Elementinventars mit zunehmender Diversität schwieriger. Dies bedingt auch steigende Kosten und Aufwand für den Naturschutz in der Entwicklung des Lebensraumes.

Die Zusammenfassung der in Tabelle 3.4-1 aufgeführten Kriterien nach dem vorgestellten Schema in die Hauptkriterien Naturnähe, Vielfalt, Seltenheit/Gefährdung und Repräsentanz soll nicht als Versuch neuerlicher Kategorisierung verstanden werden. Die Kriterien und Teilkriterien können im Gegenteil nicht losgelöst voneinander betrachtet werden. Gefährdung führt zu Seltenheit, Seltenheit bedingt automatisch eine Gefährdung. Die Unersetzbarkeit oder Reproduktionsfähigkeit eines Biotops ist automatisch auch ein Hinweis auf dessen Gefährdung - besonders in Hinsicht auf die heute überall erlebte Überformung der Landschaft durch anthropogene Nutzung.

BUCHWALD (1980) weist in seinen Ausführungen zu den Bewertungskriterien noch darauf hin, dass die Kriterien „Bedeutung für Forschung und Kultur“ und „Bedeutung für die Erholung“, welche beispielweise in Ausscheidungsverfahren für Schutzgebiete behandelt werden, als additive (zusätzliche) oder kumulative (aus anderen Kriterien abgeleitete) Kriterien zu betrachten sind.

Er beschreibt weiterhin einen Bedarf zur Differenzierung und Quantifizierung der Kriterien und fordert eine fallweise Abstimmung derselben zu einem Kriteriensystem.

Im Rahmen der vorliegenden Untersuchung zu Erstaufforstungen wird dieser Forderung auf andere Weise entsprochen. Des besseren Überblicks wegen (Abb. 3.4-I), werden vier Kriterien unterschieden:

4) Biodiversität

5) Natur

6) Kulturlandschaft - Elemente

7) Kulturlandschaft - Strukturierung

Diese Kriterien sind jedoch nicht als strenge Definition zu verstehen, da sie kaum voneinander unabhängig wären. Soweit diese im Rahmen der Systemanalyse die entscheidenden Wirkungszusammenhänge wiedergeben können, erscheint eine Differenzierung der Kriterien nicht notwendig. Stattdessen wird auf eine genaue Trennung zwischen den Indikatoren und deren Unabhängigkeit voneinander geachtet. Auch die Zuordnung der Indikatoren wird vor allem aus Gründen der Übersichtlichkeit und Nachvollziehbarkeit des Verfahrens durchgeführt. 

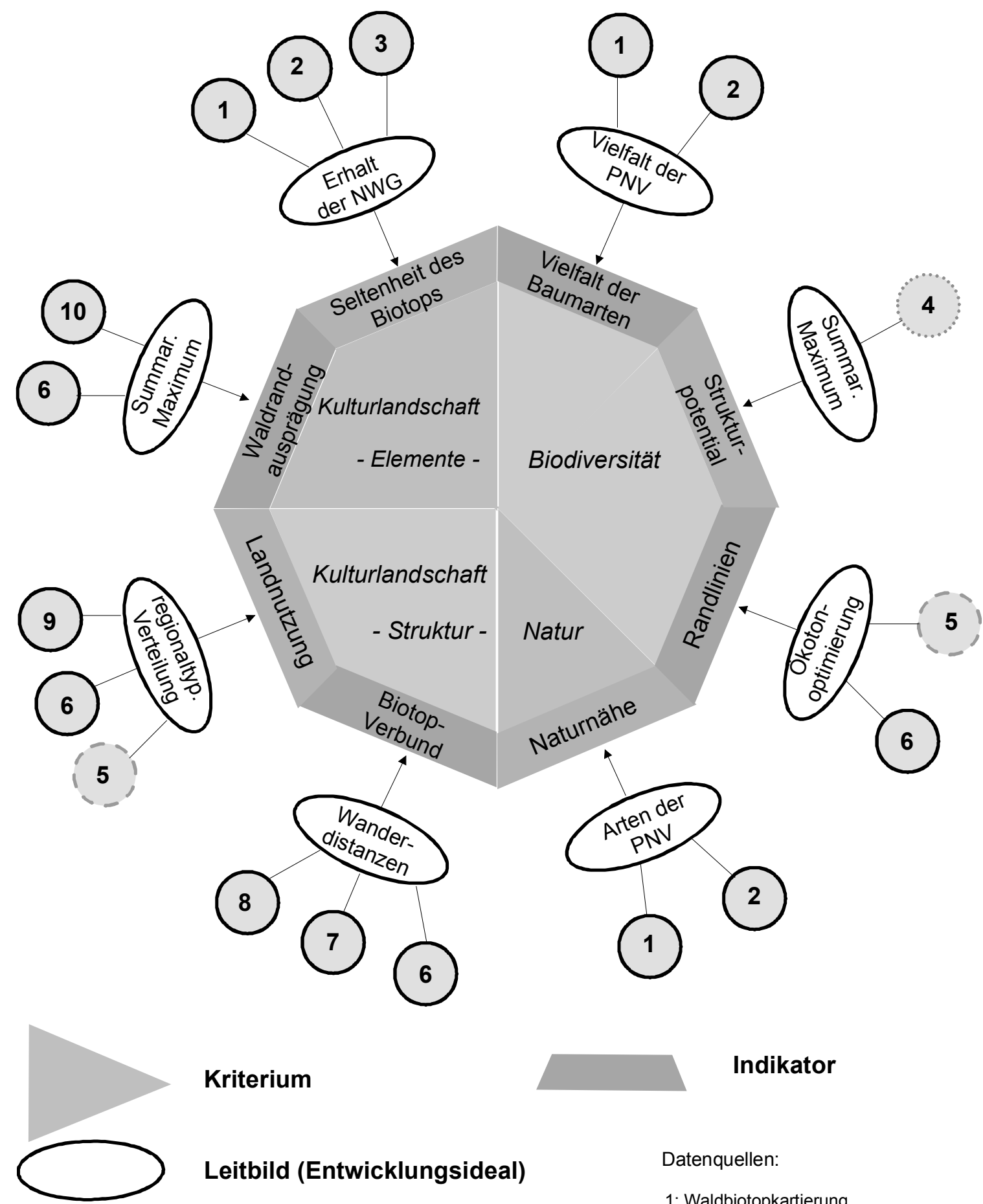

Kriterium

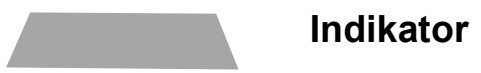

\section{Leitbild (Entwicklungsideal)}

Datenquellen:

1: Waldbiotopkartierung

2: Standortkartierung

3: Rote Liste Biotope

4: Struktur-Potential-Klassifikation

5: Landnutzungskartierung

6: Luftbild

7: Biotopkartierung (mit Offenland)

8: Artenkartierung

9: Regionale Raumplanung

10: Waldrandbeschreibung

Abb.3.4-I: Übersicht zu den Kriterien, Indikatoren, Leitbildern und Datenquellen des Evaluationsverfahrens (PNV=pot. nat. Vegetation; NWG=Nat. Walgesellschaft) 
Jedem Indikator steht ein Leitbild im Sinne eines Entwicklungsideales (nicht als Entwicklungsziel zu missverstehen) gegenüber. Dieses Ideal verkörpert die optimale Ausprägung eines Systemelementes im Sinne des Naturschutzes. Obwohl die Leitbilder teilweise für vollkommen unterschiedliche Ziele stehen, können sie manchmal nebeneinander auf derselben Fläche angestrebt werden. Schließlich werden zur Ermittlung des Evaluationswertes eine Reihe von Datenquellen herangezogen. Diese sind über verschiedene Behörden (Forstverwaltung, Naturschutzverwaltung, Landwirtschaftsämter, u.a.) zugänglich. Eine Landnutzungskartierung liegt derzeit erst für wenige Regionen aktuell vor. Für beide Untersuchungsgebiete dieser Arbeit musste sie anhand von Luftbildern und Außenaufnahmen durchgeführt werden. Mittelfristig ist allerdings zu erwarten, dass derartige Daten in größerem Umfang zur Verfügung stehen, da das neu gefasste Naturschutzgesetz die Erstellung von Landschaftsplänen für alle Regionen vorsieht. In diesem Zuge werden regelmäßig auch die Landnutzungen erfasst und kartiert.

Eine Neuerung des Verfahrens besteht in der Entwicklung eines Systems zur Klassifizierung von Baumarten nach deren Beitrag zur Bestandesstruktur (vgl. Kap. 3.5.4). Hier wurde ein neuer Schlüssel entworfen, welcher durch weitere Untersuchungen zu bestätigen ist, dann aber wertvolle Aussagen für die Entwicklung der Bestandesstruktur liefern kann. 


\subsection{Indikatoren}

Im Folgenden werden die Indikatoren, die im vorgestellten Verfahren Verwendung finden, einzeln vorgestellt. Dies erfolgt stets nach einem einheitlichen Aufbau. Der Indikator wird zunächst mit Hinsicht auf das Verfahren und dessen Zielsetzung definiert. Dabei handelt es sich jeweils um eine Definition mit Bezug zur Erstaufforstung, nicht um eine generelle, naturschutzfachliche Definition des Begriffes. Anschließend wird, mit Bezug zur wissenschaftlichen Literatur, die Bedeutung des jeweiligen Indikators für den Naturschutz allgemein dargestellt. Hier finden sich nähere Erläuterungen zur Definition und eine verkürzte Darstellung, welche Rolle der Indikator im Naturschutz und in Bezug auf dessen Bewertungskriterien spielt.

Der Abschnitt Bestimmbarkeit des Indikators geht auf die Möglichkeiten der Erfassung ein. Er schlägt die Brücke zur Situationsanalyse, die vor jedem Variantenstudium zu erfolgen hat. Der Abschnitt zeigt außerdem auf, welche Bewertungsmaßstäbe angewandt werden können. Die Ableitung des Indikators aus der Matrix stellt den Bezug zum betrachteten System und der Wirkungsanalyse her. Sie bezieht sich auf die in Kap. 3.3 vorgestellten Verflechtungsmatrizen. Die tatsächliche Erfassung des Indikators im vorliegenden Verfahren wird schließlich im Abschnitt Parameter erläutert. Zu jedem Merkmal gehört auch ein Bewertungsschema. Dieses sieht eine Punktespanne bis zu einem Maximalwert von 5 Punkten vor. Die raumbezogenen Indikatoren Biotopverbund, Landnutzungsverhältnisse und Randlinien-Vorkommen können auch negative Werte ergeben.

Die Erhebungsbogen mit Bewertungsschema zu den einzelnen Indikatoren finden sich gesammelt in Anhang 1.

\subsubsection{Baumartenvielfalt}

\section{Definition}

Die Artenvielfalt oder Diversität eines Lebensraumes bezeichnet die Anzahl der darin tatsächlich, zumindest im Jahreslauf temporär vorkommenden Arten von Pflanzen, Tieren und Pilzen.

Unter der Baumartenvielfalt ist im Sinne des vorliegenden Evaluationsverfahrens die Anzahl jener Gehölzarten zu verstehen, die im Rahmen der Bewirtschaftung langfristig (hier: 50 Jahre) eine Rolle spielen sollen und deshalb gegebenenfalls durch entsprechende Pflegeund Kultureingriffe in ihrem Bestand zu sichern sind. Unter Bewirtschaftung wird dabei jedes zielgerichtete Handeln verstanden. Die Ziele müssen dabei nicht ökonomischer Natur sein, sondern können beispielsweise auch eine besonders breite Baumartenpalette anstreben (vgl. SPEIDEL 1984).

Der Begriff Begleitarten bezeichnet in diesem Zusammenhang alle Pilz- und Pflanzenarten, die im betrachteten Bestand ebenfalls vorkommen, ohne jedoch im Rahmen der ordentlichen Bewirtschaftung eine Rolle zu spielen.

Die faunistische Artenvielfalt soll ebenfalls als Begleitinventar des Lebensraums gelten und stellt keine Wirtschaftsgröße dar.

\section{Bedeutung der Baumartenvielfalt für den Naturschutz}

Die Artenvielfalt kann neben der Naturnähe generell als eines der beiden großen Ziele des Naturschutzes gelten. Als Legalkriterium wurde sie explizit als Ziel des Naturschutzes in den Gesetzen verankert. Diese Stellung weist das Ziel der Artenvielfalt gleichzeitig als gesellschaftlichen Wert aus. Der Mensch übernimmt aus freiem Willen Verantwortung für die ihm anvertraute Schöpfung, zu der alles Leben gehört, unabhängig von dessen Rolle im Ökosystem. Eine optimale Ausübung dieser Verantwortung kann attestiert werden, wenn die Anzahl der wildlebenden Arten, also die Diversität maximal ist. Einer funktionalen 
Begründung dieses Anspruches bedarf es ebenso wenig wie beispielsweise im Bezug auf den Schutz von Kulturdenkmälern (MEINEKE 1992).

Eine Einschränkung ist allerdings zu machen, was die Feststellung der maximalen Diversität betrifft. Diese muss sich an den natürlichen Bedingungen des Betrachtungsraumes orientieren, also etwa am Naturraumes oder an den Standortsbedingungen. Ein Freilandmuseum mit unterschiedlichsten, eventuell standortsfremden oder eingebürgerten Arten besitzt zwar einen höheren Nominalwert der Diversität, ist jedoch schwerlich mit der Naturnähe, dem zweiten Primärziel des Naturschutzes, in Einklang zu bringen ist. Die Festlegung einer bestimmten Diversität in Übereinstimmung mit einer angestrebten, größtmöglichen Naturnähe legt daher den Schluss nahe, den ersten Maßstab auf der Grundlage des zweiten Zieles herzuleiten. Demnach wäre für einen Wald jene Diversität anzustreben, die der Artenvielfalt (an Baumarten) der potentiellen natürlichen Vegetation entspricht.

Wie sich ein Bewaldungsprozess in der Natur gestaltet, und welche Rolle dabei insbesondere angrenzende Bestände als Samenquellen spielen, zeigt sich beispielsweise auf (überwiegend) sich selbst überlassenen Sturmwurfflächen. Hierzu liegen aus unterschiedlichen Räumen Deutschlands Erfahrungswerte vor, die einander sehr gut entsprechen. Für Südwestdeutschland wird berichtet, dass die Baumartenanzahl auf Wurfflächen der Winterstürme 1990/91 stets zwischen 3 und 17, durchschnittlich bei 7 bis 9 lagen (SCHÖLCH 2000; ALDINGER/KENK in HuSS et al. 2000). OTTO (ebd.) bestätigt die hohe Baumartenvielfalt auf natürlich wiederbewaldeten Flächen für das Sturmereignis 1972 in Norddeutschland.

Liegt die Aufforstungsfläche inselartig in einem intensiv landwirtschaftlich genutzten Gebiet, so ist das Pendant eher in einer entsprechend isolierten Sukzessionsfläche zu sehen. Diese wird, bedingt durch den schwierigeren Anflug von Samen, mehr Zeit zur Entwicklung der waldtypischen Artenzusammensetzung benötigen. Gerade in den ersten Jahrzehnten, wird der Anteil an Pionieren, also an Bäumen mit leichten, durch den Wind transportierbaren Samen, besonders hoch sein.

Bezüglich der Begleitflora gibt es ebenfalls mehrere Angaben aus der Literatur. Wiederum in Zusammenhang mit wiederbewaldeten Sturmwurfflächen stellen REIF et al. (in Huss et al. 2000) fest, dass auf basenreichen Böden die Pflanzendiversität bereits im zweiten Sommer nach dem Sturmwurf ihr Maximum erreicht. Gleichzeitig weisen sie darauf hin, dass die Anzahl von (laut Roten Listen) seltenen und gefährdeten Arten anfangs recht gering ist. Erst mittelfristig entstehen auf diesen Flächen Lebensgemeinschaften mit seltenen Arten. Eine Bewertung des Merkmals Vielfalt sollte dementsprechend stets einen mittelfristigen Betrachtungszeitraum berücksichtigen.

Die Bedeutung des Artenschutzes und der biologischen Vielfalt geht bereits aus deren Förderung durch unterschiedliche internationale Abkommen und Vereinbarungen hervor (Agenda 21; Washingtoner Artenschutz-Abkommen; u.a.). Die Beschränkung auf die Baumartenvielfalt im Rahmen der Evaluation von Erstaufforstungen erfolgt aus rein pragmatischen Gründen. Im Gegensatz zu den floristischen und faunistischen Begleitarten eines Waldbestandes, sind die Gehölzarten sehr gut prognostizierbar. Im Regelfalle werden sie direkt bei der Kulturbegründung gepflanzt. Mehr oder weniger umfangreiche Pflegemaßnahmen sollen in den folgenden Jahrzehnten die Entwicklung und Stabilität des Bestandes fördern. Unter den in Kapitel 3.3.1 genannten waldbaulichen Prämissen kann deshalb davon ausgegangen werden, dass die Baumartenvielfalt der Fläche langfristig gesichert ist.

Andererseits stellt die Begleitartenvielfalt des Biotops ein sehr wichtiges Merkmal dar. Sie wird vermutlich auch immer ein Vielfaches der Baumartenanzahl ausmachen. Dennoch bleibt sie in Art und Umfang im Bereich des Spekulativen. Sie wird im Rahmen der Indikatoren Strukturpotential und Waldrandausprägung mit berücksichtigt. 


\section{Bestimmbarkeit des Indikators}

Die Baumartenvielfalt in einer natürlichen Waldgesellschaft, die entsprechend dem Leitbild der PNV zu bestimmen ist ${ }^{4}$, wird aus Sicht des Naturschutzes nicht nur aus Klimaxarten bestehen. Im Urwald treten nebeneinander verschiedene Sukzessionsstadien und Altersphasen auf. Demnach sind dort Baumarten unterschiedlichster Lichtansprüche und Konkurrenzstärken nebeneinander anzutreffen. Dies ist wiederum Voraussetzung für eine entsprechende Nischenvielfalt im Ökosystem. Nach diesem Vorbild, ist auch für den naturgemäßen Waldbau zu fordern, dass sämtliche, am Sukzessionsprozess einer Waldgesellschaft beteiligten Arten, mitberücksichtigt werden (REIF 2000; KAISER/ZACHARIAS 1999). Entsprechend ist bei der Bestimmung des Bewertungsmaßstabs für den Indikator Baumartenvielfalt auch die Beteiligung von Pionieren und semitoleranten Baumarten zu berücksichtigen. Selbst Bäume zweiter Ordnung (bspw. Wildobst oder Feldahorn) finden im natürlichen Sukzessionsverlauf ihren Platz - zumindest wenn die Fläche nicht gänzlich im Wald liegt, sondern Waldränder aufweist. Auch sie sind in die Artenzählung mit aufzunehmen (vgl. Kap. 3.5.2).

\section{Ableitung des Indikators aus der Matrix}

Eine eingehende Betrachtung der Verflechtungsmatrix (Tab. 3.3-1) zeigt verschiedene Verdichtungszentren (Punktewolken). Hier bestehen besonders viele Verknüpfungen zwischen einzelnen Elementen der Aufforstungsfläche, als erste Systemebene,. Im Schaubild links oben findet sich eine Punktewolke V1, die sich aus der Konkurrenz zwischen unterschiedlichen Baumarten ergibt. Markiert ist jeweils die Abhängigkeit des ZeilenElementes vom Spalten-Element. Konkurrenzschwache Arten haben - ohne waldbauliche Einflussnahme - gegen konkurrenzstarke Arten das Nachsehen und scheiden früher oder später aus. Die Beziehung ist umgekehrt auch vorhanden, jedoch bei weitem nicht so zwingend. Für den schwächeren Baum, geht es um ein Überleben im Bestand, der stärkere erfährt lediglich einen Einfluss durch die Konkurrenz um Wasser, Nährstoffe und Licht. Daher formiert sich eine Punktewolke mit zehn Verknüpfungspunkten. Mittel- bis langfristig ergibt sich aus den Konkurrenzkämpfen eine bestimmte Baumartenmischung und somit auch eine bestimmte Vielfalt. Im bewirtschafteten Bestand ist diese Vielfalt gleichbedeutend mit der Vielfalt der Wirtschaftsarten.

Unter gleichen Bedingungen ist die Existenz von Sträuchern und Begleitarten zu sehen. Großwüchsige Pflanzen sind häufig im Vorteil gegenüber kleinwüchsigen. Für Sträucher ergibt sich eine besondere Situation am Waldrand, wo sie einen festen Platz besitzen, da dieser gesondert bewirtschaftet wird. Innerhalb des Bestandes sind sie dagegen von ausreichendem Lichtgenuss abhängig. Begleitarten sind von allen anderen Arten abhängig, besonders was den Lichtgenuss betrifft.

Die waldbaulichen Pflegemaßnahmen sollen die funktionellen Ziele der Aufforstung langfristig sichern. Der Naturschutz fordert in diesem Zusammenhang, die Baumartenvielfalt als Ziel aufzunehmen. Die Einflussgrößen Mischungsform und Pflanzverband müssen dann so gewählt werden, dass die Vielfalt ohne unverhältnismäßigen Aufwand erhalten werden kann. Die Beziehungen zwischen den Baumarten und den technischen Voraussetzungen sind in der Matrix innerhalb einer Punktewolke V2 am oberen, rechten Rand wiedergegeben.

\section{Parameter}

Die Baumartenvielfalt ist aus dem Pflanzplan direkt abzulesen. Mischungsform und -anteile sollen der Konkurrenzkraft der beteiligten Arten angemessen sein. Die angestrebte Baumartenvielfalt ergibt sich aus der standortsbedingten natürlichen Waldgesellschaft (Abb. 3.5-I). Dabei ist zu beachten, dass eine Aufforstung die Möglichkeit bietet, Bäume unterschiedlichster Sukzessionsstadien zu beteiligen. Demnach gibt die gesamte Bandbreite an Haupt-, Misch- und Nebenbaumarten das quantitative Ziel an.

\footnotetext{
${ }^{4}$ Die Begriffe natürliche Waldgesellschaft (NWG) und potentiell natürliche Vegetation (PNV) werden im Hinblick auf Waldbestände in der vorliegenden Arbeit synonym verwendet
} 


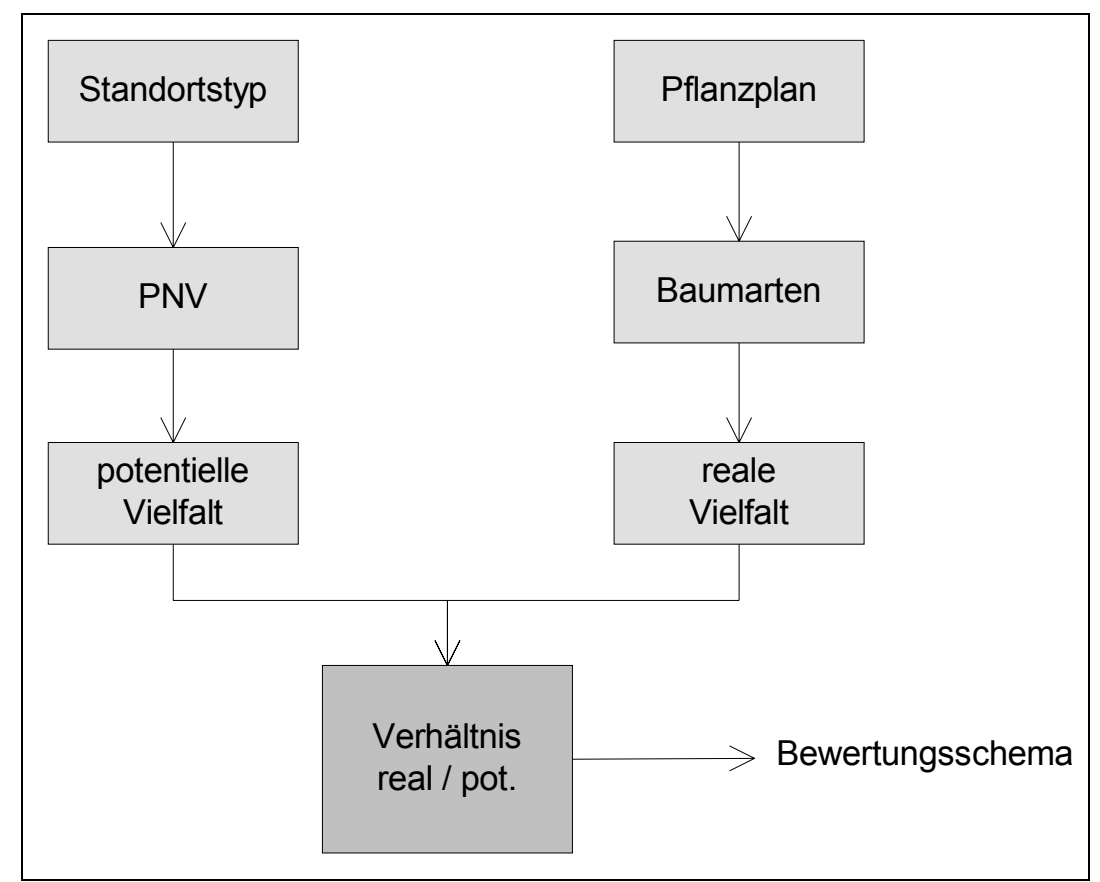

Abb. 3.5-l: $\quad$ Bewertungsschema des Indikators Baumartenvielfalt; Vergleich der realen Vielfalt mit der potentiellen Vielfalt der natürlichen Waldgesellschaft

Methodisch wird zunächst festgestellt, welche Standortsbedingungen auf der Aufforstungsfläche herrschen. Standortskartierungen forstlicher oder landwirtschaftlicher Prägung liegen in der Regel bereits vor. Anschließend wird mit Hilfe eines technischen Schlüssels die PNV des jeweiligen Standorts bestimmt. Das vorliegende Verfahren bedient sich dabei jeweils jener Herleitungsmethode, welcher durch das landesspezifische Waldbiotopkartierungsverfahren zur Ermittlung der natürlichen Waldgesellschaft verwendet wird. Dadurch ist das Verfahren auch flexibel hinsichtlich einer Veränderung dieses Schlüssels. Außerdem ist die Herleitung aus den Standortklassen auf diese Weise besonders einfach und transparent möglich.

Kommen auf einer Aufforstungsfläche zwei verschiedene Standorte vor, so werden diese getrennt evaluiert und das Ergebnis wird entsprechend der Flächenanteile gewichtet (Erhebungsbogen im Anhang 1).

Bei der Ermittlung der realen Baumartenzahl werden alle Bäume berücksichtigt, die einen Flächenanteil von mindestens $5 \%$ an der Pflanzung einnehmen. Fremdländische Baumarten werden nicht berücksichtigt. Ihr Beitrag zum Artenschutz ist deutlich geringer als jener der heimischen Baumarten, da die Anzahl der auf sie angewiesenen oder mit ihnen vergesellschafteten Arten besonders klein ist. Die eingeführten Baumarten mögen eine Rolle im Sinne der Landschaftsästhetik oder als wirtschaftliche Ergänzung spielen - für den Artenund Biotopschutz besitzen sie keine nennenswerte (positive) Bedeutung und werden deshalb bei diesem Indikator nicht mitgewertet.

Die Einteilung der sechs Bewertungsklassen (Abb. 3.5-II) erfolgt symmetrisch von der Mittleren Stufe aus. Beträgt die reale Baumartenvielfalt die Hälfte der potentiellen Vielfalt, so ist dies ein mittlerer Wert. Von diesem Zentrum aus finden sich jeweils 3 Stufen zur vielfältigen und zur monotonen Zusammensetzung hin. Die Stufen besitzen eine Breite von 15 Prozent, wobei die jeweils letzte Stufe 20 Prozent beträgt. Zu beiden Extremwerten hin nimmt der Grenznutzen ab, wodurch die Breite der obersten und untersten Klasse zu rechtfertigen ist. 


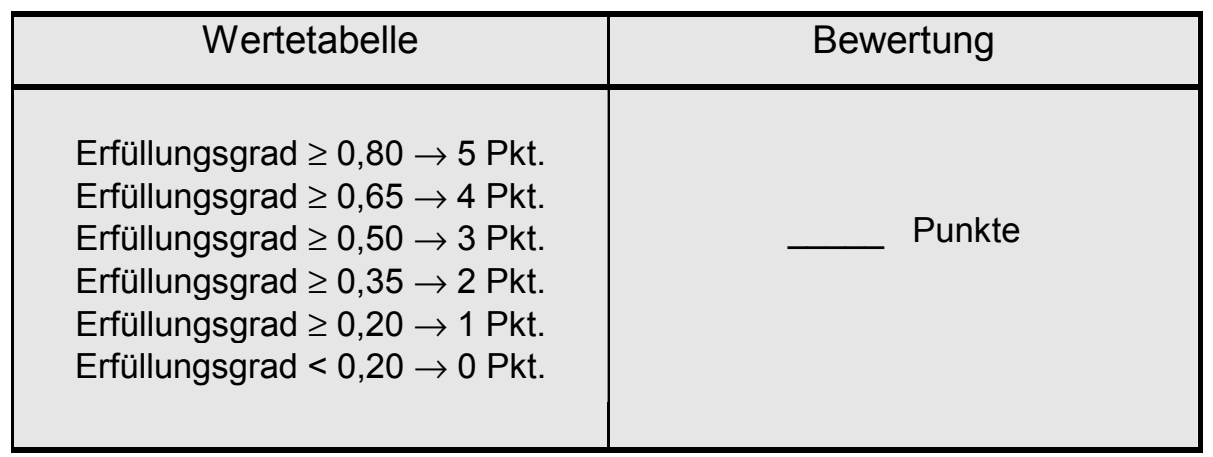

Abb. 3.5-II: $\quad$ Bewertungsstufen des Indikators Baumartenvielfalt

\subsubsection{Naturnähe}

\section{Definition}

Naturnähe der Vegetation ist die Annäherung der realen Vegetation an eine gedachte potentielle natürliche Vegetation. Die (heutige) potentielle natürliche Vegetation stellt dabei jene Pflanzengesellschaft dar, welche sich unter den heute gegebenen Bedingungen optimal ausprägen würde.

Die Naturnähe beruht demnach auf dem Naturleitbild (vgl. Kap. 3.2) und wird am Optimum, der heutigen potentiellen natürlichen Vegetation gemessen. Diese wurde in der jüngeren Vergangenheit im Rahmen einer Fachtagung von Experten aus Wissenschaft und Praxis neu definiert (KAISER/ZACHARIAS 1999). Die neue Definition begründet sich weitgehend auf der bis dahin bereits breit akzeptieren Fassung von KoWARIK (1987). Demnach ist die PNV ein Gedankenmodell und „beschreibt die höchstentwickelte Vegetation, die sich unter gegenwärtigen Standortbedingungen einstellen würde, wobei im Zuge eines gedachten Regenerationszyklus auftretende Anreicherungsprozesse (z.B. Humus-Akkumulation) und das biotische Besiedlungspotential an autochthonen Arten der naturräumlichen Region berücksichtigt werden." In den Konstruktionsprozess fließen auch nachhaltige anthropogene Standortveränderungen ein, soweit diese nicht innerhalb eines Regenerationszyklus unter PNV ausgleichbar sind. An gleicher Stelle wird darauf hingewiesen, dass bei der Gehölzauswahl für Pflanzungen das gesamte Baumartenspektrum zu berücksichtigen ist, welches sich in verschiedenen Sukzessionsphasen zum Schlusswald hin zeigt.

\section{Bedeutung der Naturnähe für den Naturschutz}

Bezüglich der Beteiligung von Pionierbaumarten und Wirtschaftsbaumarten, diese simplifizierte Unterscheidung wird im Waldbau häufig angewandt, zeigen insbesondere die Erfahrungen mit der natürlichen Wiederbewaldung von Sturmflächen interessante Ergebnisse. In Süddeutschland haben die Orkane Vivien und Wiebke im Winter 1990/91 für einen Reichtum diesbezüglicher Beobachtungsflächen gesorgt. Für Baden-Württemberg wird eine Beteiligung von Pionierpflanzen am neu angekommenen Wald von durchschnittlich 25, bzw. 33 \% festgestellt (SCHÖLCH 2000, ALDINGER/KENK in HUSS et al. 2000). BUNGENSTAB (in HuSs et al. 2000) bestätigt für das Forstamt Eberbach einen „geringen Anteil forstlich nicht erwünschter Pionierbaumarten" auf wiederbesiedelten Sturmwurfflächen. Die negative Besetzung der Pioniere in diesem Zitat wird allerdings von den anderen Autoren stark relativiert, da die Funktion der schnellwachsenden, jedoch relativ kurzlebigen und konkurrenzschwachen Baumarten als Füll-, Treib-, und Schutzholz für eine positive Konkurrenzsituation sorgt. Ihr rasantes Jugendwachstum garantiert in der Regel eine schnelle Vorwaldbildung, in dessen Schutz die „Wirtschaftsbaumarten“ gut gedeihen. EDER (ebd.) weist zudem auf ein zusätzliches Wertschöpfungspotential hin, welches durch eine frühzeitige Freistellung bestimmter Pionierbaumarten (z.B. Eberesche) erreichbar ist. Die 
südwestdeutschen Erfahrungen zur Rolle der Pionierbaumarten bei der Wiederbewaldung von Sturmwurfflächen decken sich mit jenen, die OTTO (in Huss et al. 2000) für die Erfahrungen mit Sturmwurfflächen vom Jahre 1972 in Norddeutschland resümiert. Insgesamt hat die waldbauliche Praxis der vergangenen Jahrzehnte im Zusammenhang mit der Wiederbewaldung von Sturmflächen gezeigt, dass die Pioniere aus dem "Mauerblümchendasein“ im Walde herausgetreten sind und zunehmend ihre wichtige, nämlich dienende Funktion Anerkennung finden (Huss et al., ebd.).

Bei der Übertragung der Erfahrungen auf Erstaufforstungen landwirtschaftlicher Flächen bleibt zunächst festzustellen, dass diesbezüglich noch wenig Untersuchungen vorliegen. Dies beruht zunächst auf der geringen Wertschätzung, die den Pionierbaumarten über Jahrzehnte entgegengebracht wurde, weshalb sie wohl selten gezielt bei einer Neuanlage von Wald eingebracht wurden. Des weiteren waren die Pflanzverbände häufig so eng gewählt, dass ein natürlicher Anflug von konkurrenzschwachen Baumarten keine Chance auf Bestand hatte. Die wenigen Beispiele, die es dennoch gibt, begründen sich auf der gezielten Vorwaldbildung durch Pflanzung oder Saat von Eberesche, Birke und Aspe (FRIEDRICHSDORF 1999, PETERSEN/FISCHER 1999, WAGNER 1995). Auch, oder vielleicht gerade, auf Neuaufforstungsflächen erfüllen die schnellwüchsigen Pioniere wichtige Funktionen als Vorwald. Dabei gilt für die mengenmäßige Beteiligung der Pionierbaumarten am Gesamtbestand ein ähnliches Prozentanteil (bis ca. $30 \%$ ) wie das für die natürliche Wiederbewaldung angegebene (vgl. obige Literaturquellen). Die Aufforstungspraxis hat sich in Bezug auf die hier proklamierten Ansprüche an Baumartenwahl, Pflanzverband und Beteiligung von Pionierbaumarten in den vergangenen Jahren sehr stark gewandelt. Ausdruck dieses Wandels sind einerseits die ökologisch orientierten Waldbauprogramme der Länder (z.B. LÖWE in Niedersachsen), andererseits aber auch die Aufforstungsbilder, die neuerdings landauf und landab zu sehen sind.

\section{Bestimmbarkeit des Indikators}

Das Konzept der PNV hat mittlerweile eine breite Anwendung in der Naturschutzpraxis und im Waldbau gefunden. Doch auch der oben erwähnte Versuch, einer gemeinsamen Definition der PNV für die Naturschutzpraxis weist darauf hin, dass die Bestimmung dieser gedachten Vegetation auf unterschiedliche Weise erfolgen kann. Unter den Fachleuten herrscht Einigkeit, dass das Konzept innerhalb der praktischen Umsetzung entsprechend den jeweiligen Fragestellungen und Zielen zu modifizieren ist (KAISER/ZACHARIAS 1999).

Das hier vorgestellte Verfahren vermeidet eine eigene Definition in Anpassung an den gegebenen, Untersuchungsrahmen. Stattdessen greift die Evaluation auf die Definition und Herleitung der PNV / NWG des jeweiligen Waldbiotopkartierungsverfahrens zurück. Ändern sich grundlegende wissenschaftliche Erkenntnisse oder Zielvorgaben, so ändert sich gleichzeitig der Bewertungsrahmen des hier verwendeten Indikators. Beispielsweise beteiligt die naturschutzfachliche und forstliche Praxis in der Bestimmung der PNV nur regional standortheimische, nicht jedoch fremdländische Baumarten. Sollte dies im Laufe der Zeit geändert werden, so wirkt sich die Änderung automatisch auch auf das hier vorgestellte Verfahren aus.

Dies gewährt ein Höchstmaß an Flexibilität. Gleichzeitig bleibt die Wertfeststellung, die durch das Verfahren getroffen wird, objektiv. Für die Bewertung der Erstaufforstung gilt es, dem natürlichen Vorbild der Waldzusammensetzung möglichst nahe zu kommen. Die Ermittlung dieser natürlichen Baumartenkombination liegt methodisch außerhalb des Verfahrens.

Benötigt wird dafür eine Kartierung der Bodenverhältnisse im jeweiligen Untersuchungsraum. Diese liegt zumindest von landwirtschaftlicher Seite flächendeckend vor. Die Forstbehörden verfügen meist über eine Kartierung der Waldflächen. Zum Transfer der landwirtschaftlichen Kartierung in forstliche Standortsbedingungen besitzen die Forstbehörden eigene Verfahren und ausreichend Erfahrungen. Häufig liegen spezielle Aufforstungsempfehlungen vor (siehe Kap. 4.1). Damit ist das Ziel klar vorgegeben. 
Die geplante Baumartenzusammensetzung geht aus dem einzureichenden Pflanzplan ebenfalls eindeutig hervor. Soweit die Pflege der Kultur eine waldbauliche Sicherung der Betriebsziele im Rahmen der in Kap. 3.3.1 erläuterten Prämissen verfolgt, kann ein Soll-IstVergleich durchgeführt werden.

\section{Ableitung aus der Matrix}

Funktional betrachtet erreicht die Naturnähe einen Optimalwert, wenn sich ein Fließgleichgewicht zwischen den biotischen und abiotischen Gegebenheiten einstellt. Die aufstockenden Pflanzen entsprechen vollständig den Standortbedingungen und stabilisieren gleichzeitig die systemimmanenten Prozesse und Kreisläufe.

In der Verflechtungsmatrix der ersten Systemebene (Tab. 3.3-1) zeigt dies die Punktewolke NN1 an. Wasser, Boden und Mikroklima werden von den aufstockenden Baumarten essentiell beeinflusst.

Die Punktewolke NN2 weist darauf hin, dass die Baum- und Straucharten in ihrem Gedeihen von den jeweiligen Boden-, Wasser- und Klimaverhältnissen abhängig sind. Selbstverständlich gilt diese Abhängigkeit auch für die Begleitarten im Bestand. Die abiotischen Faktoren Boden, Wasser und Klima beeinflussen sich aber auch gegenseitig. Dadurch ergibt sich ein zusätzliches Stabilisationselement in diesem Operanden. Die Größe der Punktewolke NN2 weist auf die komplexe Beschaffenheit dieses Systembereiches bereits hin. Sie zeigt auch an, dass unregelmäßige oder auch ständige Umwelteinflüsse die Naturnähe (nicht die PNV!) beeinflussen können.

Tab. 3.5-1: Vergleich der geplanten Baumartenmischung mit der potentiell natürlichen des angesprochenen Standortes; Auszug aus dem Erhebungsbogen (vgl. Anhang 1)

\begin{tabular}{|c|l|l|}
\hline Zeile & Parameter & Daten der Aufforstungsfläche \\
\hline 1 & Größe der Aufforstungsfläche & \\
\hline 2 & Standort-Typ 1 & \\
\hline 3 & $\begin{array}{l}\text { Größe } \\
\text { (ha und \%) }\end{array}$ & \\
\hline 4 & pnV & \\
\hline 5 & $\begin{array}{l}\text { BA der pnV } \\
\text { (laut pnV-Schlüssel aus der WBK) }\end{array}$ & \\
\hdashline 6 & $\begin{array}{l}\text { BA real } \\
\text { (laut Pflanzplan) }\end{array}$ \\
\hline 7 & $\begin{array}{l}\text { Übereinstimmungen } \\
\ldots\end{array}$ \\
\hdashline 8 & $\begin{array}{l}\text { Übereinstimmungen } \mathrm{X} \text { Prozent } \\
\text { Prozentanteil des Standortes an der } \\
\text { Aufforstungsfläche }\end{array}$ \\
\hline
\end{tabular}

\section{Parameter}

Die landesspezifischen Waldbiotopkartierungen streben eine Kartierung natürlicher Waldgesellschaften an. Hierzu benötigen sie einen Schlüssel, welcher den standörtlichen Bedingungen eine natürliche Waldgesellschaft zuordnet. Dieser Schlüssel (in Thüringen: Stamm-Standorts-Gruppe) ist auf landwirtschaftliche Bodenverhältnisse übertragbar. Allerdings ist dabei zu beachten, dass landwirtschaftlich genutzte Böden zum Zeitpunkt der 
Umwandlung relativ nährstoffreich sind. Ein Großteil der Nährstoffe geht in den ersten Jahren durch Auswaschung verloren, soweit sie nicht durch die Begleitvegetation in einen Kreislauf eingebunden werden kann. Die stattfindenden Bodenprozesse stabilisieren den Nährstoffhaushalt anschließend innerhalb der ersten Waldgeneration. Damit bewegen sich die Verhältnisse innerhalb der oben gegebenen Definition der PNV (KAISER/ZACHARIAS 1999).

Zur Bewertung der Naturnähe erfolgt ein Vergleich der geplanten Baumartenmischung mit der potentiell natürlichen (Tab. 3.5-1). Umfasst die Aufforstungsfläche zwei derart unterschiedliche Standorte, dass deren PNV voneinander abweicht, werden beide Teilbereiche getrennt bewertet und das Ergebnis entsprechend der Flächenanteile gewichtet (vgl. Anhang 1).

Zur Ermittlung der Bewertungszahlen wird die relative Übereinstimmung mit der PNV bestimmt. Die Zusammensetzung dieser Waldgesellschaft wird aus vorhandenen Kartierungsanleitungen als Idealziel übernommen. Der Prozentanteil, der von diesen Baumarten tatsächlich angepflanzten Gehölze, wird schließlich mit Hilfe des bereits für den Indikator Baumartenvielfalt verwendeten Bewertungsschemas (Abb. 3.5-II) in Wertziffern transformiert. Diesem Vorgehen liegt die Überlegung zu Grunde, dass ein Biotop seinen vollen Wert als naturgemäßer Waldbestand erst dann erreicht, wenn er auch über das entsprechende Baumarteninventar verfügt.

\subsubsection{Seltenheit des Biotops}

\section{Definition}

Ein Biotop ist der Lebensraum einer Biozönose im Sinne einer regelmäßig wiederkehrenden Artengemeinschaft und zeichnet sich durch besondere Merkmale (Arteninventar, Standortbedingungen, Struktur) aus. Die Abgrenzung zur Umgebung erfolgt anhand dieser Merkmale.

Der Indikator Seltenheit des Biotop(typ)s beschäftigt sich mit der Wertigkeit des durch die Aufforstung entstehenden Waldtyps in Bezug auf den regionalen und überregionalen Biotopschutz.

\section{Bedeutung der seltenen Biotope für den Naturschutz}

Der Schutz bestimmter Biotoptypen, also die Wiederherstellung und Wahrung eines günstigen Erhaltungszustandes, ist ein Hauptziel des Naturschutzes (KAULE 1991). Er dient direkt der Sicherung der Biotopvielfalt auf unterschiedlichen räumlichen Ebenen. Indirekt ist er eine wichtige Grundlage für den Artenschutz, da er diesem die notwendigen Rahmenbedingungen für einen Erfolg liefert. Daraus ergibt sich wiederum eine Bedeutung für den Schutz der biologischen und genetischen Vielfalt innerhalb von Ökosystemen und -regionen.

Eine Abgrenzung des Biotopschutzes gegenüber dem Artenschutz ist relativ leicht möglich. Ein Biotop ist ein komplexes Gebilde, welches durch sein Inventar und die Wechselbeziehungen der Elemente charakterisiert wird. Eine einzelne Art stellt darin nur ein (bestimmtes) Element dar. Zur Existenz eines Biotops und dessen Funktionserfüllung, ist das Vorhandensein aller potentiell darin vorkommenden Pflanzen- und Tierarten keine zwingende Bedingung. Umgekehrt ist für das Vorkommen einer Art das Vorkommen der für sie notwendigen Lebensraumcharakteristika unbedingte Voraussetzung. Daraus ist ersichtlich, dass der Biotopschutz eine grundlegende Bedeutung für den Artenschutz besitzt.

Biotope sind aufgrund ihrer räumlichen Erscheinung leichter kartierbar als Arten. Zwar müssen neben dem visuellen Erfassen auch noch bestimmte Merkmale eines Biotops bestimmt werden, bevor dieses bestätigt werden kann. Dafür ist das Biotop aber ganzjährig kartierbar. In der Reaktion auf eine Veränderung der Umweltbedingungen zeigen sich 
Biotope stabiler als viele der in innen vorkommenden Arten. Die Kartierung von Arten ist daher wesentlich stärker von Schwankungen der Umweltbedingungen abhängig als die Kartierung von Biotopen. Daraus ergibt sich eine höhere Praxisfreundlichkeit des Biotopschutzes für die Umsetzung im Rahmen der Naturschutzplanung.

Hinsichtlich der Bedrohung der Biotope sind direkte Vernichtung und allmähliche Degradation als Gefährdungsursachen zu unterscheiden. Direkte Vernichtung erfolgt beispielsweise durch eine Umwandlung oder Intensivierung der Nutzung, bzw. durch Beseitigung eines Biotops im Rahmen der Flurbereinigung oder anderer landschaftsverändernder Prozesse. Sie wird durch landschaftsbeschreibende Aufnahmen über die Zeit dokumentiert.

Die Degradation von Biotopen ist ein schleichender Prozess, der sich durch allmähliche Veränderung der Bewirtschaftung oder eine ebensolche Veränderung der Umweltbedingungen ergeben kann. Sie ist schwieriger feststellbar, da sie eine Reihe detaillierter Aufnahmen erfordert. Mit ihr einher geht eine Veränderung der Lebensbedingungen für die im Biotop vorhandenen Arten. Letztere drohen dann entsprechend ihrer Empfindlichkeit gegenüber der stattfindenden Veränderung abzuwandern bzw. auszufallen. Die großflächigste Bedrohung der Biotope in diesem Sinne geht von Immissionen und landwirtschaftlicher Düngung aus. Die Überversorgung mit Stickstoff gilt als Schlüsselfaktor für die Verschiebung der standörtlichen Verhältnisse und darauf folgenden Verschiebungen im Artengefüge (ELSAßER 1991).

Vernichtung und Degradation werden im Rahmen der Roten Liste der gefährdeten Biotoptypen der Bundesrepublik Deutschland-RLB (RIECKEN et al. 1994) unter den Gefährdungskriterien Flächenverlust und qualitative Veränderung berücksichtigt. Diese RLB bietet eine grundlegende Systematik der in Deutschland vorkommenden Biotoptypen. Sie liefert eine Einschätzung des Gefährdungsgrades der Biotoptypen auf der Basis von Expertenbefragungen und wissenschaftlichen Erhebungen. Im Rahmen der Naturschutzpolitik ist die RLB als strategische Ergänzung der Roten Listen der gefährdeten Pflanzen- und Tierarten zu sehen.

Trotz der verhältnismäßig hohen Stabilität gegenüber Umwelteinflüssen, führt eine andauernde Veränderung der Umweltbedingungen mittel- bis langfristig auch zu einer Veränderung der Biotopqualität. Dies wird im Rahmen der Diskussion zur Roten Liste nur für die negativen Einflüsse besprochen, gilt aber auch mit umgekehrtem Vorzeichen für eine Progradation durch Verbesserung der Verhältnisse oder Modifikation der Bewirtschaftung. Andererseits benötigt die Natur relativ lange, bis sich unter bestimmten Bedingungen ein typisches Biotop ausbildet. Dieser benötigte Entwicklungszeitraum ergibt im Rahmen der oben genannte Roten Liste ein Zusatzkriterium zur Einschätzung der Biotopgefährdung: die Regenerationsfähigkeit/ Regenerierbarkeit. Für die Landschaftsplanung ergeben sich aus der Regenerierbarkeit von Biotoptypen deutliche Hinweise auf die Notwendigkeit einer langfristigen Pflege von Biotopen, wenn diese geschützt oder gar geschaffen werden sollen. Allerdings erfolgte die Einschätzung der oben genannten Roten Liste unter einer anderen Prämisse, nämlich aus der Perspektive der Ersetzbarkeit bestehender Biotopqualitäten. Gerade für Wälder ergibt sich daraus ein methodisches Problem. Die RLB unterscheidet zwischen Wäldern (natürlicher oder naturnaher Zustand; inklusive Pflanzungen mit weitgehend der PNV entsprechender Baumartenzusammensetzung) und Forsten (mehr als ein Drittel nicht bodenständiger oder nicht einheimischer Gehölze und Monokulturen auf Mischwaldstandorten) bei der Klassifikation von Biotopen. Eine Feststellung der Regenerierbarkeit wird lediglich für die Wälder durchgeführt. Dabei werden Moorwälder als nicht regenerierbar, Hartholzauenwälder mit ungestörter Überflutungsdynamik als kaum bis nicht regenerierbar eingestuft. Weitere 53 von insgesamt 59 Waldbiotoptypen werden als kaum regenerierbar bezeichnet. Den Ausführungen von RIECKEN et al. (1994) folgend, wären demnach die meisten der in Deutschland potentiell vorkommenden Waldbiotope nur in historischen Zeiträumen (> 150 Jahre) regenerierbar, wobei eine unvollständige Ausprägung der Biotope erwartet wird. Das methodische Problem besteht in der Frage, ob ein Biotop erst dann eine Einordnung erlaubt, wenn es ein gewisses Alter erreicht hat (im Falle der meisten 
Wälder also mindestens 150 Jahre), oder ob die Einordnung bereits zu einem früheren Zeitpunkt stattfinden kann. Im Rahmen der Bewertung von Erstaufforstungen macht die Einordnung natürlich nur dann Sinn, wenn die zweite Möglichkeit akzeptiert wird. Die Evaluation folgt damit jenem Ansatz der Walddefinition, der die Pflanzung von PNVorientierten Baumartenzusammensetzungen als alleinige Voraussetzung akzeptiert. Unter Berücksichtigung der in Kap. 3.3.1 beschriebenen waldbaulichen Prämissen scheint dieses Vorgehen legitim, da die Entwicklung der wichtigsten Biotopcharakteristika im Laufe der Jahre erwartet werden kann.

Von den in der Bundesrepublik Deutschland vorkommenden Biotoptypen werden mehr als zwei Drittel als gefährdet eingestuft (RATHS et al. 1995). Diese Gefährdung wirkt sich direkt auch auf jene Pflanzen- und Tierarten aus, die auf das Vorkommen dieser Biotope, bzw. auf die dort vorkommenden Lebensraumcharakteristika angewiesen sind. Die Auswertung der RLB weist auf die grundlegende Bedeutung des Biotopschutzes hin. Die Zerstörung und Beeinträchtigung der Lebensräume wirkt sich so stark aus, „dass allein mit Einzelartenschutz keine effektive Verbesserung der Situation der Natur zu erreichen ist" (RATHS et al. 1995, S. 211). Eine langfristige Sicherung der biologischen Vielfalt von Lebensraum- und Ökosystemtypen bedarf allerdings vielfältiger Anstrengungen auf unterschiedlichen Ebenen des Natur- und Umweltschutzes sowie der Raumplanung. Alleingänge einer Planungsebene können keine dauerhafte Verbesserung bringen. Die Vereinheitlichung der Entwicklungsziele mit maßstabsgerechten Leitbildern ist daher einzufordern.

Auf internationaler Ebene bildet die FFH-Richtlinie (92/43/EWG) ein Beispiel für die Umsetzung der Ambitionen internationaler Vereinbarungen zum Natur- und Artenschutz (Agenda 21; Biodiversitätskonvention). Entsprechend den internationalen Handlungsrahmen finden dabei allerdings nur großflächige Biotope und Biotopkomplexe Berücksichtigung. In Deutschland zeigt die Praxis, dass vor allem bestehende und geplante Schutzgebiete, Nationalparke und Biosphärenreservate in das neu entstandene Schutzgebietsnetz Natura 2000 aufgenommen wurden (BROCKSIEPER/WOIKE 1999). Flächen kleineren Umfangs und Einzelbiotope fallen durch das Erfassungsraster. Sie sind aber auf regionaler Ebene unbedingt von Bedeutung und müssen folglich auf nachgeordneten Raumplanungsebenen Berücksichtigung finden.

Die regionale Landschaftsplanung kann aufgrund ihres größeren Maßstabes auch kleinere Biotope gut erfassen und berücksichtigen. Zusätzlich bietet der kleinere Planungsraum mehr Möglichkeiten zur Umsetzung der Planungsziele und damit einen effektiveren Schutz der Biotope. Eine Mitberücksichtigung übergeordneter Pläne steht dem nicht entgegen. Die Evaluation von Erstaufforstungen ist ein geeignetes Instrument zur Umsetzung der Biotopschutzziele auf der regionalen Ebene der Landschaftsplanung.

Die Berücksichtigung des angelegten Waldbiotoptyps im Rahmen einer ex-ante Evaluierung ist aus oben dargelegter Bedeutung für den Naturschutz begründbar. Allerdings muss darauf hingewiesen werden, dass die Bewertung davon ausgeht, dass kein schutzwürdiges oder gefährdetes Biotop aufgeforstet werden soll. Die angewandte Methode bezieht sich nur auf die Aufforstung landwirtschaftlich genutzter Flächen. Die Auswahl der Untersuchungsflächen erfolgt ebenfalls unter dieser Prämisse.

Eine Aufforstung gefährdeter Biotope ist nicht statthaft. Eine entsprechende Aufforstungsgenehmigung wäre zu versagen. Aus diesem Grunde wird eine Abwägung zwischen einem bestehenden, gefährdeten Biotop und einem neu anzulegenden, gefährdeten Biotop nicht erörtert, obwohl unter Umständen, z.B. in Auenbereichen, auch eine Aufforstung geschützter Flächen zur Entwicklung noch höherwertiger Biotope führen könnte (ERLBECK 1993).

\section{Bestimmbarkeit des Indikators}

Die Feststellung oder Kartierung eines Biotoptyps erfolgt im Allgemeinen anhand der vorgefundenen Vegetation und der geologischen/pedologischen Verhältnisse. Zusätzlich werden nachgewiesene faunistische Vorkommen sowie Zufallsnachweise im Rahmen der 
Kartierung berücksichtigt. Die Einteilung erfolgt nach einem landesspezifischen Schlüssel, welcher zumindest die per Naturschutzgesetz geschützten Biotope enthält. In anderen Fällen, insbesondere bei flächendeckenden Kartierungen, werden auch nicht gefährdete Biotope erfasst. Das qualitative Verfahren basiert auf dem jeweiligen, landesspezifischen Katalog der Biotoptypen und der Roten Liste der gefährdeten Biotope für Deutschland.

Im Rahmen des Indikators Seltenheit des Biotops kann im Falle von Erstaufforstungen nur der Typ des entstehenden Biotops prognostiziert und bewertet werden. Der Grad der Vollständigkeit ist nicht vorhersehbar. Hierzu wäre eine genaue Inventarliste der vorzufindenden Tier-, Pflanzen- und Pilzarten notwendig. Außerdem müssten genaue Aussagen zur typischen Struktur, zur Größe und zur Verknüpfung des Biotops mit umliegenden Flächen gemacht werden. Für jede vorkommende Art sind aber unterschiedliche Elemente in variierender Bedeutung zwingend. Es müsste ebenfalls festgestellt werden, welche Elemente unter welchen Umständen ersetzbar wären - wie im Beispiel des Neuntöterhabitats, bei dem Dornsträucher zum Aufspießen der Beutetiere durch Stacheldraht ersetzt werden können (vgl. KAULE 1991).

Mit dem Indikator Seltenheit des Biotops kann daher lediglich der gegenständliche Charakter des Biotoptyps abgefragt werden. Dazu gehört in erster Linie die Zusammensetzung der Gehölzflora. Unter der Prämisse einer nicht verfälschenden Einflussnahme auf die Krautund Begleitvegetation durch Einbringung von Düngemitteln und Pestiziden ist eine naturgemäße Entwicklung zu erwarten. Die Beachtung der Mischungsformen entsprechend den waldbaulichen Prämissen gewährleistet die Entwicklung einer der Baumartenzusammensetzung entsprechenden Struktur. Das Waldbiotop wird sich folglich so entwickeln, dass es eine gute Grundlage für einen spezifischeren Arten- und Biotopschutz bildet.

Der Nutzungswandel von der landwirtschaftlichen Fläche zum Waldbestand schränkt die Möglichkeit für entstehende Biotope auf Waldbiotope ein. Eine weitere Spezifizierung wird durch den aufgeforsteten Bodentyp sowie die sonstigen Standortsbedingungen vorgegeben. Aufgrund einer land- oder forstwirtschaftlichen Bodenkartierung wird anhand eines regionalen Schlüssels jeder Fläche eine natürlichen Waldgesellschaft zugeordnet. Der Schlüssel wurde für die vorliegende Arbeit den Waldbiotopkartierungsverfahren der jeweiligen Bundesländer (Niedersachsen und Thüringen) entnommen, bzw. so verändert, dass er für landwirtschaftliche Bodenkartierungen anwendbar ist (siehe Kap. 4).

Vorkommen und Zustand der Waldbiotope werden regelmäßig im Rahmen der Waldbiotopkartierung überprüft. In Niedersachsen wie in Thüringen bedeutet dies eine Überprüfung der Situation in zehnjährigem Abstand. Die Grenzen der Erfassungseinheiten sind jeweils identisch mit jenen des Forstbezirks und damit in der Regel nicht mit jenen des Naturraumes. Dennoch liefert die Feststellung ein hinreichend genaues Bild für die Einschätzung der Biotopschutzsituation. Die Aufforstung spielt im statistischen Sinne lediglich für die Vermehrung von Waldbiotopen eine Rolle. Aus diesem Grunde kann die Offenlandbiotopkartierung für diesen Indikator außer Acht gelassen werden.

Die Waldbiotopkartierung wird für den jeweiligen Wuchsbezirk ausgewertet. Von Seiten des Naturschutzes wird gefordert, dass alle natürlichen Waldgesellschaften eines Gebietes in repräsentativem Umfang vorhanden sein sollen. Darüber hinaus gilt es aber als erstrebenswert, den Zustand des Waldes soweit als möglich am natürlichen Vorbild zu orientieren. Aus diesem Grunde wird für die Bewertung der Aufforstung im vorliegenden Verfahren kein Erfüllungsgrad angegeben. Selbst wenn eine bestimmte Waldgesellschaft bereits mit $15 \%$ am aktuellen Waldbestand beteiligt ist, erscheint die Neuanlage eines naturnahen Waldes desselben Biotoptyps noch förderungswürdig im Sinne des Naturschutzes, zumindest entsprechend der gewählten Einschätzung anhand der Gefährdungskategorie gemäß der RLB. 


\section{Ableitung des Indikators aus der Matrix}

Der Biotoptyp vermittelt im Sinne des betrachteten Wirkungssystems zwischen den einzelnen Ebenen. Seine Ausprägung wird durch die Elemente der Aufforstungsfläche (1. Ebene) bestimmt. Als Operand tritt das Biotop aber auf der 2. und 3. Ebene (Aufforstungsumfeld und erweiterter Planungsraum) in Erscheinung. Hier wird es - als Teil des Biotoptypeninventars des Aufforstungsumfeldes - auch bewertet. Die Rolle des Biotoptyps erscheint in der Verflechtungsmatrix der besagten Ebenen als Punktewolke SB (Tab. 3.3-2).

Das Biotoptypeninventar hat Auswirkungen auf verschiedene Phänomene, die in der unmittelbaren und mittelbaren Umgebung der Aufforstungsfläche auftreten. Dazu gehört erstens die funktionale Vernetzung verschiedener Biotoptypen. Zweitens prägt es das landschaftliche Erscheinungsbild durch die Biotoptypen selbst und durch die entstehenden Randlinien.

Schließlich nimmt die Existenz verschiedener Biotope auch Einfluss auf den Gen-Austausch innerhalb einer Population und auf die Größe der artspezifischen Minimumareale. Sind keine ergänzenden Biotoptypen da (z.B. feuchtes Weidengebüsch als Ergänzung zum ErlenBruchwald), so beschränkt sich der Aktionsraum einer auf diesen Biotoptyp angewiesenen Art auf dessen Vorkommen. Der Gen-Austausch wird behindert. Die Population ist standörtlich stärker eingeschränkt.

\section{Parameter}

Die Übereinstimmung der Baumartenzusammensetzung mit jener der NWG zeigt, ob der entstehende Bestand einem gefährdeten Biotop nach diesem einen Gesichtspunkt der gesetzlichen Definitionen entspricht. Dabei muss ein hoher Grad der Entsprechung vorliegen. Allein dieser Anspruch kann eine Bewertung als potentiell wichtiges Biotop im Sinne des Naturschutzes unter Berücksichtigung der Entwicklungsunsicherheit rechtfertigen. Die landesspezifischen Biotopkartierungsverfahren zeigen, dass der Anspruch an einen besonders naturnahen Zustand eines Waldbiotops sich in Bezug auf die Baumarten aus einer weitgehenden Entsprechung des realen Bestandes mit der Zusammensetzung der PNV ergibt. Gleiches gilt für die Ansprache der Biotoptypen nach dem Klassifikationsschlüssel der RLB. Folglich wird auch für die Evaluation der Erstaufforstung eine weitgehende Entsprechung verlangt. Dies bedeutet im vorliegenden Falle, dass nur einer Aufforstung, welche unter dem Kriterium Naturnähe eine Bewertung von mindestens 4 Punkte - und damit 65 Prozent Übereinstimmung - aufweist, auch eine potentielle Entwicklung zu einem wertvollen Biotop zugesprochen wird.

Diese Bewertung folgt den Verfahren der Waldbiotopkartierung Thüringen (LAWUF 1996) für naturnahe und sehr naturnahe Wälder, sowie der Einstufung von Wäldern als „besonders gut ausgeprägte Biotope" nach dem Kartierschlüssel für Biotoptypen in Niedersachsen (DRACHENFELS 1994). Die Affinität der Methodik mit den Waldbiotopkartierungen der behandelten Bundesländer ist mit Blick auf die Praxisnähe gewählt. Schließlich ist in beiden Ländern die Forstverwaltung für die Betreuung der Waldbiotope zuständig und wird die entstehenden Wälder folglich in der Zukunft auch nach den ihr eigenen Kartierungsverfahren erfassen und dokumentieren.

Die Biotopkartierungsverfahren leiten die Listen besonders geschützter Waldbiotope auf der Basis des $\S 20 \mathrm{c}$ des Bundesnaturschutzgesetzes her, erweitert durch die landesspezifischen Gesetzgebungen. In Thüringen wird die Liste durch $\S 18$ VorlThürNatG erweitert, für Niedersachsen findet die Ergänzung durch $\S 18 a$ NNatG statt. Die Kartierungsverfahren fügen schließlich noch weitere, aus Landessicht schützenswerte Waldbiotoptype hinzu. Einige Beispiele für kartierte Biotope sind in Tab 3.5-2 zu sehen. 
Tab. 3.5-2: Synopse der festgestellten Biotoptypen in den Kartierungsverfahren Thüringen sowie der Biotoptypen der Roten Liste der gefährdeten Biotoptypen der Bundesrepublik Deutschland

\begin{tabular}{|l|l|l|l|}
\hline $\begin{array}{l}\text { Rote Liste der gefährdeten Biotoptypen } \\
\text { der Bundesrepublik Deutschland }\end{array}$ & \multicolumn{2}{c|}{ Wadbiotopkartierung Thüringen } \\
\hline Name & Code & Name & Code \\
\hline Traubeneichen-Hainbuchenwald & 43.08 .01 & $\begin{array}{l}\text { Eichen-Hainbuchenwald auf } \\
\text { eutrophen frischen bis mäßig } \\
\text { trockenen Standorten }\end{array}$ & N 202 \\
\hline Eschenwald (an Flißgewässern) & 43.04 .02 & $\begin{array}{l}\text { Erlen-Eschenwald in Bach- und } \\
\text { Flußauen sowie in Niederungen im } \\
\text { kollinen bis submontanen Bereich }\end{array}$ & $\begin{array}{l}\S \\
\text { N } 701\end{array}$ \\
\hline $\begin{array}{l}\text { Weichholzauenwald ohne } \\
\text { Überflutung }\end{array}$ & 43.04 .04 .02 & $\begin{array}{l}\text { Weiden-Auenwald in Flußauen } \\
\text { (Weichlaubholz-Auenwald) im } \\
\text { kollinen bis submontanen Bereich }\end{array}$ & N 702 \\
\hline Vorwälder & 42.06 & Birken-Pionierwald & P 102 \\
\hline $\begin{array}{l}\text { Rhitral (sommerkalte Bäche und } \\
\text { Flussoberläufe) }\end{array}$ & 23.01 & $\begin{array}{l}\text { Schnell fließende Bäche und } \\
\text { Flüsse, beschattet }\end{array}$ & B 112 \\
\hline $\begin{array}{l}\text { Artenarmes, frisches } \\
\text { Intensivgrünland der planaren bis } \\
\text { submontanen Stufe }\end{array}$ & 34.07 .02 & $\begin{array}{l}\text { Frischwiesen (Fettwiesen) im } \\
\text { Flach- und Hügelland }\end{array}$ & G 112 \\
\hline $\begin{array}{l}\text { Artenreiche, frische } \\
\text { Grünlandbrache der planaren bis } \\
\text { submontanen Stufe }\end{array}$ & 34.07 .01 .03 & $\begin{array}{l}\text { Aufgelassenes Grasland frischer } \\
\text { Standorte }\end{array}$ & G 132 \\
\hline- & - & Parkanlagen und Friedhöfe & U 100 \\
\hline
\end{tabular}

Als Vorstufe zur Wertermittlung erfolgt ein Abgleich der festgestellten Waldtypen nach Landesliste mit den Biotoptypen der Roten Liste (vgl. Tab. 3.5-2). Die Bewertung selbst geschieht entsprechend dem in Abb. 3.5-III gezeigten Erhebungsbogen aus einer Kombination der von der RLB vorgegebenen Gefährdungskategorie (maximal 3 Punkte), zuzüglich je eines Punktes für ein geschütztes Biotop nach Landesrecht ( 18 VorlThürNatG, bzw. $\S 18$ a NNatG) und eines Punktes für eine Nennung als schützenswertes Biotop nach den Kartierhinweisen des jeweiligen landesspezifischen Verfahrens.

Die Verwendung der Gefährdungskategorien nach landesspezifischem Schlüssel impliziert eine Berücksichtigung der regionalen Gefährdungssituation. Eigene Erhebungen zur Feststellung der Biotopschutzsituation sind im Rahmen einer Aufforstungsbewertung nicht möglich, da sie den Erfassungszeitraum über Gebühr verlängern würden. Somit wird auch dieser Indikator „extern“ gesteuert. Durch eine Abstufung eines bestimmten Biotoptyps in eine geringere Gefährdungskategorie, erhält der neu zu schaffende Waldtyp automatisch ebenfalls eine geringere Bewertung. Dasselbe gilt selbstverständlich für den komplementären Fall, in dem ein Biotoptyp eine höhere Gefährdungsstufe zugesprochen erhält. 


\section{Indikator 3:}

\section{Seltenheit des Biotops}

Aufforstungszielbestand entspricht einer Waldgesellschaft, welches nach heutiger Rechts- und Definitionslage folgende Schutzkategorien erfüllen würde:

Nennung in der nationalen Rote Liste Biotope (RIECKEN et al. 1994):

Biotop-Typ:

Code:

Gefährdungskategorie:

Einschätzung der Schutzwürdigkeit durch die Waldbiotopkartierung des Landes:

Biotop-Typ:

Code:

Geschütztes Biotop nach Landesrecht:

Schützenswertes Biotop nach WBK:

\begin{tabular}{|c|c|c|}
\hline Bewertung & maximal & vergeben \\
\hline Gefährdungskategorie (3 $\rightarrow$ 1Pkt., $2 \rightarrow 2$ Pkt., $1 \rightarrow 3$ Pkt.) & 3 & \\
\hline Geschütztes Biotop nach Landesrecht & 1 & \\
\hline Schützenswertes Biotop nach WBK & 1 & \\
\hline Gesamt: & 5 & \\
\hline
\end{tabular}

Abb. 3.5-III: $\quad$ Die Einordnung des potentiellen Waldbiotops in die Liste der gefährdeten und schützenswerten Biotope; Auszug aus dem Erhebungsbogen

\subsubsection{Strukturpotential}

\section{Definition}

Ein Bestand kann nach seiner horizontalen und vertikalen Struktur definiert werden.

Dabei verstehen wir unter der horizontalen Struktur die Mischungsform und -art der Gehölzarten. Sie wird gebildet aus Bäumen des Hauptbestandes und des Nebenbestandes. In lückigen Beständen spielen auch die Sträucher und kleineren Bäume des Zwischen- und Unterstandes eine zunehmend wichtige Rolle. Als Hinweis auf deren Bedeutung kann das gruppenweise Auftreten und eine voluminöse, blattreiche Wuchsform betrachtet werden.

Die Vertikalstruktur eines Bestandes ergibt sich aus dem Übereinander verschiedener Vegetationsschichten (Kraut-, Strauch-, Baumschicht), sowie der Differenzierung der Baumschicht in Ober-, Zwischen- und Unterstand.

Die Altersstruktur eines Bestandes ist ein weiteres, unter Umständen sehr wichtiges Merkmal. Eine sinnvolle Einteilung der Gehölzpflanzen erfolgt entsprechend ihres physiologischen Entwicklungsstandes in die Gruppen juvenil, adult, reif, überaltert und abgestorben,.

\section{Bedeutung der Struktur für den Naturschutz}

Die Diversität eines Waldbestandes steht in einem engen Zusammenhang mit der dort vorgefundenen Struktur (BROKAV/LENT 1999). Dies begründet sich aus der mehr oder 
weniger engen Bindung von Tieren, insbesondere der Insekten und Wirbellosen an spezifische biotische sowie abiotische Umweltbedingungen. Ein Individuum sucht nach einem artspezifischen Habitat zur Verwirklichung seiner ökologischen Nische. Das Habitat fasst als Monoplex verschiedene Lebensfaktoren-Komplexe einer Spezies zusammen, beispielsweise Temperatur, Feuchtigkeit, Pflanzenwelt und Nistmöglichkeiten (BERNDT/WINKEL in LIESEN 1996). Da sich die ökologischen Nischen der verschiedenen Arten unterscheiden, ist die Existenz unterschiedlicher Monoplexe eine Vorbedingung für eine Diversität der nischenbesetzenden Tier- und Pflanzenwelt. Der Reichtum an derartigen Monoplexen wird auch als Habitatdiversität bezeichnet (SCHÄFER 2001).

Die Habitatdiversität hängt wiederum von unterschiedlichen Merkmalen hab. So bestimmen die vorhandenen Baumarten durch Kronenaufbau und Lichtdurchlässigkeit die Lichtqualität im Kronenraum und in den unteren Bestandesschichten. PALIK und ENGSTROM (1999) haben hier einen Zusammenhang zwischen der sukzessionalen Stellung von Baumarten und der Beeinflussung der Lichtqualität nachgewiesen. Außerdem beeinflusst die vertikale Bestandesstruktur den Gradienten von Feuchtigkeit, Temperatur und Wind vom Kronendach zum Boden hin (BROKAW/LENT 1999), mit entsprechenden Veränderungen der physikalischen Bedingungen. Ein komplexeres Bestandesprofil führt daher zu einem komplexeren Lebensraum mit verschiedenen Mikroklimaten.

Auch für Wirbeltiere, sind die Strukturen eines Waldbestandes ein wichtiges Kriterium dafür, diesen in ihr Habitat zu integrieren. Vogelarten richten sich neben der Zusammensetzung der Vegetation sowie der Größe und Gestalt der Waldflächen nach der Struktur (DIERSCHKE in LIESEN 1996). Bei Zoophagen erfüllt die Struktur eine Doppelfunktion durch Diversifizierung des Beutetierangebots und Erhöhung der Versteckmöglichkeiten vor höheren Prädatoren der Nahrungskette.

BROKAW und LENT (1999) führen als Indikator für den avifaunistischen Artenreichtum eines Bestandes die Verteilung der Blätter in unterschiedlichen Ebenen des Bestandes ein (FHD = Foliage Height Diversity). Dies spricht ebenfalls für die Bedeutung der vertikalen Struktur für die faunistische Diversität, wobei zu bedenken ist, dass die Vielfalt zusätzlich durch Faktoren wie Baumartenzusammensetzung, Standort und Meereshöhe beeinflusst werden kann.

Die Konvergenz zwischen Vogel- und Pflanzengemeinschaften ist in keinem Landschaftstyp so groß, wie in Laubwäldern, Mooren und Verlandungszonen (FLADE 1994). Allerdings ergibt sich dieser Zusammenhang nicht direkt aus den vorhandenen Pflanzenarten, als vielmehr aus der durch sie bestimmten, typischen Vegetationsstruktur (SCHERZINGER 1985, ZENKER 1982). Die Beachtung der zukünftigen Bestandesstruktur über die Pflanzenauswahl bietet somit eine gute Möglichkeit, den Naturschutzwert einer Fläche bereits in der Planungsphase positiv zu beeinflussen.

Die Bestimmung der strukturellen Heterogenität eines Bestandes erfolgt üblicherweise über die Merkmale (1) Ausbildung verschiedener Stufen, (2) Mischung verschiedener Altersklassen und (3) Vorhandensein einer diversen/natürlich differenzierten Krautschicht. SCHÄFER (2001) sieht in dieser strukturellen Heterogenität einen hinreichenden Indikator für die Zoodiversität eines Waldbiotops, welches als Ergebnis einer naturnahen Waldbewirtschaftung erzielbar ist und einen Umweg über avifaunistische Indikatorarten überflüssig macht.

Die Altersstruktur spielt in diesem Konzept eine wichtige Rolle. Sie trägt unmittelbar zur Habitatvielfalt des Biotops bei, da manche Erscheinungen auf bestimmte Altersphasen eines Bestandes oder Baumes angewiesen sind, beispielsweise die Totholzbildung. Der Totholzreichtum eines Bestandes spielt für den Artenschutz eine wichtige Rolle, da viele gefährdete Käferarten auf Totholz als Lebensraum angewiesen sind.

Für eine Aufforstung scheint die Altersstruktur dagegen zunächst von nachrangiger Bedeutung. Selbstverständlich beträgt der Altersunterschied zwischen den Kulturpflanzen nur wenige Jahre. Durch die Auswahl der Baumarten mit unterschiedlicher natürlicher und wirtschaftlicher Lebensspanne, wird aber die natürliche Entwicklung im Bestand schon auf 
Jahrzehnte hinaus vorherbestimmt. Der Waldbau greift hier lediglich korrigierend, im negativen Falle nivellierend ein.

Die Vertikalstruktur verändert sich mit dem Bestandesalter und mit ihr die Biodiversität. Die Artenvielfalt (einschließlich faunistischer und floristischer Begleitarten) ist im Jugendstadium zunächst hoch, nimmt dann im Stangenholzalter ab und steigt schließlich mit zunehmender Bestandesreife wieder an, bis zu einem Maximum in einem überalterten Bestand (BROKAW/LENT 1999). Die waldbauliche Beeinflussung der Vertikalstruktur ist zum Zeitpunkt der Aufforstung sehr schlecht vorherzusagen. Aus Sicht des Arten- und Naturschutzes ist eine Nivellierung hinsichtlich der Strategietypen (z.B. durch Monokultur oder durch Kombination von Baumarten gleicher Wuchskraft) abzulehnen. Wird jedoch eine Baumartenvielfalt zusammengestellt, die unterschiedlichste Strategietypen (schnell langsam, lichtbedürftig - schattenertragend, dichte Krone - lichte Krone) berücksichtigt, und wird diese waldbauliche Vielfalt auch langfristig erhalten, so führt eine Kombination von Baumarten unterschiedlicher Umtriebszeit nicht nur zu einer zeitlichen Verteilung von Pflegeaufwand und Erlösmöglichkeiten (PREEN 1996), sondern auch zu einer hohen Vertikalstruktur in verschiedenen Altersstadien des Bestandes.

Neben der Förderung der Vertikalstruktur durch die Baumartenwahl, ist die Förderung der Horizontalstruktur durch Dichte und Form der Baumartenmischung zu berücksichtigen (WAGNER 1995). Auch hier stellt die strukturelle Vielfalt das Ziel dar, welches zugleich eine entsprechende Habitatvielfalt gewährleistet. Gleichzeitig ist die Art der horizontalen Mischung aber auch eine waldbauliche Einflussnahme auf die Konkurrenzverhältnisse im Bestand. Gruppierung und Mischung bestimmen den Ablauf der biologischen Automation. Langfristige Baumartenmischung muss als Zielsetzung auch waldbaulich verfolgt und ermöglicht werden (siehe Kap. 3.3.1), indem der Standraum des hiebsreifen Baumes als Mindestgröße einer homogenen Gruppe gesetzt wird.

\section{Bestimmbarkeit des Indikators}

Die Bewertung beruht auf der Annahme, dass der Strukturreichtum eines Bestandes, wie er sich insbesondere nach dem Dichtschluss allmählich ausbildet, bereits im Stadium der Aufforstung prognostizierbar ist und dass er ein Indiz für die Habitatvielfalt und damit für eine mögliche Vielfalt des (faunistischen) Biotopinventars ist (vgl. SCHÄFER 2001, BROKAV/LENT 1999).

In der Entwicklung der Struktur eines Bestandes (horizontal, vertikal und Altersstruktur) kommen verschiedene, baumartenspezifische Charakteristika zum Tragen. Dazu gehören sicherlich der Habitus, die Lichtdurchlässigkeit der Krone und die Dauer der Belaubungszeit, da diese Faktoren die Lichtverhältnisse im Bestand beeinflussen. Darüber hinaus spielt die Rindenbeschaffenheit für zahlreiche Wirbellose eine wichtige Rolle (HUNTER 1990). Und schließlich bestimmen Abstand und Gruppierung der Bäume die Entwicklungsvorgänge des Bestandes. Hinzu kommt - unter dem Gesichtspunkt der langfristigen Dynamik - die Umtriebszeit der beteiligten Baumarten, abhängig vom artspezifischen Wachstumsgang.

Diese Faktoren sind im Rahmen des Indikators Strukturpotential zu berücksichtigen. Eine Ableitung der Strukturvielfalt auf der Grundlage der artenspezifischen Charakteristika würde bei einer Vielfalt von ca. 35 bis 40 forstlich relevanten Baumarten sehr aufwendig werden. Da sich viele Baumarten aber in verschiedenen Merkmalen sehr ähneln, können einige zu Gruppen zusammengefasst werden. Bei genauerer Betrachtung stellt sich heraus, dass sich die Baumarten einer sukzessionalen Stufe häufig auch in den genannten Charakteristika ähneln. Auf der Grundlage eines Literaturstudiums wurde deshalb ein Klassifizierungsverfahren entwickelt, welches eine Einteilung der Baumarten anhand ihrer Merkmale in sogenannte Strukturpotentialklassen ermöglicht. Mit Hilfe dieser Klassifizierung soll eine Prognose der Strukturentwicklung vorbereitet werden. In der hier vorgestellten Art und Weise der Einteilung ist dieser Ansatz neu, obwohl auch OTто (1994) eine Typisierung anhand der sukzessionalen Stellung als Hinweis auf die Bestandesdynamik verwendet (Tab. 
3.5-3). Hierzu ist anzumerken, dass die Vertikalstruktur bei Mischung unterschiedlich konkurrenzfähiger Baumarten in den ersten Jahrzehnten groß ist. Sie bleibt umso länger erhalten, je größer die Gruppen eingestellter (Licht-)Baumarten sind.

\section{Ableitung des Indikators aus der Matrix}

Die Verflechtungsmatrix der 1. Systemebene (Tab. 3.3-1) zeigt eine wechselseitige Abhängigkeit der Baumarten untereinander (Punktewolke SR1). Bereits in Kapitel 3.5.1 wurde beschrieben, dass die Auswirkungen des Konkurrenzkampfes zwischen den einzelnen Baumarten zur Verdrängung von Individuen oder Arten führen kann. Auf alle Fälle führt sie aber zur Differenzierung des Bestandes und damit zu einer vertikalen Strukturierung. Bestehen deutliche Konkurrenzunterschiede, so kommt es zur Ausbildung von Zwischenschichten im Bestandesraum.

Sträucher spielen als Konkurrenz zu den Baumarten lediglich in der ersten Wachstumsphase der neu begründeten Kultur eine Rolle. Von einer Beeinträchtigung der Bäume ist allerdings im Rahmen einer Aufforstung nicht auszugehen.

Tab. 3.5-3: $\quad$ Auswirkung der Mischung von Baumarten unterschiedlicher sukzessionaler Stellung (nach OTTO 1994)

\begin{tabular}{|c|c|}
\hline Mischungsart & Dynamik \\
\hline nur Lichtbaumarten & $\begin{array}{c}\text { rasche Aufgabe von Vertikalstrukturen und z.T. } \\
\text { auch von Mischungen; führt zu einschichtigen } \\
\text { Wäldern }\end{array}$ \\
\hline $\begin{array}{c}\text { Lichtbaumarten (herrschend) und } \\
\text { Schattbaumarten (dienend) }\end{array}$ & $\begin{array}{c}\text { walddynamisch labile Bestände; } \\
\text { Schattbaumarten erkämpfen sich langfristig die } \\
\text { Herrschaft }\end{array}$ \\
\hline Schattbaumarten und Lichtbaumarten & $\begin{array}{c}\text { selten dauerhafte Mischung; nur auf } \\
\text { störungsanfälligen Flächen mit langfristiger } \\
\text { Dynamik; ansonsten dominieren langfristig die } \\
\text { Schattbaumarten }\end{array}$ \\
\hline Schattbaumarten & $\begin{array}{c}\text { dynamisches Gleichgewicht; Plenter- und } \\
\text { Femelstrukturen halten länger an; langfristige } \\
\text { Tendenz zur Entmischung und Entstrukturierung }\end{array}$ \\
\hline
\end{tabular}

Darüber hinaus berücksichtigt die waldbauliche Planung einer Aufforstung die ökologischen Eigenschaften der Baumarten bei der Festsetzung der Baumartenanteile sowie der Mischungsform und des Pflanzverbandes. Dies wirkt sich wiederum auf die vertikale Differenzierung aus und legt bereits die horizontale Bestandesstruktur fest. Diese Zusammenhänge spiegeln sich in einer Punktewolke SR2 am unteren, linken Rand der Verflechtungsmatrix wieder.

In den ersten Jahren der Aufforstung ist das Höhenwachstum der Bäume in der juvenilen Phase entscheidend für die Entwicklung einer vertikalen Struktur. Alle Bäume stehen zu diesem Zeitpunkt in vollem Lichtgenuss. Das Nährstoffangebot ist ebenfalls für alle Bäume optimal. Im Laufe der Jahrzehnte verändern sich die Wuchskräfte einzelner Arten relativ zueinander. Gleichzeitig verändern sich die Lichtverhältnisse im Bestand. Mit derartig veränderten Bedingungen kommen die Arten unterschiedlich zurecht.

Die ökologischen Eigenschaften der Pflanzen und die sich daraus ergebenden Konkurrenzkräfte sind die entscheidenden Einflussgrößen für die Differenzierungs- und Automationsprozesse im Bestand. Sie werden ihrerseits beeinflusst durch die technische Anlage der Pflanzung (Anteile, Mischungsform, Pflanzverband). Der Ausfall ganzer Artengruppen und damit eine Nivellierung des Bestandes soll durch waldbauliche Pflege verhindert werden. Aus diesem Grunde stellt das Differenzierungspotential der Aufforstung die zu erfassende Schlüsselfunktion des Systems dar. 


\section{Parameter}

Auf der Grundlage der ökologischen Einordnung von Bäumen nach verschiedenen Autoren (BURSCHEL/HUSS 1997, OTTO 1994, RÖHRIG/BARTSCH 1992, KNAPP 1971) erfolgte eine Einteilung von in Deutschland heimischen Baumarten in vier Strukturpotentialklassen (Tab. 3.5-4). Die Baumarten sind innerhalb der Gruppen alphabetisch geordnet. Die Zusammenstellung ist in dieser Art ein Novum und soll als Möglichkeit zur Einschätzung im Rahmen einer Potentialbewertung der Entwicklung der Bestandesstruktur vorgeschlagen werden. Die Einschätzung der Baumarten beruht auch in der zitierten Literatur weitgehend auf empirischen Erkenntnissen und nur in Einzelfällen auf wissenschaftlichen Forschungsergebnissen. In den artspezifischen Aussagen weichen die Quellen auch geringfügig von einander ab. Beispielsweise wird die Wuchshöhe von Winter- und Sommerlinde bei OTTO (1994) einheitlich als „hoch“ beschrieben, während KNAPP (1971) der Winterlinde lediglich eine „mittlere“ Wuchshöhe attestiert, die damit hinter jener der Sommerlinde zurück bleibt. Nichtsdestotrotz erfährt die für das vorliegende Evaluationsverfahren entwickelte Einteilung der Baumartenklassen eine Plausibilitätsbestätigung durch die Einschätzung der genannten Waldbau-Literatur und den Vergleich mit Angaben zur Höhenentwicklung von Baumarten aus Ertragstafeln (Anhang 7).

In der Klasse der Pioniere stehen jene Arten, die sich neben einem raschen Jugendwachstum vor allem durch einen hohen Lichtbedarf in der Jugend wie im Alter auszeichnen. Neben dem frühen Erreichen des maximalen Höhenwachstums, ist für sie außerdem eine lichtdurchlässige Krone charakteristisch, welche auch dem Unterstand und der Begleitvegetation einen relativ hohen Lichtgenuss ermöglicht. Viele Pionierbaumarten haben nur geringe Ansprüche an den Nährstoffvorrat der Fläche. Da das Nährstoffangebot auf landwirtschaftlichen Flächen aber zumindest in den ersten Jahren im Allgemeinen sehr gut ist, wurde die Anspruchslosigkeit als klassische Eigenschaft von Pionierarten nicht zum restriktiven Merkmal dieser Baumartenklasse erhoben. Daher finden sich auch Silberweide und Roterle trotz deren verhältnismäßig geringer Nährstoffmangel-Toleranz (OTTO 1994) aufgrund ihrer sonstigen Eigenschaften in dieser Gruppe.

Die Bäume der Klasse intermediäre Pioniere besitzen ebenfalls ein rasches Jugendwachstum. Durch eine höhere Schattentoleranz im Alter oder eine größere Wuchshöhe können sich diese Bäume aber länger im Bestand behaupten, bevor sie von anderen Arten ausgedunkelt werden. In der Samenproduktion und im Kronenaufbau unterscheiden sich die intermediären Pioniere teilweise stark. Als Gemeinsamkeit kann gelten, dass alle Arten sich für die erste Waldgeneration auf einer Freifläche sehr gut eignen, dabei eine schnelle Bestandesbildung gewährleisten und gleichzeitig Hoffnungen auf eine langfristige Beteiligung im Bestand auch bei natürlicher Wiederverjüngung machen. Die Europäische Lärche findet sich in dieser Gruppe wieder, weil ihre besondere Wuchshöhe (KNAPP 1971) eine langfristige Beteiligung im Herrschenden erwarten lässt. Viele Baumarten in dieser Gruppe verfügen über eine geringe Nährstoffmangel-Toleranz (OTTO 1994). Aufgrund des hohen Nährstoffangebotes auf ehemals landwirtschaftlich bewirtschafteten Flächen ist dennoch von einem erfolgreichen Anwachsen der Bäume auszugehen.

Die intermediären Klimaxarten benötigen während ihres gesamten Lebenszyklus ein bestimmtes Maß an Licht. Sie haben Schwierigkeiten, sich unter geschlossenem Schirm zu entwickeln, ertragen andererseits jedoch mehrjährig schwierige Bedingungen. Ihre relativ gute Konkurrenzkraft ergibt sich aus der Kombination von Wuchshöhe und Schattentoleranz im Alter. Durch deutliche Beteiligung der Baumart(en) im Rahmen einer Bestandesbegründung kann diese Gruppe mit geringem Aufwand langfristig gesichert werden. Sie eignet sich auch für eine natürliche Verjüngung im Rahmen langer Umtriebszeiten zur zweiten Waldgeneration hin. 
Tab. 3.5-4: $\quad$ Strukturpotential der Baumarten nach artspezifischen Charakteristika

\begin{tabular}{|c|c|c|}
\hline Klasse & Beschreibung & Baumarten \\
\hline Pioniere & $\begin{array}{l}\text { - } \text { i.d.R. besondere Genügsamkeit } \\
\text { hinsichtlich Nährstoff- und } \\
\text { Wasserbedarf } \\
\text { - } \text { i.d.R. agressive } \\
\text { Vermehrungsstrategie } \\
\text { - } \quad \text { sehr hoher Lichtbedarf in der } \\
\text { Jugend und im Alter } \\
\text { - } \quad \text { rasches Höhenwachstum in der } \\
\text { Jugend } \\
\text { - frühe Kulmination des } \\
\text { - Höhenwachstums } \\
\text { häufig lichtdurchlässige Krone }\end{array}$ & $\begin{array}{l}\text { Aspe } \\
\text { Grauerle } \\
\text { Moorbirke } \\
\text { Salweide } \\
\text { Sandbirke } \\
\text { Schwarzpappel } \\
\text { Silberpappel } \\
\text { Roterle } \\
\text { Weide }\end{array}$ \\
\hline $\begin{array}{l}\text { Intermediäre } \\
\text { Pioniere }\end{array}$ & 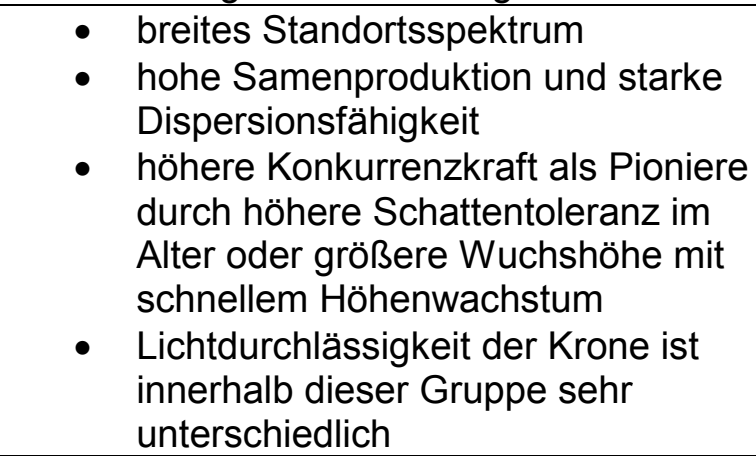 & $\begin{array}{l}\text { Bergahorn } \\
\text { Eberesche } \\
\text { Esche } \\
\text { Europäische Lärche } \\
\text { Fichte } \\
\text { Hainbuche } \\
\text { Mehlbeere } \\
\text { Spitzahorn } \\
\text { Vogelkirsche } \\
\text { Waldkiefer }\end{array}$ \\
\hline $\begin{array}{l}\text { Intermediäre } \\
\text { Klimaxarten }\end{array}$ & $\begin{array}{ll}\text { - } & \text { Mittelmäßiger Lichtbedarf in der } \\
\text { Jugend } \\
\text { - Stärkere Konkurrenzkraft / größere } \\
\text { Wuchshöhe ermöglicht langfristige } \\
\text { Beteiligung am Kronendach } \\
\text { - } \quad \begin{array}{l}\text { Relativ hoher Lichtbedarf im Alter } \\
\text { erfordert großen Standraum }\end{array} \\
\end{array}$ & $\begin{array}{l}\text { Bergulme } \\
\text { Elsbeere } \\
\text { Feldulme } \\
\text { Flatterulme } \\
\text { Speierling } \\
\text { Stieleiche } \\
\text { Traubeneiche }\end{array}$ \\
\hline Klimaxarten & $\begin{array}{l}\text { - Hohe Schattentoleranz bereits in } \\
\text { der Jugend } \\
\text { - Teilweise langes } \\
\text { Verharrungsvermögen im } \\
\text { Unterstand } \\
\text { - Geringe Lichtdurchlässigkeit der } \\
\text { Krone } \\
\text { - Wuchshöhe sehr unterschiedlich }\end{array}$ & $\begin{array}{c}\text { Eibe } \\
\text { Rotbuche } \\
\text { Sommerlinde } \\
\text { Weißtanne } \\
\text { Winterlinde }\end{array}$ \\
\hline Landschaftselemente & $\begin{array}{l}\text { - Bäume der Feldhecken und } \\
\text { Waldränder, aber auch als Solitäre } \\
\text { oder in bestimmten } \\
\text { Waldnutzungsformen } \\
\text { - Im Bestand sehr pflegebedürftig } \\
\text { - Hoher Lichtbedarf } \\
\text { - } \quad \text { Geringe Gesamtwuchshöhe } \\
\text { - Ökologische Bereicherung des } \\
\text { Biotops durch Fruchtangebot } \\
\text { - Spätfrostresistenz mittel bis hoch } \\
\text { (für Waldrand ausreichend) }\end{array}$ & $\begin{array}{l}\text { Feldahorn } \\
\text { Murraykiefer } \\
\text { Traubenkirsche } \\
\text { Walnuss } \\
\text { Wildapfel } \\
\text { Wildbirne } \\
\text { Zirbelkiefer }\end{array}$ \\
\hline
\end{tabular}

Schließlich umfasst die Gruppe der Klimaxarten all jene Baumarten, die sich durch eine besondere Schattentoleranz auszeichnen. Als weiteres Charaktermerkmal dieser Gruppe ist die dichte Krone der Bäume zu nennen. Dadurch dominieren die hier vertretenen Arten 
langfristig den Bestand und verdrängen im Laufe der Generationen die schwächeren Gruppen. Bei Tanne, z.T. auch bei Buche, ist von einer deutlichen Spätfrostgefährdung auszugehen. Dadurch eignen sich diese Arten nur bedingt für Aufforstungsmaßnahmen. Eine Kombination dieser Arten mit Pionieren oder intermediären Pionieren ist angeraten, da in diesem Falle die schnellwachsenden Baumarten für eine rasche Überwindung des gefährlichen Freiflächenstadiums sorgen. Eibe und Linden sollten in dieser Hinsicht keine Probleme bereiten, bedürfen jedoch einer höheren Pflege im Alter, falls sie mit höherwüchsigen Bäumen in Konkurrenz geraten.

Ein Miteinander verschiedener Konkurrenzgruppen ist durchaus möglich (OTTO 1994), wenn innen entsprechender Wuchsraum zur Verfügung gestellt wird (vgl. auch Abb. 3.5-I). Dadurch wird einerseits die Vertikalstruktur gefördert, andererseits kann auch die Lichtdurchlässigkeit des Kronendaches beeinflusst werden. Und letztlich schafft eine Anordnung der Bäume in Wuchsgruppen auch eine horizontale Struktur des Bestandes.

Bleibt noch zu ergänzen, dass eine weitere Gruppen von Baumarten in den Wäldern eine Rolle spielen kann. Zu ihr gehören insbesondere Bäume mit geringer Wuchshöhe und teilweise hohem Lichtbedarf. Diese Bäume werden als Landschaftselemente angesprochen, da sie nicht nur im Walde - und dort vor allem am Waldrand und in lichten Beständen sondern gerade auch in der freien Landschaft als Einzelbäume oder im Verbund mit Feldhecken eine prägende Rolle spielen. Diese Gruppe kann im Bestandesaufbau ebenfalls beteiligt werden, entweder in entsprechend großen Aggregationen oder durch Anpflanzung im peripheren Bereich.

Fremdländische Baumarten wurden im Rahmen der Potential-Einschätzung nicht berücksichtigt, da über deren ökologisches Potential in Bezug auf die Strukturbildung nur wenig Erkenntnisse vorliegen. Am ehesten wäre eine Einordnung der Arten Douglasie, Roteiche, Edelkastanie, Japanische Lärche und Weymouthskiefer möglich, da diese bereits heute eine weite Verbreitung gefunden haben. Da die nichtheimischen Baumarten jedoch im Vergleich zu den heimischen Baumarten eine unbedeutende Rolle als Wirts-, Nahrungs- und Brutpflanzen für die Fauna spielen, könnten sie im Rahmen der Strukturpotential-Bewertung nicht gleichermaßen berücksichtigt werden. Fremdländische Baumarten werden daher im Rahmen des vorliegenden Bewertungsverfahrens ausgeklammert.

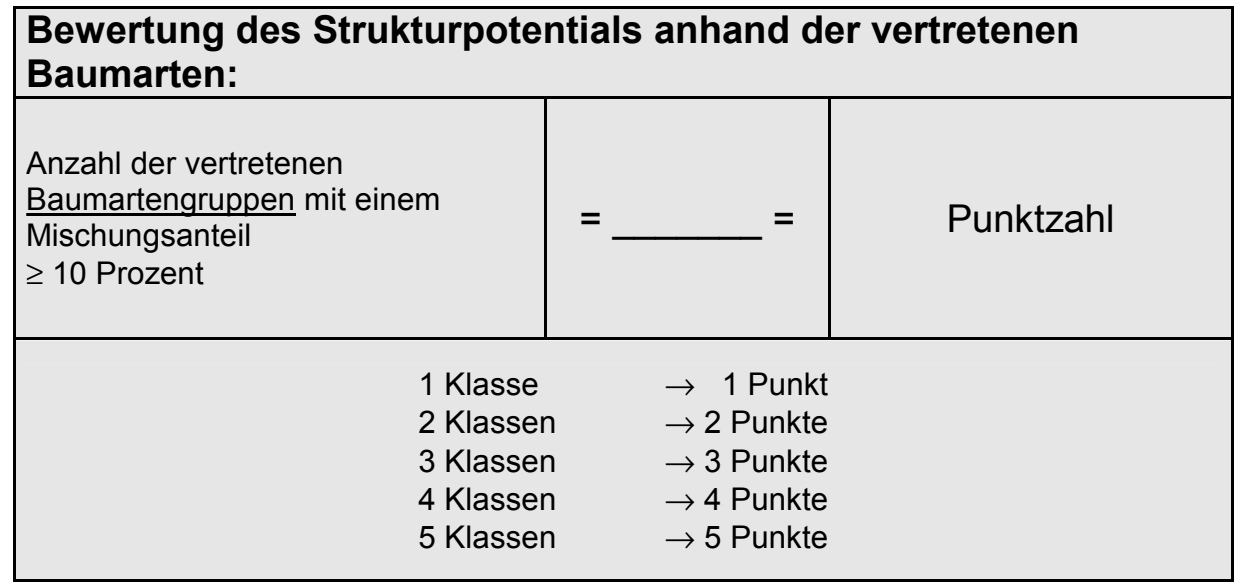

Abb.3.5-IV Bewertungsstufen zu den Strukturpotential-Klassen im Indikator Strukturpotential

Für die Bewertung ist entscheidend, dass möglichst viele der fünf Klassen am Bestand beteiligt sind, da daraus die zukünftige Bestandesstruktur erwächst. Für die Beteiligungsstärke wird ein Mindestmaß von zehn Prozent an der Aufforstungsfläche erwartet. Die Mischung sollte in Gruppen erfolgen, zur Minimierung der interspezifischen Konkurrenz. Der Höchstwert der quantitativen Bewertung ergibt sich aus der Beteiligung aller fünf Strukturpotential-Klassen am Bestand. Daraus ergibt sich ein ordinales Bewertungsschema (Abb. 3.5-IV). 


\subsubsection{Waldrandausprägung}

\section{Definition}

Der Waldrand ist der Übergangsbereich vom geschlossenen Wald zu einer anderen Landnutzungsform oder einem Gewässer. Er wird strukturell gegliedert in die Bereiche Trauf, Mantel und Saum. Er sorgt für einen allmählichen Übergang von der Freifläche zum Bestandesinneren.

\section{Bedeutung für den Naturschutz}

Der Waldrand ist, zumindest soweit er obige Strukturen erkennbar enthält, ein typisches Ökoton, das heißt ein Landschaftselement, welches einen Übergang zwischen zwei unterschiedlichen Biotoptypen herstellt. In inm treffen unterschiedliche Lebensgemeinschaften aufeinander. Dabei beherbergt der Waldrand zumindest temporär Tiere und Pflanzen aus beiden Bereichen. Das Ökoton schließt keine Arten aus sondern vermittelt vielmehr. Daher ist die Diversität im Waldrand auch besonders hoch (edge effect). Gerade lückige und unregelmäßige Waldränder ermöglichen eine hohe Anzahl unterschiedlicher Nischen und bieten dadurch vielen Arten Unterschlupf. Letzteres zum Beispiel für Tiere des Feldes, welche durch die Ernte ihre Deckung und u.U. Nahrung verlieren und sich dann temporär in den Waldrand zurückziehen.

Der große Artenreichtum eines Waldrandes ist auf dessen Heterogenität in Zusammensetzung (unterschiedlicher Pflanzentypen) und Vegetationsstruktur zurückzuführen. Selbst die einzelnen Straten, beispielsweise der Krautsaum, sind in sich stark strukturiert. Dies führt in Zusammenhang mit den Einflüssen Sonneinstrahlung, Niederschlägen und Wind zu einem Mosaik wechselnder Bedingungen. Die Vielfalt an Stellen unterschiedlicher Luftfeuchte, Temperatur und Strahlungsintensität ziehen durch die enge Verzahnung eine ebenso hohe Anzahl unterschiedlicher Arten an (vgl. CoCH 1995).

Im allgemeinen ist die Artenvielfalt auf den West- und Südseiten aufgrund der höheren Sonneneinstrahlung und der stärkeren Einflüsse durch die Windexposition größer als auf den schattigen Nord- und Ostseiten. Diese Faktoren beschleunigen den Wechsel zwischen Befeuchtung und Abtrocknung wesentlich und unterstützen dadurch beispielsweise die Entstehung trockener Stellen.

Auch für den Bestand besitzt der Waldrand eine wichtige klimatische Funktion, da Mantel und Trauf die Einwirkungen des Freiflächenklimas abschwächen. Sie setzen die Strahlungsintensität im randnahen Bereich herab und kann so Sonnenbrand an randständigen Waldbäumen verhindern. Gleichzeitig wird der Wind gebremst oder über das Kronendach geleitet. Angrenzend an eine landwirtschaftliche Nutzung verringert er die Stofffracht der Luft und verhindert somit den Eintrag von Dünge- und Pflanzenschutzmitteln in den Wald.

Schließlich ist die Form des Waldrandes, also die Buchtung und Unregelmäßigkeit in der Linienführung ein wichtiges Charakteristikum. Tiere, die von einem Baumbestand in den anderen wechseln, bevorzugen kurze Übergangswege, wo z.B. der Wald zungenförmig in eine landwirtschaftliche Nutzfläche hineinragt (FORMAN/COLLINGE 1996). Die Buchtung des Waldrandes schafft einen überschaubaren Rückzugsraum, verlängert zusätzlich die Waldrandlänge und steigert somit deren Wirkung.

Der Waldrand zeichnet sich durch lichtreiche Standortsbedingungen aus und ermöglicht konkurrenzschwachen Pflanzenarten eine Existenz. Gleichzeitig ist die Abmilderung des Freiflächenklimas aber bereits so deutlich, dass sich im Waldmantel auch in der Jugend frostgefährdete Arten ansiedeln und erfolgreich diesem Stadium entwachsen können. Die dadurch entstehende Konkurrenzsituation mündet mittel- bis langfristig in eine Dominanz der Waldarten und somit in die Entstrukturierung des Waldrandes. Die strukturelle und 
zahlenmäßige Vielfalt des Ökotons kann langfristig nur durch Pflegemaßnahmen gesichert werden (FRIEDRICHSDORF 1999; WAGNER 1995). Die Art der Pflegemaßnahmen muss dabei nicht besonders diffizil sein. Schließlich kommt es nur auf die Vielfalt der Arten und die Struktur des Waldrandes an. Qualitätsmaßstäbe, wie sie beispielsweise im Rahmen einer Auslesedurchforstung im Bestand angelegt werden, also Geradschaftigkeit, Volumen und Vitalität spielen hier keine Rolle. Lediglich eine Ausrichtung auf konkurrenzschwache Arten scheint sinnvoll.

In Sonderfällen sind auch offene Waldränder von besonderer Bedeutung für den Artenschutz. Dies ist zum Beispiel für Sandmagerrasen unter Kiefer belegt, der einen hohen Lichteinfall benötigt. Auch ein Vorkommen von Völkern der Waldameise am offenen Bestandesrand kann für dessen besonderen Wert sprechen. Allerdings ist zu bedenken, dass die Ameise auch in einem breiten Waldrand mit Freistellen, bzw. Lücken ihren Platz findet. Der Sandmagerrasen wird in einem lichten Kiefernbestand ebenfalls einen Blühaspekt ausbilden können. Im Rahmen einer Aufforstung sind derartige Sonderformen nicht zu realisieren, da sie ein mindestens mittleres Bestandesalter voraussetzen. Die Wirkung kann daher in den ersten zwei bis vier Jahrzehnten nicht erreicht werden. Eine Schaffung derartiger Waldränder ist eher an existierenden Bestandesgrenzen durch entsprechende Pflegeeingriffe zu erzielen.

\section{Bestimmbarkeit des Indikators}

Der Aufbau des Waldrandes mit den Strukturelementen Trauf, Mantel und Saum ergibt eine gute Gliederungsmöglichkeit bei der Beschreibung des Ökotons. Bezüglich der Tiefe der einzelnen Straten gibt es Anhaltswerte aus der Praxis, die eine Funktionserfüllung für Waldschutz und Naturschutz gleichermaßen erwarten lassen.

Die Forderungen nach einer wirksamen Mindestbreite eines anzulegenden Waldrandes von 15 bis 30 Metern (je nach Exposition) liegen im machbaren und ökologisch sinnvollen Bereich. WAGNER (1995) fordert darüber hinaus noch Pufferzonen zu landwirtschaftlichen Flächen. Auf jeden Fall sollen aber die drei genannten Strukturelemente Saum, Mantel und Trauf bei der Anlage berücksichtigt werden.

Ein Waldrand von zu geringer Breite kann lediglich in den ersten Jahren nach der Pflanzung eine ästhetische und ökologische Funktion erfüllen. Wird er nicht ständig gepflegt, so überschirmen bald auslandende Traufbäume die randständigen Gehölze und der strauchreiche Waldmantel geht verloren (KRAUSE 2000).

WAGNER (1995) fordert, Raum für die natürliche Ansamung von Pflanzen im Rahmen der Sukzession vorzusehen. Dem gegenüber verweist FRIEDRICHSDORF (1999) auf Erfahrungen aus Schleswig-Holstein, nach denen eine Sukzession unter Umständen lange auf sich warten lässt und schließlich auch Baumarten bringt, die unter ökologischen Gesichtspunkten nicht erwünscht sind (z.B. spätblühende Traubenkirsche, Sitka-Fichte). Eine gezielte Anlage des Waldrandes mit heimischen, möglichst lokal gewonnenen Arten ist demnach vorzuziehen.

In diesem Zusammenhang ist allerdings auf die geringe Verfügbarkeit von regional gezogenem Pflanzgut gesicherter Herkünfte hinzuweisen (REIF/NICKEL 2000). Gerade bei forstökonomisch nicht relevanten Arten, also Bäumen zweiter Ordnung und Sträuchern, ist die geringe Verfügbarkeit beklagenswert.

Auf die ökologische Bedeutung der Buchtung von Waldrändern wird an vielen Stellen hingewiesen (ZUNDEL 2000, FORMAN/COLLINGE 1996, COCH 1995, WAGNER 1995), allerdings werden keine Aussagen zur Art und Stärke der Buchtung gemacht. Sicherlich müssen diese auch im Kontext des Landschaftsbildes betrachtet werden, in dem sie stehen. Häufig geben die Eigentumsgrenzen einen recht starren und geraden Verlauf vor. In jedem Falle kann aber durch eine lückige und abwechslungsreiche Pflanzung der optische Eindruck einer geraden Linie vermieden und die Verzahnung der angrenzenden Biotope gefördert werden. 


\section{Ableitung aus der Beziehungsmatrix}

Wie bereits erwähnt, ist der Waldrand in aller Regel ein Konstrukt anthropogener Einflussnahme. Zur Erhaltung der floristischen Artenvielfalt und der benannten Strukturmerkmale bedarf es einer regelmäßigen Pflege oder Bewirtschaftung (vgl. $\mathrm{COCH}$ 1995). Neben der Standortsgerechtigkeit verwendeter Baum- und Straucharten, verlangt das Entwicklungsziel deshalb nach einer auf Arten und Struktur abgestimmten Bewirtschaftung durch Mahd, Mulchen, auf-den-Stock-setzen oder ähnliche Maßnahmen. In der Verflechtungsmatrix (Tab. 3.3.-1) sind drei Punktewolken zu erkennen, die in direktem Zusammenhang mit dem Waldrand stehen:

Das Zentrum WR1 zeigt, dass die verwendeten Strauch- (und Baum-)Arten sowie die erwarteten Begleitarten Einfluss nehmen auf die Bewirtschaftungsweise und die notwendige Waldrandbreite.

Das Zentrum WR2 steht für die wechselseitigen Einflüsse von Erscheinungsbild und anthropogener Beeinflussung. In diesem Zusammenhang ist auch der Verbissschutz zu sehen, welcher durch den Menschen auf unterschiedliche Weise zu erreichen ist (am Wild und an der Pflanze).

Das dritte Zentrum, WR3 bildet quasi das Gegengewicht zu WR1. Das Vorkommen verschiedener Pflanzen und Sträucher, ob gepflanzt oder natürlich angesamt, hängt sehr stark von den vorgegebenen Bedingungen ab. Dazu gehört der Platz, der innen eingeräumt wird ebenso, wie die Pflege, die sie mittel- und langfristig erhalten.

\section{Parameter}

Die Bewertung des Indikators Waldrandausprägung erfolgt gutachtlich anhand einer einfachen Checkliste (Tab. 3.5-5), die jene Verknüpfungen wiedergeben soll, die im Rahmen der Erstanlage bedacht werden können: Pflanzenwahl sowie Form und Breite des Waldrandes. Autochthones Pflanzenmaterial ist zu bevorzugen. Die Zusammensetzung soll sich an den natürlichen Bedingungen orientieren. Der strukturierte Aufbau mit Saum, Mantel und Trauf ergibt eine weitere Zielsetzung. Sie bedingt eine entsprechende Tiefe des Waldrandes. Somit ergeben sich fünf Merkmale, die nacheinander geprüft werden. Nicht alle Merkmale sind für einen naturschützerisch wertvollen Bestandesrand notwendig, sie ergänzen sich aber gegenseitig. Die Unabhängigkeit der Merkmale untereinander ist gegeben, mit Ausnahme der Berücksichtigung des Strauchanteiles, wo zwei Stufen in der Klassifizierung ausgewiesen wurden.

Tab. 3.5-5: Checkliste zum Indikator Waldrandausprägung; Bewertung nach der Summe der festgestellten Merkmale bei Waldrandbegründung

\section{Checkliste}

\begin{tabular}{|c|c|}
\hline Merkmal & $\checkmark$ \\
\hline Waldrandbreite mit mind. 15 Metern eingeplant & \\
\hline Lückige oder gebuchtete Grundform des Waldrandes & \\
\hline $\begin{array}{c}\text { Artzusammensetzung des Waldrandes zu mindestens } \\
90 \% \text { Sträucher oder Bäume 2. Ordnung }\end{array}$ & \\
\hline Strauchanteil mindestens 60\% & \\
\hline Verwendung von autochthonem Pflanzenmaterial & \\
\hline
\end{tabular}

Die anthropogene Beeinflussung des Waldrandes wird nicht berücksichtigt. Als künstliche geschaffenes Biotop ist die initiale Beeinflussung selbstverständlich. Die Erhaltungs- und Entwicklungspflege wird vorausgesetzt. Sie kann in die übliche Bestandespflege integriert werden und ist im Rahmen der finanziellen Förderung abzudecken. Auch der Verbissschutz 
muss nicht explizit aufgeführt werden, da er in den üblichen Maßnahmen (Zäunung, Wildmanagement) inbegriffen ist.

Die Bewertung des Indikators leitet sich aus der Summe der festgestellten Merkmale ab.

\subsubsection{Biotopverbund}

\section{Definition}

Der Biotopverbund bezeichnet eine räumliche Verknüpfung zwischen Lebensräumen, die jedoch nicht durch eine unmittelbare Nachbarschaft gleichartiger Flächen gewährleistet sein muss (JEDICKE 1994). Vielmehr kommt es darauf an, dass die Bewohner des einen Lebensraumes den anderen erreichen können, dass also ein Austausch von Individuen (und damit von Genen) zwischen zwei Populationen stattfinden kann.

Die Habitat-Ergänzung bezeichnet in diesem Zusammenhang eine Erweiterung eines Biotops durch eine angrenzende Fläche, welche in ihrer Struktur und Zusammensetzung geeignet ist, einem Großteil der Biotopbewohner temporär als Aufenthaltsort zu dienen.

\section{Bedeutung des Biotopverbunds für den Naturschutz}

Nach JEDICKE (1994) zielt der Naturschutz heute darauf ab, einen Großteil der Arten, die um 1850 in der mitteleuropäischen Kulturlandschaft heimisch waren, zu schützen. Ein Hauptziel dieses Vorhabens ist der Artenschutz, welcher mit Hilfe von Reglementierungen (z.B. Artenschutzabkommen, Naturschutzgesetze), Roten Listen und politischen Programmen einzelne Arten erhalten will. Die Förderung einer oder auch vieler Arten erfolgt zusätzlich über die Erhaltung und Pflege geeigneter Biotope. Daraus folgt das zweite Hauptziel des Naturschutzes, der Biotopschutz. Die Instrumente gleichen jenen des Artenschutzes: Internationale Vereinbarungen (Agenda 21, FFH-Richtlinie), nationale Gesetze, Rote Listen und politische Programme. Der Erfolg des Artenschutzes drückt sich in der Abundanz der Individuen aus. Die Ergebnisse des Biotopschutzes werden statistisch in der Gesamtfläche betrachteter Biotoptypen festgehalten.

Nun ist der Artenschutz vom Biotopschutz abhängig, da eine Population ein Biotop benötigt und die Vergrößerung und Vermehrung einer Population nur möglich ist, wenn adäquate Biotope in ausreichender Nähe vorhanden sind. Umgekehrt, ist die komplette Ausstattung eines Lebensraumes mit (fast) allen vergesellschafteten Arten ein wichtiges Teilziel des Biotopschutzes. Die entsprechenden Arten können aber nur einwandern, wenn Populationen in räumlicher Nähe vorhanden sind, welche einen Überschuss an Individuen produzieren. Der Biotopverbund steht somit als Bindeglied zwischen dem Arten- und dem Biotopschutz.

Das Konzept des Biotopverbundes besteht laut JEDICKE (ebd.) darin, ein System großflächiger Schutzgebiete (mind. 100 ha) aufzubauen, welche als Dauerlebensraum stabiler Populationen fungieren. Trittstein- und Korridorbiotope sollen den Individuenaustausch zwischen den einzelnen Gebieten ermöglichen. Und schließlich müssen diese Schutz- und Pflegebemühungen ergänzt werden durch eine flächendeckende Extensivierung der Flächennutzung. Planerisch resultiert aus diesen Vorgaben die Biotopverbundplanung, welche regionale Leitbilder generiert und umsetzt. Sie kann, neben der Landschaftsplanung, ein bedeutendes Instrument des Naturschutzes mit transdisziplinären Auswirkungen darstellen (NITSCHE/NITSCHE 1994).

Einige Arten benötigen eine Reihe unterschiedlicher Biotoptypen in enger räumlicher Verzahnung. Dazu gehören beispielsweise Fledermäuse, die einen Biotopkomplex aus Gebäuden, Hecken, Obstgärten, Wäldern, Alleen, Waldrändern und Feuchtflächen gleichzeitig nutzen (MAYER/GEIGER 1996). Daher muss sich eine Biotopverbundplanung nicht nur mit der Verknüpfung gleichartiger Biotoptypen beschäftigen, sondern sollte auch entsprechende funktionale Vernetzungen berücksichtigen. Weil die benötigten 
Biotopkomplexe sich aber sehr stark von einer Art zur anderen unterscheiden, greift auch die Biotopverbundplanung auf Indikatorarten zurück.

\section{Bestimmbarkeit des Indikators}

Die Verknüpfung von gleichartigen oder einander ergänzenden Biotopen muss bestimmten Regeln folgen. Die Populationen der verbundenen Biotope sollen miteinander im Austausch stehen. Dies bedeutet, dass sie nicht zu weit von einander entfernt sein dürfen. Insbesondere für terrestrische Tiere ist darüber hinaus auch die Qualität der zwischenliegenden Flächen von Bedeutung, da sie sich bei den Wanderungen zwischen den Biotopflächen nicht aus der Deckung, bzw. aus einem bestimmten klimatischen Milieu herausbewegen.

JEDICKE (1994) fordert eine Konzentration der Bemühungen auf die Verknüpfung von Biotoptypen gleicher oder ähnlicher Art, beispielsweise Hecken untereinander und mit Feldholzinseln. Dieser Ansatz begründet sich aus der Verwendung von Indikatorarten für die Planung und beschränkt sich in seinen Maßstäben auf die Ansprüche weniger Arten. Diese sollen selbst so hohe Qualitätsansprüche an das jeweilige Biotop stellen, dass deren Vorkommen quasi Gewähr ist für eine gleichzeitige Besiedelung mit vergesellschafteten Arten. Eine Berücksichtigung von Biotopkomplexen ist damit nicht durchführbar, weil die Arten eines Biotops jeweils unterschiedliche Zusatzbiotope benötigen und nutzen.

Die Biotopverbundplanung kann mit diesem Ansatz eine Reihe von Biotoptypen erfassen und mit speziellen Maßnahmen fördern. Können die wichtigsten Typen eines Landschaftsraumes auf diese Weise gesichert werden, so ist die Wahrscheinlichkeit sehr hoch, dass davon auch die darin vorkommenden Arten profitieren.

Für den Fall der Aufforstungsevaluation muss eine andere Betrachtungsweise gewählt werden, da die Art des entstehenden Biotoptyps von vornherein feststeht. Der entstehende Wald kann demnach lediglich zwischen Wäldern und Gehölzflächen vermitteln. Bei Entwicklung eines ausgeprägten Waldrandes kommt bestenfalls noch die Verknüpfung mit Gehölzinseln und Hecken hinzu. Die Beurteilung der ökologischen Auswirkungen auf die Tier- und Pflanzenarten der Feuchtgebiete und offenen Flächen, fällt bei der Eingrenzung der Betrachtung auf gleichartige Flächen unter den Tisch.

Ein praktikabler Ansatz zur Berücksichtigung von Verknüpfungen zwischen unterschiedlichen Biotoptypen, sind die von FLADE (1994) definierten Landschaftstypen. Nach dem Leitartenmodell werden aussagekräftige Vogelarten und bestimmte Landschaftsausschnitte sowie Landschaftsqualitäten zueinander in Beziehung gebracht. Dadurch kann eine Landschaft nach Strukturbildern klassifiziert und anhand der vorkommenden Vogelarten qualitativ beschrieben werden. Die Vögel stehen dabei jeweils für bestimmte Biotopkomplexe, z.B. Neuntöter, Grauammer, Ortolan und Wachtel für die halboffene, reichstrukturierte Feldflur oder Knicklandschaft mit Kleingehölzen, halboffenen Agrarflächen und linienhaften Strukturelementen. Jene Arten, die in einem bestimmten Landschaftstyp mit besonders hoher Stetigkeit auftreten, werden als Leitart bezeichnet. Ihr Vorkommen ist im Rahmen der Landschaftsplanung festzustellen. Je nach Landschaftstyp, kommen so Leitartengruppen von bis zu 24 Vogelarten vor. Dabei ist es weder notwendig, dass alle Leitarten in einer bestimmten Fläche vertreten sind, noch dass die Arten eine überdurchschnittliche Abundanz aufweisen. Da eine Art allerdings für verschiedene Landschaftstypen als Leitart verstanden werden kann, ist ein Umkehrschluss nicht möglich.

Der Landschaftstyp ist über die charakteristischen Strukturelemente und Biotoptypen definiert. Anhand dieser Elemente wird der Typ über eine Luftbildinterpretation und im Gelände festgestellt. Die Betrachtung der Avifauna im betroffenen Landschaftstyp dient der Verifizierung und kann eine qualitative Einschätzung der Biotopqualität ermöglichen, wenn die Leitarten entsprechend häufig und vollständig vertreten sind.

Im Zusammenhang mit den Landschaftstypen und deren typischen Vogelgemeinschaften stellt sich die Frage, welche Rolle die Erstaufforstung auf landwirtschaftlichen Flächen für bestimmte Tierarten spielen kann. Neben der allgemeingültigen Feststellung, dass sich das 
typische Arteninventar eines Waldes umso schneller einstellt, je größer die Kontaktfläche zu einem Altbestand ähnlichen Typus ist, kann auch die Verbundsituation mit anderen Biotopen wichtig sein. Etwa die Hälfte der ca. 210 mittel- und norddeutschen Vogelarten brütet in Laubwäldern. Immerhin ein Drittel der Arten lebt bevorzugt oder ausschließlich in innen oder in parkähnlichen Landschaften. Der größte Teil der auf den Laubwald spezialisierten, gut 70 Vogelarten kommt also mit den Bedingungen der halboffenen Landschaft und der Siedlungen sehr gut zurecht (FLADE 1994). Dazu gehören vor allem jene Waldarten, die im Naturwald auch die kurzfristigen Kahl- und Sukzessionsflächen nutzen können, z.B. Neuntöter, Heidelerche, Haselhuhn, Grünspecht, Gartengrasmücke und Fitis.

Schwieriger stellt sich die Situation bei den gefiederten Bewohnern des Nadelwaldes dar. Die dort angesiedelten ca. 25 Arten (FLADE 1994) konnten sich den Lebensraum der halboffenen Landschaft und der Siedlungen noch nicht erschließen. Eine entsprechend positive Verbundwirkung neuer Flächen müsste daher auf eine Vergrößerung der Nadelwaldfläche zielen.

\section{Ableitung des Indikators aus der Matrix}

Im Zentrum der Verflechtungsmatrix (Tab. 3.3-2) steht eine Punktewolke BV, die sich aus den vielseitigen, teils umkehrbaren Verknüpfungen zwischen dem Erhalt der genetischen Ressourcen und der Verknüpfungs-/Vernetzungsform ergibt. Die genetische Vielfalt innerhalb einer Population ist immer davon abhängig, wie viel Austausch zwischen der betroffenen und benachbarten Populationen stattfinden kann. Dieser Austausch steht wiederum in Zusammenhang mit der Entfernung zwischen den Teilpopulationen und den Möglichkeiten der Überbrückung. Eine Verinselung von Biotopen hemmt den genetischen Austausch und führt zur Homogenisierung des genetischen Reservoirs. Gleichzeitig werden Inzesterscheinungen begünstigt (ELSAßER 1991).

Je geringer die Mobilität der Tier- oder Pflanzenarten ist, desto entscheidender ist die Verbindung der Habitate durch Strukturelemente bzw. Biotope ähnlicher Beschaffenheit. Die funktionale Vernetzung zwischen gleichartigen oder unterschiedlichen Biotopen wird durch einen räumlichen Verbund ebenfalls gefördert oder erst ermöglicht. Das Minimumareal einer Population, also jene Mindesthabitatgröße, die eine Population zum Überleben benötigt, ohne dass die Gefahr der Inzucht besteht, ist seinerseits abhängig von der Diversität des Gen-Pools, vom stattfindenden genetischen Austausch und von der Art der Vernetzung und des Verbundes mit gleichen, ähnlichen oder ergänzenden Biotoptypen.

Die vielfältigen Beziehungen dieses Systembereiches werden durch den Indikator Biotopverbund zusammengefasst.

\section{Parameter}

Zur Einschätzung des Indikators Biotopverbund/Habitatergänzung findet zunächst eine gutachtliche Feststellung statt, welche der von FLADE (1994) beschriebenen Landschaftstypen (Anhang 1) im Untersuchungsgebiet vorkommen. Dies erfolgt unabhängig von einem Leitbild. Die Ergebnisse könnten also sowohl Richtung Strukturreichtum als auch Richtung Strukturarmut der Landschaft weisen. Ziel ist vielmehr, die aktuelle Situation des Biotopverbundes möglichst objektiv zu erfassen.

Anschließend wird anhand von Experteninterviews und schriftlichen Quellen festgestellt, welche der von FLADE (ebd.) genannten Leitarten dieser Lebensraumtypen in der Untersuchungsregion tatsächlich vorkommen. Diese Vorgehensweise dient der Vereinfachung des Verfahrens. Eigene Untersuchungen zur Bestätigung der Vorkommen, etwa durch Linientaxierungen, waren im Rahmen des Projektes nicht realisierbar. Es wird davon ausgegangen, dass die ornithologischen Gruppen vor Ort einen hinreichend genauen Überblick über die Populationen haben, um die Stabilität derselben einschätzen zu können. Für den Zweck der Förderung eines potentiellen Lebensraumes, reicht dies aus.

Durch einen Vergleich der vorkommenden Landschaftstypen mit den bestätigten Vorkommen von Leitarten (Abb. 3.5-V), wird für das jeweilige Untersuchungsgebiet 
festgestellt, welche Landschaftstypen förderungswürdig sind. An diesen wird schließlich die Aufforstung gemessen. Eine positive Bewertung erfährt die Umwandlung, wenn sie im Sinne der Lebensraumerweiterung der Leitarten vorkommender Landschaftstypen einen Zuwachs bedeutet. Dazu gehört selbstverständlich, dass die Entfernung zu den Strukturelementen desselben Landschaftstyps innerhalb der von den Leitarten überbrückbaren Distanzen liegt (siehe Kap. 4).

Ergänzend wird im Rahmen der Aufforstungsevaluation festgestellt, ob die Maßnahme einer Erweiterung des Lebensraumes einer Zielart aus dem regionalen / landesweiten Biotop- und Artenschutzprogramm dient. Dabei stehen dann artspezifische Biotopansprüche im Vordergrund. Es kann sein, dass diese Arten auch als Leitarten für einen vorhandenen Landschaftstyp relevant sind. Dieses muss aber nicht der Fall sein. Die Bewertung nach beiden Gesichtspunkten steht nebeneinander. Eine Höherbewertung einer Fläche, die sowohl einer Zielart des Schutzprogramms als auch der Erweiterung eines Landschaftstyps dient, ist nicht möglich. Für den Anspruch an die Vernetzung mit anderen Strukturelementen, die dem artspezifischen Habitat angehören, gilt das oben Gesagte entsprechend.

Betrachtungsfläche: $\quad 4$ km$^{2}$

Potentielles Waldbiotop:

Angrenzende Biotope:

\begin{tabular}{|c|c|c|c|c|}
\hline $\begin{array}{c}\text { Himmels- } \\
\text { richtung }\end{array}$ & $\begin{array}{c}\text { Biotoptyp } \\
\text { (It. Typenschlüssel nach } \\
\text { RIECKEN oder Offenland- } \\
\text { BK) }\end{array}$ & $\begin{array}{c}\text { Landschaftselement } \\
\text { (Acker, Grünland, } \\
\text { Sonderkultur, Hecke, } \\
\text { Fließgewässer, Wald }\end{array}$ & $\begin{array}{c}\text { Landschaftstyp } \\
\text { nach FLADE }\end{array}$ & angrenzend \\
\hline N & & & & \\
\hline NO & & & & \\
\hline O & & & & \\
\hline SO & & & & \\
\hline S & & & & \\
\hline SW & & & & \\
\hline W & & & & \\
\hline NW & & & & \\
\hline
\end{tabular}

\begin{tabular}{|c|c|}
\hline Bewertungsmerkmale: & $\checkmark$ \\
\hline Biotopentwicklung & \\
\hline Biotopergänzung & \\
\hline Biotopverknüpfung & \\
\hline Ergänzter Lebensraumtyp & \\
\hline Vergrößerter Lebensraumtyp & \\
\hline Summe: & \\
\hline
\end{tabular}

Abb. 3.5-V: $\quad$ Feststellung der Biotopverbundwirkung und Habitatergänzung durch die Aufforstung; Auszug aus dem Erhebungsbogen (vgl. Anhang 1) 


\subsubsection{Landnutzungsverhältnis}

\section{Definition}

Das Erscheinungsbild einer Landschaft wird einerseits durch die topographische Grundform geprägt, andererseits auch durch die stattfindende Landnutzung und die damit zusammenhängenden Vegetationsformen. Der Flächenanteil einzelner Landnutzungsarten ergibt zusammen mit der Parzellengröße und der Mischungsform eine Grundgliederung der Landschaft, die durch Strukturelemente, geologische Gebilde und saisonalen Fruchtwechsel ergänzt wird.

\section{Bedeutung des Landnutzungsverhältnisses für den Naturschutz}

Die Landschaftsästhetik nimmt im Naturschutz im Allgemeinen nur einen geringen Teil der Anstrengungen und Ziele ein. Der gesetzliche Auftrag an den Naturschutz spricht von der Aufgabe, die Schönheit der Landschaft zu schützen. Diese wird aber nicht definiert oder eingegrenzt und auch selten umgesetzt. Die Landschaftsästhetik steht zudem vor ungeklärten Fragen bezüglich eines verallgemeinerbaren Schönheitsempfindens. Zu wenige Untersuchungen bieten ausreichende Hinweise darauf, wie die Bevölkerung gewisse Landschaftsbilder annimmt, welche Elemente sie bevorzugt oder ablehnt (ein Beispiel gibt ASSEBURG 1985).

Auswertungen von touristischen Zielen sind müßig, da die dort anzutreffenden Besucher den vorhandenen Landschaftstyp selbstverständlich als attraktiv empfinden. Unklar bleibt jedoch, ob der Landschaftstyp auch die ideale Erscheinungsform bereits angenommen hat, oder ob der Charakter noch verstärkt werden könnte/sollte. Auch ist fraglich, welche Rolle das Landschaftsbild für das Aufsuchen selbst spielte und welche Alternativen bestanden hätten.

Diese Zweifel an der Verallgemeinerungsfähigkeit des ästhetischen Empfindens lassen sich übertragen auf die Suche nach einer idealtypischen Gliederung der Landschaft. Aus diesem Bereich der Wissenschaft liegen noch weniger Untersuchungen vor. Es gibt allerdings Hinweise in unterschiedlichen Quellen (COCH 1995, JEDICKE 1994, u.a.) nach denen eine starke Strukturierung der Landschaft nicht als negativ empfunden wird und Landschaftselemente das Erscheinungsbild eher bereichern. Die Abwechslung wird vom Besucher in der Regel geschätzt, die Eintönigkeit wird gemieden.

In Bezug auf die Artenvielfalt ist allgemein festzustellen, dass reine Waldgebiete eine geringere Bandbreite an Tier- und Pflanzenarten aufweisen, als strukturreichere Gegenden. Die traditionelle bäuerliche Kulturlandschaft gilt mit ihrem hohen Vorkommen an Übergangszonen zwischen Wäldern, Wiesen, Hecken, Einzelbäumen und anderen Landschaftselementen als besonders nischenreich und vielfältig (ELSAßER 1991).

Die Erhöhung des Waldanteils im Rahmen von Aufforstungskampagnen ruft bei ihren Kritikern immer wieder das "Gespenst“ der geschlossenen Landschaften hervor. Tatsache ist allerdings, dass selbst die kostenintensivsten Aufforstungsprogramme bislang kaum nennenswerte Aufforstungsraten bewirkten. Nach Einführung eines neuen Programms kommt es zwar regelmäßig zu einem Anstieg der Anträge, der allerdings meist wieder sehr schnell verebbt. Selbst ein längerfristiger Aufforstungsboom würde nur einen marginalen, landesweit kaum feststellbaren Anstieg der Bewaldung bewirken (vgl. AMMER 1997). Nichtsdestotrotz können auch geringe Aufforstungsraten einen prägnanten Einfluss auf das Landschaftsbild besitzen, wenn sie gezielt oder unbedacht durchgeführt werden.

Ein allgemeines Werturteil für oder gegen eine Erhöhung des Waldanteils ergibt sich aus diesen Betrachtungen nicht. ELSAßER (1991) kommt nach Auswertung unterschiedlicher Untersuchungen und Literaturquellen $\mathrm{zu}$ dem etwas ungenauen Ergebnis, dass die Bevölkerung ein Bewaldungsoptimum bei etwa 30 bis $80 \%$ Flächenanteil sehen muss. Daraus lässt sich keine brauchbare Zielvorgabe für ein allgemein anwendbares Verfahren ableiten. Vielmehr ist die Bewaldungsquote regional festzulegen, da auch das 
Landschaftsempfinden einer regionalen Differenzierung unterliegt. Es kann lediglich darauf geschlossen werden, dass gewisse Mindestbedürfnisse des Menschen hinsichtlich der Bewaldung, aber auch hinsichtlich der verbleibenden Freifläche bestehen.

Verallgemeinerungsfähig ist hingegen sicherlich die negative Empfindung gegenüber Verkehrslinien oder technischen Einrichtungen, die das Landschaftsbild zerteilen oder anderweitig überprägen. Diese spielen allerdings für die vorliegende Betrachtung von Erstaufforstungen keine Rolle und werden daher nicht weiter erörtert.

\section{Bestimmbarkeit des Indikators}

Kriterien für eine Bewertung der landwirtschaftlich geprägten Kulturlandschaft unter Berücksichtigung von Biotop- und Artenschutz sowie der Landschaftsästhetik schlagen ROTH et al. (1996) vor. Dabei liegt das Hauptaugenmerk auf der Feststellung von ökologischen und landeskulturellen Vorrangflächen (ÖLV). Diese umfassen Flächen, auf denen zukünftig keine landwirtschaftliche Nutzung mehr stattfinden soll (Brachen, Hecken, Gehölze), aber auch Biotope, die auf eine extensive Nutzung angewiesen sind (Halbtrockenrasen, Feucht- und Nasswiesen, Säume). Ein zweiter Indikator wäre nach dieser Untersuchung die Vielfalt der Flächennutzung, die sich quantitativ nach Anzahl und Flächengröße der landwirtschaftlichen Kulturen bemessen und über einen Diversitätsindex errechnen lässt.

Im vorliegenden Verfahren sind die Teilbereiche Arten- und Biotopschutz bereits durch andere Indikatoren weitgehend abgedeckt. Nun soll die Diversität der Nutzungen betrachtet werden. Dabei sind die Flächenanteile und die Größe der Landnutzungseinheiten zu unterscheiden. Im Indikator Landnutzungsverhältnis sollen die Anteile einzelner Nutzungsarten erfasst, die Veränderung aufgezeigt und im Sinne des regionalen Leitbildes oder ähnlicher interdisziplinärer Planungsvorgaben evaluiert werden.

Dazu bedarf es einer möglichst genauen Erfassung der aktuellen Situation. Zur Erstellung von Landschaftsplänen werden solche Daten häufig gewonnen. Mit Hilfe geografischer Informationssysteme ist dies, zum Teil auch halbautomatisch, schnell und mit ausreichender Genauigkeit möglich. Das novellierte Bundesnaturschutzgesetz sieht eine Erstellung von Landschaftsplänen mit entsprechenden Daten und Aussagen flächendeckend vor. Für die Zukunft ergibt sich daraus eine wertvolle Datenquelle für das hier vorgestellte Evaluationsverfahren. Neben der Ist-Situation bedarf es aber auch regionaler Zielvorgaben, die die angestrebte Entwicklung in Landnutzungsanteilen beschreiben. Solche Zielangaben integrieren ökologische, ökonomische, aber auch soziale Ansprüche an die räumliche Entwicklung. Idealerweise ergeben sie sich aus einer konsensorientierten, interdisziplinären Regional-/Raumplanung unter Beteiligung aller betroffenen Nutzergruppen.

\section{Ableitung des Indikators aus der Matrix}

Präsenz und Umfang der Landnutzungsarten ergeben die Existenzgrundlage für die auf sie angewiesenen Tier- und Pflanzenarten. Die Art der Abhängigkeit von der Nutzungsart kann unterschiedlich sein. Entscheidend ist, dass die Nutzungsfläche zeitweise oder dauernd als Lebensraum, zur Brut, Aufzucht oder Nahrungssuche genutzt wird. Der Flächenanteil der Landnutzungsart wirkt determinierend auf die Größe der Population, da sie die Abundanz innerhalb der Arten und damit auch die Möglichkeit des Gen-Austausches, den Umfang des genetischen Pools und die notwendigen Minimal-Areale zum Überleben einer Population beeinflussen oder bedienen. Das Neben- und Miteinander der diversen Nutzungen bestimmt darüber hinaus das Landschaftsbild im engeren Sinne, wie oben bereits erwähnt und auch aus der Verflechtungsmatrix (Tab. 3.3-2) ersichtlich. Auf die Bestimmung der Flächengröße, die natürlich auch ein Merkmal für die Nutzungstypenkonkurrenz und die damit zusammen hängenden Systemelemente Gen-Pool, Minimum-Areal und genetischer Austausch ist, wird verzichtet, da die Flächengrößen historisch bedingt und somit regional geprägt sind. Außerdem nimmt eine einzelne Aufforstung keinen nennenswerten Einfluss auf die durchschnittliche Flächengröße. 


\section{Parameter}

Der optische Eindruck einer Landschaft wird sehr stark durch die Anteile der Landnutzungsarten geprägt. Für Binnenlandschaften kann eine Unterteilung in die Nutzungsarten Wald, Acker, Grünland, Obstbau, Brache, Wasser und Siedlung Aufschluss geben. Der Wald steht nur einseitig in Nutzungskonkurrenz zu Wasser und Siedlung, da er von diesen verdrängt werden kann, sie aber kaum ersetzen wird. Diese beiden Landnutzungen werden deshalb nicht erfasst.

Für die anderen Nutzungsarten wird eine Zielaussage zur angestrebten Veränderung der Landnutzungsverhältnisse aus übergeordneten Raumplanungen übernommen. Dabei reicht eine Angabe der Entwicklungstendenz aus ${ }^{5}$. Benennen die Raumpläne keine derartigen Ziele, so kann behelfsweise auf Landschaftspläne und Grünordnungspläne zurück gegriffen werden, obwohl diese als Fachpläne keinen interdisziplinären Konsens darstellen. Liegt für eine Region eine genauere Zielaussage mit Angabe aktueller und angestrebter Landnutzungsanteile vor, so könnte das Bewertungsverfahren darauf abgestimmt werden. Diese Situation wird aber wohl eher eine Ausnahme darstellen.

Im Rahmen der Bewertung wird für jede Nutzungsart ermittelt, ob die geplante Aufforstung eine positive, neutrale oder negative Änderung der Anteile im Sinne des Entwicklungszieles (Differenz Soll-Ist-Wird) erbring $[$. Entspricht die tatsächliche Veränderung der angestrebten, so wird dies mit einem Punkt bewertet. Verhält sie sich neutral zu einer beabsichtigten Änderung, so wird kein Punkt vergeben. Letzteres ist beispielsweise der Fall, wenn eine Steigerung des Brachflächenanteiles angestrebt wird und nun ein Acker aufgeforstet werden soll. Gibt der Raumplan aber eine Erhaltung des Obstbauanteiles als Ziel an, so stabilisiert die gleiche Aufforstung diesen Anteil durch das neutrale Verhalten und wird ebenso mit einem Punkt berücksichtigt. Eine Veränderung der Landnutzungsanteile die dem im Plan festgestellten Entwicklungsziel entgegen läuft, wird als doppelt negativ betrachtet, da die Zielsetzung torpediert wird.

Der Soll-Ist-Wird-Vergleich zeigt somit den Beitrag der Maßnahme zur Zielerreichung an. Er wird summarisch ermittelt (vgl. Anhang 1).

\subsubsection{Randlinien-Vorkommen}

\section{Definition}

Das Randlinien-Vorkommen bezeichnet die quantitative Präsenz von Übergangszonen (Ökotonen) zwischen unterschiedlichen Landnutzungsarten bzw. Biotopen. Für die vorliegende Arbeit werden die Nutzungsarten zusammengefasst. Eine Unterscheidung zwischen einzelnen Feldfrüchten oder Waldtypen findet nicht statt. Auch eine Reihung der vorkommenden Ökotontypen unterbleibt (s.u.).

Die raumgliedernde Funktion einer Fläche ergibt sich aus deren Beitrag zur Strukturierung des lokalen Umfeldes, im ästhetischen wie im ökologischen Sinne. Diese Gliederung kann sanft oder eher abrupt erfolgen. Eine sanfte Gliederung erfolgt durch einen allmählichen Wechsel von einem Ökosystem zum anderen, z.B. durch einen breiten, gut strukturierten Waldrand, oder durch einen Wechsel zwischen zwei Biotoptypen mit ähnlichem Arteninventar, z.B. Trockenrasen und Feldhecke.

\footnotetext{
${ }^{5}$ Ein Beispiel für eine solche Aussage: „Förderung des Bewaldungsanteils und der Brachflächen bei gleichzeitiger Verringerung der Ackerfläche und des Intensivgrünlandes“. Damit ergibt sich folgende, angestrebte Entwicklungstendenz: Wald (+), Brache (+), Acker (-), Grünland (-), Obstbau (=)

6 Tatsächliche Veränderung bei Aufforstung einer Ackerfläche: Wald (+), Brache (=), Acker $(-)$, Grünland (-), Obstbau (=)

${ }^{7}$ Bewertung des Beispielfalles: bei Wald, Acker und Obstbaufläche stimmt die Entwicklung mit dem Ziel überein $\rightarrow$ je 1 Punkt; bei Brachfläche und Grünlandanteil verhält sich die Umwandlungsmaßnahme neutral zum vorgegebenen Ziel
} 
Eine abrupte Gliederung erfolgt durch aneinander stoßen intensiv genutzter Flächen unterschiedlicher Art oder durch Zerschneidung von Flächen durch Verkehrslinien, Siedlungen oder ähnliches.

\section{Bedeutung der Randlinien für den Naturschutz}

Neben den quantitativen Anteilen der Landnutzungsformen Wald, Feld, Grünland, Obstbau, usw. kommt auch der Mischungsform und der Gestaltung der Übergänge eine wichtige Bedeutung zu. Folgen die Grenzen der Nutzungstypen den natürlich vorgegebenen Strukturen, etwa Wasserläufen oder dem Relief, so wirkt sich dies positiv auf die ästhetische Wirkung der Landschaft aus. Künstliche „Ordnung“, durch geometrische Formen, Monokulturen, Wildschutzzäune und andere Expressiva der flurordnerischen, anthropogenen Einflussnahme stören häufig das Erscheinungsbild (ELSAßER 1991). Bei der Anlage neuer Wälder kann dem durch entsprechende Waldrandgestaltung, bezogen auf Zusammensetzung und Form, Rechnung getragen werden.

An der Grenze zwischen zwei Biotopen treffen unterschiedliche Reviere aufeinander. Dadurch ist die Artenvielfalt im Grenzbereich besonders hoch. Wenn es einen echten Übergangsbereich zwischen den benachbarten Ökosystemen gibt, also zum Beispiel einen breiten und strukturierten Waldrand als Übergang von einer Grünlandfläche zum Bestand, so können wir für diesen Bereich drei unterschiedliche Nutzertypen (Abb. 3.5-VI) unterscheiden (vgl. COCH 1995, HUNTER 1990).

1. Tiere die beide angrenzenden Biotope im Sinne eines Biotopkomplexes nutzen

2. Tiere und Pflanzen die an die Bedingungen des Ökotons angepasst sind und dieses als Lebensraum nutzen.

3. Arten, die eigentlich in einem der angrenzenden Ökosysteme wohnen, aber mit den Bedingungen im Übergangsbereich zurecht kommen und dort ebenfalls vorkommen

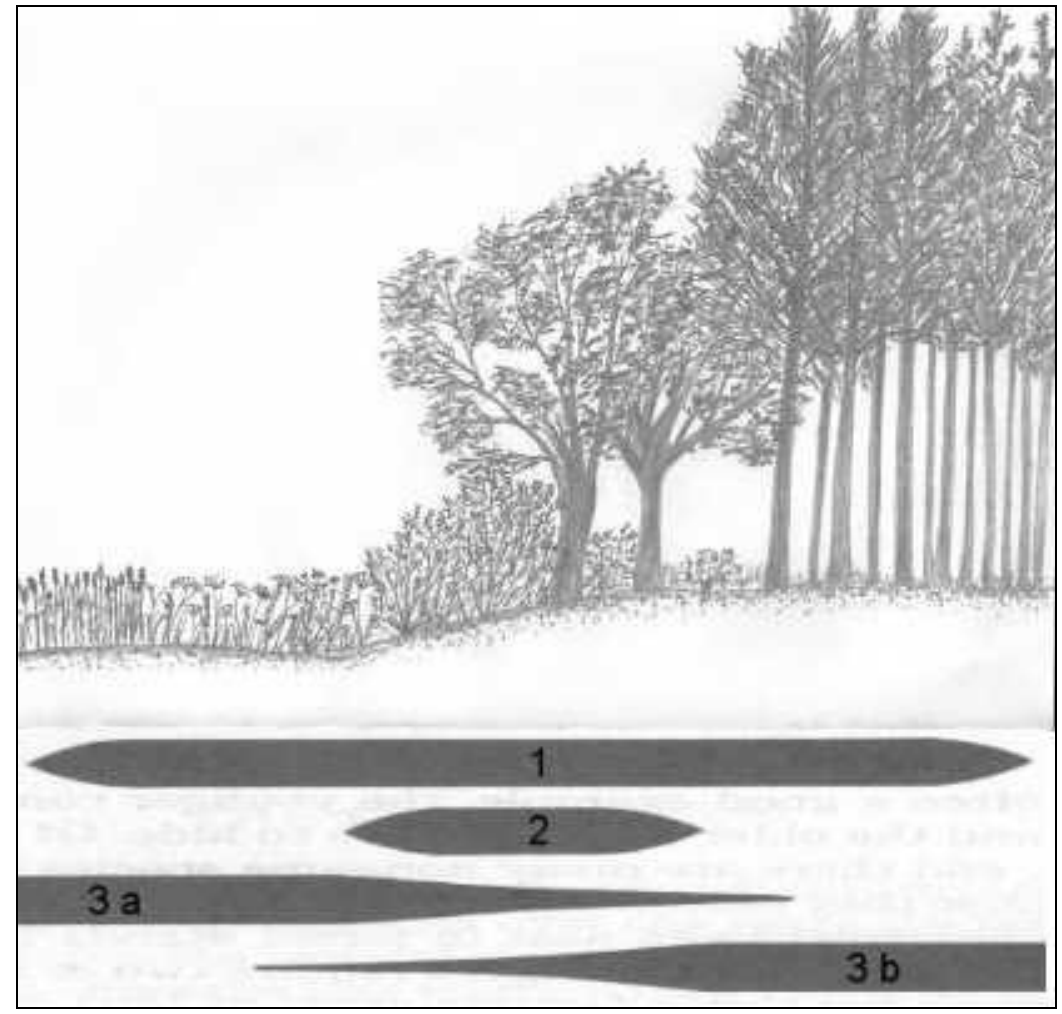

Abb. 3.5-Vl: Die Nutzung des Waldrandes als Lebensraum durch Tierarten des Offenlandes, des Waldes und der Übergangsbereiche; Gruppe 1: Wald, Feld und Waldrand; Gruppe 2: nur Waldrand; Gruppe 3: Waldrand und (a) Feld oder (b) Wald 
Der Randlinieneffekt (edge effect) bezeichnet die Verdichtung von einzelnen Revieren an der Grenze zwischen zwei Nutzungstypen oder Biotopen. Aus ihr folgt eine höhere Artenzahl im Vergleich zum Kernbereich eines bestimmten Biotoptyps. Damit ist die Bedeutung des Randlinieneffektes für die Biodiversität, einem Hauptziel des Naturschutzes, hinreichend belegt. Zusätzliche Bedeutung erlangen die Grenzen als markante Geländelinien, die von vielen Tierarten zur Abgrenzung von Revieren genutzt werden. Eine höhere Anzahl von Grenzlinien ermöglicht daher, in Zusammenhang mit der entsprechenden Biotopvielfalt, eine kleinere Abgrenzung von Revieren und damit eine höhere Populationsdichte.

\section{Bestimmbarkeit des Indikators}

Der Indikator ist durch ein einfaches, quantitatives Verfahren zu bestimmen. Dazu werden die Grenzlinien in einem betrachteten Raum ausgemessen und die Veränderung der Grenzlinienhäufigkeit durch die Nutzungsänderung ausgewertet.

Eine qualitative Berücksichtigung der Grenzverläufe wäre nur dann möglich, wenn eine Reihung der Ökotone nach deren Bedeutung für den Naturschutz konsensfähig wäre. Von Seiten der Landschaftsästhetik ist diese Reihung nicht objektiv zu begründen. Seitens des Artenschutzes sind nur vereinzelte Untersuchungen mit besonderer Betrachtung weniger oder singulärer Arten verfügbar. Ist ein Obstbau-Wald-Ökoton wertvoller als ein BracheWald-Ökoton? Eine generell gültige Reihung der Übergänge ist nicht möglich. Nicht einmal innerhalb der Waldränder, als einer besonderen Art der Ökotone, könnte eine solche Reihung objektiv erfolgen ( $\mathrm{COCH}$ 1995). Maximal kann von einer generell gültigen Tendenz gesprochen werden, dass die süd- und westexponierten Ränder durch den Einfluss der Sonneneinstrahlung und teilweise auch des Wetters ein häufigerer Wechsel der Bedingungen und eine höhere Habitatdiversität entsteht (vgl. Kap. 3.5.5).

Letztlich kann nur eine quantitative Erhebung der Grenzlinienvielfalt und deren Veränderung durch die Aufforstung erfolgen. Die Maxime lautet, dass der Reichtum an Grenzen zum Wohle der landschaftlichen wie biologischen Vielfalt zu erhöhen ist. Dabei ist zu beachten, dass dies nicht immer für eine Aufforstung spricht. Bei Arrondierungen eines Waldbesitzes wird dies im Gegenteil meist zu einer Verringerung der Grenzlinien und damit zu einer Verschlechterung der Situation unter den oben genannten Gesichtspunkten führen. In waldreichen Gebieten wird ein ähnliches Phänomen auftreten, wenn die Aufforstungen auf Freiflächen stattfinden. Derartige Fälle führen $z u$ einer negativen Bewertung im besprochenen Indikator.

\section{Ableitung des Indikators aus der Matrix}

Abgesehen von den offensichtlichen Zusammenhängen zwischen der Situation der Biotopvernetzung und dem Auftreten von Randlinien, zeigt die Verflechtungsmatrix der zweiten Systemebene (Tab. 3.3-2) weitere Zusammenhänge in diesem Systembereich: Randlinien sind sowohl ein Ausdruck funktionaler Vernetzung als auch der Konkurrenz zwischen unterschiedlichen Nutzungsarten. Umgekehrt fördert eine besondere Gestaltung der Randlinien auch die Vernetzung, den Verbund oder die Nutzungstypenkonkurrenz. Dass diese Elemente Auswirkungen auf das Erscheinungsbild der Landschaft haben, liegt auf der Hand. Dies gilt insbesondere für die Waldränder (ZUNDEL 1994), deren Übergang ganzjährig und deutlicher in Erscheinung tritt, als der zwischen verschiedenen landwirtschaftlichen Nutzungsarten.

Die Auswirkungen der Randlinien und der Nutzungstypenkombination auf Artenvielfalt und Landschaftsbild wird in den Punktewolken RV1 und RV2 angedeutet.

\section{Parameter}

Die Unterbrechungs- oder Gliederungswirkung wird durch die Randlängen zwischen unterschiedlichen Nutzungsarten indiziert. Zur Feststellung der Randlängen werden die Nutzungsarten im Untersuchungsquadrat (2x2 km über dem Zentrum der Aufforstungsfläche) mit Hilfe von Luftbildern und im Gelände kartiert. Anschließend werden die Grenzlinien im geographischen Informationssystem (GIS: ArcView 3.2) digitalisiert und vermessen. Daraus 
ergibt sich die Randlänge vor der Aufforstung, getrennt nach Ökotontypen. Da es über die ökologische Wertigkeit der Ökotone im gegenseitigen Vergleich bisher keine stichhaltigen Untersuchungsergebnisse gibt, werden sie als gleichwertig behandelt. Eine qualitative Auswertung nach Ökotontypen nicht gerechtfertigt.

Dennoch wird die Grenzlänge getrennt für die Hauptnutzungstypen Wald, Acker, Grünland, Obstbau und Brache bestimmt. Dies erfolgt jedoch nur zur zusätzlichen Information. Anschließend wird die durchschnittliche Randlinienlänge pro Hektar für das Untersuchungsquadrat und für die Aufforstung selbst bestimmt. Der Vergleich zwischen beiden Werten dient nur der Illustration der Verhältnisse. Er fließt nicht in die Bewertung ein. Über das GIS lässt sich sodann die Veränderung der Randliniendichte (in Ifm./ha) durch den Nutzungswandel berechnen. Dieser Entwicklungswert wird ins Verhältnis zum Ist-Wert gesetzt. Daraus ergibt sich ein Steigerungsprozent, der nach dem unten stehenden Schema (Abb. 3.5-VII) in einen Wert umgesetzt wird. Dies bedeutet Veränderungen in randlinienarmen Bereichen werden weitaus stärker gewichtet als in randlinienreichen Gebieten. Wie beim Indikator Landnutzungsverhältnisse wird auch hier eine negative Veränderung der Situation durch Punktabzug berücksichtigt, wobei z.B. bei Arrondierungen ein negativer Indikatorwert ermittelt werden kann.

Die Waldrandanteile werden gesondert ausgewertet, allerdings lediglich für die Aufforstungsfläche selbst. Vorher existente Waldränder können durch eine vorgelagerte Aufforstung an Wert verlieren. Dies hängt von Form, Struktur und Exposition des neuen Waldrandes im Vergleich zum vorherigen ab. Form und Struktur wurden im Indikator Waldrandausprägung bereits erfasst und bewertet. Im hier beschriebenen Indikator geht es um die Erfassung der potentiellen Waldrandfunktion die sich aus Lage und Länge der neu geschaffenen Ränder ergibt. Die bisherigen Waldränder an der Aufforstungsfläche werden mit Hilfe des GIS gemessen und deren Orientierung festgestellt. Diagonal verlaufende Waldränder (SO-NW und NO-SW) werden jeweils hälftig den entsprechenden Expositionen zugeordnet.

Nach dieser Methode werden auch die neu geschaffenen Waldränder bestimmt und ins Verhältnis zu den vorherigen gesetzt. Im Sinne deutlicher Ergebnisse wird dann ein Bewertungsschema angewandt, welches mit den Multiplikatoren zwei und drei sowie den Teilern eine Hälfte und ein Drittel arbeitet (Abb. 3.5-VII). Die Zunahme von Waldrand wird bei entsprechender Deutlichkeit - belohnt, die Abnahme durch Punktabzug bestraft.

Da aus naturschutzfachlichen Gründen den süd- und west-exponierten Waldrändern eine höhere Wertigkeit zugesprochen wird (COCH 1995, ZUNDEL 1994), wichtet das hier angewandte Verfahren die entsprechenden Waldränder mit dem Faktor 2 zur Bestimmung der Gesamtbedeutung. Eine Vergleichsuntersuchung mit einheitlich gewichteten Waldrandlängen soll zeigen, ob die vorher genannte Vorgehensweise einen nennenswerten Effekt auf die Bewertung hat (vgl. Kap. 4.2.2). 


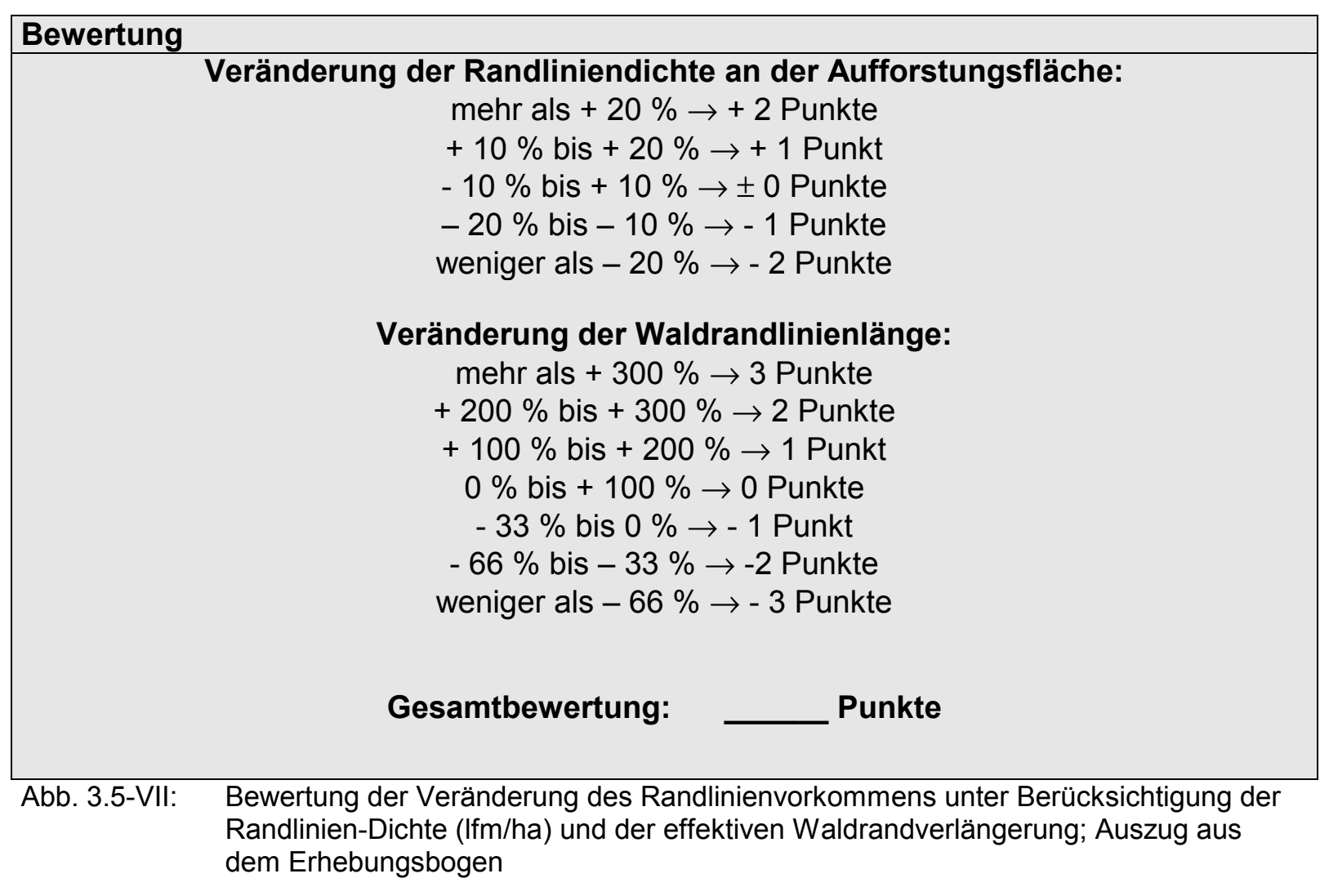




\section{Ergebnisse im USG 1 - Region Apolda (Thüringen)}

\subsection{Planungsgrundlagen}

\section{Lage und Landschaft}

Das Untersuchungsgebiet 1 befindet sich im Landkreis Weimarer Land und erstreckt sich vom Landschaftsschutzgebiet Schöten (südlich von Apolda) nach Osten bis zur Gemeinde Stobra und dann nach Norden bis zur Gemeinde Niedertreba. Der tiefste Punkt des Gebietes befindet sich mit etwa $145 \mathrm{~m}$ NN im Norden, in der Gemeinde Niedertreba. Der höchste Punkt liegt mit $280 \mathrm{~m} \mathrm{NN}$ in der Gemeinde Stobra, in der südöstlichen Ecke des Untersuchungsgebietes (USG).

Der betrachtete Landschaftsteil gehört nach der naturräumlichen Gliederung von HIEKEL et al. (1994) zum „Innerthüringer Ackerhügelland“. Entsprechend der Einteilung in Agrargebiete (TMLNU 1995) ist das Gebiet auch dem „Thüringer Keuper-Becken“ zuzuordnen. Es handelt sich um ein flachwelliges Hügelland, welches im NO und $\mathrm{O}$ von breiten, gefällearmen Niederungen durchzogen wird. Im Untersuchungsgebiet ist es die $\mathrm{IIm}$, die sich in das Hügelland einschneidet. Im Flusstal sind naturgemäß die niedrigsten topographischen Höhen zu messen. Für das Relief sind weiterhin die Seitentäler der IIm von Bedeutung. Innerhalb des USG sorgt beispielsweise der Utenbach für Geländeeinschnitte. Ökologisch sind diese Einschnitte von herausragender Bedeutung, da dort andere Landnutzungstypen und somit auch andere Biotoptypen vorherrschen, als auf den benachbarten, bis zu $30 \mathrm{~m}$ höher gelegenen Ackerflächen.

Das Innerthüringer Ackerhügelland wird optisch geprägt durch die Dominanz der landwirtschaftlichen Bewirtschaftung. Der Bewaldungsanteil liegt unter $5 \%$. Die Grünlandnutzung spielt mit weniger als $12 \%$ eine untergeordnete, wenn auch nennenswerte Rolle. Prägend für das Landschaftsbild sind neben dem geologischen Relief, den Alleen und linienförmigen Gehölzen auch die teils großflächigen Obstbau-Anlagen. Letztere wurden einst als Streuobstwiesen angelegt und bewirtschaftet, werden heute jedoch zunehmend durch Niederstamm-Anlagen verdrängt oder wandeln sich im Rahmen der Verbuschung und Sukzession allmählich in waldartige Bestände um (TLL 1997).

Nach der forstlichen Einteilung gehört das USG zum Wuchsgebiet Thüringer Becken, genauer zum Wuchsbezirk 4.3.0 Thüringer Keuper-Becken (LAWUF 1997). Hier finden sich heute nur noch geringe Restwaldflächen. Größere, zusammenhängende Waldungen gibt es in diesem Wuchsbezirk kaum. Der nächstgelegene Waldkomplex ist mit ca. 100 ha der Wald am Galgenberg in Dornburg. Er liegt ca. 2700 m südöstlich des Untersuchungsgebietes und gehört bereits zum Nachbarwuchsbezirk IIm-Saale-Platten. Der Wuchsbezirk Thüringer Keuper-Becken besitzt einen Waldanteil von 4,4 \% (LAWUF 1997). Die landschaftlichen Verhältnisse innerhalb des USG, spiegeln die Verhältnisse im übrigen Wuchsbezirk sehr gut wieder, obwohl es geographisch an dessen südöstlichem Rand gelegen ist.

\section{Klima}

Das Klima im USG ist wie im forstlichen Wuchsbezirk kontinental geprägt. Die Jahresdurchschnittstemperatur liegt bei $8,1^{\circ} \mathrm{C}$. Während der Vegetationsperiode wird eine durchschnittliche Temperatur von $14,9{ }^{\circ} \mathrm{C}$ erreicht. Im Juli steigt die durchschnittliche monatliche Temperatur auf $17^{\circ} \mathrm{C}$. Auch der geringe Winter-Niederschlag mit $207 \mathrm{~mm}$ (dies entspricht $38 \%$ des Jahresniederschlages) betont den kontinentalen Charakter des Makroklimas. 
Die Kombination von Niederschlagsarmut und Temperaturbegünstigung ergibt ein vorteilhaftes Klima für die Landwirtschaft. Auf sonnigen, südexponierten Hängen ist sogar Weinanbau möglich.

\section{Geologie und Böden}

Das geologische Ausgangsgestein wird im gesamten Naturraum hauptsächlich von den Ablagerungen des Unteren und Mittleren Keupers gebildet. Innerhalb der Folgen treten zusätzlich in ständigem Wechsel Mergel, Sandstein und Gips auf. In den Flusstälern steht zum Teil auch der Obere Muschelkalk an. Das gesamte Gebiet ist von Löß bedeckt oder zumindest beeinflusst. Die Lößschicht kann mehrere Meter mächtig sein.

Bedingt durch das karbonatreiche Ausgangsmaterial herrschen im Keuperbecken Fahlerden, Parabraunerden und Schwarzerden vor. Im Untersuchungsgebiet lässt sich der dominierende Bodentyp für die landwirtschaftlichen Flächen anhand der Bodenkarte (Mittelmaßstäbige landwirtschaftliche Bodenkartierung, AKAD. DER LANDWIRTSCHAFTSWISSENSCHAFTEN DER DDR, 1978) weiter eingrenzen. Demnach dominiert im gehobenen Gelände die Löss- und Lösskerf-Grieserde mit -Schwarzerde, z.T. mit Lösstieflehm. An den Unterhängen, noch im vernässungsfreien Bereich, schließen sich Berglehm- und Bergsandlehm-Rendzina und -Braunerde an. Im grundwasserbeeinflussten Bereich der Bachtäler sind laut landwirtschaftlicher Standortskartierung Auenschluff-Vega, z.T. Auenlehm zu suchen.

Die forstliche Standortskartierung liegt nur für die geringen Flächenanteile vor, auf denen bereits heute Wald stockt. Für diese Flächen widerspricht sie allerdings der landwirtschaftlichen Standortskartierung. Frische Bachtälchenstandorte kommen laut Standortseinheitenkarte (LAWUF 2000) nur selten vor. In den Taleinschnitten findet man dagegen häufig nährstoffreiche Karbonatgesteinsstandorte, die nicht vernässt sind. Hangaufwärts gehen diese Bodentypen in mäßig frische und schließlich mäßig trockene Schlufflehmstandorte über, zeigen also zunehmende Beeinflussung durch die Löss- und Lösslehmüberdeckung. Die Differenz in den Bodenkartierungen landwirtschaftlicher und forstwirtschaftlicher Prägung dürfte zumindest teilweise auf die unterschiedlichen Maßstäbe der Karten zurückzuführen sein. Während die forstliche Standortseinheitenkarte im Maßstab 1:10.000 vorliegt, ergibt die mittelmaßstäbige landwirtschaftliche Bodenkartierung (1: 100.000) ein wesentlich groberes Bild.

\section{Natürliche Waldgesellschaften}

In der Beschreibung des Wuchsbezirkes wird die natürliche Waldgesellschaft des terrestrischen Bereiches als Eichenwald beschrieben. Flächenmäßig ist der LabkrautEichen-Hainbuchenwald (Galio-Carpinetum) dominant, der z.T. einen hohen Lindenanteil besitzt. Ein anderes Bild ist in den Niederungen und Flußauen zu erwarten, mit EichenEschen-Ulmen-Auenwäldern (Querco-Ulmetum) und auf nasseren Standorten Traubenkirschen-Erlen-Eschenwäldern (Pruno-Fraxinetum).

Ein Vergleich der somit definierten potentiellen natürlichen Vegetation (PNV) mit den Ergebnissen der Waldbiotopkartierung ergibt ein stimmiges Bild. Als natürliche Waldgesellschaften wurden auf Bachtälchenstandorten mit alluvialen Schwemmlehmen innerhalb des USG lediglich Ahorn-Eschenwälder ausgewiesen. Wo alluviale Schwemmlehme fehlen sowie an Oberhängen, im Karbonatgesteinsboden oder auf Schlufflehmstandorten weist die Kartierung „Eichen-Hainbuchenwald auf eutrophen frischen bis mäßig trockenen Standorten" aus. Nasse und sehr feuchte Standorte, auf welchen die Roterlen-Eschenwälder zu erwarten sind, liegen laut forstlicher Standortseinheitenkarte im USG nicht vor.

Eine weitere Verifikation der PNV ist durch Anwendung des Stamm-StandortsgruppenSchlüssels möglich, welcher als Bestandteil der Waldbiotopkartierung in Thüringen jedem 
möglichen Standortstyp die entsprechend der potentiell natürlichen Vegetation zu erwartenden Baumarten zuweist (Anhang 3). Danach wäre wiederum in den Bachtälchen mit Eschenwald zu rechnen, angereichert mit Roterle und Stieleiche.

Diese Quelle ordnet den Karbonatgesteins- und Schlufflehmstandorten den edellaubbaumreichen Linden-Eichen-Hainbuchenwald zu. Es ergibt sich ein einfacher Schlüssel zur Ermittlung der PNV auf der Grundlage der landwirtschaftlichen und forstwirtschaftlichen Bodenkartierung, die durch die Waldbiotopkartierung und deren Verfahrenselemente gestützt wird (Tab. 4.1-1).

\section{Heutige Nutzung}

Die Region Niedertreba gehört zum traditionellen Ackerbaugebiet des Thüringer Beckens. Bereits um 1900 war der Wald weitgehend zurück gedrängt. Der Ackerflächenanteil entsprach etwa den heutigen Verhältnissen. Lediglich in den Auenbereichen, erfolgte seither eine weitere Umwandlung von Wald zu landwirtschaftlicher Fläche. Die Ackerparzellierung wurde in den vergangenen Jahrzehnten den Bewirtschaftungsformen angepasst. Schlagform und -größe bieten in aller Regel günstige Voraussetzungen für den Einsatz von Großtechnik. Die mittlere Feldgröße liegt bei etwa 16 ha (TLL 1997). Diese steht allerdings nicht in direktem Zusammenhang mit den Eigentumsverhältnissen an den Flächen. Für die Agrargenossenschaft „Ilmtal“ liegt die mittlere Flächengröße je Grundstückseigentümer bei lediglich 0,8 ha Ackerland. Die Bewirtschaftung erfolgt zumindest innerhalb des Untersuchungsraumes häufig durch Großbetriebe. Der Agrarraumnutzungs- und -pflegeplan (ANP) für die Region Niedertreba (TLL 1997), im Folgenden kurz „ANP Niedertreba“ genannt, gibt an, dass die drei größten Betriebe zusammen etwa zwei Drittel der gesamten landwirtschaftlichen Nutzfläche bewirtschaften. Im Gebiet ist ein recht engmaschiges Wegenetz vorhanden.

Getreide und Futterfrüchte stellen die Hauptanbaukulturen im Untersuchungsraum. Sonderkulturen spielen keine nennenswerte Rolle. Das Weinbaugebiet „IIm-Saale“ liegt mit seinen knapp 12 Hektar Rebfläche (dies entspricht ca. $88 \%$ des Thüringer Weinanbaus) nordöstlich des Untersuchungsgebietes bei Bad Sulza und Kaatschen.

Obwohl die Rebfläche um knapp 130\% auf 26 Hektar ausgebaut werden soll (TLL 1997), ergibt sich daraus keine Flächenkonkurrenz innerhalb des Untersuchungsgebietes.

Die Gründlandnutzung ist derzeit in weiterem Rückgang begriffen. Die landwirtschaftliche Tierhaltung wird ebenfalls weitgehend von den oben angesprochenen Großbetrieben abgedeckt. Diese stellten den Betrieb bereits in den vergangenen Jahren weitgehend auf ganzjährige Stallhaltung um, weshalb die Weiterführung der bislang extensiven Weidewirtschaft des Auengrünlandes in Frage gestellt ist. Den Flächen droht großteils die Verbuschung und Wiederbewaldung. Innerhalb des USG ist davon vor allem das Landschaftsschutzgebiet Utenbacher Schweiz (Flächen AP-3 und AP-4) betroffen. Hier sind mehrere offen gelassene Weideflächen mit älterem Streuobstbestand vorhanden. Eine Intensivweide mit 4,71 ha und eine Schafweide mit 6,66 ha liegen ebenfalls innerhalb dieses Biotopkomplexes. Darüber hinaus findet sich am Oberhang des Pfuhlgrabens ein weiteres Grünland mit ca. 3 ha Fläche, welches ebenfalls sich selbst überlassen wird und der Sukzession anheim fällt.

In Bezug auf den Wald ist festzustellen, dass einige Restwaldflächen noch in naturnahem Zustand vorhanden sind. Teilweise sind aber auch standortsfremde Anbauten, vor allem mit Nadelholz erfolgt. In den Jahren um 1950/60 wurden einige Aufforstungen mit Pappeln in den Auen und Niederungen getätigt. Die Mischung der hier vorzufindenden Baumarten ist sehr groß und beschränkt sich nicht auf heimische Arten. Bereits die Wuchsgebietsbeschreibung durch die Landesanstalt für Wald und ForstwirtschafT (LAWUF 1997) weist auf diesen unbefriedigenden Zustand hin und propagiert den Waldumbau in den Niederungen mit Edellaubhölzern, Stieleiche und Roterle. 
Tab. 4.1-1: Übersicht zu den vorkommenden Bodentypen im Untersuchungsgebiet und den zugehörigen natürlichen Waldgesellschaften

\begin{tabular}{|c|c|c|c|}
\hline $\begin{array}{l}\text { Landwirtschaft- } \\
\text { liche Standorts- } \\
\text { kartierung })^{1}\end{array}$ & $\begin{array}{c}\text { Forstliche } \\
\text { Standortskartierung ) }\end{array}$ & $\begin{array}{c}\text { NWG, ausgewiesen im USG } \\
\text { durch die } \\
\text { Waldbiotopkartierung })^{3}\end{array}$ & $\begin{array}{l}\text { NWG nach } \\
\text { Stamm- } \\
\text { Standorts- } \\
\text { gruppe })^{4}\end{array}$ \\
\hline $\begin{array}{l}\text { Lö1C1 } \\
\text { „Löß- und } \\
\text { Lößkerf-Griserde } \\
\text { mit } \\
\text {-Schwarzerde, } \\
\text { z.T. mit } \\
\text { Lößtieflehm; } \\
\text { vorw. } \\
\text { vernässungsfrei“ }\end{array}$ & $\begin{aligned} \text { RLL3 }= & \text { reicherer Schlufflehm } \\
& \text { (Lößlehm und Löß) mäßig } \\
& \text { trocken) }\end{aligned}$ & \multirow{8}{*}{$\begin{array}{l}\text { N202 } \\
\text { Eichen-Hainbuchenwald auf } \\
\text { eutrophen frischen bis mäßig } \\
\text { trockenen Standorten } \\
\text { Standorte: Reiche und kräftige } \\
\text { vernässungsfreie Kalk-, } \\
\text { Lößlehm, Lehm-, Ton-, Sand- } \\
\text { oder seltener auch Basalt- und } \\
\text { Diabasstandorte mit Mull bis } \\
\text { mullartigem Moder als } \\
\text { Humusformen }\end{array}$} & \multirow{8}{*}{$\begin{array}{l}\text { edellaubbaum- } \\
\text { reicher Linden } \\
\text { Eichen- } \\
\text { Hainbuchen- } \\
\text { wald }\end{array}$} \\
\hline \multirow{4}{*}{$\begin{array}{l}\text { V2a4 } \\
\text { "Berglehm- und } \\
\text { Bersandlehm- } \\
\text { Rendzina und } \\
\text {-Parabraunerde; } \\
\text { vernässungsfrei“" }\end{array}$} & $\begin{aligned} \text { RLL1 } & \text { reicherer Schlufflehm, } \\
& \text { sehr frisch bis frisch }\end{aligned}$ & & \\
\hline & $\begin{aligned} \text { RLL2 } & =\text { reicherer Schlufflehm, } \\
& \text { mäßig frisch) }\end{aligned}$ & & \\
\hline & $\begin{array}{ll}\text { RT2 } & \begin{array}{l}\text { = mäßig frischer, reicherer } \\
\text { Tonstandort }\end{array} \\
\end{array}$ & & \\
\hline & $\begin{array}{l}=\text { mäßig trockener, } \\
\text { reicherer Tonstandort }\end{array}$ & & \\
\hline \multirow{7}{*}{$\begin{array}{l}\text { Al3a } \\
\text { "Auenschluffvega } \\
\text { und -vegagley; } \\
\text { tw. Grundwasser } \\
\text { anstehend" }\end{array}$} & 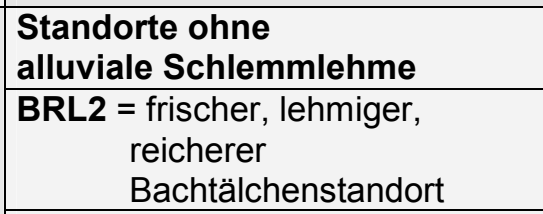 & & \\
\hline & $\begin{array}{c}\text { BRL3 = frischer, lehmiger, } \\
\text { reicherer } \\
\text { Bachtälchenstandort }\end{array}$ & & \\
\hline & $\begin{array}{|ll|}\text { RC2 } & \begin{array}{l}\text { = reicheres } \\
\text { Karbonatgestein, mäßig } \\
\text { frisch }\end{array} \\
\end{array}$ & & \\
\hline & $\begin{array}{l}\text { Standorte mit } \\
\text { alluvialen Schlemmlehmen }\end{array}$ & \multirow{4}{*}{$\begin{array}{l}\text { N704 Ahorn-Eschen-Wald in } \\
\text { Trockentälern, } \\
\text { Schwemmmulden und } \\
\text { grundwasserferneren } \\
\text { Bachauen; kolliner bis } \\
\text { submontaner Bereich } \\
\text { Standorte: Reichere Standorte } \\
\text { in grundwasserbeeinflussten } \\
\text { Schwemmmulden und } \\
\text { Trockentälern sowie } \\
\text { Bachauen mit tief } \\
\text { eingeschnittenem Bachlauf mit } \\
\text { überwiegend alluvialen } \\
\text { Schwemmlehmen } \\
\end{array}$} & \multirow{4}{*}{$\begin{array}{l}\text { stieleichen- } \\
\text { reicher } \\
\text { (Roterlen-) } \\
\text { Eschenwald }\end{array}$} \\
\hline & $\begin{aligned} \text { BRL2 } & \text { = frischer, lehmiger, } \\
& \text { reicherer } \\
& \text { Bachtälchenstandort }\end{aligned}$ & & \\
\hline & $\begin{aligned} \text { BRL3 = frischer, lehmiger, } \\
\text { reicherer } \\
\text { Bachtälchenstandort }\end{aligned}$ & & \\
\hline & $\begin{array}{l}=\text { reicheres } \\
\text { Karbonatgestein, mäßig } \\
\text { frisch }\end{array}$ & & \\
\hline
\end{tabular}

Quellen:

)$^{1}$ Akademie der Landwirtschaftswissenschaften der DDR, 1978; Mittelmaßstäbige landwirtschaftliche Standortkartierung - GERA, Blatt 49; Maßstab 1:100.000

)$^{2}$ Landesanstalt für Wald und Forstwirtschaft, Gotha, 2000; Standortseinheitenkarten 4935 NW, NO, SW, SO; Maßstab 1:10.000

)$^{3}$ Landesanstalt für Wald und Forstwirtschaft, Gotha, 2000; Waldbiotopkartierung im Forstbezirk Bad Berka

)$^{4}$ Landesanstalt für Wald und Forstwirtschaft, Gotha, 1997; Stamm-Standortsgruppen und Baumarten potentiellnatürlicher Wälder auf der Basis von Stamm-Vegetationsformen im forstlichen Wuchsgebiet Thüringer Becken 
Wo noch natürliche Eichenwälder vorkommen, weisen sie Spuren der früheren Mittelwaldbewirtschaftung auf. Obwohl diese Wälder unter naturschutzfachlichen Gesichtspunkten besonders wertvoll sind, sucht die Landesforstverwaltung nach einer ökonomisch tragfähigeren Nutzungsalternative. Sie schlägt die Verjüngung der Eichenwälder über Kernwüchse vor. Das Produktionsziel sind Eichen-Wertholz-Bestände mit Beimischung weiterer, ökologisch wie ökonomisch wertvoller Baumarten, beispielsweise Esche und Feldulme (LAWUF 1997).

Nach Auskunft des Landwirtschaftsamtes Umpferstedt werden 91,4 \% der derzeit landwirtschaftlich kultivierten Fläche (ca. 60.000 ha) im Landkreis Weimarer Land als Ackerland genutzt. Sonderkulturen spielen mit einem Umfang von weniger als 100 ha keine nennenswerte Rolle. 8,3 \% der landwirtschaftlichen Fläche bestehen aus Grünland. Für die Zukunft ist eine Stillegung von 10 \% der Flächen geplant (RITSCHEL, briefliche Mitteilung im März 2002).

Die aktuellen Landschaftspflegepläne für das USG (DANE 1999, JANETZ 1998, BETTINGER 1995) geben leider keine näheren Informationen zur aktuellen Verteilung der Flächennutzungen. Lediglich die nächst höhere Ebene, der Regionale Raumordnungsplan Mittelthüringen macht wieder entsprechende Angaben, demzufolge das Innerthüringer Ackerhügelland mit $95 \%$ landwirtschaftlicher Nutzung einseitig geprägt ist. Der Grünlandanteil liegt mit $5 \%$ unterhalb demjenigen, der für die ANP-Region angegeben wird (5,57 \%; TLL 1997). Aus pragmatischen Gründen wird die Flächennutzungsangabe des ANP Niedertreba für das vorliegende Verfahren verwendet. Dazu zwingt die planungstechnisch ungünstige Lage des Untersuchungsgebietes, dessen fünf Bezugsräume in die Planungsgebiete von drei unterschiedlichen Landschaftsplänen fallen. Auch im übergeordneten Planungsraum des Raumordnungsplanes nimmt das USG eine Randstellung im Teilbereich Innerthüringer Ackerland ein. Die Verhältnisse des USG werden daher durch den ANP Niedertreba ausreichend genau wiedergegeben. Außerdem ergänzt dieser die Planungsvorgaben durch Angaben zu propagierten Veränderungen in der Flächennutzung (s.u., Abschnitt „Vorhandene Planungsgrundlagen“).

Auf Grund der Daten des ANP Niedertreba (TLL 1997) ergeben sich folgende Flächennutzungsanteile (näherungsweise bestimmt):

Tab. 4.1-2 Flächennutzungsanteile in der Region Apolda / Niedertreba, berechnet auf der Grundlage des Agrarraumnutzungsplanes Niedertreba (TLL 1997)

\begin{tabular}{|c|c|c|}
\hline \multirow{2}{*}{$\begin{array}{l}\text { Nutzungs- } \\
\text { form }\end{array}$} & \multicolumn{2}{|c|}{ Ist } \\
\hline & ha & $\%$ \\
\hline Wald & 337,30 & 5,57 \\
\hline Acker & 4088,80 & 56,12 \\
\hline Grünland & 451,30 & 6,32 \\
\hline Obstbau & 148,80 & 2,48 \\
\hline Brache & & \\
\hline
\end{tabular}

\section{Landschaftstypen}

Die Einordnung des USG Apolda in die von FLADE (1994; Anhang 2) vorgegebenen Landschaftstypen erfolgt im vierstufigen Verfahren. Dabei steht zunächst die Feststellung vorhandener Strukturen im Vordergrund. Dies erfolgt durch Beurteilung im Gelände, Auswertung von verbalen und quantitativen Gebietsbeschreibungen und schließlich unter Zuhilfenahme von Luftbildern. Anschließend soll eine Verifikation anhand der zugehörigen avifaunistischen Leitarten Sicherheit bringen.

Zur Verifizierung der Landschaftstypen bedarf es der Informationen zu nicht besonders geschützten oder seltenen Arten, da sich Leitarten über die Stetigkeit im entsprechenden Landschaftstyp definieren, nicht über Rote-Liste-Kriterien. Die stichhaltigste Überprüfung der Populationen ergäbe sich aus gezielten Taxierungen der Vogelpopulationen im 
Untersuchungsgebiet, eventuell beschränkt auf die Leitarten der vorhandenen oder potentiell entwickelbaren Landschaftstypen. Aus zeitlichen Gründen, war diese Möglichkeit im Rahmen der vorliegenden Arbeit nicht realisierbar. Solche Aufnahmen könnten auch leicht den Rahmen eines Genehmigungsverfahrens zu einem Aufforstungsantrag sprengen. Daher soll auf andere Quellen ausgewichen werden.

Die Auswertung der für das USG relevanten Landschaftspläne, gibt hinsichtlich der benötigten Aussagen zur Populationssituation der Vogelarten ein unterschiedliches Bild. Von den Bearbeitern der Pläne wurden jeweils verschiedene Quellen ausgewertet. Hierzu gehören behördliche Unterlagen (z.B. Arten- und Biotopschutzkonzept Mittelthüringen) ebenso wie die Aufzeichnungen ehrenamtlicher Kartierer. Dabei konzentrieren sich die Landschaftspläne Ost-Abschnitt des Kreises Weimarer Land und Mellingen-Apolda auf die geschützten und gefährdeten Vogelarten. Der Landschaftsplan Unteres IImtal berücksichtigt dagegen, unter Berufung auf Auskünfte der Fachgruppe Ornithologie und Vogelschutz, Apolda und sonstigen Gebietskennern eine Reihe weiterer, weniger "privilegierter“ Vogelarten.

Eine persönliche Anfrage bei der Fachgruppe Ornithologie und Vogelschutz in Apolda ergab, dass der augenblickliche Kenntnisstand über die gebietstypische Avifauna nicht ausreichend detailliert ist, um die Populationen einschätzen zu können. Diese Situation lässt sich vermutlich mit der Arbeitsweise dieser ehrenamtlichen Ornithologiegruppe erklären, deren Mitglieder sich über Zufallsbeobachtungen regelmäßig austauschen, jedoch keine systematischen Kartierungen durchführen. Im Mittelpunkt des Interesses stehen besonders seltene oder besonders auffällige sowie durchziehende Vogelarten. Die Aussagekraft solcher Beobachtungslisten ist für das hier angewandte Verfahren aber sehr eingeschränkt.

Konkrete Aussagen zur Bestandessituation der gebietstypischen Vogelarten waren aber von den ornithologisch bewanderten und ortskundigen Mitarbeitern der unteren Naturschutzbehörde (Herr Weise, Umweltamt Kreis Weimarer Land, Apolda; mdl., 2001) zu erhalten. Zwar beruhen auch diese Aussagen weitgehend auf Zufallsbeobachtungen und nicht auf systematischen Untersuchungen, jedoch konnten sie das von dem Landschaftsplan Unteres Ilmtal skizzierte Bild auf gleichem Niveau vervollständigen. Hierzu gehört die Einschätzung der Vorkommen in die Kategorien a) Nachweis ohne Bruthinweis, b) Brutverdacht, c) Brutnachweis.

Die Auswertung der Landschaftspläne und die mündlichen Aussagen der Behördenmitarbeiter ergab eine Datengrundlage (Anhang 2 a), die keine grundlegenden Aussagen über das vorhandene, avifaunistische Arteninventar zulässt, die jedoch für eine Bestätigung der vorhandenen und entwickelbaren Landschaftstypen ausreicht.

Das Landschaftsbild des Untersuchungsgebietes wird dominiert von landwirtschaftlicher Nutzfläche, insbesondere vom Ackerbau. Grünland spielt eine untergeordnete Rolle, großflächige Wälder fehlen; sonstige Gehölze nehmen ebenfalls nur geringe Flächen ein. In manchen Bereichen entsteht eine offene Landschaft mit wenigen, aber doch vorhandenen Strukturelementen. Mit einem Gehölzanteil von knapp über $5 \%$ (Tab. 4.1-2) entspricht das Untersuchungsgebiet formal nicht vollständig dem Landschaftstyp (LT) gehölzarme Felder, bewegt sich derzeit aber noch im Grenzbereich. Da das regionale Leitbild ebenso wie der ANP Niedertreba eine Erhöhung des Gehölzanteiles vorsieht, scheinen die Charaktermerkmale dieses Landschaftstyps nicht als Evaluationsmerkmale geeignet. Die Leitarten Wachtel und Grauammer kommen im USG wohl vor, es fehlt aber die Großtrappe. Sie benötigt besonders weitläufige Landschaften mit unbegrenztem Horizont. Diese Bedingungen liegen hier aber nicht vor. Eine Entwicklung der Landschaft in Richtung dieses Lebensraumtyps würde eine Reduktion der vorhandenen Gliederungselemente verlangen und entspräche nicht dem regionalen Leitbild. Da das Arteninventar für diesen Lebensraum nicht vorhanden ist, wäre der Erfolg einer solchen Maßnahme auch sehr fraglich.

Wachtel und Grauammer zählen auch als Leitarten für den Landschaftstyp halboffene, reichstrukturierte Felder. Neuntöter, Steinkauz und Ortolan gehören ebenfalls zu den steten Bewohnern dieses Lebensraumes, der sich durch viele Strukturelemente (Hecken, 
Feldgehölze, Allen und Feldraine) auszeichnet. Die Strukturen im Untersuchungsgebiet entsprechen dieser Beschreibung. Der Anteil linienartiger Strukturelemente ist besonders hoch. Die regionalen Leitbilder und die Vorgaben des ANP Niedertreba sehen eine weitere Anreicherung mit entsprechenden Elementen vor. Die Bestände des Ortolan haben in den vergangenen Jahren im Untersuchungsgebiet stark abgenommen. Ob er derzeit vorkommt, ist zumindest fraglich (WEISE, Umweltamt LK Weimarer Land, mdl., 2001). Die anderen Leitarten kommen hingegen nachweislich vor und brüten auch im USG. Eine Förderung dieser seltenen und teilweise geschützten Arten durch die Weiterentwicklung und Ergänzung des Lebensraumes ist für sie und die anderen Arten des Biotops sinnvoll und erfolgversprechend.

Eine eingehende Betrachtung der LTs halboffene reichstrukturierte Niedermoore, Fluss- und Bachauen sowie feuchte und nasse Brachen und Sukzessionsflächen unterbleibt, da deren Elemente nur sehr kleinflächig im USG auftreten und eine Ausweitung derselben durch eine Aufforstung mäßig trockener, landwirtschaftlicher Flächen nicht möglich ist. Dagegen sind die Obstplantagen erwähnenswert, die in unmittelbarer Nachbarschaft zum USG zu finden sind. Der LT Obstbau teilt sich mit den oben beschriebenen halboffenen, reichstrukturierten Feldern die Leitarten Ortolan und Neuntöter. Mit innen leben Stieglitz, Girlitz, Gelbspötter und Turteltaube vergesellschaftet, die im USG ebenfalls mehrfach gesichtet wurden. Für den Neuntöter und den hoffentlich bald zurückkehrenden Ortolan sind demnach Synergismen zwischen beiden Habitattypen zu erwarten.

Aufforstungen inmitten landwirtschaftlicher Flächen haben zumindest temporär den Charakter von Feldgehölzen. Eine positive Auswirkung von Aufforstungen auf die Populationen der typischen Gehölzbewohner ist zu erwarten, wenn die Flächen inselartig angelegt sind oder als Waldfortsatz in die landwirtschaftliche Kulturfläche hineinragen. Feldgehölze kommen im Untersuchungsgebiet mehrfach vor. Die Leitarten Aaskrähe (bzw. Rabenkrähe), Turmfalke und Waldohreule brüten im betrachteten Regionalausschnitt. Auch die Turteltaube wurde bereits gesichtet. Der Sprosser, der ebenfalls als Leitart dieses Landschaftstyps gilt, wäre hier außerhalb seines natürlichen Verbreitungsgebietes.

Bei der Bewertung von Aufforstungen ist es von besonderem Interesse, ob die neu entstehenden Biotope schnell von Waldvogelarten besiedelt werden können. Dies wird naturgemäß einige Jahre dauern, da sich die Lebensraummerkmale nur langsam entwickeln. Dennoch kann von einer baldmöglichsten Besiedelung ausgegangen werden, wenn die Arten bereits heute im Gebiet vorkommen.

Der lindenreiche Eichen-Hainbuchenwald, der als dominierende NWG im Gebiet seinen Platz findet, zeichnet sich durch stetes Vorkommen verschiedener Vogelarten aus. Davon brüten nachweislich Waldlaubsänger, Gartenbaumläufer, Sumpfmeise, Trauerschnäpper und Pirol bereits heute im USG (Anlage 2 a). Nach den Angaben der Landschaftspläne kommen Mittelspecht und Grauspecht ebenfalls in der Region vor. Allerdings sind sie im Untersuchungsgebiet derzeit kaum zu beobachten (WEISE, mdl.). Diese Angaben stimmen zuversichtlich für eine positive Bestandesentwicklung, wenn der Landschaftstyp EichenHainbuchenwälder entwickelt oder ergänzt werden kann.

Schließlich ist darauf hinzuweisen, dass auch sämtliche Leitarten des LT Parks im USG vorkommen. Obwohl Blumenrabatten und weitläufige Rasenflächen als Elemente dieses LT kaum vorkommen, werden die vorhandenen Strukturen offensichtlich gerne von den entsprechenden Arten angenommen (FLADE 1994). Eine Entwicklung, die die vorhandenen Strukturen bewahrt und fördert, sollte demnach auch für die Bewohner von parkartigen Landschaften vorteilhaft sein.

\section{Regionale Leitbilder}

Das Landschaftsprogramm Thüringen gibt regionale Leitbilder für einzelne Naturräume vor. So macht es beispielsweise Zielaussagen für das Innerthüringer Ackerhügelland als Teil des Thüringer Beckens (HIEKEL 1997). Nur einige Aussagen des dortigen Leitbildes sind für 
Aufforstungsplanungen relevant und werden deshalb hier wiedergegeben. Weitere Ziele sind der untenstehenden Tabelle (Tab. 4.1-3) zu entnehmen.

Der offene Landschaftscharakter des landwirtschaftlich geprägten Hügellandes soll weitgehend erhalten werden. Dennoch ist eine Anreicherung mit Gehölzflächen auf mindestens $2 \%$ der landwirtschaftlichen Nutzfläche beabsichtigt. Flächenhafte Aufforstungen können als Maßnahme bis zu 10 \% der Ackerfläche ablösen.

Tab. 4.1-3: $\quad$ Regionales Leitbild für das Innerthüringer Ackerhügelland

\begin{tabular}{|c|c|c|}
\hline Beschreibung des Leitbildes & $\begin{array}{l}\text { Zu schützende } \\
\text { Biotope }\end{array}$ & $\begin{array}{l}\text { Hinweise für } \\
\text { Erstaufforstungen }\end{array}$ \\
\hline 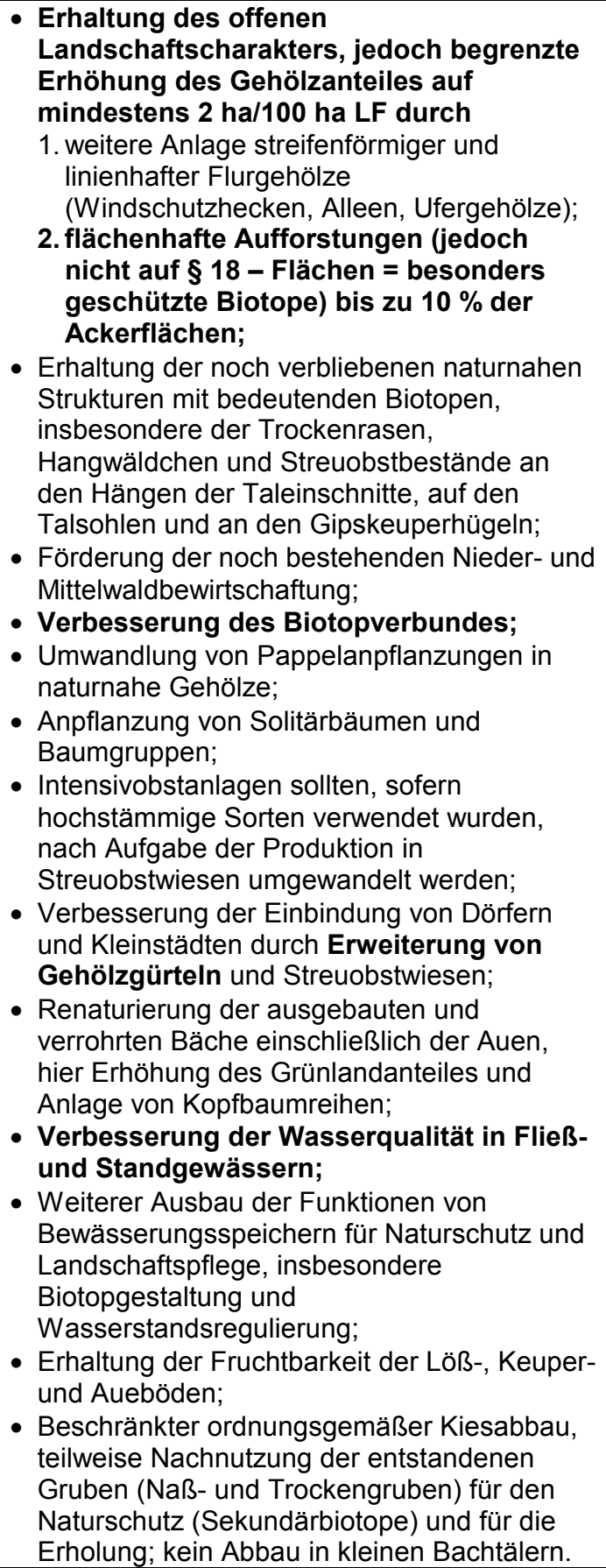 & $\begin{array}{l}\text { - Restwälder, kontinentale } \\
\text { Trockenrasen, } \\
\text { Streuobstwiesen, } \\
\text { Karstquellen, Erdfälle und } \\
\text { Bewässerungsspeicher } \\
\text { südlich exponierten } \\
\text { Kuppen und Hängen der } \\
\text { kleinen Bachtäler } \\
\text { auftretende Trockenrasen } \\
\text { sind aufgrund ihrer } \\
\text { Bedeutung für bestimmte } \\
\text { Arten, welche im Thüringer } \\
\text { Becken ihre } \\
\text { Verbreitungsgrenze } \\
\text { besitzen und damit } \\
\text { zumindest eine nationale } \\
\text { Bedeutung haben, zu } \\
\text { erhalten, zu entwickeln und } \\
\text { zu vernetzen. Dieses Ziel } \\
\text { muss absoluten Vorrang } \\
\text { vor jeder anderen Nutzung } \\
\text { haben } \\
\text { - Bachtäler, teilweise mit } \\
\text { kleinen Auewaldresten, } \\
\text { stellen nicht ersetzbare } \\
\text { Biotope der Landschaft dar } \\
\text { und sind daher } \\
\text { schützenswert }\end{array}$ & $\begin{array}{l}\text { - Erhöhung des Waldanteils } \\
\text { (von derzeit weniger als } 5 \\
\% \text { ) auf mindestens } 2 \% \text { der } \\
\text { Landwirtschaftlichen } \\
\text { Fläche ( bis zu } 10 \% \text { der } \\
\text { Ackerflächen) } \\
\text { - Zielkonflikt zwischen der } \\
\text { Erhaltung des offenen } \\
\text { Landschaftsbildes und der } \\
\text { Erhöhung des } \\
\text { Gehölzanteils erfordert } \\
\text { eine sorgfältige } \\
\text { Abstimmung in der } \\
\text { Planung } \\
\text { - Eine Aufforstung der } \\
\text { stärker geneigten } \\
\text { Hangmitten würde eine } \\
\text { deutlich Minderung der } \\
\text { Wassererosion bewirken } \\
\text { und die Weiträumigkeit der } \\
\text { Landschaft erhalten, die } \\
\text { hangparallelen Strukturen } \\
\text { würden eine Betonung der } \\
\text { Landschaftsformen } \\
\text { bewirken } \\
\text { - Gehölzelemente der } \\
\text { Landschaft sollen im } \\
\text { Rahmen des } \\
\text { Biotopverbundes } \\
\text { miteinander verknüpft } \\
\text { werden } \\
\text { Beschattung von } \\
\text { Fließgewässern durch } \\
\text { Aufforstungen als } \\
\text { Unterstützung der } \\
\text { Renaturierung und } \\
\text { Verbesserung der } \\
\text { Gewässergüte }\end{array}$ \\
\hline
\end{tabular}

Quelle: $\quad$ HIEKEL, W. (1997), entspricht den Vorgaben des Landschaftsprogramms Thüringen 
Außerdem soll der Biotopverbund verbessert werden, in dem neben den bisherigen auch die künftige Waldflächen eine tragende Rolle spielen sollen. Um die Siedlungen herum liegende Gehölzgürtel sind, ebenso wie Streuobstwiesen, auszuweiten. Schließlich können Aufforstungen im Bereich von stehenden und fließenden Gewässern, durch ihre Bodenschutz- und Klimafunktion noch ein weiteres, regionales Ziel verfolgen: die Verbesserung der Wasserqualität in Oberflächengewässern.

Die regionalen Leitbilder weisen Ziele aus, die für die Planung und Bewertung von Erstaufforstungen wichtig sind. Bezüglich der bestehenden Waldflächen, wird beispielsweise eine Förderung von Nieder- und Mittelwäldern und eine Umwandlung von Pappelbeständen in naturnahe Gehölze angestrebt. Auch wenn dieser Punkt keinen direkten Bezug zur Aufforstung besitzt, zeigt er dennoch den Willen zum ökologisch orientierten Wald(um)bau und begründet somit ebensolche Ansprüche an neue Wälder.

Die im Gebiet noch fragmentarisch vorhandenen, naturnahen Restwälder sind zu erhalten, zu ergänzen und zu vernetzen. Solche Bemühungen sind erfolgversprechend, wenn das derzeitige regionale Arteninventar für eine schnelle Besiedelung der neuen Flächen spricht und die aktuellen Populationen noch über einen ausreichend großen Gen-Pool verfügen.

Andere Biotope (Trockenrasen, Streuobstwiesen) sind ebenfalls zu erhalten, dürfen also nicht aufgeforstet werden. Dies bedingt gleichzeitig eine Erhaltungsnutzung zum Schutz vor natürlicher Wiederbewaldung. Bei der Auswahl von Aufforstungsflächen ist die Erhaltung des offenen Landschaftscharakters $\mathrm{zu}$ berücksichtigen. Aufforstungen von Mittel- und Unterhängen der Hügel engen den Horizont nicht ein und sind unter diesen Gesichtspunkten unbedenklich. Darüber hinaus leisten sie einen wichtigen Beitrag zum Boden- und Wasserschutz durch Verringerung von Erosion und Oberflächenabfluss.

\section{Vorhandene Planungsgrundlagen}

Das variabel einsetzbare Verfahren ist über die Bewertungsmaßstäbe innerhalb der Indikatoren an die regionalen Verhältnisse anzupassen. Landespflegerische Zielvorgaben werden aus übergeordneten Plänen abgeleitet. Für das Untersuchungsgebiet Apolda ist das Landschaftsprogramm Thüringen maßgebend, welches die regionalen Leitbilder liefert (s.o.). Dessen Vorgaben werden im Indikator Landnutzungsverhältnis, bei der Bewertung der Flächenverhältnisse berücksichtigt. Des weiteren wirkt das regionale Leitbild in die Entwicklung anderer Pläne hinein. Der ANP Niedertreba (TLL 1997) greift das Leitbild auf und ergänzt es. Er schlägt Maßnahmen zur Nutzungsverteilung und Strukturierung der Landschaft vor. Daraus ergeben sich weitere Eckdaten zur Kalibrierung der Bewertungsmaßstäbe in den Indikatoren Landnutzungsverhältnis und RandlinienVorkommen. Die übergeordneten Pläne sind interdisziplinär erstellte Vorgaben und berücksichtigen unterschiedliche Interessen. Sie propagieren die strukturreich gestaltete Agrarlandschaft als Erscheinungsbild des Raumes. Der Indikator Biotopverbund berücksichtigt diese Vorgabe bei der Definition der regional relevanten Landschaftstypen.

Eine weitere, essentielle Vorlage für die regionale Anpassung des Bewertungsmaßstabes ergibt sich aus dem Verfahren zur flächendeckenden Waldbiotopkartierung in Thüringen (LAWUF 1996). Dieses enthält einen Schlüssel zur Ableitung der natürlichen Waldgesellschaft aus den Standortstypen. Es liefert darüber hinaus Angaben zur hypothetischen Zusammensetzung dieser Gesellschaft (LAWUF 1997a). Damit ergibt sich für jeden Standort die Zusammensetzung und Vielfalt der natürlichen Waldgesellschaft als Ziel des ökologisch orientierten Waldbaus und somit der Bewertungsmaßstab für die Indikatoren Baumartenvielfalt und Naturnähe.

Neben diesen Planungsgrundlagen wurden auch die Ergebnisse der Waldfunktionenkartierung, der Offenland-Biotopkartierung (TMUL 1993), Luftbilder aus der Region und eigene Feldaufnahmen bei bestimmten Indikatoren berücksichtigt. 


\section{Vorstellung der Untersuchungsflächen}

Mit Hilfe von Luftbildern und topographischen Karten wurden im Untersuchungsraum ApoldaNiedertreba-Stobra insgesamt fünf landwirtschaftlich genutzte Felder als potentielle Aufforstungsflächen ausgewählt. Die Auswahl erfolgte systematisch, um möglichst unterschiedliche Anbindungsvarianten an existierende Biotope gleicher und anderer Art zu berücksichtigen. Die kleine Stichprobenauswahl ist durch das Ziel der Arbeit zu rechtfertigen, das Verfahren in seiner Umsetzung zu proben. Es war nicht beabsichtigt, einen Stichprobenumfang zu erhalten, der statistische Berechnungen zulässt.

Der Untersuchungsraum stellt sich als hügeliges Gelände mit kleineren, teilweise bewaldeten Bachtälchen dar. Die landwirtschaftliche Nutzung dominiert das Erscheinungsbild. Zahlreiche, vor allem linienhafte Strukturen gliedern den Raum. Die Hügelkuppen sind unbewaldet und nur im Einzelfall mit Hecken und anderen Strukturen besetzt. Dadurch wirkt die Landschaft übersichtlich.

Die Erschließung der Flächen ist entsprechend der intensiven Nutzung sehr gut. Praktisch alle Wege sind mit Maschinen befahrbar. Alleen, vornehmlich mit Obstbäumen, begleiten die Erschließungslinien. Größere Wasserflächen fehlen, doch der Utenbach verläuft als Seitenarm der IIm durch das Untersuchungsgebiet und stellt somit die Verbindung zu diesem Fluss her.

Entsprechend der Kategorisierung der Landschaftstypen nach FLADE (1994) ist das Untersuchungsgebiet zwischen den Typen gehölzarme Felder und halboffene, reichstrukturierte Felder einzuordnen, wobei der Anteil und vor allem die flächige Verteilung gehölzartiger Strukturelemente eher für den zweiten Typ spricht. Der erste Typ steht darüber hinaus auch im Widerspruch zu den landschaftsplanerischen Zielaussagen übergeordneter und externer Planungen (s.o.).

Eine größere Obstbaumplantage kommt im Südwesten des Untersuchungsgebietes vor. Daneben existieren einige Kleingärten und Randgebiete der Bebauung innerhalb des zu besprechenden Raumes. Diese spielen allerdings keine Rolle als Alternative zu einer Aufforstung, da sie weder dem regionalen Leitbild noch anderen Planvorgaben entsprechen. In der Bewertung läuft dies auf die Frage hinaus, welchen Beitrag eine Aufforstung für die Entwicklung des LT halboffene, reichstrukturierte Felder leisten kann.

\section{Aufforstungsvarianten}

Die Entwicklung des Waldbiotops und damit die ex-ante Bewertung der Erstaufforstungsfläche ist zu großen Teilen von der Art der Aufforstung abhängig. Baumartenwahl und -mischung beeinflussen die ablaufenden natürlichen Prozesse und determinieren Vielfalt, Naturnähe, Bestandesstruktur und den Typus des entstehenden Biotops. Somit werden vier der hier behandelten Indikatoren maßgeblich durch die Art der Aufforstung bestimmt, die sich wiederum planerisch aus dem zugehörigen Pflanzplan ergibt.

Für die Aufforstungsflächen in Thüringen ist allen berücksichtigten Standorten ein edellaubbaumreicher Linden-Hainbuchen-Eichenwald als natürliche Waldgesellschaft zuzuordnen. Dementsprechend wird eine Reihe von Aufforstungsvarianten mit unterschiedlicher Baumartenanzahl und unterschiedlichem Naturnähegrad vorgeschlagen (Anlage 3). Sie werden im Nachfolgenden als Aufforstungsvarianten (AV) mit zugehörigem Kürzel (I-2 bis III-12) bezeichnet. Die darin enthaltenen Baumarten ergeben sich aus dem Baumarten-Standortsgruppen-Schlüssel des Verfahrens zur Waldbiotopkartierung in Thüringen (Tab. 4.1-4; LAWUF 1996, LAWUF 1997a):

Zusätzlich wird eine Reihe von Erstaufforstungszieltypen (EAZT) berücksichtigt (Anhang 3), die von der Landesforstverwaltung Thüringen für die Aufforstung von landwirtschaftlichen Flächen vorgeschlagen werden (LAWUF 2001a). Diese Vorschläge berücksichtigen die Anlage unterschiedlicher Waldtypen, die wiederum verschiedene wirtschaftliche Interessen 
verfolgen. Diese Erstaufforstungszieltypen werden spezifischen

Standortsklassen zugeordnet und listen zughörige Baumarten auf.

Tab. 4.1-4 Baumarten potentiell natürlicher Wälder im forstlichen Wuchsgebiet Thüringer Becken (Auszug)

\begin{tabular}{|c|c|c|c|c|}
\hline $\begin{array}{c}\text { potentiell natürliche } \\
\text { Waldgesellschaft }\end{array}$ & $\begin{array}{c}\text { Haupt- } \\
\text { BA }\end{array}$ & Misch-BA & Neben-BA & Bemerkungen \\
\hline $\begin{array}{c}\text { edellaubbaumreicher } \\
\text { Linden-Hainbuchen- } \\
\text { Eichenwald }\end{array}$ & $\begin{array}{c}\mathrm{TEi}, \\
\mathrm{SEi}\end{array}$ & $\begin{array}{c}\mathrm{HBu}, \mathrm{WLi}, \\
\mathrm{SLi}, \mathrm{Es}, \mathrm{BAh}\end{array}$ & $\begin{array}{c}\mathrm{Bu}, \mathrm{BUI}, \mathrm{FUI}, \\
\text { SAh, FAh, El, } \\
\mathrm{Kb}, \mathrm{Sp}, \mathrm{As}\end{array}$ & $\begin{array}{c}\text { auf Tonstandorten } \\
\text { z.T. auch SEi } \\
\text { dominieren }\end{array}$ \\
\hline $\begin{array}{c}\text { Stieleichenreicher } \\
\text { (Roterlen-) } \\
\text { Eschenbachwald }\end{array}$ & $\mathrm{Es}$ & $\begin{array}{c}\text { REr, SEi, } \\
\text { (TEi) }\end{array}$ & $\begin{array}{c}\text { BAh, SAh, BUI, } \\
\mathrm{HBu}, \mathrm{WLi}, \mathrm{As}, \\
\mathrm{FUI}\end{array}$ & Bachtalstandorte \\
\hline
\end{tabular}

Die Erstaufforstungszieltypen benennen die Haupt- und Mischbaumarten direkt und mit Flächenanteilen. Nebenbaumarten erreichen je nach Zieltyp 10 oder $20 \%$, werden aber artspezifisch nicht näher bestimmt. Ihre Auswahl erfolgt immer nach Kriterien der Standortsverträglichkeit. Die vorliegende Arbeit nutzt den entstehenden Entscheidungsspielraum aus, um unterschiedliche Naturschutzziele zu verfolgen.

Nebenbaumarten werden innerhalb dieser Arbeit bei den EAZT grundsätzlich mit einem Flächenanteil von jeweils $5 \%$ berücksichtigt. Somit sind je nach EAZT zwei oder vier Baumarten auszuwählen. Für den ersten Standort (RLL2) sind sie der Baumartenliste für die potentiell natürlichen Wälder entnommen (Tab. 4.1-4), stehen also für angestrebte Naturnähe. Außerdem wurden sie so gewählt, dass der entstehende Bestand möglichst früh an Strukturreichtum gewinnt, die Erstaufforstung also einen hohen Wert nach dem Indikator Strukturpotential erhält.

Gegenteilig erfolgte die Benennung der Nebenbaumarten für den etwas trockeneren Standortstyp RLL3. Sie entsprechen nicht der PNV und versprechen auch keine frühe Strukturierung.

Für den dritten Standort (RC2) wurden die Nebenbaumarten entsprechend ihrer Reihenfolge in der Baumartenempfehlung (LAWUF 2001b) übernommen, ohne Berücksichtigung von Struktur oder Naturnähe.

Für jeden Standort wird somit eine unterschiedliche Strategie verfolgt, die in der Bewertung auch zu unterschiedlichen Bewertungsergebnissen führen soll.

\section{Waldrandgestaltung}

Die Waldrandgestaltung nimmt im Rahmen des Evaluationsverfahrens eine besondere Rolle ein. Unabhängig vom Standort erlaubt sie die Berücksichtigung wichtiger Strukturparameter. Der Aufbau des Waldrandes steht nicht zwingend in Zusammenhang mit Art, Vielfalt und Mischungsform des Bestandes. Im Rahmen des Evaluationsverfahrens entwickelt sich der Indikator Waldrandausprägung daher zu einem Aktionsraum, dessen Berücksichtigung immer ein Bewertungspotential von 5 Punkten ermöglicht. Diese Punkte könnten auch als Zusatzleistung betrachtet werden, die ein Eigentümer bei der Waldanlage einbringen kann, zur Aufwertung des Vorhabens.

\subsection{Teilergebnisse}

\subsubsection{Flächenspezifische Ergebnisse - Aufforstungsvarianten und Erstaufforstungszieltypen}

Die Gestaltung der Erstaufforstung mit unterschiedlichen Baumarten und bestimmten Mischungstypen legt den Grundstein für die Entwicklung der Flächen in den ersten Jahrzehnten. Anhand der Indikatoren Baumartenvielfalt, Naturnähe, Strukturpotential und 
Gefährdung des Biotops erfolgt eine Einschätzung und Bewertung dieser potentiellen Entwicklung. Die in der vorliegenden Arbeit untersuchten Aufforstungsvarianten (AV) und Erstaufforstungszieltypen (EAZT) geben ein breites Spektrum unabhängiger Zielvorstellungen wieder. Im Sinne eines Variantenstudiums wird die potentielle Entwicklung der Waldfläche prognostiziert und bewertet. Im Folgenden werden die Ergebnisse vorgestellt und erläutert.

Für alle betrachteten Aufforstungsflächen im Untersuchungsgebiet Apolda stellt der edellaubbaumreiche Linden-Hainbuchen-Wald die potentiell natürliche Waldgesellschaft dar (siehe Kap. 4.1). Diese Vegetationsgesellschaft liefert damit eine Vorgabe für die anzustrebende Vielfalt in der Baumschicht des neu entstehenden Waldes. Dies stellt die Regionalisierung des Bewertungsmaßstabes für den Indikator Baumartenvielfalt sicher. Die Zusammensetzung der entstehenden Baumschicht wird in Zusammenhang mit der Kartieranleitung zur Waldbiotopkartierung in Thüringen (LAWUF 1997a) mit 1 Haupt-, 4 Misch- und 7 Nebenbaumarten angegeben. Im Rahmen des Evaluationsverfahrens werden Gattungen nur als einzelne Arten gezählt, mit Ausnahme des Feldahorns, der aufgrund seiner niedrigen Wuchshöhe und deutlich anderen sukzessionalen Rolle eine Sonderstellung einnimmt.

Innerhalb der eigenen Aufforstungsvarianten, zeichnet sich eine stetige Zunahme des Indikatorwertes von AV I-2 zu I-12 und von AV II-2 zu II-12 ab. Diese folgt logisch aus der Erstellung der Variantenvorschläge durch Berücksichtigung steigender Baumartenzahlen. Da die oberste und die unterste Bewertungsklasse jeweils etwas breiter sind, als die mittleren Klassen, ergibt sich eine nicht vollständig lineare Bewertungsreihe (Abb. 4.2-I, auch Anhang 4).

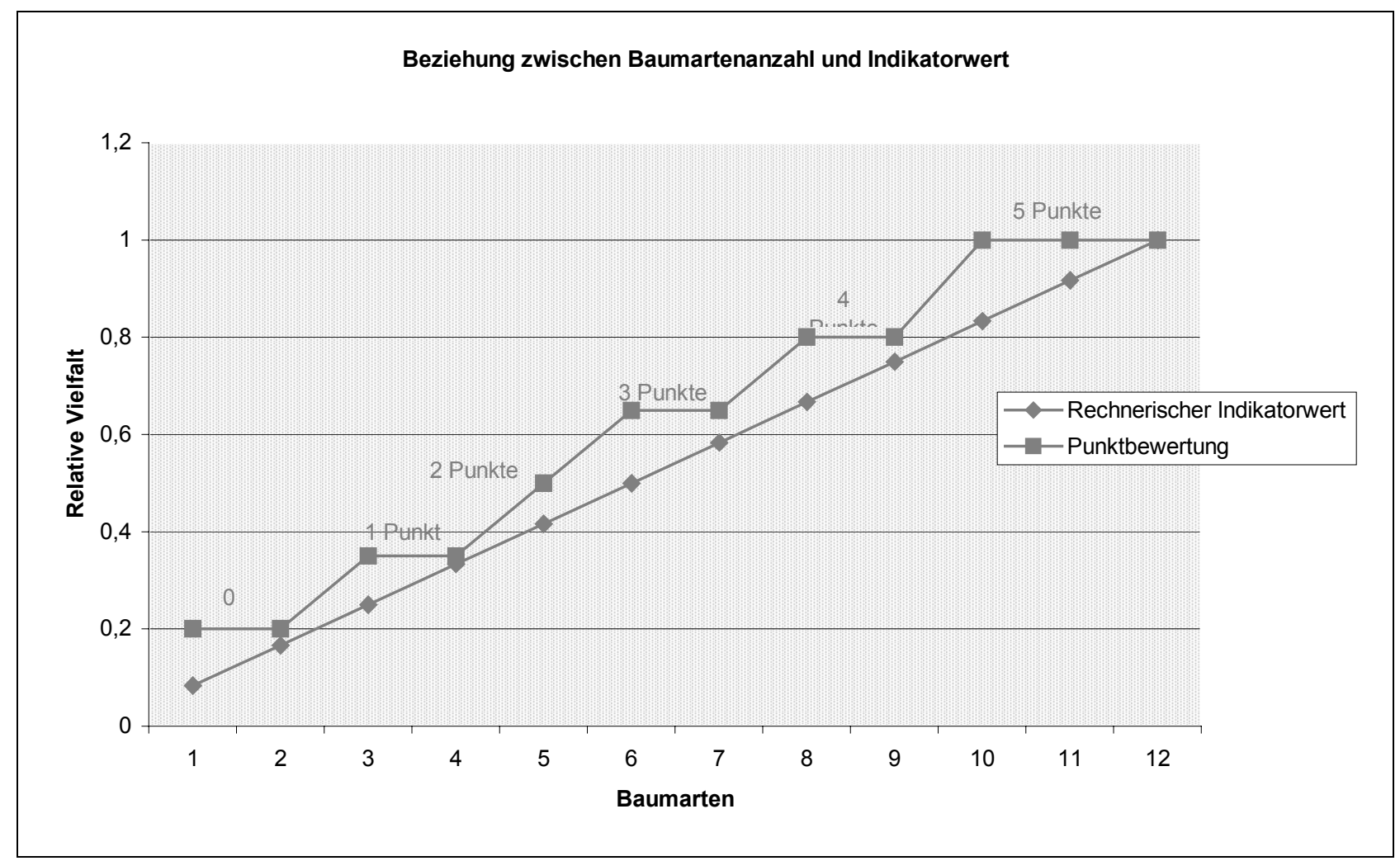

Abb. 4.2-1: $\quad$ Die Beziehung zwischen der Baumartenzahl und dem Indikatorwert Baumartenvielfalt

Bei den Erstaufforstungszieltypen Thüringen (EAZT) zeigt sich ein ungleich differenzierteres Bild (Abb. 4.2-II). Bei der Zusammenstellung dieser Aufforstungsvorschläge, stand die Baumartenvielfalt nicht im Vordergrund, sondern offensichtlich die Berücksichtigung unterschiedlicher wirtschaftlicher Interessen. Der 1. Traubeneichen-Nadelbaum-Typ (1.TEiNb-Typ) mit 6 und der Nadelbaum-Laubbaumtyp mit 7 Baumarten erreichen hier die höchsten Werte, die sich aber mit 3 Punkten knapp über der Mitte des Möglichen befinden. 
Auch für den Indikator Naturnähe gibt die natürliche Waldgesellschaft das Entwicklungsziel vor. Erneut wird die relative Übereinstimmung mit diesem Idealziel festgestellt und anhand desselben Bewertungsschema (vgl. Abb. 3.5-III) in Wertziffern übertragen. Somit liegt auch in diesem Falle eine nichtlineare Bewertungsreihe vor, die sich aus den ungleichen Bewertungsklassen ergibt.

Für die erste Reihe der vorgeschlagenen Aufforstungsvarianten (Standort RLL2/3) ist die Bewertung identisch mit jener für die Zielartenvielfalt, da alle gewählten Baumarten aus der natürlichen Waldgesellschaft stammen. Bei der zweiten Variantenreihe (Standort RC2) wurde nur jede zweite Baumart der PNV-Vorgabe entnommen. Hieraus resultiert ein langsameres, dennoch stetiges Ansteigen der Bewertungszahl innerhalb der Reihe.

Ein direkter Vergleich der AV und der EAZT für die Standorte RLL2 und RLL3 (Tab. 3.2-2) deutet an, wie sehr eine gezielte Beteiligung natürlich vorkommender Baumarten (wie im Beispiel für RLL2) die Naturnähe eines Bestandes fördert. Die hohen Bewertungsziffern für die EAZT zum Standort RC2 weisen darauf hin, dass natürliche Nebenbaumarten auch in der Auflistung der Baumartenempfehlungen für bestimmte Standorte (LAWUF 2001b) zuerst genannt werden.

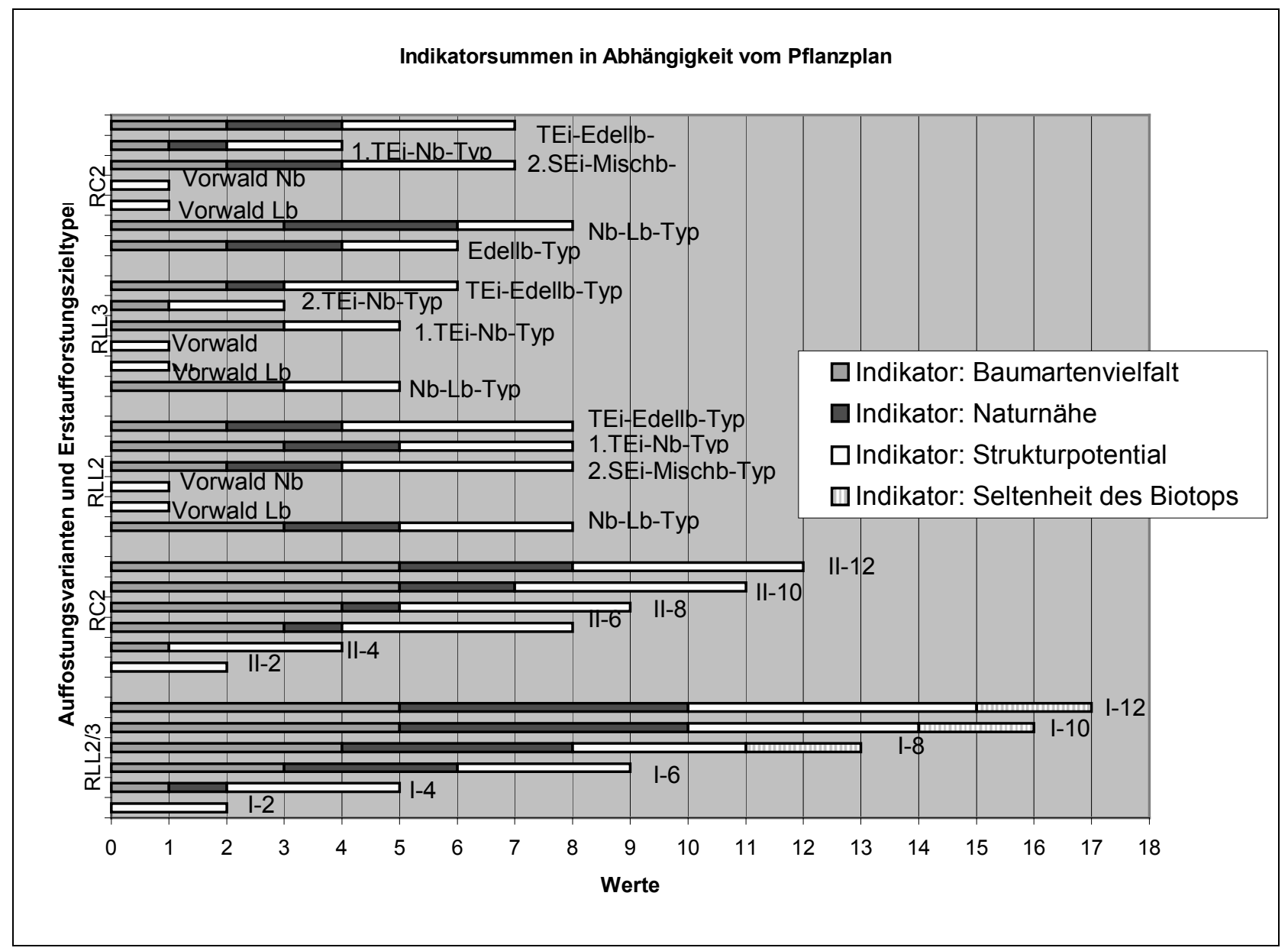

Abb. 4.2-II: $\quad$ Indikatorsummen in Abhängigkeit vom Pflanzplan (EAZT und AV für Apolda)

Die Beteiligung von Baumarten unterschiedlicher Konkurrenzstärke, Wuchshöhe und Standortsansprüche ermöglicht eine schnelle vertikale Strukturierung des Bestandes. Auch für die horizontale Strukturierung kann dieser Faktor von Bedeutung sein. Der eigene Vorschlag verschiedener Aufforstungsvarianten für die relevanten Standorte berücksichtigt auch die Kombination von Baumarten möglichst unterschiedlicher Strukturpotential-Gruppen (Tab. 3.5-4 und Abb. 3.5-IV). Daraus resultiert erneut ein Bild stetig steigender Werte innerhalb der beiden Variantengruppen, diesmal für den Indikator Strukturpotential. Für 
den Standort RC2 wird der optimale Strukturwert allerdings nicht erreicht, da hier - im Vergleich zur komplementären Variante des Standorts RLL2/3 - die PNV-Baumart Birke (Pionier) durch die Robinie ersetzt wurde (intermediärer Pionier).

Die sehr hohen Bewertungsziffern der EAZT für den Standort RLL2, mit Baumartenauswahl nach Strukturpotentialklassen, verdeutlicht erneut die Möglichkeit, bereits bei der Waldanlage positiv auf die Strukturierung Einfluss zu nehmen. Ein Blick auf die Ergebnisse für die Standorte RLL3 und RC2 zeigt außerdem, dass die Werte dieses Indikators im Vergleich zu den vorher besprochenen Indikatoren relativ hoch liegen, obwohl hier bei der Auswahl der Nebenbaumarten nicht mehr auf deren Strukturklassen geachtet wurde.

Die Bewertung des Indikators Seltenheit des Biotops zeigt ein vollständig anderes Bild als die übrigen, von der Aufforstungsplanung abhängigen Indikatoren (Anhang 4). Dies liegt zunächst darin begründet, dass eine Aufforstung nur dann eine Würdigung im Sinne des Biotopschutzes erfährt, wenn der entstehende Biotoptyp gefährdet ist. Die Höhe der Bewertung richtet sich wiederum nach dem Gefährdungsgrad, der in der Roten Liste für Biotope (RIECKEN et al. 1994) ausgewiesen ist (vgl. Kap 3.5.3). Weitere Bewertungspunkte erhält das potentielle Biotop, wenn es seiner Art nach durch Landesgesetz geschützt oder im Sinne der Waldbiotopkartierung als schützenswert deklariert ist.

Per Definition, muss eine Waldgesellschaft auf einem bestimmten Standort, bzw. einer bestimmten Standortsgruppe stehen, um ein geschütztes Biotop im Sinne einer NWG darzustellen. Für die besprochenen Bezugsräume bedeutet dies, dass ein neu entstehender Bestand nur dann für diese Funktionen ex-ante honoriert werden kann, wenn er diese Bedingungen erfüllt. Da auch im Sinne der Waldbiotopkartierung nur die natürlichen Waldgesellschaften als besonders schutzwürdig gelten, ergibt sich daraus eine feste Bewertungszahl für jeden Standort. Die Frage, ob eine Aufforstungsvariante diese Bewertung erfährt, ist dann mit ja oder nein zu beantworten. Eine prozentuale Annäherung an die Bedeutung als schützenswertes Biotop ist nicht möglich. Daher zeigt das Ergebnisdiagramm (Anhang 4) keine Funktion, sondern eine Bild mit einzelnen Bewertungsspitzen. Diese Bewertungsspitzen liegen dort, wo sowohl eine bestimmte Naturnähe als auch eine hohe Baumartenvielfalt gegeben ist. Von den untersuchten Aufforstungsvarianten ist dies nur für drei Beispiele der Fall: I-8, l-10 und I-12

In der summarischen Darstellung der Indikatorwerte für die Indikatoren Baumartenvielfalt, Naturnähe, Strukturpotential und Seltenheit des Biotops (Abb. 4.2-II) spiegeln sich die Einzelergebnisse und deren Zusammenspiel wieder. Die mit Abstand höchsten Bewertungsergebnisse erreichen die drei baumartenreichsten, eigenen Aufforstungsvarianten für den Standort RLL2/3. Entscheidend könnte dabei die bereits angesprochene Kombination von Baumartenvielfalt und Naturnähe sein, die auf diesem Standort zusätzlich eine hohe Bewertung über den Indikator Seltenheit des Biotops erreicht.

Die Grafik verdeutlicht ebenfalls, dass Reinbestände (auch Vorwälder aus nur 1 Baumart) sowie Bestände mit zwei Baumarten innerhalb dieses Verfahrens und auf diesen Standorten keine hohen Punktzahlen erreichen. Von den 20 untersuchten Aufforstungsvarianten erreichen immerhin zwölf eine Bewertung von 8 Punkten. Diese Varianten verfügen gleichzeitig über mindestens fünf Baumarten (im Vergleich zu zwölf in der natürlichen Waldgesellschaft). Mindestens die Hälfte der optimal erreichbaren Punkte erzielen allerdings nur besonders vielfältige Bestände (mindestens zehn Baumarten oder mindestens acht Baumarten bei gleichzeitig hoher Naturnähe).

Der Indikator Waldrandausprägung erfüllt, wie bereits erwähnt, eine Sonderstellung in der Bewertung. Die Struktur und Erscheinung des Waldrandes ist über die Merkmale Waldrandbreite, Grundform, Artenzusammensetzung, Strauchanteil und Herkunft bei jeder Waldanlage frei zu wählen. Diese Faktoren sind nicht an bestimmte Standorte gebunden. $\mathrm{Ob}$ und in welchem Maße diese Elemente bei der Erstaufforstung Berücksichtigung finden, liegt im Ermessen des Grundeigentümers. Die Aufforstung kann aber durch sie immer eine Aufwertung im Sinne des Naturschutzes erfahren. Selbst Aufforstungsvarianten mit geringen 
Punktzahlen aus den bisher vorgestellten Indikatoren, können dadurch noch deutlich aufgebessert werden.

\subsubsection{Flächenspezifische Ergebnisse - Raumbezug}

Für die Bewertung der Beziehungen zwischen den neu entstehenden Aufforstungsflächen und der unmittelbaren bis mittelbaren Umgebung (Aufforstungsumfeld und erweiterter Planungsraum) werden die Aufforstungsflächen eingebettet in Bezugsräume (BR). Diese besitzen eine quadratische Grundform mit zwei Kilometern Seitenlänge. Die Ausweisung erfolgt über Luftbilder in einem geographischen Informationssystem (GIS; ArcView 3.2), wobei das Zentrum des BR mit dem mathematischen Zentrum der jeweiligen Aufforstungsparzelle übereinstimmt.

Innerhalb dieser Räume werden anschließend die notwendigen Informationen zur Beschreibung der aktuellen Situation und zur Prognose der Entwicklung gemessen. Die Beurteilung der Biotop-Verbund-Wirkung bedarf zunächst der Feststellung der im BR vorhandenen, geschützten Biotope. Die vorliegende Untersuchung übernimmt diese nachrichtlich aus der Waldbiotopkartierung für den Forstbezirk Bad Berka und aus dem Arten- und Biotopschutzkonzept für Mittelthüringen. Die Biotope werden entsprechend den Quellen flächig, bzw. punktuell in das GIS übertragen und qualitativ ausgewertet.

Eine Aufforstung führt zur Entstehung eines neuen Biotops, ungeachtet dessen naturschutzfachlicher Wertigkeit. Dies ist dann zu würdigen, wenn das Biotop im Sinne der regionalen Biotopdiversität eine Bereicherung darstellt. Im landwirtschaftlich dominierten Raum Apolda wird dies für jede der hier behandelten Aufforstungsvarianten in Ablösung einer intensiv ackerbaulichen Nutzung vermutet.

Die Aufforstung kann darüber hinaus bestehende Waldbiotope oder Gehölze erweitern oder sogar miteinander verbinden. Diese Verbundfunktionen sind gesondert festzustellen und zu bewerten.

Mit dem Indikator Biotop-Verbund soll der Beitrag der Aufforstung zur Ergänzung und Erweiterung des Landschaftstyps beurteilt werden. Dies erfordert die Definition des vorhandenen Landschaftstyps und der Entwicklungsmöglichkeiten. Wie in Kapitel 4.1 erläutert, können Aufforstungen im USG Apolda am ehesten einen Beitrag zur Entwicklung des Landschaftstyps halboffene, reichstrukturierte Felder leisten. Ein Beitrag zur Ausweitung von Eichen-Hainbuchenwäldern kann ebenso geleistet werden, wobei dann der Anschluss an entsprechende Restwälder notwendig ist. Wie oben ausgeführt, fehlen die Leitarten Mittel- und Grauspecht noch im Untersuchungsgebiet, während andere Leitarten (z.B. Waldlaubsänger, Gartenbaumläufer, Trauerschnäpper) wohl vorhanden sind. Daher ist zum gegenwärtigen Zeitpunkt das Augenmerk auf die Förderung der halboffenen Landschaft zu richten, wobei parallel auf eine naturgemäße Zusammensetzung der neuen Wälder zu achten ist. Eine Förderung der Arten des LT Gehölze (bis ca. 7 ha) kann als Nebenwirkung von Aufforstungen erwartet werden.

Die Erweiterung des LT halboffene, reichstrukturierte Felder ist dann zu attestieren, wenn der deutlich strukturierte Raum offensichtlich und auf Kosten des strukturarmen Raumes ausgedehnt wird. Dies ist zur Zeit nur gutachtlich feststellbar, da quantitative Analyseverfahren zu diesem Sachverhalt fehlen. Die hier betrachteten, unten erläuterten Flächenbeispiele sollen diesen Bewertungsschritt demonstrieren und nachvollziehbar machen.

Für die Bewertung des Indikators Landnutzungsverhältnis ist die Feststellung der derzeitigen Flächennutzungsverteilung notwendig. Auf deren Grundlage und unter Berücksichtigung raumplanerischer Vorgaben für den Untersuchungsraum, wird die Umwandlung der landwirtschaftlichen Nutzungsart zu Wald bewertet. Diese Angaben werden in der Regel für ganze Naturräume oder - noch häufiger - für bestimmte Verwaltungsbezirke 
erhoben. Leider werden dabei in aller Regel nur bestimmte Daten erhoben. Im Beispiel des Landkreises Weimarer Land verfügt das Landwirtschaftsamt Umpferstedt zwar über flächengenaue Angaben zu unterschiedlichsten (landwirtschaftlichen) Nutzungsformen, jedoch beziehen sich diese nur auf jene Flächen, welche im Rahmen von Mehrfachanträgen erfasst wurden. Bestimmte Nutzungsformen, etwa der Obstbau, tauchen nicht auf. Angaben zu Gehölzanteilen fehlen vollständig. Auch das Regionale Raumordnungsprogramm Mittelthüringen macht hier keine genaueren Angaben.

Für die vorliegende Untersuchung wurde deshalb auf die Angaben des ANP Niedertreba (TLL 1997) zurück gegriffen. Dieses Dokument liefert detaillierte Flächenangaben, nicht nur zur aktuellen Situation, sondern auch zu den angestrebten Veränderungen. Einschränkend muss erwähnt werden, dass der ANP von der Thüringer Landesanstalt für Landwirtschaft erstellt wurde und somit eine Fachplanung darstellt. Für die konsensorientierte Anwendung des Evaluationsverfahrens und zur reibungslosen Integration in die Raumplanung wäre es von Vorteil, wenn das Verfahren auf interdisziplinär erarbeitete Ist- und Soll-Werte zurück greifen könnte.

Unter der oben genannten Prämisse zur Übertragung der im ANP Niedertreba genannten Flächennutzungsanteile stellen sich Situation und Ziel für den Untersuchungsraum wie folgt dar:

Tab. 4.2-1: Aktuelle und angestrebte Flächennutzungsanteile in der Region Apolda / Niedertreba, berechnet auf der Grundlage des Agrarraumnutzungsplanes Niedertreba (TLL 1997)

\begin{tabular}{|c|c|c|c|c|}
\hline \multirow{2}{*}{$\begin{array}{c}\text { Nutzungs- } \\
\text { form }\end{array}$} & \multicolumn{2}{|c|}{ Ist } & \multicolumn{2}{c|}{ Soll } \\
\cline { 2 - 5 } Wald & 337,30 & 5,57 & 421,13 & ha \\
\hline Acker & 4088,80 & 56,12 & 3762,30 & 52,40 \\
\hline Grünland & 451,30 & 6,32 & 624,32 & 10,40 \\
\hline Obstbau & 148,80 & 2,48 & 179,03 & 3,00 \\
\hline Brache & & & & \\
\hline
\end{tabular}

Der Indikator Randlinien-Vorkommen geht auf die quantitative und qualitative Veränderung der Ökotone ein. Die Randlinien werden über den Luftbildern im GIS digitalisiert und statistisch ausgewertet. Die Aufforstung führt zu einer Veränderung der Randlinienlänge bei den betroffenen Biotopen. Je nach Schlussgrad der Bewaldung ist eine Zu- oder Abnahme der Ökotonlängen zu prognostizieren. Die Bewertung erfolgt summarisch nach den Klassen, die von der Veränderung profitieren, da der hier festgestellte Ziellandschaftstyp eine Vielfalt an Randlinien erfordert.

Zusätzlich wird die Veränderung der Waldrandsituation betrachtet. Süd- und westexponierte Waldränder stellen besonders wertvolle Biotope aus Sicht des Artenschutzes dar (ZUNDEL 2000, $\mathrm{COCH}$ 1995), weshalb deren Vermehrung hier honoriert wird. Eine Feststellung der aktuellen und zukünftigen Situation wird ebenfalls mit Hilfe des GIS für jeden Bezugsraum durchgeführt.

Je ein Luftbild zu jeder Aufforstungsfläche mit eingezeichneten Ökotonen und Biotopen findet sich in Anhang 4

\section{Bezugsraum Apolda-1 / Aufforstungsfläche AP-1}

Die Aufforstung der Fläche AP-1 schafft ein neues Biotop, welches als Laubgehölz den bachbegleitenden Wald (östlich der Aufforstungsfläche) mit einer relativ neu angelegten Laubwaldfläche verbindet, die südwestlich an die Parzelle angrenzt. Damit entsteht eine Erweiterung und Verknüpfung gleichartiger Biotope. Die Aufforstung eines 
Nadelholzreinbestandes (vgl. EAZT Thüringen Vorwald Nadelbaum) wird diese Funktion allerdings kaum gewährleisten, da dessen Lebensraummerkmale sich von den vorigen deutlich abheben. Der LT halboffene, reichstrukturierten Felder gewinnt zusätzlich an Strukturelementen und Biotopen, wird also ergänzt. Von einer Ausweitung des Lebensraumes kann nicht gesprochen werden, da der betrachtete Bezugsraum mit Strukturelementen locker durchzogen ist und keine Lücke geschlossen wird. Eine negative Bewertung durch das entstehende Biotop entsteht nicht, da kein bedeutungsvolles Biotop verloren geht, keine Zerschneidungswirkung stattfindet und auch der Landschaftstyp keine Beeinträchtigung erfährt (vgl. Abb. 4.2-III).

Hinsichtlich der Änderung in den Flächennutzungsanteilen (Indikator Landnutzungsverhältnis) ist festzustellen, dass die Aufforstung den beabsichtigten Trend der Waldmehrung auf Kosten der landwirtschaftlichen Nutzfläche unterstützt. Gleichzeitig sollen aber auch Grünland, Brache und Obstbau ausgeweitet werden. Die Aufforstung kann naturgemäß keinen Beitrag hierzu leisten, läuft diesem Ziel aber auch nicht zuwider. Die Neutralität bezüglich der letztgenannten Nutzungsarten verhindert einen Punktabzug.

In Bezug auf das Landschaftsbild ist die Aufforstungsfläche integriert in Strukturelemente und Siedlung, weshalb eine negative Auswirkung nicht zu erwarten ist. Der entstehende Gehölzgürtel um den Siedlungsbereich herum ist sogar positiv zu beurteilen. 
Tab. 4.2-2: Daten zum Bezugsraum Apolda-1 und zur Aufforstungsfläche AP-1

\begin{tabular}{|c|c|c|c|c|}
\hline \multicolumn{5}{|l|}{ Beschreibung } \\
\hline Lage: & \multicolumn{4}{|c|}{$\begin{array}{l}\text { zwischen Apolda (nördlich) und Schöten (südlich), unmittelbar angrenzend } \\
\text { an das LSG Schöten (östlich) }\end{array}$} \\
\hline Flächengröße: & \multicolumn{4}{|l|}{8,48 ha } \\
\hline Standort(e): & \multicolumn{4}{|c|}{$\begin{array}{l}\text { unter Wald vermutlich: } \\
\text { Schlufflehm, mäßig frisch bis mäßig trocken }(R L L 2 / 3) \text { und reicherer } \\
\text { Karbonatgesteinsstandort mäßig frisch (RC2) }\end{array}$} \\
\hline $\begin{array}{l}\text { Ausgewiesene } \\
\text { Biotope: }\end{array}$ & $\begin{array}{ll}\text { - } & \mathrm{F} 112 \\
\text { - } & \mathrm{G} 112(2 \mathrm{x}) \\
\text { - } & \mathrm{L} 110 \\
\text { - } & \mathrm{L} 140 \\
\text { - } & \mathrm{L} 170(3 \mathrm{x}) \\
\text { - } & \mathrm{L} 180(4 \mathrm{x}) \\
\text { - } & \mathrm{U} 100\end{array}$ & \multicolumn{3}{|c|}{$\begin{array}{l}\text { Schnell fließender Bach } \\
\text { Frischwiese } \\
\text { Fläch. Feldgehölz/Restwald } \\
\text { Allee } \\
\text { Flächiges Obstgehölz } \\
\text { Streifenförm. Obstgehölz } \\
\text { Parkanlage/Friedhof }\end{array}$} \\
\hline Nutzungsanteile & Ist & & Soll & Veränderung \\
\hline $\begin{array}{l}\text { Acker } \\
\text { Grünland } \\
\text { Wald / Gehölze } \\
\text { Obstbau } \\
\text { Brache } \\
\text { Siedlung }\end{array}$ & $\begin{array}{r}261 \text { he } \\
3 \text { he } \\
48 \text { he } \\
19 \text { he } \\
0 \text { he } \\
69 \text { he }\end{array}$ & & $\begin{array}{l}- \\
+ \\
+ \\
+ \\
+\end{array}$ & $\begin{array}{l}- \\
= \\
+ \\
= \\
=\end{array}$ \\
\hline Randlinien & $\begin{array}{l}\text { Länge ir } \\
\text { Bezugsra }\end{array}$ & & $\begin{array}{l}\text { Länge an der } \\
\text { Aufforstungsfläche }\end{array}$ & $\begin{array}{l}\text { Veränderung an der } \\
\text { Aufforstungsfläche }\end{array}$ \\
\hline $\begin{array}{l}\text { Feldlinie } \\
\text { Waldlinie } \\
\text { Grünlandlinie } \\
\text { Obstbaulinie } \\
\text { Siedlungslinie } \\
\text { Allee } \\
\text { Relation }\end{array}$ & $\begin{array}{r}10.145 \\
7.447 \\
1.138 \\
3.811 \\
13.651 \\
1.921 \\
\cong 95,28\end{array}$ & $\begin{array}{l}\mathrm{m} \\
\mathrm{m} \\
\mathrm{m} \\
\mathrm{m} \\
\mathrm{m} \\
\mathrm{m} \\
\mathrm{lfm} / \mathrm{ha}\end{array}$ & $\begin{array}{r}706 \mathrm{~m} \\
625 \mathrm{~m} \\
0 \mathrm{~m} \\
0 \mathrm{~m} \\
81 \mathrm{~m}\end{array}$ & $\begin{array}{r}-54 \mathrm{~m} \\
+109 \mathrm{~m}\end{array}$ \\
\hline Waldränder & ex-ante & & ex-post & Differenz \\
\hline $\begin{array}{l}\text { Nordexposition } \\
\text { Ostexposition } \\
\text { Südexposition } \\
\text { Westexposition }\end{array}$ & $\begin{array}{r}199 \\
0 \\
0 \\
327\end{array}$ & $\begin{array}{l}\mathrm{m} \\
\mathrm{m} \\
\mathrm{m}\end{array}$ & $\begin{array}{r}364 \mathrm{~m} \\
0 \mathrm{~m} \\
0 \mathrm{~m} \\
374 \mathrm{~m}\end{array}$ & $+165 \mathrm{~m}$ \\
\hline
\end{tabular}

Bei den Randlinien ist nur eine geringfügige Zunahme durch die Aufforstung festzustellen. Der relative Anteil Ifm. Randlinie pro Hektar steigt nur geringfügig, um etwa 3,7 Prozent (Tab. 4.2-2). Eine deutliche Verbesserung (von 10 oder 20 Prozent) ist also nicht feststellbar. Auch nominal erhöht sich der Randlinienanteil kaum. Somit ist die Wirkung für den Raum vernachlässigbar. Eine negative Veränderung ist allerdings auch nicht zu erwarten, weshalb kein Punktabzug notwendig ist.

Die Waldrandsituation ist von einer Veränderung einer bislang herrschenden Nord-WestExposition hin zu einem deutlichen Nord- und einem noch längeren Ost-Rand geprägt. Die Länge nimmt ebenfalls etwas zu. Im Bewertungsschema (Abb. 3.5-VIII) ist damit allerdings lediglich der 1. positive Sektor (bis $100 \%$ Steigerung) beansprucht, weshalb nur ein Punkt zugeteilt wird. Der Indikator Randlinien-Vorkommen schlägt folglich bei dieser Aufforstung nur mit einem Punkt zu Buche. 


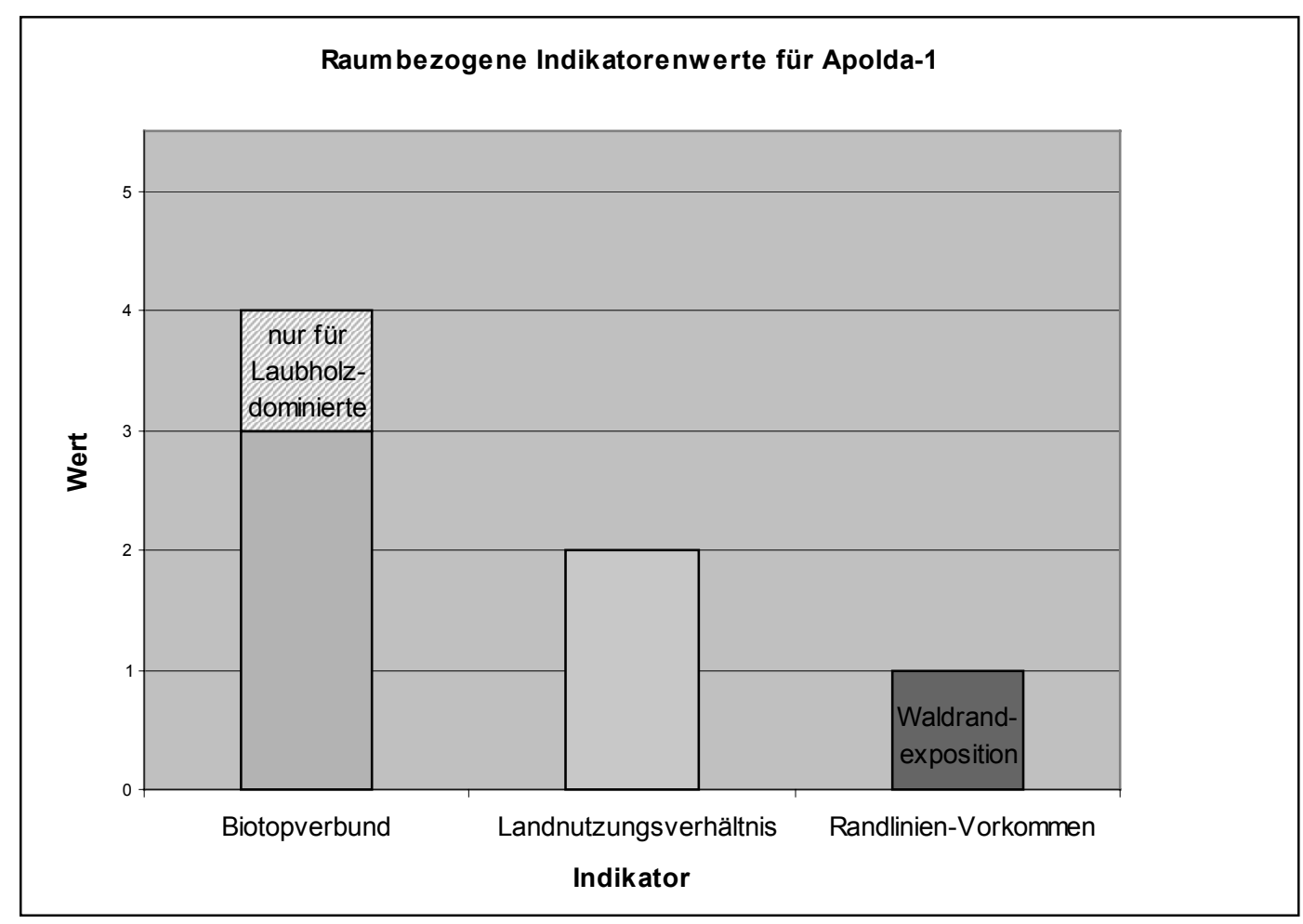

Abb. 4.2-III: $\quad$ Raumbezogene Indikatorenwerte für Apolda-1 


\section{Bezugsraum Apolda-2 / Aufforstungsfläche AP-2}

Tab. 4.2-3 Daten zum Bezugsraum Apolda-2 und zur Aufforstungsfläche AP-2

\begin{tabular}{|c|c|c|c|}
\hline \multicolumn{4}{|l|}{ Beschreibung } \\
\hline Lage: & \multicolumn{3}{|c|}{ zwischen Apolda (westlich) und Kösnitz (östlich), Kuppenlage } \\
\hline Flächengröße: & \multicolumn{3}{|l|}{13,14 ha } \\
\hline Standort(e): & \multicolumn{3}{|c|}{$\begin{array}{l}\text { Ackerfläche: } \\
\text { Löss-/Lösskerf-Grieserde } \\
\text { unter Wald vermutlich: } \\
\text { reicherer Schlufflehm, mäßig trocken(RLL2/3) }\end{array}$} \\
\hline \multirow{8}{*}{$\begin{array}{l}\text { Ausgewiesene } \\
\text { Biotope: }\end{array}$} & - G111 Fri & \multicolumn{2}{|c|}{ Frischweide } \\
\hline & - $\mathrm{G} 112(3 \mathrm{x})$ & \multicolumn{2}{|c|}{ Frischwiese } \\
\hline & - L110 & \multicolumn{2}{|c|}{ Fläch. Feldgehölz/Restwald } \\
\hline & - L132 & \multicolumn{2}{|c|}{ Streifenförmiges Gehölz } \\
\hline & - L150 (3x) & \multicolumn{2}{|c|}{ Solitärbäume } \\
\hline & - L160 & \multicolumn{2}{|c|}{ Kopfbäume } \\
\hline & - L170 & \multicolumn{2}{|c|}{ Fläch. Obstgehölze } \\
\hline & - L180 (8x) Str & \multicolumn{2}{|c|}{ Streifenförm. Obstgehölze } \\
\hline Nutzungsanteile & Ist & Soll & Veränderung \\
\hline Acker & 379 ha & - & - \\
\hline Grünland & \multirow{2}{*}{$\begin{array}{r}18 \text { ha } \\
2 \text { ha }\end{array}$} & + & $=$ \\
\hline Wald / Gehölze & & + & + \\
\hline Obstbau & $\begin{array}{l}2 \text { ha } \\
0 \text { ha }\end{array}$ & + & $=$ \\
\hline $\begin{array}{l}\text { Brache } \\
\text { Siedlung }\end{array}$ & 0 ha & + & $=$ \\
\hline Randlinien & $\begin{array}{l}\text { Länge im } \\
\text { Bezugsraum }\end{array}$ & $\begin{array}{c}\text { Länge an der } \\
\text { Aufforstungsfläche }\end{array}$ & $\begin{array}{l}\text { Veränderung an de } \\
\text { Aufforstungsfläche }\end{array}$ \\
\hline Feldlinie & $584 \mathrm{~m}$ & & $+1505 m$ \\
\hline Waldlinie & $633 \mathrm{~m}$ & & $+1505 m$ \\
\hline Grünlandlinie & $422 \mathrm{~m}$ & Keine Randlinie & \\
\hline Obstbaulinie & $0 \mathrm{~m}$ & vorhanden & \\
\hline $\begin{array}{l}\text { Siedlungslinie } \\
\text { Allee }\end{array}$ & $\begin{array}{r}0 \mathrm{~m} \\
5000 \mathrm{~m}\end{array}$ & & \\
\hline $\begin{array}{l}\text { Allee } \\
\text { Relation }\end{array}$ & $\cong 16,59 \mathrm{Ifm} / \mathrm{ha}$ & $\cong 0,00 \mathrm{lfm} / \mathrm{ha}$ & $\cong 229,00 \mathrm{lfm} / \mathrm{ha}$ \\
\hline Waldränder & ex-ante & ex-post & Differenz \\
\hline Nordexposition & $0 \mathrm{~m}$ & $322 \mathrm{~m}$ & $+322 \mathrm{~m}$ \\
\hline Ostexposition & $0 \mathrm{~m}$ & $496 \mathrm{~m}$ & $+496 \mathrm{~m}$ \\
\hline Südexposition & $0 \mathrm{~m}$ & $305 \mathrm{~m}$ & $+305 \mathrm{~m}$ \\
\hline Westexposition & $0 \mathrm{~m}$ & $382 \mathrm{~m}$ & $+382 \mathrm{~m}$ \\
\hline
\end{tabular}

Auf der Aufforstungsfläche Apolda-2 wird die Entstehung eines isolierten Waldbestandes betrachtet, der inselartig in einer landwirtschaftlich genutzten Umgebung liegt. Die bedeutendsten Biotope des Bezugsraumes sind die Alleen aus Obstbäumen und die Grünlandnutzungen am östlichen Rand des BR. Das neu entstehende Biotop kann somit weder eine Ergänzung noch einen Verbund von Biotopen leisten. Allerdings ergänzt und erweitert es den LT halboffene, rechstrukturierte Felder, durch die Schaffung wesentlicher Strukturen in einem bis dato ausgeräumten Teil der Feldflur. Eine Beeinträchtigung oder Zerschneidung von Biotopen liegt nicht vor.

Die Umwandlung der Ackerfläche in Wald ist ganz im Sinne des angestrebten Landschaftswandels. Wiederum bleiben Grünland-, Brache- und Obstbestände unberührt. 


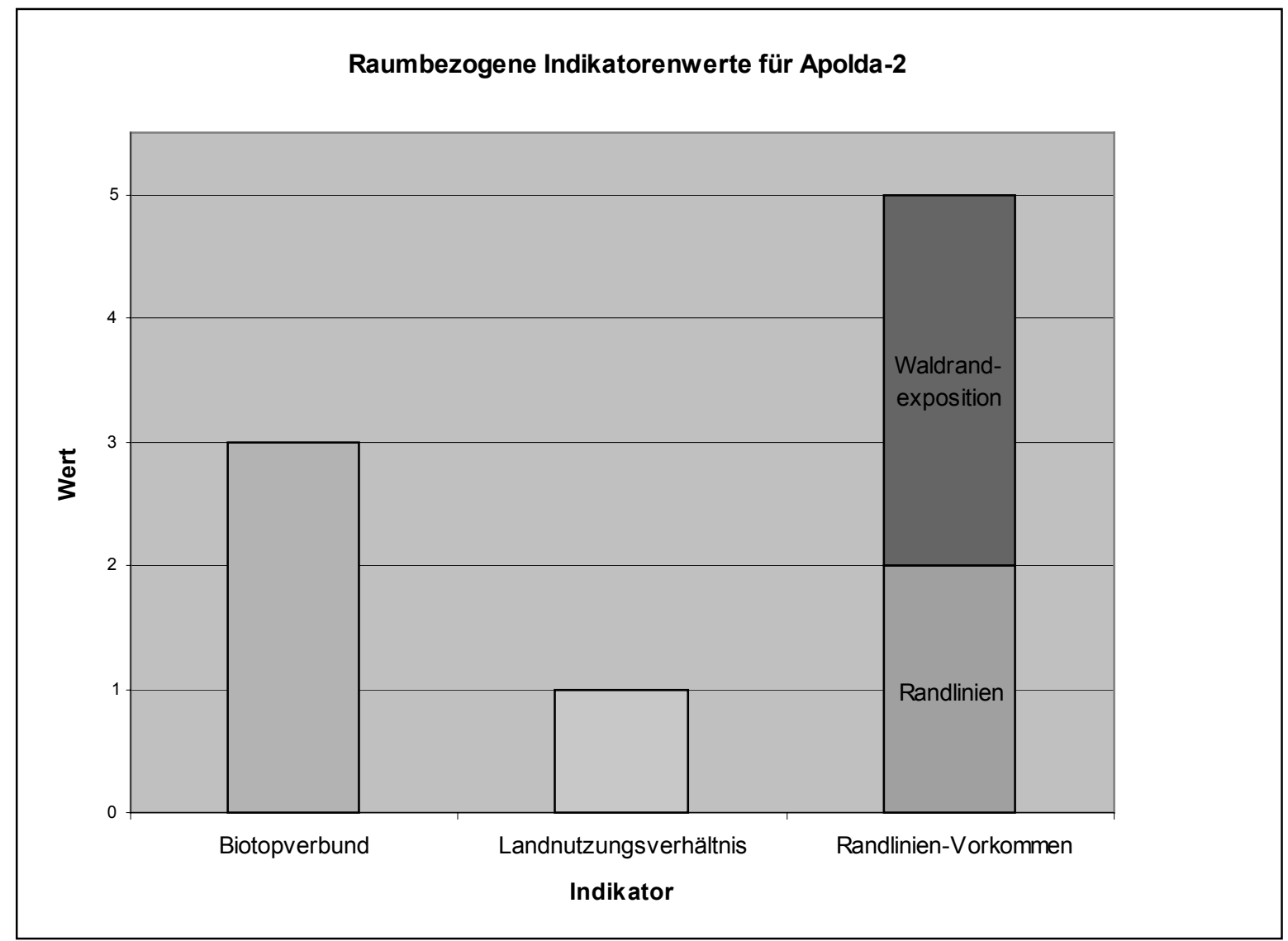

Abb. 4.2-IV: $\quad$ Raumbezogene Indikatorenwerte für Apolda-2

Der Bezugsraum ist, mit Ausnahme der Alleen, besonders arm an Randlinien, also an Übergängen zwischen unterschiedlichen Nutzungsarten. Dies drückt sich auch in der geringen Dichte von 16,59 Ifm/ha aus. Die Aufforstungsfläche selbst grenzt unmittelbar nur an andere Ackerflächen. Der betrachtete Nutzungswandel würde dementsprechend eine deutliche Anreicherung an Randlinien für die Fläche wie für die Umgebung bedeuten.

Bedingt durch die beschriebene Lage, geht kein Waldrand verloren. Stattdessen wird Waldrand jeder Exposition neu geschaffen. Daher ist von einer deutlichen Verbesserung der Randlinien-Effekte im betrachteten Bezugsraum auszugehen (Abb. 4.2-IV). 


\section{Bezugsraum Apolda-3 / Aufforstungsfläche AP-3}

Tab. 4.2-4: Daten zum Bezugsraum Apolda-3 und zur Aufforstungsfläche AP-3

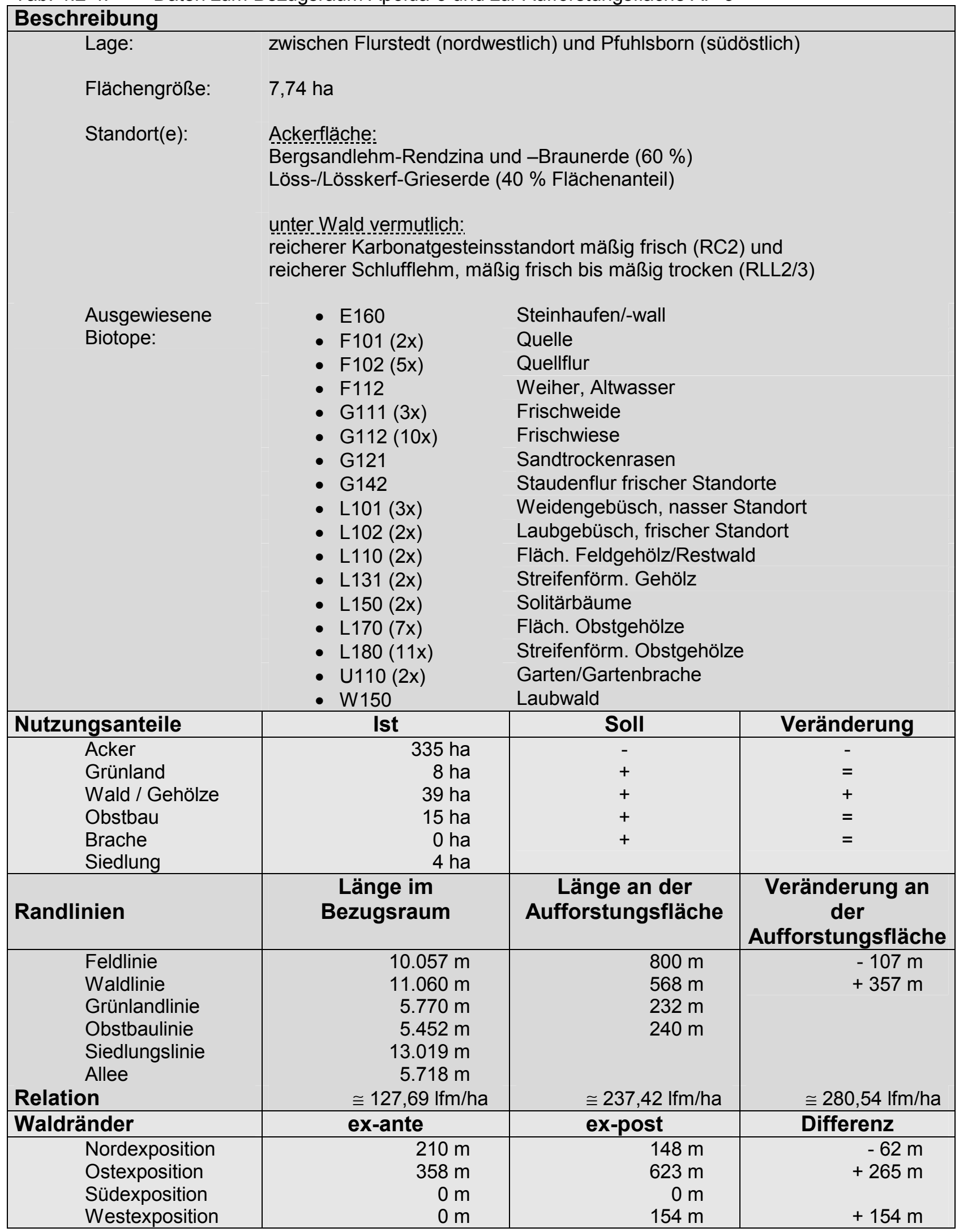

Das durch eine Aufforstung der Fläche AP-3 entstehende Waldbiotop stellt eine Ergänzung der vorhandenen Baumbestände dar. Letztere wurden im Rahmen der Waldbiotopkartierung erfasst als naturnaher Eichen-Hainbuchenwald (Restwaldfläche, südlich der Parzelle), 
kulturbestimmter Pappelwald und ebensolcher Eschenwald (westlich). Eine Bewertungsausnahme stellt eine mögliche Aufforstung mit einem Nadelholzreinbestand dar, da sich dessen Lebensraummerkmale von jenen der Laubwaldbiotope deutlich unterscheiden und deshalb nicht von einer Erweiterung dieses Biotoptyps gesprochen werden kann. Eine echte Verbundwirkung ist von der Aufforstung nicht zu erwarten, obwohl die Distanz zwischen dem südlichen und einem etwas weiter nördlichen, nicht direkt angrenzenden Rest Eichen-Hainbuchenwald durch eine entsprechende Aufforstung verringert werden könnte. Die an diese Fläche angrenzenden Streuobstbestände werden aktuell nicht gepflegt oder bewirtschaftet und sind von fortgeschrittener Verbuschung bedroht.

Geographisch liegt die Aufforstungsfläche am Oberhang und weist andere Standortsaspekte auf, als die benachbarten Flächen des Bacheinschnittes. Eine Aufforstung würde deshalb den Landschaftstyp ergänzen. Von einer Ausweitung desselben wird allerdings nicht gesprochen, da sich die Situation aus dem Luftbild heraus eher als Arrondierung darstellt, denn als Ausdehnung in ein unstrukturiertes Gebiet.

Die Anbindung an die bestehenden Gehölzbiotope und die standörtlichen Gegebenheiten versprechen eine optische Integration in das bisherige Landschaftsbild. Der Landnutzungswandel ist wiederum vollständig im Interesse des regionalen Leitbildes und der Vorgaben des ANP. Er bleibt auch für den Bezugsraum Apolda-3 ohne Auswirkung auf die Nutzungsarten Grünland, Obstbau und Brache.

Der Bezugsraum ist mit 127,69 Ifm/ha Randlinien bereits relativ reich an Ökotonen, zumindest im Vergleich zu den Flächen Apolda-1 und Apolda-2. Die Parzelle selbst verfügt sogar über fast die doppelte Randlinienlänge an Feld/Wald- und Feld/Obstbau-Ökotonen. Die Aufforstung würde diesen Reichtum aber nochmals um annähernd 20 Prozent steigern. Dabei wird die Feld/Obstbau-Grenze von einer Wald/Obstbau-Grenze ersetzt.

Auch bei der Betrachtung der Waldrandexpositionen ist eine beträchtliche Wertsteigerung feststellbar. Nach Anwendung des Bewertungsschemas aus Kap. 3.5.8 ist fast eine Verdoppelung des Wertes festzustellen.

Der Indikator Randlinien-Vorkommen erreicht somit eine Bewertung von 2 Punkten (Abb. 4.2-V). 


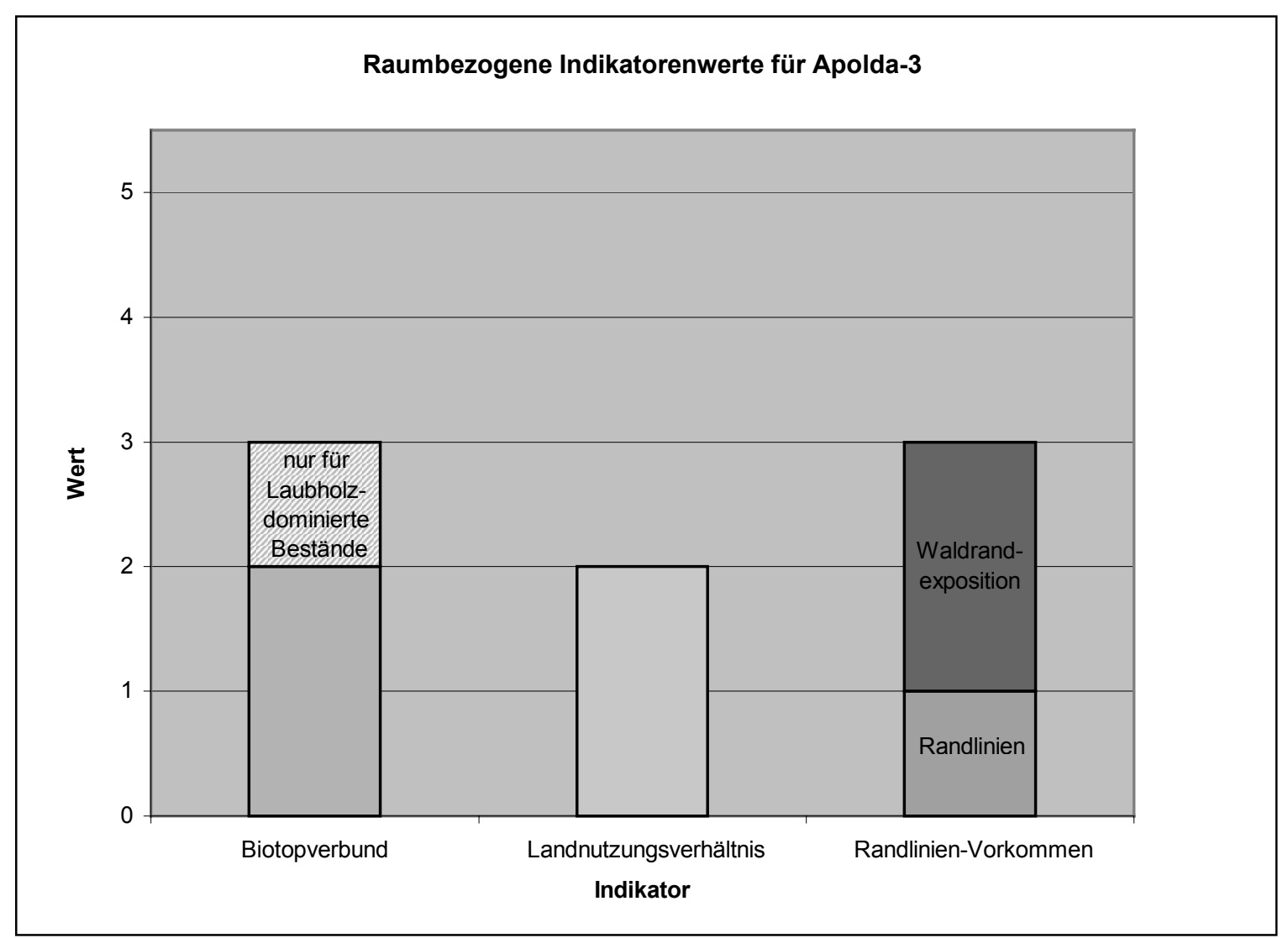

Abb. 4.2-V: Raumbezogene Indikatorenwerte für Apolda-3

\section{Bezugsraum Apolda-4 / Aufforstungsfläche AP-4}

Durch eine Aufforstung der Fläche AP-4 entstet eine Biotop, welches die westlich angrenzende Laubwaldfläche erweitert. Darüber hinaus entsteht eine Verbindung zur Allee, die den Kammweg der Erhebung begleitet. Diese Allee verknüpft ihrerseits wieder verschiedene Biotope miteinander. Deshalb trägt die Aufforstung mit einem Laubholzbestand zum Biotopverbund bei. Zusätzlich schlägt sie eine Brücke zum östlichen Gehölzbogen des Landschaftsschutzgebietes Utenbacher Schweiz. Zwar sind die Waldflächen im Falle der Durchführung noch immer $320 \mathrm{~m}$ voneinander entfernt, jedoch könnten sie leicht durch eine lineares Strukturelement oder ein Trittsteinbiotop verbunden werden. Kritischer ist die Entwicklung bei Nadelholz-dominierten Beständen zu betrachten, da diese die Biotopverbundwirkung über derart große Flächen kaum leisten können. Bei der Anlage eines Nadelreinbestandes, kann nicht von einer Erweiterung des Laubwaldbiotops gesprochen werden, da die beiden Lebensräume zu verschieden wären.

Hinsichtlich des Landschaftstyps ist nicht nur eine Ergänzung desselben festzustellen, sondern in diesem Falle auch eine tastende Ausweitung des Strukturbereiches in den wenig strukturierten Rücken hinein. Damit wird der dort vorhandene, ausgeräumte Landschaftstyp durch den halboffenen, reichstrukturierten Typ verdrängt. 
Tab. 4.2-5: Daten zum Bezugsraum Apolda-4 und zur Aufforstungsfläche AP-4

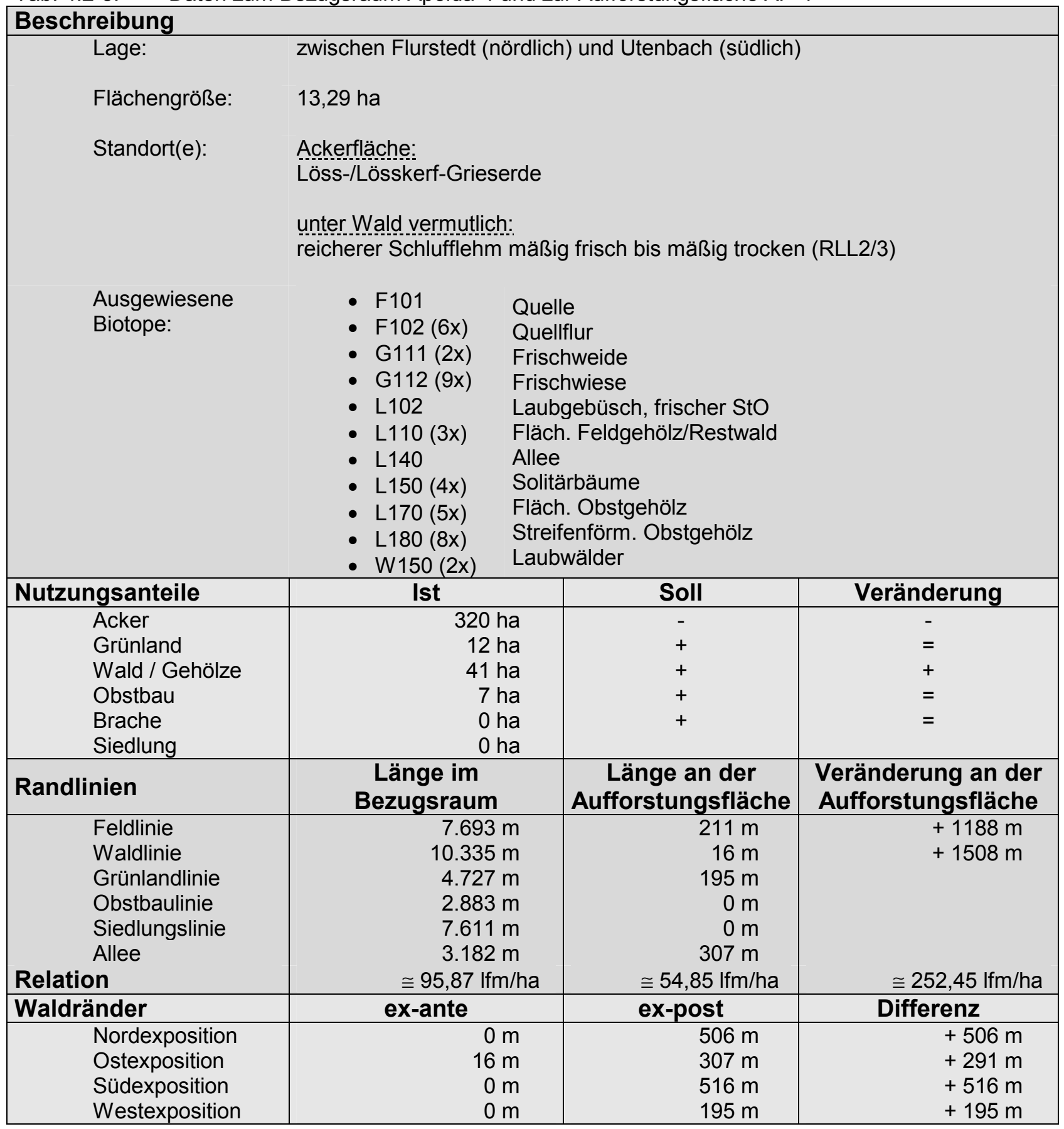

Die Bewertung der Raumgliederung erfolgt analog zu den anderen Bezugsräumen, da der Nutzungswandel lediglich Acker- und Waldfläche betrifft (Abb. 4.2-VI). Eine Beeinträchtigung des Charakters der weiträumigen Landschaft kann verneint werden, da die Angliederung an die bestehenden Bestände und im Hang selbst, nicht auf der Kuppe erfolgt, der Horizont zudem bereits durch die galerieartig wirkenden Allee beschränkt wird. 


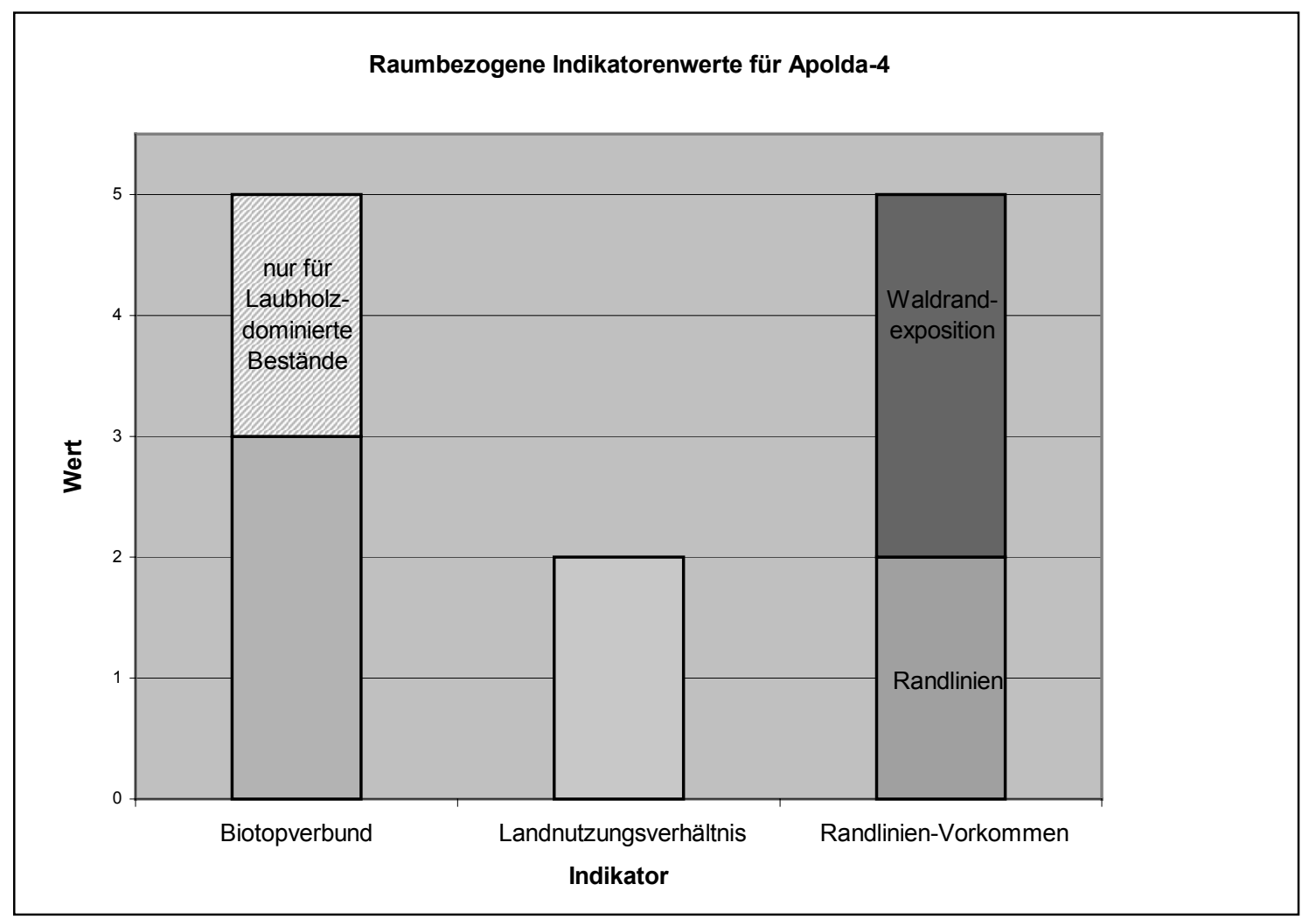

Abb. 4.2-VI: Raumbezogene Indikatorenwerte für Apolda-5

Bezüglich der Randlinien ist festzustellen, dass die Parzelle vor der Aufforstung eine Randliniendichte (Ifm/ha) besitzt, die nur etwa die Hälfte dessen beträgt, was sich für den gesamten BR Apolda-5 berechnen lässt. Nach der Erstaufforstung kehrt sich dieses Verhältnis um, da die Maßnahme den Randlinienanteil fast verfünffacht.

Ebenso deutlich stellt sich der Zugewinn an Waldrändern dar. Insbesondere der lange, südexponierte Waldrand sollte eine Bedeutung für den Artenschutz erlangen. 


\section{Bezugsraum Apolda-5 / Aufforstungsfläche AP-5}

Tab. 4.2-6: Daten zum Bezugsraum Apolda-5 und zur Aufforstungsfläche AP-5

\begin{tabular}{|c|c|c|c|}
\hline \multicolumn{4}{|l|}{ Beschreibung } \\
\hline Lage: & \multicolumn{3}{|c|}{ zwischen Niedertreba (nördlich) und Pfuhlsborn (südlich) } \\
\hline Flächengröße: & \multicolumn{3}{|l|}{13,59 ha } \\
\hline Standort(e): & \multicolumn{3}{|c|}{$\begin{array}{l}\text { Ackerfläche: } \\
\text { Löss-/Lösskerf-Grieserde }\end{array}$} \\
\hline & \multicolumn{3}{|c|}{ unter Wald vermutlich: } \\
\hline & \multicolumn{3}{|c|}{ RLL2/3 (reicherer Schlufflehm mäßig frisch bis mäßig trocken) } \\
\hline $\begin{array}{l}\text { Ausgewiesene } \\
\text { Biotope: }\end{array}$ & $\begin{array}{l}\text { - } \mathrm{E} 160 \\
\text { - } \mathrm{F} 102 \\
\text { - } \mathrm{G} 122 \\
\text { - } \mathrm{G} 132 \\
\text { - } \mathrm{G} 133(4 \mathrm{x}) \\
\text { - } \mathrm{G} 142 \\
\text { - } \mathrm{G} 143 \\
\text { - } \mathrm{L} 103 \\
\text { - } \mathrm{L} 110(3 \mathrm{x}) \\
\text { - } \mathrm{L} 131(2 \mathrm{x}) \\
\text { - } \mathrm{L} 140 \\
\text { - } \mathrm{L} 170(7 \mathrm{x}) \\
\text { - } \mathrm{L} 180(4 \mathrm{x}) \\
\text { - } \mathrm{U} 110\end{array}$ & \multicolumn{2}{|c|}{$\begin{array}{l}\text { Steinhaufen/-wall } \\
\text { Quellflur } \\
\text { Kalktrockenrasen } \\
\text { Aufgelass. Grasland, frischer Standort } \\
\text { Aufgelass. Grasland, trock. Standort } \\
\text { Staudenflur frisch. Standort } \\
\text { Staudenflur trock. Standort } \\
\text { Laubgebüsch trockenwarm. StO } \\
\text { Fläch. Feldgehölz/Restwald } \\
\text { Streifenförm. Gehölz } \\
\text { Allee } \\
\text { Fläch. Obstgehölz } \\
\text { Streifenförm. Obstgehölz } \\
\text { Garten/Gartenbrache }\end{array}$} \\
\hline Nutzungsanteile & Ist & Soll & Veränderung \\
\hline $\begin{array}{l}\text { Acker } \\
\text { Grünland } \\
\text { Wald / Gehölze } \\
\text { Obstbau } \\
\text { Brache } \\
\text { Siedlung }\end{array}$ & $\begin{array}{r}329 \text { ha } \\
7 \text { ha } \\
22 \text { ha } \\
17 \text { ha } \\
14 \text { ha } \\
13 \text { ha }\end{array}$ & $\begin{array}{l}- \\
+ \\
+ \\
+ \\
+\end{array}$ & $\begin{array}{l}- \\
= \\
+ \\
= \\
=\end{array}$ \\
\hline Randlinien & $\begin{array}{c}\text { Länge im } \\
\text { Bezugsraum }\end{array}$ & $\begin{array}{c}\text { Länge an der } \\
\text { Aufforstungsfläche }\end{array}$ & $\begin{array}{l}\text { Veränderung an der } \\
\text { Aufforstungsfläche }\end{array}$ \\
\hline $\begin{array}{l}\text { Feldlinie } \\
\text { Waldlinie } \\
\text { Grünlandlinie } \\
\text { Obstbaulinie } \\
\text { Siedlungslinie } \\
\text { Allee } \\
\text { Relation }\end{array}$ & $\begin{array}{c}9.091 \mathrm{~m} \\
5.893 \mathrm{~m} \\
1.778 \mathrm{~m} \\
4.252 \mathrm{~m} \\
7.780 \mathrm{~m} \\
2.787 \mathrm{~m} \\
\cong 78,95 \mathrm{lfm} / \mathrm{ha}\end{array}$ & $\begin{array}{r}675 \mathrm{~m} \\
603 \mathrm{~m} \\
0 \mathrm{~m} \\
72 \mathrm{~m} \\
0 \mathrm{~m} \\
0 \mathrm{~m} \\
\cong 99,34 \mathrm{lfm} / \mathrm{ha}\end{array}$ & $\begin{array}{r}+492 \mathrm{~m} \\
+564 \mathrm{~m}\end{array}$ \\
\hline Waldränder & ex-ante & ex-post & Differenz \\
\hline $\begin{array}{l}\text { Nordexposition } \\
\text { Ostexposition } \\
\text { Südexposition } \\
\text { Westexposition }\end{array}$ & $\begin{array}{r}0 \mathrm{~m} \\
0 \mathrm{~m} \\
0 \mathrm{~m} \\
603 \mathrm{~m}\end{array}$ & $\begin{array}{r}224 \mathrm{~m} \\
72 \mathrm{~m} \\
263 \mathrm{~m} \\
608 \mathrm{~m}\end{array}$ & $\begin{array}{r}+224 \mathrm{~m} \\
+72 \mathrm{~m} \\
+263 \mathrm{~m} \\
+5 \mathrm{~m}\end{array}$ \\
\hline
\end{tabular}

Die Aufforstungsfläche AP-5 verspricht die Entwicklung eines neuen Biotops, das ebenfalls den angrenzenden Laubwaldstreifen erweitern kann, soweit es überwiegend aus Laubholz aufgebaut ist. Eine Verbundwirkung geht von der neuen Waldfläche allerdings nicht aus, obwohl sie den Abstand zwischen dem bewaldeten Pfuhlsgraben und der westlich gelegenen, jungen Waldfläche deutlich verkürzt auf ca. 450 Meter. Die Anlage weiterer 
Strukturelemente zwischen den beiden und weiteren Biotopen, würde die Situation allerdings schnell verbessern.

Für den LT halboffene, reichstrukturierte Feldflur bietet der Nutzungswechsel sowohl eine Ergänzung als auch eine Erweiterung des strukturierten Raumes. Eine Zerschneidung oder Beeinträchtigung von Offenlandbiotopen besteht nicht.

Die Vermehrung der Waldfläche auf Kosten der bisher landwirtschaftlich genutzten Felder ist auch bei diesem Beispiel im Sinne des regionalen Leitbildes, ohne dem offenen Landschaftsbild zu schaden.

Die Lage der Parzelle am Rande des Pfuhlsgrabens bewirkt bereits vor der Aufforstung einen relativ (zur Umgebung) hohen Anteil an Randlinien. Diese gewinnen durch die Maßnahme noch deutlich an Länge hinzu und erreichen deutlich mehr als das Doppelte des Untersuchungsflächendurchschnitts.

Auch die Waldrandsituation ändert sich deutlich. Während der westliche Rand im wesentlichen nur ein Stück weiter nach Westen verlagert wird, kommt ein nordexponierter und vor allem auch ein gutes Stück südexponierter Waldrand hinzu. Dies führt zu einem Indikatorwert von insgesamt 4 Punkten für das Randlinien-Vorkommen ( Abb. 4.2-VII).

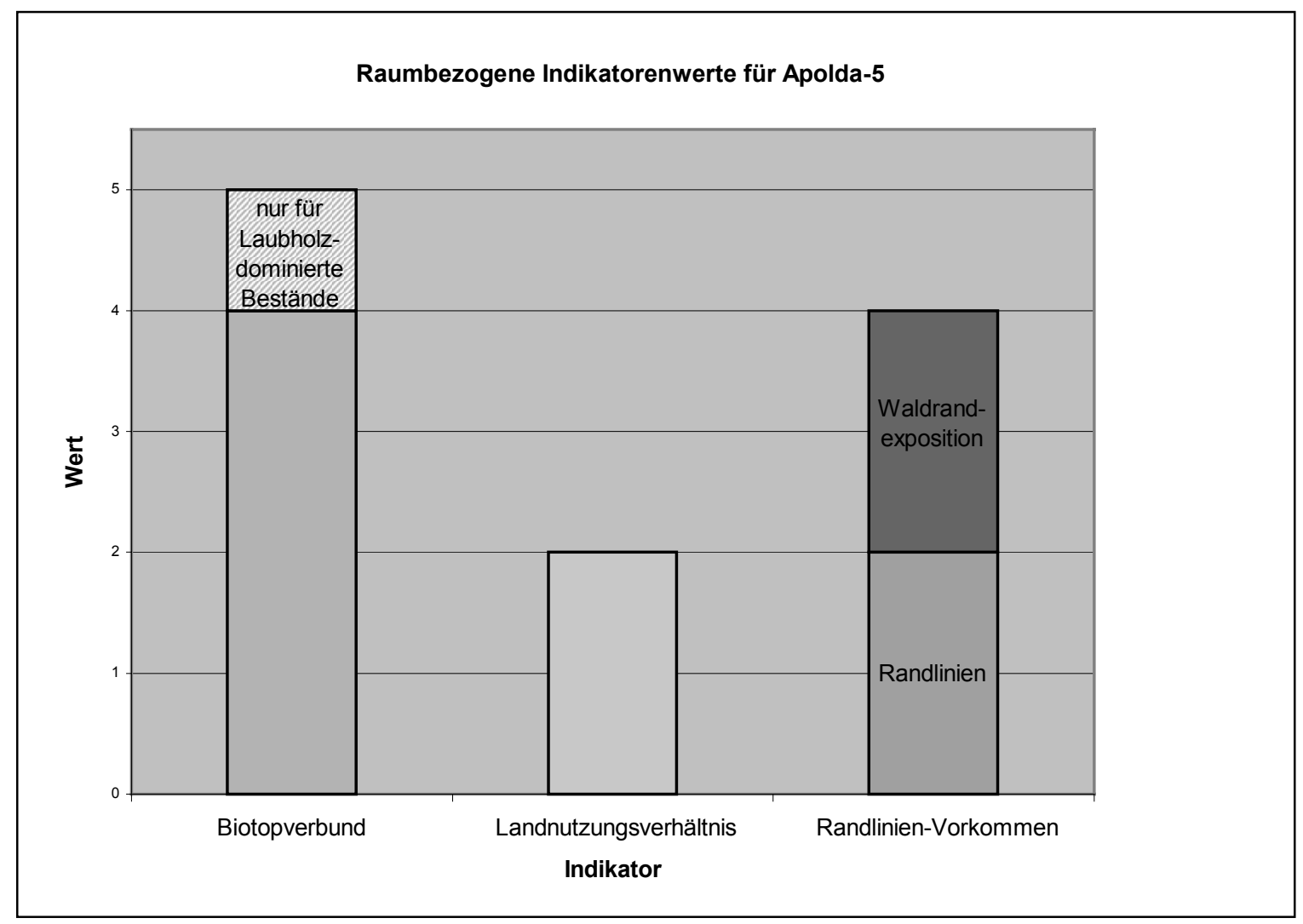

Abb. 4.2-VII: Raumbezogene Indikatorenwerte für Apolda-5

\subsection{Gesamtergebnisse für die Untersuchungsflächen in der Region Apolda}

\section{Gegenüberstellung der raumbezogenen Bewertungen}

Die Werte für die Indikatoren Biotop-Verbund, Landnutzungsverhältnis und RandlinienVorkommen ergeben zusammen die Eignung der unterschiedlichen Aufforstungsflächen für einen Nutzungswandel. Hinsichtlich des Landnutzungsverhältnisses sind wenig Unterschiede 
zu erwarten, da stets vom gleichen Nutzungswandel - Acker zu Wald - ausgegangen wird und die Flächennutzungsvorgaben der übergeordneten Pläne für alle Untersuchungsflächen gelten.

Die Bewertung des Biotop-Verbunds zeigt, dass alle behandelten Aufforstungsvarianten zu einem wertvollen Biotop, zumindest in Relation zur intensiven landwirtschaftlichen Nutzung führen. Bei der Betrachtung der Biotopergänzung und -verknüpfung präsentiert sich der „EAZT Vorwald Nadelbaum“ als Sonderfall. Der Laubholzanteil an der Bestockung im Untersuchungsgebiet dominiert deutlich den Nadelholzanteil. Typische Nadelwaldbiotope fehlen, während Laubgehölze und -wälder in mehr oder weniger anthropogen überprägter Zusammensetzung häufig sind. Aus diesem Grunde können Aufforstungen mit (überwiegend) Laubholz die bestehenden Biotope besser ergänzen und - in einem Fall sogar räumlich wie funktionell verknüpfen.

Davon abgesehen sind Aufforstungen prinzipiell geeignet, den LT halboffene, reichstrukturierte Felder zu ergänzen. Dies gilt, zumindest unter den im Untersuchungsgebiet vorgefundenen Bedingungen, auch für Nadelholzbestände. Die Erweiterung des Landschaftstyps in offene, unstrukturierte Bereiche hinein ist allein von der aktuellen Strukturierung und der Lage der Fläche abhängig. Die Luftbilder geben aufgrund der fehlenden Tiefe nur einen unvollständigen Eindruck der Situation wieder. Derzeit sind Gehölze vornehmlich in den Senken und Bachtälchen zu finden. Die Erhöhungen bilden dagegen ein Biotopmosaik mit Alleen, Heckenreihen und wenig Grünland. Neue Waldflächen können die Verbindung zwischen den Biotopen optisch wie funktional herstellen. Dadurch dehnt sich der Landschaftstyp in der Fläche aus. Für die Bezugsräume Apolda-2, -4 , und -5 scheint dies prognostizierbar.

Die Aufforstung einer landwirtschaftlichen Fläche führt zur Schaffung neuer Ökotone. Je mehr gleichartig bewirtschaftete Flächen an die Aufforstungparzelle angrenzen, desto mehr neue Randlinien entstehen. Umgekehrt kann allerdings nicht geschlossen werden, dass in einer strukturreichen und somit Ökoton-reichen Landschaft ein Nutzungswandel keine nennenswerte Zunahme an solchen Übergangslinien schafft. Ohne dass die Untersuchung statistische Ergebnisse liefern möchte, zeigen die fünf Beispielfälle doch, dass es in einem ungegliederten Landschaftsteil zwar häufig, aber nicht immer zu einer bedeutsamen Steigerung an Ökotonen kommt. Vielmehr ist die unmittelbare, örtliche Grenzsituation, bzw. die Integration der Aufforstung in den umgebenden Biotopkomplex entscheidend.

Auch in Bezug auf den vorkommenden und neugeschaffenen Waldrand ist die spezielle Aufforstungssituation maßgebend für die naturschutzfachliche Bewertung. Die höhere Wertschätzung für südlich und westlich exponierte Waldränder schafft dem Grundeigentümer mehr Möglichkeiten, den Artenschutz schon bei der Planung zu berücksichtigen. Abb. 4.3-I zeigt, dass in den vorliegenden Untersuchungen die Aufforstungen stets zu einem Gewinn an Biotopwerten durch einen hohen Anteil süd- und westexponierter, neuer Waldränder führen. 


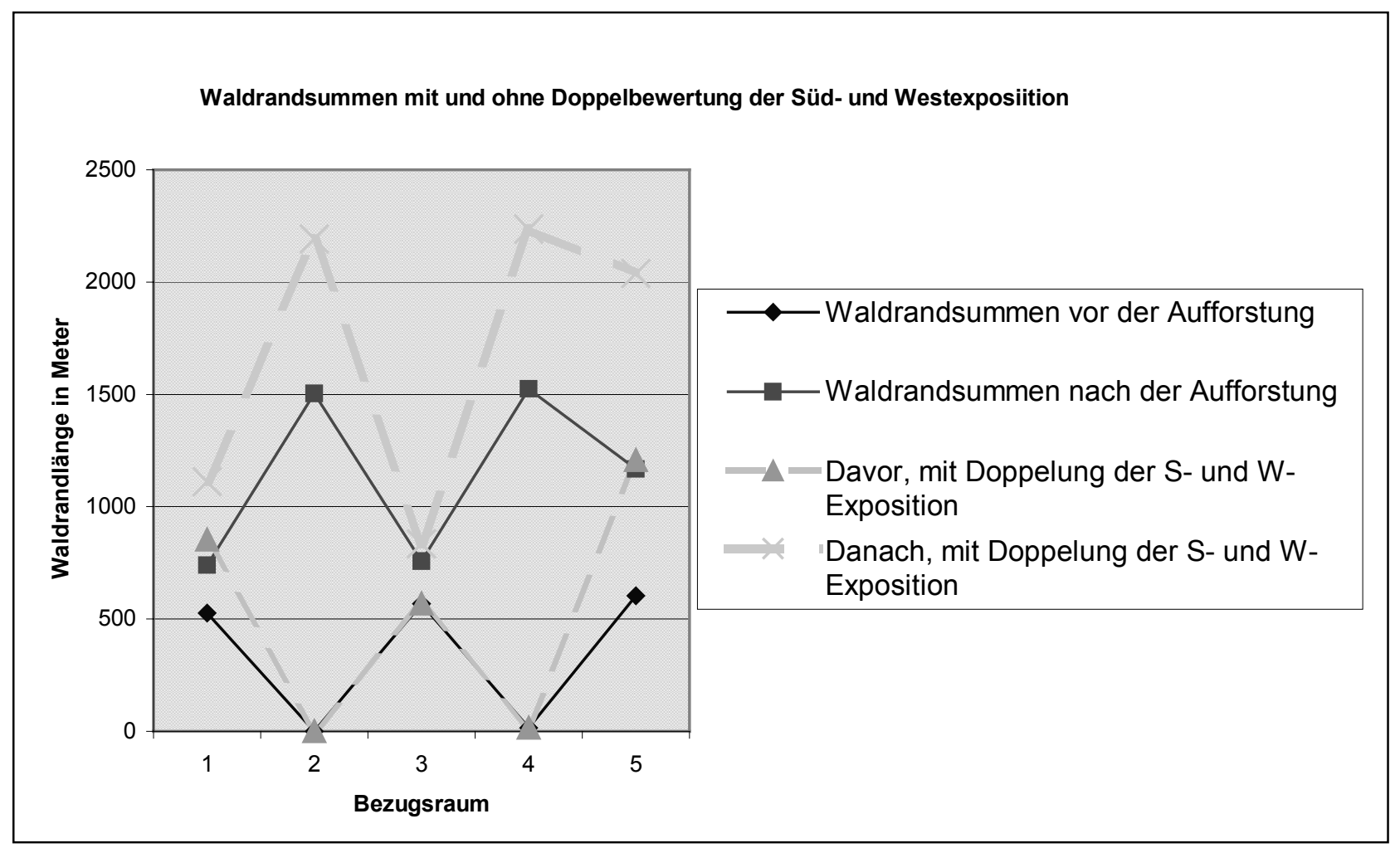

Abb. 4.3-I: Indikatorwerte für die Waldrandlänge; Vergleich vor und nach der Aufforstung; mit und ohne doppelter Gewichtung der Süd- und West-exponierten Waldränder

Wie im Methodikteil angekündigt (vg. Kap. 3.5.8) ist das angewandte Bewertungsschema für die Waldrandlinienlänge zu überprüfen. Insbesondere die doppelte Wertung der Süd- und West-exponierten Waldränder, welche aufgrund deren besonderer ökologischen Wertschätzung eingeführt wurde, ist kritisch zu betrachten. Die Ergebnisse für die Aufforstungsflächen im USG Apolda zeigen, dass die Verdoppelung sich teilweise verstärkend, teilweise vermindernd auf die Differenzbeträge im Vorher-Nachher-Vergleich der Waldrandlänge auswirkt. Die Indikatorenwerte verändern sich in den betrachteten Fällen jedoch nicht. Die qualitative Unterscheidung zwischen S-/W-exponierten und N-/Oexponierten Waldrändern bringt auf den ersten Blick keinen Vorteil. Dieses Ergebnis ist aufgrund der kleinen Stichprobenmenge nicht abgesichert und kann nur als Hinweis gewertet werden.

\section{Gesamtbewertung durch Kombination der flächen- und der raumbezogenen Indikatoren}

Erwartungsgemäß zeigen die Ergebnisse für die einzelnen Aufforstungsflächen ähnliche Verhältnisse (Anhang 4). Innerhalb einer Aufforstungsparzelle unterscheiden sich die erzielten Werte der einzelnen Varianten nach den im Kapitel 4.2.1 dargelegten Merkmalen. Dabei wurden der Einfachheit halber jene Aufforstungstypen gewählt, die für den vorherrschenden Standortstyp einer Parzelle vorgesehen sind. Da die Standorte verwandt sind und die gleiche natürliche Waldgesellschaft erwarten lassen, ist dieses Vorgehen als vertretbar und praxisnah zu bezeichnen. Zwei Standorte (RLL2 und RC2) kommen lediglich auf den Parzellen Apolda-1 und Apolda-3 vor, wobei bei ersterer der Schlufflehm-Standort überwiegt, während es bei der zweiten umgekehrt ist. Eine deutliche Unterscheidung des naturschutzfachlichen Wertes der Erstaufforstungen ist für alle Flächen zu konstatieren. Die Punktesummen reichen von 13 bis 33 Punkte. Abb. 4.3-II zeigt, dass der Spielraum des Grundeigentümers auf den Untersuchungsflächen in Apolda sehr groß ist. Teilweise kann die Punktzahl durch eine naturgemäße und vielfältige Mischung in Zusammenhang mit der Anlage eines strukturreichen Waldrandes zu einer Verdoppelung der naturschutzfachlichen Bewertung führen. So kann selbst der unter Gesichtspunkten des Raumbezuges und der 
Biotopvernetzung noch „ungünstigste“ Standort so aufgewertet werden, dass die Maßnahme mit manchen Szenarien auf günstiger gelegenen Flächen konkurrieren kann.

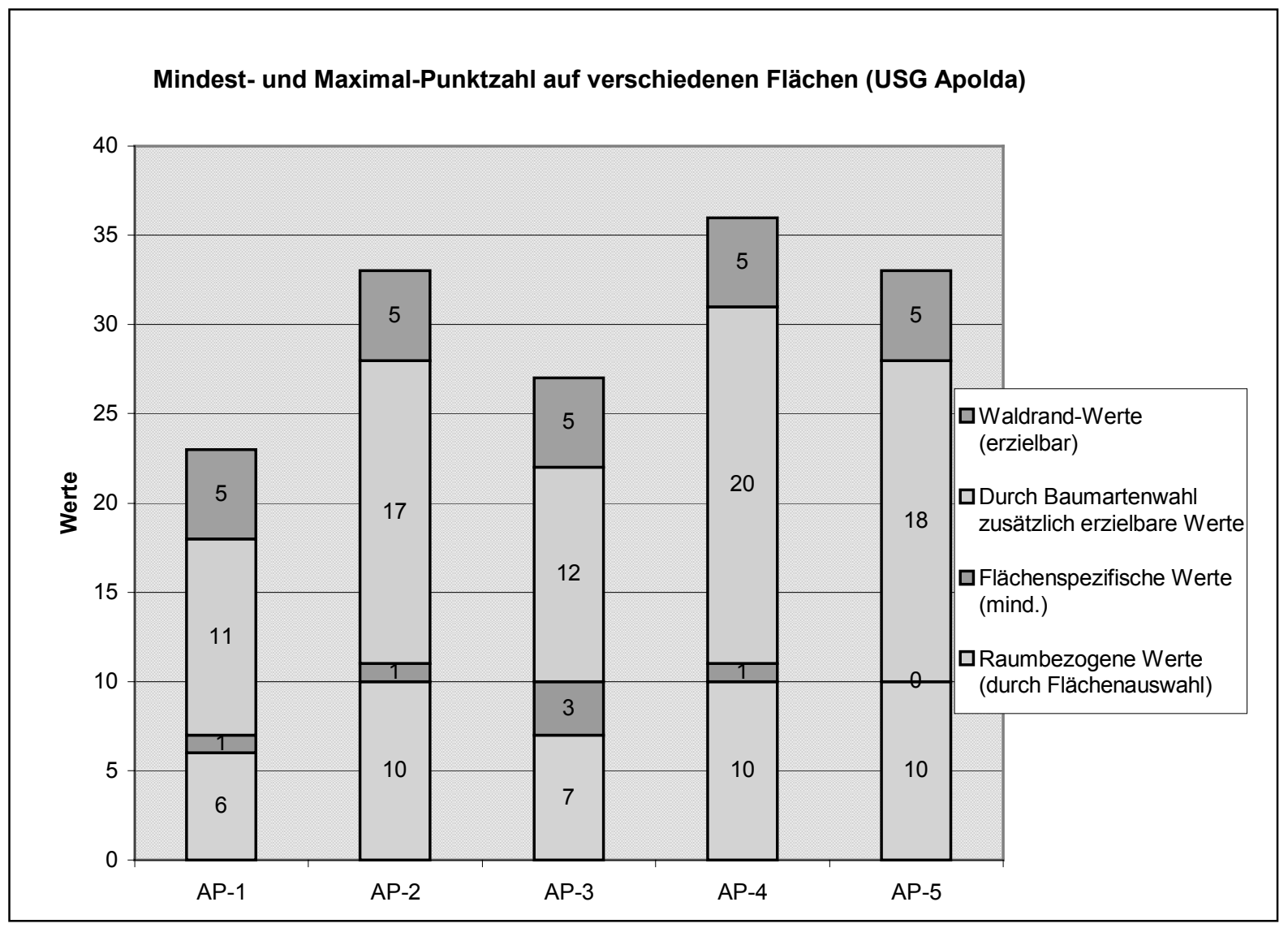

Abb. 4.3-II: Erreichbare Gesamtbewertungen auf den einzelnen Aufforstungsflächen (USG Apolda) 


\section{Ergebnisse im USG 2 - Region Dassel (Niedersachsen)}

\subsection{Planungsgrundlagen}

\section{Lage und Landschaft}

Das Untersuchungsgebiet 2 befindet sich im Nordwesten des Landkreises Northeim und erstreckt sich von dessen Verwaltungsgrenze am Kahle-Berg (bei Lüthorst) in östlicher Richtung bis nach Vardeilsen und Avendshausen (Abb. 5.1-I). Es stellt geometrisch betrachtet ein Rechteck dar, mit einer Kantenlänge von ca. $7 \mathrm{~km}$ (als ungefähre N-S-Achse) und $9 \mathrm{~km}$ (als ungefähre O-W-Achse).

Das Untersuchungsgebiet umfasst mehrere kleine Erhebungen, die sich als Band in OstWest-Richtung hinziehen. Außerdem gehören im wesentliche Teile der Hügelkette Elfas (im Norden) und der Amtsberge (im Westen) an.

Der tiefste Punkt des Untersuchungsgebietes liegt mit ca. $145 \mathrm{~m} \mathrm{NN}$ im Südwesten, südlich von Vardeilsen an den in der Niederung der Ilme. Der Helleberg ist mit 409,6 m NN die höchste Erhebung der Hügelkette Elfas und stellt damit auch den höchsten Punkt des Untersuchungsgebietes dar.

Nach der naturräumlichen Gliederung gehört der betrachtete Landschaftsteil zur Region „Weser- und Leinebergland“, darin zur Einheit „Sollingvorland“. Es weist eine hohe geomorphologische Vielfalt auf, die durch den Wechsel von Becken und Senken mit Erhebungen und Hochflächen geprägt wird. Während in den Beckenlagen die landwirtschaftliche Nutzung dominiert, zeigen die meisten Erhebungen eine Bewaldung im Bereich der Hochflächen und Kuppen. Für den Betrachter wird der Horizont an den meisten Beobachtungspunkten von Wald gesäumt. Die Unterbrechung der Waldflächen ist visuell kaum feststellbar. Erst in der kartographischen Darstellung und im Luftbild zeigt sich die Insellage der kleineren Erhebungen und damit auch der darauf stockenden Gehölzgruppen oder Wälder.

Die geomorphologischen Bedingungen stellen die Grundlage für eine gewisse Vielfalt an landschaftsökologischen Einheiten dar. Im Elfas und am südlich gelegenen Auls-Berg steht Buntsandstein an, während die übrigen Erhebungen dem Kalkgestein zuzuordnen sind. In den Senken und an den Unterhängen des Berglandes werden die Standortsbedingungen von einer Lößüberdeckung dominiert. Azonale Gesellschaften finden ihren Raum in den Bachniederungen der Bewer und ihrer Seitenarme (Reißbach, Allerbach, u.a.), die südlich des USG bei Markoldendorf in die IIme mündet.

\section{Geologie und Böden}

Das Untersuchungsgebiet besitzt eine landschaftliche und geologische Vielfalt, die auf einen mitunter komplizierten geologischen Aufbau hinweist. Der Naturraum Sollingvorland erscheint als wechselvoll gestaltete Schichtstufenlandschaft. Lössbedeckte Becken und Senken liegen im ständigen Wechsel mit Hügeln und Bergen aus mesozoischen Kalk- und Sandsteinen, im USG speziell Muschelkalk und Buntsandstein.

Der Leinegraben, an dessen nördlichem Ende das USG liegt, ist Teil einer Verwerfungszone und verursachte die Absenkung der Sedimentschichten und das Einbrechen der benachbarten Schollen (Göttinger Wald, Solling) zum Graben hin. Das Ergebnis der tektonischen Vorgänge ist in einer weiten, flachen Tallandschaft zu sehen. Die Keuperschichten im Untergrund sind von mehrschichtigem Löss überlagert (AK STOK 1985).

\section{Natürliche Waldgesellschaften}

Das USG gehört nach der forstlichen Einteilung zum Wuchsgebiet Südniedersächsisches Bergland. Der größte Teil des Untersuchungsgebietes gehört nach der weiteren Untergliederung in Wuchsbezirke zur Leine-Ilme-Senke. Lediglich der westlichste Teil, etwa 
bis zum Wende-Berg gehört noch zum Solling-Vorland. Da sich alle Aufforstungsflächen aber auf die, für die Leine-Ilme-Senke typischen, vereinzelten Hügel konzentrieren, reicht die Beschreibung des bedeutenderen Wuchsbezirkes aus. Die Leine-Ilme-Senke stellt den wärmsten und am stärksten kontinental getönten Wuchsraum innerhalb der südniedersächsischen Berglandschaften dar. Die Jahresniederschlagssumme liegt im USG mit ca. 800-850 mm deutlich unter jenen des südwestlich gelegenen Hochsollings (bis 1050 $\mathrm{mm}$ ). Die vorkommenden Höhenstufen können planar bis kollin angegeben werden. Nach der Wuchsgebietsbeschreibung sind die Waldreste auf den Randhügeln des Leinegrabens den anspruchsvolleren Traubeneichen-Buchenwald-Gesellschaften zuzuordnen. In den heute großflächig landwirtschaftlich genutzten Tallagen waren dagegen EichenmischwaldGesellschaften bis zum Hainbuchen-Stieleichenwald verbreitet (AK STOK 1985). Bedingt durch die im Verhältnis zum restlichen Wuchsgebiet noch recht hohen Niederschläge, dürften im Bereich Elfas auch noch die für das Solling-Vorland typischen, reicheren Buchenwaldgesellschaften eine Rolle spielen.

Die potentielle natürliche Vegetation im Untersuchungsgebiet und im Landkreis Northeim (ohne die landschaftsökologische Einheit der Talniederungen größerer Flüsse) wird in Tab. 5.1-1 detailliert aufgeschlüsselt. Im Rahmen der vorgenommenen Untersuchungen sind lediglich die Verhältnisse der landwirtschaftlich bewirtschafteten, durch Lössbedeckung geprägten Böden sowie der Erhebungen aus Muschelkalk, bzw. Buntsandstein relevant. Hier können im Einzelnen Perlgras- und Flattergras-Buchenwald (auf Lössboden), HainsimsenBuchenwald (Buntsandstein-Erhebungen) und Perlgras-Buchenwälder (auf MuschelkalkErhebungen) erwartet werden. Mancherorts stellen diese Pflanzengesellschaften auch die reale Vegetation dar und sind dann als natürliche Waldgesellschaft besonders schützenswert. Darüber hinaus bilden sie wertvolle Ausgangsbiotope für eine Neuwaldentwicklung, da sie einen wichtigen Beitrag zur Besiedelung durch Waldarten leisten.

Im Sinne des Naturschutzes verdient die Sicherung möglichst großer repräsentativer Bestände des Perlgras- und Hainsimsen-Buchenwaldes in seinen verschiedenen Ausprägungen eine besondere Beachtung (RROP NOM 1996). Dies schließt eine Förderung der Biotopquantität durch Flächenausdehnung im Rahmen von Erstaufforstungen ein. Daneben schützt das Gesetz Wälder und Gebüsche trocken-warmer Standorte (§ 28a NNatG), wie sie auch vereinzelt im USG auftreten könnten.

Im Untersuchungsgebiet selbst wurden nur wenige Waldflächen als geschützte Biotope nach $\S 28$ a NNatG ausgewiesen. Dazu gehört der Oberlauf der Bewer als naturnaher Bachlauf mit wertvoller Begleitvegetation (Waldgebiet Elfas) sowie der Biotopkomplex Kurzeberg / Dickenberg (Gmd. Rengershausen). Letzteren beschreibt die Biotopkartierung als „Kalkrücken mit artenreichem Laubmischwald, teilweise ehemaliger Nieder- u. Mittelwald, totholzreich und mit Vorkommen gefährdeter Pflanzenarten“. Die Freiflächen dieses Biotopkomplexes wurden gleichzeitig im Rahmen der Waldfunktionenkartierung als "von Wald freizuhaltende Flächen" kartiert.

Bemerkenswert ist außerdem ein Biotop, welches bei Vardeilsen im Süd-Osten unmittelbar an das USG angrenzt. Dabei handelt es sich um einen Perlgras-Buchenwald mit Orchideenvorkommen. Orchideen-Buchenwälder können kaum geplant werden und die Einbringung von Orchideen bei der Neuanlage von Wald stellt keine übliche Maßnahme dar. Dennoch gibt die Existenz des genannten Biotops einen Hinweis auf das standörtliche Potential ähnlicher Flächen des USG. Vergesellschaftet mit dem Perlgras-Buchenwald kommt, in trockeneren Bereichen, der Seggen-Buchenwald vor. Dieser Orchideen-reiche Waldtyp stößt in dieser Region an die nördliche Grenze seines Verbreitungsgebietes (FISCHER 1995). 
Tab. 5.1-1: Die potentielle natürliche Vegetation (PNV) im Untersuchungsraum Dassel und ihre forstlichen Ersatzgesellschaften

\begin{tabular}{|c|c|c|c|c|}
\hline $\begin{array}{l}\text { Ökologische } \\
\text { Landschafts- } \\
\text { einheit }\end{array}$ & Geologie & Böden & PNV & $\begin{array}{c}\text { forstliche } \\
\text { Ersatz- } \\
\text { gesellschaften }\end{array}$ \\
\hline $\begin{array}{c}\text { Staufeuchte } \\
\text { Mulden der } \\
\text { Lößgebiete, } \\
\text { Tongruben und } \\
\text { Bach- } \\
\text { niederungen }\end{array}$ & $\begin{array}{l}\text { Mesozoische } \\
\text { Tone, Mergel } \\
\text { (Röt, Jura, } \\
\text { Kreide) }\end{array}$ & $\begin{array}{l}\text { Pseudogley, } \\
\text { Gley, } \\
\text { Pseudogley- } \\
\text { Pelosol } \\
\text { (mäßig bis gut } \\
\text { basenversorgt) }\end{array}$ & $\begin{array}{l}\text { Feuchter Eichen- } \\
\text { Hainbuchenwald (Stellario- } \\
\text { Carpinetum); } \\
\text { Bach-Erlen-Eschenwald } \\
\text { (Carici remotae-Fraxinetum) } \\
\text { entlang der Bäche; } \\
\text { Weiden-Auwald (Salicion } \\
\text { albae) an größeren Bächen } \\
\text { und Hainmieren-Erlenwald } \\
\text { (Stellario-Alnetum) in } \\
\text { Quellgebieten }\end{array}$ & $\begin{array}{l}\text { forstlich: keine, } \\
\text { bzw. selten } \\
\text { Pappel-, Erlen-, } \\
\text { Eschenwälder } \\
\text { u.a. }\end{array}$ \\
\hline $\begin{array}{l}\text { Lössgebiete in } \\
\text { Senken und } \\
\text { auf } \\
\text { Unterhängen } \\
\text { des } \\
\text { Berglandes }\end{array}$ & $\begin{array}{c}\text { Kolluviale } \\
\text { Löss- } \\
\text { ablagerungen }\end{array}$ & $\begin{array}{c}\text { Parabraunerde, } \\
\text { Pseudogley, } \\
\text { seltener } \\
\text { Braunerde } \\
\text { (mäßig bis gut } \\
\text { basenversorgt) }\end{array}$ & $\begin{array}{l}\text { Perlgras-Buchenwald (Melico- } \\
\text { Fagetum); } \\
\text { Flattergras-Buchenwald } \\
\text { (Milio-Fagetum) }\end{array}$ & $\begin{array}{c}\text { Kleinflächig } \\
\text { Fichtenwälder } \\
\text { u.a. }\end{array}$ \\
\hline $\begin{array}{l}\text { Hügel und } \\
\text { Berge aus } \\
\text { Sandgestein }\end{array}$ & $\begin{array}{l}\text { Erhebungen } \\
\quad \text { aus } \\
\text { Sandgestein } \\
\text { (Buntsand- } \\
\text { stein, Keuper) } \\
\text { ohne oder mit } \\
\text { dünner } \\
\text { Lössdecke } \\
\end{array}$ & $\begin{array}{c}\text { Ranker, } \\
\text { oligotrophe } \\
\text { Braunerde, } \\
\text { Podsol, } \\
\text { Pseudogley und } \\
\text { Übergangstypen }\end{array}$ & $\begin{array}{l}\text { Hainsimsen-Buchenwälder } \\
\text { (Luzulo-Fagion); seltener } \\
\text { Eichen-Buchenwald (Fago- } \\
\text { Quercetum molinietosum); } \\
\text { bodensaure } \\
\text { Eichenmischwälder (Luzulo- } \\
\text { Quercetum petreae) auf } \\
\text { Sandsteinklippen }\end{array}$ & $\begin{array}{l}\text { Eichen-Hude- und } \\
\text { Niederwälder } \\
\text { (historische } \\
\text { Nutzungsformen), } \\
\text { Fichtenwälder, } \\
\text { Kiefernwälder, } \\
\text { Lärchenwälder, } \\
\text { Douglasienwälder }\end{array}$ \\
\hline $\begin{array}{l}\text { Hügel und } \\
\text { Berge aus } \\
\text { Kalkgestein }\end{array}$ & $\begin{array}{l}\text { Erhebungen } \\
\text { aus } \\
\text { Kalkgestein } \\
\text { (Muschelkalk, } \\
\text { Jura) ohne } \\
\text { oder mit } \\
\text { dünner } \\
\text { Lössdecke }\end{array}$ & $\begin{array}{c}\text { Rendzina, } \\
\text { Braunerde- } \\
\text { Rendzina, } \\
\text { seltener } \\
\text { eutrophe } \\
\text { Braunerde }\end{array}$ & $\begin{array}{l}\text { Perlgras-Buchenwälder } \\
\text { (Melico-Fagetum elymetosum, } \\
\text { allietosum, u.a.); } \\
\text { kleinflächig Seggen- } \\
\text { Buchenwald (Carici-Fagetum), } \\
\text { Ahorn-Eschen-Schluchtwald } \\
\text { (Aceri-Tilietum) und Eichen- } \\
\text { Elsbeerenwald (Lithospermo- } \\
\text { Quercetum); kleinflächig } \\
\text { Felsvegetation (Seslerion } \\
\text { variae, Galeopsietum } \\
\text { angustifoliae, u.a.) }\end{array}$ & $\begin{array}{l}\text { Eichen- } \\
\text { Hainbuchen- } \\
\text { Nieder- und } \\
\text {-Mittelwälder } \\
\text { (historische } \\
\text { Nutzungsformen), } \\
\text { Fichtenwälder, } \\
\text { Eschenwälder, } \\
\text { Kiefernwälder }\end{array}$ \\
\hline
\end{tabular}

Weitere geschützte Biotope innerhalb des betrachteten Raumes sind in erster Linie Halbtrockenrasen auf Kalkböden, häufig begleitet von Trockengebüschen oder in einem Falle auch vergesellschaftet mit Eichen-Hainbuchenwald. Im Westen, zwischen Amtsberge und Wende-Berg, treten Kalkquellsümpfe mit natürlicher Begleitvegetation auf, die als geschützte Bereiche von Bewaldung freizuhalten sind.

Neben den geschützten Biotopen und den darin enthaltenen Waldgesellschaften, sind weitere Waldtypen als vorrangig schutz- und entwicklungsbedürftig zu bezeichnen (RROP NOM 1996). Diese sind bei der Baumartenwahl im Rahmen von Aufforstungen möglichst zu berücksichtigen. Sie beinhalten

- Perlgras-Buchenwälder mittlerer Standorte (Melico-Fagetum)

- Eichen-Hainbuchenwälder mittlerer Standorte (Carpinion betuli)

- bodensaure Eichenmischwälder (Luzulo-Quercetum)

- (bodensaure) Hainsimsen-Buchenwälder (Luzulo-Fagetum)

- frischen Kalkbuchenwald (Elymo-Fagetum) 
- frischen Eichenwald auf Kalk

- trockenen Kalkbuchenwald (Seggen-Buchenwald - Carici-Fagetum)

- trock. Eichenmischwald auf Kalk (Eichen-Elsbeerenwald - Lithospermo-Quercetum)

- Ahorn-Lindenwälder trockener Felsschutthänge und Schluchten (Aceri-Tilietum)

- Erlen-Eschenwälder der Auen

- Erlen-Bruchwälder (Carici elongatae-Alnetum) und

- Birken-Bruchwälder

Eine generelle Aufforstungsempfehlung kann mit Bezug zum USG für Buchen- und EichenMischwälder ausgesprochen werden. Zusätzlich stellt die Förderung von trockenwarmen Gebüschen im Rahmen der Waldrandgestaltung ein Instrument zur Erhöhung des ökologischen Landschaftswertes dar.

Für die hier zu behandelnden Aufforstungsflächen werden die Baumarten der natürlichen Waldgesellschaften festgestellt. Als Anhaltspunkt dient die Kurzbeschreibung der natürlichen Waldgesellschaften Deutschlands (AK Forstl. Landespflege 1996) (Tab. 5.1-2). Detailliertere Aufstellungen könnten diese Quelle ersetzen, sind jedoch für den kollinen bis montanen Bereich Niedersachsens derzeit noch in Bearbeitung (SCHARPER, Nds. Forstplanungsamt, mdl., 2002).

Tab. 5.1-2 Die den Aufforstungsflächen zuzuordnenden natürlichen Waldgesellschaften mit Haupt- und Nebenbaumarten (Quelle: AK FORSTL. LANDESPFLEGE1996)

\begin{tabular}{|c|c|c|}
\hline $\begin{array}{c}\text { potentiell natürliche } \\
\text { Waldgesellschaft }\end{array}$ & Haupt-BA & Neben-BA \\
\hline $\begin{array}{c}\text { Perlgras-Buchenwald } \\
\text { (Melico-Fagetum) }\end{array}$ & $\mathrm{Bu}$ & $\begin{array}{c}\text { BAh, Es, BUI, Ei, HBu, Ta, Fi, } \\
\text { Eberesche, Aspe, Bi }\end{array}$ \\
\hline $\begin{array}{c}\text { Seggen-Buchenwald } \\
\text { (Carici-Fagetum) }\end{array}$ & $\mathrm{Bu}$ & $\begin{array}{c}\text { BAh/SAh, Es, BUI, SLi, TEi, Wild- } \\
\text { Obst, Eibe, FAh, Mehlb }\end{array}$ \\
\hline $\begin{array}{c}\text { Hainsimsen- } \\
\text { Buchenwald } \\
\text { (Luzulo-Fagetum) }\end{array}$ & $\mathrm{Bu}, \mathrm{TEi}$, (SEi) & $\begin{array}{c}\mathrm{Ah}, \mathrm{Li}, \mathrm{HBu}, \mathrm{Fi}, \mathrm{Ta}, \mathrm{Kie}, \mathrm{Bi}, \\
\text { Eberesche }\end{array}$ \\
\hline
\end{tabular}

\section{Heutige Nutzung}

Im Landkreis Northeim dominiert die Landwirtschaft mit $52 \%$ der Flächennutzung das Landschaftsbild. Mit immerhin $37 \%$ Waldanteil, erreicht die Bewaldung einen höheren Anteil als im Bundes- $(30 \%)$ und Landesdurchschnitt (23 \%). Hinsichtlich der Struktur landwirtschaftlicher Betriebe ist auch in diesem Landkreis eine Tendenz zu flächenstarken Betrieben zu erkennen, während kleine Betriebe zahlenmäßig stark abnehmen.

Die Zielsetzung der Raumplanung (RROP NOM 1996) sieht eine Erhaltung der landwirtschaftlichen Struktur zur Sicherung der besonderen Funktionen dieser Flächennutzung vor. In diesem Zusammenhang wird die Entwicklung von Förderungsinstrumenten für den ökologischen Landbau gefordert. Eine agrarstrukturelle Neuordnung ist im USG weder im Gange noch geplant. Daher sind für die unmittelbare Zukunft keine gravierende Eingriffe in die Betriebs- und Flächenstruktur zu erwarten. Lediglich im Rahmen von flächenbeanspruchenden Großvorhaben (Wasserwirtschaft, Straßenbau, Bahnstrecken-Ausbau) kommt es im Landkreis zu einzelnen, flurordnerischen Neuordnungen.

Bei den landwirtschaftlichen Nutzungen dominiert im Kreis NOM mit 30798 ha der Getreideanbau. Dies entspricht ca. 63,5 \% der Ackerfläche. Innerhalb dieser Kategorie dominieren wiederum Weizen und Gerste mit fast $92 \%$ die Produktion. Immerhin ein Viertel der landwirtschaftlichen Nutzfläche unterliegt der Grünlandnutzung. Die im Rahmen der Untersuchungen durchgeführte Kartierung der Flächennutzungen bestätigt diese Aussage 
für das USG. Der hohe Anteil der Grünlandnutzung weist auf eine ebenso hohe Bedeutung der Nutzviehhaltung hin.

Die Flächennutzungsdaten für die Gemeinden Dassel und Einbeck zeigen die Verhältnisse im USG an (Tab. 5.1-3). Im Vergleich mit den Flächennutzungsanteilen im Kreis zeigt sich der rurale Charakter des Untersuchungsgebietes mit einem besonders hohen Anteil landwirtschaftlicher Nutzfläche und einem ebenfalls deutlich über dem Schnitt des Landkreises liegenden Grünlandanteil. Diese Zahlen sollen nicht darüber hinwegtäuschen, dass auch im Kreis NOM, respektive im USG, sich ein allmählicher Strukturwandel vollzieht. Dem gemäß ist zur Sicherung der nachhaltigen Landnutzung eine Förderung der Grünlandbewirtschaftung, insbesondere auf nassen und auf erosionsgefährdeten Standorten vorgesehen (RROP NOM 1996). Stützungsprogramme werden, wie allgemein in Deutschland, auch für die Erhaltung extensiv genutzter Flächen eine zunehmende Bedeutung erlangen. Welche Rolle eine Stärkung des ökologischen Landbaus in diesem Zusammenhang spielen kann, ist derzeit noch fraglich.

Tab.5.1-3:Landwirtschaftliche Nutzfläche in Relation zur Gesamtfläche der Verwaltungseinheiten (Stand 1992; Quelle: RROP NOM 1996; Statistisches Landesamt Nds.)

\begin{tabular}{|c|c|c|c|c|c|c|}
\hline \multirow{2}{*}{ Gemeinde } & \multicolumn{2}{|c|}{ Ackerland } & \multicolumn{2}{c|}{ Grünland } & \multicolumn{2}{c|}{ Brachland } \\
\cline { 2 - 7 } & real (ha) & $\%$ & real (ha) & $\%$ & real (ha) & $\%$ \\
\hline Dassel & $5.124,72$ & 45,34 & $2.031,92$ & 17,98 & 24,43 & 0,22 \\
\hline Einbeck & $8.939,65$ & 53,90 & $2.200,89$ & 13,27 & 5,19 & 0,03 \\
\hline $\begin{array}{c}\text { Landkreis } \\
\text { Northeim }\end{array}$ & $48.556,07$ & 38,33 & $14.563,11$ & 11,50 & 123,67 & 0,10 \\
\hline
\end{tabular}

Für die Waldflächen liegen keine gemeindescharfen Angaben vor. Mit $53 \%$ ist gut die Hälfte der Waldbestände im Kreis NOM dem Laubwald (mind. 70 \% Laubholzanteil) zuzuordnen. In weiteren $13 \%$ der Wälder kommen Nadel- und Laubbäume in ähnlicher Gewichtung vor. Die restlichen $34 \%$ werden von Nadelbäumen (v.a. Fichte) dominiert (RROP NOM 1996). Zur Sicherung der Waldfunktionen sowie zur Stabilisierung und Förderung der Artenvielfalt in Wald und Landschaft sollen die Waldvorkommen erhalten und zum Teil ausgedehnt werden. In diesem Zusammenhang kommt neben der Aufforstung auch der ökologisch orientierten Bewirtschaftung eine wichtige Rolle zu. Diesbezüglich ist das Niedersächsische Programm zur langfristigen ökologischen Waldentwicklung in den Landesforsten (LÖWE) für Wald der öffentlichen Hand maßgeblich. Für andere Waldbesitzarten dient das gleiche Programm als Empfehlung und Leitlinie. Derzeit sind etwa die Hälfte der bestehenden Wälder im USG dem Privatwald oder den Genossenschaftsforsten zuzuordnen. Nach Auskunft der zuständigen Forstbehörden (ENGELL, Forstamt Dassel, mdl., 2002) ist im Gebiet mittelfristig keine Aufforstung landeseigener Flächen zu erwarten. Der Schwerpunkt potentieller Aufforstungen liegt damit bei der privaten Hand. Dies korreliert mit der Absicht der regionalen Raumplanung, den Privat- und Körperschaftswald in seiner wirtschaftlichen Struktur zu stärken. Insbesondere kleine, verinselte Waldflächen sind nur unter hohem Kostenaufwand zu bewirtschaften, weshalb eine Ausdehnung oder Verbindung derartiger Flächen gefördert werden soll. 
Öffentlich geförderte Erstaufforstungen sollen nach Angaben des Regionalen Raumordnungsprogramms im Landkreis Northeim „insbesondere in den „Vorsorgegebieten für Forstwirtschaft“ und in "Gebieten zur Vergrößerung des Waldanteils“ durchgeführt werden, sind aber auch außerhalb dieser Gebiete wegen der wichtigen ökologischen Funktionen des Waldes ausdrücklich erwünscht und förderungswürdig“ (RROP NOM 1996 / Erläuterungen - S. 106). Demnach sind Aufforstungen auch außerhalb dieser Ausweisungsbereiche, eventuell gar in anderen Vorrang- oder Vorsorgegebieten genehmigungsfähig, wenn sie den Belangen des Naturschutzes nicht entgegen stehen. Lediglich in den besonders ausgewiesenen Freiflächen - „Von Aufforstung freizuhaltende Gebiete" - sollen Aufforstungsanträge keine Aussicht auf Erfolg haben. Für alle Vorrang-, Vorsorge- und Verweigerungsgebiete gilt allerdings eine regelmäßige Überprüfung durch die regionale Raumordnung als notwendig.

\section{Landschaftstypen}

Wie schon für das Untersuchungsgebiet Apolda beschrieben (vgl. Kap. 4.1) erfolgte auch für das USG Dassel eine Einordnung in die von FLADE (1994; vgl. Anhang XXe) vorgegebenen Landschaftstypen in vier Schritten: 1) Feststellung vorhandener Strukturen durch Geländebegehung, 2) Auswertung von Gebietsbeschreibungen, 3) Luftbildinterpretation sowie 4) eine Verifikation der festgestellten Landschaftstypen durch Überprüfung der Präsenz zugehöriger Leitarten.

Für das USG Dassel waren keine Landschaftspläne mit entsprechenden Artenkartierungen verfügbar. Die Durchführung von Taxierungen zur Feststellung der Artvorkommen war auch in diesem Falle aus zeitlichen Gründen nicht realisierbar. Daher mussten andere Quellen genutzt werden. Die Recherche vor Ort führte schließlich zur Ortsgruppe Dassel-Einbeck des Naturschutzbundes Deutschland e.V.. Die ehrenamtlichen Mitarbeiter der ornithologischen Arbeitsgruppe stellten ihr Wissen über die lokalen Vogelvorkommen bereitwillig zur Verfügung (Anhang 2 b). Dadurch konnte eine behelfsmäßige Überprüfung der festgesetzten Landschaftstypen durchgeführt werden.

Das USG Dassel wird landschaftlich geprägt durch die offenen, landwirtschaftlichen Felder in den Senken. Die meisten Hügel tragen dagegen eine bewaldete Kuppe. Entlang der Fließgewässer findet sich noch Grünlandbewirtschaftung und ein galerieartiger Bestand, meist aus Weiden und Erlen bestehend. Auwälder sind nicht vorhanden. In der Tiefe des Raumes bilden die Waldgebiete Elfas im Norden und Amtsberge / Wende-Berg im Westen einen optischen Riegel. Wie bereits geschildert, scheint der Horizont für den Betrachter auch an jenen Stellen durchgängig bewaldet, wo eigentlich nur eine Bewaldung der Kuppen besteht. Das Landschaftsbild der offenen Felder scheidet daher aus optischen Gründen von vornherein aus. Die Auswertung der Artvorkommen bestätigt diese Einschätzung.

Das Vorkommen von einzelnen kleinen Wäldchen und die gewässerbegleitende Gehölzvegetation erweckt andererseits aber auch nicht den Eindruck halboffener, reichstrukturierter Felder. Für diesen Landschaftstyp fehlen insbesondere die linienartigen Strukturelemente, die zwar vereinzelt, keineswegs aber nach einem ausgewogenen Verteilungsmuster vorkommen. Von den sechs Leitarten dieses Landschaftstyps brütet lediglich der Neuntöter bestimmt, eventuell auch noch die Wachtel im Untersuchungsgebiet. Grauammer, Raubwürger, Steinkauz und Ortolan tauchen dagegen nicht regelmäßig auf. Auch wenn die Schaffung linienhafter Elemente, beispielsweise einer Feldhecke, wohl keinen Nachteil auf die gegenwärtige Artengesellschaft bedeuten dürfte, kann die halboffene Feldflur dennoch nicht als pragmatisches Ziel betrachtet werden.

Eine bessere Ausgangsposition ist für die Fauna der Feldgehölze gegeben. Von den fünf Leitarten dieses LT brüten immerhin zwei nachweislich (Rabenkrähe und Turmfalke), zwei weitere vermutlich (Turteltaube und Waldohreule) im USG. Die fünfte Leitart, der Sprosser, kommt in dieser Region generell nicht vor, da sein natürliches Verbreitungsgebiet nicht so weit südlich reicht. Auch die Aufteilung der Landschaft entspricht ganz dem von FLADE 
gezeichneten Bild, mit Gehölzen zwischen 1 und 7 ha sowie deren großräumiger Umlagerung mit offenen Feldern und Grünland. Aufforstungen könnten diesen Landschaftstyp - und damit auch die Habitate der vorkommenden Tier- und Pflanzenarten auf zweierlei Weise ergänzen: einerseits durch die Schaffung neuer Gehölzinseln, andererseits durch flächenmäßige Ausdehnung der Gehölze.

Bezüglich der Einteilung der im USG tatsächlich und potentiell vorkommenden Laubwälder ist anzumerken, dass in erster Linie Buchenwälder vorhanden sind. Diese liegen oberhalb von $150 \mathrm{~m}$ NN und zeigen sich hallenartig, mit wenig Unterholz (Hainsimsen-Buchenwald und Perlgras-Buchenwald). Sie passen daher sehr gut in die Beschreibung des LT kolline und montane Buchenwälder. Diese Gruppe umfasst zusätzlich die etwas strukturreicheren Traubeneichen-Buchenwälder sowie die häufig ebenfalls strukturreichen Kalkbuchenwälder. Nach den avifaunistischen Unterlagen zum USG (vgl. Anhang 2b) sind alle für diesen Landschaftstyp festgestellten Leitarten auch tatsächlich präsent. Sumpfmeise, Grauspecht, Kleiber, Trauerschnäpper, Waldlaubsänger und Hohltauben brüten in den umliegenden Wäldern. Lediglich beim Rauhfußkauz steht ein Fragezeichen hinter der Bruttätigkeit. Eine Ausdehnung der Waldflächen, bzw. die Vergrößerung der kleinen Waldinseln auf eine Fläche von mindestens 10 ha verspricht daher eine schnelle Annahme durch die Populationen. Anders ausgedrückt ist mit einer „schnellen“ Besiedelung der neuen Flächen zu rechnen.

Die Wälder der Bachniederungen fehlen im USG, mit Ausnahme des Bach-ErlenEschenwaldes am Oberlauf der Bewer. Dieser Waldtyp fällt sozusagen durch das Raster der von FLADE (1994) definierten Landschaftstypen. Seine linienförmige Ausprägung führt zu einer Beeinflussung durch die auf breiter Fläche angrenzenden Waldtypen. Dies verhindert wohl eine spezifische Vogelgemeinschaft für diese Waldgesellschaft. Ihre Präsenz ist daher eher als Ergänzung der angrenzenden Lebensräume zu betrachten. Außerhalb der Waldgebiete Elfas und Amtsberge fehlen die bachbegleitenden Wälder. Sollten sie geschaffen werden, so wäre auf eine ausreichende Flächengröße (in der Summe mindestens 7 ha) zu achten, damit sich ein deutlicher Waldcharakter ausbildet. Ansonsten würden die Flächen eher dem oben beschriebenen LT Feldgehölze zuzuordnen sein.

Auch der feuchte Eichen-Hainbuchenwald (Stellario-Carpinetum) fehlt im USG. Er wäre in den staufeuchten Mulden der Lössgebiete zu suchen. Diese Flächen werden aber landwirtschaftlich, i.d.R. als Grünland genutzt. Eine Förderung des zugehörigen LT EichenHainbuchenwald wäre generell durch entsprechende Maßnahmen möglich. Die augenblicklichen Vogelvorkommen lassen dies auch sinnvoll erscheinen, da von den acht Leitarten dieses LT bereits sechs im USG brüten. Die Leitarten Sumpfmeise, Grauspecht, Kleiber, Trauerschnäpper und Waldlaubsänger hat der Eichen-Hainbuchenwald mit dem LT kolline und montane Buchenwälder gemeinsam. Der Gartenbaumläufer ist ebenfalls präsent. Lediglich bei Mittelspecht und Pirol ist eine Bruttätigkeit fraglich. Für die Ausdehnung der Aufforstungsflächen sollte auch in diesem Fall die oben genannte Größenordnung beherzigt werden, da kleine Waldinseln kaum den typischen Charakter des Waldverbandes erwarten lassen. Strategisch kann die Aufforstung als Ergänzungsmaßnahme zum Bewer-Programm gelten, welches die Erhaltung der bachnahen Feuchtgrünland-Bewirtschaftung anstrebt.

Bezüglich der Vorkommen von Nadelwäldern ist insbesondere der LT Fichten-Forste im USG vertreten. Dies gilt sowohl für Amtsberge und Elfas, als auch - allerdings in einer unter der Flächengrenze von 7 ha gelegenen Ausdehnung - für zwei Hügelkuppen. Alle Leitarten dieses LT brüten nachweislich in der Region: Sommer- und Wintergoldhähnchen, Haubenund Tannenmeise sowie Misteldrossel. Angesichts der ausgedehnten Fichtenbestände in den angrenzenden Waldgebiete ist dies auch kaum verwunderlich. Eine Berücksichtigung dieses Landschaftstyps als Zielvorstellung bei Erstaufforstungen kommt allerdings kaum in Frage. Einerseits weil die Bedingungen für die mit den Fichten-Forsten vergesellschafteten Tierarten bereits ausreichend gesichert scheinen. Andererseits weil die potentiell aufzuforstenden landwirtschaftlichen Böden ganz andere natürliche Waldgesellschaften besitzen und daher aus ökologischen Gründen nicht mit Fichte bestockt werden sollten. 
Bei der Auswertung der Informationen zu den vorkommenden besonders geschützten Biotopen sowie der Waldfunktionenkartierung, fallen die Kalk-Halbtrockenrasen auf. Auch für diese Vegetationsform hat FLADE einen Landschaftstyp beschrieben. Ein Großteil der von inm untersuchten Flächen zum LT Trocken- und Halbtrockenrasen besteht auch aus südoder sudwestexponierten Kuppen der Region Südniedersachsen, bietet also einen guten Referenzrahmen für die Einschätzung des hier betrachteten USG. Bezüglich der Avifauna weist FLADE (1994) darauf hin, dass die Untersuchungen für Halbtrockenrasen noch einen unbefriedigenden Umfang aufweisen. Lediglich der Hänfling ist für diese Region als Leitart zu verstehen. Er brütet auch im USG. Die Sperbergrasmücke tritt als Leitart dieses LT erst östlich des Harzes in Erscheinung. Auch der im USG brütende Neuntöter bekommt von FLADE erst viel weiter östlich die Kennzeichnung Leitart verliehen. Das Verbreitungsgebiet der Zippammer liegt dagegen deutlich südwestlich der betrachteten Untersuchungsregion. Der LT ist geprägt durch eine mehr oder weniger extensive Grünlandbewirtschaftung. Die Flächen stehen in engem Verbund mit Gebüschsäumen und werden teilweise von Verbuschung bedroht. Eine Aufforstung derselben oder angrenzender Flächen kann lediglich einen negativen Effekt auf die Erhaltung dieses Landschaftsbildes haben. Die KalkHalbtrockenrasen sind bereits per Gesetz gegen eine Umwandlung gesichert. Darüber hinaus weist die Waldfunktionenkartierung diese Flächen sowie deren unmittelbare Umgebung als „von Bewaldung freizuhalten“ aus.

\section{Regionale Leitbilder}

Die regionalen Leitbilder werden von den übergeordneten Leitbilder des LandesRaumordnungsprogramms abgeleitet, im Rahmen des regionalen Raumordnungsprogramms aufgegriffen und erläutert. Jene Punkte, die direkt oder indirekt für landschaftsgestalterische Maßnahmen wie eine Erstaufforstung relevant erscheinen, werden hier wiedergegeben. Beispielsweise wird der Erhaltung und Entwicklung des ländlichen und landschaftstypischen Charakters, des Gemeinwesens sowie der soziokulturellen Eigenart von Dörfern und Siedlungen besonderes Gewicht beigemessen. Naturnahe Flächen sollen als funktionales und räumlich zusammenhängendes System gefördert werden.

Speziell auf den Naturschutz abgestimmt ist die Forderung, Tier- und Pflanzenarten, bzw. -gesellschaften in den für sie charakteristischen Ökosystemen zu sichern. Dies beinhaltet die Förderung der Lebensräume nach Flächenausdehnung, Qualität, räumlicher Verteilung und funktionaler Vernetzung. Im Sinne der Raumordnung stützen entsprechende Maßnahmen auch die Eigenart der Landschaft sowie die Leistungsfähigkeit des Naturhaushalts.

Mit einem Hinweis auf die Bedeutung der Landschaft als Attraktion für den Fremdenverkehr wird die Sicherung und Entwicklung der für Weser- und Leinebergland typischen Wälder als vorrangiges Ziel genannt.

Das Raumordnungsprogramm sieht vor, für Flächen, die dauerhaft aus der landwirtschaftlichen Nutzung ausscheiden, zu prüfen, ob sie aufgeforstet, zu Zwecken des Biotopschutzes gestaltet oder sich selbst überlassen werden sollen. In diesem Zusammenhang sind mehrere Fragen zu prüfen, wie etwa die Frage nach der Eingliederung der Fläche in das angestrebte, evtl. schon vorhandene Landschaftsbild. Als Beitrag zur Biotopentwicklung kann die zukünftige Flächennutzungsform nur dann gelten, wenn der gleiche oder ein ähnlicher Biotoptyp bereits in mittelbarer Nähe (weniger als $200 \mathrm{~m}$ entfernt) vorhanden ist. Im Sinne des Biotopverbundes sollen existente Waldflächen, insbesondere Restwälder und Feldgehölze räumlich wie funktional durch Aufforstungen vernetzt werden. Dadurch kann einer Verinselung von Tier- und Pflanzenvorkommen wirksam vorgebeugt werden.

Das RROP NOM äußert die Hoffnung, durch Förderprogramme im Rahmen der Gemeinsamen Agrarpolitik (GAP) die Extensivierung und Stillegung von Flächen zum Zwecke des Biotopverbunds nutzen zu können. Insbesondere die Dauergrünlandnutzung auf staunassen Böden und in den Auenbereichen liegt hier im Visier der Raumplaner. Wo extensive Bewirtschaftung keine Lösung für einen Betrieb darstellt, sollen Aufforstungen für eine Diversifizierung der betrieblichen Produktion, Entzerrung der Arbeitsaufkommen sowie 
eine langfristige Investitionsplanung sorgen. Hier steht eine Förderung des Privat-, Körperschafts- und Genossenschaftswaldes im Sinne der Raumplaner. Dies geht so weit, dass bereits im Raumordnungsplan angemahnt wird, bei öffentlichen Bauvorhaben bevorzugt Holz aus der Region zu verwenden, um die Forstwirtschaft im Landkreis zu unterstützen. Für den Waldbesitzer ist insbesondere auch die Größe der Waldungen ein entscheidendes wirtschaftliches Kriterium. Aus diesem Grunde muss eine Ausweitung und Arrondierung bestehender Wälder angestrebt werden. Gegebenenfalls sind vorbereitend zum Landnutzungswandel auch Flurneuordnungsmaßnahmen durchzuführen. Auch die Erschließung der Waldflächen mit Wegen ist bei der Neuanlage zu bedenken, stellt allerdings im gut erschlossenen USG der vorliegenden Arbeit kein restriktives Element dar.

In Bezug auf die Waldbewirtschaftung verweist das RROP NOM auf eine ökologisch ausgerichtete Waldpflege. Die Zielaussagen sind kongruent zu jenen der landesweiten Waldbaurichtlinien im Rahmen des LÖWE-Programmes. Da ein beträchtlicher Anteil der Wälder noch nicht die angestrebte, naturnahe Baumartenbestockung aufweist, diese jedoch im Zuge der allmählichen Nutzung und Verjüngung der Bestände nur sehr langsam zu verändern ist, muss ein entsprechend langfristiges Ziel gesetzt werden. Andererseits kann bei der Neuanlage von Wäldern bereits in der Planungsphase auf eine frühzeitige Erfüllung der unterschiedlichen Waldfunktionen Rücksicht genommen werden.

Das regionale Raumordnungsprogramm bringt die Anforderungen an neu anzulegende Wälder in engen Zusammenhang mit den späteren Funktionen des Waldes (RROP NOM 1996 / Ziele der Raumordnung, S. 56). Sie fordert eine Berücksichtigung von Standort, Baumartenvielfalt, naturgemäßer Zusammensetzung und zukünftiger Strukturierung des Waldes. Hier spielen auch Aufbau, Zusammensetzung und Gestaltung des Waldrandes eine bedeutende Rolle. Aufgrund der im Waldrand auftretenden Vielfalt an Strukturen, Temperatur-, Nährstoff-, Feucht- und Lichtverhältnisse und der Lage zwischen unterschiedlichen Nutzungsformen, besitzen gut ausgebildete Waldrandbiotope eine hohe Artenvielfalt. Der Pflege existenter, aber auch der Anlage neuer Waldränder ist daher besondere Aufmerksamkeit zu widmen. Dies gilt gerade in der räumlichen Nähe mit den bereits erwähnten Kalk-Halbtrockenrasen, die ihrerseits bereits heute vielfach vergesellschaftet mit Trockengebüschen vorkommen, also eine Biotopvernetzung über Waldränder ermöglichen.

Nutzungswandel, insbesondere zwischen land- und forstwirtschaftlicher Nutzung, hat stets einen sichtbaren Effekt auf das Landschaftsbild. Dieses kann auch gezielt entwickelt werden. Das Raumordnungsprogramm Northeim sieht beispielsweise eine Vergrößerung der Waldfläche im Landkreis zur Neugliederung ausgeräumter Landschaftsteile explizit vor. So kann eine Doppelfunktion gestützt werden: die Steigerung der Vielfalt in Natur und Landschaft sowie die Sicherung der Holzversorgung zukünftiger Generationen. Aufforstungsinitiativen werden insbesondere für jene Bereiche des Landkreises erwartet, in denen der Bewaldungsanteil unter dem Landesdurchschnitt (22,6\%) liegt. Sie kann aber auch in anderen Gegenden bedeutsam sein, wenn etwa der Waldanteil zwar hoch, die Verteilung aber räumlich nicht ausgeglichen ist. Als angestrebtes Bewaldungsprozent wird das Bundesmittel in Höhe von $30 \mathrm{v}$. H. angegeben. In leicht überdurchschnittlich bewaldeten Gebieten kann darüber hinaus eine Aufforstung dringlich erscheinen, wenn sie der Verknüpfung von Feldgehölzen und/oder Restwaldflächen im Sinne des Biotopverbundes dient.

In den waldreichen Gebieten des Landkreises, namentlich im Solling und im Leinebergland, soll dagegen einer Nivellierung der Landschaft durch Aufforstung der unterrepräsentierten Freiflächen vorgebeugt werden. Daher wurden in diesen Bereichen „von Aufforstung freizuhaltende Gebiete" ausgewiesen. Zusätzlich verbietet sich eine Aufforstung auf Flächen mit besonderer Bedeutung für den Artenschutz, beispielsweise solchen, die nach $\S 28$ a und b NNatG geschützt sind. 


\section{Vorhandene Planungsgrundlagen}

Das Evaluationsverfahren wird nach den Untersuchungen in Thüringen auch im Raum Dassel (Niedersachsen) angewandt, um die Übertragbarkeit zu testen. Die hier verfügbaren Daten und Planungsgrundlagen unterscheiden sich nach Art, Umfang und Quelle teilweise deutlich von jenen in Thüringen. Dennoch reicht die Informationsgrundlage zur Bewertung von Aufforstungen aus.

Eine zentrale Rolle für die Ableitung der landespflegerischen Ziele spielt das Regionale Raumordnungsprogramm für den Kreis Northeim (RROP NORTHEIM 1996). Dieses liefert eine differenzierte Analyse der Nutzungsschwerpunkte und beschreibt die regionalen Leitbilder in wirtschaftlicher und landschaftlicher Hinsicht (s.o. - Regionale Leitbilder). Darüber hinaus werden nähere Angaben zum Schutz von Biotopen und Landschaftselementen gemacht. Auch zur Bedeutung potentieller Aufforstungen bezieht das Programm Stellung. Hinsichtlich der Bewirtschaftung von Waldbeständen wird dagegen auf das LÖWE-Programm der Landesforsten verwiesen.

Bezüglich der anzustrebenden Flächennutzungsanteile wird das Programm kaum konkret. Für Grünland wird eine Sicherung des aktuellen Bestandes angestrebt. Waldarme Bereiche sollen durch Aufforstungen und Pflanzungen an Attraktivität gewinnen. Die im RROP Northeim geäußerten Hoffnungen, die im Rahmen der GAP stattfindende Strukturreform zur Erhöhung der Nutzungstypenvielfalt zu nutzen, weist darauf hin, nicht nur Grünland zu erhalten und Wälder anzulegen, sondern auch die Zahl der Brachflächen auf bisher ackerbaulich genutzten Flächen zu erhöhen. Die Signale sind damit gesetzt, werden allerdings nicht durch Zahlen konkretisiert. Diese Information reicht aber aus, um den Indikator Landnutzungsverhältnis anzuwenden.

Die Beschreibung der landschaftlichen Reize und typischen Erscheinungsbilder findet Eingang in die Feststellung der Landschaftstypen, die im Indikator Biotopverbund eine tragende Rolle spielen. Die festgestellten Landschaftstypen sind auch für den Untersuchungsraum Dassel anhand der aktuellen Vorkommen von avifaunistischen Leitarten auf Entwicklungsfähigkeit und Planungsrelevanz zu überprüfen. Die bereits früher angesprochene Knappheit an Zeit und Ressourcen verhinderte allerdings auch hier die Durchführung von Taxierungen. Einmal mehr erwies sich die Zusammenarbeit mit den Behörden und dem ehrenamtlichen Naturschutz als hilfreich. Ausreichend detaillierte Aussagen zur Bestandessituation der betroffenen Leitvogelarten konnten die Mitarbeiter der NABU-Ortsgruppe Dassel-Einbeck liefern.

Die Herleitung der PNV erfolgt über die Grundlage der Bodenkarte (NDS. LANDESVERWALTUNGSAMT 1981). Hier wären aktuellere Aufnahmen, insbesondere unter Berücksichtigung der Aufforstungsfähigkeit, wünschenswert. Da neuere Daten jedoch nicht zur Verfügung stehen, mussten Wasserhaushalts- und Nährstoffzahl behelfsweise ermittelt werden (s.u. - Aufforstungsvarianten). Die Aufforstungsflächen bewegen sich demnach im gut bis sehr gut nährstoffversorgten Bereich. Es handelt sich bei allen Flächen um Hangoder Kuppenlagen. Der Wasserhaushalt reicht von frisch bis mäßig sommertrocken.

Die Kartierung von Wald- und Offenlandbiotopen ist im Untersuchungsraum abgeschlossen. Sie wird von der Waldfunktionenkarte Niedersachsen dargestellt und im Textteil näher beschrieben.

Zusätzlich zu den erwähnten Unterlagen wurde auch für diesen Raum eine Luftbildinterpretation (Flächennutzung, Flächengrößen, Ökotone) mit anschließender Überprüfung im Feld durchgeführt.

\section{Vorstellung der Untersuchungsflächen}

Der Untersuchungsraum Dassel ist gekennzeichnet durch die kleinen, einzelnen Waldstücke, welche die Kuppen der Erhebungen bedecken. Neue Waldflächen können in diesem Zusammenhang unterschiedliche Auswirkungen auf den Landschaftscharakter und den Biotopverbund besitzen. Sie können bestehende Waldflächen vergrößern oder miteinander 
verbinden. Letzteres auch im Sinne von Trittsteinbiotopen, wenn kein unmittelbarer Anschluss gegeben ist. Letztlich können sie auch in Einzellage neue Wald- oder Gehölzflächen schaffen. In diesem Sinne wurden auch für den Raum Dassel potentielle Aufforstungsflächen demarkiert. Die Auswahl erfolgte wiederum systematisch, um die unterschiedlichen Anbindungsvarianten zu berücksichtigen.

Der Raum selbst tritt weder als besonders strukturarm noch als offensichtlich gut vernetzt in Erscheinung. Das Bild wird durch die intensive Landwirtschaft geprägt. Der Wald ist auf Kuppenlagen und Oberhänge beschränkt, bzw. auf die höheren Erhebungen mit geschlossenen Waldgebieten. Hecken und Baumreihen treten nur vereinzelt in Erscheinung. Dennoch ist der Horizont von Wald gesäumt, da sich die Waldflächen für den Betrachter in der seitlichen Perspektive miteinander verbinden. Entsprechend der intensiven landwirtschaftlichen Nutzung ist eine gute Erschließung der gesamten Fläche vorhanden. Auch die Anbindung der Aufforstungsflächen mit maschinentauglichen Wegen an das Wegenetz ist gegeben.

Wie oben beschrieben, finden vor allem die Landschaftstypen Feldgehölze und Wälder ihre Anwendung bei der Betrachtung der räumlichen Zusammenhänge. Die inselartigen Waldbestände liegen häufig unter der Flächengrenze von 7 ha, die von FLADE (1994) als Unterscheidungsmerkmal zwischen Gehölz und Wald bestimmt wurde. Aufforstungen können hier allerdings dazu beitragen, dass die passenden Lebensbedingungen auch für Waldarten zunehmen. Landwirtschaftliche Landschaftstypen (offene Felder; halboffene, reichstrukturierte Felder) sind derzeit nicht in typischer Ausprägung (s.o. - Landschaftstypen) festzustellen. Die ex-ante-Bewertung der Aufforstungen konzentriert sich daher auf deren Beitrag zur Verknüpfung und Schaffung von Wäldern, bzw. Gehölzen.

\section{Aufforstungsvarianten}

Wie bereits in Kap. 4.1 erläutert, bestimmen die Baumarten einer Anpflanzung ganz wesentlich die Entwicklung des Bestandes in den ersten Jahrzehnten. Die Aufforstungsflächen in der Untersuchungsregion Dassel weisen unterschiedliche Bodenverhältnisse auf, weshalb drei unterschiedliche natürliche Waldgesellschaften zuzuordnen ist. Lediglich die Flächen 1 und 4 liefern diesbezüglich das gleiche Ergebnis. Um die Methode des Variantenstudiums in ähnlicher Weise durchzuführen, wie in der Untersuchungsregion Apolda, wurde wiederum eine Reihe von Aufforstungsvarianten mit unterschiedlicher Baumartenvielfalt vorgeschlagen (Anhang 5). Die Baumartenanzahl nimmt dabei von einer Variante zur nächsten um jeweils 2 Baumarten zu. In Abhängigkeit von der standortspezifischen PNV wurden die Baumarten ausgewählt und die Variantenzahl soweit erhöht, bis jene Vielfalt erreicht war, die auch in der PNV zu vermuten ist. Die Ermittlung der PNV erfolgte über die Bodenverhältnisse (NDS. LANDESVERWALTUNGSAMT 1981) unter Berücksichtigung der klimatischen Bedingungen und der jeweiligen Exposition der Aufforstungsfläche. Als Quelle für die Zusammensetzung der PNV wurde die Beschreibung der Waldgesellschaften Deutschlands (AK FORSTL. LANDESPFLEGE 1996) herangezogen.

Da Niedersachsen kein offizielles Pendant zu den Erstaufforstungszieltypen Thüringens besitzt, wurden zusätzlich die in Frage kommenden Waldentwicklungstypen (WET) ermittelt. Hierzu war es notwendig, Nährstoff- und Wasserhaushaltszahl für die Aufforstungsflächen zu bestimmen. Unter Berücksichtigung der Bodenverhältnisse und der Nutzungsart erfolgte diese Ermittlung im Gelände. Es wurde davon ausgegangen, dass landwirtschaftlich genutzte Flächen generell eine hohe Verfügbarkeit von Nährstoffen aufweisen. Im direkten Vergleich auf gleichartigen Standorten, ist der Aufforstung auf ackerbaulich genutzten Flächen eine höhere Nährstoffzahl zuzuweisen als auf Grünland. Aufgrund dieser Daten lassen sich den Standorten eine Reihe von Waldentwicklungstypen zuordnen. Der entsprechende Schlüssel liegt für Niedersachsen vor (NDS. FORSTPLANUNGSAMT 1996) und wurde hier übernommen (Anhang 5). Anhand der WET wurde eine Reihe von Aufforstungsvarianten definiert, die entsprechend der Empfehlungen der Landesforsten auf den passenden Standorten betrachtet werden. Im Gegensatz zu den Aufforstungsvarianten in Thüringen wurde hier bei der Zusammenstellung der Baumarten nicht auf deren 
Strukturpotential im Sinne des entsprechenden Indikators geachtet. Die resultierenden Indikatorwerte für Naturnähe und Strukturpotential wurden nicht bewusst beeinflusst.

\section{Waldrandgestaltung}

Das Regionale Raumordnungsprogramm für den Landkreis Northeim (RROP NOM 1996) weist an mehreren Stellen auf die Bedeutung von Waldrändern für den Artenschutz und die Landschaftsgestaltung hin. Dabei werden die für Waldränder typischen, vielgestaltigen Lebensbedingungen hervorgehoben, die durch eine auf engstem Raum verzahnten Wechsel von Vegetationsstruktur, Temperatur-, Nährstoff-, Feuchtigkeits- und Lichtbedingungen beruhen. Auch die Bedeutung von Ökotonen an sich wird in diesem Zusammenhang betont. Als Bedingung für die Funktionserfüllung - sowohl in ästhetischer als auch in ökologischer Sicht, ist die Zusammensetzung und Struktur des Waldrandes von Bedeutung. Als natürliche Grundlage wurde die Exposition des Waldrandes schon mehrfach in dieser Arbeit angesprochen. Das Raumordnungsprogramm weist zusätzlich darauf hin, dass schädliche Einflüsse von Waldrändern abzuweisen sind, bzw. dass entsprechende Nutzungen einen Mindestabstand von $100 \mathrm{~m}$ zum Waldrand einzuhalten haben.

\subsection{Teilergebnisse}

\subsubsection{Flächenspezifische Ergebnisse - Aufforstungsvarianten und Erstaufforstungszieltypen}

Auch für die Untersuchung im Raum Dassel wurde zunächst eine Reihe von Erstaufforstungsvarianten definiert, die den unterschiedlichen Standorten und damit der hypothetischen PNV entsprechen. Gemäß der Verhältnisse auf den Aufforstungsflächen wären drei unterschiedliche, natürliche Waldgesellschaften zu erwarten. Dazu gehören auf basenreichen Standorten der Perlgras-Buchenwald und der Seggen-Buchenwald. Eine Aufforstungsfläche (DA-2) wird dagegen vom anstehenden Buntsandstein beeinflusst, so dass dort eine Entwicklung zu einem Hainsimsen-Buchenwald zu erwarten wäre. Daneben wurden gemäß der von den Niedersächsischen Landesforsten definierten WET einige Aufforstungsvarianten erstellt. Diese berücksichtigen in erster Linie das Nährstoffangebot und den Wasserhaushalt der Fläche. Die Nebenbaumarten wurden für diese Waldentwicklungstypen aus den standortsgerechten Baumarten, aber ohne besonderes Augenmerk auf das Strukturpotential oder die PNV-Zugehörigkeit ausgewählt (Anhang 5)

Im Sinne der Regionalisierung des anzuwendenden Verfahrens, sind landesübliche Methoden zur Ermittlung der PNV anzuwenden. Das Niedersächsische Biotopkartierungsverfahren (vgl. DRACHENFELS 1994) bietet diesbezüglich aber weniger Durchführungshinweise, als beispielsweise das Thüringer Verfahren zur Waldbiotopkartierung. Forstliche Standortsinformationen lagen weder für die Aufforstungsflächen, noch für die zunächst gelegenen Waldinseln vor. Daher musste die Definition der PNV einen Umweg über die Beschreibungen im Raumordnungsprogramm (RROP NORTHEIM 1996), die Auswertung der Bodenkarten (NDS. LANDESVERWALTUNGSAMT 1981), die Beschreibung der Waldgesellschaften in verschiedenen Quellen (AK FORSTL. LANDESPFLEGE 1996; NDS. MELF 1996; FISCHER 1995) und eine einfache Ansprache im Gelände nehmen.

Bezüglich der Indikatoren Baumartenvielfalt und Naturnähe muss jeweils die standortspezifische PNV berücksichtigt werden, da sie als Idealziel angenommen wurde. Perlgras-Buchenwald und Seggen-Buchenwald sind durch die Dominanz der Buche charakterisiert. Daneben kommen 10, bzw. 9 weiteren Baumarten vor, die dieser natürlichen Waldgesellschaft zuzuordnen sind. Im Hainsimsen-Buchenwald ist die Eiche als zusätzliche Hauptbaumart zu nennen. Auch hier ist der Nebenbestand mit 8 weiteren Arten recht vielfältig.

Die Reihe der PNV-orientierten Erstaufforstungsvarianten (EAV) zeigt eine parallel verlaufende und stetige Zunahme der Indikatorwerte für Baumartenvielfalt und Naturnähe 
(Abb.5.2-I und Anhang 5). Dies folgt logisch aus der Definition der Pflanzenmischungen, bei denen von einer Variante zur nächsten jeweils zwei Baumarten aus der PNV hinzu kommen. Die graphische Darstellung zeigt deutlich, dass es nicht notwendig ist, alle Baumarten einer PNV zu beteiligen, um die höchste Bewertung für die Vielfalt der Zielarten und die Naturnähe zu erhalten.

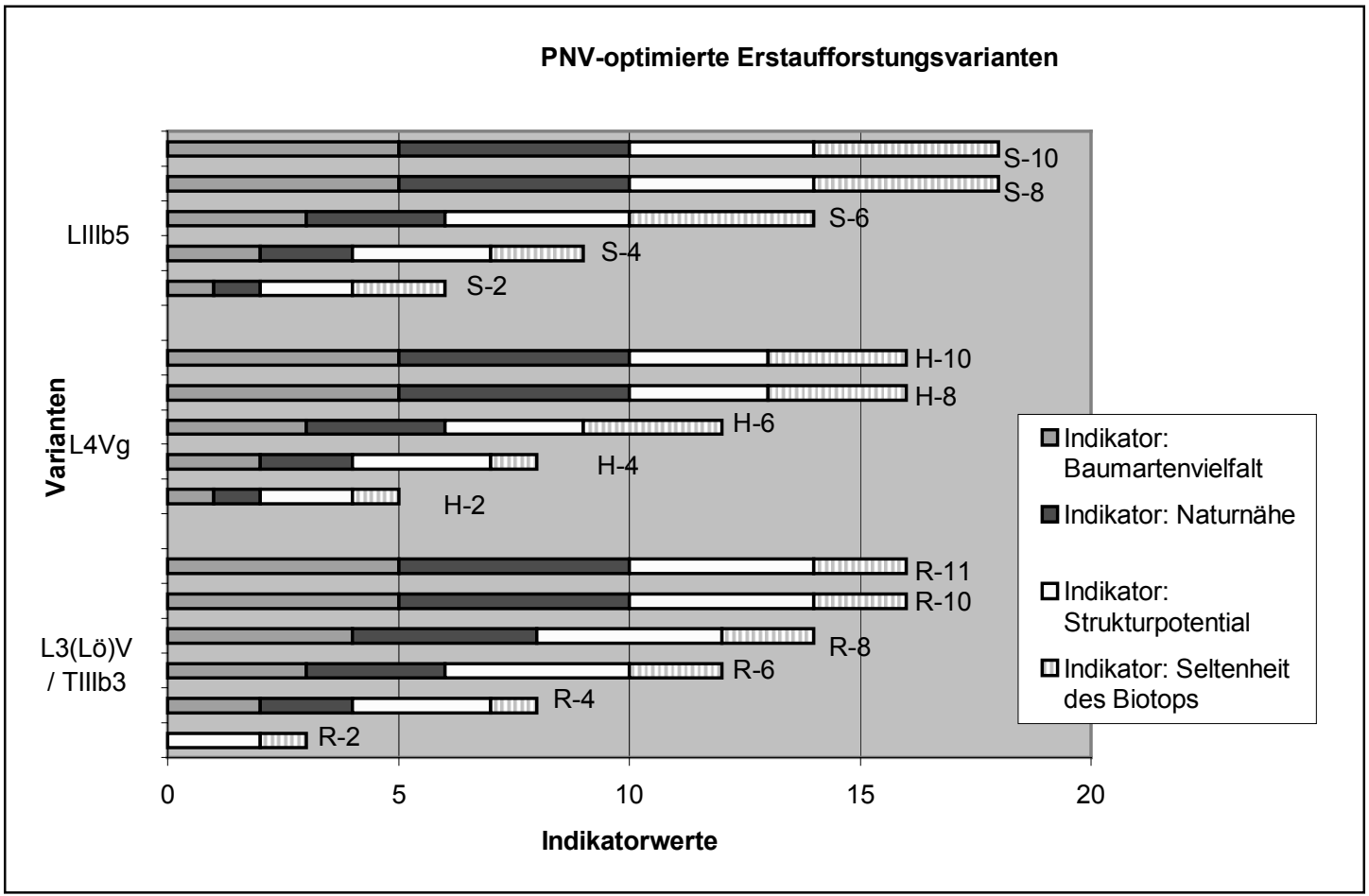

Abb. 5.2-I: Flächenbezogene Indikatorwerte für die PNV-optimierten

Erstaufforstungsvarianten (USG Dassel)

Die Waldentwicklungstypen (WET) beinhalten jeweils mindestens vier Baumarten. Typische Vorwaldmodelle und Reinkulturen fehlen. Dadurch entsteht hinsichtlich der Baumartenvielfalt in den WET ein ausgeglichenes Bild. Die Naturnähe spielt hingegen bei der Zuordnung eines WET zu einem Standort nicht zwangsweise eine Rolle. Obwohl die Anwendung der WET auf die Aufforstungsfläche den Empfehlungen der Niedersächsischen Landesforsten folgt, kommen deshalb sehr unterschiedliche Werte für den Indikator Naturnähe heraus (Abb.5.2-II).

Bezüglich der zu erwartenden Bestandesstruktur lassen sowohl EAV als auch WET ein Mindestmaß erwarten. Seitens der PNV-orientierten Baumartenzusammensetzungen liegt dies im Konzept der natürlichen Waldgesellschaft begründet, die die Arten verschiedener Sukzessionsstadien in sich vereint. Es kommen Licht- und Schattenarten, schnell- und langsamwüchsige Arten nebeneinander vor. Bei den WET liegt der hohe Strukturwert eher in der waldbaulichen Überlegung begründet, dienende Baumarten zu integrieren. Beide Überlegungen führen zu einem Mindestmaß an vertikaler und horizontaler Struktur. Die höchsten Strukturpotentialwerte erreichen die PNV-orientierten Varianten mit besonders hoher Baumartenzahl. Der Maximalwert von 5 Punkten wird nicht erreicht. Dies beruht auf der zu Grunde gelegten natürlichen Baumartenzusammensetzung, die beispielsweise für den Perlgras-Buchenwald und den Hainsimsen-Buchenwald keine Beteiligung von Bäumen vorsieht, die in der vorliegenden Arbeit als "Landschaftselemente" eingestuft wurden. 


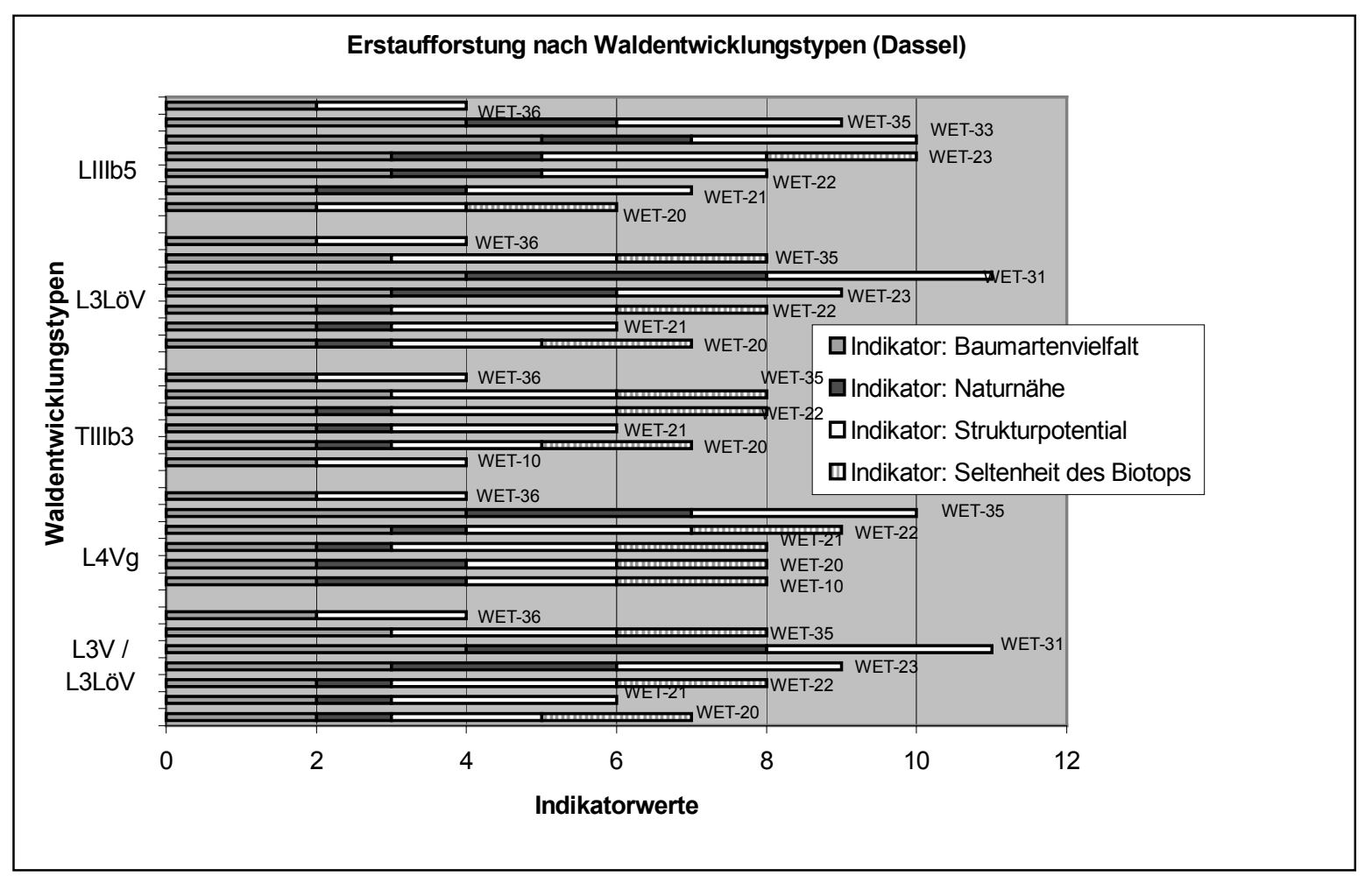

Abb. 5.2-II Flächenbezogene Indikatorwerte für die Erstaufforstung nach Waldentwicklungstypen (USG Dassel)

Beim Indikator Seltenheit des Biotops zeigt sich für den Untersuchungsraum Dassel ein ähnliches Phänomen wie für den Raum Apolda. Ein entstehender Waldbestand erfährt nur dann eine Würdigung im Sinne dieses Indikators, wenn der sich entwickelnde Waldtyp eine Beachtung durch die landesweite Kartierung schutzwürdiger Bereiche erfährt, einen Bestandesschutz nach $\S 28$ a oder b NNatG besitzt oder in der Roten Liste der gefährdeten Biotoptypen für die Bundesrepublik Deutschland aufgeführt ist. Generell gilt, dass ertragsstarke Flächen in intensiv landwirtschaftlich genutzten Gebieten selten bewaldet sind. Daher liegt eine kulturell begründete Seltenheit vor, die sich in den genannten Listen und Verfahren niederschlägt. Für alle hier behandelten Aufforstungsflächen besteht grundsätzlich die Möglichkeit, einen aus Biotopschutzgründen wertvollen Wald zu schaffen. Der PerlgrasBuchenwald geht in die RLB beispielsweise als Buchenwald basenreicher Böden (colline bis montane Stufe) ein und wird bei der landesweiten Kartierung als mesophiler Kalkbuchenwald erfasst. Ähnliche Beachtung erfahren auch der Hainsimsen-Buchenwald (bodensaurer Buchenwald der collinen Stufe) und der Seggen-/Orchideen-Buchenwald. Dass im Untersuchungsraum Dassel keine Maximalbewertungen erreicht werden, liegt schlicht daran, dass die betrachteten Standorte keine Ausgangslage für die Schaffung von Wäldern bieten, welche gleichzeitig in dieser Region als "von vollständiger Vernichtung bedroht" einzustufen wären.

Aus den Grafiken zur Bewertung der Seltenheit geht leider nicht hervor, dass die Kartierung schützenswerter Biotope in Niedersachsen wesentlich lockerer mit der Zuweisung von Biotoptypen umgeht, als dies die Erläuterungen zur bundesweiten RLB vorsehen. Während letztere einen Waldzustand erwartet, der zumindest in seiner Zusammensetzung der PNV entspricht (RIECKEN et al. 1994), nimmt die niedersächsische Kartierung einen EichenReinbestand unter Umständen auch in die Obergruppe „Eichen- und Hainbuchen-Mischwald“ auf (DRACHENFELS 1994). Eine einheitliche Vorgehensweise wäre hier aus methodischen Gründen wünschenswert. Ein Anspruch, nach dem ein Bestand nur dann als schutzwürdig aufgenommen wird, wenn er mindestens zwei Drittel der entsprechenden PNV-Baumarten enthält, würde allerdings in der vorliegenden Untersuchung zu deutlich geringeren Werten führen. 
Die summarische Darstellung der hier besprochenen Indikatorwerte Vielfalt der Zielarten, Naturnähe, Strukturpotential und Seltenheit des Biotops zeigt einen deutlichen Unterschied zwischen den PNV-orientierten Varianten und den WET. Die Spanne der zugeteilten Werte liegt für die EAV zwischen 3 und 18 Punkten. Die große Anzahl an Baumarten und die naturgemäße Baumartenzusammensetzung der Bestände schlägt sich hier nieder. Die WETErgebnisse liegen enger beieinander. Sie beginnen etwas höher in der Skala, bei 4 Punkten, reichen allerdings auch nicht über 11 Punkte hinaus. Dies kann nur zum Teil damit begründet werden, dass die WET maximal neun Baumarten für die Bestandesbegründung vorsehen. Schließlich schafft es die EAV S-8 (Seggen-Buchenwald auf flachgründiger Rendzina) auch, mit nur acht Baumarten ein Ergebnis von 18 Punkten zu erzielen.

Die Entwicklung eines Waldbiotops ist in starkem Maße von den örtlichen Bedingungen abhängig. Ein bestimmter Aufforstungstyp wird daher, wenn er auf verschiedene Ausgangssituationen trifft, zu unterschiedlichen Ergebnissen führen. Dies zeigt sich an den Ergebnissen der Waldentwicklungstypen (Abb. 5.2-II). Für die Indikatoren Naturnähe und Vielfalt der Zielarten liegt dieser Zusammenhang auf der Hand, da sie direkt auf die PNV als Bewertungsmaßstab zurückgreifen. Auch für die Chance, einen als gefährdet eingestuften Biotoptyp zu entwickeln, spielt die Übereinstimmung von gepflanzter und natürlicher Baumartenzusammensetzung eine entscheidende Rolle. Die Entwicklung des Strukturpotentials ist hingegen ein recht stabiles Element in der Bewertung. Hier erreichen die Variantenvorschläge und die Waldentwicklungstypen jeweils konstante Werte für alle Flächen, auf denen sie als geeignet gelten.

Für den Indikator Waldrandausprägung gilt das bereits im Zusammenhang mit der Untersuchung in Apolda Gesagte. Der Grundeigentümer kann den ökologischen Wert des Waldrandes durch dessen Breite, Grundform, Artenzusammensetzung, Strauchanteil und Herkunft bereits bei der Anlage entscheidend beeinflussen. Die lokalen Bedingungen sollen Beachtung finden. Die Bewertung folgt einer eindeutigen Checkliste und muss daher an dieser Stelle nicht simuliert werden. 


\subsubsection{Flächenspezifische Ergebnisse - Raumbezug}

Für die Feststellung der raumbezogenen Werte wurden zunächst die Informationen über vorhandene Biotope in den Bezugsräumen (BR) digital aufgenommen. Als Quelle diente die Waldfunktionenkarte Niedersachsen (Blatt 4124 Einbeck), die neben den Waldbiotopen auch Offenlandbiotope darstellt. Es sei in diesem Zusammenhang darauf hingewiesen, dass die Biotopkartierung in diesem Landesteil nur selektiv durchgeführt wurde. Im direkten Vergleich zu den Aufnahmen im Untersuchungsraum Apolda fällt auf, dass die Dichte festgestellter Biotope in Dassel deutlich niedriger ist. Dies liegt einerseits daran, dass die "Kartierungsschwelle“ offensichtlich in Thüringen niedriger liegt (z.B. bei Alleen und Restwaldflächen). Andererseits sind im dortigen Untersuchungsgebiet auch deutlich mehr Strukturelemente vorhanden.

Der Indikator Biotop-Verbund würdigt die Entstehung eines neuen Biotops, welches eine Bereicherung im Sinne der Biodiversität darstellt. In Ablösung der intensiven landwirtschaftlichen Nutzung (Ackerbau oder Intensivgrünland), die in allen hier behandelten Fällen zu prognostizieren ist, scheint diese Art der Aufwertung gegeben. Die Erweiterung eines Biotops im Sinne des Indikators ist dann gegeben, wenn die Aufforstung an einen (Laub-)Wald, ein Feldgehölz oder ein Gebüsch anschließt. Verbindet es zwei dieser Elemente direkt, so ist auch ein Biotop-Verbund zu bestätigen, der in aller Regel gar zur Vernetzung wird.

Der zweite Teil dieses Indikators beschäftigt sich mit den Landschaftstypen, die für bestimmte Biotopkomplexe und -verhältnisse stehen. Es wurde bereits darauf hingewiesen, dass der LT offene Felder und der LT halboffene, reichstrukturierte Felder im Untersuchungsraum nicht ohne weiteres festzustellen sind. Ein Beitrag von Aufforstungen zu diesen Landschaftstypen ist daher auszuschließen. Stattdessen spielen der LT Gehölze (bis ca. 7 ha) und Laubwälder (v.a. LT kolline Buchenwälder) eine wichtige Rolle. Aufforstungen können einerseits neue Gehölze oder Wälder schaffen, können gleichzeitig aber auch Gehölze zu Wäldern verbinden oder den Anschluss an geschlossene Waldkomplexe herstellen. Die betrachteten Fallbeispiele bewegen sich teilweise genau im Übergangsbereich zwischen den beiden Landschaftstypen. Funktional ist die Trennung letztlich nicht an der Flächengröße selbst festzumachen. Die Ähnlichkeit der aneinander stoßenden Biotope, die zwischengelagerten Nutzungen und die Entfernung zu den nächsten, größeren Waldkomplexen können ebenfalls von großer Bedeutung sein. Der Erfolg des Biotop-Verbundes zeigt sich letztlich nur in einer möglichst schnellen Besiedelung durch die erwarteten Tier- und Pflanzenarten.

Das Regionale Raumordnungsprogramm für den Kreis Northeim (1996) liefert die Vorlagen für die Bewertung des Indikators Landnutzungsverhältnis. Es ist das einzige, noch dazu interdisziplinär erstellte Dokument, welches verlässliche Angaben sowohl zur derzeitigen Flächennutzung als auch zur angestrebten Entwicklung macht. Sicherlich könnten die Daten noch etwas genauer sein, insbesondere was die Unterscheidung zwischen landwirtschaftlichen Kulturformen angeht. Doch mit einer Besserung dieser Situation ist bereits mit der Erstellung eines Landschaftsplanes für die Region zu erwarten.

Neben den Daten des RROP Northeim wurden zusätzliche Daten zu den Bezugsräumen benötigt. Diese wurden über die Interpretation von Luftbildern mit anschließender Verifizierung im Gelände erhoben. Die Daten wurden elektronisch gespeichert und verarbeitet. In diesem Zusammenhang wurden auch die existenten Ökotone erfasst und digitalisiert. Der Indikator Randlinieneffekt setzt die gewonnenen Informationen in eine Bewertung um. Erneut spielt in diesem Zusammenhang die Beeinträchtigung und die Neuschaffung von Waldrändern eine wichtige Rolle.

Je ein Luftbild zu jeder Aufforstungsfläche mit eingezeichneten Ökotonen und Biotopen findet sich in Anhang 6 
Bezugsraum Dassel-1 / Aufforstungsfläche DA-1

Tab. 5.2-1 Daten zum Bezugsraum Dassel-1 und zur Aufforstungsfläche DA-1

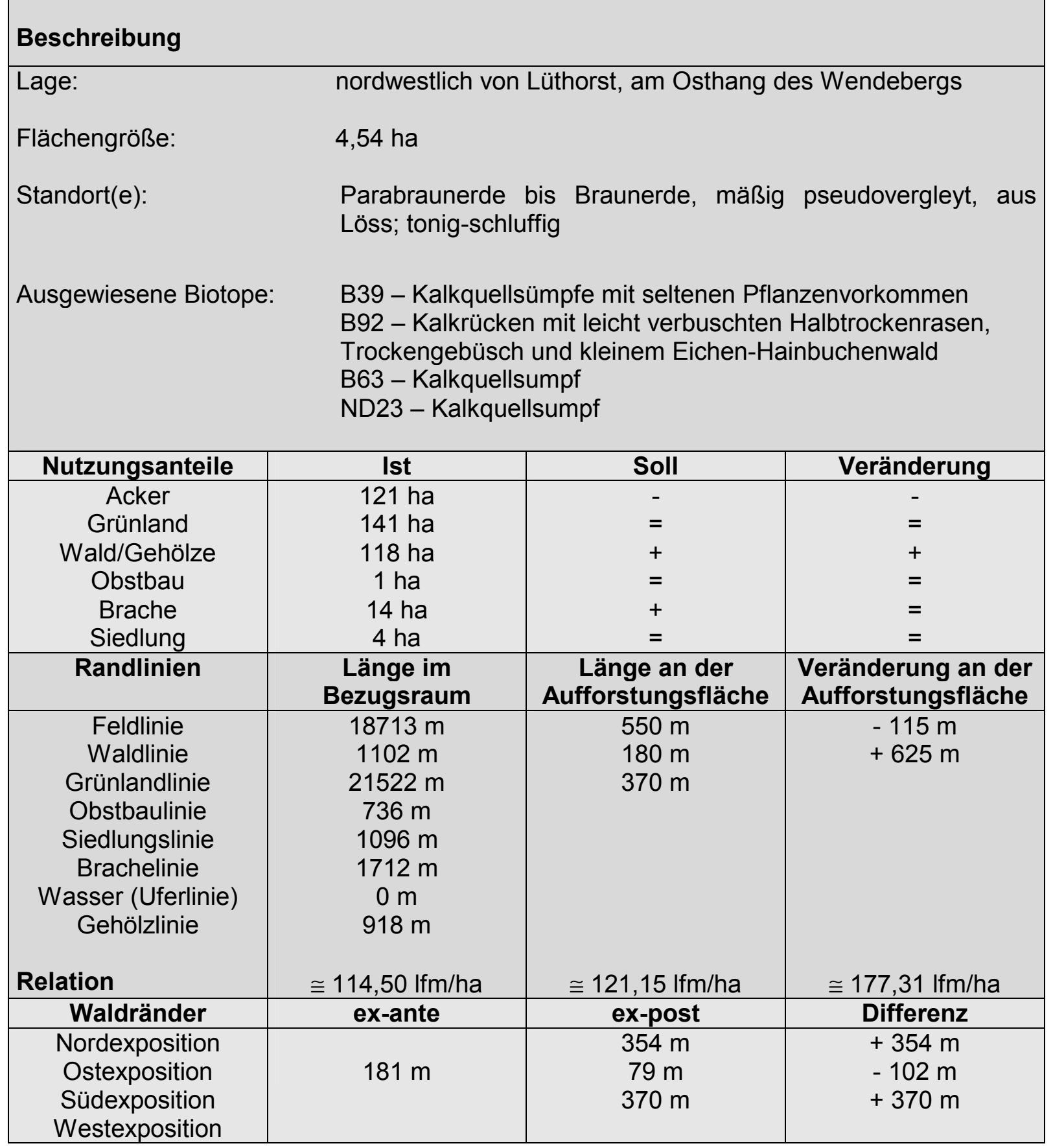

Eine Aufforstung der Fläche DA-1 nach einer der vorgeschlagenen Varianten führt zur Entstehung eines Laubwald-Biotops. Dieses ergänzt den vorhandenen, westlich angrenzenden Waldkomplex (Wendeberg). Die neue Fläche reicht bis zur hangabwärts gelegenen Landstraße. Sie grenzt an Ackerbau- und Grünlandflächen an. Ca. $130 \mathrm{~m}$ in nördlicher Richtung liegt eine kleine Streuobstwiese (0,6 ha). Eine Biotopverbundwirkung zwischen Wald-/Gehölzflächen ist nicht gegeben.

Der BR Dassel-1 weist mit 29,5 \% Flächenanteil bereits eine hohe Bewaldung auf. Die Aufforstungsmaßnahme führt zu einer Ergänzung und Ausdehnung des Landschaftstyps 
Laubwälder, insbesondere des Untertyps kolliner Buchenwald. Der Indikator Biotopverbund erhält damit eine Bewertung von 4 Punkten (Abb. 5.2-III).

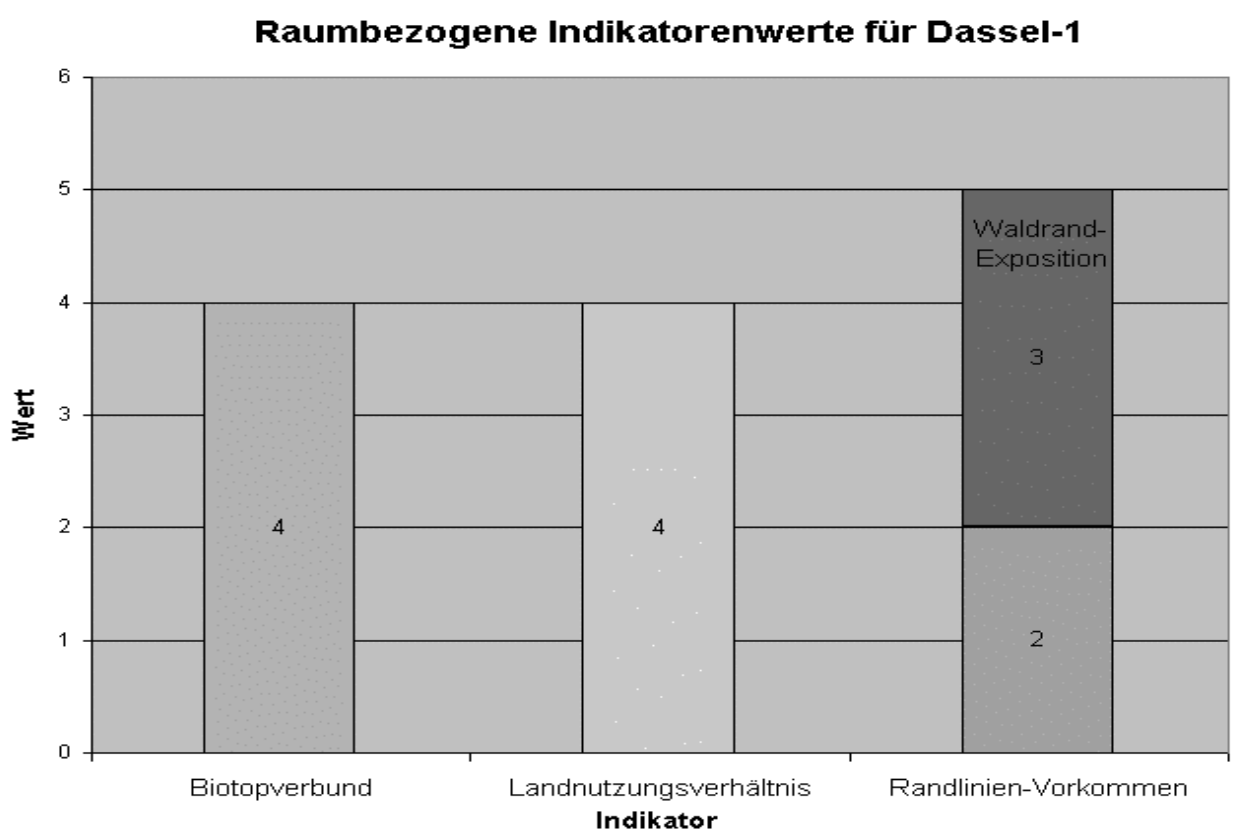

Abb. 5.2-III: Raumbezogene Indikatorenwerte für den Bezugsraum Dassel-1

Das Regionale Raumordnungsprogramm (RROP Northeim 1996) erwähnt eine beabsichtigte Waldmehrung im Rahmen des landwirtschaftlichen Strukturwandels, bei gleichzeitiger Erhaltung der Grünlandnutzung. Die Aufforstung einer Ackerfläche, wie im Fall DA-1, setzt diese Ziele direkt um. Die Waldfläche nimmt auf Kosten der Ackerfläche zu. Grünland und Obstbau sind von der Maßnahme nicht betroffen. Die Aufforstung verhält sich neutral zu dem, ebenfalls im RROP genannten, Ziel der Brachflächenmehrung. Der Indikator Landnutzungsverhältnis erhält folglich den Wert 4.

Die betrachtete Fläche weist bereits vor der Aufforstung eine Ökotonlänge auf, die über dem Durchschnitt des Bezugsraums liegt. Die Maßnahme schafft dennoch eine Steigerung der Randlinienlänge um 46 Prozent. Auch die Waldrandsituation verbessert sich deutlich. Zwar nimmt der ostexponierte Waldrand der Länge nach ab, jedoch werden gleichzeitig $370 \mathrm{~m}$ südexponierter Waldrand mit Übergang zum Grünland geschaffen. Des weiteren entsteht ein nordexponierter Waldaußenrand, der auf einer Länge von $347 \mathrm{~m}$ an eine Ackerfläche angrenzt. Damit erhält der Indikator Randlinien-Vorkommen den Maximalwert von 5 Punkten. 


\section{Bezugsraum Dassel-2 I Aufforstungsfläche DA-2}

Tab. 5.2-2 Daten zum Bezugsraum Dassel-2 und zur Aufforstungsfläche DA-2

\begin{tabular}{|c|c|c|c|}
\hline \multicolumn{4}{|l|}{ Beschreibung } \\
\hline Lage: & \multicolumn{3}{|c|}{$\begin{array}{l}\text { westexponierter Oberhang am Aulsberg (269,6 m), nördlich von } \\
\text { Lüthorst }\end{array}$} \\
\hline Flächengröße: & \multicolumn{3}{|l|}{2,46 ha } \\
\hline Standort(e): & \multicolumn{3}{|c|}{ Braunerde mittlerer Entwicklungstiefe; über Buntsandstein } \\
\hline Ausgewiesene Biotope: & \multicolumn{3}{|c|}{$\begin{array}{l}\text { ohne Nr. - kleinere Restwaldfläche mit „besonderer Bedeutung für } \\
\text { die Landschaftsökologie, das Landschaftsbild und das Lokalklima“ }\end{array}$} \\
\hline Nicht ausgewiesen: & \multicolumn{3}{|c|}{ je 1 Gehölz (< 7 ha) nördl. und südl. der Aufforstungsparzelle } \\
\hline Nutzungsanteile & Ist & Soll & Veränderung \\
\hline Acker & 173 ha & - & $=$ \\
\hline Grünland & 130 ha & $=$ & - \\
\hline Wald/Gehölze & 92 ha & + & + \\
\hline Obstbau & 2 ha & $=$ & $=$ \\
\hline Brache & 1 ha & + & $=$ \\
\hline Siedlung & 3 ha & $=$ & $=$ \\
\hline Randlinien & $\begin{array}{l}\text { Länge im } \\
\text { Bezugsraum }\end{array}$ & $\begin{array}{l}\text { Länge an der } \\
\text { Aufforstungsfläche }\end{array}$ & $\begin{array}{l}\text { Veränderung an der } \\
\text { Aufforstungsfläche }\end{array}$ \\
\hline Feldlinie & $25998 \mathrm{~m}$ & $731 \mathrm{~m}$ & \\
\hline Waldlinie & $7301 \mathrm{~m}$ & $28 \mathrm{~m}$ & $+1009 m$ \\
\hline Grünlandlinie & $29316 \mathrm{~m}$ & $969 \mathrm{~m}$ & $-806 m$ \\
\hline Obstbaulinie & 1243 m & $193 \mathrm{~m}$ & \\
\hline Siedlungslinie & $1067 \mathrm{~m}$ & & \\
\hline Brachelinie & 89 m & & \\
\hline Wasser (Uferlinie) & 309 m & & \\
\hline Gehölzlinie & 1701 m & & \\
\hline Relation & $\cong 167,56 \mathrm{Ifm} / \mathrm{ha}$ & $\cong 390,45 \mathrm{lfm} / \mathrm{ha}$ & $\cong 435,16 \mathrm{Ifm} / \mathrm{ha}$ \\
\hline Waldränder & ex-ante & ex-post & Differenz \\
\hline Nordexposition & & $372 \mathrm{~m}$ & $+372 m$ \\
\hline Ostexposition & & $37 \mathrm{~m}$ & $+37 m$ \\
\hline Südexposition & & $306 \mathrm{~m}$ & $+306 m$ \\
\hline Westexposition & $28 \mathrm{~m}$ & $93 \mathrm{~m}$ & $+65 \mathrm{~m}$ \\
\hline
\end{tabular}

Die Aufforstung der bislang als Grünland bewirtschafteten Fläche DA-2 lässt ein Waldbiotop entstehen, welches die südlich und nordöstlich angrenzenden Gehölze verbindet. Die bisherige Situation war nicht geeignet, Waldarten ein Zuhause zu bieten, da die beiden Flächen mit 1,9 bzw. 0,7 ha zu klein waren. Die Aufforstung führt nun zu einer Gesamtgröße von ca. 5,1 ha. Im Norden der Aufforstung, in einer Distanz von knapp 180 m, befindet sich bereits der große Waldkomplex des Elfas. Daher ist trotz des geringen Umfangs des neuen Laubwaldes mit einer baldigen Besiedelung durch waldbewohnende Tierarten zu rechnen. FLADE (1994) fasst Wälder, die weniger als $200 \mathrm{~m}$ voneinander entfernt liegen, zu Einheiten zusammen. Neben der Verbindung von gleichartigen Biotopen bewirkt die Aufforstung also auch eine Ergänzung und Ausdehnung des LT Laubwald.

Eine neutrale Bewertung erhält die Maßnahme für den Indikator Landnutzungsverhältnis. Zwar wird die Waldfläche entsprechend den raumplanerischen Zielsetzungen gemehrt, 
jedoch geht dafür auch Grünlandfläche verloren. Da die Aufforstung keine negative Auswirkung auf die Obstbaufläche besitzt, was wiederum den im RROP genannten Zielen entspricht, gleicht sich die Bewertung aus.

Die Auswertung des Luftbildes hinsichtlich der im BR vorhandenen Ökotone ergab eine hohe Dichte der Randlinien. Durch die unregelmäßige Grundform der Aufforstungsparzelle und die Nachbarschaft zu unterschiedlichen Landnutzungsarten, ist die Fläche DA-2 besonders reich an Grenzlinien. Dennoch nimmt die Länge pro Flächeneinheit durch die Aufforstung nochmals um $11,45 \%$ zu. Ein noch deutlicheres Bild zeigt die Zunahme an Waldrändern. Durch die Aufforstungsmaßnahme werden insgesamt $780 \mathrm{~m}$ Waldrandlinie neu geschaffen, wobei knapp die Hälfte nach Süden oder Westen exponiert ist. Damit erhält auch die Fläche DA-2 den Maximalwert von 5 Punkten für den Indikator Landnutzungsverhältnis (Abb. 5.2IV).

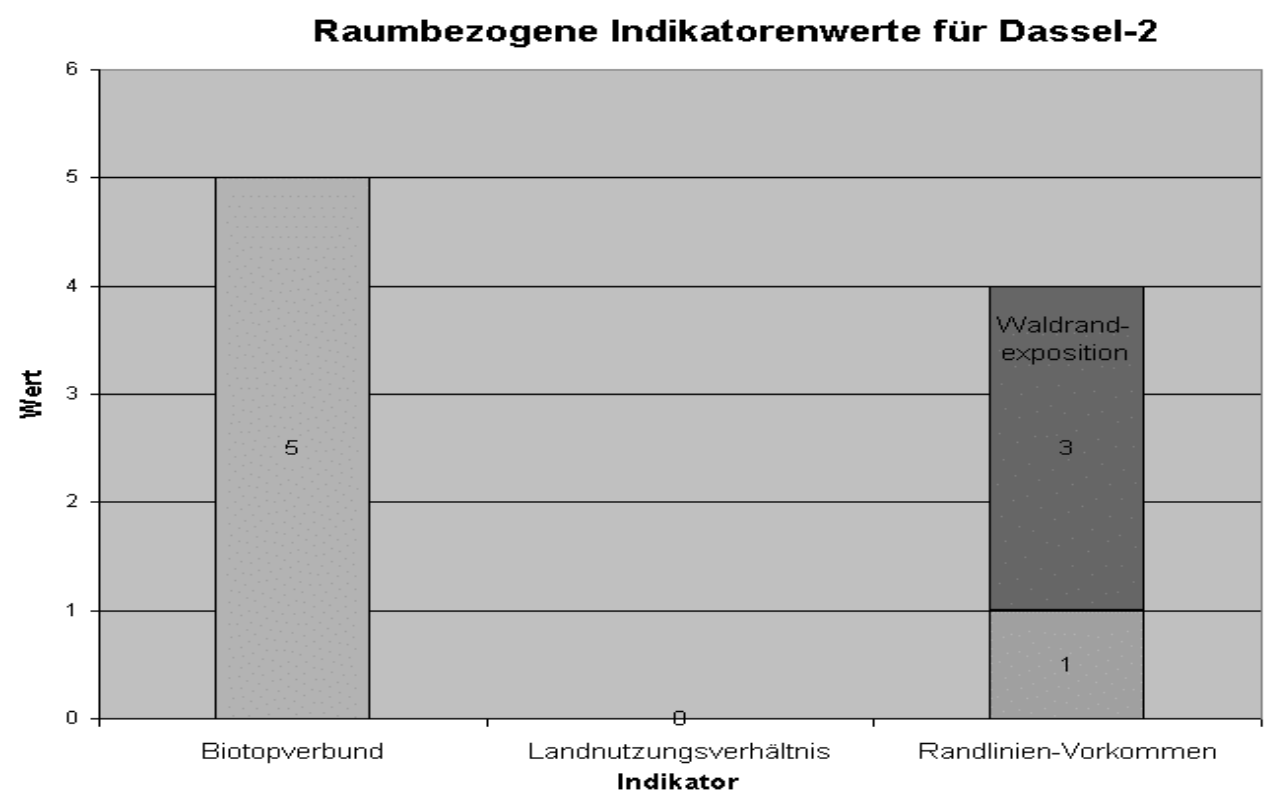

Abb. 5.2-IV: Raumbezogene Indikatorenwerte für den Bezugsraum Dassel-2 


\section{Bezugsraum Dassel-3 / Aufforstungsfläche DA-3}

Tab. 5.2-3: Daten zum Bezugsraum Dassel-3 und zur Aufforstungsfläche DA-3

\begin{tabular}{|c|c|c|c|}
\hline \multicolumn{4}{|l|}{ Beschreibung } \\
\hline Lage: & \multicolumn{3}{|c|}{ Hügelkuppe (ca. 245 m ü. NN) nördlich von Lüthorst } \\
\hline Flächengröße: & \multicolumn{3}{|l|}{7,62 ha } \\
\hline Standort(e): & \multicolumn{3}{|c|}{ Rendzina mittl. Entwicklungstiefe über Muschelkalk (80 \%) } \\
\hline & \multicolumn{3}{|c|}{ Parabraunerde, mäßig pseudovergleyt, erod.; aus Löss (20 \%) } \\
\hline Ausgewiesene Biotope: & \multicolumn{3}{|c|}{$\begin{array}{l}\text { ohne Nr. - kleinere Restwaldfläche mit „besonderer Bedeutung für } \\
\text { die Landschaftsökologie, das Landschaftsbild und das Lokalklima“ } \\
\text { B71 - zwei ungenutzte Senken mit Teichen, floristisch wertvoll } \\
\text { B124 - naturnaher Bachoberlauf (Bewer) }\end{array}$} \\
\hline Nicht ausgewiesen: & \multicolumn{3}{|c|}{2 Gehölze (< 7 ha), in westlicher Richtung gelegen (siehe DA-2) } \\
\hline Nutzungsanteile & Ist & Soll & Veränderung \\
\hline Acker & 183 ha & - & - \\
\hline Grünland & 125 ha & $=$ & - \\
\hline Wald/Gehölze & 79 ha & + & + \\
\hline Obstbau & 2 ha & $=$ & $=$ \\
\hline Brache & & + & $=$ \\
\hline Siedlung & 10 ha & $=$ & $=$ \\
\hline Randlinien & $\begin{array}{l}\text { Länge im } \\
\text { Bezugsraum }\end{array}$ & $\begin{array}{l}\text { Länge an der } \\
\text { Aufforstungsfläche }\end{array}$ & $\begin{array}{l}\text { Veränderung an der } \\
\text { Aufforstungsfläche }\end{array}$ \\
\hline $\begin{array}{l}\text { Feldlinie } \\
\text { Waldlinie }\end{array}$ & $\begin{array}{l}25203 \mathrm{~m} \\
5366 \mathrm{~m}\end{array}$ & $1150 \mathrm{~m}$ & $\begin{array}{l}-454 \mathrm{~m} \\
+942 \mathrm{~m}\end{array}$ \\
\hline Grünlandlinie & $27636 \mathrm{~m}$ & $1150 \mathrm{~m}$ & $-904 m$ \\
\hline Obstbaulinie & $1152 \mathrm{~m}$ & & \\
\hline Siedlungslinie & $2297 \mathrm{~m}$ & & \\
\hline Brachelinie & 89 m & & \\
\hline Wasser (Uferlinie) & 309 m & & \\
\hline Gehölzlinie & 2003 m & & \\
\hline Relation & $\cong 160,14 \mathrm{lfm} / \mathrm{ha}$ & $\cong 150,92 \mathrm{lfm} / \mathrm{ha}$ & $\cong 123,62 \mathrm{lfm} / \mathrm{ha}$ \\
\hline Waldränder & ex-ante & ex-post & Differenz \\
\hline $\begin{array}{l}\text { Nordexposition } \\
\text { Ostexposition } \\
\text { Südexposition } \\
\text { Westexposition }\end{array}$ & & $\begin{array}{l}336 \mathrm{~m} \\
190 \mathrm{~m} \\
388 \mathrm{~m} \\
217 \mathrm{~m}\end{array}$ & $\begin{array}{l}+336 m \\
+190 m \\
+388 m \\
+217 m\end{array}$ \\
\hline
\end{tabular}

Eine neue Situation ist für die Aufforstungsfläche DA-3 zu betrachten. Das entstehende Biotop liegt mit einer Größe von ca. 7,6 ha am oberen Grenzwert dessen, was von FLADE noch als Feldgehölz angesprochen wird. Die Distanz zum nächsten Waldkomplex beträgt $600 \mathrm{~m}$ und erschwert damit die Zuwanderung von Waldarten. In südlicher Richtung liegt eine Restwaldfläche, die mit einer Fläche von 2,3 ha ebenfalls als Feldgehölz zu bezeichnen ist. Die Entfernung beträgt $200 \mathrm{~m}$ und stellt eine überwindbare Hürde dar. Dem Bezugsraum wird aus diesen Gründen der LT Feldgehölze zugeordnet. Das entstehende Biotop dient der Ergänzung und Ausdehnung des Landschaftstyps. 
Die Bewertung der Veränderung der Landnutzungsanteile ergibt ein leicht positives Bild. Die Verringerung der Ackerfläche, die Mehrung der Waldfläche und das neutrale Verhalten gegenüber dem Obstbau, werden positiv eingestuft. Der Verlust an Grünlandfläche wird dagegen negativ bewertet (Abb. 5.2-V)

Die Randlinienlänge pro Hektar ist an der Aufforstungsfläche niedriger als im zugehörigen Bezugsraum, obwohl die Ökotone zum Teil quer über die Parzelle verlaufen. Durch die Aufforstung nimmt die Randliniendichte dann nochmals um ca. $18 \%$ ab. Dieser Verlust schlägt sich negativ in der Bewertung nieder, wird gleichzeitig aber durch die Würdigung der entstehenden Waldränder ausgeglichen. Insgesamt werden $1131 \mathrm{~m}$ Waldrandlinie geschaffen, wovon mehr als die Hälfte nach Süden oder Westen exponiert sind.

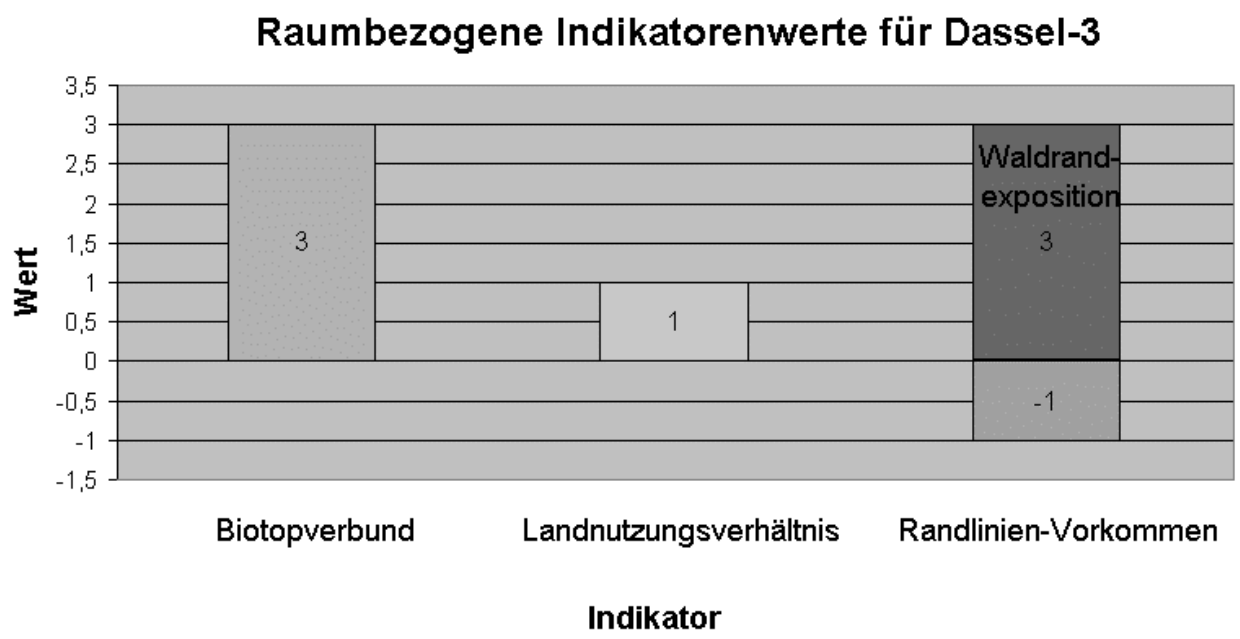

Abb. 5.2-V Raumbezogene Indikatorenwerte für den Bezugsraum Dassel-3 


\section{Bezugsraum Dassel-4 / Aufforstungsfläche DA-4}

Tab. 5.2-4: Daten zum Bezugsraum Dassel-4 und zur Aufforstungsfläche DA-4

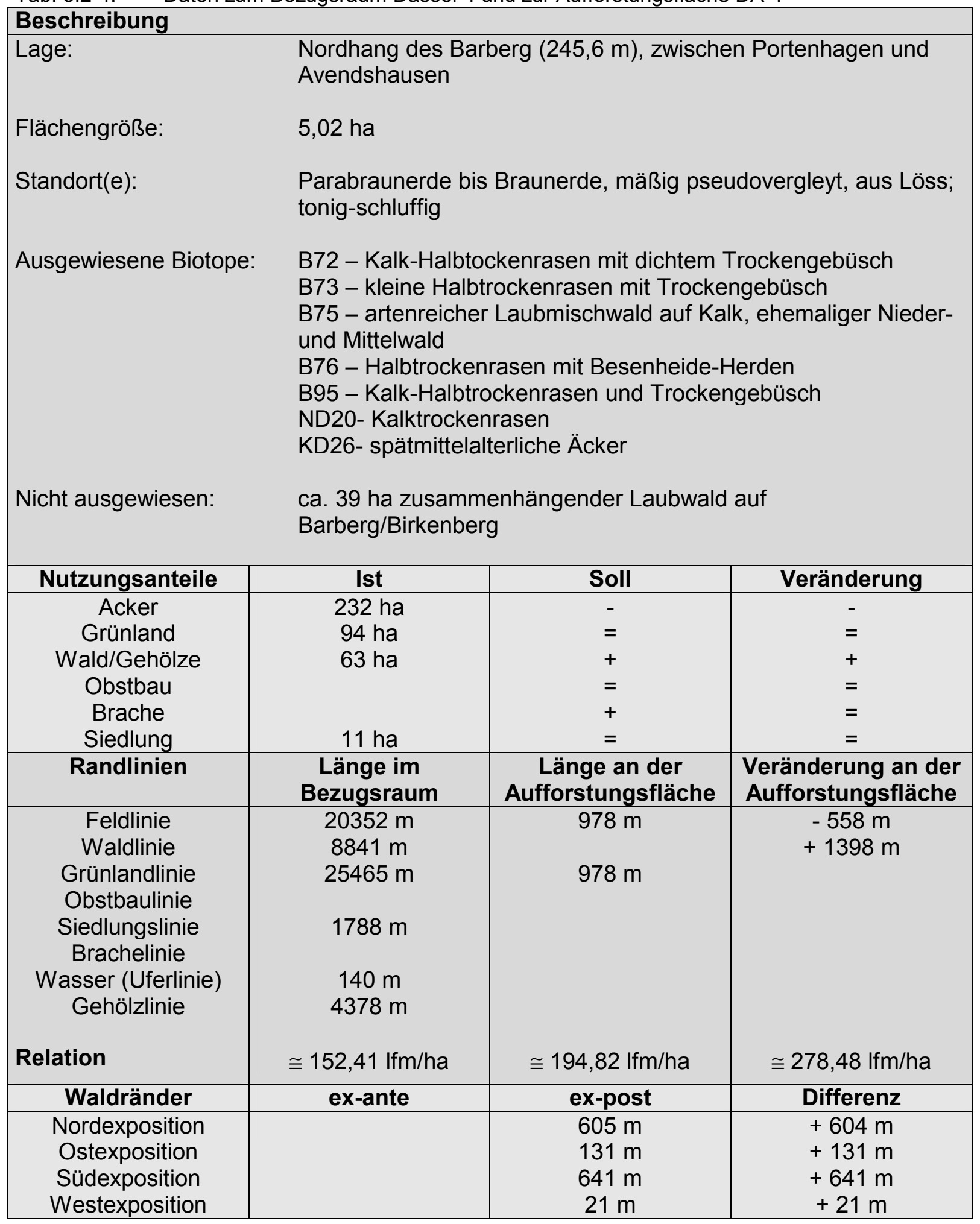

Die Fläche DA-4 liegt in mittelbarer Nachbarschaft zu einem knapp 40 ha großen Waldgebiet, einigen Halbtrockenrasen und Trockengebüschen. Das entstehende Biotop verbindet keine Gehölze miteinander, fügt sich aber gut in den Lebensraumkomplex aus den genannten Biotopen ein. Aus diesem Grunde wird eine Biotopergänzung angenommen. Darüber hinaus trägt die Aufforstung zur Ergänzung und Ausweitung des LT Laubwald bei. 
Eine frühe Besiedelung der neuen Waldfläche durch Tier- und Pflanzenarten aus dem benachbarten Wald am Barberg ist zu vermuten.

Hinsichtlich der Einflussnahme auf die Landnutzungsverhältnisse ist die gleiche Situation vorhanden, wie im Bezugsraum DA-1. Die Waldmehrung erfolgt ausschließlich auf Kosten der Ackerbauflächen. Die Entwicklung ist konform mit den Erhaltungsbestrebungen für die Gründlandbewirtschaftung und den Obstbau.

Die Aufforstung führt zu einer deutlichen Zunahme (+43\%) der Randliniendichte. Diese liegt vor, aber insbesondere auch nach der Maßnahme ganz deutlich über dem Schnitt des Bezugsraumes. Noch eindrucksvoller zeigt sich die Situation mit Blick auf die Waldaußenränder. Hier werden knapp 1,4 km Waldrandlinie geschaffen. Besonders die etwa $500 \mathrm{~m}$ lange, südwestlich exponierte Grenzlinie kann sich zu einem wertvollen Übergangsbiotop entwickeln. Hier trennt ein schmaler Grünlandstreifen zwischen den benachbarten Waldflächen. Der Indikator Randlinien-Vorkommen führt auch in diesem Fall zu einer maximalen Bewertung (Abb. 5.2-X).

\section{Raumbezogene Indikatorenwerte für Dassel-4}

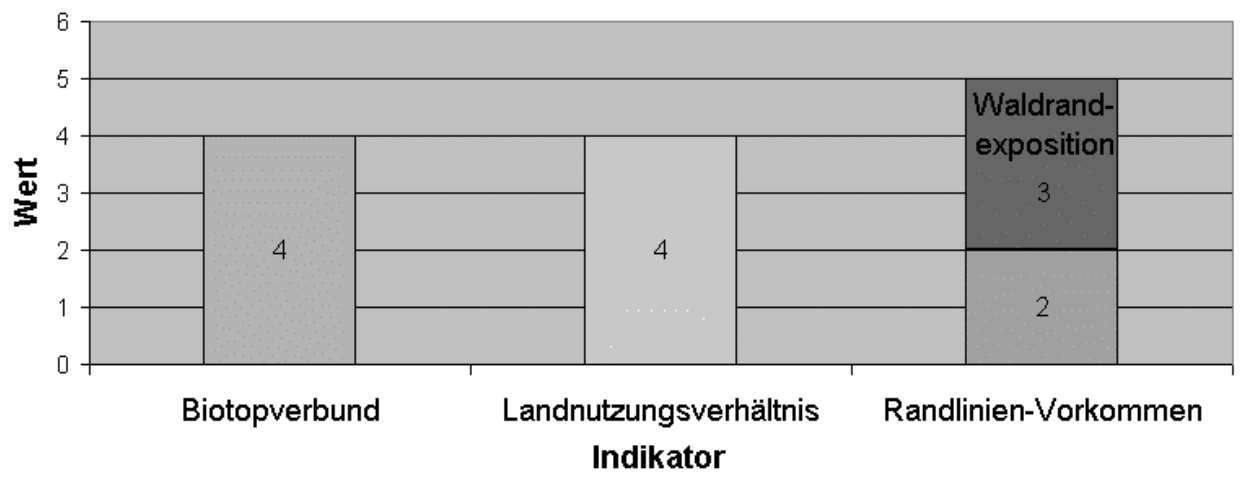

Abb. 5.2-VI Raumbezogene Indikatorenwerte für den Bezugsraum Dassel-4 


\section{Bezugsraum Dassel-5/ Aufforstungsfläche DA-5}

Tab. 5.2-5 Daten zum Bezugsraum Dassel-5 und zur Aufforstungsfläche DA-5

\begin{tabular}{|c|c|c|c|}
\hline \multicolumn{4}{|l|}{ Beschreibung } \\
\hline Lage: & \multicolumn{3}{|c|}{ Kuppenlage auf der Erhebung „Platte“ (266,6 m) } \\
\hline Flächengröße: & \multicolumn{3}{|l|}{5,12 ha } \\
\hline Standort(e): & \multicolumn{3}{|c|}{ Rendzina, sehr flachgründig, aus Kalkstein } \\
\hline Ausgewiesene Biotope: & \multicolumn{3}{|c|}{$\begin{array}{l}\text { B215 - Perlgras-Buchenwald mit Orchideenvorkommen (19,3 } \\
\text { ha) }\end{array}$} \\
\hline Nicht ausgewiesen: & \multicolumn{3}{|c|}{$\begin{array}{l}\text { Mehrere Gebüsche/Gehölze bis } 1,5 \text { ha südl. und nördl. der } \\
\text { Aufforstungsfläche }\end{array}$} \\
\hline & \multicolumn{3}{|c|}{$\begin{array}{l}\text { Laubmischwald ( } 26 \text { ha) westlich und südlich ( } 5 \text { ha, teils noch in } \\
\text { Kultur) der Aufforstungsfläche }\end{array}$} \\
\hline Nutzungsanteile & Ist & Soll & Veränderung \\
\hline $\begin{array}{c}\text { Acker } \\
\text { Grünland } \\
\text { Wald/Gehölze } \\
\text { Obstbau } \\
\text { Brache } \\
\text { Siedlung }\end{array}$ & $\begin{array}{l}230 \text { ha } \\
86 \text { ha } \\
57 \text { ha }\end{array}$ & $\begin{array}{l}- \\
= \\
+ \\
= \\
+ \\
=\end{array}$ & $\begin{array}{l}- \\
= \\
+ \\
= \\
= \\
=\end{array}$ \\
\hline Randlinien & $\begin{array}{l}\text { Länge im } \\
\text { Bezugsraum }\end{array}$ & $\begin{array}{l}\text { Länge an der } \\
\text { Aufforstungsfläche }\end{array}$ & $\begin{array}{l}\text { Veränderung an der } \\
\text { Aufforstungsfläche }\end{array}$ \\
\hline $\begin{array}{c}\text { Feldlinie } \\
\text { Waldlinie } \\
\text { Grünlandlinie } \\
\text { Obstbaulinie } \\
\text { Siedlungslinie } \\
\text { Brachelinie } \\
\text { Wasser (Uferlinie) } \\
\text { Gehölzlinie }\end{array}$ & $\begin{array}{c}20252 \mathrm{~m} \\
5691 \mathrm{~m} \\
18565 \mathrm{~m} \\
190 \mathrm{~m} \\
3782 \mathrm{~m}\end{array}$ & $\begin{array}{l}776 \mathrm{~m} \\
248 \mathrm{~m} \\
392 \mathrm{~m}\end{array}$ & $\begin{array}{l}-545 \mathrm{~m} \\
+511 \mathrm{~m}\end{array}$ \\
\hline Relation & $\cong 127,00 \mathrm{lfm} / \mathrm{ha}$ & $\cong 151,56 \mathrm{lfm} / \mathrm{ha}$ & $\cong 148,24 \mathrm{lfm} / \mathrm{ha}$ \\
\hline Waldränder & ex-ante & ex-post & Differenz \\
\hline $\begin{array}{l}\text { Nordexposition } \\
\text { Ostexposition } \\
\text { Südexposition } \\
\text { Westexposition }\end{array}$ & $248 \mathrm{~m}$ & $\begin{array}{l}231 \mathrm{~m} \\
294 \mathrm{~m} \\
215 \mathrm{~m}\end{array}$ & $\begin{array}{l}+231 \mathrm{~m} \\
+294 \mathrm{~m} \\
-33 \mathrm{~m}\end{array}$ \\
\hline
\end{tabular}

Die Aufforstungsfläche DA-5 ist geprägt durch eine sehr flachgründige Rendzina. Derzeit noch ackerbaulich genutzt, könnte dieser Standort bald einen ökologisch wertvollen Laubwald tragen. Das dabei geschaffene Biotop würde den angrenzenden PerlgrasBuchenwald ergänzen. Eine echte Verbindung mit dem westlich gelegenen Wald wird nicht erreicht. Die Distanz zwischen den Waldflächen verringert sich aber von $350 \mathrm{~m}$ auf $85 \mathrm{~m}$, wodurch der Austausch zwischen den dort lebenden Populationen deutlich erleichtert wird. Der LT Laubwald, bzw. kolliner Buchenwald wird ergänzt durch den neuen Wald. Aus der Vogelperspektive ist zu erkennen (Luftbild im Anhang 6), dass die Entwicklung eher als Anreicherung des Biotopkomplexes anzusprechen ist, denn als Ausdehnung eines Landschaftstyps. 
Die Veränderung der Landnutzungsverhältnisse entspricht dem für DA-1 und DA-4 geschilderten Fall. Die Bewertung erreicht ebenfalls 4 Punkte.

Die Randliniendichte nimmt an der Aufforstungsfläche geringfügig (-0,2 \%) ab. Angesichts der Tatsache, dass die Dichte an der Fläche dennoch deutlich über jener des Bezugsraumes liegt, scheint diese Veränderung marginal. Dies gilt insbesondere bei Betrachtung der Waldrandsituation. Die Waldrandlinie vergrößert sich um $492 \mathrm{~m}$. Der westlich exponierte Rand nimmt um $33 \mathrm{~m}$ ab. Gleichzeitig werden $294 \mathrm{~m}$ südlicher Rand neu geschaffen. Gerade für diesen potentiellen Standort für einen Seggen-Buchenwald könnte dies eine gute Entwicklungschance darstellen. Werden süd- und westexponierte Ränder stärker gewichtet wie in Kap. 3.5.7 beschrieben - so geht daraus eine Multiplikation der Wertungszahl um den Faktor 2,52 hervor. Unter Berücksichtigung der negativen Veränderung der Ökotonlänge pro Fläche, erzielt DA-5 einen Wert von 2 Punkten für das Randlinienvorkommen.

\section{Raumbezogene Indikatorenwerte für Dassel-5}

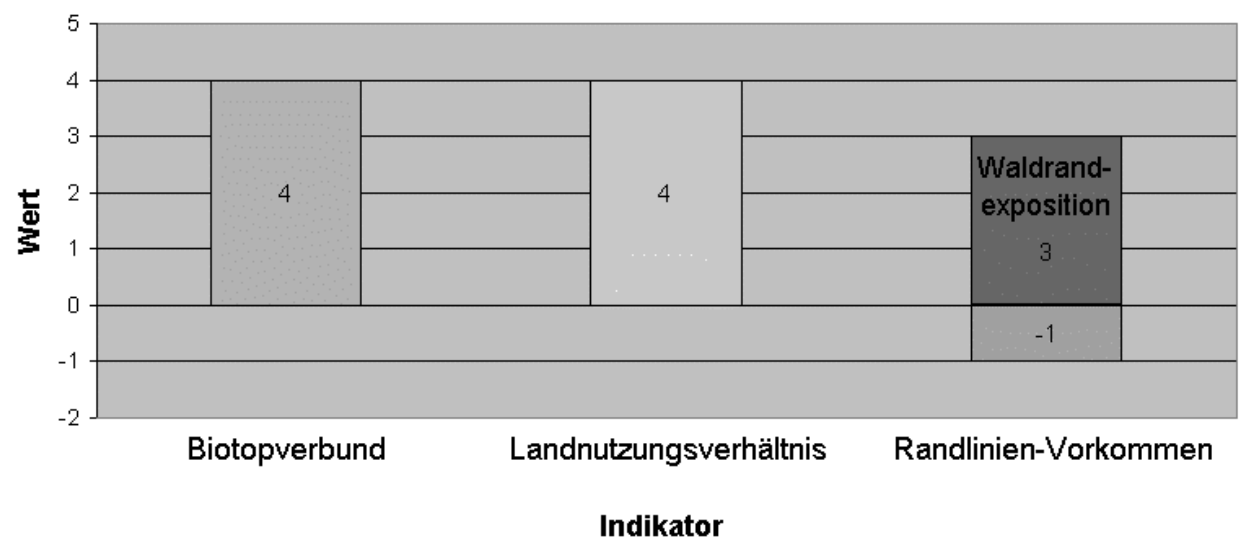

Abb. 5.2-VII Raumbezogene Indikatorenwerte für den Bezugsraum Dassel-5 


\subsection{Gesamtergebnisse für die Untersuchungsflächen in der Region Dassel}

\section{Gegenüberstellung der raumbezogene Bewertungen}

Die Schaffung von Waldbiotopen im landwirtschaftlich geprägten Raum führt zu einer Anreicherung der Landschaft mit Elementen. Die Vielfalt der Landschaft wird dabei visuell wie funktionell gefördert. Es entstehen neue Biotope. Die Dauer bis zu deren Besiedelung ist stark davon abhängig, wie nahe andere Waldbiotope vergleichbaren Typs liegen. Die Aufforstungen im Untersuchungsraum Dassel konzentrieren sich auf die Muschelkalk- und Buntsandstein-Hügel zwischen Lüthorst und Elfas. Diese weisen bereits ein weites Mosaik kleiner Waldbestände auf. Die Aufforstungen ergänzen in der Regel angrenzende Flächen im Sinne des Biotop-Verbundes. Eine echte Verbund-Wirkung zwischen Gehölzbiotopen bleibt jedoch die Ausnahme.

Die relevanten - und durch eine Aufforstung erweiterbaren - Landschaftstypen im Untersuchungsraum stellen die Laubwälder (insbesondere der kolline Buchenwald) und die Feldgehölze dar. In Abhängigkeit von der Größe des neuen Waldes und der eventuell miteinander verbundenen Gehölze, wirkt hier die Aufforstung als Ergänzung und Ausweitung des Landschaftstyps. Dabei dominiert bei den hier behandelten Fällen der LT Laubwald, da die kritische Größe von 7 ha durch den entstehenden Biotop-Verbund i.w.S. meist erreicht wird.

Einen wichtigen Beitrag zur ökologischen Aufwertung der Landschaft leisten die Aufforstungen mit der Schaffung von Randlinien. Ökotone sind als Übergangsbereiche zwischen unterschiedlichen Biotopen auch der Lebensraum für viele Tierarten. Sie können temporär als Rückzugsgebiete dienen, wenn die Flächenbiotope akut gestört werden. Die Bezugsräume in Dassel zeigen im Durchschnitt eine wesentlich höhere Randliniendichte, als jene in Apolda. Die neuen Waldflächen können sogar noch weitere Impulse setzen. Dies gelingt insbesondere durch die Schaffung von Waldaußenlinien. Deren strukturreiche Gestaltung ist Aufgabe des Grundeigentümers und sollte entsprechend berücksichtigt werden. Im Rahmen der vorliegenden Arbeit erfolgt dies bereits bei den flächenbezogenen Bewertungen.

Die mit einer Aufforstung einhergehende Umwidmung einer Fläche führt kleinräumig zu Verschiebungen im Landnutzungsverhältnis. In der Raumpolitik wird immer wieder darauf hingewiesen, dass im Rahmen der Agrarstrukturreform aus der Nutzung ausscheidende Bestände sich selbst überlassen oder in Wald überführt werden sollten. Zusätzlich beschreibt das Regionale Raumordnungsprogramm Northeim (1994) für seinen Geltungsbereich das Ziel, die Grünlandwirtschaft in ihrem heutigen Umfang zu erhalten. Aufforstungen sind also vor allem dann zielkonform, wenn sie bisher ackerbaulich genutzte Flächen betreffen. Wird Grünland aufgeforstet, so führt dies im vorliegenden Fall zu einem geringeren Evaluationsergebnis im entsprechenden Indikator.

\section{Gesamtbewertung durch Kombination der flächen- und der raumbezogenen Indikatoren}

Sie Summierung der flächenbezogenen und der raumbezogenen Indikatorwerte für die jeweiligen Aufforstungsflächen zeigt ein harmonisches Bild (Anhang xxk). Alle Aufforstungsvarianten versprechen die Entwicklung eines Laubmischwaldes. Dessen Beitrag zur Landschaftsstruktur und zum Biotop-Verbund ist daher nur von der Flächenauswahl selbst abhängig. Die raumbezogenen Werte sind im Variantenvergleich flächenspezifisch konstant. Baumartenvielfalt, Naturnähe und Strukturentwicklung werden dagegen durch die Baumartenwahl und -mischung bestimmt. Auch die Frage, ob sich ein Biotop entwickeln kann, welches nach derzeitigem Erhebungsstand als schützenswert oder gefährdet gilt, wird in diesem Zusammenhang entschieden. Ein Vergleich der Aufforstungsvarianten 
untereinander zeigt, dass sie alle zu einem Mindestmaß an Bewertungspunkten führen. Durch gezielte Baumartenzusammenstellung können die flächenbezogenen Indikatorwerte aber stark angehoben werden. Der Handlungsspielraum des Grundeigentümers ist sehr hoch (Abb. 5.3-I). Hinzu kommt die Möglichkeit, die Aufforstung durch einen breiten, vielfältigen und strauchreichen Waldrand ökologisch aufzuwerten. Damit kann die Bewertung einer Aufforstung nach dem vorliegenden Verfahren gezielt beeinflusst werden. In den Fallbeispielen ist eine Erhöhung auf die doppelte bis dreifache Bewertungszahl möglich.

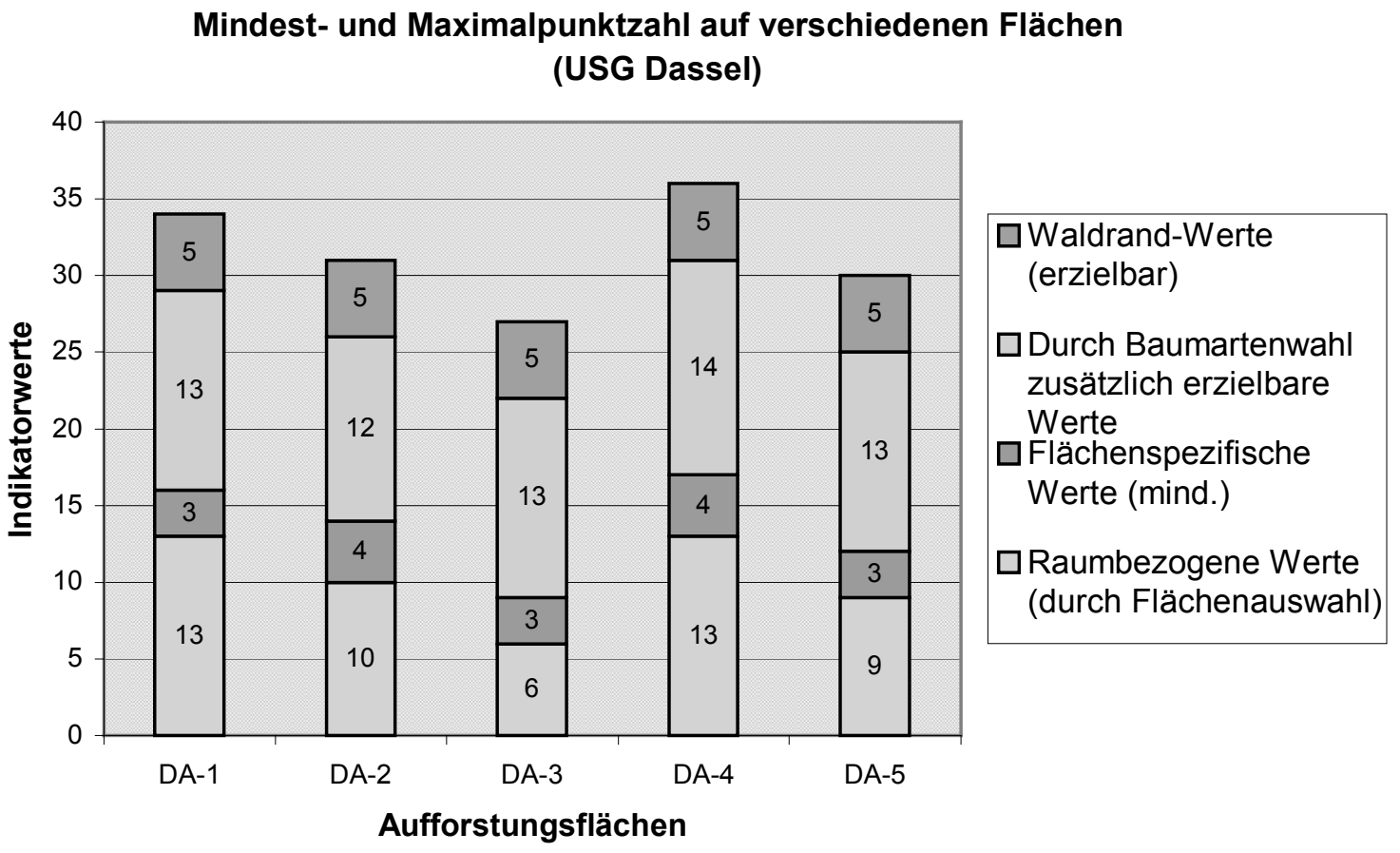

Abb. 5.3-I: Mindest- und Maximalpunktzahl auf verschiedenen Flächen (USG Dassel) 


\section{Diskussion}

\subsection{Instrumentalisierung der naturschutzfachlichen Bewertung}

Das vorliegende Verfahren zur ex-ante Bewertung von Erstaufforstungen folgt dem Prinzip einer Wirkungsanalyse. Die Betrachtung der Wirkungszusammenhänge erfolgt für das entstehende Waldbiotop und für die mittelbare Umgebung, den Bezugsraum. Einer abstrakten Wiedergabe des Systems folgt die Ableitung von Indikatoren. Diese stehen für wichtige, häufig verwendete Bewertungskriterien des Naturschutzes. Dazu gehören Naturnähe, Eigenart, Vielfalt und Seltenheit. Im Gegensatz zu den Kriterien können die Indikatoren losgelöst von Zielkonflikten betrachtet werden. Im vorgestellten Verfahren stehen sie deshalb gleichwertig nebeneinander. Eine Aufforstungsmaßnahme kann dadurch hinsichtlich ihrer Kompatibilität mit verschiedenen Leitbildern (visionäre Zielrichtungen) bewertet werden.

Die Indikatoren unterscheiden sich durch ihren räumlichen Bezug. Variantenbezogene Indikatoren behandeln die Entwicklung auf der spezifischen Aufforstungsfläche. Raumbezogene Indikatoren zeigen die Beziehungen mit den Flächen der unmittelbaren und mittelbaren Umgebung auf. Das Verfahren ermöglicht sowohl den Vergleich einer identischen Aufforstungsvariante auf unterschiedlichen Flächen, als auch den Vergleich unterschiedlicher Varianten für dieselbe Fläche. Die Darstellung der Bewertungsergebnisse erfolgt anhand einer ordinalen Skala. Dies fördert die Vergleichbarkeit der Ergebnisse und ermöglicht eine Verwendung des Verfahrens zur Ermittlung der Förderungsfähigkeit. Extern definierte Wertmaßstäbe gewährleisten eine hohe Flexibilität in der Anwendung, die Übertragbarkeit auf andere Regionen sowie die Adaption an veränderte Rahmenbedingungen.

Das beschriebene Verfahren muss zwei unterschiedlichen Personengruppen gerecht werden: Den für eine Aufforstungsgenehmigung zuständigen Behörden und den antragstellenden Grundeigentümern. Die Aufgabe der Behörden ist es, Eigentümerinteresse und öffentliche Belange gegeneinander abzuwägen. In diesem Sinne werden regionale Zielvorgaben zur Entwicklung der Flächennutzungsanteile und rechtliche Vorgaben zum Biotopschutz in das Evaluationsverfahren integriert. Darüber hinaus ermöglichen die gewählten Indikatoren eine Berücksichtigung weiterer Faktoren, die bei Genehmigungsverfahren bislang nicht systematisch beachtet wurden. Dazu gehören Naturnähe, Baumartenvielfalt und Strukturentwicklung. Art und Anzahl der Indikatoren wurden unter praktischen Gesichtspunkten ausgewählt. Dies trägt zur Anwenderfreundlichkeit bei. Ein Genehmigungsverfahren soll einen geringen Aufwand zur Aufnahme und Verarbeitung der Daten erfordern. Das Verfahren greift deshalb auf bereits vorhandene Datenquellen (Biotopkartierung, Standortskarten, PNV-Schlüssel) zurück. Soweit diese Datenquellen unmittelbar verfügbar sind, ist eine Bearbeitung des Aufforstungsgesuchs innerhalb weniger Stunden möglich. Im Rahmen der vorliegenden Arbeit mussten einige Daten zunächst aufbereitet werden (z.B. durch Digitalisierung), bevor sie mit Hilfe eines Geographischen Informationssystems verarbeitet werden konnten. Das Verfahren setzt voraus, dass der Anwender über ein GIS-Programm verfügt. Es impliziert auch einen Schulungsaufwand für die Behördenmitarbeiter. Die Entwicklung eines einfachen Computerprogramms könnte diesbezüglich eine deutliche Steigerung der Anwendungsfreundlichkeit bewirken. Ein solches Programm erleichtert darüber hinaus den Austausch der Informationen und Ergebnisse zwischen den Behörden. Dies ist wichtig, wenn mehrere Behörden in die Entscheidungsfindung einbezogen werden. Die für das Evaluationsverfahren eindeutig definierten Indikatoren ermöglichen aber bereits heute einen schnellen Austausch präziser Informationen. Der Entscheidungsgang ist damit transparent und nachvollziehbar.

Die verwendete Ordinalskala zur Darstellung der Einzelwerte erhöht die Anwendungsmöglichkeiten des Verfahrens zusätzlich. Sie erleichtert den Vergleich 
unterschiedlicher Aufforstungsmaßnahmen und eröffnet Möglichkeiten zur statistischen Auswertung der Anträge. Potentiell können die Ergebnisse auch im Sinne einer Förderungswürdigkeit der geplanten Maßnahme interpretiert werden. Dazu wird allerdings ein Referenzwert benötigt, der im Rahmen einer langfristigen, statistischen Auswertung oder durch eine modellhafte Durchführung des Verfahrens mit großer Stichprobenmenge zu ermitteln ist.

Der Antragsteller profitiert gleich mehrfach von der Transparenz des Verfahrens. Das Ergebnis des Genehmigungsverfahrens wird kalkulierbar. Verständnisschwierigkeiten zwischen Grundeigentümer und bearbeitender Behörden werden minimiert. Gleichzeitig enthält der Antragsteller indirekte, strategische Empfehlungen. Das Verfahren verdeutlicht, wie der ökologische Wert der Aufforstung durch gezielte Baumartenwahl zu beeinflussen ist. Auch die Bedeutung der Flächenauswahl für die Biotopverbundwirkung wird erläutert. Damit öffnen sich neue Möglichkeiten zum Austausch zwischen Behörden und Bürger über die ökologischen Entwicklung der Kulturlandschaft.

Schließlich kann das Verfahren unabhängig von der Vorgabe durch Landschaftspläne oder Forstliche Rahmenpläne eine naturschutzkonforme Beurteilung von Aufforstungsmaßnahmen sichern. Kann ein interdisziplinärer Konsens über die Verwendung des Verfahrens und die enthaltenen Wertmaßstäbe erreicht werden, so erübrigt sich die Ausscheidung von Aufforstungsgewannen und Freihaltezonen oder die Definition starrer Erstaufforstungsleitbilder. Die Raumplanung wird damit flexibler für veränderte Rahmenbedingungen und Eigentümerinteressen.

\subsection{Indikatoren}

Die verwendeten Indikatoren stehen für naturschutzfachliche Aspekte bei der Bewertung einer Aufforstung, die aus dem abstrakten System im Rahmen einer Wirkungsanalyse abgeleitet wurden. Sie sind voneinander unabhängig und können daher einzeln betrachtet werden. Die Ergebnisse der Fallstudien sind unter Beachtung der jeweiligen Indikatorenmerkmale zu würdigen.

Für den Indikator Naturnähe wird der Bewertungsmaßstab dem jeweils landesspezifischen Verfahren der Waldbiotopkartierung entliehen. Die Odinalskala gibt die Spannweite zwischen Naturferne (kaum PNV-Baumarten) und Natürlichkeit (vollständiges Baumartenspektrum der PNV) wieder.

Der Indikator Baumartenvielfalt orientiert sich ebenfalls an dem natürlichen Idealzustand. Wieder kommt der landesspezifische Schlüssels zur Herleitung der PNV in Anwendung. Auch das Bewertungsschema entspricht jenem des Indikators Naturnähe. Dennoch erfolgt eine klare Trennung zwischen der Vielfalt und der Natürlichkeit eines Bestandes.

Die Wertschätzung der Biotoptypen im Rahmen des Indikators Seltenheit des Biotops wird ebenfalls aus fremden Quellen (Waldbiotopkartierung, Roten Liste der gefährdeten Biotoptypen) übernommen und in eine Ordinalskala transformiert.

Der Indikator Landnutzungsverhältnisse greift auf Zielvorgaben der Raumplanung zurück. Die betrachteten Landnutzungstypen ergeben sich aus der Nutzungstypenkonkurrenz.

Die genannten Indikatoren beruhen auf qualitativen Feststellungen und Wertschätzungen, die außerhalb des Evaluationsverfahrens bereits zur Anwendung kommen. Sie werden durch das vorgestellte Verfahren übernommen und mittels eines indikatorspezifischen Bewertungsschemas operationalisiert. Zur Aufforstungsevaluation sind nunmehr nur noch quantitative Daten zu erheben. Durch die Verwendung „externer“ Definitionen verändert sich das Evaluationsverfahren mit jeder Weiterentwicklung der verwendeten Schlüssel oder einer revidierten Wertschätzung. Ein weiterer, verfahrenstechnischer Vorteil dieser Indikatoren besteht in der Übernahme externer Daten, die teilweise bereits digital vorliegen und dementsprechend einfach bearbeitet werden können. 
Für den Indikator Strukturpotential wurde eine Klassifikation zur Einteilung der Baumarten in Strukturpotentialklassen entwickelt. Grundlage der Entwicklung ist die Auswertung der Fachliteratur zu Konkurrenzkraft und anderen Charakteristika der betrachteten Baumarten. Der Ansatz einer prognostischen Bewertung der Strukturentwicklung ist neu, weshalb Vergleiche mit anderen Untersuchungen nicht möglich sind. Die Baumartenklassifikation muss im Rahmen von Folgeuntersuchungen überprüft werden. Wird sie bestätigt, so kann sie zu einem praktikablen Instrument für die Bestandesplanung weiter entwickelt werden.

Die Anwendung des Indikators Waldrandausprägung erfolgt anhand einer einfachen Checkliste zur gutachtlichen Feststellung von Waldrandmerkmalen. Die Merkmale wurden aus der Literatur / wissenschaftlichen Diskussion abgeleitet und beziehen sich auf Struktur und Form des Waldrandes. Die Checkliste führt direkt zu einem Bewertungsschema mit gleicher Gewichtung der aufgenommenen Parameter.

Zur Betrachtung des Biotop-Verbundes wird auf die Beschreibung von Landschaftstypen (FLADE 1994) zurückgegriffen, die auf die Betrachtung von Biotop-Komplexen zurück geht. Die Ermittlung der Landschaftstypen erfolgt gutachtlich und wird anhand ornithologischer Daten zum Vorkommen der entsprechenden Leitarten verifiziert. Bezüglich der avifaunistischen Informationen ist das Verfahren auf fremde Quellen angewiesen. Im vorliegenden Fall konnten ortskundigen Ornithologen die notwendigen Daten liefern. Generell ist die Verfügbarkeit von Daten zur Population bestimmter Vogelarten eine Vorbedingung zur Anwendbarkeit des vorgestellten Verfahrens.

Der Indikator Randlinien-Vorkommen betrachtet die Randlinien-Dichte sowie die Waldrandlänge vor und nach der Aufforstung. Für die Randlinien-Dichte wird keine Gewichtung zwischen unterschiedlichen Ökotonen vorgenommen. Beurteilt wird die generelle Veränderung des Mittelwertes an der Aufforstungsfläche. Zusätzliche Daten (Ökotontypen, Randlinien-Dichte des Bezugsraumes) werden ermittelt und ergänzen die Informationen. Das Bewertungsschema wurde intuitiv erstellt und bedarf somit der Bestätigung durch weitergehende Untersuchungen. Bei der Ermittlung der Waldrandsituation wurde nach Exposition der Randlinien unterschieden. Eine höhere Gewichtung der S- und W-exponierten Ränder wurde probeweise durchgeführt, führte aber zum gleichen Bewertungsergebnis wie die ungewichtete Variante. Die Vermischung der quantitativen mit einer qualitativen Bewertung in diesem Indikator bringt offensichtlich keinen Vorteil. Darüber hinaus können die qualitativen Merkmale eines Waldrandes (siehe Indikator Waldrandausprägung) eine höhere Bedeutung besitzen als die Exposition. Die Konzentration auf die messbare Länge der Randlinien ist daher anzuraten.

Alle Indikatoren zeigen eine gute Erfassbarkeit und führen zu nachvollziehbaren Ergebnissen. Soweit die Merkmale und Bewertungsmaßstäbe von fremden Verfahren abgeleitet wurden, bleibt das Verfahren objektiv und flexibel. Wo eigene Klassifikationen und intuitive Bewertungsschemata entwickelt wurden, müssen die Ergebnisse durch weitere Untersuchungen gestützt werden. Gutachtliche Einschätzungen wurden weitgehend vermieden. Wo sie dennoch erfolgen (Waldrandmerkmale, Landschaftstypen), bleibt das Verfahren durch klare Grundregeln nachvollziehbar.

Die raumbezogenen Indikatoren Biotopverbund, Landnutzungsverhältnis und RandlinienVorkommen können zu negativen Bewertungsergebnissen führen. Dadurch wird das Verfahren der Einschätzung gerecht, dass eine Aufforstung den Arten- und Biotopschutz auch negativ beeinflussen kann. Die anderen Indikatoren besitzen ausschließlich eine positive Bewertungsskala.

Das Verfahren kombiniert quantitative und qualitative Merkmale der Aufforstung, wobei der Schwerpunkt auf den quantitativen liegt. Dieses Vorgehen fördert die Objektivität der Evaluation und entspricht den Ansprüchen der Landschaftsplanung (KIRBY 1994). Die Indikatoren sind darüber hinaus voneinander unabhängig und werden gleich gewichtet weitere wichtige Voraussetzungen für ein objektives Vorgehen in der Landschaftsplanung. 


\subsection{Planungsvoraussetzungen}

Die Flexibilität und Übertragbarkeit des Verfahrens sollen durch eine Reihe externer Zielvorgaben und Bewertungsmaßstäbe gewährleistet werden. Hierzu gehören raumplanerische Ziele ebenso wie die Verfahren zur Ableitung der potentiell natürlichen Vegetation (PNV) oder die Liste der zu schützenden Biotoptypen. Das Evaluationsverfahren versucht dadurch eigene, womöglich stärker subjektiv geprägte Wertmaßstäbe soweit als möglich zu umgehen. Lediglich die Skalenbreite und die Gleichbewertung der verwendeten Indikatoren wurden vorgegeben.

Das entwickelte Verfahren greift externe Zielsetzungen, etwa aus Raumplänen und Biotopschutzkonzepten, auf und integriert sie. Dies bedingt zunächst das Vorhandensein derartiger Grundlagen. Darüber hinaus müssen die Angaben zu den Entwicklungszielen konkret sein. Für den Indikator Landnutzungsverhältnisse ist eine Aussage zu angestrebten Flächennutzungsanteilen notwendig. In den behandelten Untersuchungsgebieten war die Informationslage diesbezüglich sehr unterschiedlich. Für den Raum Apolda waren konkrete Aussagen erhältlich, jedoch stammen diese aus einem Fachplan der Landesanstalt für Landwirtschaft, stellen also keinen interdisziplinären Konsens dar. Für das Untersuchungsgebiet Dassel war ein Raumordnungsprogramm vorhanden. Dieses lieferte hinreichend genaue Angaben zu den angestrebten Flächennutzungsanteilen.

Bei der Ermittlung der Naturnähe und der Bewertung der Baumartenvielfalt wird die PNV des jeweiligen Standorts als Maßstab verwendet. Dabei greift das Verfahren die jeweils landesübliche Methode zur Herleitung dieser hypothetischen Waldgesellschaft auf. Für Thüringen ist diese Herleitung mit Hilfe des Kartierungsverfahrens zur Waldbiotopkartierung und einem separaten Baumartenschlüssel zur PNV sehr gut dokumentiert. Für Niedersachsen mussten zur Ermittlung der NWG weitere Quellen hinzugezogen werden. In beiden Fällen ist die Verfügbarkeit von Standortsinformationen eine Vorbedingung. Eine forstliche Standortskartierung liegt in der Regel nur für bereits bewaldete Flächen und häufig auch nur für solche der öffentlichen Hand vor. Landwirtschaftliche Bodenkartierungen sind verfügbar. Die Veränderung der Bodenverhältnisse im Verlaufe der ersten Waldgenerationen muss nicht im konzeptionellen Widerspruch zu einer PNV stehen. Dennoch wäre ein Schlüssel zur Herleitung der PNV für Acker- und Grünlandflächen wünschenswert. Dies gilt insbesondere, da die Übertragung der Verhältnisse von Waldflächen auf landwirtschaftliche Flächen nicht ohne weiteres möglich ist.

Das Evaluationsverfahren greift verschiedentlich auf das Verfahren und die Ergebnisse der Biotopkartierungen zurück. Diesbezüglich ist der Erfassungsstand in Deutschland sehr weit fortgeschritten. Je besser die Wald- und Offenlandbiotopkartierung aufeinander abgestimmt sind, desto einfacher ist deren Integration in das vorgestellte Verfahren. Für das Gebiet Apolda lag eine flächendeckende Waldbiotopkartierung digital vor. Die Biotope des Offenlandes mussten über die Karten der Landschaftspläne digitalisiert werden. Für den Raum Dassel mussten die Biotopflächen ebenfalls in digitale Form gebracht werden. Hier genügte die Waldfunktionenkarte als Informationsquelle, wobei die Kartierungsdichte deutlich unter der thüringischen Variante lag. Bei der Frage, ob durch die Aufforstung ein Waldbiotop entsteht, welches als besonders wertvoll gelten kann, greift das Verfahren ebenfalls auf die Biotopkartierung zurück. Die Unterscheidung zwischen besonders geschützten und schützenswerten Biotopen konnte von dort übernommen werden. Ergänzend erfolgte eine Gefährdungseinschätzung des entstehenden Biotops anhand der Roten Liste der gefährdeten Biotoptypen für die Bundesrepublik Deutschland. Hier wäre es hilfreich, wenn die Biotopkartierungen einen Hinweis für den entsprechenden Biotoptyp der RLB gäben. Leider geben sie diesen nur für die Entsprechung bezüglich der FFH-Richtlinie.

Zur Bewertung der Biotopverbundwirkung wird der Beitrag der Aufforstung zu den im Bezugsraum vorherrschenden Landschaftstypen prognostiziert. Dabei werden die Landschaftstypen so angesprochen, wie sie von FLADE (1994) für landschaftsplanerische Zwecke beschrieben wurden. Die Ansprache erfolgt über das Luftbild und im Gelände. Eine 
Verifizierung der Ergebnisse erfolgt über die den Landschaftstypen zugehörigen, avifaunistischen Leitarten. Eine Taxierung von Vogelpopulationen im Gelände war im Rahmen der vorliegenden Arbeit nicht realisierbar und würde den Aufwand für ein Bewertungsverfahren unverhältnismäßig stark erhöhen. Bei Biotopkartierungen werden Vogelvorkommen nur unregelmäßig erwähnt und dies auch nur dann, wenn es sich um gefährdete Arten handelt. Dieses Merkmal muss aber auf Leitarten nicht zutreffen, da diese nur über ihr stetes Vorkommen in einem bestimmten Landschaftstyp definiert sind. Daher wurde der Kontakt zu lokal aktiven Ornithologen gesucht. Mit deren Hilfe konnte in beiden Untersuchungsräumen eine Einschätzung der Bestandessituationen erfolgen. Die Verifizierung der Landschaftstypenansprache war damit möglich.

Der Ansatz, die Biotopverbundwirkung einer Fläche mit Hilfe von Landschaftstypen zu bewerten, dient der realitätsbezogenen Planung. Der Betrachtungsraum ist mit vier Quadratkilometern überschaubar. Der Ist-Zustand wird erfasst und qualitativ überprüft. Wenn die für den Lebensraumkomplex typischen Vogelarten vorhanden sind, kann von einer schnellen Besiedelung der neu zu schaffenden Flächen ausgegangen werden. Sind die entsprechenden Arten nicht vorhanden, so ist der Erfolg der Biotopbegründung in Frage gestellt. Schwieriger ist die Situation zu bewerten, wenn die Aufforstungsmaßnahme zu einer Zerschneidung eines anderen Landschaftstyps führt. Soweit die entsprechende Tierartengemeinschaft dadurch benachteiligt wird, muss eine negative Bewertung möglich sein. In diesem Zusammenhang wären weitere Untersuchungen hilfreich, um die Bedeutung von Landschaftstypen für die praktische Raumplanung zu bestätigen. Denkbar wäre auch, die Liste der bislang definierten Typen zu verlängern und auch den süddeutschen Raum einzuschließen.

Die Auswirkung des Nutzungswandels wird auch in Bezug auf die Landnutzungsverhältnisse betrachtet. Hier wird eine Zielvorgabe, beispielsweise aus einem Raumordnungsprogramm, als fremdbestimmter Maßstab in das Bewertungsverfahren integriert. Auf die unbefriedigende Genauigkeit derartiger Zielvorgaben wurde bereits hingewiesen. Die Angaben über die Landnutzungsverhältnisse sind auf regionaler Ebene zwar meist vorhanden, jedoch nicht immer vollständig. Für den Raum Apolda lagen die Daten nur für Flächen vor, die im Rahmen von Anträgen auf landwirtschaftliche Subventionen erfasst wurden. Für kleine Gehölze fehlten die Daten vollständig. Die Nutzungstypenunterscheidung im Raumordnungsprogramm für den Kreis Northeim ist hinreichend differenziert, lässt aber ebenfalls Daten zu den Gehölzen vermissen. Anlass zur Hoffnung gibt in diesem Zusammenhang das novellierte Bundesnaturschutzgesetz, welches die Entwicklung von Landschaftsplänen für alle Regionen vorsieht. Falls diese digital erstellt werden, sollten genaue und differenzierte Aussagen zu Flächennutzungsverhältnissen künftig erhältlich sein. Bis dahin müssen die Daten für den Bezugsraum durch Digitalisierung aus Luftbildern ermittelt werden. Dies wurde für die untersuchten Flächen durchgeführt. Die anschließende Überprüfung der Nutzungsansprache im Gelände zeigte sich vor allem für den Raum Dassel als dringend notwendig, da hier nur monochromatische Aufnahmen erhältlich waren.

Schließlich zählt der Pflanzplan zu den Planungsvoraussetzungen, die eine Bewertung der Aufforstungsmaßnahme ermöglichen. Aus inm sollen die Baumarten, deren Mischungsanteile und die Mischungsform hervorgehen. Die Baumartenkombination ist entscheidend für die Naturnähe und die Baumartenvielfalt. Diese wird am Ideal der standortspezifischen PNV gemessen. Die beiden Indikatoren lassen sich einfach und präzise erfassen.

Der Pflanzplan sollte neben den Angaben zum Bestand auch die beabsichtigte Gestaltung des Waldrandes beinhalten. Form, Breite und Zusammensetzung des Waldrandes bestimmen seine Struktur. Die Literatur (COCH 1995, ZUNDEL 1994) geht von einer allgemeinen Höherwertigkeit der strukturreichen Bestandesränder aus. Im Rahmen der Aufforstung können hier wertvolle Lebensräume geschaffen werden. Deren Bewertung erfolgt ex-ante anhand einer kurzen Checkliste. Sie ist daher einfach und gut nachvollziehbar. 


\subsection{Transferierbarkeit des Verfahrens}

$\mathrm{Zu}$ den Voraussetzungen der Verfahrensanwendung gehört das Vorhandensein der genannten Planungsgrundlagen. Von Vorteil sind raumplanerische Zielvorgaben, die mit einem interdisziplinären Konsens beschlossen wurden. Sind diese nicht vorhanden, so kann behelfsweise auf Fachplanungen mit detaillierten Informationen zurück gegriffen werden. Die üblichen Informationsquellen zu nutzungs- und raumbezogenen Daten, einschließlich Biotopkartierung, Standortkartierung und Forsteinrichtung sollten verfügbar sein. Wenn sie digital vorliegen, erleichtert dies die Verarbeitung der Daten erheblich. Aus einem detaillierten Landschaftsplan können ebenfalls wertvollen Informationen entnommen werden. Liegen darüber hinaus auch noch Daten zu avifaunistischen Populationen vor, kann dies bereits als solide Grundlage für eine schnelle Bewertung einer Aufforstungsmaßnahme gelten.

Das Verfahren wurde bislang in zwei Untersuchungsgebieten angewandt. Davon ist der eine (Apolda / Thüringer Becken) stark unterbewaldet. Der andere (Dassel / Leine-llme-Senke) weist einen annähernd (bundes-)durchschnittlichen Bewaldungsanteil auf. Für beide Gebiete erwies sich das Verfahren als praktikabel. Obwohl die Agrarstrukturpolitik der EU in erster Linie auf eine Veränderung in den intensiv landwirtschaftlich genutzten Regionen abzielt, kommt es auch in stark bewaldeten Landesteilen immer wieder zu Aufforstungsanträgen. Aus Naturschutzgründen ist dort jedoch in der Regel eine Offenhaltung der Freiflächen geboten. Dass das Verfahren auch unter diesen Bedingungen plausible Ergebnisse liefert, kann bislang nur vermutet werden. Generell kann die Evaluation der Aufforstungsmaßnahmen nach den vorgestellten Indikatoren jedoch für alle Räume und alle Standorte durchgeführt werden. Die regionalen Bedingungen werden vom Verfahren aufgegriffen. Regionale Entwicklungsziele und landes- oder bundesweite Reglementierungen, etwa im Biotopschutz, werden berücksichtigt. Eine Erweiterung um weitere Aspekte (z.B. FFH-Richtlinie) ist denkbar.

Die Prinzipien des Verfahrens könnten im Rahmen einer wissenschaftlichen Untersuchung auf andere Landnutzungsarten übertragen werden. Für die raumbezogenen Indikatoren Biotopverbund, Landnutzungsverhältnisse, Randlinien-Vorkommen - ist eine Übertragbarkeit ohne weiteres gegeben. Für die flächenbezogene Werte wären teilweise alternative Indikatoren zu suchen. Im Sinne der Agrarstrukturreform ist insbesondere eine Übertragung des Verfahrens auf Brachflächen und extensive Grünlandbewirtschaftung interessant.

Eine Bewertung von Aufforstungen auf besonders geschützten Flächen ist nicht möglich. Dies scheint aber auch nicht notwendig, da ein Aufforstungsantrag in solchen Fällen aufgrund des Bestandesschutzes der wertvollen Biotope kaum Aussicht auf Erfolg hätte.

\subsection{Entwicklungsempfehlungen}

Das Verfahren zur Evaluation von Erstaufforstungen bietet einen neuen, nach Ansicht des Verfassers wertvollen Ansatz zur Prognose der ökologischen Werte auf der Fläche und in Bezug zu deren Umfeld. Die bisherigen Ergebnisse sind ermutigend. Die Übertragbarkeit auf andere Regionen und Bedingungen scheint gegeben. Die Anwendung des Verfahrens im Genehmigungsverfahren setzt neben der interdisziplinären Verständigung über deren Anerkennung noch einige Erfahrungen und Erweiterungen voraus.

Geht es um die generelle Bewertung einer Aufforstungsmaßnahme, und nicht um die Entscheidung zwischen mehreren Varianten, so wird ein allgemeiner Bezugswert benötigt. Dieser kann objektiv ermittelt werden durch die mehrfache Anwendung des Verfahrens mit zufälligen oder systematisch verteilten Stichproben. Hierbei kommt es insbesondere auf die unterschiedlichen Grade der Verflechtung zwischen Aufforstung und anderen Biotoptypen an. Die Anzahl der notwendigen Untersuchungen ist stark von der Heterogenität des Planungsgebietes abhängig. Da sich die zur Erstaufforstung anstehenden landwirtschaftlichen Flächen aber in der Regel sehr ähneln, dürften schon wenige Untersuchungen ein Ergebnis liefern, welches eine eindeutige Einschätzung der Maßnahme ermöglicht. Denkbar wäre beispielsweise die Durchführung anhand eines 
Stichprobenrasters. Der Aufwand für ein derartiges Modellprojekt wäre sicherlich zu rechtfertigen, wenn ein Aufforstungsanstieg in der Region erwünscht und zu erwarten ist. Liegen die Planungsgrundlagen bereits digital vor, so reduziert sich der Aufwand erheblich.

Als Kontrolle $\mathrm{zu}$ den wissenschaftlichen Untersuchungen bietet sich eine Akzeptanzforschung bei den Planungsträgern und Genehmigungsbehörden an. Diese konnte im vorliegenden Untersuchungsrahmen nicht realisiert werden, würde aber sicherlich schnell zeigen, ob das Verfahren konsensfähig ist. Eine Erfassung von Expertenmeinungen zur Evaluation könnte auch einen Hinweis geben, ob die intuitiven Bewertungselemente (Randlinien-Vorkommen, Landnutzungsverhältnisse) allgemein nachvollziehbar sind. Diesbezüglich kann das Verfahren auch schnell angepasst werden, wenn die Erfahrung oder die Statistik neue Werte ergibt.

Politisch betrachtet fordert die angestrebte Konsensfähigkeit eine interdisziplinäre Weiterentwicklung des Verfahrens. In diesem Zusammenhang wäre auch die Übertragbarkeit auf andere Nutzungsarten zu erörtern. 


\section{Zusammenfassung}

Die vorliegende Arbeit entwickelt ein Verfahren zur ex-ante Evaluation von Erstaufforstungen nach naturschutzfachlichen Aspekten. Die Ziele überregionaler Planungen und Programme werden integriert. Die Verwendung externer Verfahrensschritte (v.a. aus der Biotopkartierung) fördert die Anpassungsfähigkeit an veränderte Rahmenbedingungen. Maßnahmenspezifische Daten werden erhoben zur Berücksichtigung regionaler Interessen und lokaler Bedingungen.

Die Verfahrensentwicklung fand vor dem Hintergrund der aktuellen, unbefriedigenden Situation bei der Berücksichtigung ökologischer Interessen in Genehmigungsverfahren statt. Diese zeigt sich in der Missachtung ökologischer Kriterien in den Förderungsinstrumenten für die Erstaufforstung, obwohl letztere als flankierende Maßnahme der Gemeinsamen Agrarpolitik durchaus eine Bedeutung besitzt. Die Situation beruht aber auch auf der schwierigen Integration divergierender Leitbilder des Naturschutzes. Dies zeigt sich in der ständig wechselnden Argumentation bei Genehmigungsverfahren. Ein neuer Ansatz war notwendig zur Berücksichtigung nationaler, regionaler und lokaler Interessen, bei gleichzeitiger Förderung der flexiblen Anwendungsfähigkeit.

Der Schwerpunkt der Arbeit liegt auf der methodischen Entwicklung des Evaluationsverfahrens. Dieses beginnt mit einer Analyse der gängigen Planungsmethoden in der Landschaftsplanung und der herrschenden Vielfalt unterschiedlicher Leitbilder. Als zielführende Methode zur Verfahrensentwicklung präsentiert sich die Wirkungsanalyse. An deren Anfang steht die Definition eines Systems, welches die Wirkungsbeziehungen innerhalb und mit der Umgebung einer Aufforstungsfläche abbildet. Das System gliedert sich in drei unterschiedliche Ebenen: die Aufforstungsfläche, deren unmittelbare Umgebung und die mittelbare Umgebung. Die systemimmanenten Beziehungen werden mit Hilfe einer Verflechtungsmatrix dargestellt. Sie ermöglicht die Ableitung und Definition von fünf flächenbezogenen und drei raumbezogenen Indikatoren: Natürliche Baumartenvielfalt, Naturnähe der Baumartenzusammensetzung, Strukturpotential, Seltenheit des Biotops, Waldrandausprägung, Biotop-Verbund, Landnutzungsanteile und Randlinien-Vorkommen. Diese Indikatoren geben Hinweise auf die systeminternen Wirkungsbeziehungen.

Die ermittelten Indikatoren werden auf ihre Aussagekraft für allgemein anerkannte Naturschutzkriterien - Naturnähe, Vielfalt, Seltenheit, Eigenart - untersucht. Sie bilden jeweils nur Teilaspekte ab, erlauben dafür aber die Bewertung einer Maßnahme im Hinblick auf unterschiedliche, teilweise konkurrierende Ziele. Die Verwendung der einzelnen Indikatoren wird anhand der wissenschaftlichen Literatur legitimiert und die Aussagekraft überprüft. Unter Berücksichtigung externer Verfahrensschlüssel werden anschließend Parameter und Bewertungsschemata entwickelt. Daraus resultiert ein Gesamtverfahren mit gleich gewichteten, unabhängigen Indikatoren und einer ordinalen Bewertungsskala.

Die Praktikabilität des entwickelten Verfahrens wird durch die Anwendung in zwei Untersuchungsgebieten (Thüringer Becken; Sollingvorland) getestet. Für beide Regionen werden standortabhängige Aufforstungsvarianten mit systematischer Baumartenwahl nach den Kriterien Vielfalt, Naturnähe und z.T. Strukturpotential vorgestellt. Zusätzlich werden die von den jeweiligen Landesforsten vorgeschlagenen Aufforstungsvarianten (Erstaufforstungszieltypen in Thüringen, Waldentwicklungstypen in Niedersachsen) einbezogen. Die Untersuchung vergleicht unterschiedliche Aufforstungstypen für eine gegebene Fläche, aber auch verschiedene Aufforstungsflächen bei gleichbleibender Baumartenmischung.

Die Ergebnisse der Verfahrensanwendung belegen die Bedeutung der Baumartenwahl und des Standorts für die ökologische Wertigkeit des entstehenden Waldbiotops. Sie zeigen darüber hinaus den großen Handlungsspielraum des Grundeigentümers auf, der die naturschutzfachliche Beurteilung der Maßnahme durch Beachtung bestimmter Kriterien gezielt beeinflussen kann. 
Aufgrund der ordinalen Bewertungsskala und des übersichtlichen Indikatorenkatalogs bietet das Evaluationsverfahren eine gute Hilfe beim Vergleich unterschiedlicher Aufforstungsmaßnahmen. Es greift auf externe Datenquellen zurück und bedient sich der technischen Hilfe eines Geographischen Informationssystems. Eigene Aufnahmen werden auf ein Minimum reduziert. Da öffentliche Interessen berücksichtigt werden, ist es zum Einsatz bei Behörden geeignet. Gleichzeitig vermittelt es dem Antragsteller wichtige Informationen über die Verfahrensinhalte.

Schließlich zeigt die Arbeit die Voraussetzungen auf, unter denen das Evaluationsverfahren angewandt werden kann. Dazu gehört die Verfügbarkeit landschaftsbezogener Daten (insbesondere Biotopkartierung), externer Verfahrensschlüssel (Herleitung der PNV über den Standort) sowie raumplanerischer Zielvorgaben für die Flächennutzungsanteile. Diese Bedingungen dürften für die meisten Regionen Deutschlands erfüllt sein. Das Verfahren kann daher an die spezielle Situation in anderen Räumen angepasst werden. Wird darüber hinaus angestrebt, es auf andere Landnutzungsarten zu übertragen oder im Rahmen der Fördermittelzuteilung anzuwenden, so sind weitere Untersuchungen notwendig. 


\section{Literaturverzeichnis}

AK StOK, 1985: Forstliche Wuchsgebiete und Wuchsbezirke in der BRD. Arbeitskreis Standortskartierung in der Arbeitsgemeinschaft Forsteinrichtung. Landwirtschaftsverlag Münster-Hiltrup. 170

AKADEMIE DER LANDWIRTSCHAFTSWISSENSCHAFTEN DER DDR ,1978: Mittelmaßstäbige landwirtschaftliche Standortkartierung 1:100.000, GERA, Blatt 49. Forschungszentrum für Bodenfruchtbarkeit Müncheberg, Bereich Bodenkunde Eberswalde.

AMMER, U., 1997: Erstaufforstung und Landschaftsplanung. Schr.-R. f. Landschaftspflege und Naturschutz, H. 49. BfN, Bad-Godesberg. 39-45

AMMER, U., PRÖBSTL, Ulrike, 1988: Erstaufforstungen und Landespflege. Forstw. Cbl. 107. Parey, Hamburg und Berlin. 60-71

ARBEITSKREIS FORSTLICHE LANDESPFLEGE, 1996: Waldlebensräume in Deutschland. . ecomed, Landsberg. 124

ASSEBURG, M. 1985: Landschaftliche Erlebniswirkungsanalyse und Flurbereinigungsmaßnahmen. Natur und Landschaft 58 (1). Kohlhammer, Köln. 9-15

BASTIAN, O. 1999: Landschaftsfunktionen als Grundlage von Leitbildern für Naturräume. Natur und Landschaft 74, (9). 361-373

BAYERISCHE AKADEMIE FÜR NATURSCHUTZ UND LANDSCHAFTSPRLEGE, 1991: Das Mosaik-Zyklus-Konzept der Ökosysteme und seine Bedeutung für den Naturschutz. Laufener Seminarbeiträge 5/1991. Akademie für Naturschutz und Landschaftspflege, Laufen.

BECHMANN, A., 1981: Grundlagen der Planungstheorie und Planungsmethodik. UTB - Haupt, Bern.

BEESE, F.O., 1996: Indikatoren für eine multifunktionelle Waldnutzung. Forstw. Cbl., 15 S.

BETTINGER, A., 1995: Landschaftsplan "Unteres IImtal". Gesellschaft für Freiraumplanung und Landschaftsökologie. Apolda-Zottelstedt.

BMELF, 1999: Übersicht zur forstlichen Rahmenplanung in der Bundesrepublik Deutschland (Stand: 30.06.1999). Arbeitspapier im NFP-Prozess (unveröffentlicht). BMELF, Bonn. 21

BMELF, 2000: Nationales Forstprogramm Deutschland. BMELF, Bonn. 71

BROCKSIEPER, R., WOIKE, M., 1999: Kriterien zur Auswahl der FFH- und Vogelschutzgebiete für das europäische Schutzgebietssystem "Natura 2000". LÖBF-Mitteilungen (2). 15-26

BROGGI, M. F., SCHLEGEL, H. 1989: Mindestbedarf an naturnahen Flächen in der Kulturlandschaft. Bericht 31 des Nationalen Forschungsprogramms "Boden". Liebefeld-Bern. 180

BROGGI, M. F., 1999: (Naturschutz-)Leitbilder für die freie Landschaft. in BBN, 1999, Naturschutz zwischen Leitbild und Praxis. Bundesverband Beruflicher Naturschutz, Bonn. 9-27

BROKAW, N. V.L., LENT, R. A., 1999: Vertical structure. Maintaining Biodiversity in Forest Ecosystems; in: M.L. HUNTER JR (ed.). Cambridge University Press. 373-399

BUCHWALD, K., 1980: Die Zuweisung ökologischer Funktionen an Räume - Chancen einer Realisierung und Restriktionen. Landschaft + Stadt 12, (1). Ulmer, Stuttgart. 1-6

BUCHWALD, K., ENGELHARDT, W., 1980: Handbuch für Planung, Gestaltung und Schutz der Umwelt - Band 3 - Die Bewertung und Planung der Umwelt. BLV, München. 754

BUNDESVERBAND BERUFLICHER NATURSCHUTZ, (Hrsg.), 1999: Naturschutz zwischen Leitbild und Praxis. Jb. Natursch. Landschaftspfl. (50). Bundesverband Beruflicher Naturschutz, Bonn. 260 
BURGGRAAFF, P., HEIN, Gertrud, 1999: Kulturlandschaftspflege - Sukzession contra Erhalten. LÖBF-Mitteilungen (1). . 63-66

BURSCHEL, P., HUSS, J., 1997: Grundriss des Waldbaus - Ein Leitfaden für Studium und Praxis. Parey, Hamburg und Berlin. 487

COCH, T., 1995: Waldrandpflege - Grundlagen und Konzepte. . Neumann, Radebeul. 240

DANE, M., 1999: Landschaftsplan "Mellingen/Apolda". Dane Landschaftsarchitekten BDLA. Weimar.

DEGRAAF, R., MILLER, R., 1996: Conservation of Faunal Diversity in Forested Landscapes. Chapman \& Hall, London. 499-502

DOHRENBUSCH, A., 1996: Ökologische und ökonomische Aspekte bei der Waldvermehrung durch erstaufforstung. LÖBF-Mitteilungen (3). 18-26

DRACHENFELS, O. von, 1994: Kartierschlüssel für Biotoptypen in Niedersachsen. Naturschutz und Landschaftspflege in Niedersachsen A/4. Nds. Landesamt f. Ökologie, Hildesheim. 192

DRL, 1997: Leitbilder für Landschaften in "peripheren Räumen". Schriftenreihe des Deutschen Rates für Landschaftspflege (67). Deutscher Rat für Landschaftspflege, Bonn. 134

ELLENBERG, H., 1982: Vegetation Mitteleuropas mit den Alpen. Ulmer, Stuttgart. 989

ELSAßER, P., 1991: Umweltwirkungen der Aufforstung ackerbaulich genutzter Flächen. Arbeitsbericht des Instituts für Ökonomie 91/2. Bundesforschungsanstalt für Forst- und Holzwirtschaft, Hamburg. 39

ERLBECK, R., 1993: Aufforstung landwirtschaftlicher Böden. AFZ 5/1993. BLV, München. 230233

FINCK, P., SCHRÖDER, E., 1997: Waldvermehrung auf der Grundlage von bundesweiten Konzepten für naturschutzfachliche Landschafts-Leitbilder. Schr.-R. f. Landschaftspfl. u. Natursch., H. 49. BfN, Bad-Godesberg. 11-25

FISCHER, A., 1995: Forstliche Vegetationskunde. . Blackwell, Berlin, Wien. 315

FLADE, M., 1994: Die Brutvogelgemeinschaften Mittel- und Norddeutschlands - Grundlagen für den Gebrauch vogelkundlicher Daten in der Landschaftsplanung. . IHW-Verlag, Eching. 879

FORMAN, R. T.T., COLLINGE, Sharon K. 1996: The 'spatial solution' to conserving biodiversity in landscapes and regionsChapman \& Hall. Conservation of Faunal Diversity in Forested Landscapes , R.M. DEGRAAF \& R.I. MILLER,. Chapman \& Hall, London. 537-568

FRIEDRICHSDORF, B., 1999: Praktische Erfahrungen bei der Erstaufforstung - am Beispiel ehemals landwirtschaftlich genutzter Flächen im Forstamt Schleswig. Forst und Holz 54 (19). Schaper-Verlag. 387-391

GRABHERR, G., KOCH, G., KIRCHMEIR, H., REITER, K., 1995: Hemerobie österreichischer Waldökosysteme - Vorstellung eines Forschungsvorhabens im Rahmen des österreichischen Beitrages zum MAB-Programm der UNESCO. Zeitschr. F. Ökologie u. Naturschutz (4). Urban \& Fischer. 105-110

GRUTTKE, H., WILLECKE, S., 1993: Tierökologische Langzeitstudie zur Besiedelung neu angelegter Gehölzpflanzungen in der intensiv bewirtschafteten Agrarlandschaft. Natur und Landschaft, 68 (7/8). . 367-376

HAAREN, Christa von, 1999: Begriffe, Vorgehen und Hierarchien bei der Zielentwicklung im Naturschutz. in: WIEGLEB et al. 1999: Naturschutzfachliche Bewertung im Rahmen der Leitbildmethode. Physica-Verlag, Heidelberg. 15-36

HERREN, G., BAUR, H., 1993: EG Regionalseminar: Aufforstung von Landwirtschaftsflächen. AFZ 5/1993. BLV, München. 234-235 
HEYDEMANN, B., 1986: Grundlagen eines Verbund- und Vernetzungskonzeptes für den Artenund Biotopschutz. Grüne Mappe 1986. Landesnaturschutzverband Schleswig-Holstein, Kiel. $11-22$

HIEKEL, W. 1994: Wissenschaftliche Beiträge zum Landschaftsprogramm Thüringens. Thüringer Landesanstalt für Umwelt, Jena. 105

HIEKEL, W., 1997: Naturschutzleitbilder für das Thüringer Becken. IN: DRL 1997. 83-88.

HOFMEISTER, Heinrich (1990): Lebensraum Wald. . Parey, Hamburg und Berlin. 275

HORNSTEIN, F.v. (1958): Wald und Mensch. . Ravensburg. 282

HUNTER, M., 1990: Wildlife, forests and forestry: principles of managing forests for biological diversity. Prentice-Hall, London. 370

HUSS, J., et al., 2000: Wiederbewaldung von Sturmschadensflächen. AFZ/Der Wald 23/2000. BLV, München. 1219-1226

IDF, 2001: Evaluation of Regulation No. 2080/92. Final report. Manuskript - Inst. f. Waldbau I, Göttingen. 201

JANETZ, G., 1998: Landschaftsplan "Ost-Abschnitt des Kreises Weimarer Land". Gesellschaft für Freiraumplanung und Landschaftsökologie. Apolda-Zottelstedt.

JEDICKE, E., 1994: Biotopverbund - Grundlagen und Maßnahmen einer neuen Naturschutzstrategie. . Ulmer, Stuttgart. 287

KAISER, T., ZACHARIAS, D., 1999: Eine anwendungsorientierte Definition der potentiellen natürlichen Vegetation als Ergebnis der Fachtagung "Die potentielle natürliche Vegetation Bedeutung eines vegetationskundlichen Konzeptes für die Naturschutzpraxis" vom 1.2.10.1998 an der NNA. in: NNA 1999. Alfred Töpfer Akademie für Naturschutz, Schneverdingen. 46-47

KAULE, G., 1991: Arten- und Biotopschutz. Ulmer, Stuttgart. 519

KEDING, W., 1994: Aufforstungswelle in Niedersachsen. Forst und Holz 49 (1). Schaper-Verlag. 3-5

KEDING, W., 1999: Rechtliche Voraussetzungen und öffentlich Förderung für die Neuanlage von Wald in Niedersachsen. Forst und Holz 54 (13). Schaper-Verlag. 401-403

KIRBY, K., 1994: Die Bewertung von Wäldern und Gehölzbeständen. in: USHER/ERZ 1994. Quelle und Meyer Verlag, Heidelberg und Wiesbaden.

KLEIN, M., 1997: Naturschutz und Erstaufforstung - eine Einführung. Schr.-R. f. Landschaftspfl. u. Natursch., H. 49. BfN, Bad-Godesberg. 7-10

KLEIN, M., 1997a: Erstaufforstung - Chancen und Risiken für Naturschutz und Landschaftspflege. Schr.-R. f. Landschaftspfl. u. Natursch., H.49. BfN, Bad-Godesberg. 167171

KNAPP, R., 1971: Einführung in die Pflanzensoziologie. Ulmer, Stuttgart. 388

KNICKREHM, Barbara; MÖNNECKE, Margit und BRINKMANN, R., 2000: Standardisierung in Naturschutz und Landschaftspflege. Naturschutz und Landschaftsplanung 32, (1). 14-19

KOCH, G., KIRCHMEIER, H., GRABHERR, G., 1999: Naturnähe im Wald - Methodik und praktische Bedeutung des Hemerobiekonzeptes für die Bewertung von Waldökosystemen. . Zentrum für Umwelt und Naturschutz, Universität für Bodenkultur, Wien. 97

KÖHL, M.; ZINGG, A., 1995: Eignung von Diversitätsindizes bei Langzeituntersuchungen zur Biodiversität in Waldbeständen. AFJZ 167 (4). . 76-85

KÖPF, E. U., 2002: Forstpolitik. Ulmer, Stuttgart. 320 
KORNECK, D., SUKOPP, H., 1988: Rote Liste der in der BRD ausgestorbenen, verschollenen und gefährdeten Farn- u. Blütenpflanzen ... Sch.R. Veg.Kunde, H. 19, Bonn, 210.

KOWARIK, I., 1987: Kritische Anmerkungen zum theoretischen Konzept der potentiellen natürlichen Vegetation mit Anregungen zu einer zeitgemäßen Modifikation. Tuexenia 7. 53-67

KRAHL, W., 1999: Leitbilddiskussion und Selbstverständnis der Naturschützer. in BBN, 1999, Naturschutz zwischen Leitbild und Praxis. Bundesverband Beruflicher Naturschutz, Bonn. 2944

KRAUSE, C. L., HENKE, H., 1980: Wirkungsanalyse im Rahmen der Landschaftsplanung. Schr.R. Landschaftspflege u. Naturschutz, H.20. Bonn. 299.

KRAUSE, A., 2000: 30-jährige Bracheaufforstung ohne Pflegeeingriffe. Forst und Holz 55 (10). Schaper-Verlag. 319-324

LAWUF, 1996: Kartieranleitung zur flächendeckenden Waldbiotopkartierung im Freistaat Thüringen. . Landesanstalt für Wald und Forstwirtschaft, Gotha; Thüringer Landesanstalt für Umwelt, Jena. 116

LAWUF, 1997: Die forstlichen Wuchsbezirke Thüringens - Kurzbeschreibung. Mitteilungen der Landesanstalt für Wald und Forstwirtschaft, Heft 13. Landesanstalt für Wald und Forstwirtschaft, Gotha. 199

LAWUF, 1997a: Stamm-Standortsgruppen und Baumarten potentiell-natürlicher Wälder auf der Basis von Stamm-Vegetationsformen im forstlichen Wuchsgebiet Thüringer Becken. unveröffentlichtes Manuskript, Anlage $3 \mathrm{~b}$ zur Kartieranleitung für die Waldbiotopkartierung; Stand Februar 1997. Landesanstalt für Wald und Forstwirtschaft, Gotha. 7

LAWUF, 1999: Erstaufforstung I . Informationen der Thüringer Landesanstalt für Wald und Forstwirtschaft. Landesanstalt für Wald und Forstwirtschaft, Gotha. 12

LAWUF, 2000: Standortseinheitenkarte 4935 NO, NW, SO, SW. Landesanstalt für Wald und Forstwirtschaft, Gotha.

LAWUF, 2001a: Erstaufforstungszieltypen (EAZT) für Erstaufforstungsflächen (1. Waldgeneration). unveröffentlichtes Manuskript. Landesanstalt für Wald und Forstwirtschaft, Gotha.

LAWUF, 2001b: Baumartenvorschläge für den subkontinentalen Klimabereich, kollin bis planare Höhenstufe, Klimastufen Vtt, Vt, Vm und Vf. unveröffentlichtes Manuskript. Landesanstalt für Wald und Forstwirtschaft, Gotha.

LEDER, B., 1996: Hinweise für die waldbautechnische Durchführung der Erstaufforstung. LÖBFMitteilungen (3). 11-18

LFU, 1992: Landschaftspflege - Quo vadis? - Kolloquium zur Standortsbestimmung und Entwicklung der Landschaftspflege. Tagungsbericht. Landesanstalt für Umweltschutz Bad.Württ., Karlsruhe. 209

LIESEN, J., 1996: Aspekte der Verwendung des Mittelspechtes (Picoides medius) als Leitart für die Bewertung ehemaliger Mittelwälder. Natur in Buch und Kunst, Verlag Dieter Prestel, Neunkirchen. 91

MAYER, F., GEIGER, H., 1996: Fledermäuse in der Landschaftsplanung - Möglichkeiten und Grenzen. Schr.-R. f. Landschaftspfl. u. Natursch., H. 46. BfN, Bad-Godesberg. 25-34

MEINEKE, J. 1992: Artenvielfalt um jeden Preis!. in LFU, 1992, Landschaftspflege - Quo vadis. Landesanstalt für Umweltschutz Bad.-Württ., Karlsruhe. 90-95

MENGEL, A., 1999: Leitbilder in der Landschaftsrahmenplanung. in BBN, 1999, Naturschutz zwischen Leitbild und Praxis. Bundesverband Beruflicher Naturschutz, Bonn. 45-55

MIRZA, Nicole, 2001: Auswirkungen und Effekte der forstlichen Maßnahmen der Verordnung EWG Nr. 2080/92. Diplomarbeit an der Fakultät für Forstwissenschaften und Waldökologie. Georg-August-Universität, Göttingen. 99+XXVII 
NABU, 1997: Waldvermehrung in einer belasteten Landschaft - Positionspapier des Naturschutzbund Deutschland zur Aufforstungsproblematik. Naturschutzbund Deutschland, Bonn. 4

NIEDERSÄCHSISCHES FORSTPLANUNGSAMT, 1996: Waldentwicklungstypen. . Nds. Forstplanungsamt, Wolfenbüttel. 3

NIEDERSÄCHSISCHES FORSTPLANUNGSAMT, 2001: Standorte und ihre standortsgerechten Waldentwicklungstypen. . Nds. Forstplanungsamt, Wolfenbüttel. 6

NIEDERSÄCHSISCHES LANDESVERWALTUNGSAMT, 1981: Bodenkarte auf der Grundlage der Bodenschätzung. Karten 4124/-14, -15, 16, -17, -23; Maßstab 1:5.000. Nds. Landesverwaltungsamt, Hannover.

NDS. MELF, 2000: Erstaufforstung - Neue Wälder, Schriftenreihe Waldinformation. Niedersächsisches Ministerium f. Ernährung, Landwirtschaft und Forsten, Hannover. 46.

NITSCHE, Sieglinde; NITSCHE, L., 1994:Extensive Grünlandnutzung.Neumann, Radebeul. 247

NNA (Hrsg.), 1999: Fachliche Konzepte für die Naturschutzpraxis - Prognosemmodelle zur Populationsentwicklung - Potentielle Natürliche Vegetation - Blaue Listen. NNA-Berichte 12 (2). Alfred Töpfer Akademie für Naturschutz, Schneverdingen. 154

OTTO, H.-J., 1994: Waldökologie. Ulmer, Stuttgart. 391

PALIK, B., ENGSTROM, R.T., 1999: Species composition. Maintaining Biodiversity in Forest Ecosystems; M.L. HUNTER JR (ed.). Cambridge University Press. 65-94

PETERSEN, R., FISCHER, H., 1999: Acker-Erstaufforstung im FA Nordfriesland. AFZ/Der Wald 5/1999. BLV, München. 215-217

PLACHTER, H., 1990: Indikatorische Methoden zur Bestimmung der Leistungsfähigkeit des Naturhaushalts. Schr.-R. f. Landschaftspfl. u. Natursch., H. 32. BfN, Bad-Godesberg. 187-199

PLACHTER, H., REICH, M. 1994: Großflächige Schutz- und Vorrangräume: eine neue Strategie des Naturschutzes in Kulturlandschaften. Veröff. PAOe Karlsruhe, Heft 8. . 17-43

PREEN, A. von, 1996: Erstaufforstung aus Sicht des Naturschutzes und der Landschaftspflege. LÖBF-Mitteilungen (3). . 33-39

PRIES, E., 1997: Ackeraufforstungen als gesellschaftliche Notmaßnahmen. Schr.-R. f. Landschaftspflege und Naturschutz, H. 49. BfN, Bad-Godesberg. 99-113

RATHS, Ulrike, RIECKEN, U., SSYMANK, A., 1995: Gefährdung von Lebensraumtypen in Deutschland und ihre Ursachen. Natur und Landschaft 70 (5). Kohlhammer, Köln. 203-212

REIF, A., 1997: Sukzession statt Erstaufforstung - eine Alternative?. Schr.-R. f. Landschaftspflege und Naturschutz, H. 49. BfN, Bad-Godesberg. 157-165

REIF, A., 2000: Das naturschutzfachliche Kriterium der Naturnähe und seine Bedeutung für die Waldwirtschaft. Z. Ökologie und Naturschutz 8 (1999/2000). Urban \& Fischer. 239-250

REIF, A., NICKEL, Elsa 2000: Pflanzungen von Gehölzen und "Begrünung". Naturschutz und Landschaftsplanung 32, (10). Ulmer, Stuttgart. 299-308

RIECKEN, U., RIES, Ulrike, SSYMANK, A., 1994: Rote Liste der gefährdeten Biotoptypen der Bundesrepublik Deutschland. . KILDA, Greven. 184

RIESS, W., 1986: Konzepte zum Biotopverbund im Arten- und Biotopschutzprogramm Bayern. Laufener Seminarbeiträge 10/1986. Akademie für Naturschutz und Landschaftspflege, Laufen. 102-115

RÖHRIG, E., BARTSCH, N., 1992: Der Wald als Vegetationsform und seine Bedeutung für den Menschen. in DENGLER, Waldbau auf ökologischer Grundlage, Band 1. Parey, Hamburg und Berlin. 350 
ROTH, D., ECKERT, H., SCHWABE, M., 1996: Ökologische Vorrangflächen und Vielfalt der Flächennutzung im Agrarraum - Kriterien für eine umweltverträgliche Landwirtschaft. Natur und Landschaft 71 (5). Kohlhammer, Köln. 199-203

RROP NORTHEIM, 1996: Regionales Raumordnungsprogramm für den Kreis Northeim. . Landratsamt Northeim.

SCHÄFER, M., 2001: Die Beziehung zwischen Waldstruktur und Diversität der Fauna. Forst und Holz 56 (15). Schaper-Verlag. 488-492

SCHERZINGER, W., 1985: Die Vogelwelt der Urwaldgebiete im Inneren Bayerischen Wald. Schr.R. Bayerischen Staatsministeriums f. Ernähring, Landwirtschaft und Forsten, Heft 12. Nationalparkverwaltung Bayerischer Wald, Grafenau. 188

SCHÖLCH, M., 2000: Natürliche Wiederbewaldung von Sturmflächen. AFZ/Der Wald 23/2000. BLV, München. 1216-1218

SCHÖLMERICH, U., 1999: Wiederbewaldung im rheinischen Braunkohlegebiet. Forst und Holz 54 (13). Schaper-Verlag. 392-397

SPEIDEL, G., 1984 Forstliche Betriebswirtschaftslehre. 2. Auflage. Parey, Hamburg u. Berlin. 226

SPEIGHT, M.R., WAINHOUSE, D., 1989: Ecology and Management of Forest Insects. . Clarendon Press, Oxford. $374+\mathrm{VI}$

SPELLERBERG, I. F., SAWYER, J. W.D. 1996: Standards for biodiversity: a proposal based on biodiversity standards for forest plantations. Biodiversity and Conservation (5). Chapman \& Hall, London. 447-459

STURM, K., 1993: Prozeßschutz - ein Konzept für naturschutzgerechte Waldwirtschaft. Z. Ökologie u. Naturschutz 2 (1993). Fischer, . 181-192

THOROE, C., 1997: Erstaufforstung aus ökonomischer Sicht. Schr.-R. f. Landschaftspfl. u. Natursch., H.49. BfN, Bad-Godesberg. 27-37

TLL, 1997: Agrarraumnutzungs- und -pflegeplan (ANP) für die Region Niedertreba. . Thüringer Landesanstalt für Landwirtschaft, Jena. 120

TMLNU, 1995: Bericht zur Entwicklung der Landwirtschaft in Thüringen. TMLNU und TLL, Erfurt und Jena. ,Eisenach.

TMUL, 1993: Arten- und Biotopschutzprogramm (Grobkonzept) Thüringen. mit Karten. Thüringer Ministerium für Umwelt und Landesplanung, Erfurt.

TRIEBEL, J., 2000: Erstaufforstung in Thüringen - Chance oder forstpolitisches Auslaufmodell?. Manuskript. Tharandt / TU Dresden. 11

USHER, M., ERZ, W., 1994: Erfassen und Bewerten im Naturschutz. UTB Große Reihe. Quelle und Meyer Verlag, Heidelberg und Wiesbaden.

VOGEL, W. von, 1999: Neuer Wald "Links der Ems". Forst und Holz 54 (13). Schaper-Verlag. 398-401

WAGNER, H., 1992: "Natur total" - Biotoppflege oder Sukzession. in LFU, 1992, Landschaftspflege - Quo vadis. Landesanstalt für Umweltschutz Bad.-Württ., Karlsruhe. 38-52

WAGNER, S., 1995: Eine waldbauliche Konzeption zur Erstaufforstung in Nordwestdeutschland. Forst und Holz 50 (21). Schaper-Verlag. 662-672

WESTHUS, W., FABER, Lieselotte, 1998: Biotopkartierungen in Thüringen im Überblick. Landschaftspflege und Naturschutz in Thüringen, (1) . . 8-13

WIEGLEB, G., SCHULZ, Friederike, BRÖRING, U.,1999: Naturschutzfachliche Bewertung im Rahmen der Leitbildmethode. . Physica-Verlag, Heidelberg. 263

WOLF, R., 1992: "Oh Schwarzwald, Oh Heimat" - ethische Aspekte der Landschaftspflege für das Landschaftsbild. "Landschaftspflege - Quo vadis", Tagungsbericht. LfU Baden-Württemberg, Karlsruhe. 96-106 
ZENKER, W., 1982: Beziehungen zwischen dem Vogelbestand und der Struktur der Kulturlandschaft. R. Beiträge zur Avifauna des Rheinlandes, Heft 15. Gesellschaft Rheinischer Ornithologen (Hrsg.), Düsseldorf. 249

ZUNDEL, R., 1994: Waldränder - gestalten und pflegen. aid-Broschüre 1010/1994. aid, Bonn. 32

ZUNDEL, R., 2000: Waldvermehrung - wo und wie?. aid-Broschüre 1394/2000. aid, Bonn. 28 


\section{Anhang}

Anhang 1: Erhebungsbogen zum Evaluationsverfahren

Indikator 1: $\quad$ Baumartenvielfalt

Baumartenvielfalt gemessen am „natürlichen Vorbild“

\begin{tabular}{|c|c|c|}
\hline Zeile & & \\
\hline 1 & Aufforstungsgröße & \\
\hline 2 & Standort-Typ 1 & \\
\hline 3 & $\begin{array}{l}\text { Größe } \\
\text { (ha und \%) }\end{array}$ & $\begin{array}{l}\text { ha } \\
\%\end{array}$ \\
\hline 4 & hpnV & \\
\hline 5 & BA-Zahl hpnV )' & \\
\hline 6 & BA-Zahl real )2 & \\
\hline 7 & Verhältnis real:hpnV & \\
\hline 8 & Verhältnis $x$ Prozent ) ${ }^{3}$ & \\
\hline 9 & Standort-Typ 2 & \\
\hline 10 & $\begin{array}{l}\text { Größe } \\
\text { (ha und \%) }\end{array}$ & $\begin{array}{c}\text { ha } \\
\%\end{array}$ \\
\hline " 11 & hpnV & \\
\hline " & BA-Zahl hpnV) & \\
\hline 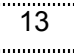 & BA-Zahl real )2 & \\
\hline 14 & Verhältnis real:hpnV & \\
\hline 15 & Verhältnis x Prozent $)^{3}$ & \\
\hline 16 & Erfüllungsgrad (Zeile $8+$ Zeile 15) & \\
\hline
\end{tabular}

\begin{tabular}{|c|c|}
\hline Wertetabelle & Bewertung \\
\hline Erfüllungsgrad $\geq 0,80 \rightarrow 5$ Pkt. & \\
Erfüllungsgrad $\geq 0,65 \rightarrow 4$ Pkt. & \\
Erfüllungsgrad $\geq 0,50 \rightarrow 3$ Pkt. & \\
Erfüllungsgrad $\geq 0,35 \rightarrow 2$ Pkt. & \\
Erfüllungsgrad $\geq 0,20 \rightarrow 1$ Pkt. & \\
Erfüllungsgrad $<0,20 \rightarrow 0$ Pkt. & \\
& \\
\hline
\end{tabular}

)$^{1}$ Baumarten-Zahl in der hpnV laut Anlage

)$^{2}$ Baumarten-Zahl im realen Waldbestand. Gezählt werden nur einheimische Baumarten und nur jene, die einen Flächenanteil an der Bestockung dieses Standortes von mind. $5 \%$ erreichen

)$^{3}$ Multiplikation der Zeile 7 (bzw. 14) mit dem Prozentwert (in Dezimalform!) aus der Zeile 3 (bzw. 10) 
Indikator 2: $\quad$ Naturnähe

\begin{tabular}{|c|c|c|}
\hline Zeile & Parameter & 10 Daten der Aufforstungsfläche \\
\hline 1 & Größe der Aufforstungsfläche & \\
\hline 2 & Standort-Typ 1 & \\
\hline 3 & $\begin{array}{l}\text { Größe } \\
\text { (ha und \%) }\end{array}$ & $-\frac{h}{h}$ \\
\hline 4 & pnV & \\
\hline 5 & BA der pnV $)^{1}$ & \\
\hline 6 & BA real $)^{2}$ & \\
\hline 7 & Übereinstimmungen & \\
\hline 8 & Übereinstimmungen x Prozent $)^{3}$ & \\
\hline 9 & Standort-Typ 2 & \\
\hline 10 & $\begin{array}{l}\text { Größe } \\
\text { (ha und \%) }\end{array}$ & $\begin{array}{c}\text { ha } \\
\%\end{array}$ \\
\hline 11 & pnV & \\
\hline 12 & BA der pnV ) & \\
\hline 13 & BA real $)^{2}$ & \\
\hline 14 & Übereinstimmungen & \\
\hline 15 & Übereinstimmungen x Prozent ) ${ }^{3}$ & \\
\hline 16 & $\begin{array}{l}\text { Gesamtwert } \\
\text { (Zeile } 8+\text { Zeile 15) }\end{array}$ & \\
\hline
\end{tabular}

)$^{1}$ laut pnV-Schlüssel aus der Waldbiotopkartierung

)$^{2}$ laut Pflanzplan

)$^{3}$ Prozentanteil des Standortes an der Aufforstungsfläche

\begin{tabular}{|l|l|}
\hline Wertetabelle & Bewertung \\
\hline Erfüllungsgrad $\geq 0,80 \rightarrow 5$ Pkt. & \\
Erfüllungsgrad $\geq 0,65 \rightarrow 4$ Pkt. & \\
Erfüllungsgrad $\geq 0,50 \rightarrow 3$ Pkt. & \\
Erfüllungsgrad $\geq 0,35 \rightarrow 2$ Pkt. & \\
Erfüllungsgrad $\geq 0,20 \rightarrow 1$ Pkt. & \\
Erfüllungsgrad $<0,20 \rightarrow 0$ Pkt. & \\
\end{tabular}




\section{Indikator 3: $\quad$ Seltenheit des Biotops}

Aufforstungszielbestand entspricht einer Waldgesellschaft, welches nach heutiger Rechts- und Definitionslage folgende Schutzkategorien erfüllen würde:

Nennung in der nationalen Rote Liste Biotope (laut Anlage A3; Referenz: RIECKEN et al. 1994):

Biotop-Typ:

Code:

Gefährdungskategorie:

Einschätzung der Schutzwürdigkeit durch die Waldbiotopkartierung des Bundeslandes:

Biotop-Typ:

Code:

Geschütztes Biotop nach Landesrecht:

Schützenswertes Biotop nach WBK:

\begin{tabular}{|l|c|c|}
\hline Bewertung & maximal & vergeben \\
\hline Gefährdungskategorie & 3 & \\
\hline Geschütztes Biotop nach Landesrecht & 1 & \\
\hline Schützenswertes Biotop nach WBK & 1 & \\
\hline Gesamt: & 5 & \\
\hline
\end{tabular}

Siehe Anlage 8

\footnotetext{
${ }^{8}$ Anlage $\mathrm{A} 3$ enthält eine Aufstellung der Biotope, die nach landesspezifischer Waldbiotopkartierung erfasst werden. Am Beispiel Thüringens gehören dazu 4 Typen:

(1) Biotope, die als schützenswert eingeschätzt werden, jedoch keinen rechtlichen Schutzstatus besitzen und auch nicht in der RLB genannt sind $\rightarrow 1$ Punkt

(2) Biotope, die nach Landesrecht geschützt sind (diese sind automatisch auch landesweit schützenswert), jedoch nicht in der RLB genannt werden $\rightarrow 2$ Punkte

(3) Biotope, die landesweit schützenswert sind und in der RLB genannt sind, jedoch keinen rechtlichen Schutzstatus besitzen $\rightarrow$ Wert ergibt sich aus der Flächengefährdung (max. 3 Pkt.) +1 Pkt.

(4) Biotope, welche durch Landesrecht geschützt (und damit auch landesweit schützenswert) sind und gleichzeitig in der RLB genannt sind $\rightarrow$ Wert ergibt sich aus der Summe der Flächengefährdung (max. 3 Pkt.) +2 Pkt.

Die Bewertung des Biotops erfolgt als absolute Potentialbewertung unter Annahme einer Bewirtschaftung, die das Ziel der natürlichen Waldgesellschaft unterstützt und gleichzeitiger Standortsanpassung. Da das Biotop sich erst entwickeln muss, ist bisher kein Schutzstatus zu vergeben.
} 
Indikator 4:

Strukturpotential

\begin{tabular}{|c|c|c|c|}
\hline Baumarten-Gruppe & Baumart & Mischungsprozent & $\begin{array}{l}\text { Mischungsprozent } \\
\text { der BA-Gruppe }\end{array}$ \\
\hline \multirow{8}{*}{ Pioniere } & Sandbirke & & \\
\hline & Roterle & & \\
\hline & Weißerle & & \\
\hline & Schwarzpappel & & \\
\hline & Aspe & & \\
\hline & Weide & & \\
\hline & Salweide & & \\
\hline & & & \\
\hline \multirow{10}{*}{ Intermediäre Pioniere } & Waldkiefer & & \\
\hline & Fichte & & \\
\hline & Europ. Lärche & & \\
\hline & Esche & & \\
\hline & Bergahorn & & \\
\hline & Spitzahorn & & \\
\hline & Hainbuche & & \\
\hline & Eberesche & & \\
\hline & Mehlbeere & & \\
\hline & $\ldots \ldots$ & & \\
\hline \multirow{8}{*}{$\begin{array}{l}\text { Intermediäre } \\
\text { Klimaxarten }\end{array}$} & Stieleiche & & \\
\hline & Traubeneiche & & \\
\hline & Bergulme & & \\
\hline & Feldulme & & \\
\hline & Flatterulme & & \\
\hline & Elsbeere & & \\
\hline & Speierling & & \\
\hline & ........ & & \\
\hline \multirow{6}{*}{ Klimaxarten } & Weißtanne & & \\
\hline & Eibe & & \\
\hline & Rotbuche & & \\
\hline & Winterlinde & & \\
\hline & Sommerlinde & & \\
\hline & & & \\
\hline \multirow{6}{*}{ Landschaftselemente } & Feldahorn & & \\
\hline & Wildkirsche & & \\
\hline & Wildapfel & & \\
\hline & Wildbirne & & \\
\hline & Walnuß & & \\
\hline & …... & & \\
\hline
\end{tabular}

\begin{tabular}{|l|l|l|}
\hline $\begin{array}{l}\text { Bewertung des Strukturpotentials anhand der vertretenen } \\
\text { Baumarten: }\end{array}$ & \\
\hline $\begin{array}{l}\text { Anzahl der vertretenen } \\
\text { Baumartengruppen mit einem } \\
\begin{array}{l}\text { Mischungsanteil } \\
\geq 10 \text { Prozent }\end{array}\end{array}$ & $=$ & Punktzahl \\
\hline
\end{tabular}


Indikator 5: $\quad$ Waldrandausprägung

\section{Checkliste}

Merkmal

Waldrandbreite mit mind. 15

Metern eingeplant

Lückige oder gebuchtete

Grundform des Waldrandes

Artzusammensetzung des

Waldrandes zu mindestens

$90 \%$ Sträucher oder Bäume

2. Ordnung

Strauchanteil mindestens 60

$\%$

Verwendung von

autochthonen Pflanzen

\section{Bewertung}

Anzahl der erfüllten Merkmale $=$ Punktzahl $=$ 


\section{Indikator 6: $\quad$ Biotopverbund}

\section{Betrachtungsfläche: $\quad 4 \mathrm{~km}^{2}$}

Potentielles Waldbiotop:

\section{Angrenzende Biotope:}

\begin{tabular}{|c|c|c|c|c|}
\hline $\begin{array}{c}\text { Himmels- } \\
\text { richtung }\end{array}$ & $\begin{array}{c}\text { Biotoptyp } \\
\text { (It. Typenschlüssel nach } \\
\text { RIECKEN oder } \\
\text { Offenland-BK) }\end{array}$ & $\begin{array}{c}\text { Landschaftselement } \\
\text { (Acker, Grünland, } \\
\text { Sonderkultur, Hecke, } \\
\text { Fließgewässer, Wald }\end{array}$ & $\begin{array}{c}\text { Landschaftstyp } \\
\text { nach FLADE }\end{array}$ & angrenzend \\
\hline N & & & & \\
\hline NO & & & & \\
\hline O & & & & \\
\hline SO & & & & \\
\hline S & & & & \\
\hline SW & & & & \\
\hline W & & & & \\
\hline NW & & & & \\
\end{tabular}

\begin{tabular}{|c|c|}
\hline Bewertungsmerkmale & $\checkmark$ \\
\hline Biotopentwicklung & \\
\hline Biotopergänzung & \\
\hline Biotopverknüpfung & \\
\hline Ergänzung des Lebensraumtyps & \\
\hline Erweiterung des Lebensraumtyps & \\
\hline
\end{tabular}

\section{Bewertung}

Anzahl der vorhandenen Merkmale $=$ $=$ Punktzahl 
Indikator 7: $\quad$ Landnutzungsverhältnis

Vorgabe: Regionalplan, Landschaftsplan, o.ä.

\begin{tabular}{|l|l|l|l|}
\hline & $\begin{array}{l}\text { Soll } \\
(+/=/-)\end{array}$ & $\begin{array}{l}\text { Wird } \\
(+/=/-)\end{array}$ & $\begin{array}{l}\text { Übereinstimmung } \\
(\text { Soll }- \text { Wird })\end{array}$ \\
\hline Wald & & & \\
\hline Acker & & & \\
\hline Grünland & & & \\
\hline Obstbau & & & \\
\hline Brache & & & \\
\hline
\end{tabular}

\begin{tabular}{|l|c|c|}
\hline Bewertung & Kombinationen & Punktzahlen \\
\hline Positive Übereinstimmungen & $\begin{array}{c}(+/+) \\
(-/-) \\
(=/=)\end{array}$ & - X 1 Pkt. $=\ldots$ Pkte. \\
\hline Negative Übereinstimmungen & $\begin{array}{c}(+/-)(-/+) \\
(=/-)(=/+)\end{array}$ & - $\times(-2$ Pkte. $)=-\ldots$ Pkte. \\
\hline $\begin{array}{l}(+/=) \\
(-/=)\end{array}$ & ohne Bewertung \\
Landnutzungswandels & \multicolumn{2}{|c|}{ Summe: } \\
\hline
\end{tabular}




\section{Indikator 8: $\quad$ Randlinien-Vorkommen}

Im Flächenquadrat $2 \mathrm{~km} \times 2 \mathrm{~km}$

Aufforstungsfläche: ha

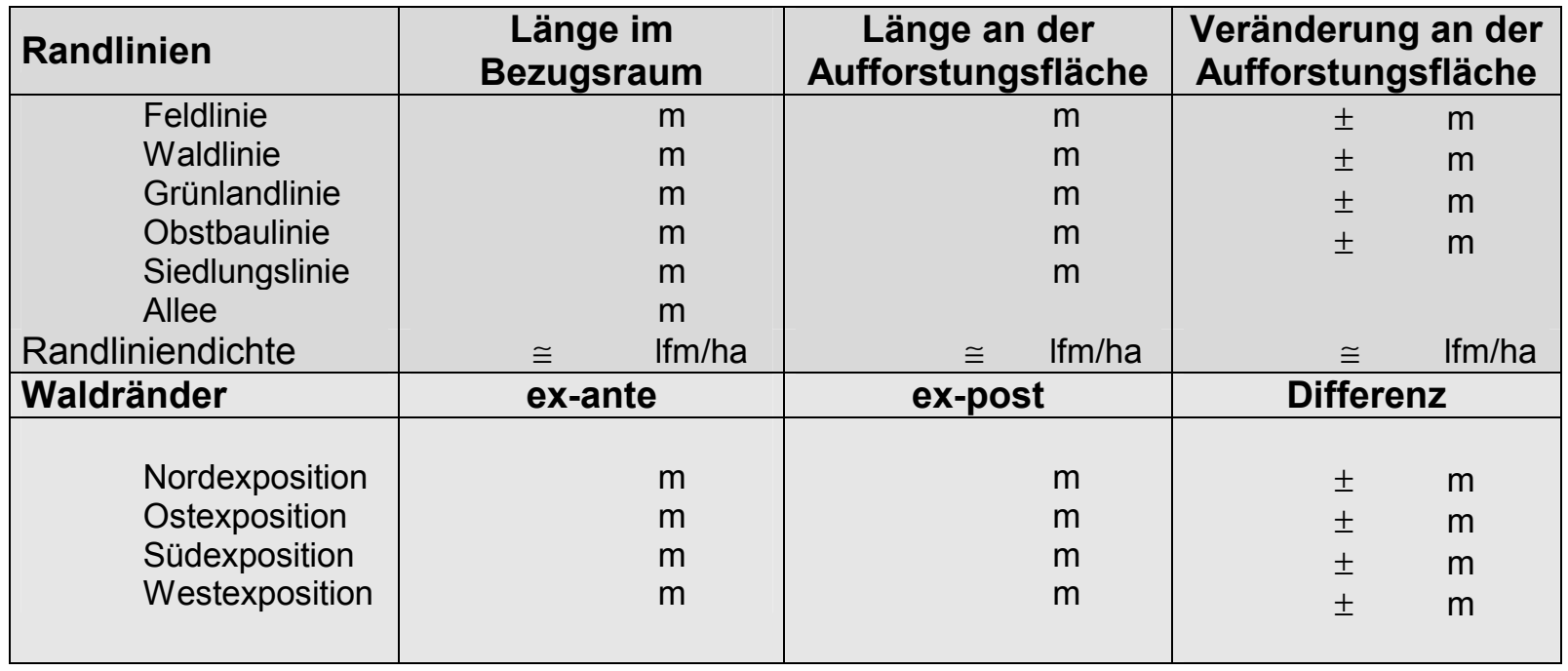

\begin{tabular}{|c|}
\hline Bewertung \\
\hline Veränderung der Randliniendichte an der Aufforstungsfläche: \\
mehr als $+20 \% \rightarrow+2$ Punkte \\
$+10 \%$ bis $+20 \% \rightarrow+1$ Punkt \\
$-10 \%$ bis $+10 \% \rightarrow \pm 0$ Punkte \\
$-20 \%$ bis $-10 \% \rightarrow-1$ Punkte \\
weniger als $-20 \% \rightarrow-2$ Punkte \\
Veränderung der Waldrandlinienlänge: \\
mehr als $+300 \% \rightarrow 3$ Punkte \\
$+200 \%$ bis $+300 \% \rightarrow 2$ Punkte \\
$+100 \%$ bis $+200 \% \rightarrow 1$ Punkt \\
$0 \%$ bis $+100 \% \rightarrow 0$ Punkte \\
$-33 \%$ bis $0 \% \rightarrow-1$ Punkt \\
$-66 \%$ bis $-33 \% \rightarrow-2$ Punkte \\
weniger als $-66 \% \rightarrow-3$ Punkte \\
Gesamtbewertung: \\
\end{tabular}




\section{Auswertung}

\begin{tabular}{|l|l|}
\hline Aufforstungsfläche (Lage) & \\
\hline Eigentümer & \\
\hline Flurstücksnummer(n) & \\
\hline Flächengröße & \\
\hline
\end{tabular}

\begin{tabular}{|l|c|l|}
\hline Indikator & Erhebungsbogen & Punktzahl \\
\hline Baumartenvielfalt & 1 & \\
\hline Naturnähe & 2 & \\
\hline Strukturpotential & 3 & \\
\hline Seltenheit des Biotops & 4 & \\
\hline Waldrand-Ausprägung & 5 & \\
\hline Biotop-Verbund & 6 & \\
\hline Landnutzungsverhältnis & 7 & \\
\hline Randlinien-Vorkommen & 8 & \\
\hline \multicolumn{1}{|c|}{ Gesamtsumme } & & \\
\hline
\end{tabular}

\begin{tabular}{|l|l|}
\hline Name des Gutachters & \\
\hline Verwendete Unterlagen & \\
\hline Datum & \\
\hline Ort & \\
\hline Unterschrift des Gutachters & \\
\hline
\end{tabular}




\title{
Definition der relevanten Landschaftstypen für die durchgeführten Untersuchungen
}

Nach: FLADE, M. (1994) „Die Brutvogelgemeinschaften Mittel- und Norddeutschlands Grundlagen für den Gebrauch vogelkundlicher Daten in der Landschaftsplanung“

\section{Landschaftstypen der Landwirtschaftlichen Felder}

\author{
Landschaftstyp: Küstennahe See- und Flussmarschen (D1, S. $200 \mathrm{ff})$
}

Beschreibung: $\quad$ Echte Marschen auf Schwemmland sowie küstennahe, zumindest ehemals überschwemmungs- und tidebeeinflusste, überwiegend als Grünland genutzte Niedermoore.

Dichtes Netz aus wechsel- oder immerfeuchten Gräben, häufig offene Wasserflächen, eventuell auch Priele. Die Grünlandgesellschaften werden ergänzt durch

Schilfbestände und Seggenriede. Gehölze treten nur vereinzelt auf und besitzen keine besondere Wuchshöhe.

Leitarten: Kiebitz (Vanellus vanellus), Bekassine (Gallinago gallinago), Rotschenkel (Tringa totanus), Uferschnepfe (Limosa limosa), Austernfischer (Haematopus ostralegus), Kampfläufer (Philomachus pugnax), Wachtelkönig (Crex crex), Sumpfohreule (Asio flammeus)

\section{Landschaftstyp: Binnenländisches Feuchtgrünland $\quad$ (D2, S. $206 \mathrm{ff})$ \\ Beschreibung: Feuchtgrünlandgebiete mit weiträumigem, offenem Charakter auf (teilentwässerten) Moorstandorten oder im Überschwemmungsbereich von Flüssen \\ Nutzungsmosaik aus Mähwiesen, Weiden und Mähweiden. Neben den Wiesengesellschaften treten Schilfröhrichte und Großseggenriede auf. Einzelne Bäume, Sträucher und Gehölzgruppen können vorkommen, erreichen jedoch maximal $5 \%$ Flächenanteil. \\ Leitarten: $\quad$ Kiebitz (Vanellus vanellus), Brachvogel (Numenius arquata), Grauammer (Milaria calandra), Wachtelkönig (Crex crex), Wachtel (Coturnix coturnix), Sumpfohreule (Asio flammeus)}

Landschaftstyp: Feldflur mit hohem Grünlandanteil, Frischwiesen und -weiden (D3, S. $216 \mathrm{ff})$

Beschreibung: Heterogene Kategorie von frischen Glatthaferwiesen und Weiden bis hin zu Acker-Grünland-Mischgebieten

Grünlandanteil schwankt in den Untersuchungsflächen von FLADE (1994) zwischen 15 und 70 Prozent, bestimmt aber in jedem Falle den Landschaftscharakter. Der Übergang zur „halboffenen Feldern“ (D5) ist fließend, lässt sich aber am ehesten am geringeren Gehölzanteil fest machen.

Leitarten: Grauammer (Milaria calandra), Wachtel (Coturnix coturnix), Raubwürger (Lanius excubitor) 


\section{Landschaftstyp: Gehölzarme Felder (D4, S. 220 ff)}

Beschreibung: Offene, weiträumige, gehölzfreie oder gehölzarme Feldlandschaften, unabhängig von Bodenart und dominierenden Feldfrüchten

Einzelne oder wenige Baumreihen und Alleen, Hecken, Gebüsche und kleine Feldgehölze können vorhanden sein, sollten jedoch weniger als $5 \%$ der Gesamtfläche ausmachen.

Im Referenzgebiet haben tiefgründige Lößlehme bzw. Schwarzerden auf Löß den größten Anteil an den Ackerböden.

An Feldfrüchten dominiert im Referenzgebiet Getreide, allerdings ist stets ein mehr oder weniger großer Anteil an Hackfrüchten, Leguminosen, Raps und anderen

Leitarten: Kulturpflanzen, sowie auch kleine Brachen und Grünlandzellen vorhanden.

Grauammer (Emberiza calandra); Wachtel (Coturnix coturnix); Großtrappe (Otis tarda)

\section{Landschaftstyp: Halboffene, reichstrukturierte Felder (D5, S. 230 ff)}

Beschreibung: Halboffene Agrarlandschaften von der mit Kleinstrukturen angereicherten offenen Landschaft bis hin zu parkartigen Wald-FeldMosaiklandschaften; lineare Strukturen (Hecken, Gehölzstreifen, Alleen) und Feldgehölze

Je geringer der Anteil der Gehölze ist, desto gleichmäßiger muss dessen Verteilung sein, damit ein halboffener Landschaftscharakter entsteht. Innerhalb der Gehölze dominieren häufig Eiche, Weißdorn, Weide, Hasel und Obstbäume die Zusammensetzung. Eine Kombination von Ackerbau und Grünland ist häufig vorhanden, aber keine Bedingung. Gehölzbewohnende Vogelarten kommen

\section{Leitarten:}

besonders häufig vor. Der Landschaftstyp kommt unabhängig von der Bodenart vor.

Neuntöter (Lanius collurio), Grauammer (Emberiza calandra), Steinkauz (Athene noctua), Wachtel (Coturnix coturnix), Ortolan (Emberiza hortulana)

\section{Landschaftstyp: \\ Halboffene, reichstrukturierte Niedermoore, Fluss- und Bachauen} (D6, S. 240 ff)

Beschreibung:

\section{Leitarten:}

Halboffene, reichstrukturierte Mosaiklandschaften mit parkartigem Charakter, in denen sich Feuchtgrünländer, Feldgehölze, Gebüsche, Hecken, Röhrichte, nasse Brachen und oft auch Gewässer kleinflächig abwechseln.

Die Landwirtschaftliche Nutzung bestimmt das Landschaftsbild. Hier herrscht wiederum die Grünlandbewirtschaftung vor, wobei vor allem die Heuwiesennutzung bestimmend ist. Weidewirtschaft ist seltener anzutreffen. Schilfröhrichte, Seggenriede und Nassbrachen sind weitere wichtige Strukturelemente dieses Landschaftstyps. Auch offene Wasserstellen gehören zum Bild.

Feldschwirl (Locustella naevia), Teichrohrsänger (Acrocephalus scirpaceus), Nachtigall (Luscinia megarhynchos), Neuntöter (Lanius collurio), Beutelmeise (Remiz pendulinus), Turteltaube (Streptopelia turtur), Schlagschwirl (Locustella fluviatilis), Sprosser (Luscinia Iuscinia), Wachtel (Coturnix coturnix), Sperbergrasmücke (Sylvia nisoria), Steinkauz (Athene noctua) 


\section{Landschaftstyp: Feuchte und nasse Brachen und Sukzessionsflächen (D7, S. $247 \mathrm{ff})$}

Beschreibung: Auf brachgefallenen Feuchtwiesen, in verbuschenden Verlandungszonen und auf künstlich von höherem Gehölzwuchs freigehaltenen Bruchwaldstandorten wechseln Hochstauden- und Schilfdickichte mit sich allmählich ausbreitenden Gebüschen.

Leitarten:

Der Gehölzanteil kann recht unterschiedlich sein (10-40\%). Aufgrund der nassen und feuchten Bereiche, treten Schilfröhricht und Seggenried regelmäßig auf. Feldschwirl (Locustella naevia), Teichrohrsänger (Acrocephalus scirpaceus), Neuntöter (Lanius collurio), Nachtigall (Luscinia megarhynchos), Rohrschwirl (Locustella luscinioides), Sperbergrasmücke (Sylvia nisoria), Blaukehlchen (Luscinia svecica), Sprosser (Luscinia luscinia), Schlagschwirl (Locustella fluviatilis), Karmingimpel (Carpodacus erythrinus), Kranich (Grus grus), Wiesenweihe (Circus pygargus)

\section{Landschaftstyp: \\ Beschreibung:}

Leitarten:

\section{Landschaftstyp:}

Beschreibung:

\section{Leitarten:}

\section{Landschaftstyp: Beschreibung:}

Leitarten:

\section{Rieselfelder (D8, S. 253 ff)}

Berieselte landwirtschaftliche Felder mit einem wesentlichen Anteil an Gewässern (Klärteiche, Gräben) mit Schlammflächen und Röhrichten. Die Flächen werden getrennt durch Gräben, Dämme und Wege, an denen Gebüsche, Hecken und Baumreihen stehen.

Feldsperling (Passer montanus), Bachstelze (Motacilla alba), Nachtigall (Luscinia megarhynchos), Teichrohrsänger (Acrocephalus scirpaceus), Gelbspötter (Hippolais icterina), Elster (Pica pica), Grauammer (Milaria calandra), Tüpfelralle (Porzana porzana), Pirol (Oriolus oriolus), Knäkente (Anas querquedula), Neuntöter (Lanius collurio), Turmfalke (Falco tinnunculus), Wachtelkönig (Crex crex), Blaukelchen (Luscinia svecica), Steinkauz (Athene noctua), Sperbergrasmücke (Sylvia nisoria)

\section{Obstbaumbestände (D9, S. 256 ff)}

Großflächige Obstbaumplantagen mit einem hohen Anteil an Hochstamm-Obstbäumen, außerhalb von Siedlungsbereichen. Typische Streuobstwiesen, wie sie vor allem aus dem süddeutschen Raum bekannt sind, spielen bei FLADE (1994) keine Rolle, da er sich in Untersuchungen und Aussagen auf den Norddeutschen Raum beschränkt.

Stieglitz (Carduelis carduelis), Gelbspötter (Hippolais icterina), Neuntöter (Lanius collurio), Girlitz (Serinus serinus), Turteltaube (Streptopelia turtur), Ortolan (Emberiza hortulana)

\section{Feldgehölze (D10, S. 259 ff)}

Wäldchen von ca. $1-7$ ha Größe die \pm isoliert in der offenen Landschaft liegen und großräumig von Feldern, Grünland oder ausnahmsweise auch Verlandungsvegetation umgeben sind. Alter und Baumartenzusammensetzung sind nachrangig, da die Struktur „Gehölz in der Agrarlandschaft" beschrieben wird. Eng beieinander liegende Gehölze können u.U. als zusammengehörig beurteilt werden. In den von FLADE untersuchten Flächen dominieren die Laubgehölze.

Aaskrähe (Corvus corone); Turteltaube (Streptopelia turtur); Turmfalke (Falco tinnunculus); Waldohreule (Asio otus); Sprosser (Luscinia luscinia) 


\section{Landschaftstypen des Waldes (Auszug)}

Im Zusammenhang mit den Landschaftstypen definiert FLADE (1994) Wälder erst ab einer Flächengröße von mindestens 7, möglichst 10 ha. Der Begriff „Forst“ wird für künstlich angelegte, i.d.R. gleichaltrige und artenarme Baumplantagen verwendet.

Waldvögel sind nach derselben Definitionsquelle Vogelarten, „die mehr oder weniger regelmäßig in Wäldern brüten und auf Gehölze als wesentliche Habitatstruktur angewiesen sind. Als echte Waldvögel im strengen Sinne können jedoch nur Arten verstanden werden, die weitgehend an großflächige, zusammenhängende Baumbestände (ab 7-10 ha Größe) gebunden sind, in Siedlungen und in der halboffenen Landschaft dagegen nur unstet siedeln oder sogar vollkommen fehlen. Eine Bindung an Einzelbäume oder Gehölzgruppen allein ist noch nicht ausreichend, um eine Vogelart als echten Waldvogel einzustufen.

FLADE weist ausdrücklich darauf hin, dass die Bindung der Vogelgesellschaften an einen bestimmten Waldtyp eher über die Struktur desselben, als über dessen Pflanzensoziologische Artenzusammensetzung erfolgt. Selbstverständlich hängt die Struktur unmittelbar, aber nicht nur, von der Baumartenzusammensetzung ab. Dementsprechend ist eine Zuordnung pflanzensoziologischer Verbände zu bestimmten Landschaftstypen möglich. (Tab. s.u.)

Eine Beschreibung der Schwarzerlen-Eschenwälder (Alno-Fraxinetum oder PrunoFraxinetum) war aufgrund fehlender Untersuchungsdaten nicht möglich. Ein weiteres Problem besteht darin, dass diese azonalen Vegetationseinheiten häufig nur kleinflächig und linear auftreten, somit mit anderen Waldtypen vergesellschaftet und flächenmäßig unterrangig sind. FLADE ordnet die Vogelgemeinschaft des Erlen-Eschenwaldes zwischen denjenigen der Erlenbruchwälder, der feuchten Eichen-Hainbuchenwälder und den Hartholzauen ein.

Die Problematik des kleinflächigen und vergesellschafteten Vorkommen tritt auch bei den Ahorn-Eschenwäldern (Aceri-Fraxinetum) auf. Auch sie wurden aus diesem Grunde von FLADE nicht flächenmäßig untersucht. Häufig ist dieser Waldtyp innerhalb von EichenHainbuchen-Wäldern oder Berg-Buchenwäldern zu finden. Die Vogelgemeinschaft dürfte derjenigen der feuchten und basenreichen Eichen-Hainbuchen-Wälder entsprechen.

\section{Landschaftstyp: Eichen-Hainbuchenwälder und Tiefland-Perlgras-Buchenwälder} (E16, S. 304 ff)

Beschreibung: Von den feuchten bis nassen, z.T. auf aperiodisch überfluteten Auenböden stockenden Ausformungen der Eichen-Hainbuchenwälder bis hin zu den sommertrockenen (Linden-) TraubeneichenHainbuchenwäldern im Regenschatten des Harzes.

Außerdem umfasst die Gruppe die im Tiefland wachsenden, etwas basenreicheren Perlgras-Buchenwälder mit hohen Eichenanteilen, die häufig durch den Übergang von der Mittelwaldbewirtschaftung auf den Hochwaldbetrieb mit Förderung entstanden sind.

Die rauhborkige Eiche dominiert den Bestandestyp. Meist besteht eine zweite Baumschicht aus Hainbuche sowie eine häufig spärlich ausgebildete Strauchschicht. Die Krautschicht ist extrem variabel und kann innerhalb eines Waldkomplexes ganz unterschiedlich ausgebildet sein.

Leitarten: $\quad$ Kleiber (Sitta europaea), Waldlaubsänger (Phylloscopus sibilatrix), Gartenbaumläufer (Certhia brachydactiyla), Trauerschnäpper (Ficedula hypoleuca), Sumpfmeise (Parus palustris), Pirol (Oriolus oriolus), Mittelspecht (Dendrocopos medius), Grauspecht (Picus canus) 


\section{Landschaftstyp: Beschreibung:}

Kolline und montane Buchenwälder (E18, S. 320 ff)

Dieser Landschaftstyp umfasst die oft hallenartig ausgeprägten, unterholzarmen Hainsimsen-Buchenwälder und PerlgrasBuchenwälder, die etwas reicher strukturierten TraubeneichenBuchen-Wälder mit z.T. hohen Eichenanteilen sowie die ebenfalls of hallenartigen, jedoch mit einer reicheren Krautschicht versehenen Waldmeister-Buchenwälder bis hin zu den etwas unterholzreicheren und vielfältiger strukturierten Orchideen-Buchenwälder des Hügel- und Berglandes.

Reliefbedingt verfügt der Landschaftstyp über Strukturelemente wie steile Hanglagen, unterschiedliche Hangexpositionen und Höhenlagen. Der Nadelholzanteil ist im Allgemeinen gering.

Leitarten: $\quad$ Waldlaubsänger (Phylloscopus sibilatrix), Kleiber (Sitta europaea), Trauerschnäpper (Ficedula hypoleuca), Sumpfmeise (Parus palustris), Grauspecht (Picus canus), Hohltaube (Columba oenas),

[Rauhfußkauz]

\section{Landschaftstypen der Nadelwälder (Auszug)}

Natürliche Vorkommen von Nadelwäldern beschränken sich in Mitteleuropa auf wenige Bereiche, etwa die höchsten Regionen der Mittelgebirge sowie einige kleinflächige, inselartig vorgeschobene Vorkommen in tieferen Lagen, etwa in Moorrandbereichen, auf extrem armen, sandigen Dünenstandorten sowie Fichtenwaldvorkommen in der Lausitz und im submontanen westlichen Harzvorland.

Darüber hinaus stehen heute auch auf Laubwaldstandorten vielerorts Nadelwälder, die im Rahmen der „ordnungsgemäßen Forstwirtschaft“ in der Vergangenheit angelegt wurden.

Die Zuordnung der pflanzensoziologischen Gemeinschaften zu den von FLADE definierten Landschaftstypen der Nadelwälder ergibt sich aus der Tabelle xxe2. Wie bei den Laubwäldern gilt auch hier, dass die Vogelgemeinschaften dieser Biotope sich entsprechend der typischen Bestandesstruktur einstellt, welche wiederum von der Baumartenzusammensetzung und Bewirtschaftung beeinflusst wird.

Eine Beschreibung einzelner Nadelwald-Landschaftstypen entfällt, da diese für die hier angestellten Untersuchungen keine Rolle spielen. Sie gehen jedoch aus der folgenden Tabelle mit Benennung der Leitarten hervor.

\section{Landschaftstyp: Beschreibung:}

Leitarten:

\section{Fichtenforste (E24; S. 363 ff)}

Der Landschaftstyp umfass einerseits die dichten, geschlossenen Fichten-Reinbestände, andererseits Fichten-Kiefernforste mit einem Fichtenanteil von mindestens 70 \%. Einzelne Laubhölzer kommen vor und sorgen schnell für eine starke Auflockerung der Kronenachse. Auch zu den Bestandesrändern hin, bewirken andere Baumarten häufig eine Auflockerung der Struktur.

Wintergoldhähnchen, Sommergoldhähnchen, Tannenmeise, Haubenmeise, Misteldrossel 


\section{Landschaftstypen der Trockenbiotope und Sonderstandorte (Auszug)}

\section{Landschaftstyp: Beschreibung:}

Leitarten:

\section{Trocken- und Halbtrockenrasen (G1; S. 465 ff)}

Durch Beweidung oder Gehölzschnitt gehölzfrei gehaltene Grünflächen unterschiedlicher Rasengesellschaften (TrespenHalbtrockenrasen, Glatthaferwiesen, Weidelgras-Weißklee-Weiden, vereinzelt auch Blaugrasrasen und Brennesselfluren). Standörtlich häufig auf süd- bis südwestexponierten Kuppen und Hanglagen mit Muschelkalk oder Devon im Untergrund.

Unterschiedlich starke Verbuschung oder Wiederbewaldung gehören zum üblichen Erscheinungsbild. Insbesondere dornenbewehrte Gebüsche kommen vor. Vereinzelt sind Bäume (Obst, Eichen, Kiefern, Buchen) vorhanden. Typisch ist außerdem eine geringe Flächengröße der Trockenrasen, die häufig unter den Minimalarealen etlicher Vogelarten liegen.

Hänfling, (außerhalb des USG auch Zippammer (im Südwesten), Neuntöter und Sperbergrasmücke (im Osten)); aber: wenig untersucht!

\section{Landschaftstypen der Siedlungen}

\section{Landschaftstyp: Beschreibung:}

Leitarten:

\section{Landschaftstyp: Beschreibung:}

Leitarten:

\section{Friedhöfe (F1, S. $410 \mathrm{ff})$}

Einheitlich strukturierte Vogellebensräume mit Gräberfeldern, dichten Hecken und Gebüschen (insbesondere aus Koniferen), einige Gebäude und eine lockere Baumschicht.

Trotz hoher Besucherfrequenz und dichtem Wegenetz insgesamt ruhig und störungsarm. Höherer Nadelholzanteil als in Parks, geringeres Baumalter, wenig Rasenfläche und ein höheres Angebot an Nistkästen.

Gartenrotschwanz (Phoenicurus phoenicurus), Feldsperling (Passer montanus), Elster (Pica pica), Girlitz (Serinus serinus), Grauschnäpper (Muscicapa stiata), Türkentaube (Streptopelia decaocto), Saatkrähe (Corvus frugilegus)

\section{Parks (F2, S. 416 ff)}

I.d.R. alter Baumbestand mit dominantem Laubholzanteil, dazwischen teilweise weitläufige Rasenflächen, Wasserflächen und teilweise Blumenrabatten.

Hohe Besucherfrequenz und weitere starke anthropogene Einflüsse (Vogelfütterungen, Nistkastenangebot, Verlärmung, Gebäude). Parzellierung ist geringer als auf Friedhöfen. Totholzanteil kann hoch sein.

Gartenrotschwanz (Phoenicurus phoenicurus), Grauschnäpper (Muscicapa stiata), Gelbspötter (Hippolais icterina), Kleiber (Sitta europaea), Türkentaube (Streptopelia decaocto), Girlitz (Serinus serinus), Grünspecht (Picus viridis), Dohle (Corvus monedula), Saatkrähe (Corvus frugilegus) 


\section{Landschaftstyp:} Beschreibung:

\section{Leitarten:}

Landschaftstyp: Beschreibung:

\section{Leitarten:}

Landschaftstyp: Beschreibung:

\section{Leitarten:}

\section{Landschaftstyp:} Beschreibung:

Leitarten:

\section{Baumschulen (F3, S. 424)}

Hohes Artenspektrum an Pflanzen und ähnliches Habitatstruktur wie auf Friedhöfen, allerdings ohne Baumschicht.

Wenig untersucht! nicht bestimmt, da zuwenig Untersuchungen vorliegen!

\section{Kleingartenkolonien (F4, S. $425 \mathrm{ff})$}

Einheitlich strukturierte Lebensräume mit kleinparzellierten Grundstücken, vielen Lauben und Schuppen, locker stehende Obstbäume und Beerensträucher sowie dichte Hecken entlang der vielen Wege.

Hohe Nutzungs- und Störungsintensität, allerdings schwankend im Wochenverlauf. Extrem hohe Anzahl an Nistkästen. Weiterhin kommen Zierrasenflächen und Blumenrabatte vor. Hohe Biozid- und Düngebelastung Feldsperling (Passer montanus), Haussperling (Passer domesticus), Gartenrotschwanz (Phoenicurus phoenicurus), Girlitz (Serinus serinus)

\section{Gartenstädte (F5, S. 429 ff)}

Meist ein- bis dreigeschossige Häuser mit durch Hecken und Gebüsche gekammerten Gärten.

Reichtum an Zierrasenflächen, Obst- und Ziergehölzen und z.T. auch Waldbäume. Nisthilfen in unterschiedlicher Anzahl vorhanden. Unterscheidung von den Kleingärten durch älteren Baumbestand, geringere Nistkastendichte, größere Gebäude sowie Straßenland. Gegenüber Dörfern fehlen hier die Scheunen und Stallungen sowie der Reichtum an Obstbäumen. Stattdessen kommen mehr Ziergartenelemente und mehr Koniferen vor. Die Entfernung zur offenen Landschaft ist größer.

Haussperling (Passer domesticus), Gartenrotschwanz (Phoenicurus phoenicurus), Girlitz (Serinus serinus), Türkentaube (Streptopelia decaocto), Grauschnäpper (Muscicapa striata), Straßentaube (Columba livia $f$. domestica), Mehlschwalbe (Delichon urbica)

\section{Dörfer (F6, S. 435)}

Kleinere Siedlungen (bis 100 ha) mit bäuerlichem Charakter, geprägt durch bewirtschaftete Bauernhöfe und landwirtschaftliche Produktionsbetriebe, Scheunen, gepflasterte oder unversiegelte Hofstellen.

Misthaufen verschieden aus dem heutigen Dorfbild. Die Dörfer werden nach und nach durch den Zuzug von Städtern in Gartenstädte umgewandelt, leiden somit unter Strukturverlust.

Vereinzelt bestehen noch Elemente wie ein Dorfteich oder eine durchs Dorf verlaufende Bachaue mit begleitendem Grünland. Auch Streuobstwiesen kommen im Dorfbereich noch vor.

Haussperling (Passer domesticus), Rauchschwalbe (Hirundo rustica), Hänfling (Carduelis cannabina), Gartenrotschwanz (Phoenicurus phoenicurus), Bachstelze (Motacilla alba), Mehlschwalbe (Delichon urbica), Hausrotschwanz (Phoenicurus ochruros), Stieglitz (Carduelis carduelis), Feldsperling (Passer montanus), Grauschnäpper (Muscicapa striata), Schleiereule (Tyto alba), Grauammer (Milaria calandra), Weißstorch (Ciconia ciconia), Steinkauz (Athene noctua) 


\section{Landschaftstyp: Beschreibung:}

Leitarten:

Landschaftstyp: Beschreibung:

\section{Leitarten:}

Landschaftstyp: Beschreibung:

\section{Leitarten:}

\section{City- und Altbau-Wohnblockzonen (F7, S. $441 \mathrm{ff})$}

Innerstädtische Siedlungstypen mit hoher bis sehr hoher Bebauungsdichte und hohem Versiegelungsgrad.

Bäume sind vor allem an Straßen und auf Plätzen vorhanden. Fremdländische Gehölze dominieren das Bild. Hoher Lärmpegel, warmes Stadtklima, starkes Verkehrsaufkommen.

Haussperling (Passer domesticus), Mauersegler (Apus apus), Türkentaube (Streptopelia decaocto), Straßentaube (Columba livia $f$. domestica), Turmfalke (Falco tinnunculus), Dohle (Corvus monedula), Mehlschwalbe (Delichon urbica), Schleiereule (Tyto alba)

\section{Neubau-Wohnblockzonen (F8, S. $447 \mathrm{ff}$ )}

Einheitlich gestaltete Zonen am Rande der Stadtzentren, mit lockerer Blockbebauung, mehr oder weniger ausgedehnten Rasenflächen und kleineren Ziergebüschen.

Wohnhäuser mehrgeschossig, Plattenbauweise und flachdächer herrschen vor. Hinsichtlich der Vegetation bestimmt der Zierrasen, ergänzt durch Blumenbeete und Strauchrabatten das Bild. Einzelne Straßen- und Parkbäume kommen vor.

Haussperling (Passer domesticus), Haubenlerche (Galerida cristata), Mauersegler (Apus apus), Türkentaube (Streptopelia decaocto), Straßentaube (Columba livia f. domestica), Mehlschwalbe (Delichon urbica)

Industriegebiete, gr. Bahnhofs- und Hafenanlagen (F9, S. 452 ff)

Weites Spektrum an Gewerbeanlagen und -gebieten, die typischerweise hoch immissionsbelastet sind.

An Strukturelementen treten große Hallen, große asphaltierte Plätze, Bahn- und Gleisanlagen, Halden, Schuppen und Garagen auf. Zwischendrin finden sich vereinzelt Rasen- und Ruderalflächen, auch spärlicher Baum- und Strauchbewuchs. Haussperling (Passer domesticus), Hausrotschwanz (Phoenicurus ochruros), Mauersegler (Apus apus), Steinschmätzer (Oenanthe oenanthe), Straßentaube (Columba livia $f$. domestica), Mehlschwalbe (Delichon urbica) 
Die Landschaftstypen nach FLADE (1994) und deren zugehörige Pflanzengesellschaften

\begin{tabular}{|c|c|c|}
\hline Code & Landschaftstyp & Pflanzensoziologische Zuordnung \\
\hline E 11 & $\begin{array}{l}\text { Birken- und Birken- } \\
\text { Kierfern-Bruchwälder }\end{array}$ & $\begin{array}{l}\text { Rhamno-Betuletum pub. } \\
\text { Vaccinio uliginosei-Betuletum pub. } \\
\text { Vaccinio uliginosi-Pinetum; Ledo-Pinetum }\end{array}$ \\
\hline E 12 & Erlenbruchwälder & Carici elongatai-Alnetum medioeruopaeum \\
\hline E 13 & Weidenwälder & Salicetum albae, Salicetum fragilis \\
\hline E 14 & Pappelforste & \\
\hline E 15 & Hartholzauen & Fraxino-Ulmetum \\
\hline E 16 & $\begin{array}{l}\text { Eichen-Hainbuchenwald } \\
\text { und Tiefland-Perlgras- } \\
\text { Buchenwald }\end{array}$ & $\begin{array}{l}\text { Stellario-Carpinetum } \\
\text { Tilio-Carpinetum } \\
\text { Galio-Carpinetum } \\
\text { Melico-Fagetum }\end{array}$ \\
\hline E 17 & $\begin{array}{l}\text { Bodensaure Tiefland- } \\
\text { Buchenwälder }\end{array}$ & $\begin{array}{l}\text { Luzulo-Fagion: } \\
\text { Avenello-Fagetum, Periclymeno-Fagetum, } \\
\text { Petraeo-Fagetum }\end{array}$ \\
\hline E 17a & Birken-Eichenwälder & Betulo-Quercetum \\
\hline E 18 & $\begin{array}{l}\text { Kolline u. montane } \\
\text { Buchenwälder }\end{array}$ & $\begin{array}{l}\text { Luzulo-Fagetum } \\
\text { Luzulo-Querco-Fagetum } \\
\text { Milio-Fagetum } \\
\text { Galio odorati-Fagion: } \\
\text { Dentario bulbiferae-Fagetum } \\
\text { Lathyro-Fagetum } \\
\text { Cephalantero-Fagetum }\end{array}$ \\
\hline E 19 & $\begin{array}{l}\text { Laubniederwälder und } \\
\text {-stangenhölzer }\end{array}$ & $\begin{array}{l}\text { Größtenteils zu: } \\
\text { Betulo-Quercetum und Luzulo-Fagion }\end{array}$ \\
\hline E 21 & $\begin{array}{l}\text { Laubholzreiche } \\
\text { Kiefernforst u. Eichen- } \\
\text { Kierfernwälder }\end{array}$ & $\begin{array}{l}\text { Pino-Quercetum / Quercetum-Pinetum } \\
\text { sowie ähnlich: } \\
\text { Luzulo-Fagion } \\
\text { Stellario-Carpinetum } \\
\text { Betulo-Quercetum }\end{array}$ \\
\hline E 22 & Reine Kiefernforste & $\begin{array}{l}\text { Dicranum-Kiefernforste } \\
\text { Cladonia-Kiefernforste } \\
\text { Sieglingia-Kiefernforste }\end{array}$ \\
\hline E 22 a & Kiefernstangenhölzer & wie E 21, E 22 \\
\hline E 23 & $\begin{array}{l}\text { Fichtenreiche } \\
\text { Kiefernwälder und -forste }\end{array}$ & $\begin{array}{l}\text { Sieglingia-Kiefernforste } \\
\text { Rubus-Kiefernforste }\end{array}$ \\
\hline E 24 & Fichtenforste & \\
\hline E 25 & $\begin{array}{l}\text { Hochmontane } \\
\text { Fichtenwälder und - } \\
\text { forste }\end{array}$ & $\begin{array}{l}\text { Calamagrostio-Piceetum } \\
\text { Piceetum subalpinum sphagnetosum }\end{array}$ \\
\hline E 25 a & $\begin{array}{l}\text { Hochmontane } \\
\text { Fichtenmoore }\end{array}$ & Piceeto-Vaccinietum uliginosi hercynicum \\
\hline E 26 & $\begin{array}{l}\text { Krüppelfichten-Bestände, } \\
\text { Baumgrenze }\end{array}$ & \\
\hline E 27 & Fichtenstangenhölzer & z.T. wie E 25 \\
\hline E 27 a & Fichtendickungen & \\
\hline E 28 & Kieferndickungen & \\
\hline
\end{tabular}


Anhang 2 a: Auswertung der avifaunistischen Artenlisten in den Landschaftsplänen für den Untersuchungsraum Apolda

\begin{tabular}{|c|c|c|c|c|c|c|c|c|c|c|c|}
\hline $\begin{array}{l}\text { Wiss. } \\
\text { Artname }\end{array}$ & $\begin{array}{l}\text { Deutsche } \\
\text { Bezeichnung }\end{array}$ & $\begin{array}{l}\text { Leitarten für } \\
\text { Landschafts- } \\
\text { typen }\end{array}$ & $\begin{array}{c}\mathrm{ABSP} \\
)^{1}\end{array}$ & BArtSchV & $\begin{array}{c}\text { EG- } \\
\text { Vogel- } \\
\text { schutz- } \\
\text { richtlinie }\end{array}$ & $\begin{array}{l}\text { RL } \\
\text { Bund }\end{array}$ & $\begin{array}{c}\mathrm{RL} \\
\text { Thü- } \\
\text { ringen }\end{array}$ & $\begin{array}{l}\text { Status } \\
\text { LP } \\
\text { Unteres } \\
\text { IImtal })^{2}\end{array}$ & $\begin{array}{c}\text { Status } \\
\text { LP } \\
\text { Mellingen- } \\
\text { Apolda ) } \\
\end{array}$ & $\begin{array}{l}\text { Status LP } \\
\text { Ost- } \\
\text { Abschnitt } \\
\text { LK AP ) }\end{array}$ & $\begin{array}{c}\text { Status } \\
\text { nach mdl. } \\
\text { Auskunft) }^{5}\end{array}$ \\
\hline Coturnix coturnix & Wachtel & D4,D5 & & & & 2 & 2 & + & $\mathrm{MN}$ & & \\
\hline Emberiza calandra & Grauammer & D4,D5 & & $?$ & $?$ & $?$ & $?$ & & -- & & $\mathrm{BN}$ \\
\hline Otis tarda & Großtrappe & D4 & & $?$ & $?$ & $?$ & $?$ & & & & \\
\hline Lanius collurio & Neuntöter & D5,D9 & & $\S$ & $!$ & 3 & & $\mathrm{BN}$ & $\mathrm{MN}$ & $\mathrm{MN}$ & \\
\hline Athene noctua & Steinkauz & D5 & & & & 2 & 1 & + & -- & $\mathrm{MN}$ & \\
\hline Emberiza hortulana & Ortolan & D5,D9 & & $?$ & $?$ & $?$ & $?$ & & & & -- \\
\hline Carduelis carduelis & Stieglitz & D9 & & & & & & $\mathrm{BN}$ & & & \\
\hline Hippolais icterina & Gelbspötter & D9,F2 & & & & & & $\mathrm{BV}$ & & & \\
\hline Serinus serinus & Girlitz & D9,F2 & & & & & & $\mathrm{BN}$ & & & \\
\hline Streptopelia turtur & Turteltaube & D9,D10 & & & & & & + & & & \\
\hline Corvus corone & Aaskrähe & D10 & & & & & & $\mathrm{BN}$ & & & \\
\hline Falco tinnunculus & Turmfalke & D10 & & & & & & $\mathrm{BN}$ & & & \\
\hline Asio otus & Waldohreule & D10 & & & & & & $\mathrm{BN}$ & & & \\
\hline Luscinia luscinia & Sprosser & D10 & & & außerh & alb des & latürliche & Verbreitı & ingsgebietes & & \\
\hline Sitta europaea & Kleiber & E18,F2 & & & & & & $\mathrm{BN}$ & & & \\
\hline Phylloscopus sibilatrix & Waldlaubsänger & E16,E18 & & & & & & BV & & & BV \\
\hline Certhia brachydactiyla & Gartenbaumläufer & E16 & & & & & & $\mathrm{BN}$ & & & $\mathrm{BN}$ \\
\hline Ficedula hypoleuca & Trauerschnäpper & E16,E18 & & & & & & $\mathrm{BN}$ & & & $\mathrm{BN}$ \\
\hline Parus palustris & Sumpfmeise & E16,E18 & & & & & & + & & & $\mathrm{BN}$ \\
\hline Oriolus oriolus & Pirol & E16 & & & & & & $\mathrm{BN}$ & & & $\mathrm{BN}$ \\
\hline Dendrocopos medius & Mittelspecht & E16 & * & $\S$ & $!$ & 3 & 3 & + & MN & $\mathrm{MN}$ & -- \\
\hline Picus canus & Grauspecht & E16,E18 & & $\S$ & $!$ & & 3 & $\mathrm{BN}$ & --- & & -- \\
\hline Columba oenas & Hohltaube & E18 & & $\S$ & $?$ & $?$ & 3 & & EN & & \\
\hline Phoenicurus phoenic. & Gartenrotschwanz & $\mathrm{F} 2$ & * & & & & 3 & $\mathrm{BN}$ & $\mathrm{MN}$ & & \\
\hline Muscicapa striata & Grauschnäpper & $\mathrm{F} 2$ & & & & & & BV & & & \\
\hline Streptopelia decaocto & Türkentaube & $\mathrm{F} 2$ & & & & & & $\mathrm{BV}$ & & & \\
\hline Picus viridis & Grünspecht & $\mathrm{F} 2$ & * & & & 3 & 3 & $\mathrm{BN}$ & $\mathrm{MN}$ & $\mathrm{MN}$ & $\mathrm{BN}$ \\
\hline Corvus monedula & Dohle & $\mathrm{F} 2$ & & & & 3 & 3 & BV & & EN & \\
\hline Corvus frugilegus & Saatkrähe & $\mathrm{F} 2$ & & & & 3 & 1 & + & $\mathrm{MN}$ & & \\
\hline
\end{tabular}

${ }^{1}$ Nennung im ABSP Mittelthüringen: Grünspecht, Mittelspecht, Rotmilan, Schwarzmilan, Gartenrotschwanz, Teichralle, Braunkelchen, Sperber, Weißstorch, Steinschmätzer, Schleiereule, Kolkrabe )$^{2}$ Landschaftsplan Unteres IImtal; 122 Arten genannt, davon 54 Brutvögel (BN); 22 Brutverdacht (BV), 44 ohne Bruthinweis (+) inkl. 6 reine Durchzügler

)$^{3}$ Landschaftsplan Mellingen Apolda; 39 (geschützte) Arten genannt, davon 1 Art flächendeckend vorkommend, 16 mal Mehrfachnachweis, 4 mal Einzelnachweis (EN), 18 mal ohne Angaben )$^{4}$ LP Ost-Abschnitt Landkreis Weimarer Land; 33 Arten genannt, davon 15 mit Mehrfachnachweis (MN) inkl. 1 Durchzügler, 16 mit Einzelnachweis (EN) inkl. 1 Durchzügler, 1 ohne Angaben $5^{5}$ mündliche Auskunft Herr Weise, Umweltamt Kreis Weimarer Land; nachgefragt wurden 10 Arten, davon brüten nachweislich 6, vermutlich 1 weitere im USG; 3 Arten sind im Bereich Apolda/Niedertreba vermutlich nicht regelmäßig vorhanden 
Anhang 2 b: Angaben zum Vorkommen bestimmter Leitarten im Untersuchungsraum Dassel

\begin{tabular}{|l|c|c|c|}
\hline \multicolumn{1}{|c|}{ Artname } & Brutnachweis & Brutverdacht & $\begin{array}{c}\text { Nicht vorhanden } \\
\text { oder kein } \\
\text { nennenswertes } \\
\text { Vorkommen }\end{array}$ \\
\hline Sumpfmeise & $\mathrm{X}$ & & \\
\hline Grauspecht & $\mathrm{X}$ & & \\
\hline Gartenbaumläufer & $\mathrm{X}$ & & \\
\hline Mittelspecht & $\mathrm{X}$ & $\mathrm{X}$ & \\
\hline Kleiber & & $\mathrm{X}$ & \\
\hline Grünspecht & $\mathrm{X}$ & & \\
\hline Trauerschnäpper & $\mathrm{X}$ & & \\
\hline Waldlaubsänger & $\mathrm{X}$ & & \\
\hline Sommergoldhähnchen & $\mathrm{X}$ & & \\
\hline Wintergoldhähnchen & & & \\
\hline Dohle & $\mathrm{X}$ & & \\
\hline Hohltaube & & $\mathrm{X}$ & \\
\hline Rauhfußkauz & $\mathrm{X}$ & & $\mathrm{X}$ \\
\hline Haubenmeise & $\mathrm{X}$ & & $\mathrm{X}$ \\
\hline Tannenmeise & & $\mathrm{X}$ & \\
\hline Misteldrossel & $\mathrm{X}$ & $\mathrm{X}$ & \\
\hline Zwergschnäpper & & $\mathrm{X}$ & $\mathrm{X}$ \\
\hline Sperlingskauz & & $\mathrm{X}$ & \\
\hline Zeisig & & & \\
\hline Tannenhäher & & & $\mathrm{X}$ \\
\hline Wachtel & & & \\
\hline Grauammer & & & \\
\hline Raubwürger & & & \\
\hline Steinkauz & & & \\
\hline Neuntöter & & & \\
\hline Ortolan & & & \\
\hline Turteltaube & & & \\
\hline Turmfalke & & & \\
\hline Rabenkrähe & & & \\
\hline Waldohreule & & & \\
\hline Sperbergrasmücke & & & \\
\hline Zippammer & & & \\
\hline Hänfling & & & \\
\hline Wanderfalke & & & \\
\hline Feldsperling & & & \\
\hline Hausrotschwanz & & & \\
\hline
\end{tabular}

Erstellt mit Hilfe und nach Aussagen des ornithologischen Arbeitskreises der Ortsgruppe Dassel-Einbeck, Naturschutzbund Deutschland e.V. 
Anhang 3: Grundlagen und Informationen für den Untersuchungsraum Apolda

Stamm-Standortsgruppen und Baumarten potentiell-natürlicher Wälder auf der Basis von Stamm-Vegetationsformen im forstlichen Wuchsgebiet Thüringer Becken

(Auszug, Quelle: LAWUF 1997 a):

\begin{tabular}{|c|c|c|c|c|c|c|}
\hline $\begin{array}{l}\text { Stamm- } \\
\text { Standortsgruppe }\end{array}$ & \multicolumn{5}{|c|}{$\begin{array}{l}\text { Baumarten potentiell-natürlicher Wälder auf der Basis von } \\
\text { Stamm-Vegetationsformen }\end{array}$} & Bemerkungen \\
\hline $\begin{array}{l}\text { R2, w, C } \\
\text { R3, C }\end{array}$ & $\begin{array}{l}\text { edellaubbaumreicher } \\
\text { Linden-Hainbuchen- } \\
\text { Eichenwald }\end{array}$ & TB 13 & TEi, SEi & $\begin{array}{l}\text { HBu, } \\
\text { WLi, } \\
\text { SLi, Es, } \\
\text { BAh, }\end{array}$ & $\begin{array}{l}\text { Bu, BUl, } \\
\text { FUl,SAh, } \\
\text { FAh, El, } \\
\text { Kb, Sp, As }\end{array}$ & $\begin{array}{l}\text { auf Tonstandorten } \\
\text { z.T. auch SEi } \\
\text { dominierend }\end{array}$ \\
\hline BR2, BK2 & $\begin{array}{l}\text { Stieleichenreicher } \\
\text { (Roterlen-) } \\
\text { Eschenbachwald }\end{array}$ & TB34 & ES & $\begin{array}{l}\text { REr, SEi } \\
\text { (TEi) }\end{array}$ & $\begin{array}{l}\text { BAh, SAh, } \\
\text { BUl, HBu, } \\
\text { WLi, As, } \\
\text { FUI }\end{array}$ & Bachtalstandorte \\
\hline
\end{tabular}

Erstaufforstungs-Varianten nach Naturnähe und Strukturpotential erstellt für das Untersuchungsgebiet Apolda

\begin{tabular}{|c|c|c|c|c|}
\hline Standort & Szenario & Hauptb. & Mischb. & Nebenb. \\
\hline \multirow{6}{*}{$\begin{array}{l}\text { Standort RLL2, RLL3 } \\
\text { Reichere Schlufflehm- } \\
\text { Standorte mäßig frisch bis } \\
\text { mäßig trocken; } \\
\text { PNV: edellaubbaumreicher } \\
\text { Linden-Hainbuchen- Eichenwald } \\
\text { (12 pnV-BA) } \\
\text { (6 Szen. mit je um } 2 \\
\text { zunehmenden Arten aus der } \\
\text { pnV) }\end{array}$} & $\mathrm{I}-2$ & $\begin{array}{l}\text { TEi, } \\
(6)\end{array}$ & $\begin{array}{l}\mathrm{HBu} \\
(4)\end{array}$ & \\
\hline & $\mid-4$ & $\begin{array}{l}\text { TEi } \\
(5)\end{array}$ & $\begin{array}{l}\text { HBu, WLi } \\
(2 / 2)\end{array}$ & $\begin{array}{l}\text { Ah } \\
\text { (1) }\end{array}$ \\
\hline & $I-6$ & $\begin{array}{l}\text { TEi } \\
(4)\end{array}$ & $\begin{array}{l}\text { HBu, WLi } \\
(2 / 2)\end{array}$ & $\begin{array}{l}\text { Ah, Kir, As } \\
(1 / .5 / .5)\end{array}$ \\
\hline & $1-8$ & $\begin{array}{r}\text { TEi } \\
(4)\end{array}$ & $\begin{array}{l}\text { HBu, WLi } \\
(2 / 1)\end{array}$ & $\begin{array}{l}\text { Ah, Kir, As, Sp, Ul } \\
(1 / .5 / .5 / .5 / .5)\end{array}$ \\
\hline & $\mathrm{I}-10$ & $\begin{array}{r}\mathrm{TEi} \\
(4)\end{array}$ & $\begin{array}{l}\text { HBu, WLi, } \\
\text { Es } \\
(1 / 1 / 1)\end{array}$ & $\begin{array}{l}\text { Ah, Kir, As, Sp, Ul, } \\
\text { FAh } \\
(6 \times 0.5)\end{array}$ \\
\hline & $\mathrm{I}-12$ & $\begin{array}{l}\text { TEi } \\
(3)\end{array}$ & $\begin{array}{l}\text { HBu, WLi, } \\
\text { Es } \\
(1 / 1 / 1)\end{array}$ & $\begin{array}{l}\text { Ah, Kir, As, Sp, Ul, } \\
\text { FAh, EL, Bi } \\
(8 \times 0.5)\end{array}$ \\
\hline \multirow{6}{*}{$\begin{array}{l}\text { Standort RC2 } \\
\text { Reicherer Karbonat- } \\
\text { gesteins-StO, mäßig frisch } \\
\text { PNV: edellaubbaumreicher } \\
\text { Linden-Hainbuchen-Eichenwald } \\
\text { (12 pnV-BA) } \\
\text { (6 Szen. mit je um } 2 \\
\text { zunehmenden BA, davon } 1 \text { aus } \\
\text { pnV) } \\
\rightarrow \text { Varianten mit nicht- } \\
\text { einheimischen BA }\end{array}$} & II-2 & $\begin{array}{l}\text { TEi; ELä } \\
(5 / 5)\end{array}$ & & \\
\hline & II-4 & $\begin{array}{l}\text { TEi, ELä } \\
(4 / 4)\end{array}$ & $\begin{array}{l}\text { Birn, Kir } \\
(1 / 1)\end{array}$ & \\
\hline & II-6 & $\begin{array}{l}\text { TEi, ELä } \\
(4 / 3)\end{array}$ & $\begin{array}{l}\text { Birn, Kir, WLi } \\
(.5 / 1 / 1)\end{array}$ & $\begin{array}{l}\mathrm{Mb} \\
(.5)\end{array}$ \\
\hline & II-8 & $\begin{array}{l}\text { TEi, ELä } \\
(4 / 2)\end{array}$ & $\begin{array}{l}\text { Birn, Kir, WLi } \\
(.5 / 1 / 1)\end{array}$ & $\begin{array}{l}\text { Mb, As, Kie } \\
(.5 / .5 / .5)\end{array}$ \\
\hline & II-10 & $\begin{array}{l}\text { TEi, ELä } \\
(3 / 2)\end{array}$ & $\begin{array}{l}\text { Birn, Kir, } \\
\text { WLi, UI } \\
(.5 / 1 / 1 / .5)\end{array}$ & $\begin{array}{l}\text { Mb, As, Kie, Vb } \\
(4 \times 0.5)\end{array}$ \\
\hline & II-12 & $\begin{array}{l}\text { TEi, ELä } \\
(3 / 1)\end{array}$ & $\begin{array}{l}\text { Birn, Kir, } \\
\text { WLi, Ul } \\
(.5 / 1 / 1 / .5) \\
\end{array}$ & $\begin{array}{l}\text { Mb, As, Kie, Vb, Sp, } \\
\text { Rob } \\
(6 x 0.5)\end{array}$ \\
\hline \multirow{4}{*}{$\begin{array}{l}\text { Standorte ARL1 u. ARL2 } \\
\text { Reich. auenart. Lehmböden } \\
\text { grundfeucht bis frisch } \\
\text { PNV: stieleichenreicher } \\
\text { (Roterlen-) Eschenbachwald } \\
\text { (8 pnV-Baumarten) } \\
\text { (4 Szen. mit je um } 2 \text { zunehm. } \\
\text { BA aus der pnV) }\end{array}$} & III-2 & $\begin{array}{l}\text { Es } \\
(6)\end{array}$ & $\begin{array}{l}\text { REr } \\
(4) \\
\end{array}$ & \\
\hline & III-4 & $\begin{array}{l}\text { Es } \\
(5)\end{array}$ & $\begin{array}{l}\text { REr } \\
(3)\end{array}$ & $\begin{array}{l}\text { Ul, } \mathrm{HBu} \\
(1 / 1)\end{array}$ \\
\hline & III-6 & $\begin{array}{l}\text { Es } \\
(4)\end{array}$ & $\begin{array}{l}\text { REr, SEi } \\
(2 / 1)\end{array}$ & $\begin{array}{l}\text { Ul, HBu, WLi } \\
(1 / 1 / 1)\end{array}$ \\
\hline & III-8 & $\begin{array}{l}\text { Es } \\
(4)\end{array}$ & $\begin{array}{l}\text { REr, SEi } \\
(2 / 1)\end{array}$ & $\begin{array}{l}\text { Ul, } \mathrm{HBu}, \mathrm{WLi}, \mathrm{Ah}, \mathrm{As} \\
(4 \times 0.5 / 1)\end{array}$ \\
\hline
\end{tabular}


Szenarien, erstellt nach den Erstaufforstungszieltypen des Landes Thüringen (EAZT)

\begin{tabular}{|c|c|c|c|c|}
\hline \multirow{3}{*}{$\begin{array}{l}\text { Standort } \\
\text { Standort RLL3 } \\
\text { Reichere Schlufflehm- } \\
\text { Standorte; } \\
\text { PnV: }\end{array}$} & EA-Zieltyp & Hauptb. & Mischb. & Nebenb. \\
\hline & $\begin{array}{c}\text { Nadelbaum- } \\
\text { Laubbaumtyp }\end{array}$ & \begin{tabular}{|l} 
ELä \\
$(5)$
\end{tabular} & $\begin{array}{l}\text { WLi, HBu } \\
(2 / 1)\end{array}$ & $\begin{array}{l}\text { Ah, Sp, Kir, Obst } \\
(4 \times 0.5)\end{array}$ \\
\hline & $\begin{array}{c}\text { Vorwaldtyp } \\
\text { Laub }\end{array}$ & $\begin{array}{l}\text { Eberesche } \\
\text { (10) }\end{array}$ & & \\
\hline \multirow{3}{*}{$\begin{array}{l}\text { edellaubbaumreicher } \\
\text { Linden-Hainbuchen- } \\
\text { Eichenwald } \\
\text { (12 pnV-BA) } \\
\text { ( } 6 \text { Szen. mit je um } 2 \\
\text { zunehmenden Arten } \\
\text { aus der pnV) }\end{array}$} & $\begin{array}{c}\text { Vorwaldtyp } \\
\text { Nadel }\end{array}$ & \begin{tabular}{|l|} 
ELä \\
$(10)$ \\
\end{tabular} & & \\
\hline & $\begin{array}{c}1 . \\
\text { Traubeneichen- } \\
\text { Nadelbaum- } \\
\text { Typ } \\
\end{array}$ & $\begin{array}{l}\text { TEi } \\
(5)\end{array}$ & $\begin{array}{l}\text { ELä } \\
(3)\end{array}$ & $\begin{array}{l}\text { WLi, HBu, Kir, Sp } \\
(4 \times 0.5)\end{array}$ \\
\hline & $\begin{array}{c}2 . \\
\text { Traubeneichen- } \\
\text { Nadelbaumtyp }\end{array}$ & $\begin{array}{r}\text { TEi } \\
(5)\end{array}$ & $\begin{array}{l}\text { Kie } \\
(4)\end{array}$ & $\begin{array}{l}\text { Kir, WLi } \\
(2 \times 0.5)\end{array}$ \\
\hline \multirow{2}{*}{$\begin{array}{l}\text { Standort RC3 } \\
\text { Reicherer } \\
\text { Kabornatgesteins- } \\
\text { Standort, mäßig } \\
\text { trocken } \\
\text { PnV: } \\
\text { edellaubbaumreicher } \\
\text { Linden-Hainbuchen- } \\
\text { Eichenwald } \\
\text { (12 pnV-BA) } \\
\text { (6 Szen. mit je um } 2 \\
\text { zunehmenden BA, } \\
\text { davon } 1 \text { aus pnV) }\end{array}$} & Vorwaldtyp & $\begin{array}{l}\text { Eberesche } \\
\text { (10) }\end{array}$ & & \\
\hline & $\begin{array}{l}\text { Traubeneichen- } \\
\text { Nadelbaum- } \\
\text { Typ }\end{array}$ & $\begin{array}{l}\text { TEi } \\
(5)\end{array}$ & $\begin{array}{l}\text { Kie } \\
(4)\end{array}$ & $\begin{array}{l}\text { Kir, WLi } \\
(2 \times 0.5)\end{array}$ \\
\hline \multirow{2}{*}{$\begin{array}{l}\text { Standorte ARL1 u. } \\
\text { ARL2 } \\
\text { Reichere auenartige } \\
\text { Lehm-Böden, } \\
\text { grundfeucht bis frisch } \\
\text { PnV: } \\
\text { stieleichenreicher } \\
\text { (Roterlen-) } \\
\text { Eschenbachwald } \\
\text { (8 pnV-Baumarten) } \\
\text { (4 Szen. mit je um } 2 \\
\text { zunehm. BA aus der } \\
\text { pnV) }\end{array}$} & $\begin{array}{l}\text { 1. Stieleichen- } \\
\text { Mischbaum- } \\
\text { Typ }\end{array}$ & $\begin{array}{l}\text { SEi } \\
(5)\end{array}$ & $\begin{array}{l}\text { REr, Es } \\
(2 / 2)\end{array}$ & $\begin{array}{l}\text { WLi, UI } \\
(2 \times 0.5)\end{array}$ \\
\hline & $\begin{array}{l}\text { 2. Stieleichen- } \\
\text { Mischbaum- } \\
\text { Typ }\end{array}$ & $\begin{array}{l}\text { SEi } \\
(5)\end{array}$ & $\begin{array}{l}\text { WLi, HBu } \\
(2 / 2)\end{array}$ & \begin{tabular}{|l}
$\mathrm{Ul}, \mathrm{Ah}$ \\
$(2 \times 0.5)$
\end{tabular} \\
\hline
\end{tabular}


Anhang 4: Ergebnisschaubilder für das USG Apolda
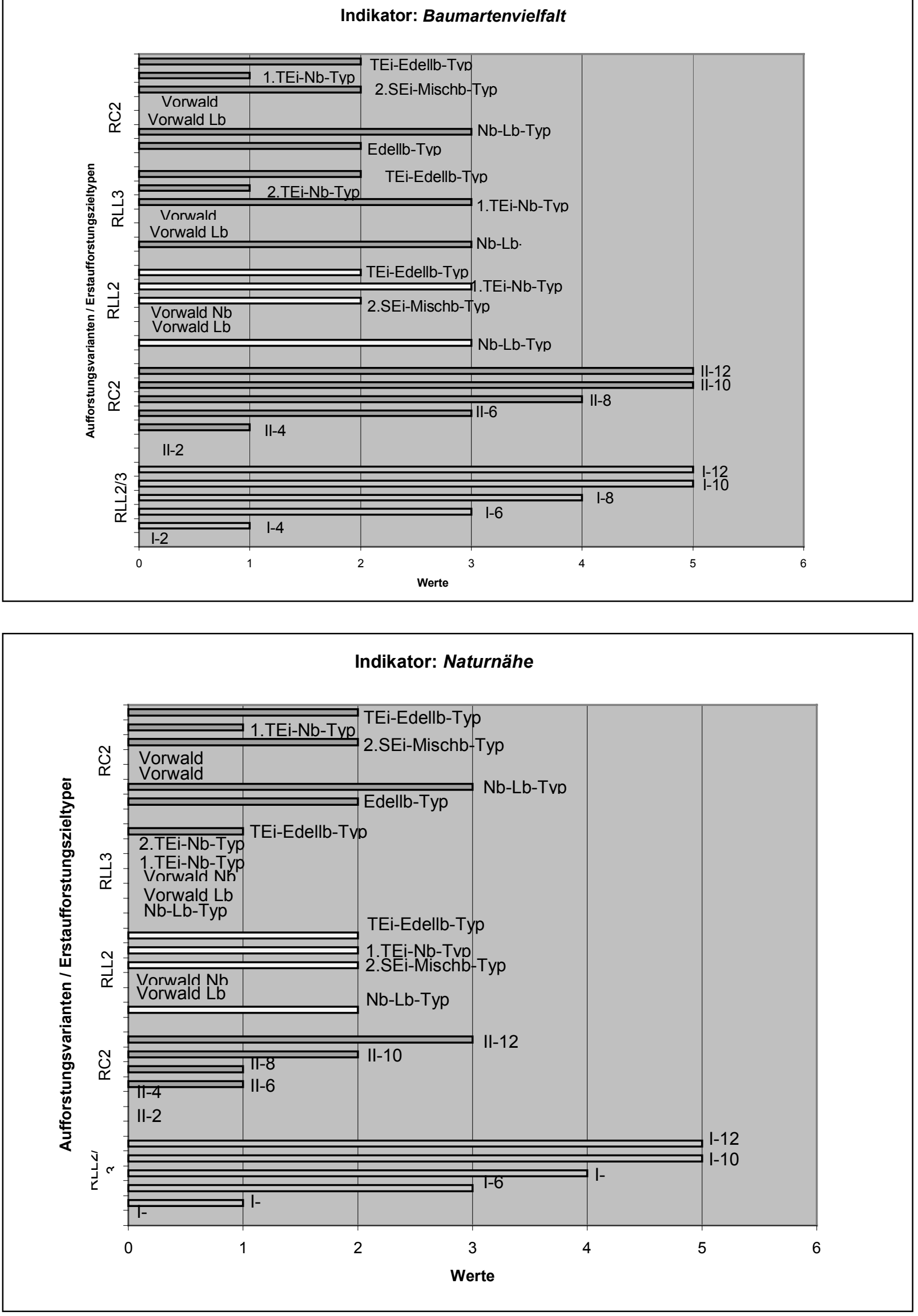


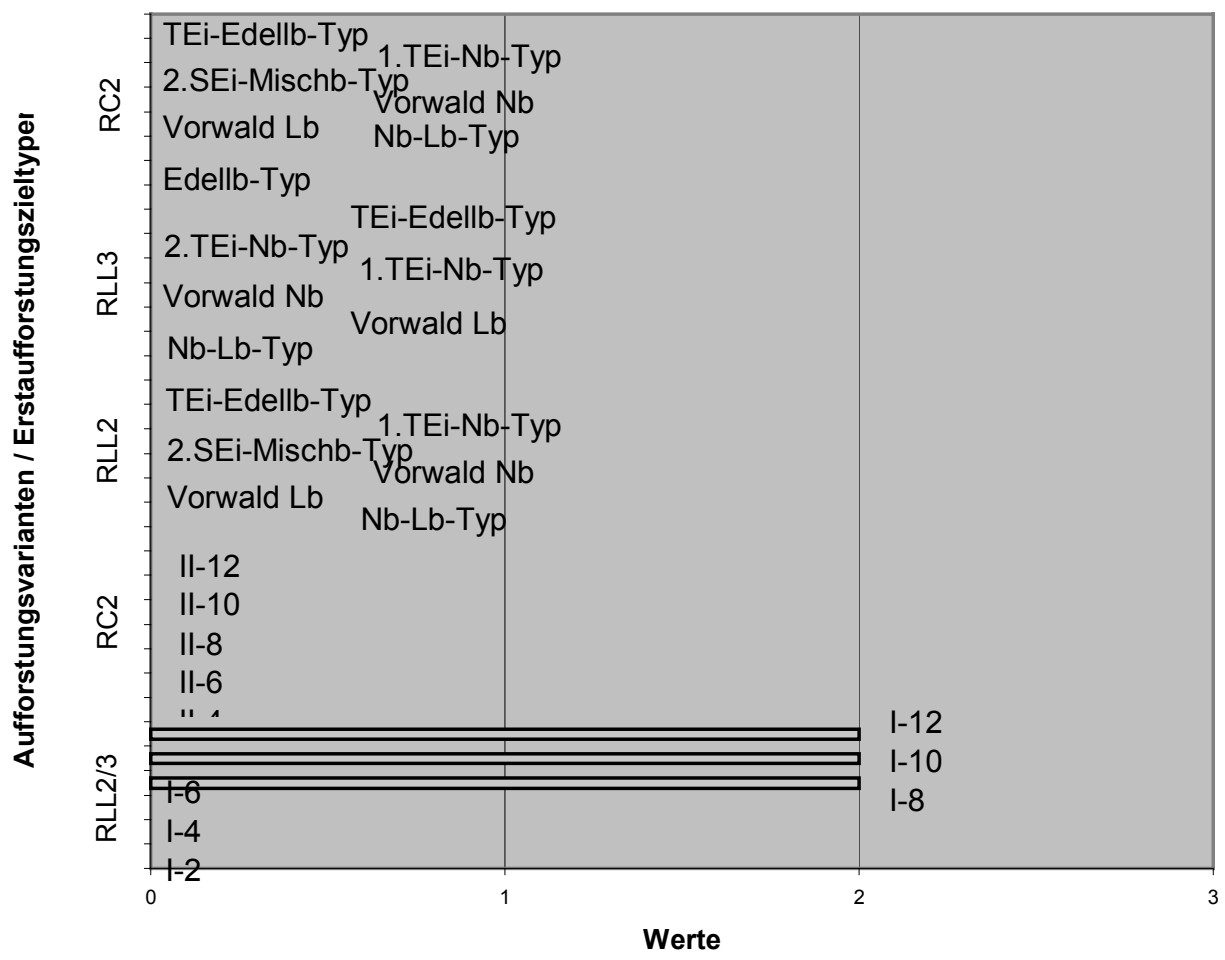

Indikator: Strukturpotential

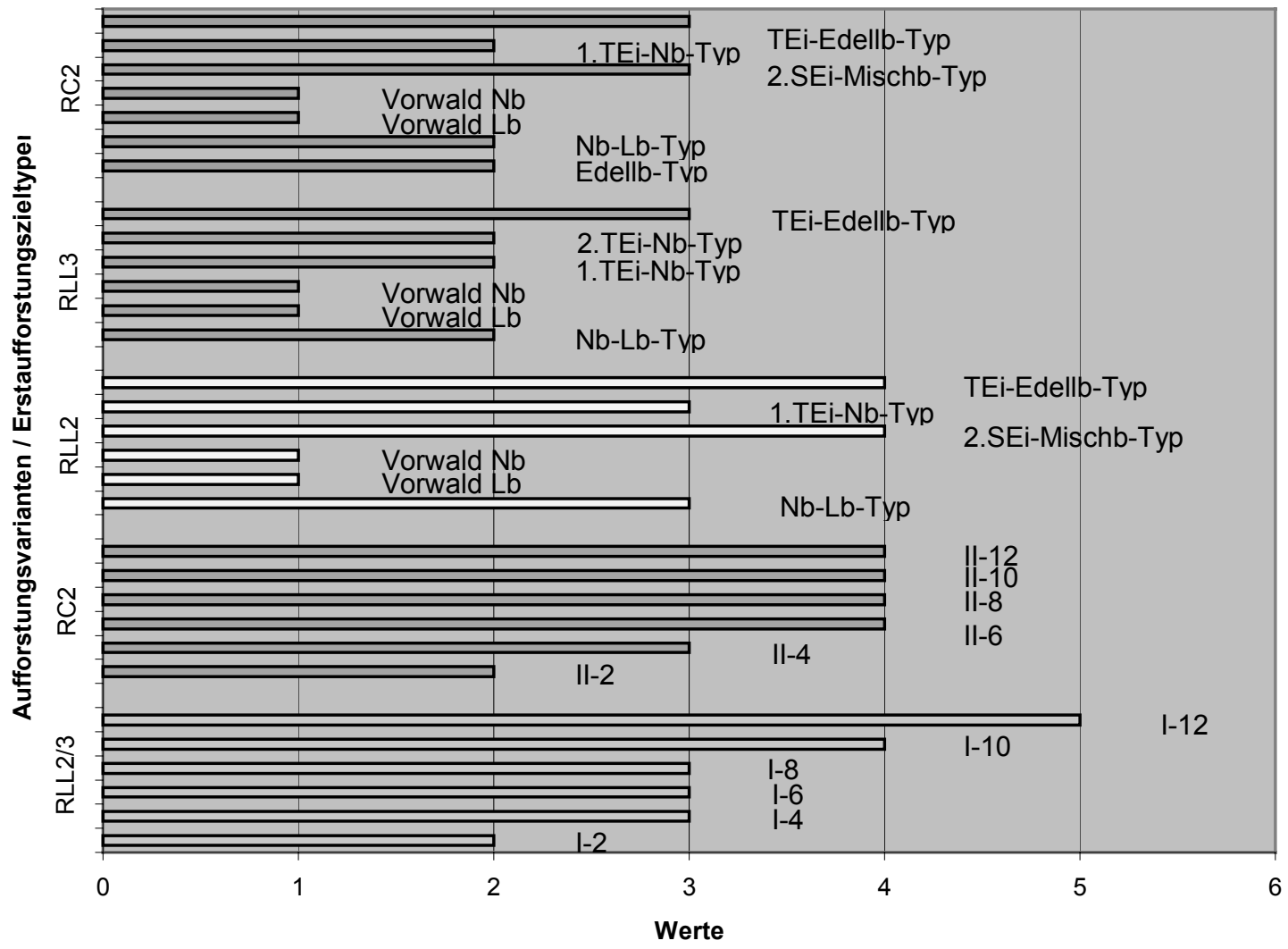




\section{AUFFORSTUNGSFLÄCHE APOLDA-1}

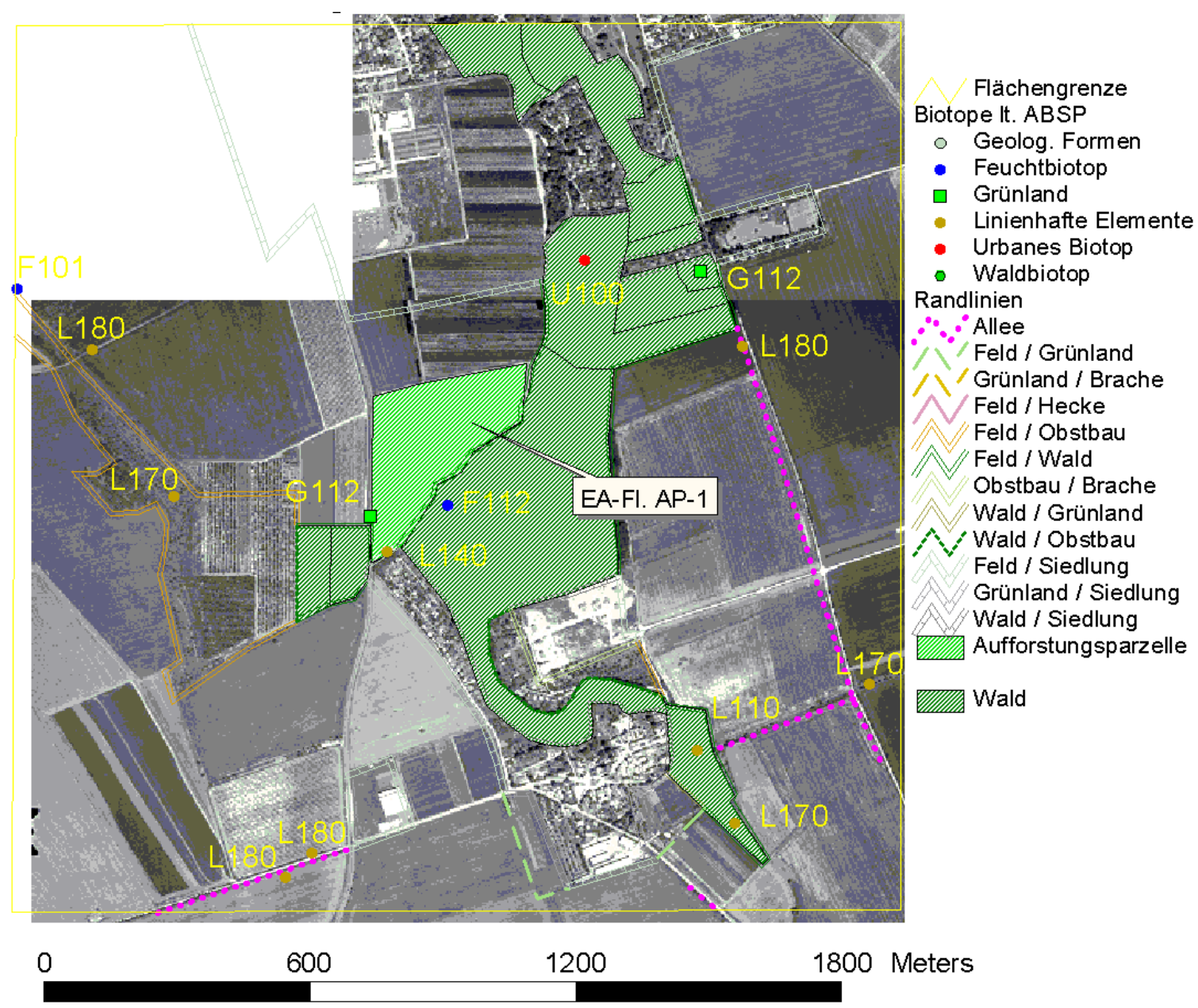

Indikatorsummen für die Aufforstungsfläche AP-1 Standort überwiegend RLL2

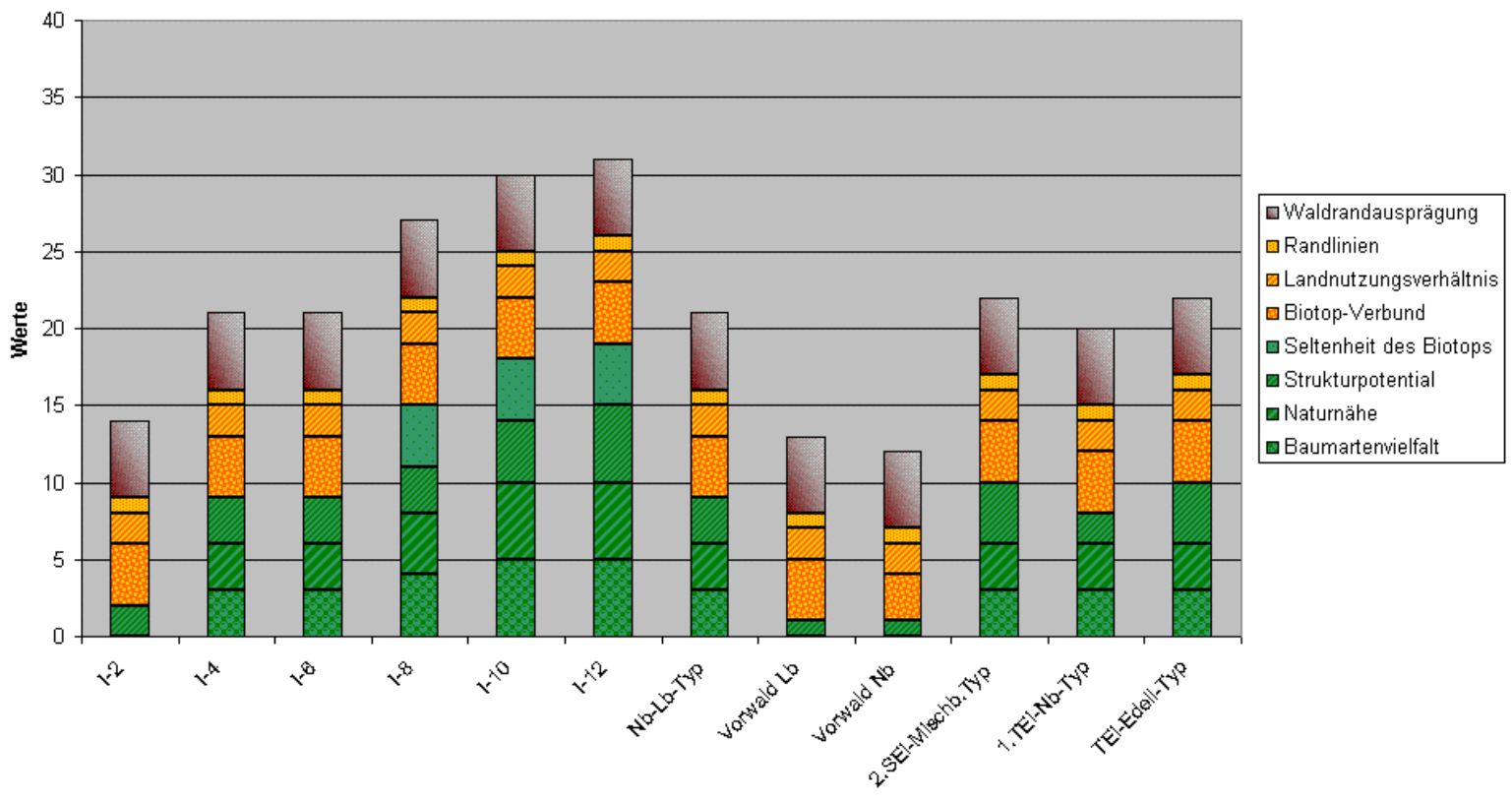

Aufforstungsvariante 


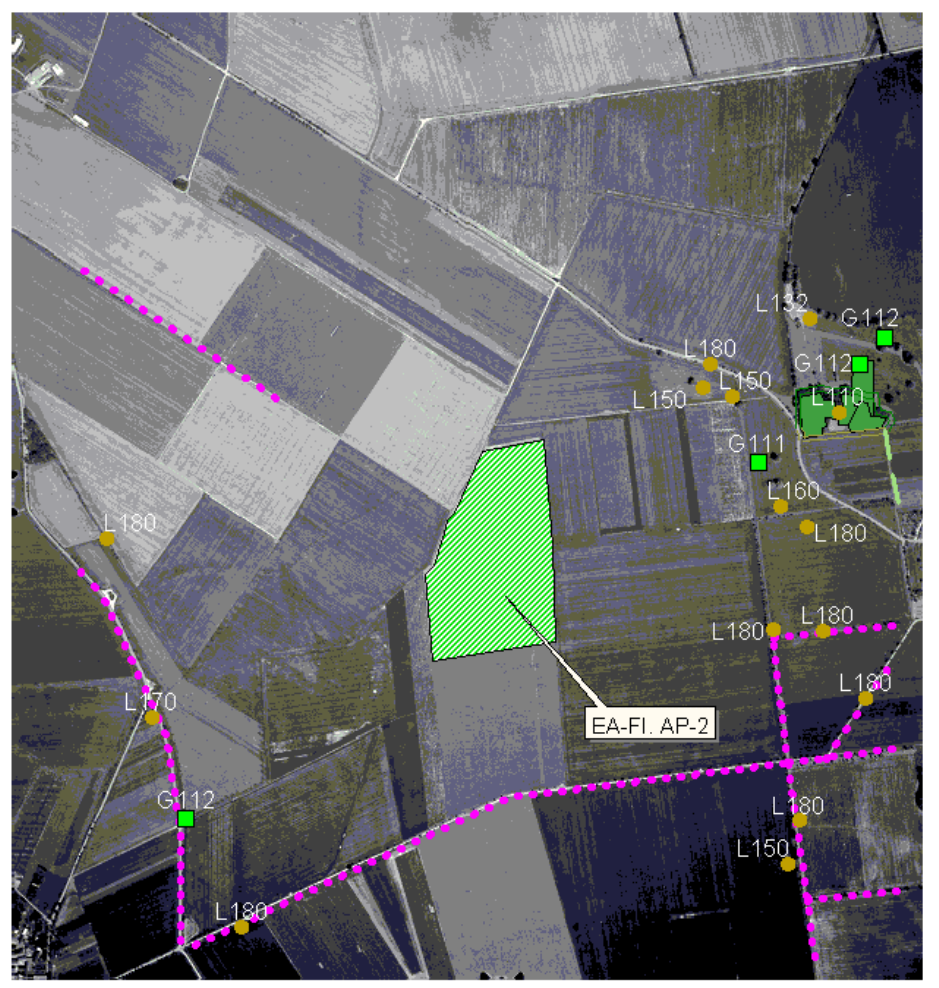

Flächengrenze

Biotope It. ABSP

- Geolog. Formen

- Feuchtbiotop

Grünland

- Linienhafte Elemente

- Urbanes Biotop

- Waldbiotop

Randlinien

$\because \therefore$ Allee

Feld / Grünland

Grünland / Brache

Feld / Hecke

Feld / Obstbau

Feld / Wald

Obstbau / Brache

Wald / Grünland

Wald / Obstbau

Feld / Siedlung

Grünland / Siedlung

Wald / Siedlung

Feldgehölz

Aufforstungsparzelle

\section{Wald}

Indikatorsummen für die Aufforstungsfläche AP-2 Standort RLL3

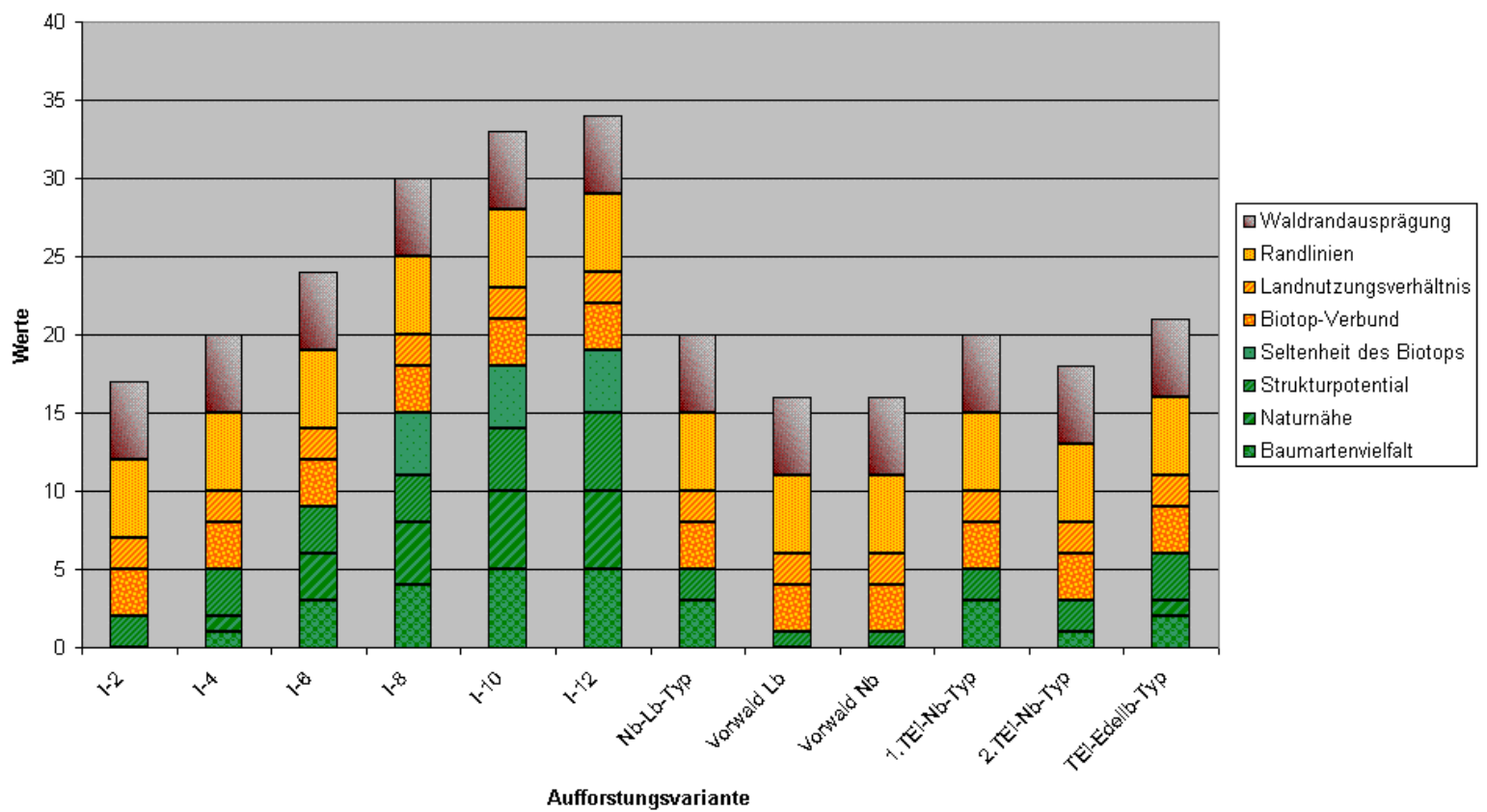




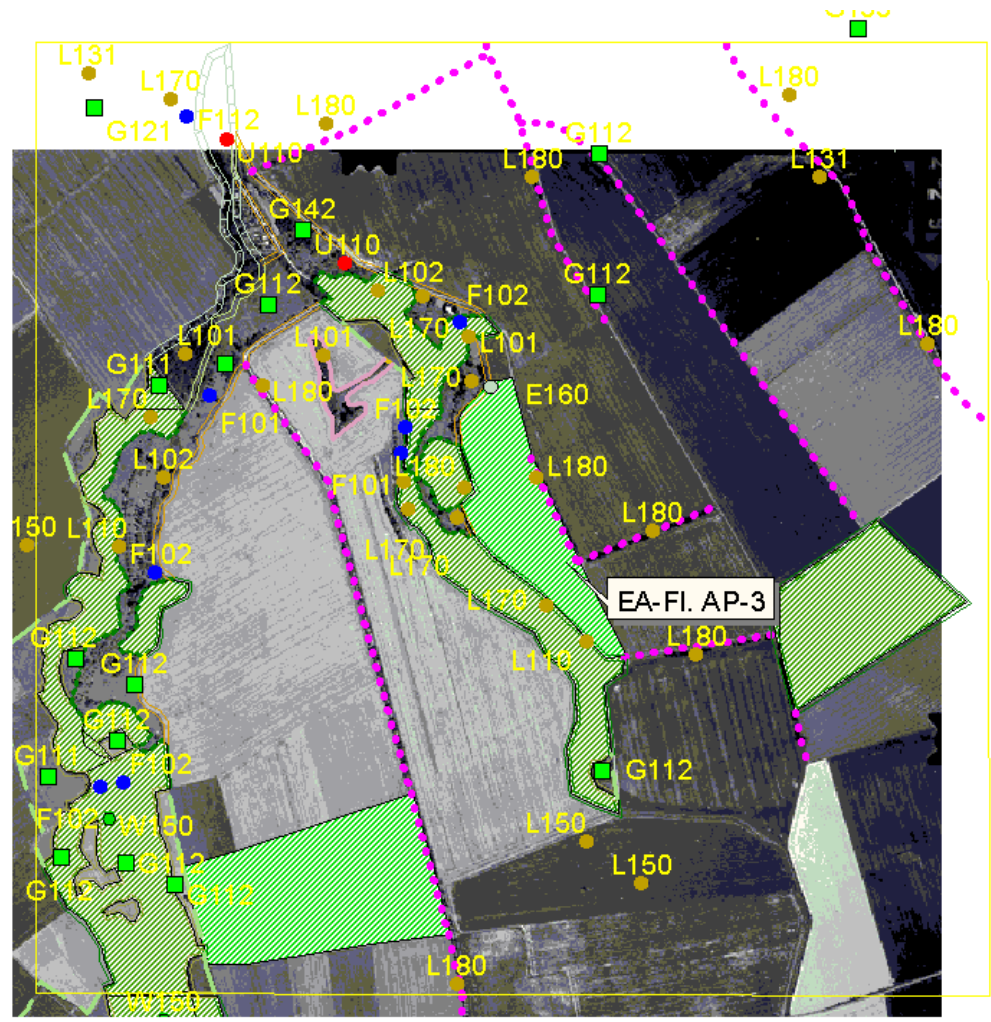

Flächengrenze

Biotope It. ABSP

- Geolog. Formen

- Feuchtbiotop

- Grünland

- Linienhafte Elemente

- Urbanes Biotop

- Waldbiotop

Randlinien

$\therefore$ Allee

$\because \because$ Feld / Grünland

Grünland / Brache

Feld / Hecke

Feld / Obstbau

Feld / Wald

Obstbau / Brache

Wald / Grünland

Wald / Obstbau

Feld / Siedlung

Grünland / Siedlung

Wald / Siedlung

Wald

Aufforstungsparzelle

0

700

1400

2100 Meters

Indikatorsummen für die Aufforstungsfläche AP 3

Standort überwiegend RC2

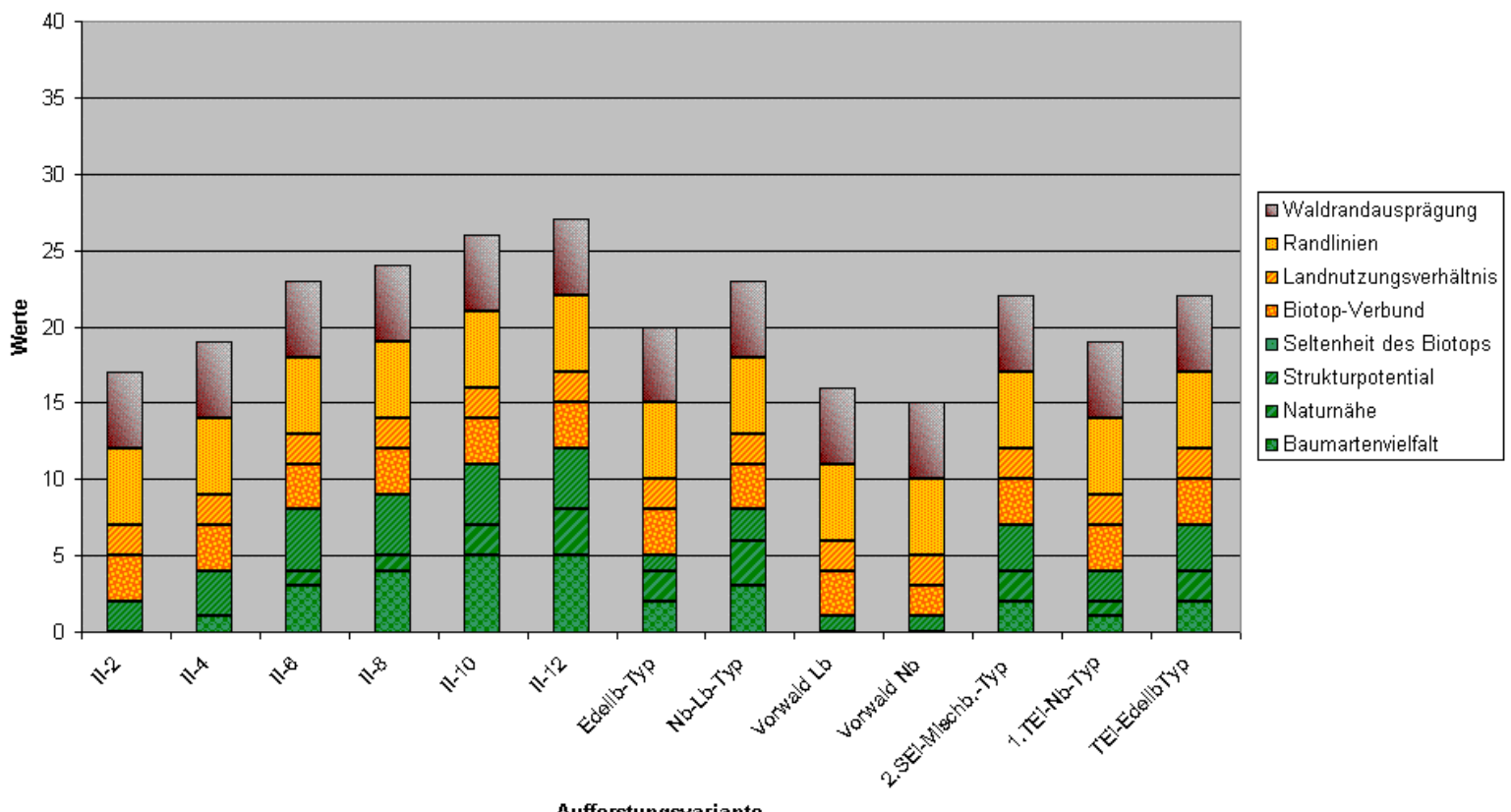

Aufforstungsvariante 


\section{AUFFORSTUNGSFLÄCHE APOLDA-4}

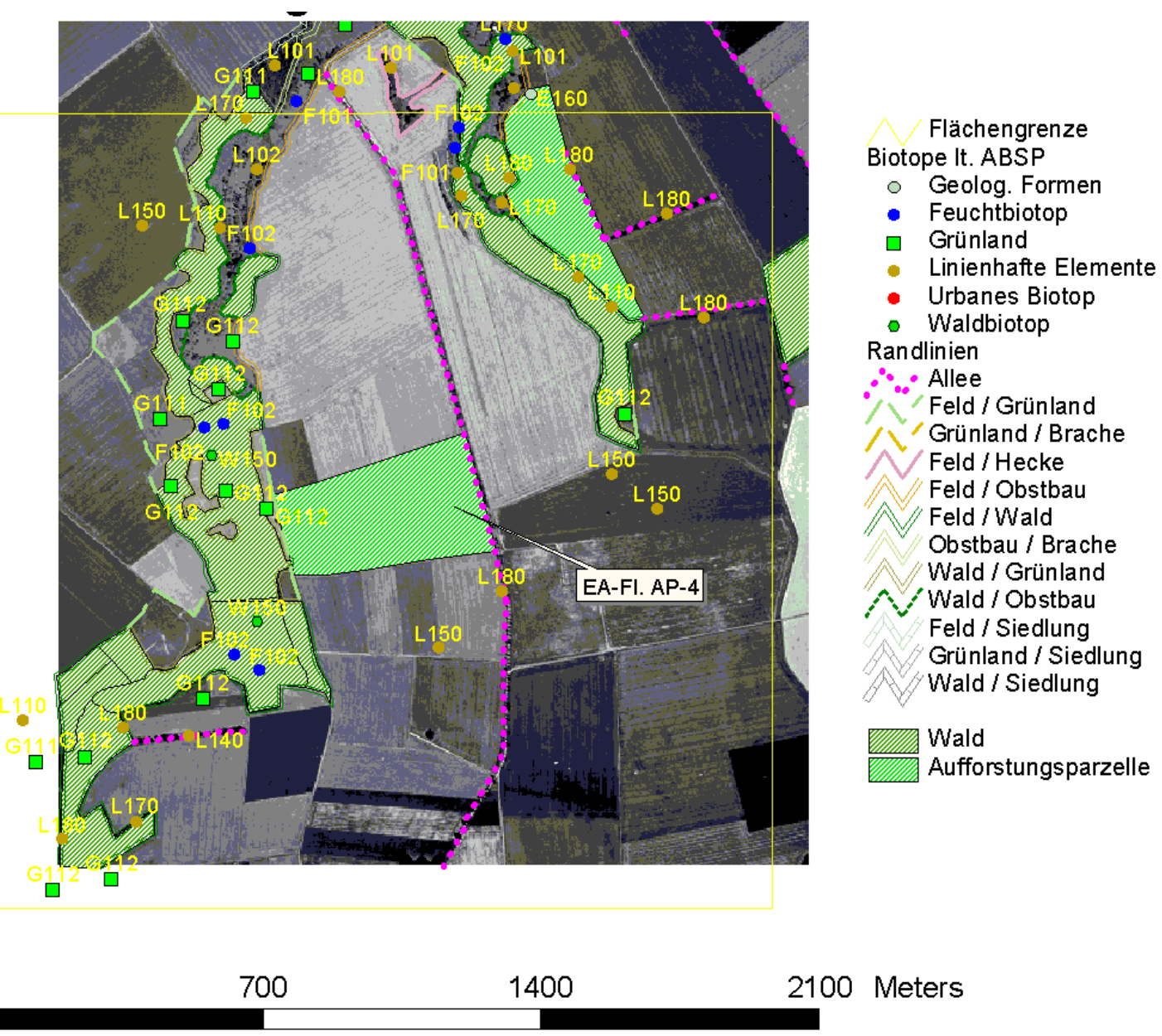

Indikatorsummen für die Aufforstungsfläche AP-4 Standort RLL3

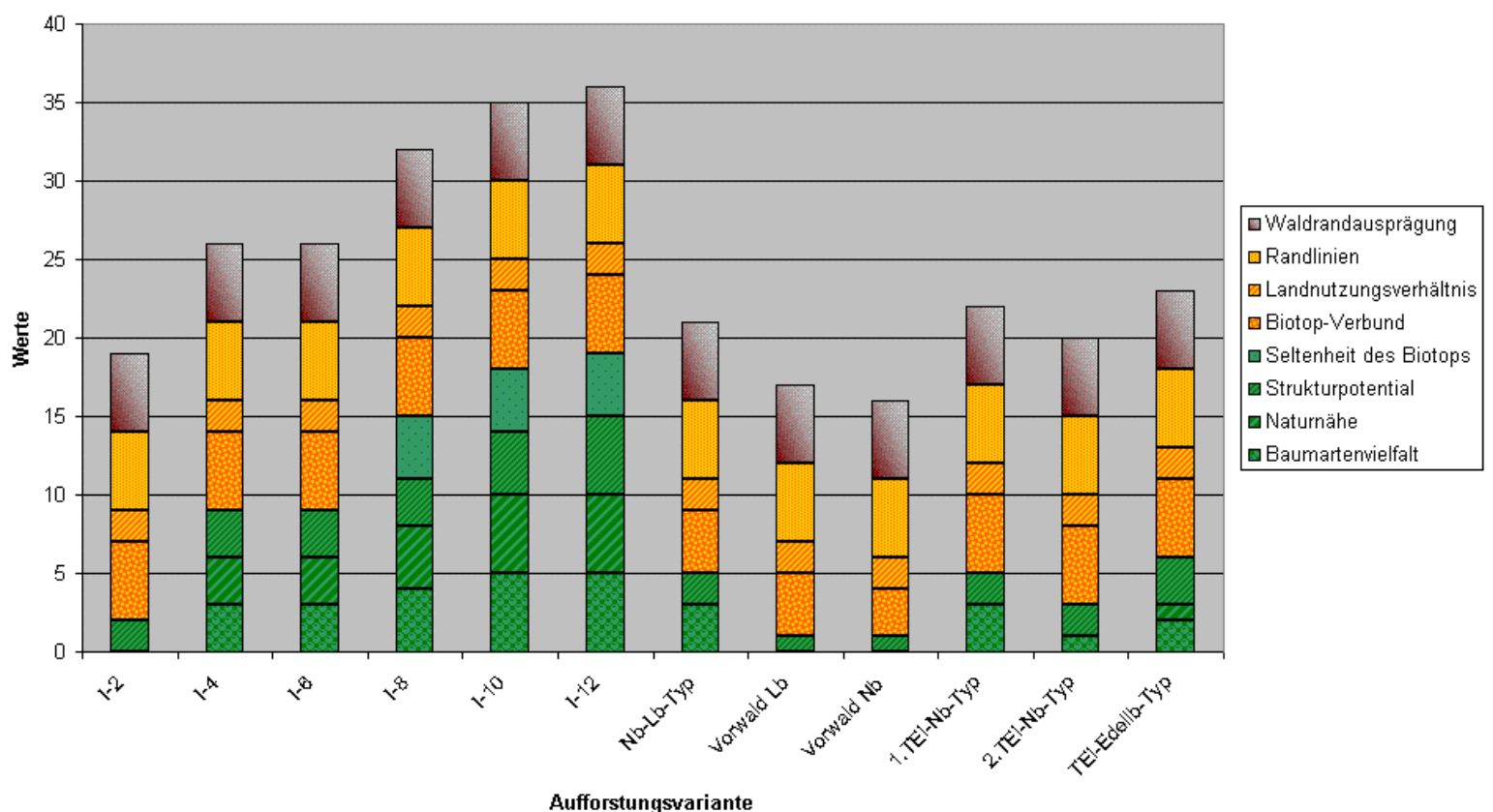




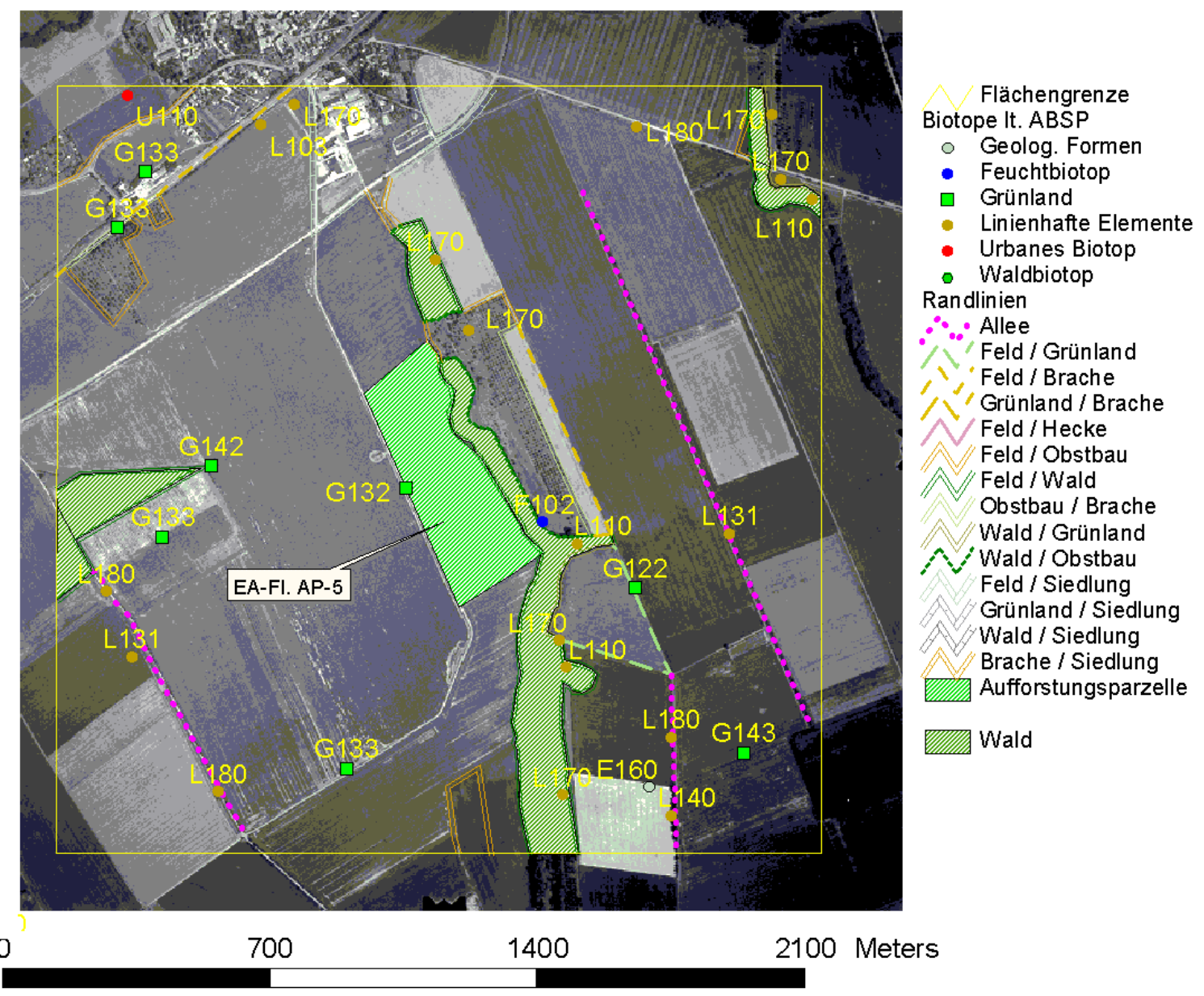

Indikatorsummen für die Aufforstungsfläche AP-5

Standort RLL2

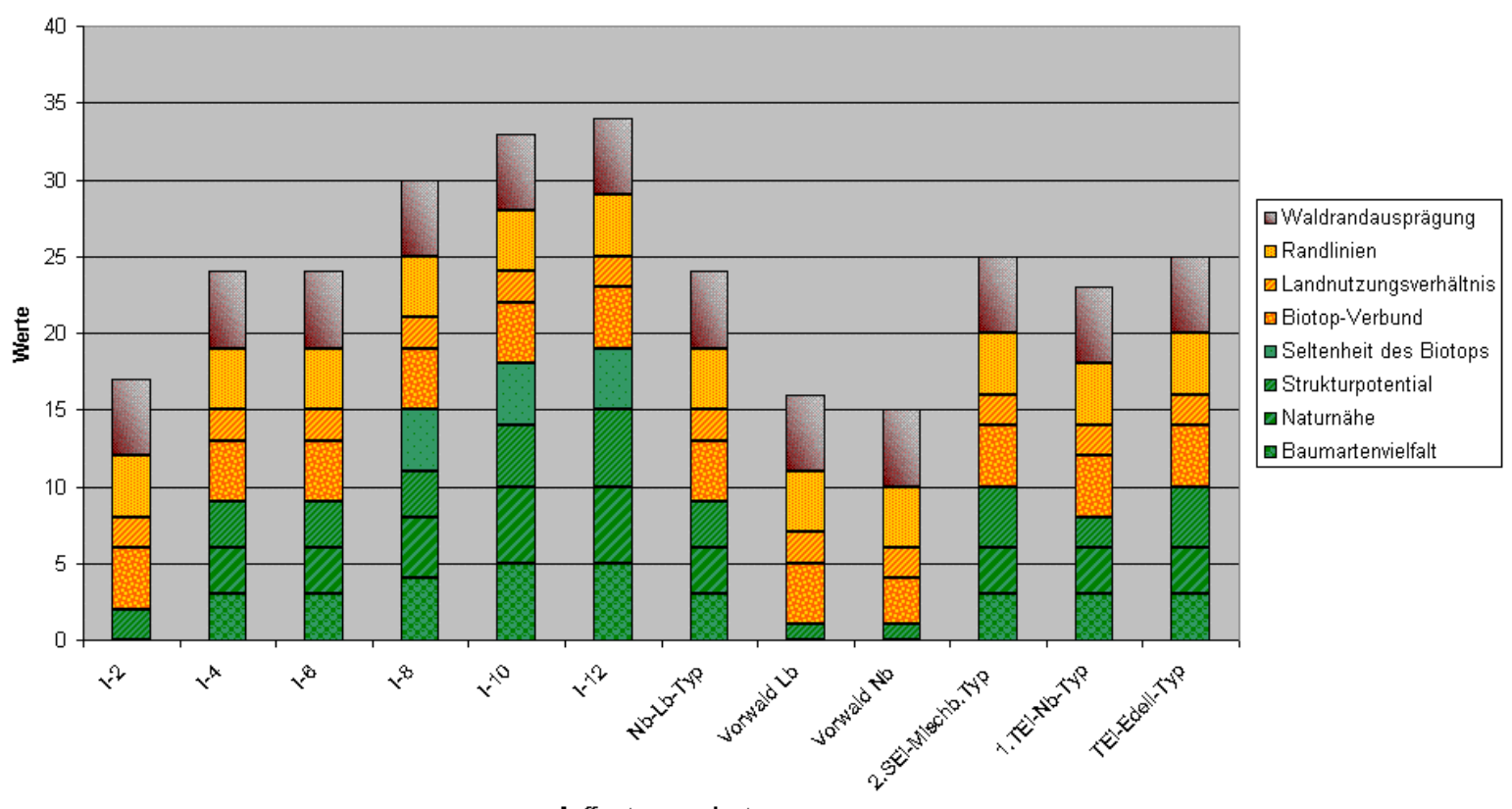

Aufforstungsvariante 
Anhang 5: Grundlagen und Informationen für den Untersuchungsraum Dassel

\section{Die relevanten Waldentwicklungstypen für die Aufforstungsflächen im Untersuchungsraum Dassel}

Der Zuordnung der WET zu den Flächen geht eine Einschätzung von Wasserhaushalts- und Nährstoffzahl anhand den standörtlichen Bedingungen im Gelände, der augenblicklichen Nutzungsart und der Bodenkarte zuvor. Die Ergebnisse dieser Einschätzung werden hier wiedergegeben:

\begin{tabular}{|l|c|c|c|c|c|}
\hline & AF-1 & AF-2 & AF-3 & AF-4 & AF-5 \\
\hline Bodentyp & L3V & L4Vg & TIIIb3 & L3LÖV & LIIIb5 \\
\hline Nutzungsart & Acker & Grünland & Acker/Grünland & Acker & Acker \\
\hline Nährstoffzahl & $5-6$ & $4+-5-$ & $4+-5-$ & $5-6$ & $5-6$ \\
\hline Wasserhaushaltszahl & 9 & 10 & 9 & 9 & 10 \\
\hline
\end{tabular}

Zuordnung der einzelnen WET zu den Aufforstungsflächen anhand der oben ermittelten Daten:

\begin{tabular}{|l|l|l|l|l|l|}
\hline WET & AF-1 & AF-2 & AF-3 & AF-4 & AF-5 \\
\hline 10 & & X & X & & \\
\hline 11 & & & X & & \\
\hline 12 & & & X & & \\
\hline 13 & X & & & X & X \\
\hline 18 & & & X & & \\
\hline 20 & X & X & X & X & X \\
\hline 21 & X & X & X & X & X \\
\hline 22 & X & X & X & X & X \\
\hline 23 & X & & & X & X \\
\hline 25 & & & X & & \\
\hline 26 & & $X$ & X & & \\
\hline 28 & & X & X & & \\
\hline 29 & & & X & & \\
\hline 31 & X & & & X & \\
\hline 33 & & & & & X \\
\hline 35 & X & X & X & X & X \\
\hline 36 & X & X & X & X & X \\
\hline
\end{tabular}

Die wichtigsten WET, also jene die entweder für eine Reihe von Flächen in Frage kommen oder zumindest für einen der betroffenen Standorte von der Quelle (NDS.

FORSTPLANUNGSAMT 2001) als vorrangig angegeben werden (gekennzeichnet durch ein großes $X$ in der Tabelle), werden in der Tabelle auf der folgenden Seite aufgeführt und mit einer bestimmten Baumartenmischung spezifiert. 
Nach zunehmender Vielfalt und Naturnähe vorgeschlagenen Aufforstungsvarianten in Abhängigkeit vom Standort:

\begin{tabular}{|c|c|c|c|}
\hline Standort & Szenario & Haupt-BA & Neben-BA \\
\hline L3V (und L3LöV) & R-2 & $\begin{array}{l}\mathrm{Bu} \\
(6)\end{array}$ & $\begin{array}{l}\mathrm{Ei} \\
(4)\end{array}$ \\
\hline \multirow{3}{*}{$\begin{array}{l}\text { "Kolluvium mittlerer Mächtigkeit, } \\
\text { kalkhaltig; örtl. Rendzina od. Braunerde, } \\
\text { aus umgelagert. Löss über Hangschutt; } \\
\text { schluffig, Lehm üb. steinig., tonig. Lehm" }\end{array}$} & R-4 & $\begin{array}{l}\mathrm{Bu} \\
(6)\end{array}$ & $\begin{array}{l}\text { Ei, BAh, } \mathrm{HBu} \\
(2 / 1 / 1)\end{array}$ \\
\hline & R-6 & $\begin{array}{l}\mathrm{Bu} \\
(6)\end{array}$ & $\begin{array}{l}\text { Ei, BAh, HBu, Aspe, Bi } \\
(2 / .5 / .5 / .5 / .5)\end{array}$ \\
\hline & $\mathrm{R}-8$ & $\begin{array}{l}\mathrm{Bu} \\
(5)\end{array}$ & $\begin{array}{l}\text { Ei, BAh, Es, HBu, Fi Aspe, Bi } \\
(2 / 6 \times 0.5)\end{array}$ \\
\hline $\begin{array}{l}\text { TIllb3 } \\
\text { „Rendzina mittl. Entwicklungstiefe, in } \\
\text { Hanglage, aus Hangschut u. Kalkstein d. } \\
\text { Muschelkalkes; steiniger, toniger Lehm } \\
\text { üb. Kalkstein“ }\end{array}$ & R-10 & $\begin{array}{l}\mathrm{Bu} \\
(4)\end{array}$ & $\begin{array}{l}\text { Ei, BAh, Es, BUI, HBu, Fi, } \\
\text { Eberesche, Aspe, Bi } \\
\text { (1/1/1/6x.5) }\end{array}$ \\
\hline $\begin{array}{l}\text { PNV: Perlgras-Buchenwald } \\
\text { (11 PNV-Baumarten) } \\
\text { (6 Szenarien mit jeweils } 2 \text { ( zuletzt um 1) } \\
\text { zunehmende Baumarten aus der PNV) }\end{array}$ & R-11 & $\begin{array}{l}\mathrm{Bu} \\
(3)\end{array}$ & $\begin{array}{l}\text { Ei, BAh, Es, BUI, HBu, Fi, Ta, } \\
\text { Eberesche, Aspe, Bi } \\
(4 \times 1,6 \times 0.5)\end{array}$ \\
\hline \multirow{3}{*}{$\begin{array}{l}\text { Llllb5 } \\
\text { „Rendzina, sehr flachgründig, in schwach } \\
\text { geneigt. Hanglagen; aus Kalkstein“ }\end{array}$} & S-2 & $\begin{array}{l}\mathrm{Bu} \\
(8)\end{array}$ & $\begin{array}{l}\text { Ah } \\
(2)\end{array}$ \\
\hline & S-4 & $\begin{array}{l}\mathrm{Bu} \\
(6)\end{array}$ & $\begin{array}{l}\text { Ah, Es, FAh } \\
(2 / 1 / 1)\end{array}$ \\
\hline & S-6 & $\begin{array}{l}\mathrm{Bu} \\
(5)\end{array}$ & $\begin{array}{l}\text { Ah, Es, TEi, Wildobst, FAh } \\
(5 \times 1)\end{array}$ \\
\hline \multirow{2}{*}{$\begin{array}{l}\text { PNV: Seggen-Buchenwald } \\
\text { (10 PNV- Baumarten) } \\
\text { (5 Szenarien mit je um } 2 \text { zunehmenden } \\
\text { Baumarten aus der PNV) }\end{array}$} & S-8 & $\begin{array}{l}\mathrm{Bu} \\
(5)\end{array}$ & $\begin{array}{l}\text { Ah, Es, BUI, TEi, Wildobst, FAh, } \\
\text { Mehlbeere } \\
(1 / 1 / 1 / 4 \times 0.5)\end{array}$ \\
\hline & S-10 & $\begin{array}{l}\mathrm{Bu} \\
\text { (4) }\end{array}$ & $\begin{array}{l}\text { Ah, Es, BUI, SLi, TEi, Wildobst, } \\
\text { Eibe, FAh, Mehlbeere } \\
(1 / 8 \times 0.5)\end{array}$ \\
\hline \multirow{4}{*}{$\begin{array}{l}\text { L4Vg } \\
\text { "Pelosol mittl. Entwicklungstiefe, meist } \\
\text { schwach verbraunt, in unterer Hanglage; } \\
\text { aus Tonstein des ob. BuSa oder Keupers } \\
\text { mit gering. Hangschuttdecke; schluffiger } \\
\text { Ton über Tonstein“" }\end{array}$} & $\mathrm{H}-2$ & $\begin{array}{c}\mathrm{Bu}, \mathrm{TEi} \\
(6 / 4)\end{array}$ & \\
\hline & $\mathrm{H}-4$ & $\begin{array}{l}\mathrm{Bu}, \mathrm{TEi} \\
(4 / 4)\end{array}$ & $\begin{array}{l}\mathrm{HBu}, \mathrm{Li} \\
(1 / 1)\end{array}$ \\
\hline & $\mathrm{H}-6$ & $\begin{array}{l}\mathrm{Bu}, \mathrm{TEi} \\
(4 / 3)\end{array}$ & $\begin{array}{l}\mathrm{HBu}, \mathrm{Li}, \mathrm{Bi}, \text { Eberesche } \\
(1 / 1 / .5 / .5)\end{array}$ \\
\hline & $\mathrm{H}-8$ & $\begin{array}{l}\mathrm{Bu}, \mathrm{TEi} \\
(4 / 2)\end{array}$ & $\begin{array}{l}\mathrm{HBu}, \mathrm{Ah}, \mathrm{Li}, \mathrm{Kie}, \mathrm{Bi} \text {, Eberesche } \\
(1 / 1 / 4 \times 0.5)\end{array}$ \\
\hline $\begin{array}{l}\text { PNV: Hainsimsen-Buchenwald } \\
\text { (10 PNV_Baumarten) } \\
\text { (5 Szenarien, um je } 2 \text { Baumarten aus der } \\
\text { PNV zunehmend) }\end{array}$ & $\mathrm{H} 10$ & $\begin{array}{l}\mathrm{Bu}, \mathrm{TEi} \\
(4 / 1)\end{array}$ & $\begin{array}{l}\mathrm{HBu}, \mathrm{Ah}, \mathrm{Li}, \mathrm{Fi}, \mathrm{Ta}, \mathrm{Kie}, \mathrm{Bi} \text {, } \\
\text { Eberesche } \\
(1 / 1 / 6 \times 0.5)\end{array}$ \\
\hline
\end{tabular}


Die für die Aufforstungsflächen im Untersuchungsraum Dassel relevanten Waldentwicklungstypen (WET) ergeben die Aufforstungstypen für das Variantenstudium:

\begin{tabular}{|c|c|c|c|c|}
\hline WET & Haupt-BA & Misch-BA & Neben-BA & $\begin{array}{c}\text { BA- } \\
\text { Anzahl }\end{array}$ \\
\hline $\begin{array}{c}20 \\
\text { Buche }\end{array}$ & $\begin{array}{l}\mathrm{Bu} \\
\text { (8) }\end{array}$ & $\begin{array}{l}\text { Ah } \\
\text { (1) }\end{array}$ & $\begin{array}{c}\text { Eberesche, Kie } \\
(.5 / .5)\end{array}$ & 4 \\
\hline $\begin{array}{c}21 \\
\text { Buche- } \\
\text { Traubeneiche }\end{array}$ & $\begin{array}{l}\mathrm{Bu}, \mathrm{TEi} \\
(5 / 4)\end{array}$ & & $\begin{array}{l}\text { Ah, Mehlb } \\
(.5 / .5)\end{array}$ & 4 \\
\hline $\begin{array}{c}22 \\
\text { Buche- } \\
\text { Bergahorn- } \\
\text { Wildkirsche }\end{array}$ & $\begin{array}{c}\text { Bu, BAh, Kir } \\
(6 / 2 / 1)\end{array}$ & & $\begin{array}{l}\mathrm{UI}, \mathrm{HBu} \\
(.5 / .5)\end{array}$ & 5 \\
\hline $\begin{array}{c}35 \\
\text { Linde-Laubbäume }\end{array}$ & $\begin{array}{l}\mathrm{Li} \\
(5)\end{array}$ & $\begin{array}{c}\mathrm{Bu}, \mathrm{HBu}, \mathrm{Ei}, \mathrm{BAh} \\
(1 / 1 / 1 / 1)\end{array}$ & $\begin{array}{c}\text { FAh, Kie } \\
(.5 / .5)\end{array}$ & 7 \\
\hline $\begin{array}{c}36 \\
\text { Wildkirsche- } \\
\text { Hainbuche }\end{array}$ & $\begin{array}{l}\text { Kir, Elsb } \\
(7 / 2)\end{array}$ & & $\begin{array}{l}\mathrm{HBu}, \mathrm{Li} \\
(.5 / .5)\end{array}$ & 4 \\
\hline $\begin{array}{c}23 \\
\text { Buche- } \\
\text { Edellaubbäume }\end{array}$ & $\begin{array}{c}\mathrm{Bu}, \mathrm{BAh}, \mathrm{Es}, \mathrm{BUI} \\
(5 / 2 / 1 / 1)\end{array}$ & & $\begin{array}{c}\mathrm{HBu} \text {, Eberesche } \\
(.5 / .5)\end{array}$ & 6 \\
\hline $\begin{array}{c}31 \\
\text { Edellaubbäume, } \\
\text { frischer Typ }\end{array}$ & $\begin{array}{c}\text { Es, BAh, BUI, TEi } \\
(3 / 2 / 1 / 1)\end{array}$ & $\begin{array}{c}\mathrm{Bu}, \mathrm{HBu} \\
(1 / 1)\end{array}$ & $\begin{array}{c}\text { Eberesche, Birke } \\
(.5 / .5)\end{array}$ & 8 \\
\hline $\begin{array}{c}33 \\
\text { Edellaubbäume, } \\
\text { trockener Typ }\end{array}$ & $\begin{array}{c}\text { Es, SLi, Elsb, Ah, Kir } \\
(2 / 2 / 1 / 1 / 1)\end{array}$ & $\begin{array}{c}\mathrm{Bu}, \mathrm{HBu} \\
(1 / 1)\end{array}$ & $\begin{array}{c}\text { Eberesche, } \mathrm{Fi} \\
(.5 / .5)\end{array}$ & 9 \\
\hline $\begin{array}{c}10 \\
\text { Traubeneiche- } \\
\text { Buche }\end{array}$ & $\begin{array}{l}\text { TEi, Bu } \\
(7 / 2)\end{array}$ & & $\begin{array}{l}\text { Ah, Li } \\
(.5 / .5)\end{array}$ & 4 \\
\hline
\end{tabular}

Bei der Festlegung der Baumartenmischungen wurde für die Haupt- und Nebenbaumarten jeweils ein mittlerer Anteil angenommen. Sieht der WET 10-Traubeneiche-Buche beispielsweise einen Anteil von Traubeneiche zwischen $60 \%$ und $80 \%$, sowie von Buche zwischen $10 \%$ und $30 \%$ vor, so wurde die Aufforstungsvariante mit einem Anteil von $70 \%$ TEi und $20 \%$ Buche (Rest: Nebenbaumarten) bestimmt. Soweit die Misch- und Nebenbaumarten nicht durch den WET-Schlüssel vorgegeben waren, wurden Mischbaumarten mit einem Anteil von je $10 \%$ und Nebenbaumarten mit einem Anteil von je $5 \%$ bestimmt. Die Auswahl orientierte sich nur bedingt an der PNV, da die Varianten für unterschiedliche Standortsverhältnisse anzuwenden waren. 
Anhang 6: Ergebnisschaubilder für das Untersuchungsgebiet Dassel

Die folgenden Diagramme zeigen die Ergebnisse der Bewertung für die flächenbezogenen Indikatoren Baumartenvielfalt, Naturnähe, Strukturpotential und Seltenheit des Biotops.

Bei diesen Diagrammen steht der Vergleich zwischen den Standorten und den Varianten in Bezug auf den jeweiligen Indikator im Vordergrund. Aus Gründen der Übersichtlichkeit wurde auf eine Beschriftung der einzelnen Balken verzichtet. Die Einzeldaten sind aus den anschließend präsentierten Diagrammen mit den gesamten Indikatorwerten für die jeweilige Aufforstungsfläche zu entnehmen, oder dem Ergebnisteil der Arbeit.

Die Baumarten der einzelnen Aufforstungsvarianten sind in Anhang 5 aufgeschlüsselt.

Für die folgenden Diagramme stehen die Balken von oben nach unten in folgender Reihenfolge für die jeweiligen WET bzw. EAV:

\begin{tabular}{|c|c|c|}
\hline Standort & WET/EAV & Kürzel \\
\hline \multirow{7}{*}{ LIIIb5 } & \multirow{7}{*}{ WET } & 36 \\
\hline & & 35 \\
\hline & & 31 \\
\hline & & 23 \\
\hline & & 22 \\
\hline & & 21 \\
\hline & & 20 \\
\hline \multirow{7}{*}{ L3LöV } & \multirow{7}{*}{ WET } & 36 \\
\hline & & 35 \\
\hline & & 31 \\
\hline & & 23 \\
\hline & & 22 \\
\hline & & 21 \\
\hline & & 20 \\
\hline \multirow{6}{*}{ TIIIb3 } & \multirow{6}{*}{ WET } & 36 \\
\hline & & 35 \\
\hline & & 22 \\
\hline & & 21 \\
\hline & & 20 \\
\hline & & 10 \\
\hline \multirow{6}{*}{ L4Vg } & \multirow{6}{*}{ WET } & 36 \\
\hline & & 35 \\
\hline & & 22 \\
\hline & & 21 \\
\hline & & 20 \\
\hline & & 10 \\
\hline \multirow{7}{*}{ L3V } & \multirow{7}{*}{ WET } & 36 \\
\hline & & 35 \\
\hline & & 31 \\
\hline & & 23 \\
\hline & & 22 \\
\hline & & 21 \\
\hline & & 20 \\
\hline \multirow{5}{*}{ LIIIb5 } & \multirow{5}{*}{ EAV } & S-10 \\
\hline & & S-8 \\
\hline & & S-6 \\
\hline & & S-4 \\
\hline & & S-2 \\
\hline \multirow{5}{*}{ L4Vg } & \multirow{5}{*}{ EAV } & $\mathrm{H}-10$ \\
\hline & & $\mathrm{H}-8$ \\
\hline & & $\mathrm{H}-6$ \\
\hline & & $\mathrm{H}-4$ \\
\hline & & $\mathrm{H}-2$ \\
\hline \multirow{6}{*}{$\begin{array}{l}\text { L3LöV I } \\
\text { TIIIb3 }\end{array}$} & \multirow{6}{*}{ EAV } & R-11 \\
\hline & & $\mathrm{R}-10$ \\
\hline & & R-8 \\
\hline & & R-6 \\
\hline & & R-4 \\
\hline & & R-2 \\
\hline
\end{tabular}



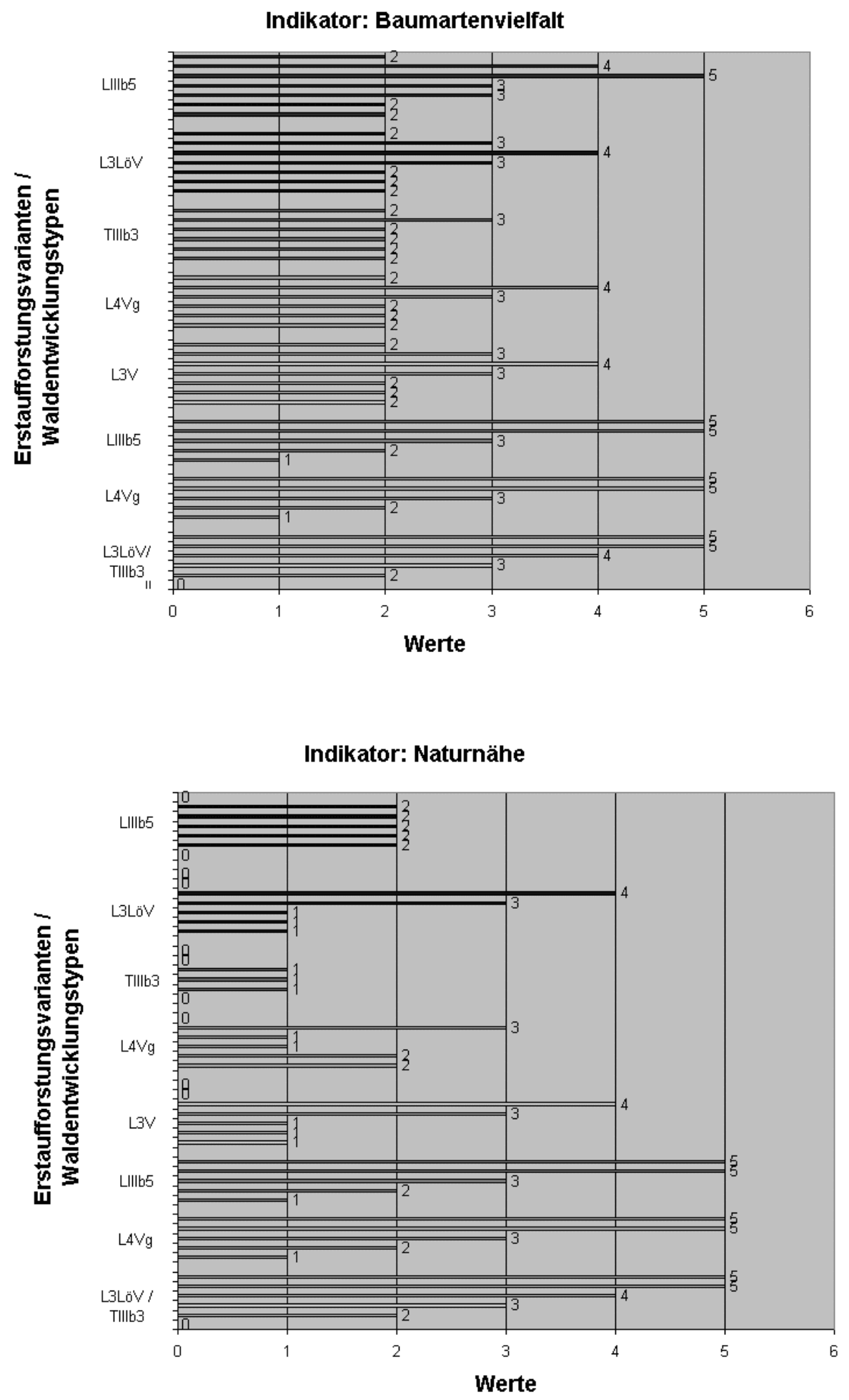

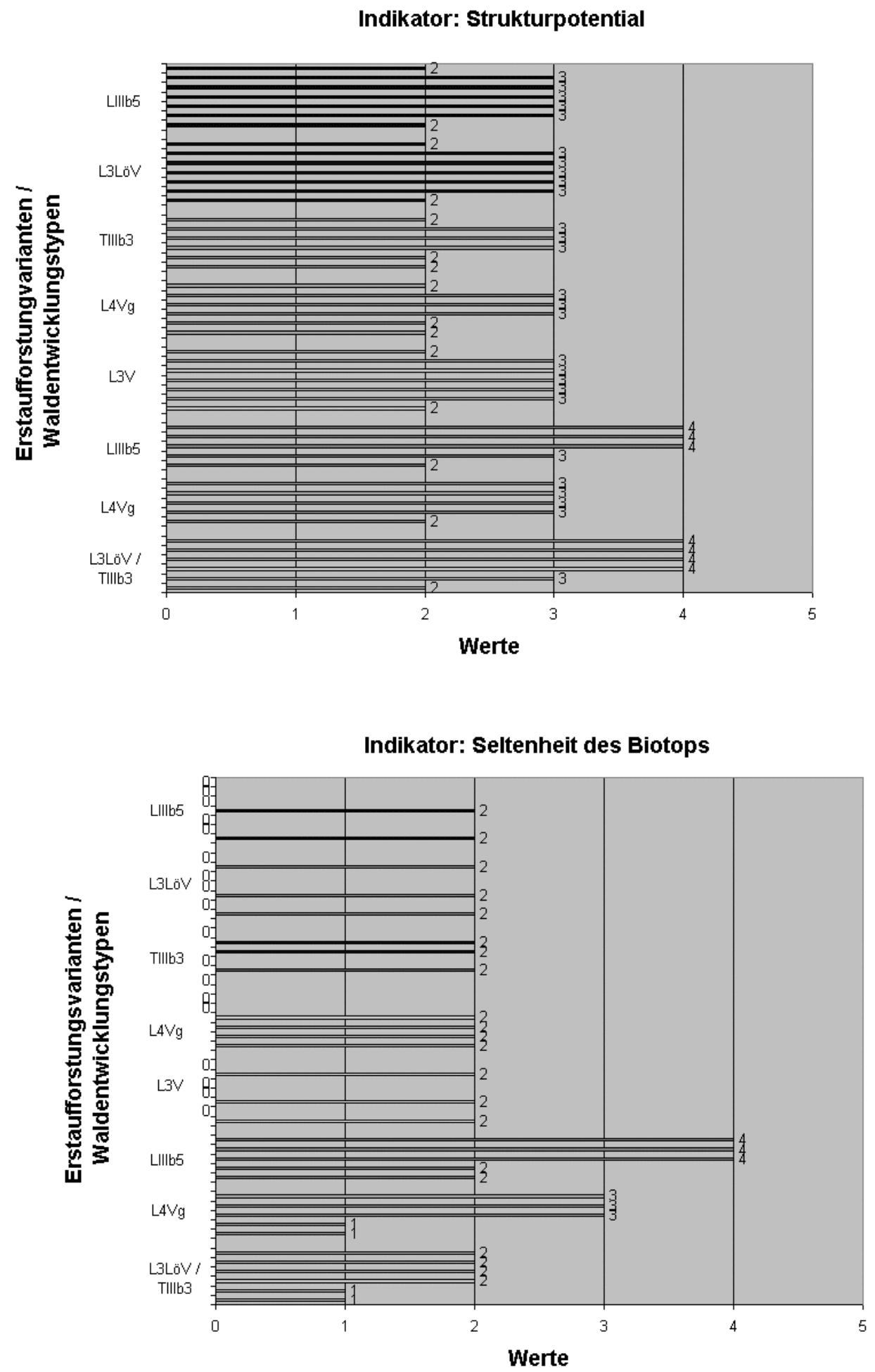


\section{AUFFORSTUNGSFLÄCHE DASSEL-1}

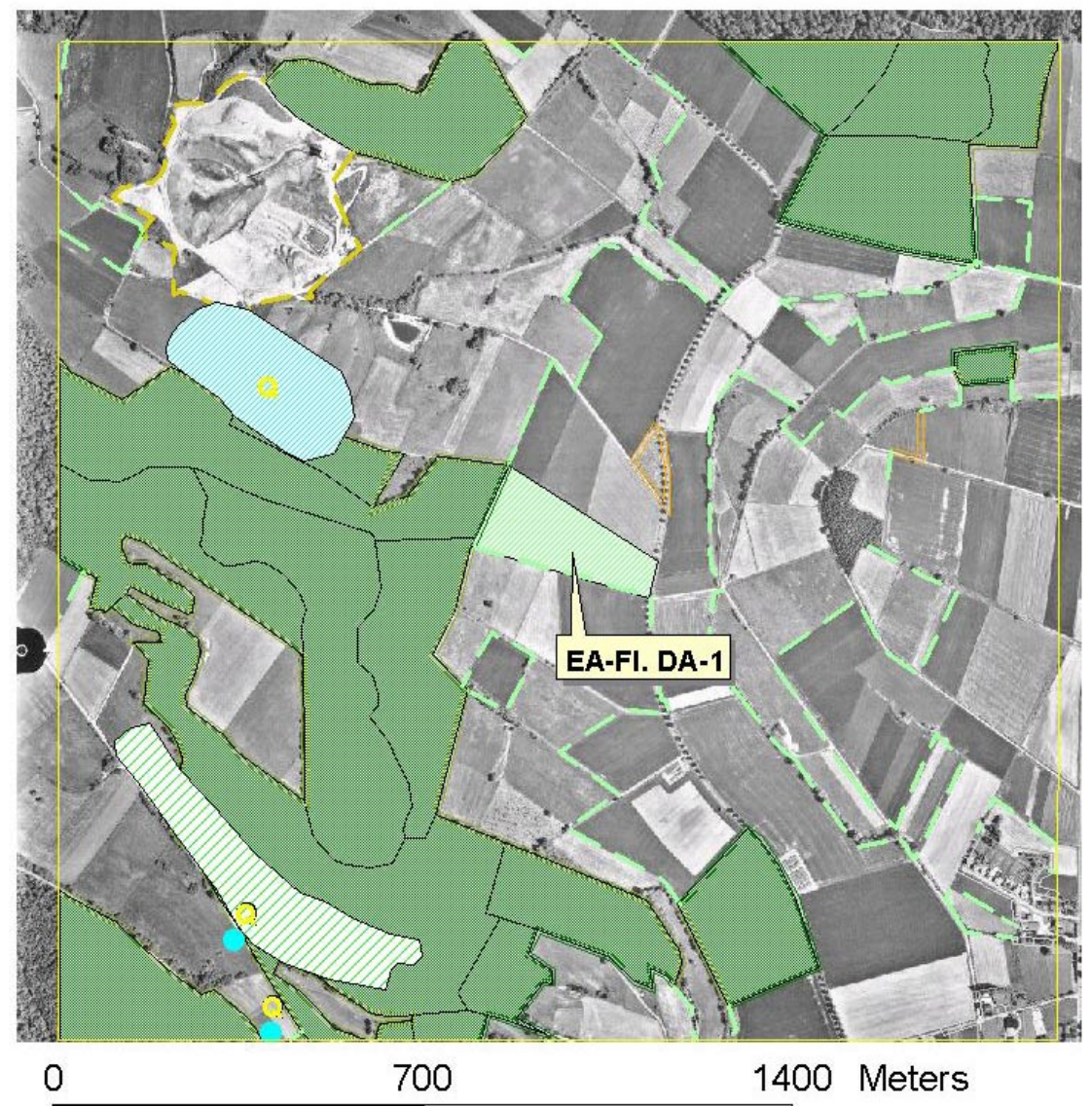

Flächengrenze

Flächenbiotope

D. Gewässer

27 Grünland

Kulturdenkma

Quelle

Z7] Wald

Punktbiotope

$\checkmark$ Geologisch

- Feuchtbiotop

- Trockenrasen

Q Quellbereich

- Wald

Randlinien

$\therefore$ Feld / Grünland

6eld/Brache

/ Grünland / Brache

/ Feld / Hecke

Feld / Obstbau

N/Feld/Wald

Obstbau / Brache

N/Wald/Grünland

A Wald/Obstbau

Feld/Siedlung

Grünland/Siedlung

N/Wald/Siedlung

Brache / Siedlung

Aufforstungsparzelle

Wald

Indikatorsummen für die Aufforstungsfläche DA-1

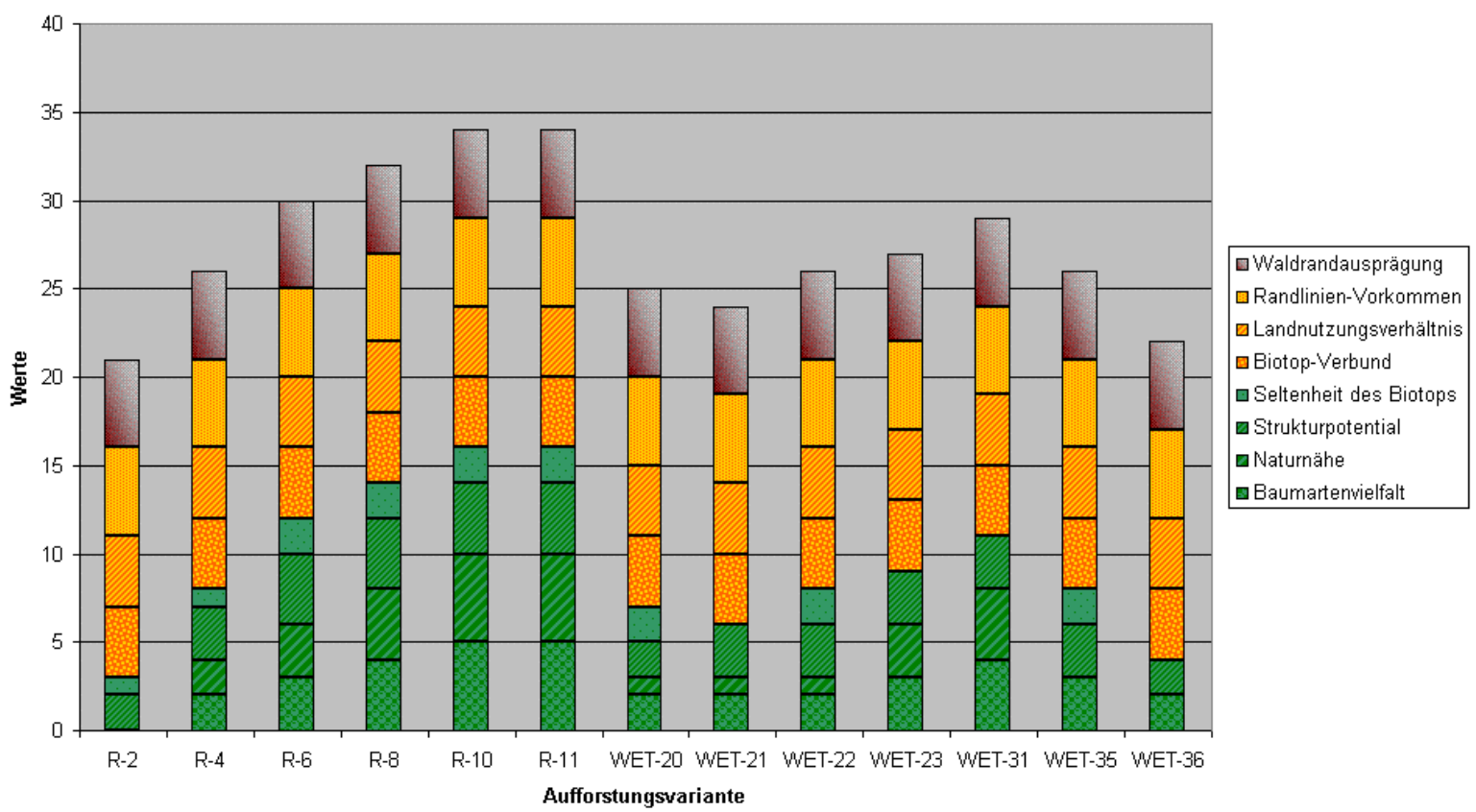




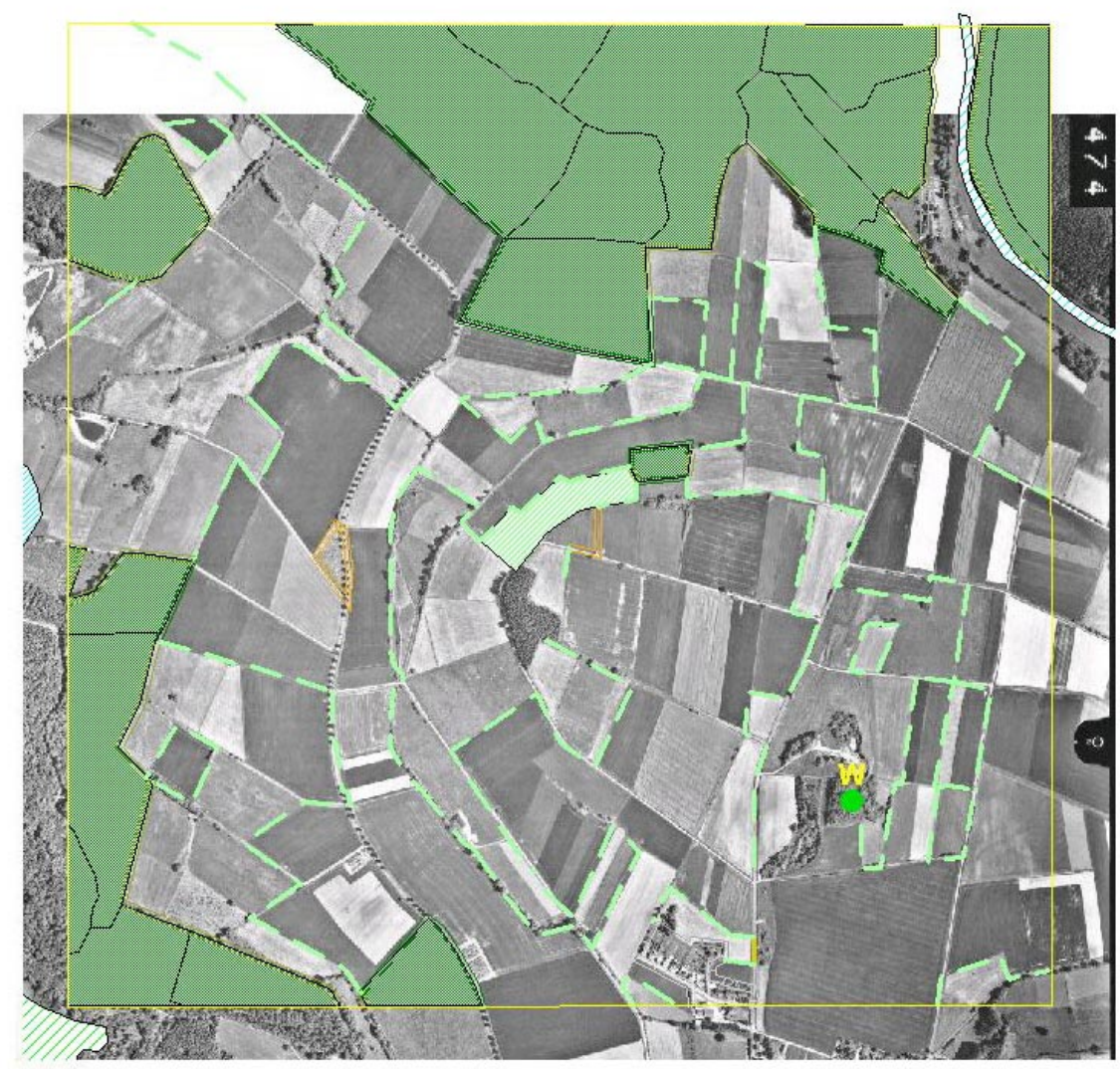

Flächengrenze

Punktbiotope

$\bigcirc \mathrm{E}$

- $F$

G

$Q$

- W

Flächenbiotope

Gewässer

Grünland

Kulturdenkmal

Quelle

Z7] Wald

Randlinien

$\therefore$ Allee

$\because$ Feld / Grünland

1) Feld / Brache

/V Grunland/Brache

A Feld/Hecke

Feld / Obstbau

N/Feld / Wald

Obstbau / Brache

Wald / Grünland

A Wald / Obstbau

Feld / Siedlung

Grünland/ Siedlung

A Wald/Siedlung

Brache / Siedlung

Aufforstungsparzelle

$\longrightarrow$ Wald

0

800

1600 Meters

Indikatorsummen für die Aufforstungsfläche DA-2

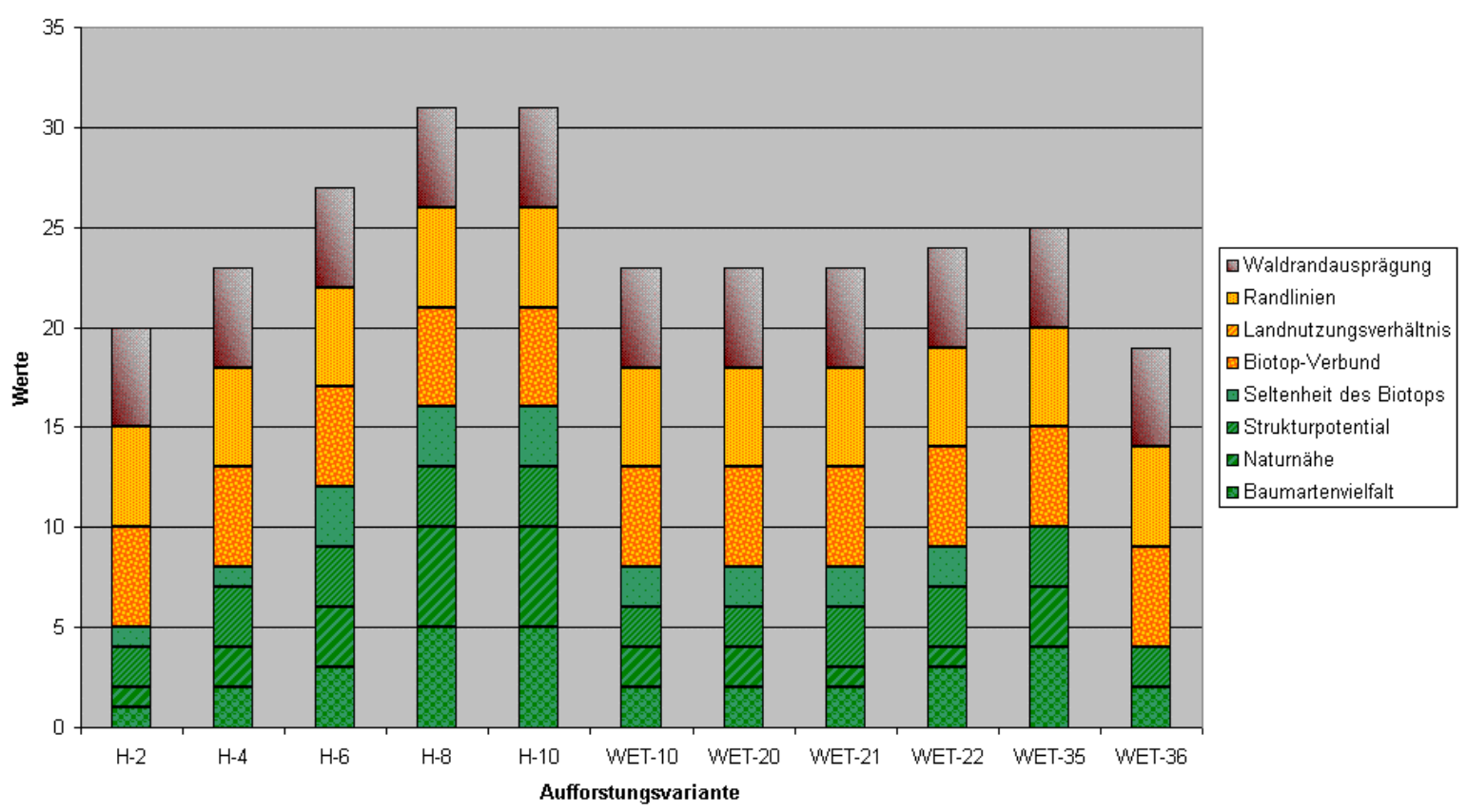




\section{AUFFORSTUNGSFLÄCHE DASSEL-3}

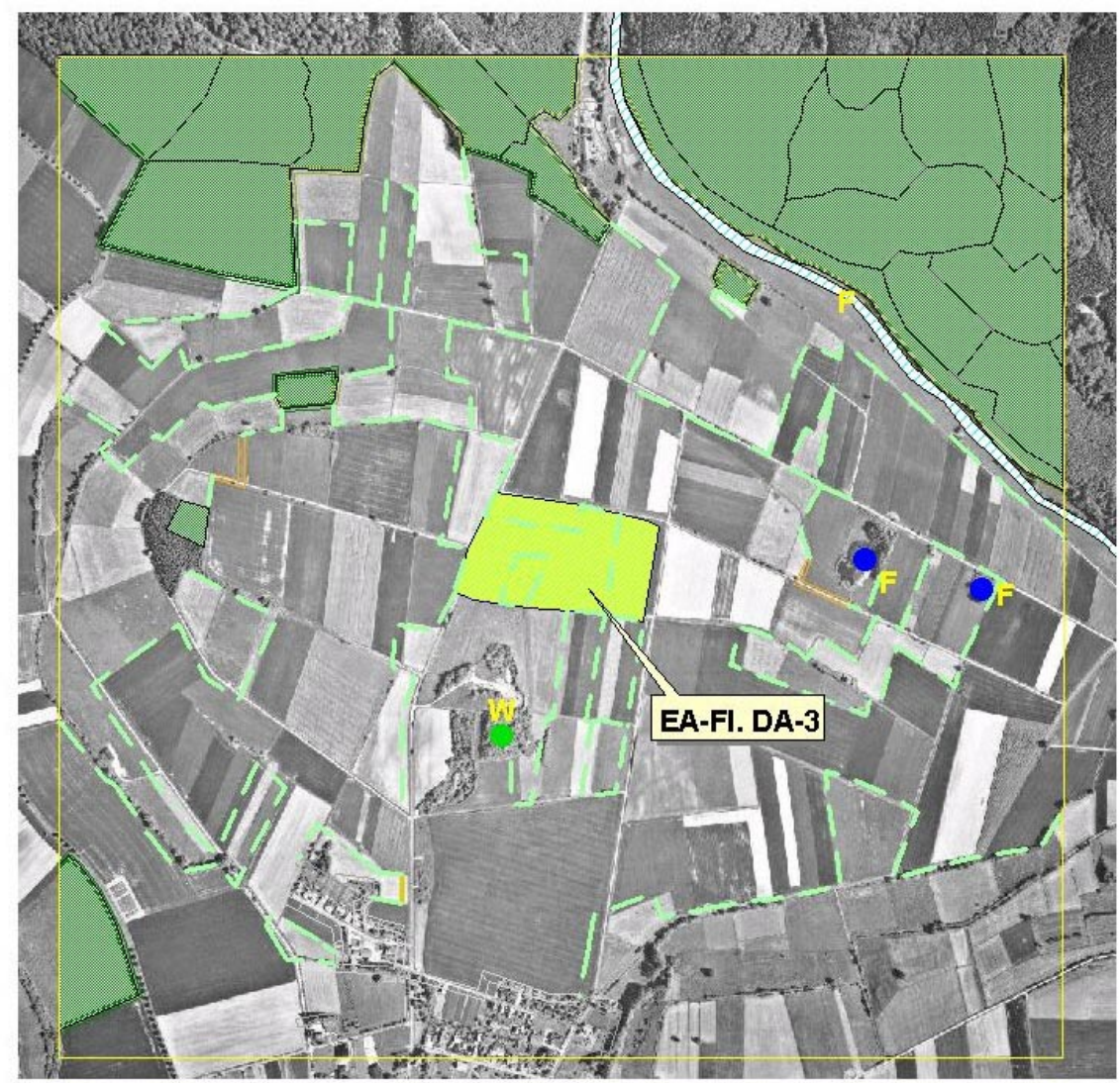

Flächengrenze

Punktbiotope

$\bigcirc \mathrm{E}$

- $F$

G

Q

- W

Flächenbiotope

Gewässe

Grünland

Kulturdenkmal

Quelle

Wald

Randlinien

$\because$ Allee
Feld / Grünland

1/Feld/Brache

Grünland/Brache

Feld/Hecke

Feld/Obstbau

N/Feld / Wald

Obstbau / Brache
Obld

W/Wald / Grünland

Wald/Obstbau

Feld / Siedlung

Grünland/Siedlung

A Wald / Siedlung

Brache / Siedlung

$\longrightarrow$ Aufforstungsparzelle

Wald

Indikatorsummen für die Aufforstungsfläche DA-3

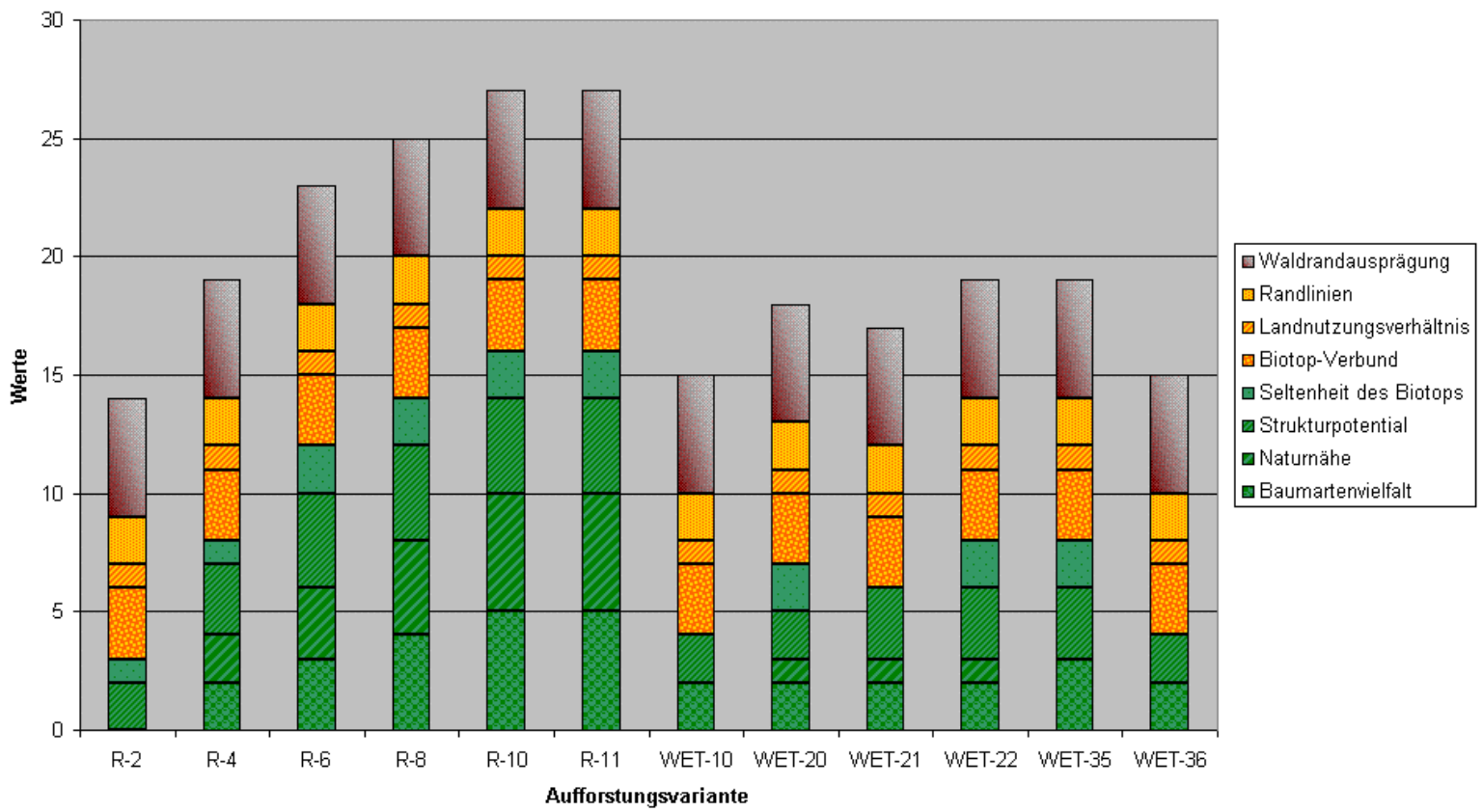




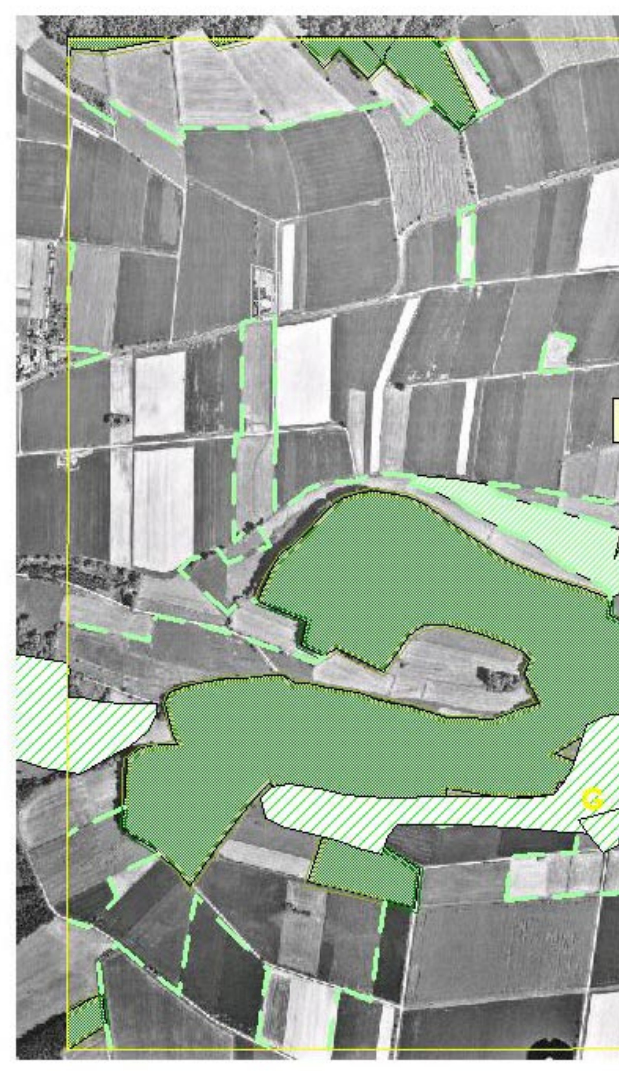

0

800

1600 Meter:

Indikatorsummen für die Aufforstungsfläche DA-4

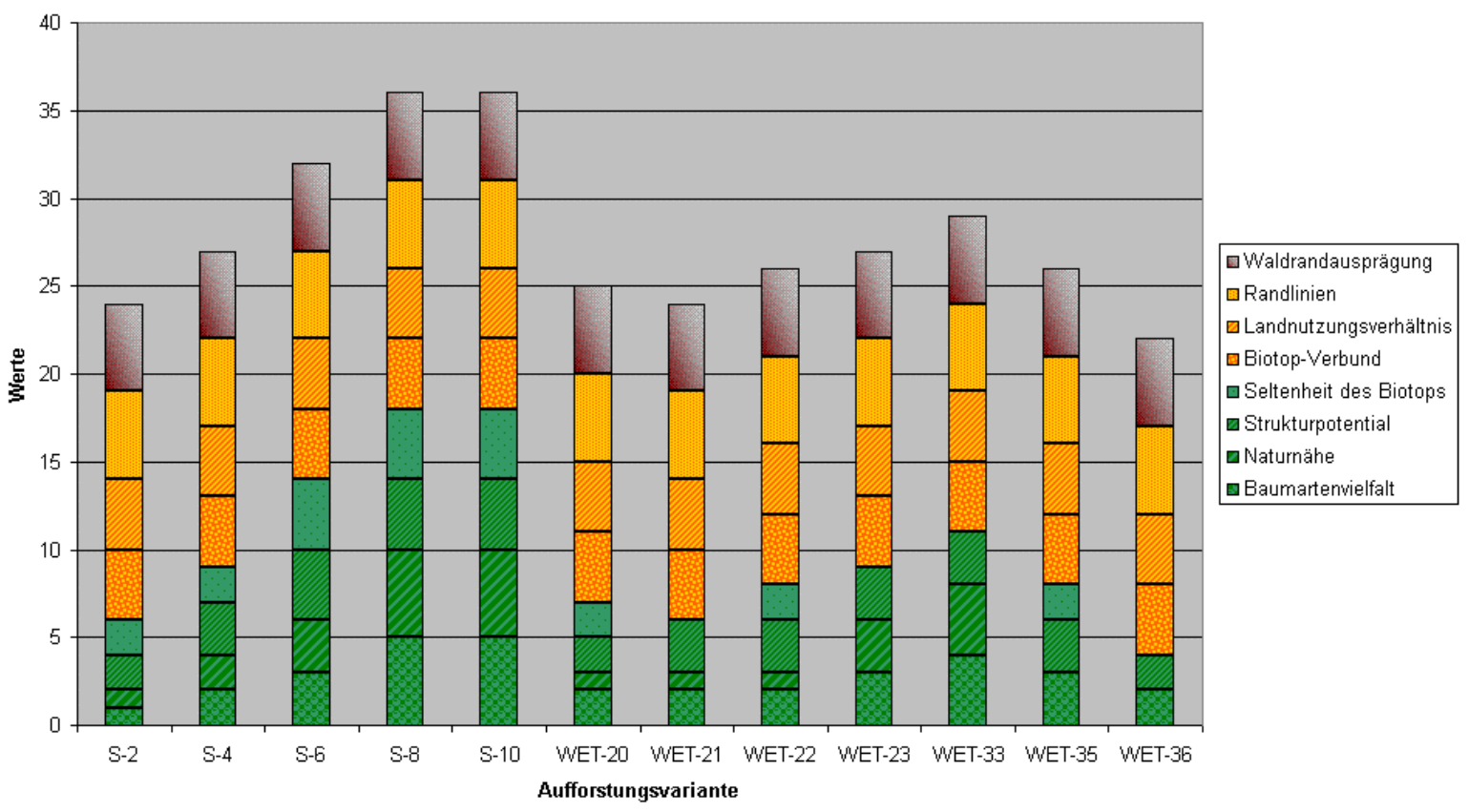




\section{AUFFORSTUNGSFLÄCHE DASSEL-5}

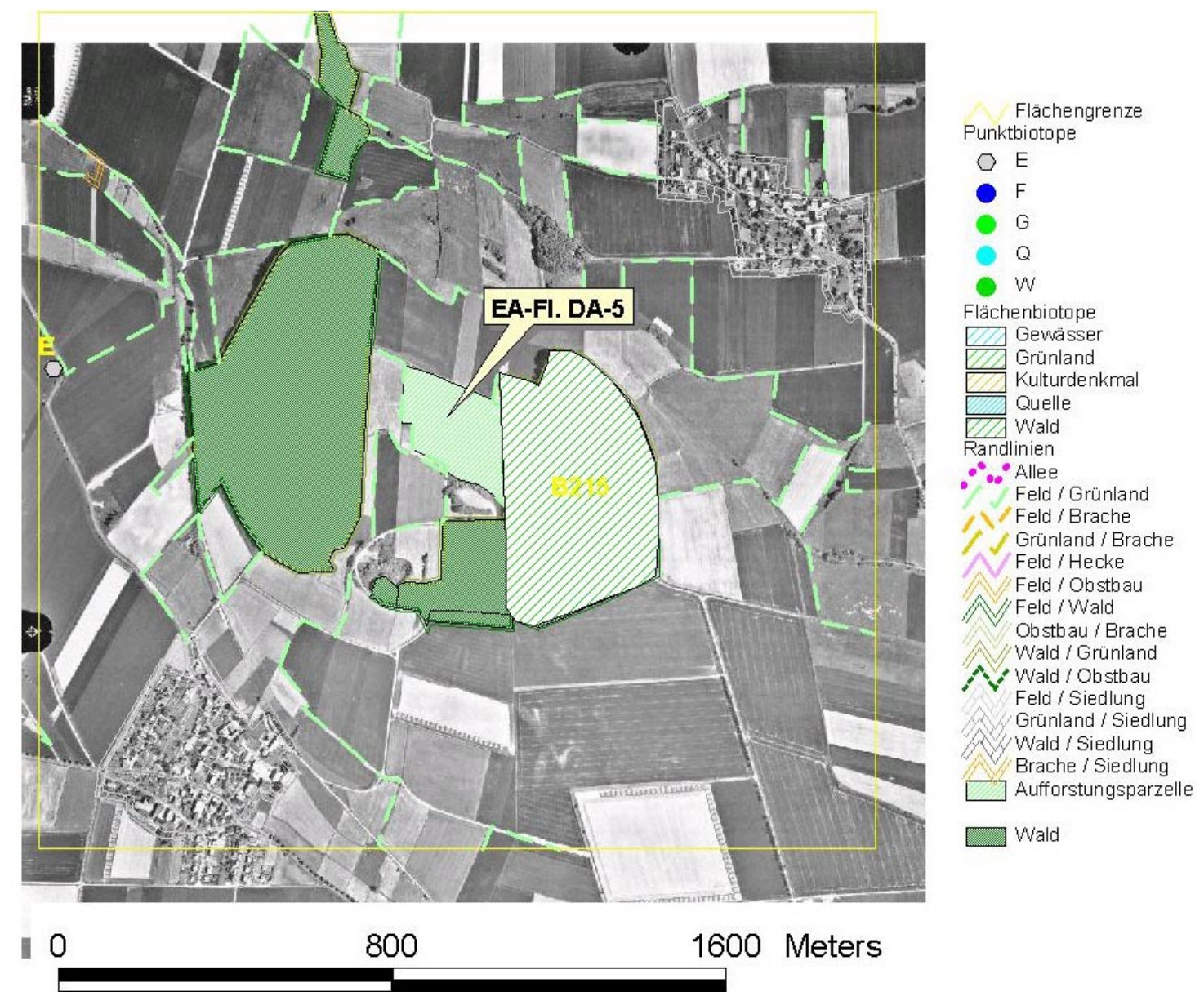

Indikatorsummen für die Aufforstungsfläche DA-5

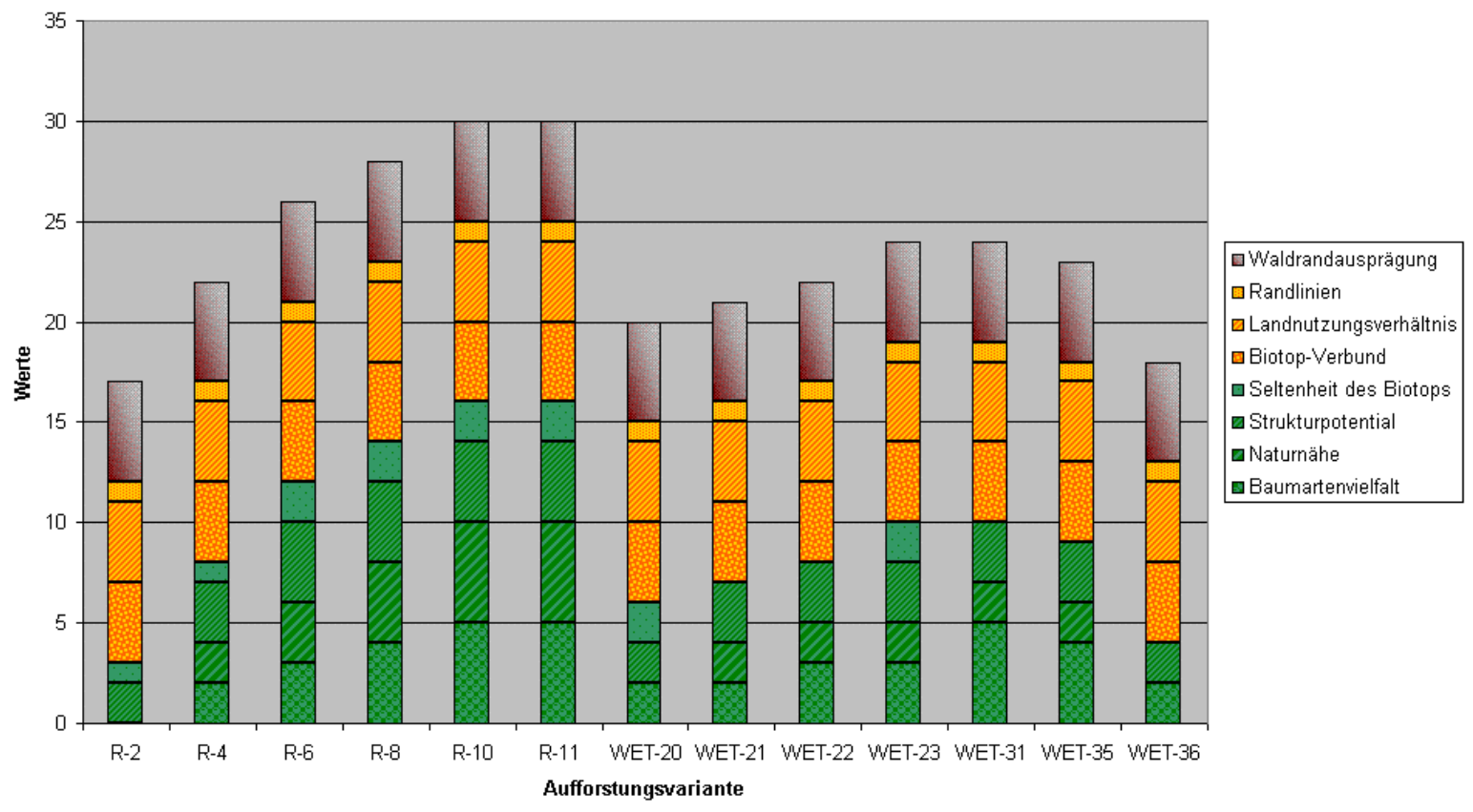




\section{Höhenkurven für mäßige Durchforstung ( aus Ertragstafeln)}

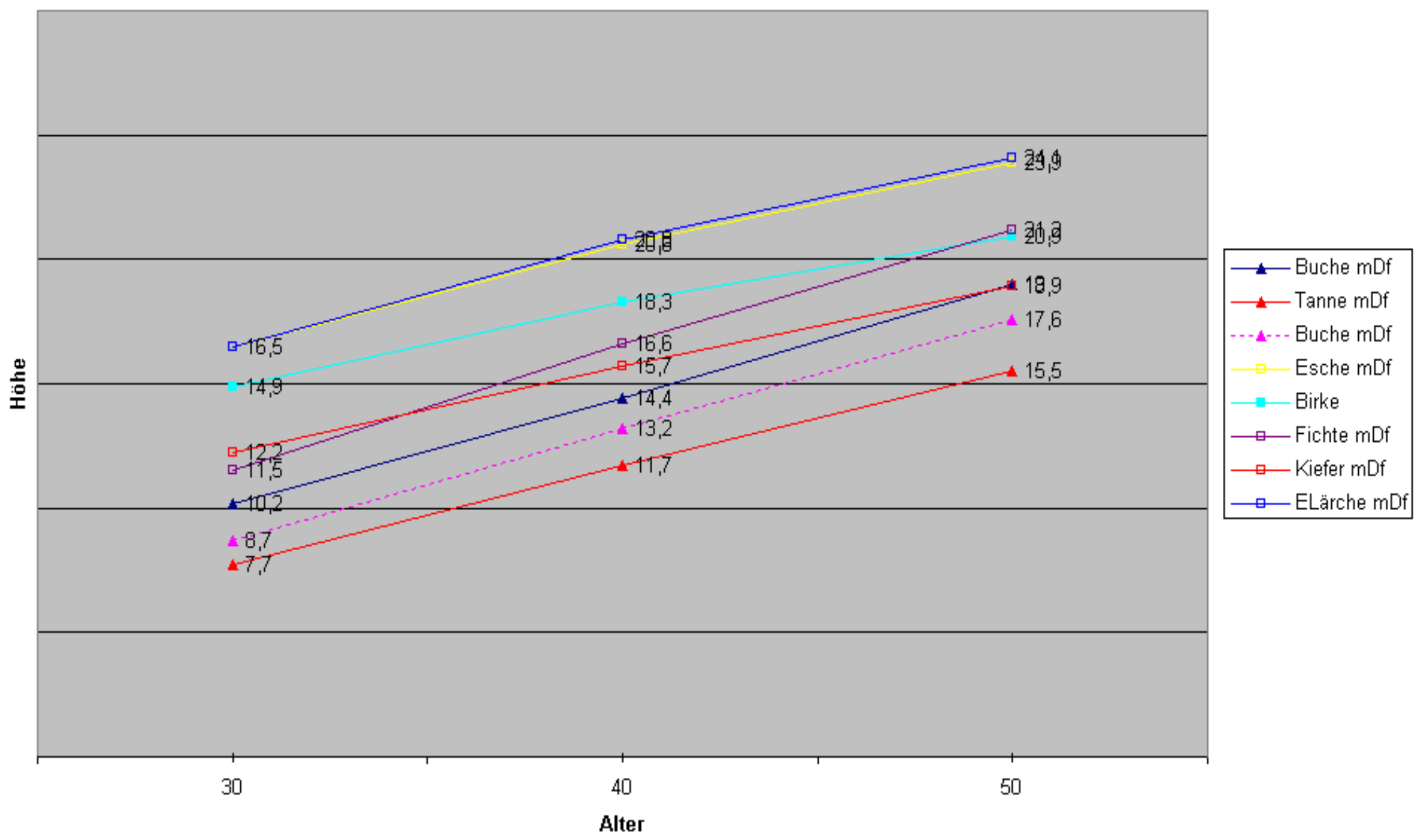




\section{Höhenkurven für starke Durchforstung (aus Ertragstafeln)}

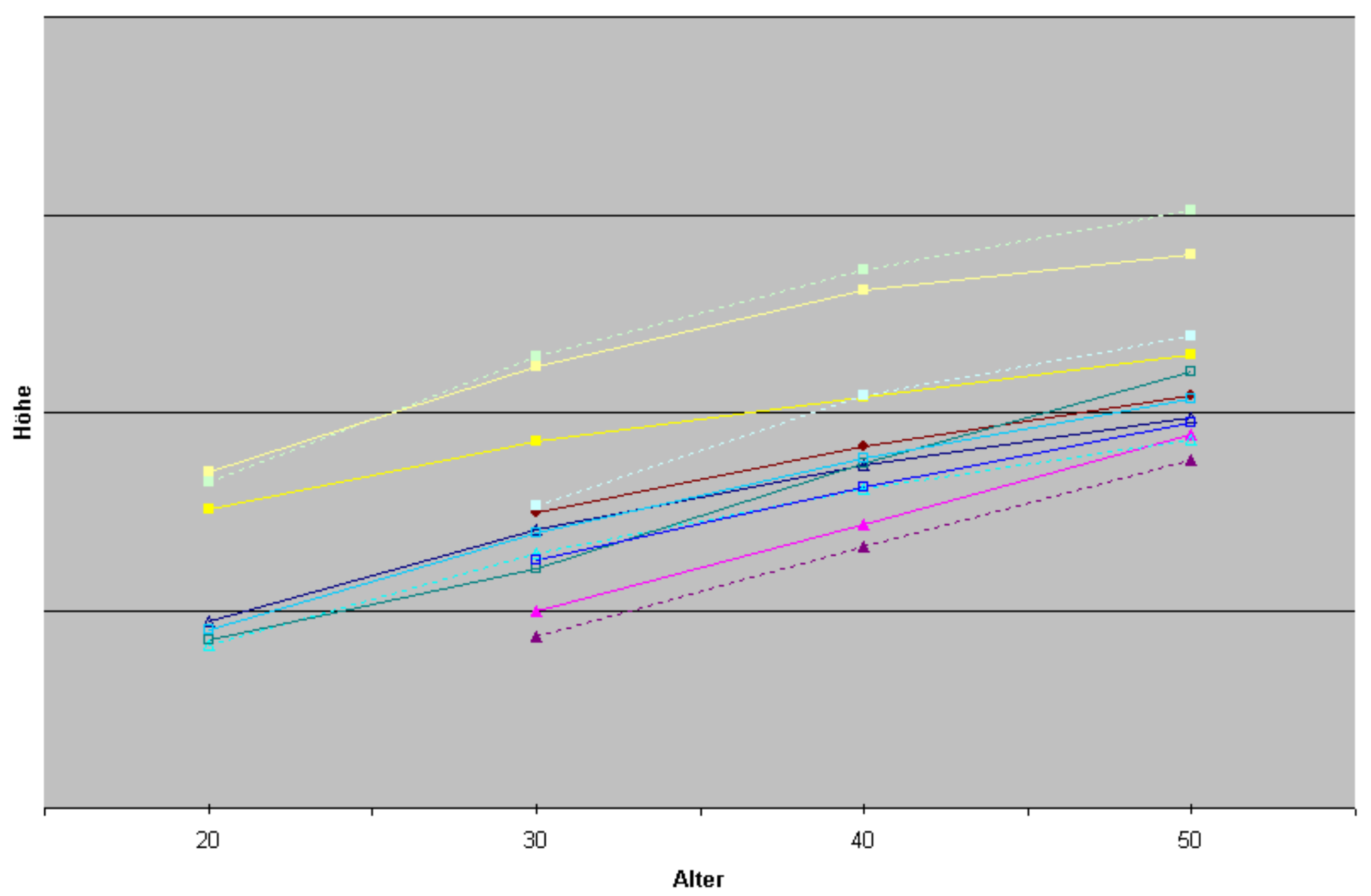

- Eiche stDF
Roterle stDf
- Biche stDF
- Bichte stDf
Kiefer stDF
ELärche stDf
Moorbirke stDf
Sandbirke stDf
Roterle stDf

Alter 


\begin{tabular}{|c|c|c|c|c|c|}
\hline BA & 20 & 30 & 40 & 50 & Ertragstafel \\
\hline Eiche stDF & 9,4 & 14,1 & 17,3 & 19,7 & OHw, Jüttner 1955 \\
\hline Buche stDF & & 10 & 14,3 & 18,9 & OHw, Schober 1967 \\
\hline Roterle stDf & 15,1 & 18,5 & 20,8 & 22,9 & MH, Mitscherlich 1945 \\
\hline Eiche stDF & 8,2 & 12,9 & 16,1 & 18,6 & MH, Jüttner 1955 \\
\hline Buche stDF & & 8,7 & 13,2 & 17,6 & MH, Schober 1967 \\
\hline Birke & & 14,9 & 18,3 & 20,9 & MH, Schwappach 1903/29 \\
\hline Fichte stDf & 8,5 & 12,1 & 17,4 & 22,1 & stDf, Wiedemann 1936/42 \\
\hline Kiefer stDF & & 12,5 & 16,2 & 19,5 & MH, Wiedemann 1943 \\
\hline ELärche stDf & 9 & 13,9 & 17,7 & 20,7 & MH, Schober 1946 \\
\hline Moorbirke stDf & & 15,3 & 20,9 & 23,9 & OH, Lockow 1996 \\
\hline Sandbirke stDf & 16,5 & 22,8 & 27,2 & 30,2 & OH, Lockow 1996 \\
\hline Roterle stDf & 17 & 22,3 & 26,2 & 28 & MH, Lockow 1994 \\
\hline Buche mDf & & 10,2 & 14,4 & 19 & OHw, Schober 1967 \\
\hline Buche mDf & & 8,7 & 13,2 & 17,6 & MH, Schober 1967 \\
\hline Esche mDf & 12 & 16,5 & 20,6 & 23,9 & MH, Volquards 1958 \\
\hline Birke & & 14,9 & 18,3 & 20,9 & MH, Schwappach 1903/29 \\
\hline Fichte mDf & 7,1 & 11,5 & 16,6 & 21,2 & MH, Wiedemann 1936/42 \\
\hline Tanne mDf & & 7,7 & 11,7 & 15,5 & MH, G.P. Schmidt 1955 \\
\hline Kiefer mDf & & 12,2 & 15,7 & 18,9 & MH, Wledemann 1943 \\
\hline ELärche mDf & 10,9 & 16,5 & 20,8 & 24,1 & MH, Schober 1946 \\
\hline
\end{tabular}




\section{LEBENSLAUF}

Name:

Ralf Eisenbeiß

Geboren:

am 29.12.1970

in Kehl am Rhein

Familienstand: $\quad$ Verheiratet seit 11. Mai 2002 mit Silke Eisenbeiß, geb. Schneider

1977-1981 Falkenhausenschule (Grundschule), Kehl am Rhein

1981-1990 Math.-naturwiss. Gymnasium (Einstein-Gymnasium), Kehl am Rhein

Abschluss: allgemeine Hochschulreife

1990-1991 Wehrdienst

1991-1997 Studium der Forstwissenschaften;

Albert-Ludwigs-Universität Freiburg i.Br.

Abschluss: Diplom-Forstwirt

Zusatzqualifikationen: Baumpflege;

Forstwirtschaft der Tropen und Subtropen

1995-1996 Studium der Umweltwissenschaften und Ressourcenwirtschaft;

Trent University, Peterborough / Kanada

Abschluss: Bachelor of Science

1997-1998 Freiberufliche Tätigkeit für:

- Institut für Forstbenutzung und Arbeitswissenschaften, Universität Freiburg: Untersuchungen zur Vermarktung von rotkernigem Buchenholz

- Landesforstverwaltung Baden-Württemberg: Forstliche Betriebsinventur

- Forstdirektion Tübingen: Erstellung von digitalen Forstkarten (mit FOGIS)

1999-2002 Institut für Forstpolitik, Forstgeschichte und Naturschutz, Universität Göttingen:

- Koordination eines internationalen Projektes (INTERREG IIC) zur Verbesserung der transnationalen Zusammenarbeit in der Raumplanung; Schwerpunkt: Nachhaltige Sicherung und Nutzung natürlicher Ressourcen im unterbewaldeten Raum

- Parallel seit Januar 2000: Promotion mit eigenem Forschungsprojekt „Biodiversität und Waldvermehrung - Ein Verfahren zur naturschutzfachlichen Evaluation von Erstaufforstungen“" 